\begin{tabular}{|l|l|}
\hline $\begin{array}{l}\text { 2. To: (Receiving Organization) } \\
\text { Distribution }\end{array}$ & $\begin{array}{l}\text { 3. From: coriginating Organization) } \\
\text { TWRS Projects/SST Retrieval }\end{array}$ \\
\hline 5. Proj./Prog./Dept./Div.: D299) & $\begin{array}{l}\text { 6. Design Authority/ Design Agent/Cog. } \\
\text { Engr.: }\end{array}$ \\
W-320 TWRS/TCPN \# Bailey, NHC. \\
\hline
\end{tabular}

8. Originator Remarks:

For approval and release of a new supporting document. This document has been generated to ensure retrievability of the Project W-320 "Electrical Calculations, Vol. 1".

11. Receiver Remarks: 11A. Design Baseline Document? [X] Yes [] No

\begin{tabular}{|c|} 
4. Related EDT No.: \\
n/a
\end{tabular}

9. Equip./Component No.:

$n / a$

10. System/Bldg./Facility: 241-C-106

12. Major Assm. Dwg. No.:

$n / a$

13. Permit/Permit Applicetion No.: $n / a$

14. Required Response Date:

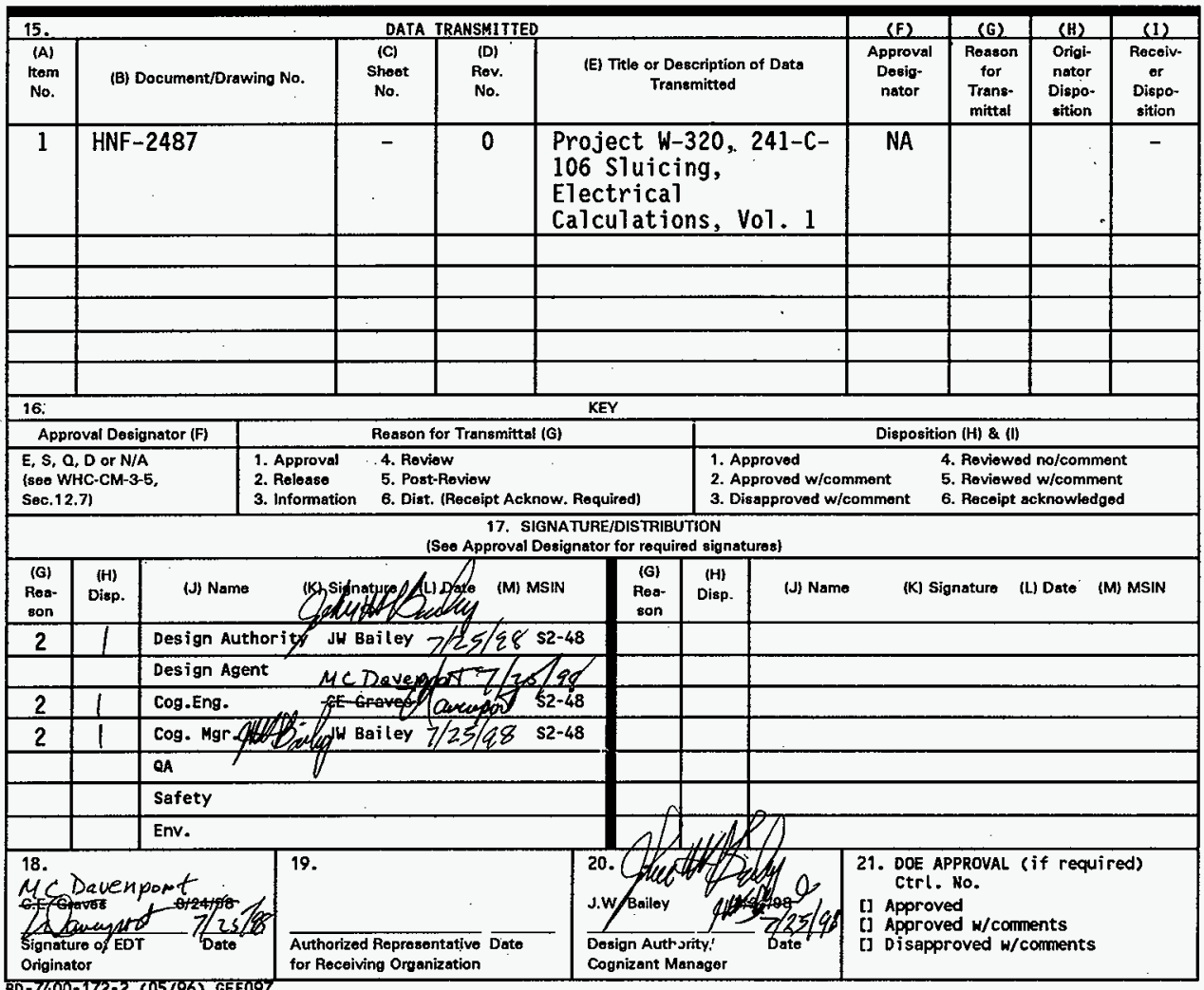




\section{Project W-320, 241-C-106 Sluicing Electrical Calculations, Vol. 1}

John W. Bailey

Numatec Hanford Co., Richland, WA 99352

U.S. Department of Energy Contract DE-AC09-96RL13200

$\begin{array}{lll}\text { EDT/ECN: } & 622245 & \text { UC: } 506 \\ \text { Org Code: } & 8 C 452 & \text { Charge Code: } \text { D2991/HANA0600 } \\ \text { B\&R Code: } & \text { EW3130010 } & \text { Total Pages: } 300\end{array}$

Key Words: W-320, Sluicing, Tank 241-C-106, Tank 241-AY-102, WRSS, calculations, Electrical.

Abstract: This supporting document has been prepared to make the FDNW calculations for Project $\mathbf{W}-320$, readily retrievable.

TRADEMARK DISCLAIMER. Reference herein to any specific conmercial product, process, or service by trade name, trademark, manufacturer, or otherwise, does not necessarily constitute or imply its endorsement, recommendation, or favoring by the United States Goverrment or any agency thereof or i ts contractors or subcontractors.

Printed in the United States of America. To obtain copies of this document, contact: Document Control Services, P.0. Box 950, Mailstop H6-08, Richland WA 99352, Phone (509) 372-2420; Fax (509) 376-4989.

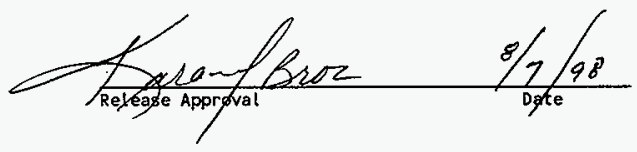

Approved for Public Release

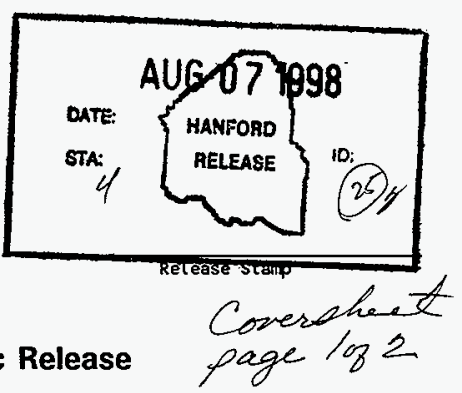




\section{APPLIGATION FOR PERMISSION TO USE}

To! Rockbestos $\begin{aligned} & \text { Attr: Donna Argento } \\ & \text { Date: } 8 / 3 / 98\end{aligned}$

Permission is requested to reproduce the fallowing copyrighted inaterial from:

your catalog

Selections from text (speeify by date of issue, page, paragraph, or illustration; if desired, attech a copy of the material in question):

see attached four (4) pages

Title of woork or projeet-in whioh this matsrial will bo included:

Control Circuit Sizing \& Voltage Drop Analysis for Seismic Shutdown 5ystem Specification Document

Estimated publication date: $8 / 8 / 98$

Author: D.E. Evans

Publisher (if applicable): Hanföd Site, Richland, Washingtion

If the copyrighted material is not to be used in a published work, please provide a brief description of how it is to be used: -

\begin{tabular}{|c|c|c|c|c|}
\hline $\begin{array}{l}\text { Requested by: } \\
\text { Contact: }\end{array}$ & $\begin{array}{l}\text { Fluor Daniel-North West } \\
\text { Mary Davenport } \\
\text { P.O.Box 1050 } \\
\text { Rith7 and, Wa } 99352-1050 \\
\text { Phone: (509) } 375-7793 \\
\text { Fax: (509) } 376-0546\end{array}$ & $\begin{array}{c}\text { MSSIN: } \\
-\end{array}$ & $\begin{array}{c}52-48 \\
\therefore\end{array}$ & Date: \\
\hline
\end{tabular}

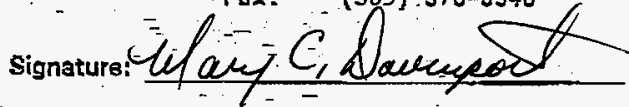

Permission is granted $X$ denied - to use-requested materiais:

Company Name: Roch bestos-Surpremant Coble Coxp Signatory's Position: CSR

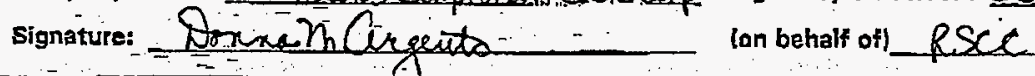


HNF-2487, Rev. 0

\title{
Project W-320, 241-C-106 Sluicing Electrical Calculations, Vol. 1
}

\author{
TABLE OF CONTENTS
}

$\begin{array}{lll}\text { Calc. No. } & \text { Title } & \text { Page } \\ \text { W320-31-001 } & \text { Fault Current Analysis } & \text { A-i } \\ \text { W320-31-002 } & \text { Standby Generator Sizing } & \text { B-i } \\ \text { W320-31-003 } & \text { Feeder Cable Sizes \& \% Voltage Drop } & \text { C-i } \\ \text { W320-31-010 } & \begin{array}{l}\text { C-106 Demand Load Analysis, } \\ \text { Size Feeders, Voltage Drop and }\end{array} & \text { D-i } \\ & \text { Fault Study } & \\ \text { W320-31-011 } & \begin{array}{l}\text { Tank C-106 Load Determination, } \\ \end{array} & \text { Feeder/Protection Sizing, Voltage } \\ & \begin{array}{l}\text { Drop, Lighting Calculations and } \\ \text { Conduit Sizing }\end{array} & \\ \text { W320-31-016 } & \begin{array}{l}\text { AY-102 Demand Load Analysis, Size } \\ \text { Feeders, Voltage Drop and Fault Study }\end{array} & \text { F-i } \\ \text { W320-31-017 } & \begin{array}{l}\text { Tank AY-102 Load Determination, } \\ \end{array} & \begin{array}{l}\text { Feeder/Protection Sizing, Voltage Drop, } \\ \text { Lighting and Conduit Sizing Calculations }\end{array}\end{array}$


HNF-2487, Rev. 0

W320-31-001

Fault Current Analysis

$A-i$ 
This sheet shows the status and description of the attached Dosign Analysis sheots.

Disciplino ELECTRICAL WO/Job No. W320/ER4289 Calculation No. W320-31-001

Projoct No. \& Name

W320

EQUNMENT REMOVAL STSTEM

Calculation Itom

FAULT CURRENT ANALYEIS

Theso caiculationa spply to:

Dwg. No.

$H-2-820748$ SH1

Dwg. No.

$H-2-820749 \mathrm{SHI}, \mathrm{SHZ}$

Rov. No.

o

Other (Study, CDR)

Rov. No.

The status of those calculations is:

$\square$ Proliminery Calculations

$\checkmark$ Finsl Calculations

WChock Calculations IOn Calculation Dated

Void Calculation (Resson Voided

Incorporeted in Final Drawings?

(1) Yos

This calculetion verified by independent "check" calculations?

Rov. No.

0

Originel and Revised Calculation Approvala:

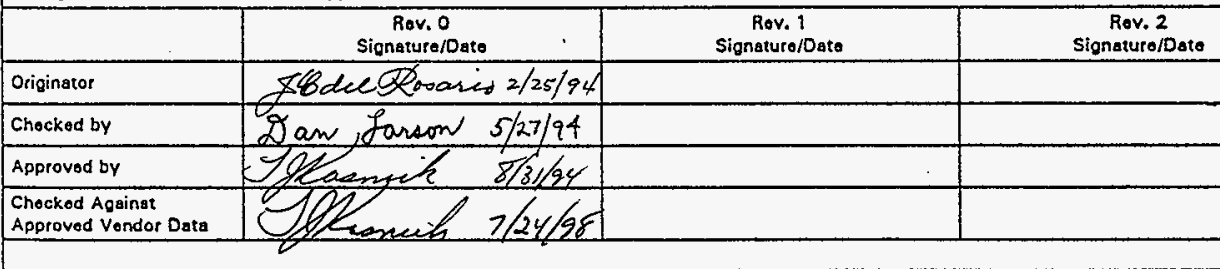

INDEX

Design Anelysis

Pago No.

$\frac{i^{2} i}{i i i}$

2

$3,4,5$

6

7,8

9,10

APFENणत्ड

A-1 THRU A- 7
INOEX

CALCHLATLON CROSS INDEX

DESISN VERIFICATILON SCREENING CRITERIA

OBJECTVE, DATA

FEEDER CABLES, TRANSFORMER BANK MUA

METHOD OF CALC, FORMULAS, SALCLLATIOMS

ONE LINE DIAGRAM, PER UNIT IMPEDANCE DIAGRAM

FAULT IMPEDANCE $Z_{F X}$, FAULT MVAFX, FAULT CURRENT IFX SUMMARY OF $Z_{F X}$, MVAFX, $I_{F X}, C B$ CLEARING TIME, $M T$, TDT; FINDINGS \& CONCLUSUN

3థ PAULT CALC. BY UTLUTIES, COND. MELTING CURVE, INS. THERMAL DAMACE CURVE

BREAKER TIME CLIRRENT CURVES. 


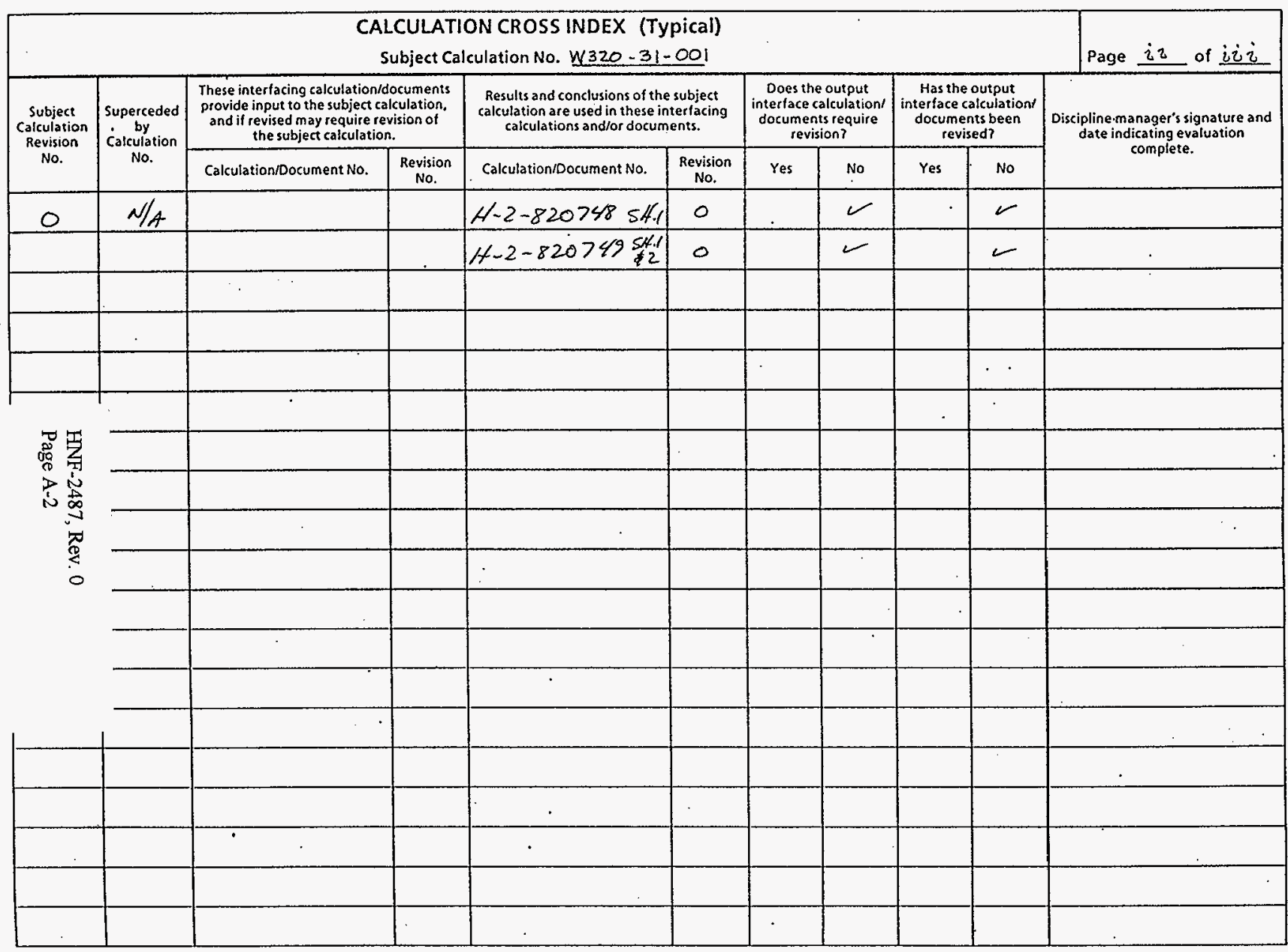




\section{DESIGN VERIFICATION SCREENING CRITERIA}

Project/Document No. W320/W320-31-001

When the design or design change affects hardware, formal design verification must be performed if one or more of the following questions must be answered affirmatively (YES).

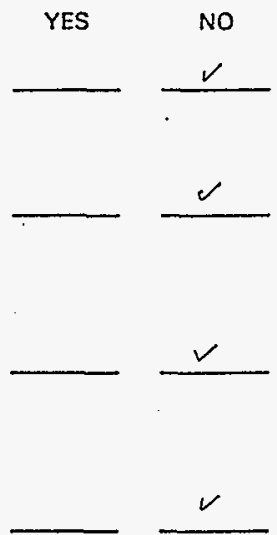

1. Does the design or design change involved meet the established criteria to be considered Safety Class 1 ?

2. Does this design or design change cause or permit changes to Safety Class 1 instrument or alarm setpoints outside of previously approved operational limits?

3. Does this design or design change significantly affect the nuclear or environmental safety consequences of a malfunction or failure of the structure, system, or component?

4. Does this design or design change involve or change design that has previously undergone formal design verification?
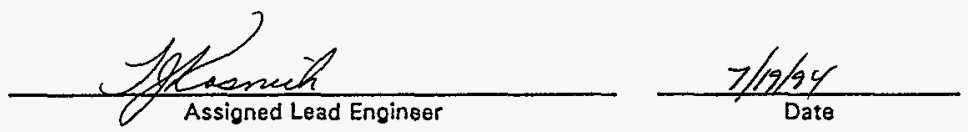

Responsible Discipline Manager

Date

Original Design Package Distribution:

Project Manager

Design Verification Officer

Engineering Document Control
HNF-2487, Rev. 0

Page A-3
Design Change Distrịbution:

Attach to Engineering Change Notice 
KAISER ENGINEERS HANFORD
DESIGN ANALYSIS
Calc. No. $10320-31-001$

Revision 0

Page No.

WO/Job No. ER $\$ 28 s$

Client $W H C$ Date 2/25/24 BY

SUbject FAULT CURZENT ANALUSIS Checked $5 / 27 / 94$ By D.E. Larson

FEEDER CABLE \& CB. SIZING

Location TANK FARMC, 200 EAST AREA Revised

By

1. OBJECTIVE: THE PURPOSE OF THIS CALCULATIONS IS TO SUPPORT W3ZO TANK Z41-C-106 IN-TANK EQUIPT, REMOVAL PHASE. THE MAXIMUM FAULT CURRENTS THAT THE CIRCUIT BEEAKER WILL BEREQUIRED TO MTERIRUVT WILL BE DETERMINED TO PROPERLY SELECT THE CIRCUIT BREAKERS THAT:

$\therefore$ WILL PROTECT THE FEEPER CABLES.

2. REFERENCES:

A. G.E. industrial power sYSTEM coOrdination

B.: WEST. CATALOS 55.000 10TH ED. 1991-1992

C. $1993 \mathrm{NEC}$

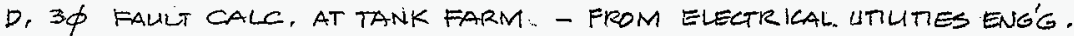

E. INDUSTRIAL POWER SYSTEM HANDBOOK BY BEEMAN

F. GE CB T-C CURVES G. CU COND MELTINE TEMP

3. DATA:

H. CABLE STAND
Se WiTH EURVE.

LOAD DATA

HPPT H HOH PRESS PUMPTRLR - (ELECTSYST BYWHC/VENDOR) INFO FURNISHED BY WHC MAIN PUMP MOTOR - $100 H P, 3 \phi, 480 \mathrm{~V}-124 \mathrm{~A}$

BOOSTER PUMP - $2 H P, 3 \phi, 480 V-3.4 A$ - $\}$ ONE USED AT

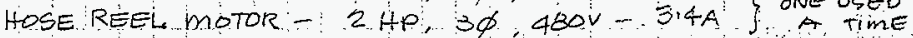
OTHER LOADS I $3 \phi$ HB $480 \mathrm{~V}-5 \mathrm{~A}$ AMPACIIES

TOTAL CONNECTEO LOAD T - I27.9A FKOM NEC

10O HP MAIN PUMP MOTOR HAS $150 \mathrm{~A}$ BRANCH CB \& $200 \mathrm{~A}$ FUSES INSTALLED, BY THE PUMP AND CONTEOLLEVE VENDOR TO LIMIT THE STARTING \& RUNNING CURRENT INPUT TO THE VARIABLE FREQLENCY CONTROLLER:

THE 100 HP INDUCTION MOTOR WUL BE STARTEDAT LOW SPEED (AND STARTING CURRENT) THEN ACLELERATED TO REQUUED PUMP OPERATING SPEED.

SBT STRONG BACK TRALER - 5OA, (UESTIMATE)

LATEST INFO FROM WEST. IS THAT $Z-10 H P, 3 \phi 46 O \mathrm{~V}$ MOTORS WILL ALTERNATELY BE USED. THE OTHER LOADS ARE NOT YET AVALLABLE.

SPT - SINGLE PHASE TRANSFORMER BANK- $1-50 \mathrm{KVA}, 1,480-120 / 240 \mathrm{~V}$ FOR CONSTRUCTION TRAILERS

$70 A, 120 / 240 V, 1 \phi$ SERVICES ROR 3 TIRAILERS FOR KEH CONSTRUCTION :FORCES

3- CONSTRUCTION TRAILERS@IEKVA CONNILOAP EN $=45 \mathrm{KVA}$. 


\section{KAISER ENGINEERS HANFORD}

\section{DESIGN ANALYSIS}

Calc. No. W320-31-00

Revision 0

Page No. 2 of 10

Client $W H C$

WO/Job No. ER 4283

Subject FAULT CURRENT ANALYSIS Date $2 / 25 / 94$ By J.C. DEL POSARI

FEEPER CABLE \& CB SI2ING Checked 5/27/94 BY Dan Larson
Location TANK FARMC,20OEAST AREARevised

3. DATA

CABLS

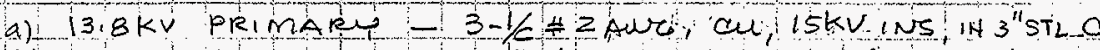

b) $4480 V$ SECOUDAR - 3 WLTH ERD COND, DIRELT BURAL

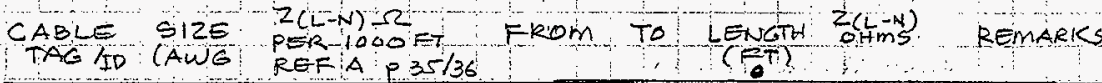

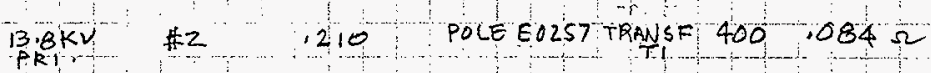

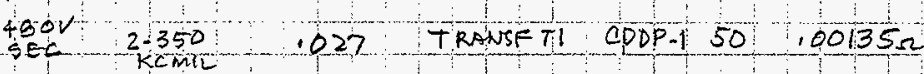

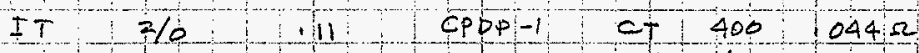

HPP $4 / 0 \quad$ H 10745 HPP 400 .03 2

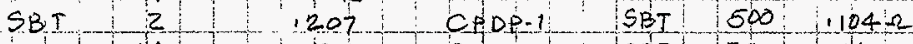

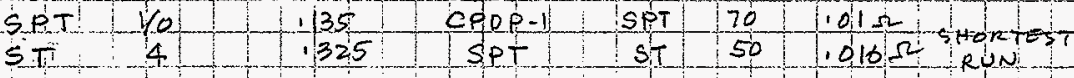

- SEE DWGS H-2-820749 SHI \& SH2 FOK QABLS LENCFL $13.8 \mathrm{KV} 3 \phi$ TRANSF - ZtPU REFA $\rho 2 z$

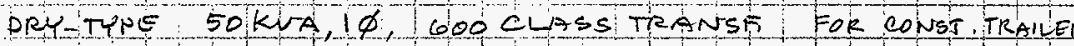

FUL WINDINE \%R\% \%X, $\%$ REF E DABLE 1.17996 $\% R=13 \% \quad, \quad \% x=2.2 \%, \quad \% z=2.6 \%$

HALE WWOING(12OV) \%R; \% $\%$ REFE PRARTS

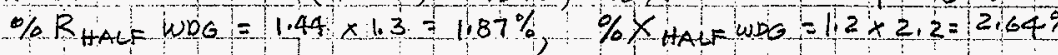
$\% z_{H+1} \sqrt{(1.87)^{2}+(2.64)^{2}=3.2 \%}=$ TRANSE. IMPEDANCE

PAD MOUNTED TRANSE BANK $3 \phi$ MVA $=.225 \mathrm{MVA}$

$\% Z=4.43$ ON 225 MYA BASE CROM TRANSF NAMEP

HNF-2487, Rev. 0

$54.4300 .037^{-}$KEH-0037.00'106/92

Page A-5 


\section{KAISER ENGINEERS HANFORD}

DESIGN ANALYSIS
Calc. No. W320-31-001

Revision 0

Page No.

\section{Client WHC WO/Job No. ER 4288}

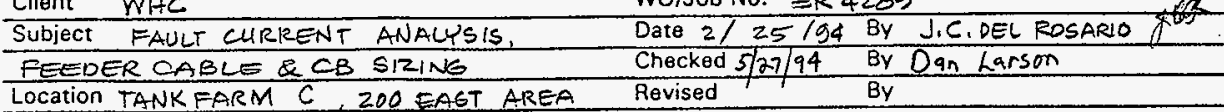

4. METHOD OF CALCMLATIONS:

MANUAL METHOP UGING PER UNIT FAULT CUIRENT CALCULATIONS PER REFEENCE A:

5. FORMULAS

PER UNIT IMPEDANCES ON MVA EASE UTILITT SYSTEM:

ZUT.SYST IN PER UNIT. = MVABASE MVA SC UTILITY SYTEM

FEEDER CABLE:

$Z P U(L-N)$, FDR: $=\frac{\text { ZOHM }(L-N) \times K V A B A S E}{K V^{2} \times 1000}$ REF A

$$
=Z_{0} M(L-N) \cdot\left(\frac{M V A B A S E}{K V}\right)
$$

\section{TRANSEORMER:}

ZT: IN PER UNIT) $=Z_{\text {ONPU }} \times \frac{\text { MVABASE }}{\text { OnVWN }}$

REF A P 34

MOTOR:

$Z_{M}$ IN PER UNIT $=Z_{m \text { INU }} \times \frac{\text { MVABASE }}{\text { MVAM BW }}$ 
KAISER ENGINEERS HANFORD
Calc. No. W 320-31-001

Revision 0

Page No. 4 of 10

Client WHC WO/Job No. ER 4289

Subject FAULT CURRENT ANALTSIS Date $2 / 25 / \% 4$ BY J.C. DEL ROSARIO

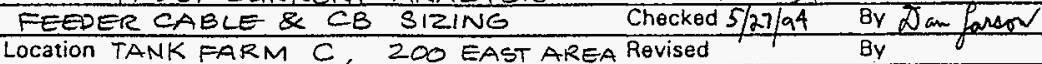

5. FORMULAS

$$
\begin{aligned}
& \text { WHERE: MVA BASE = } 10 \text { (CHDSEN BASE - SEEREF A) } \\
& \text { MVASC }=50 \quad \text {... (ELECT UTLITIES CALC-REF D) } \\
& \text { KV = PREPAULT VOLTAGE IN KILOVOLT AT PONTT } \\
& \text { OF. CALQULATION. } \\
& \text { ZOHM }(L-N)=\text { LINE TO NEUTRAL IMPEDANCE } \\
& \text { of FEeDER cable in oHMS }
\end{aligned}
$$

$\% \mathrm{Zm}=$ mOTOR \% IMPEDANCE ON ITS OUN MVA

Z. IN $\mathrm{NO}$ = MOTOR PER UNIT IMPEDANEE ON 10 MVA BASE

ZUT SYSTINP.U. $=\frac{M V A \text { BASE }}{\text { MVASC (UT,SYST) }}$

NDTE THAT ZUT SUST PUI DECREASES AS THE AVAILARLE

SC M MVASC FROM UTILITT SYSTEM INCREASES . FOR INFINITS UTLITY BUS ZUT STST IS 16 NOR=O (ZUT =0)

FOR:INFINITE UTILITT BUS:

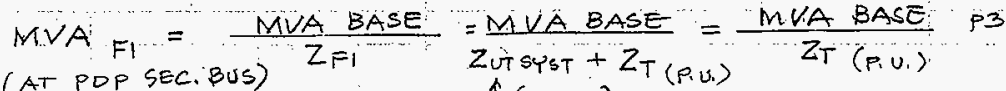
(AT POP SEC.BUS) ZFI RUTSYST

$13.8 \mathrm{KV}$ "INE IMPEDAUCE IS NEGLCCTED,

MVAFI $=\frac{M V A B A S E}{\frac{\% Z_{T}}{100}\left(\frac{M V A B A S E}{M V A T}\right)}=\frac{M V A_{T}}{\% Z_{T}}=\frac{M V A T}{100}=\frac{Z_{T}}{\left(P_{V}\right)}$

FAULT MVAFI AT TRANSFORMER SECONDARY TERMINIAL (EI) NEGLECTING ZUT SUST (INFINITE UTILITT BUS) IS DIRECTLT PROPORTIONAL TO THE BANK CAPACITT AND INVERSEL

HNF-2487, Rev. 0

Page A-7 PROPORTIONAL TO \%ZT OR ZT(RU)

FOR $225 \mathrm{KVA}$ BANK, MVAFI $=\frac{.225}{.0443}=5.08 \mathrm{MVA}$ 


\section{KAISER ENGINEERS HANFORD}

\section{DESIGN ANALYSIS}

Calc. No. $w 320-31.00$

Revision 0

Page No. 5 of 10

Client

WHC

SUbjeCt FAULT CURRENT ANAUYSIS

FEEDER CAOLE\& CB SIZINR

Location TANK FARM C $2 D$ EAST AREA

WO/Job No. ER 4289

Date $2 / 25 / 94$ BY Jic, DEL ROSARLOU Checked 5/21/94

Revised

By Dar forcon

6 CAL CULATLONS.

CONSIDER WORST CASE OE SHORT URCUIT CONDITON $3 \not$ BOL

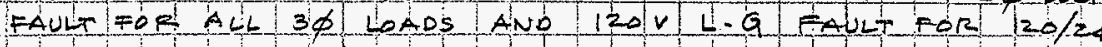
1. $3 W$ CONSTRUCTION TRALER SERVICEL,

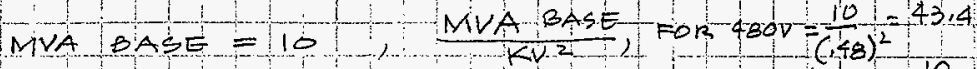
FoR $13.8 \mathrm{kU}=\frac{10}{(13.8)^{2}}=0,052$

a) ZuTiuT SYSTEm $=\frac{\text { MVA BASE }}{\text { MVASCUTSLST }}=\frac{10}{50}=2$ pu

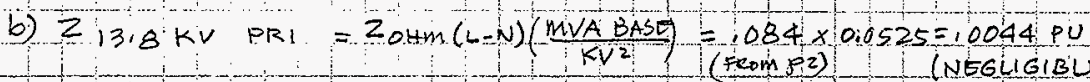

(c) $Z 3 \phi$ TKANSF $=\frac{\% 2}{100} \times \frac{\text { MUA BASE }}{\text { mVAT }}=\frac{443}{100} \times \frac{10}{225}=1.97 .9$.

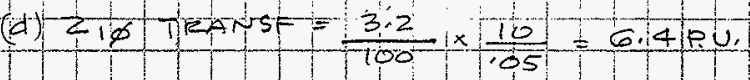

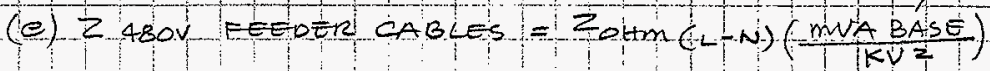

ZSEC (L-N) $=.00135 \times 43,4=059$ OU. NEGLIGIBL

$2 I T(L-N)=044 \times 43.4=1.910 u$

$Z_{-1}+P(L-N)=03 \times(43,4=1,3, P U /$

$Z \cdot 3 B r(-N-N)=104+434=4.5-P .4$

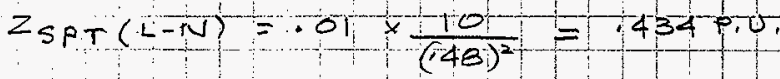

$\sum_{1}=(L-N)=0.016 \times \frac{10}{(12)^{2}}=1111 p u$

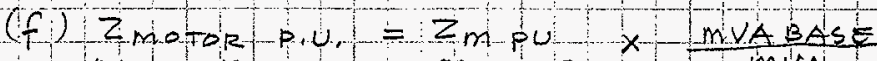
ONIOMVABAS ON MOROR

MVAT

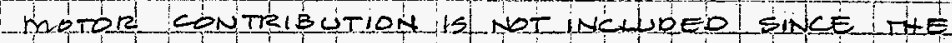

HNF-2487, ReV. 0 ․ VARABLE FREQUENCR OONTROLER WLLL NOT PASS Page A-8

THE mOTOR CONTRBBTION TO TH SUPPLY SIOE. 


\section{KAISER ENGINEERS HANFORD}

Calc. No. $\omega 320.31 .001$

Revision

Page No.

WO/Job No. ER $428 \mathrm{~g}$

Client

WHC

DESIGN ANALYSIS

Date $2 / 25 / 94$ BY J.L. DEL ROSARLO

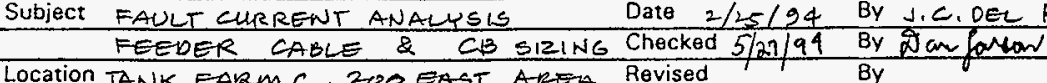

Location TANK FARMC, 200 EAST AREA Revised BY

$$
\text { ONE LINE DIAGRAM }
$$

(1) STANOBY DIESEL

(6) ENEINE GENERATOK
$480 / 277 \times$, $60+2$

$60-3 / c .350$

Kcmitripe

ma mit TPPE

$275 \mathrm{KVN}, 344 \mathrm{KVA}$

$275 \mathrm{KWN}$
$80 \mathrm{PF}$

GEN POWERUURE WAY $480 V, 3 \phi, 3 W$ $\frac{1}{I T-F B(S)}$

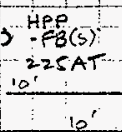

$$
\int_{0 . F B(5)}^{S B T} \text { 100AT }
$$

J) $F B(5)$
UinLins śstem avaLABLE

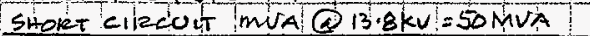
$13.6 \mathrm{kV} P \mathrm{PA}$ 4807277 , in $400^{\prime}=3+2,15 \mathrm{kV}, \mathrm{z}_{3,3,0040}$ AN 3 STELL COND PRI CNECE ZEC, $=.05 \mathrm{SOU}^{\circ}$ (NEGCECTO)

$C-P D P-1$ HOV $3 \%$ 21.350 $\eta_{20} Z p u(T$ TANSE $)=1.97 p$ $\frac{2.350}{\mathrm{kimiL} / \phi}$ KamiLlo CoNDU (QTL)

TTFB(N) $/ H P P-F B(N) S B T-F B(N)$ SPT-FB

J175AT D 2 $5 A T$ DIOOAT P WO $3 \mathrm{KE} \mathrm{TT}$

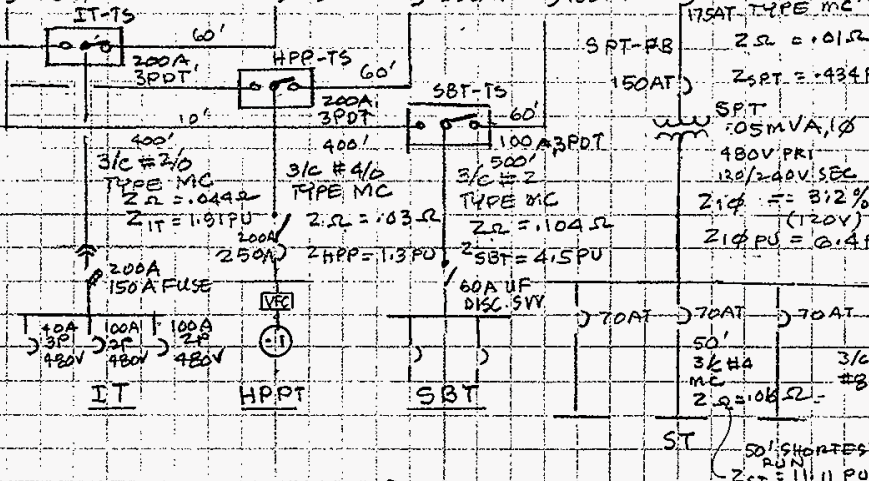

PEK UNIT IMPEPANCE (ZPU) DIASRAM

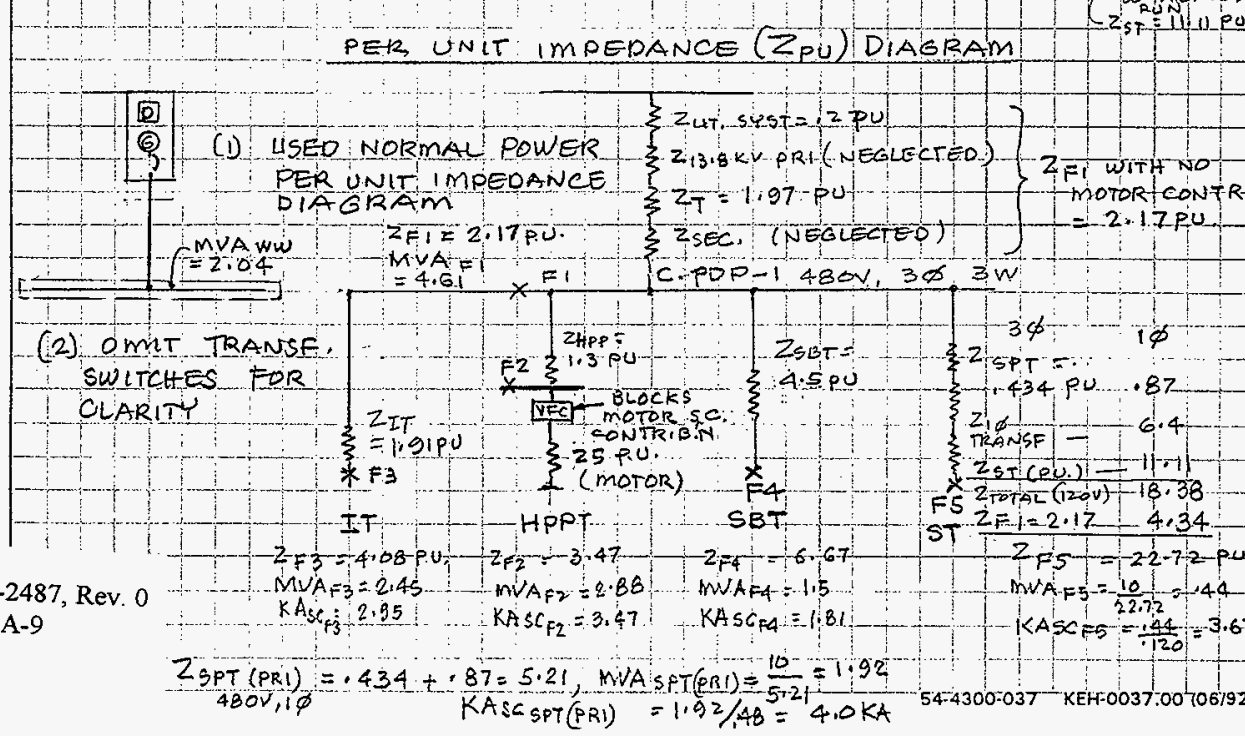


KAISER ENGINEERS

HANFORD

DESIGN ANALYSIS
Calk. No. $\frac{W 320-31-00}{0}$

Revision

Page No. 7

of 10

Client WHC

WO/Job No. ER 4289

SUbjeCT FAULT CURRENT ANALYS 15

Date $2 / 25 / 94$ BY IC, DEL ROSARIO

FEEDER CABLE \& CB SI INS Checked 5/27/94 BY Douforson
Location TANK FARM C, 200 EAST AREA Revised By

TOO HP MOTOR WII NOT PASS MOTOR CONTRIBUTION TO FAULT THRU J VARIABLE ERERUENGT CONTROLLER.

FAULT AT CPDP-I MAIN CB (PI)

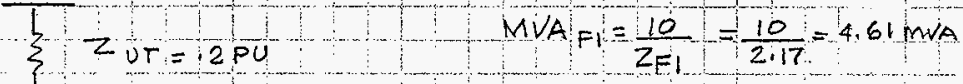

$$
\begin{aligned}
& \left\{\begin{array}{l}
z_{T}=1.97 \beta U \\
\times z_{F}=2.178 U
\end{array}\right. \\
& I_{F_{1}}=\frac{M V A F_{1}}{\sqrt{3} \times \cdot 48}=5.5 \mathrm{KA}
\end{aligned}
$$

FAULT AT APT $\left(F^{2}\right)$

$$
\left\{\begin{array}{r}
Z_{F 1}=2.17 P U \\
Z_{H P P}=1.3 P U \\
x-Z_{F 2}=3.47 . P 0
\end{array}\right.
$$

PFC WILL NOT PASS

I MOTOR CONTRIBUTION

(100)

FAULT AT IT (FE)

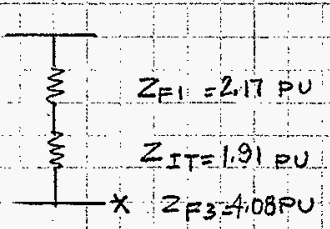

HNF-2487, Rev. 0

Page A-10

$$
M \mathrm{MA}=\frac{10}{Z_{F 2}}=\frac{10}{3.47}-2.88 \mathrm{MVA}
$$$$
I=2=\frac{M V A F 2}{\sqrt{3} \times 4 B}=3.47 \mathrm{KA}
$$

IVA $3=\frac{10}{Z_{F 3}}=\frac{10}{4.08}=2.45 \mathrm{MVA}$

$I_{F 3}=\frac{M V A_{F}}{\sqrt{3} \times 48}=-2.95 \mathrm{KA}$ 
KAISER ENGINEERS

HANFORD

DESIGN ANALYSIS
Calc. No. W320-31-001

Revision $\frac{0}{8 \text { of } 10}$
Page No.

Client WHC WO/Job No. ER 4289

Subject FAULT CURRENT ANAWYSIS Date $2 / 25 / 94$ BY JCDEL ROSARIO FEEDER CABLE\& CB SIZING Checked 5/27/94 By Danfaroon

Location TANK FARM C 200 EAST AREA Revised BV

FAULT AT SET (FA)

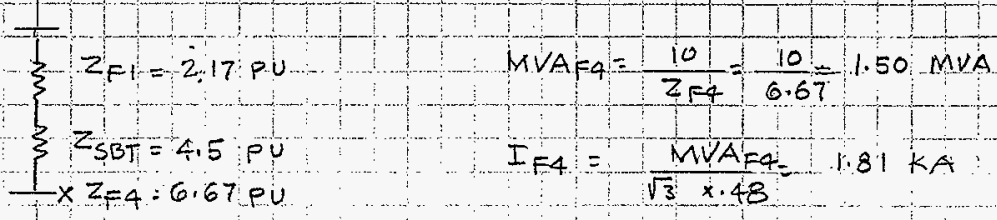

IQFAULT AT ST PR (FF)

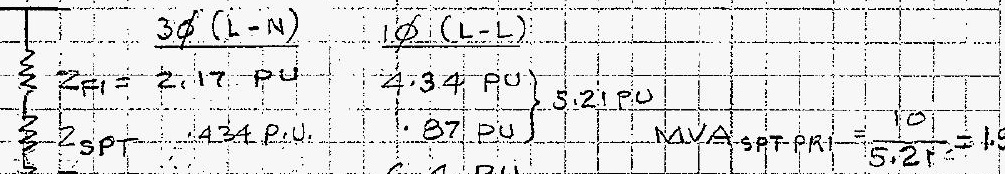

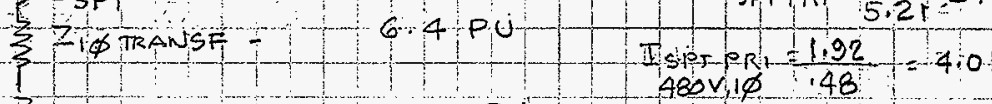

$$
\begin{aligned}
& \sum_{x \mid F S} Z_{S T}=\frac{1111 p u}{Z_{F S}=22.72 p u} \\
& M V A F 5=\frac{10}{Z_{F 5}}=\frac{10}{22.72}=44 M V A \\
& I_{F 5}=\frac{M V A F S}{.12}=\frac{.44}{.12}=3.67 \mathrm{KA}
\end{aligned}
$$

STANDBY GENERATOR:

AVALABLE MVASO AT GEN, POWER WIRELUAY WITH $275 k W\left(344 \mathrm{kVA}\right.$ ) STANDBY GEN WITH $60^{\circ}-3 / \mathrm{C} 350 \mathrm{kam}$ FEEDER TO WIREWTY ASSUME ZN gen $=15 \%$

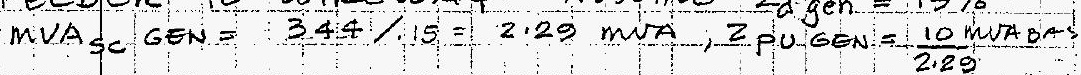
$z \Omega \quad=60^{\prime} 3 / \mathrm{c} 350 \mathrm{kmil}=.06 \times .21=0126 \Omega$ $=4.366$ $Z_{p}=.0126 \times 43.4=.547 p u$

HNF-2487, Rev. 0

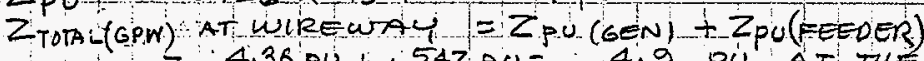

Page A-11

MVASC AT THE WIREWAT $=\frac{10 \text { MVABASE }=\frac{10}{4.9}=0.04 \mathrm{MV}}{2 \text { TOLL }}$

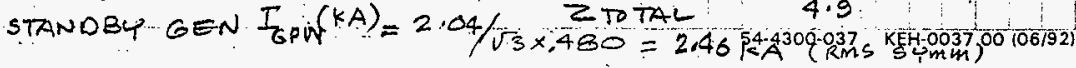


Client WHC

SUbject FAULT CURRENT ANALYSIS FEEDER CABLE \& CB SIRING Location TANK FARM $C$
WO/JOb No. ER 4289 Date 2/25/94 BY J,C,DEL ROSAR10 Gis Revised $5 / 27 / 94$ By Dom fousen

SUMmARY OF $3 \phi$ SHORT CIRCUI mUA (MUAse) FAUL duRRENTS IN KLLOAMPS (KASC) ANR PER UNLT IMPEDANCE (ZF PU. AT PONT OF FAULT. BASED ON NORMAL POWER (13.8KV TRANSE) SOURCE

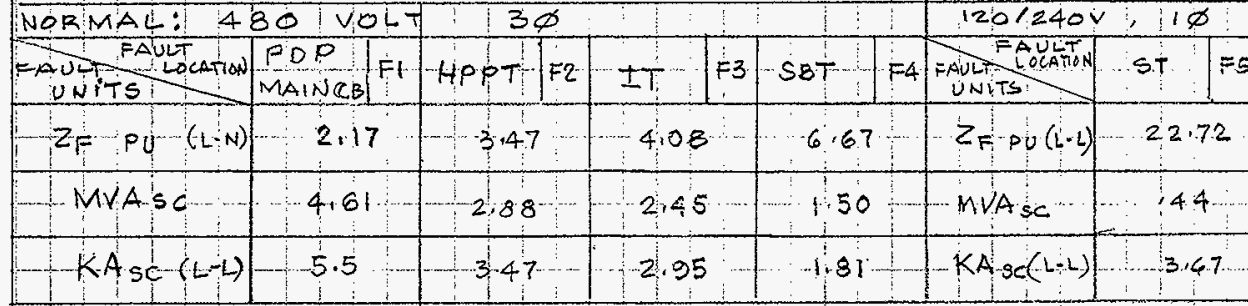

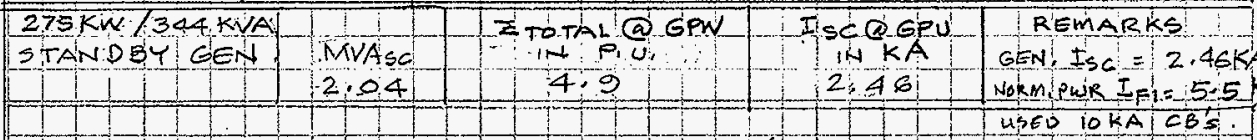

BREARER ELEARING TINE (CT) USINGEOE MOLDED CASE CIRCUIT BREAKERS TO PROTECT FEEDER CABLES TO TRALERS GROUND CONDUUTOR MELTING TIME (MT) FOR GROUND FAULT CUREENT MAGN LTUDE ASSUMED EQUAL TO $3 \phi$ FAULT INSULATION WLTHSTAND TIME CITERMAL DAMAGEITIMETIN SECLIDT AES SUMMARIZEO BELOW:

(1) (2) $\frac{10 \times 1000}{(2)}$

LOAD $:$ : HPPT (F2)

IT $(-5)$

SBT $(F 4)$

- 1 - 4

$4 \quad 225$

3

2

4.
175

100

18

17

17

20

25 :

150

70 52
FAULT CB MULT.OF WIRE SIZE KA

TRIP TRIP

$S T$ (FS) $\quad 3.67$

$A-4, A-5, A-6: A-2$ PHASE GRD CT(S) $4 / 0$ a A $4(10325)>105$ $2 / 0|6| A-4(10305) \mid>10.5$ $2 \quad 6 \quad A .5(.0215) \quad 3.5$ $1 / 06$ A.6(.025) $\because 9$ 4 - $6-5(30195), 131$
A-B PHASE
WIREINS REMARKS
TDT(S) 810 ok OK 5 3 ok OK.

2
ok

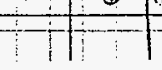

HINF-2487, Rev. 0

Page A-12 


Client WHC
Subject FAULT CURRENT ANALYSIS
FEEDER CAISLE \& CB S SIINC
Location TANK FARMC

WO/Job No. ER 4289

Page No. 10 of 10 Date $2 / 25 / 94$ BY J.C,DEL ROSARID Checked 5/27/74 8y Dar forrum

5 FINDINOS AND CONCLUSIONS:

$T H O$ SUMHARY OF $3 \phi$ FAULT CURRENTS SHOWS MAXIMUM FAULT OF APPROXIMATELY $5.5 \mathrm{KA}$ AT THE $225 \mathrm{KVA}$ TRANSFORMER SECONDARU TERMINALS. THAE FAULT MAGNLTUDE VARLES FROM 1.81 TO $3.47 \mathrm{KALAT}$ THE: DIFFERENT ER TRALERS AT THENEAREST CONSTRUCTION TRALER FAULT ACROSS THE IZOUAC TERMUNALS IS $3.67 \mathrm{KA}$

MOLDED CASE CB WITH MINIMUM INTERRUPTINTC RATING OFIOKA AT 4 BOVISHAU BEUSED THE TRANSFER SWITCHES SHAL HAVE THE SAME INTERRUPTING IRATINE AS THEICB'SITHE USE OF HGHER IC ON THE REJUSABLE TRAWSEER SWITCHES/ BREAKERS SKID WILL PERMIT ITS LSE LW LOCATIONS WITHNLITS IC PUTY. FOR THE SINGLE PHASE I2O/24O TRAILERS $240 V$ I $2 P$ MOLDED CASE CB'S WITH $5 K A$ INTERRUPTINO RATINOS SHAL BEUSED

THE BREAKERS WLL CLEAR THE FAULT BEFORE INSULATION ARE DAMAGED DUE TO OUERHEATINC ANO THE GROUNO CONDUCTORS WELT IAS INOICATED INTHE TABLE SUMMARZINTE BREARER CLEARINO TIME, INSULATION THERMAL DAMÁGE TIME ANO GROUNO WIRE mELTINC TIme.

HNF-2487, Rev. 0

Page A-13 


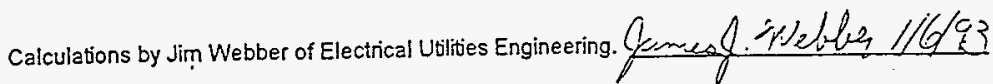

3-Phase Fault Duty at AY Tank Farm. Fault duty is calculated at the $13.8 \mathrm{kV}$ side of the 3-25KVA transformers.

$$
I_{f}:=2200 \cdot a m p \quad X_{R}:=2.97
$$

MVA $_{B}:=100 \cdot 10^{6} \cdot$ volt amp $\quad k V_{B}:=13.8 \cdot 10^{3} \cdot$ rolt

$$
I_{B}:=\frac{M_{B}}{\sqrt{3} \cdot k V_{B}} \quad Z_{B}:=\frac{k V_{B}^{2}}{M V A_{B}}
$$

$$
\begin{aligned}
& Z_{9}:=0.00841+0.01846 j \quad Z_{10}:=0.01137 \div 0.02497 j \\
& Z_{96}:=0.01256+0.01893 j \quad Z_{97}:=0.02746 \div 0.04141 j \\
& Z_{126}:=0.12516+0.03578 \mathrm{j}
\end{aligned}
$$$$
Z_{\mathrm{pu}}=\left(\frac{\mathrm{I}_{\mathrm{f}}}{\mathrm{I}_{\mathrm{B}}}\right)^{-1}
$$$$
. Z_{F B}:=\sqrt{\frac{Z_{p u}{ }^{2}}{1+X_{R}{ }^{2}}}+X_{R} \cdot \sqrt{\frac{Z_{p u}{ }^{2}}{1+X_{R}{ }^{2}}} \cdot j
$$$$
Z_{\text {Tpu }}:=Z_{F B}+Z_{9}+Z_{10}+Z_{96}+Z_{97}+\dot{Z}_{126}
$$

$$
\begin{gathered}
I_{\mathrm{Tpu}}:=\left(\mid \mathrm{Z}_{\mathrm{Tpu}}\right)^{-1} \\
\mathrm{Z}_{\mathrm{Tpu}}=0.79178+1.9418 \mathrm{lj}
\end{gathered}
$$$$
\mathrm{F}_{3 \Phi}=\mathrm{I}_{\mathrm{Tpu}} \mathrm{I}_{\mathrm{B}}
$$$$
F_{3 \Phi}=1.99505 \cdot 10^{3} \text { amp }
$$

Three phase fault current and X/R ratio at point previously calculated (node 6500 of H-2-818278 Sheet 6, Rev.0).

Positive sequence impedances of line segments between the calculation point and the point of previous calculation.
Per unit impedance at the upstream fault box.
Total positive sequence per unit impedance at calculation point (100MNA base).

$13.8 \mathrm{kV}$ three phase fault current at calculation point.

HNF-2487, Rev. 0

Page A-14 


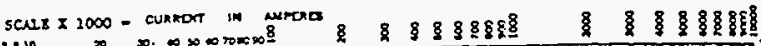

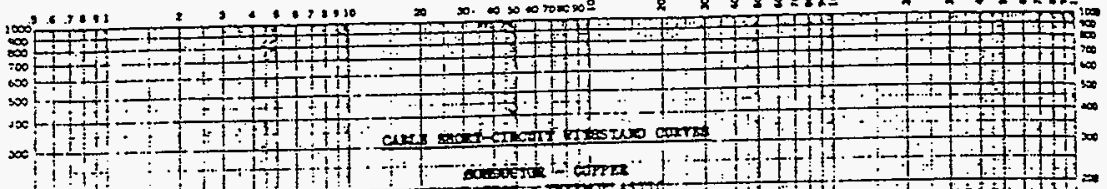

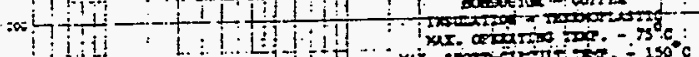
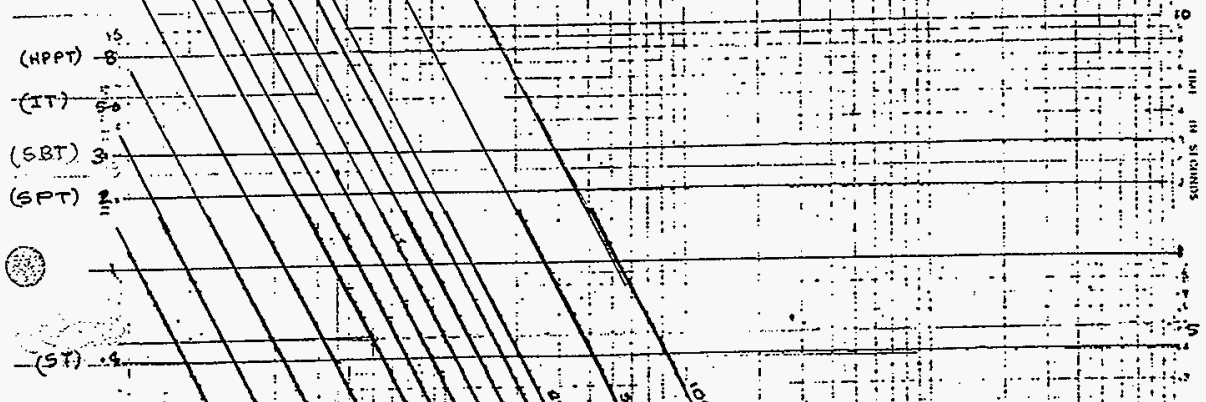
$-(5+)$

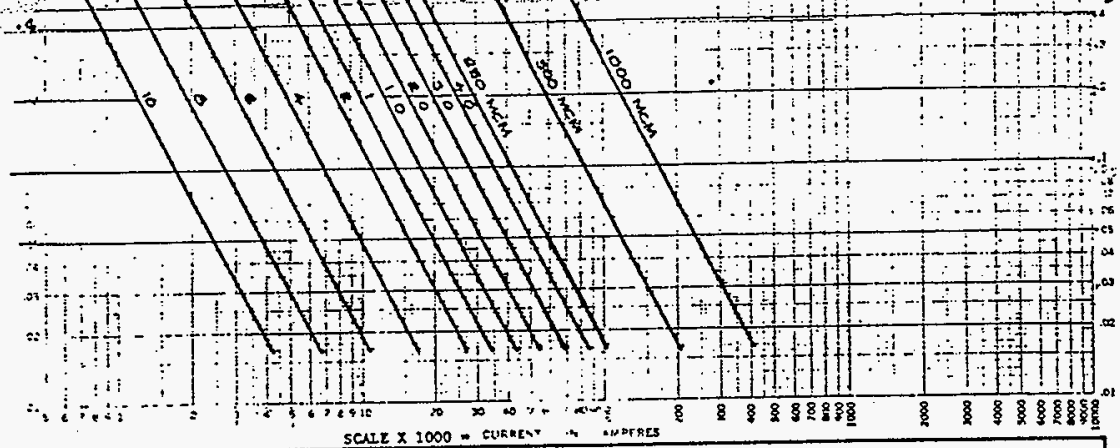

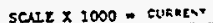

OVERCURRENT DEVICE COORDINATION TIME-CURRENT CHARACTERISTIC CURVES

JOB TILE:

LOCATION:

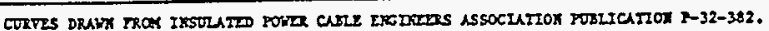

HNF-2487, Rev. 0

Page A-16
3WO. NO:

SATE: 
70-250 Amperes, 2 and 3 Poles, 600 Volts Ac Max. 250 Volts DC

CURRENT IN PERCENT OF BREAKER TRIP UNIT RATING

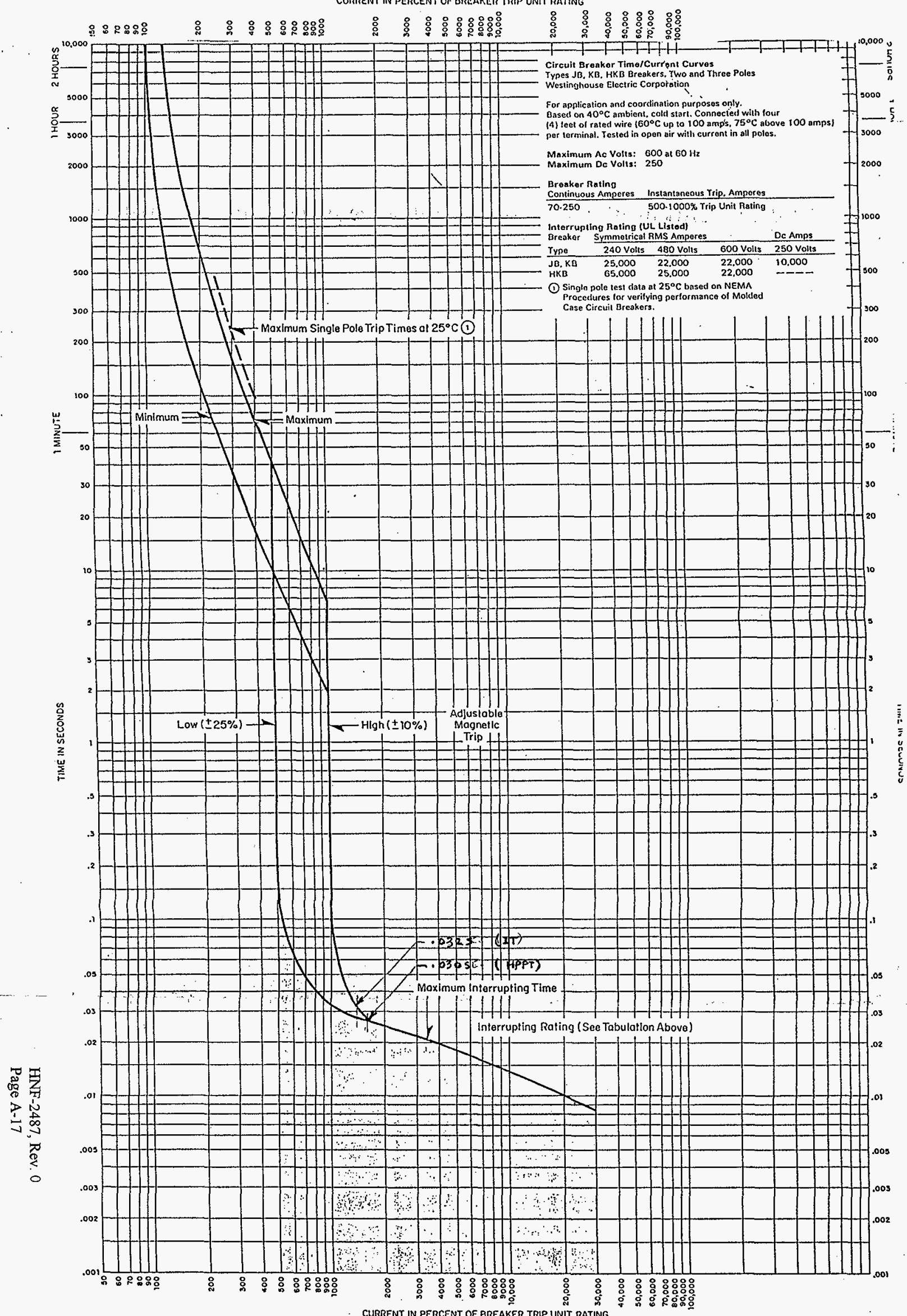
क क के 
Type EB: $50-70$ Amperes, 2 and 3 Poles, 240 Voits Ac Max.

Type EHB: 50-70 Amperes, 2 and 3 Poles, 480 Volts Ac Max.

Type FB: $50-70$ Amperes, 2 and 3 Poles, 600 Volts Ac Max.

Type HFB: 50-70 Amperes, 2 and 3 Poles, 600 Volts Ac Max.

CURRENT IN PERCENT OF QREAKER TRIP UNIT RATING

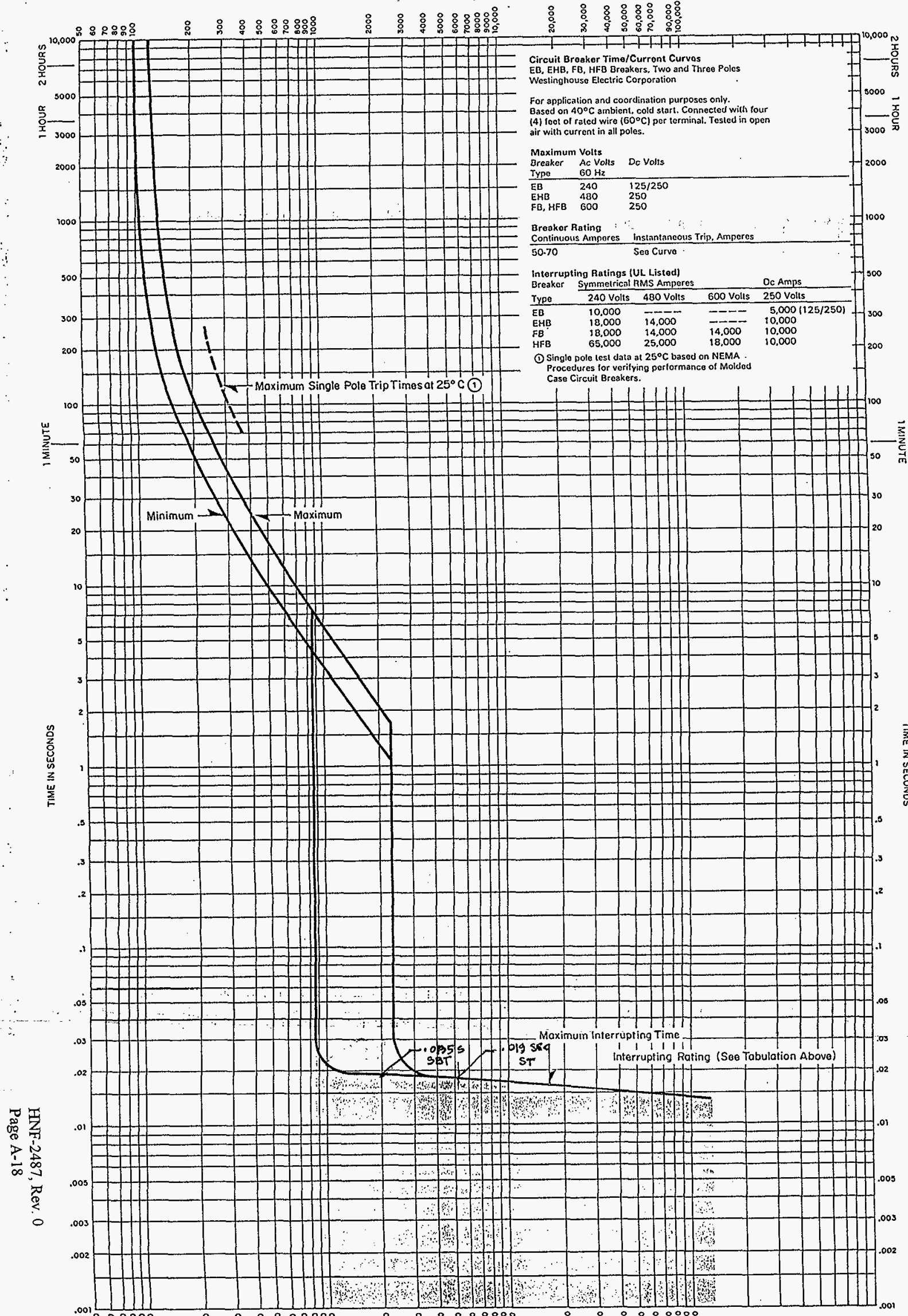

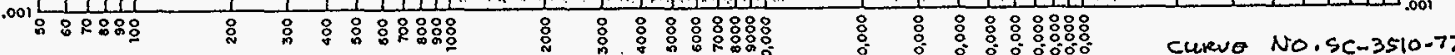
CURRENT IN PERCENT OF BREAKER TRIP UNIT RATING 


\section{Type EB: 30-100 Amperes, 2 and 3 Poles, 240 Volts Ac Max. \\ Type EHB: $90-100$ Amperes, 2 and 3 Poles, 480 Volts AC Max. \\ Type FB: 90-150 Amperes, 2 and 3 Poles, 600 Volts Ac Max. \\ CURRENT IN PEACENT Type HFB: $90-150$ Amperes, 2 and 3 Poles, 600 Volts Ac Max.}

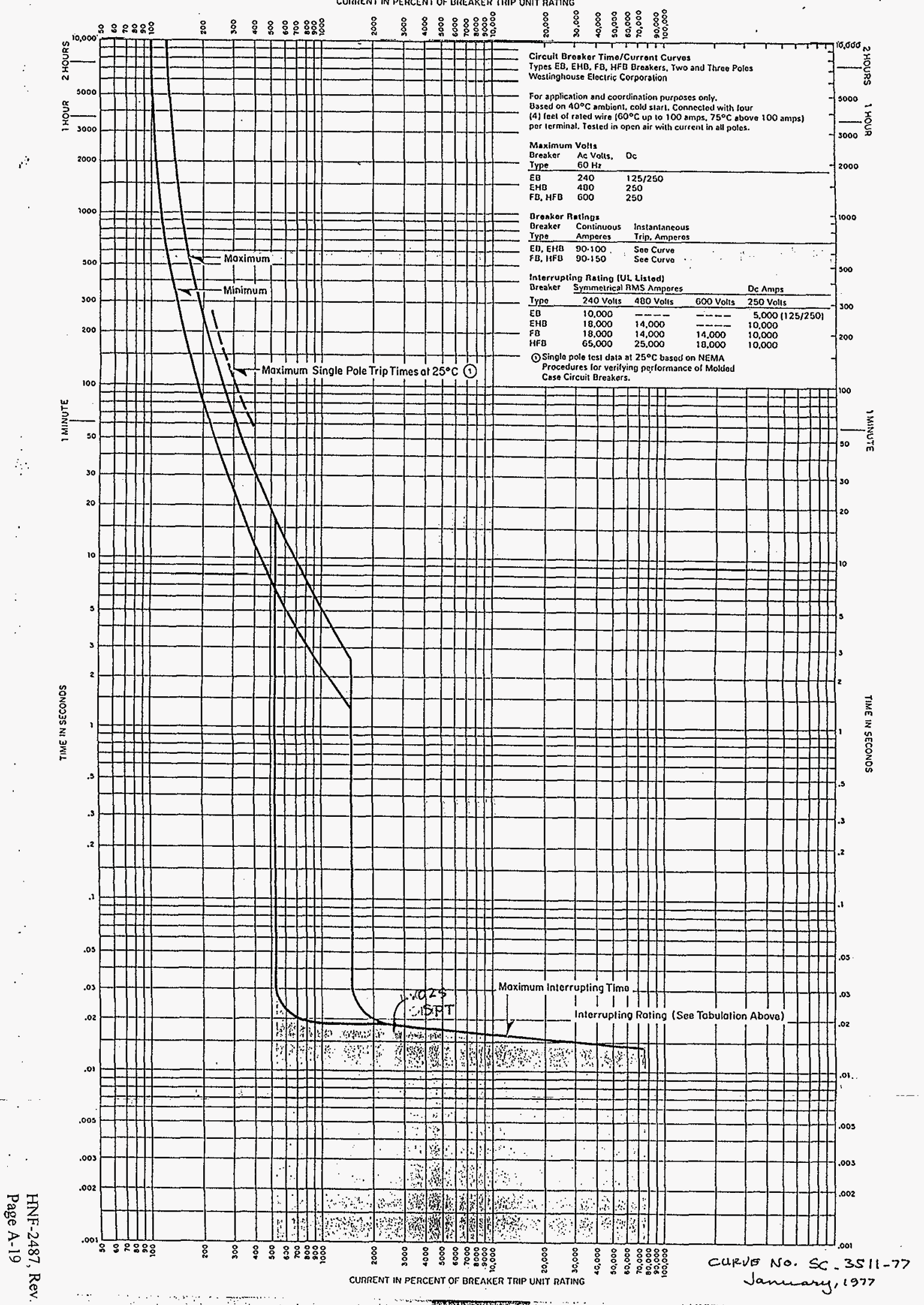


HNF-2487, Rev. 0

\section{W320-31-002}

Standby Generator Sizing 
This sheet shows the stakus and description of the attachod Dasign Analysis sheots.

Disciplino ELECTRLEL WO/Job No. W320/0124289 Caiculation No. W320-31-002

Projoct No. \& Nama W320 EQUUPMENT REMOVAL SYSTEM

Calculation Itom STANOBL SENERATOR SIZING

These caiculations apply to:

Dwo. No. $H-2-820748 \quad S H I$

Dwo. No. $H-2-820>49 \quad S H 2$

Rev. No.

Rev, No.

0

Other (Study, CDR)

The ststus of these calculstions is:

$\square$ Proliminary Calculations

$\square$ Final Calculations

$\square$ Chack Calculations IOn Calculation Datod

Void Calculation (Resson Voided

$\begin{array}{ll}\text { Incorposatod in Final Drawings? } & \square \text { Yos } \\ \text { This calculation verifiod by indepondent "chock" calculations? }\end{array}$

Original and Revised Calculation Approvals:

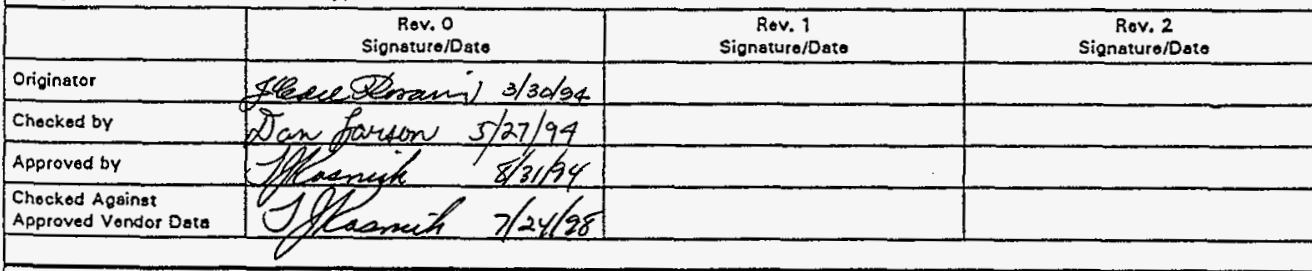

INDEX

\section{Dosign Analysig} Paga No.

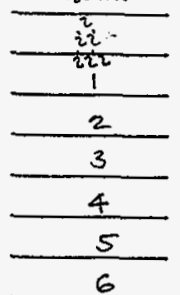

APPENक्यद: $A-1$ THRU A-E6

INPEX

Description

EALCULATION CROSS INDEX

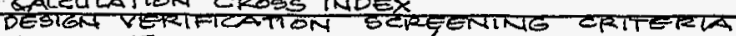

OBUECTIVE, REFERENCES/SOURCES, PATA

DATA, ASSUMPTLONS

CALCULATLONS

SUMmARY OF RESULTS

KU/KVA STRRTING \& RUNNING LOAD PROFILE

FINDINGS \& CONCLUSLON

\% motor STARTIAG KVA, ALTITUDE CORR. FACTOR, GEN. VOLTAGE BEHAVIOR DURING MOTOR STARTING, GEN, SIZING (KOHLER QSIZE) INSTRUMENT TRAILETR (IT) LOAD, HPPT DATA 
Project/Document No. W320/W320-31.002

When the design or design change affects hardware, formal design verification must be performed if one or more of the following questions must be answered affirmatively (YES).

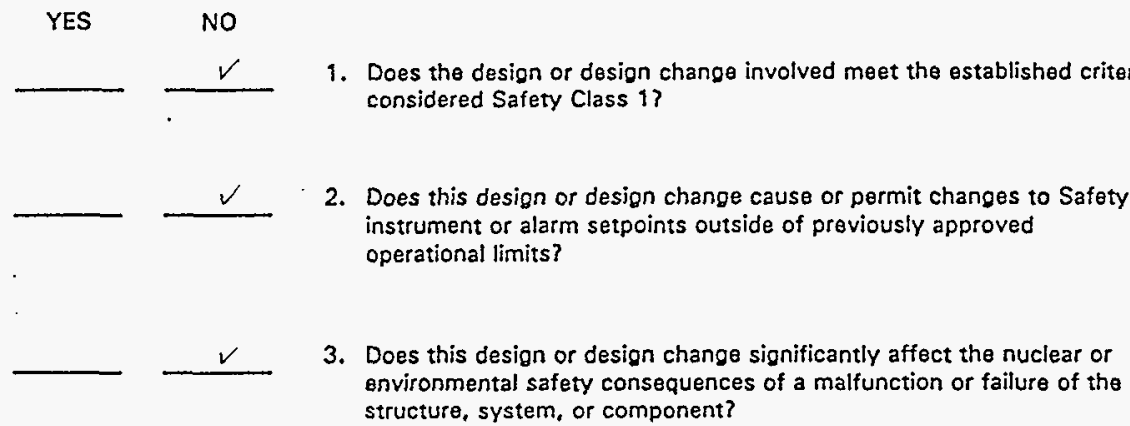

4. Does this design or design change involve or change design that has previously undergone formal design verification?
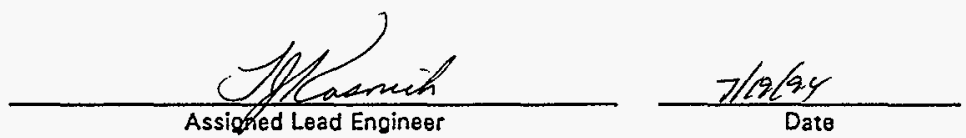

Responsible Discipline Manager

Date

Original Design Package Distribution:

Project Manager

Design Verification Officer

Engineering Document Control
Design Change Distribution:

Attach to Engineering Change Notice

HNF-2487, Rev. 0

Page B-2 


\begin{tabular}{|c|c|c|c|c|c|c|c|c|c|c|}
\hline \multicolumn{10}{|c|}{$\begin{array}{l}\text { CALCULATION CROSS INDEX (Typical) } \\
\text { Subject Calculation No. W320-31-002 }\end{array}$} & Page il of iii \\
\hline \multirow{2}{*}{$\begin{array}{l}\text { Subject } \\
\text { calculation } \\
\text { Revison } \\
\text { No. }\end{array}$} & \multirow{2}{*}{$\begin{array}{l}\text { Superceded } \\
\text { by } \\
\text { cakcuation } \\
\text { No. }\end{array}$} & \multicolumn{2}{|c|}{ 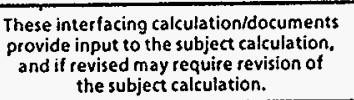 } & \multicolumn{2}{|c|}{ 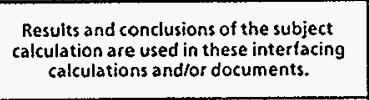 } & \multicolumn{2}{|c|}{\begin{tabular}{|c|}
$\begin{array}{c}\text { Dees the output } \\
\text { intertacece calculation } \\
\text { documents } \\
\text { revision? }\end{array}$ \\
\end{tabular}} & \multicolumn{2}{|c|}{ 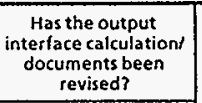 } & \multirow{2}{*}{$\begin{array}{l}\text { Discipline.manager's signature and } \\
\text { date indicating evaluation } \\
\text { complete. }\end{array}$} \\
\hline & & Cakculation/Document No. & $\begin{array}{c}\text { Revision } \\
\text { No. } \\
\text { No }\end{array}$ & Calculation/Document No. & $\begin{array}{l}\text { Revision } \\
\text { No. }\end{array}$ & Yes & No & Yes & No & \\
\hline \multirow[t]{2}{*}{0} & $N / A$ & & & $4-2-820748$ 54.1. & 0 & & $\sim$ & & 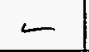 & \\
\hline & & & & $4 A-2-82074954.2$ & 0 & & - & & - & \\
\hline & & & & & & & & & & \\
\hline & & & & & & & & & & \\
\hline & & & & & & & & & . & \\
\hline & & & & & & & & & & \\
\hline \\
\hline 学䆓 & & & & & & & & & & \\
\hline 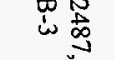 & & & & & & & & & & \\
\hline \multicolumn{11}{|l|}{ 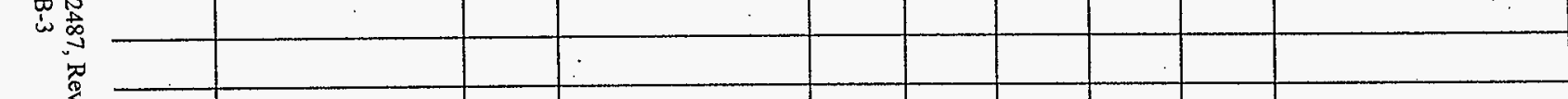 } \\
\hline & & & & & & & & & & \\
\hline & & & & & & & & & & \\
\hline & & & & & & & & & & \\
\hline & & 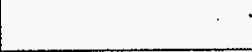 & & & & & & & & \\
\hline & 1 & & & & & & & & & \\
\hline & & & & . & & & & & & \\
\hline & & & & - & & & & & & \\
\hline & & & & & & & & & & \\
\hline & & & & & & & & & & \\
\hline
\end{tabular}


KAISER ENGINEERS

HANFORD

Client WHC
Subject STANDBY GENERATOR

SIZINE

Location JANK FARMS

I OBJECTVE:

DETERM WN THE MINIMUM REQULEO CAPACLTY OE THE TEMPORARY GENER ADEQUATE TO SUPRORT A ZWWEEK EQLUTTUENT REMOVAL OPERATION TO BE PERFORMEO WU SUGLST BEFORE THE START OF THE SLUICING PHASE
Calc. No.

$\omega 320-31-00:$

DESIGN ANALYSIS

WO/Job No. ER 4289 Date 3/30/94 By J,C, DEL ROSARLA) Chacked $5 / 27 / 94$ By Don faram

8y
Revision $o$

Page No. 1 of 6

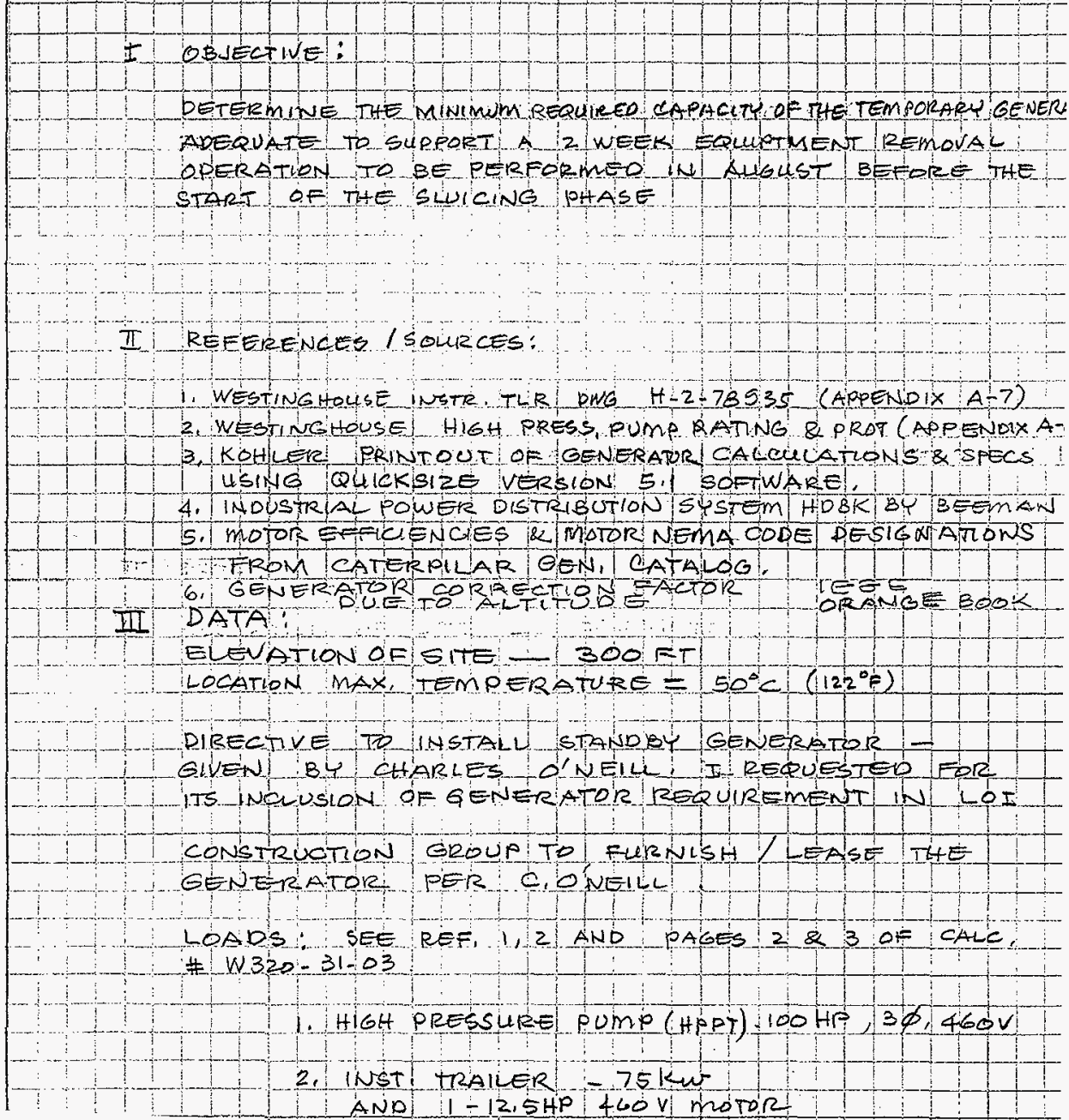

HNF-2487, Rev. 0 Page B-4
3. STRONG BACK TRALLER WITA REDUNDANT $10 \mathrm{HP} 3 \phi, 460 \mathrm{~V}$ HYD POMP - ONE SHAW BE OPERATEO AT A TIME 
KAISER ENGINEERS

HANFORD

Client

XHC

Subject

STANDBY GENETRATOR

SIZING

Location TANK FARMS C \& AY

200 EAST AREA Revised

\section{DESIGN ANALYSIS}

WO/JOb No. ER 4289

Date $3 / 30 / 94$ By J.C.DELROSAR10 J63K

By
Calc. No. W320-31-00:

Revision 0

Page No. 2 of 6

II DATA : CONTINUED

A DIESEL GENERATOK SHAL SUPPLT STANDBY

POWER TO THE EQULPMENT REMOVAL TRALERS CLOADS SHOLLD THE NORMAL POWER LOSS OLCUE DURNG THE EQLIPMENT REMOVAL OPERATION.

THE STANDBY GENERATOR WIL BE MANUALLOSTARTEK AFTER THE EEEDER COS IN THE TRANSFER SWITH BREAKERS SKID ARE CHECKED TO BE IN OPEN

POSITIDN.:

THE TRANSFER SWITCH FOR HPPT IS MANUALLY SWITAHE TO THE STANDSY SENGRATOR OTHE HHP-FB IS ELOSE STANDBY EERERATOR POWER IS SIMLLRLY TRPNSEEA IN STEPS TO TIHE NSTRODENT JRALER ANDLTHENTO THE STRONG-BACL TKAILER

\section{iv Assumptons:}

1. THE HPPT IS STARTED EIRST AFTER THE SENERATOR HAS ATTAINED SPEED TO RECEIVEI FUL LOAP

MAXIMUM GEN VOLTAGE DIN DUE TO STARTING OF THE

LARGEST MOTOR (IDOHP) IS LIMITED TO $20 \%$ OF GEN VOL

2. WORST IPPT UNDUCTION MOTOR STARTLNG CONDLTLON IS ASSUMED - HPPT IS STARTED AT FULL SPEEF

CIHAT TTE ZOOA EUSESATTHE DKSONNEET SWLTCH \& THE SETINIO OE THE ZSOAF VAR UABL FREQUENCY DRUE WULL PASS TAL STARTING CURRENT IOOHP IS FULYYLOADEO!

3. NO DEMAND FACTOR IS APPLLED ON ANY OF TH LOADS CONNECTED LOANS ARE LUEO $\mathrm{NA}$ THE CALCLLARLONS

4. TKAILER MOTOR WOAD DATA:

\begin{tabular}{|c|c|c|c|c|c|}
\hline$\angle O A D$ & $E F=$ & $P F R$ & NEMA COO & KVA $/ A P$ & \\
\hline $100 \mathrm{HP}$ HPe & $92^{\prime}$ & .80 & $\frac{5 E_{i} T T}{F}$ & 5.6 & 30 \\
\hline $12.5 \mathrm{HP}$ pump & 89 & .80 & $G$ & 6.29 & .53 \\
\hline $10 \mathrm{HP}$ & 89 & .80 & $G$ & 6.29 & 53 \\
\hline
\end{tabular}

HNF-2487, Rev. 0

Page B-5

$10 \mathrm{HP}$

89
6.29
53

5. OTHER INSTRUMENT TRALER LOADS $2-25 \mathrm{KVA}, 1$. TRANSFORMER, ASSUMED 75 KVA BALAWCED $3 \phi_{\text {KE LOAD }} .8511$ 
KAISER ENGINEERS

DESIGN ANALYSIS
Calc. No. W 320-31-00

Revision

Page No. 3 of 6

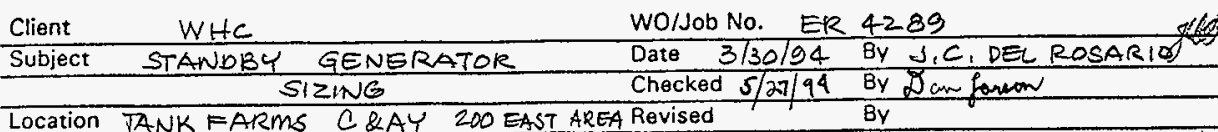

Location TANK FARMS C\&AY 200 EAST AREA Revised

Br

- Calculations: I manual)

$$
\begin{aligned}
& \text { (A KWR (RUNNING) }=H P X .746 \\
& \mathrm{KW}_{\mathrm{R}}(\mathrm{HPOH})=\frac{100 \times 746}{92}=81 \mathrm{~kW} \\
& K W_{R}\left(\begin{array}{c}
12.5 \mathrm{HP} \\
\text { YPPP }
\end{array}\right)=\frac{12,5 \times 1746}{89}=10,5 \mathrm{ku} \\
& K W_{R}(S T B H Y P P)=\frac{10 \times 1746}{85}=814 t u
\end{aligned}
$$

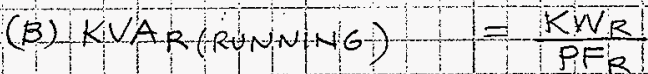
WHERE PER = RUNNNG PF

$$
\begin{aligned}
& \text { KVAR ( } 100 \% \text { HPP })=81 / 80=101 \mathrm{KVA} \\
& K V A R\left(\begin{array}{c}
125 H P \\
H Y D P
\end{array}\right)=10.5 / 80=13 \mathrm{KVA} \\
& K V A R(S T B H P D P)=8,4 / 80=10,5 \mathrm{KVA}
\end{aligned}
$$

(c)

$$
\begin{aligned}
& \text { KVAS (STARANG) }=H P \times \mathrm{KVA} / H P \\
& \text { KVAS }(100 \mathrm{HP})=100 \times 5.6=560 \mathrm{kVA} \\
& \text { KVAS (1250) }=12,5 \times 6,29=78.63 \mathrm{kVA} \\
& \text { KVAS (10HP }
\end{aligned}
$$

HNF-2487, Rev. 0

Page B-6

$54-4300=037-K E F=0037.000679$ 
KAISER ENGINEERS

DESIGN ANALYSIS
Cali. No. W320-31.00

Revision $\frac{0}{4}$ of 6

Client

THC WO/Job No. ER 4289

Subject STANDBY GENERATOR Date $3 / 30 / 94$ BY IC. DEL ROSARIO SIZING Checked $5 / 27 / 94$ By 5 an forworn

Location TANK FARMS C\&AY, 200 EAST AREA Revised

By

IV CALCULATING (CONT)

(D)

$$
\begin{aligned}
& \text { KXYS(STARTING) }=K V A_{R} \times P F_{S} \\
& \text { Kos }\left(\begin{array}{c}
100+P \\
1+90
\end{array}\right)=560 \times 3=168 \mathrm{~kW} \\
& \text { aWS }\left(\frac{12.51+P}{40 P p}\right)=72.63 \times .53=41.67 \\
& K W S(S H B H 0.8 P)=62.9 \times 153=33 \mathrm{kw}
\end{aligned}
$$

(E) FROM REF 4 FIG $4,42 \%$ KNA DF mOTOR $X-6 E N K \nu A$ FOR $20 \%$ DIP GO N HTAL LOAD RATED GEN VOLTAGE =1. FOR 250|kVA GENITHIS IS $=1250 \times 1 \mathrm{~W}=425 \mathrm{kVA}$

USE KOHLER 200ROZD MAX KVAS@20\%DIP=560kVA

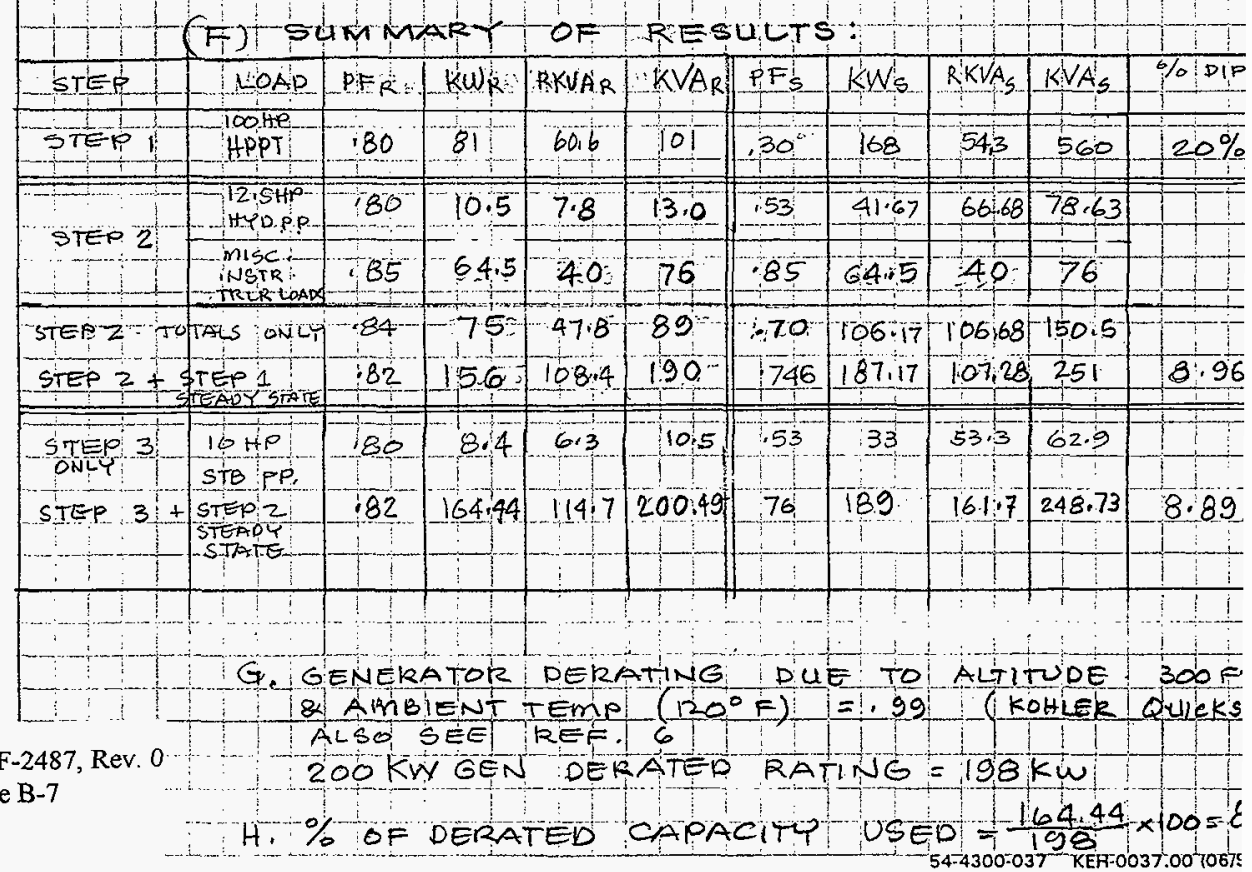

HNF-2487
Page B-7

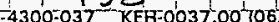




\section{KAISER ENGINEERS HANFORD}

DESIGN ANALYSIS
Calc. No. W320-31-002 Revision 0

Page No. 5 of 6

Client

WHC

Subject STANDBY GENERATOR

WO/Job No. ER 4235

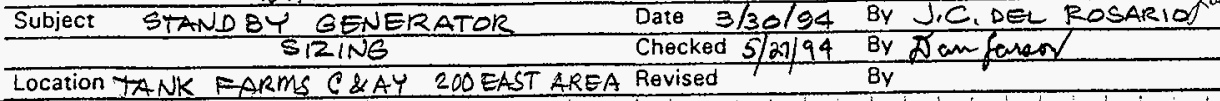

8

$560 \mathrm{kNASS}$

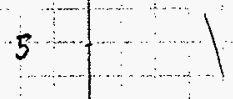

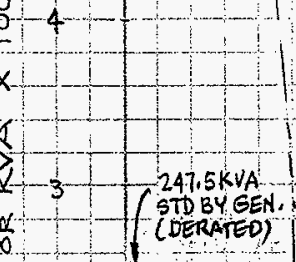

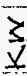

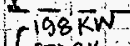

$=251 \mathrm{kVA}_{\mathrm{s}}$

(5)

$-220 \mathrm{~kW} \times 217.5$

2

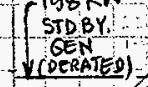

$168 \mathrm{~kW}$
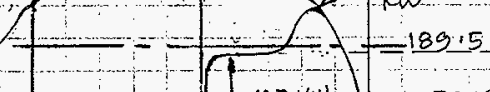

\begin{tabular}{c}
1 \\
$101 \mathrm{kVA}$ \\
\hline $81 \mathrm{~kW}$
\end{tabular}

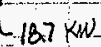

$156 \mathrm{~kW}$

$248 \cdot 7 \cdot 3$

KVA'S:

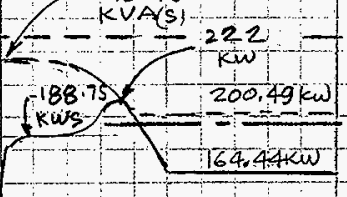

$10 \mathrm{HP}$

MOTOR

D. STARTINE" RUNNUG5 STARTING RUNNING 70 STARTING RUNNING STEP T STEP 2 $>$ STEO 3, $\Rightarrow$ TIMEI IN SECONDS

HNF-2487, Rev. 0

Page B-8

KW/KVA STARTING \& RUNNING LOAD PROFILE 
KAISER ENGINEERS

HANFORD

Client

WHO

DESIGN ANALYSIS
Calc. No. W $320-31-00$

Revision 0

Page No. 6 of 6

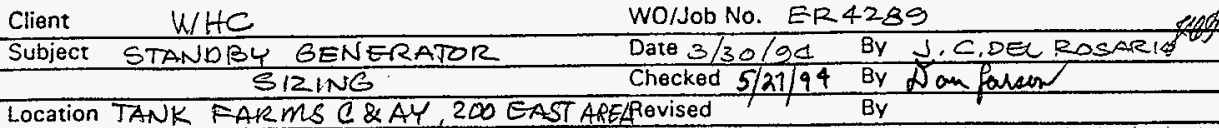

Location TANK FARMS C \& AY, 200 EAST ARELRevised

By

FINDINGS \& CONCLUSIONS:

THE SUMMARY OE RESULTS \& THE LOAD PROFILE SHOW A STEADY STATE (RUNNING) LOAD OF $164.44 \mathrm{~kW}$ OR $200 \mathrm{KVAL}$ LOAD AT $82 \%$ PF, MAX MUM STARTINg KWA $(560)$ OCCURS DURING FULL SPEED START OF $10 O H P$ HG PRESSURE PUMP CAUSING MAXIMUM VOLTAGE DIE OF $20 \%$. (PLOWABLE VOLTAGE DIP IS UP TO $30 \%$ )

A $200 \mathrm{KW}$ GENERATOR (aERATED TO $198 \mathrm{KW}$ ), $250 \mathrm{KVA}$ AT $80 \%$ PE NL BE SUFFICIENT TO SUPPLY THE EQUIPMENT REMOVAL LOADS AND LIMIT THE VOLTAGE DIP TO LESS THAN $20 \%$ IF STARTED IN 3 STEPS As SHOW WI IN THE Summary OF RESULIS

KOHLER GENERATOR COMPUTER CALCULATIONS USIA "QUIKSSIRE $5: 1$ "SOFT ARE IS ALSO ATTACHED F COMPARISON. (APPENDIX AC)

DIESEL GENERATOR PRESENTLY IN COLDEST FACILITY AS OOF $8 / 24 / 94$

CATERPLAR 3406

$275 \mathrm{KW}, 344 \mathrm{KVA}, 80 \% \mathrm{PE}, 3 \phi \quad Y$

HNF-2487, Rev. 0

Page B-9

$54=4300=037^{2}$ KEF $0037.00706 / 9$ 


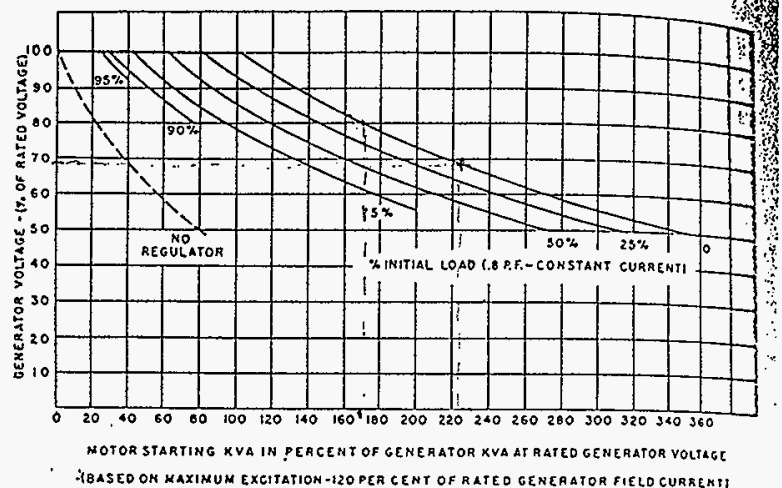

NOTE: AESTOREO VOLTAGE COUALS VALUE REAO FGON CURVE OR THE IHITAL VOLTAGE (ACGULATOR SETTIMG) WMICKEYER IS LOWCR

FIG. 4.42 Restored generolor volloge.

Advantages of Voltage Regulators. Figures 4.38, 4.39, and 4.42 show dashed curves, marked $N$, which indicate the results to be expected if no regulator is used. It is apparent that regulators are very bencficial. They practically always justify their cost whenever the starting of latic molors is involved. For example, consider a 450 -voll 12.5-kva 1200-rpm gencrator. From Fig. 1.40 this may have a performance factor $k$ of about 1.7 with a regulator. From $l i j g .439,110$ per cent notor-starting load or $138 \mathrm{kva}$ will cause a 28 per cent voltage dip.

This load would correspond to starting a 25 -hp motor at lull roltagc.

To obtain the same motor-starting performance without a regulator would require a 438 -kva generator, because the curve $N$ shows that about 32 per cent motor-starting load will cause a 28 per ccut voltage drop if no regulator is used. (133 kva is about 32 per cent of $135 \mathrm{kva}$.) The 438-kva gencrator would cost over twice as much as a 125-kva machine. The best and least cxpensive arrangement would be to pruvide a regulator adding less than 15 per cent to the cost of the 125 -kva gencratur. This would permit successful starting of the $25 \cdot \mathrm{hp}$ motor cven itgainst full-load torque and would improve normal generator performance.

In Fig. 4.39 are curves, marked $E$, which show the performance available when using an cle excitation system. It s olher very high-responsc imit to the improvement 
From REF 6

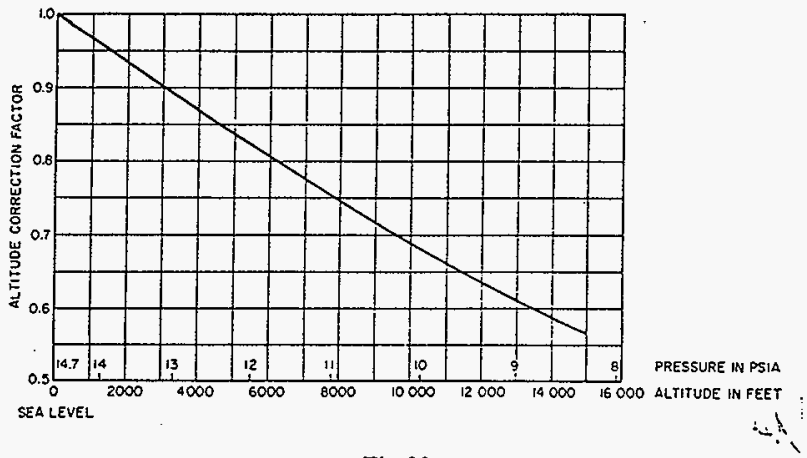

Typical Performance Correction Factor for Altitude

p131

it:

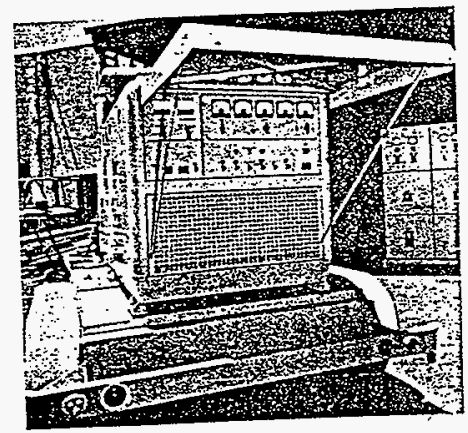

Fig 28 Modular Packaged Gas-Turbine-Generator Set Mounted on Trailer $P 131$

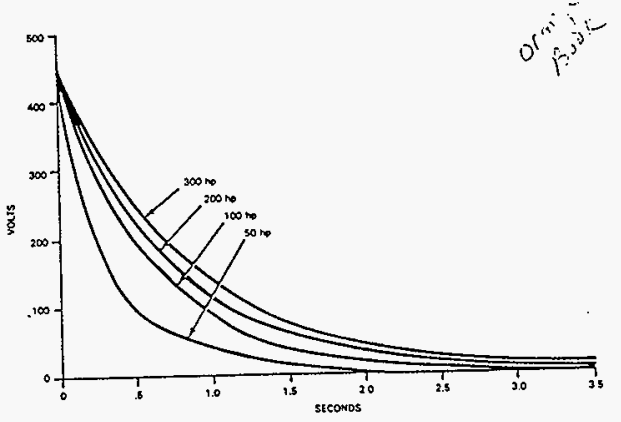

Fig 19 Induction Motor Open Circuit Voltage Decay (Based on Constant Speed)

HNF-2487, Rev. 0

Page B. 11 
Fig 21

Minimum Generator Voltage Due to Full-Voltage Starting of a Motor

(because it is not influenced by a regulator as in the generator case) and does not recover until the motor approaches full speed. Since the transformer is usually the largest single impedance in the distribution system, it takes almost the total arop. Figure 22 has been plotted in terms of motor starting load in kilovolt-amperes that would be drawn if rated transformer secondary voltage were maintained.

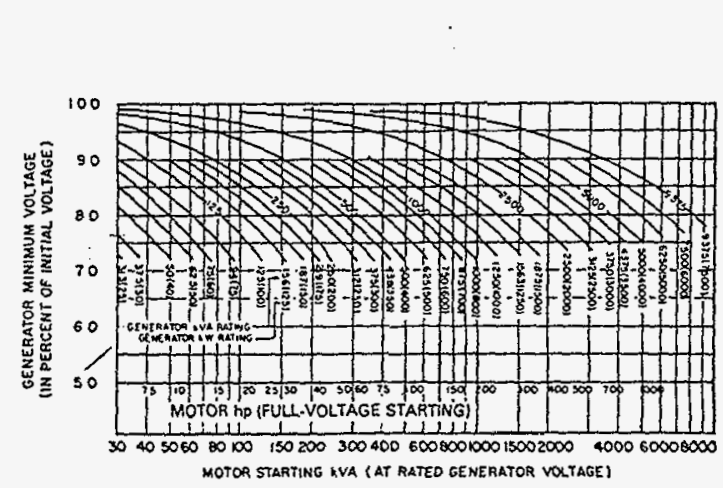

NOTES: (1) The scale of motor horsepower is based on the starting current being equal to approximately 5.5 times normal.

(2) If there is no initial load, the voltage regulator will restore voltage to $100 \%$ after dip os values given by curves.

(3) Initial load, if any, is assumed to be of constant-current type.

(4) Generator characteristics are assumed as follows: (a) Generators rated 1000 KVA or less: Performance factor $k=10$; transient reactance $X_{d}=25 \%$; synchronous reactance $X_{d}=120 \%$. (b) Generators rated above $1000 \mathrm{kVA}$. Characteristics for $3600 \mathrm{r} / \mathrm{min}$ turbine generators.

HNF-2487, Rev. 0

Page B-12 
VOLTAGE CONSIDERATIONS

ANSI/IEEE

Std 141-1986

Table 14

Comparison of MotorStarting Methods

\begin{tabular}{|c|c|c|c|}
\hline $\begin{array}{l}\text { Type of Starer } \\
\text { (Settings Given Are } \\
\text { the More Common for } \\
\text { Each Type) }\end{array}$ & $\begin{array}{c}\text { Motor } \\
\text { Terminal } \\
\text { Voitage } \\
\text { (Percent Line } \\
\text { Voltage) }\end{array}$ & $\begin{array}{c}\text { Starting } \\
\text { Torque } \\
\text { (Percent Full- } \\
\text { Voltage } \\
\text { Starting } \\
\text { Torque) } \\
\end{array}$ & $\begin{array}{c}\text { Line- } \\
\text { Current } \\
\text { (Percent Fuld- } \\
\text { Voltage } \\
\text { Starting } \\
\text { Current) } \\
\end{array}$ \\
\hline Full-voltage starter & 100 & 100 & 100 \\
\hline $\begin{array}{l}\text { Autotransformer } \\
80 \% \text { tap } \\
65 \% \text { tap } \\
60 \% \text { tap }\end{array}$ & $\begin{array}{l}80 \\
65 \\
50\end{array}$ & $\begin{array}{l}64 \\
42 \\
25\end{array}$ & $\begin{array}{l}68 \\
46 \\
30\end{array}$ \\
\hline $\begin{array}{l}\text { Resistor starter, single step (adjusted for motor } \\
\text { voitage to be } 80 \% \text { of line voltage) }\end{array}$ & 80 & 64 & 80 \\
\hline $\begin{array}{l}\text { Reactor } \\
50 \% \text { tap } \\
45 \% \text { tap } \\
37.5 \% \text { tap }\end{array}$ & $\begin{array}{l}50 \\
45 \\
37.5\end{array}$ & $\begin{array}{l}25 \\
20 \\
14\end{array}$ & $\begin{array}{l}50 \\
45 \\
37.5\end{array}$ \\
\hline $\begin{array}{l}\text { Part-winding starter (low-speed motors only) } \\
75 \% \text { winding } \\
50 \% \text { winding }\end{array}$ & $\begin{array}{l}100 \\
100 \\
\end{array}$ & $\begin{array}{r}75 \\
50 \\
\end{array}$ & $\begin{array}{l}75 \\
50 \\
\end{array}$ \\
\hline
\end{tabular}

Fig 20

Typical Generator Voltage Behavior Due to

Full-Voltage Starting of a Motor

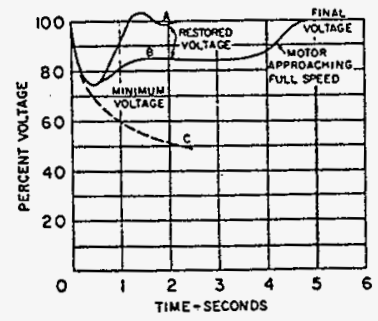

$$
\operatorname{Sim}_{+1}(\therefore)
$$

Motor-starting kVA $=100 \%$ of generator rating

$A-$ No initial load on generator

$\mathrm{B}-50 \%$ initial load on generator

$\mathrm{C}-$ No regulator

HNF-2487, Rev. 0

Page B-13 
Solution. Enter the chart on the horizontal scale at $2000 \mathrm{kVA}$, extend a line (v) vertically to its intersection with the 0.85 power factor curve. Extend a line horizon1 tally from this point to the left to its intersection with the vertical scale. This point on the vertical scale gives the percent voltage drop for rated load. Multiply this value by the ratio of actual load to rated load:

$$
\begin{aligned}
\text { percent drop at rated load } & =3.67 \\
\text { percent drop at } 1500 \mathrm{kVA} & =3.67 \cdot \frac{1500}{2000} \\
& =2.75
\end{aligned}
$$

actual voltage drop $=2.75 \% \cdot 480$

$$
=13.2 \mathrm{~V}
$$

Figure 19 applies to the $34.5 \mathrm{kV}$ insulation class power transformer in ratings from 1500-10000 kVA. These curves can be used to determine the voltage drop for transformers in the 46 and $69 \mathrm{kV}$ insulation classes by using appropriate multipliers at all power factors except unity.

To correct for $46 \mathrm{kV}$, multiply the percent voltage drop obtained from the chart by 1.065 , and for $69 \mathrm{kV}$, multiply by 1.15 .

3.11.5 Motor-Starting Voltage Drop. It is characteristic of ac motors that the current that they draw on starting is much higher than their normal running current. Synchronous and squirrel-cage induction motors started on full voltage may drawa current as high as seven or eight times their full-load running current. This sudden increase in the current drawn from the power system may result in excessive drop in voltage unless it is considered in the design of the system. The motor-starting load in kilovolt-amperes, imposed on the power supply system, and the available motor torque are greatly affected by the method of starting used. Table 14 gives a comparison of several common methods.

3.11.6 Effect of Motor Starting on Generators. Figure 20 shows the behavior of the voltage of a generator when an induction motor is started. Starting a synchronous motor has a similar effect up to the time of puil-in torque. The case used for this illustration utilizes a full-voltage starting device, and the full-voltage motor starting load in klovolt-amperes is about $100 \%$ of the generator rating. It is assumed for curves A and B that the generator is provided with an automatic voltage regulator.

The minimum voltage of the generator as shown in Fig 20 is an important quantity because it is a determining factor affecting undervoltage devices and contactors connected to the system and the stalling of motors running on the system. The curves of Fig 21 can be used for estimating the minimum voitage occurring at the terminals of a generator supplying power to a motor being started.

3.11.7 Effect of Motor Starting on Distribution System. Frequently in the case of purchased power, there are transformers and cables between the starting motor and the generator. Most of the drop in this case is within the distribution equipment. When all the voltage drop is in this equipment, the voltage falls immediately

HNF-2487, Rev. 0

Page B-14 
ER4289 STANDBY GENERATOR

Data for model - 200ROZD

03/18/94 08:20:09

\section{GENERATOR DATA}

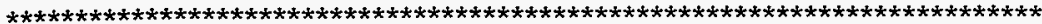

Kohler genset model

Alternator model

Voltage and phase selected

Fuel selected

Altitude (meters)

Temperature (C)

Generator's KW Standby rating

Generator's derated running KW's

Percent of available KW's used

Generator's KVA rating.

Maximum starting KVA at $20 \%$ voltage dip ..........

Generator's power factor.................
200ROZD

$4 U 13$

$277 / 480$ volts, 3 phase $60 \mathrm{hz}$

Diesel

300

50

200.0

197.8

$90 \%$

250.0

560.0

0.8

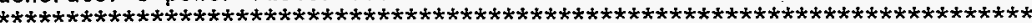

This generator meets the minimum requirements for a $20 \%$ voltage dip

When loads are started as specified by the loads report.

Largest actual voltage dip is $15.0 \%$

N O T E: When the available KW's used exceeds $85 \%$, please read the following three notes before you specify this equipment:

1. During actual operation, the sequence of load application may vary from the data you input.

2. If motor code 'Tetters were assumed, actual motor starting could vary from the output data.

3. Reserve capacity for abnormal conditions or expansion is limited.

Kohler Company offers the full line of generator sets and accessories as well as other equipment which may be required for this application SUCH AS:

- Automatic Transfer Switches

- Bypass Isolation Switches

- Synchronizing Switchgear

- Weather Proof Housings

If you have any questions regarding this application call your local distributor or the Kohler Company Generator Division, Applications Engineering Department, at 414-565-3381.

HNF-2487, Rev. 0

Page B-15 
$03 / 18 / 94$

ER4289 STANDBY GENERATOR

DATA FOR MODEL - 200ROZD

KOHLER GENERATORS

03/18/94 08:20:09

\section{LOADS REPORT}

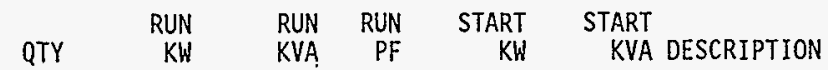

STEP - 1

Loaded $100.00 \mathrm{hp}$ code $\mathrm{F}$ motor

\begin{tabular}{|c|c|c|c|c|c|}
\hline 0 & $\begin{array}{r}\text { code } \\
84.00\end{array}$ & $\begin{array}{l}\text { totor } \\
105.00\end{array}$ & 0.80 & 126.00 & 420.00 High Pressure Pump \\
\hline TEP TOTAL - & 8 & & 0.80 & 126.00 & V. DIP \\
\hline
\end{tabular}

Loaded 12.50 hp code G motor

$\begin{array}{llllll} & 10.90 & 13.63 & 0.80 & 35.31 & 74.38 \text { Hyd. Pumps }\end{array}$

Miscellaneous Load ${ }_{1} 75.00 \quad 75.00 \quad 1.00 \quad 75.00 \quad 75.00$ Instrument Traiter Load

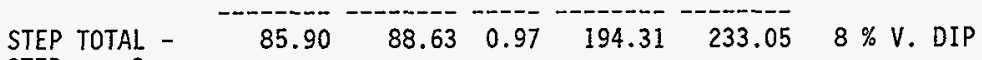

STEP - 3

Loaded $10.00 \mathrm{hp}$ code $\mathrm{G}$ motor

\begin{tabular}{|c|c|c|c|c|c|c|}
\hline 1 & 8.80 & $11.00^{\circ}$ & 0.80 & 28.54 & 59.50 & Strongback Hyd. Pp \\
\hline STEP TOTAL - & 8.80 & 11.00 & 0.80 & 198.44 & 241.27 & $9 \%$ V. DIP \\
\hline TOTALS & 178.70 & 204.63 & 0.87 & & & \\
\hline
\end{tabular}

HNF-2487, Rev. 0

Page B-16 
HNF-2487, Rev. 0

W320-31-003

Feeder Cable Sizes \& \% Voltage Drop

$c-i$ 
This sheat shows the status and description of the attachod Dosign Analysis shoots.

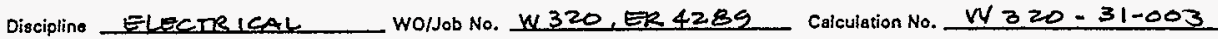
Project No. \& Nam DQ EQUPMENT REMOVAL PROLECT

Csiculation Itom FEFDER CABLE SIZES \& \% VOLTAGE DROD

These calculations apply to:

Dwo. No. H-2-820749 SHI, SH

Dwğ. No.

Rov. No.

0

Other (Study, CDR)

Rov. No.

The status of these calculations is:

$\square$ Preliminary Calculations

UT Finat Calculations

$\square$ Check Calculations IOn Calculation Dated

[ Void Colculation (Roeson Voided

Incorporstod in Final Drawings?

VYos

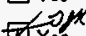

Rov. No.

This calculation verified by independent "check" calculations?

Original and Rovised Calculation Approvals:

\begin{tabular}{|c|c|c|}
\hline & $\begin{array}{c}\text { Rev. } 0 \\
\text { Signatute/Oate }\end{array}$ & $\begin{array}{c}\text { Rov. } 1 \\
\text { Signature/Date }\end{array}$ \\
\hline \multicolumn{3}{|l|}{ Originator } \\
\hline \multicolumn{3}{|l|}{ Checked by } \\
\hline \multicolumn{3}{|l|}{ Approved by } \\
\hline $\begin{array}{l}\text { Chocked Against } \\
\text { Approvod Vondor Data }\end{array}$ & & \\
\hline
\end{tabular}

INDEX
HNF-2487, Rev. 0

Page C-1

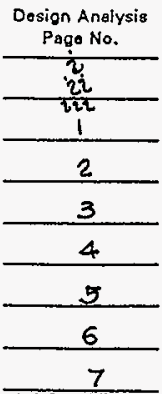

APPENDICES

A-1 THRU A-4
INDEX

Description

CALCULATION CROSS INDEX

DESICA VERाFLATION SCREENTINS CRITERA OQJECTIVE, DATA

ASSUMPTIONS, METHEDS TO BE USED, REF, CALCS. (HPPT LOAD) CALCS. HPPT LOAD (OONT.), IT LOAD, STB TRAILER LOAD CONSTRUCILN IRAULER LOAD DERSTED AMPACITIES OF TYPE MC CABLES, TOTAL ER LOAD CABLE TATA, \% VOLTAGE DBDP, MAXIMUM CABLE LENGTHS FINDINGS \& CONCLUSIONS

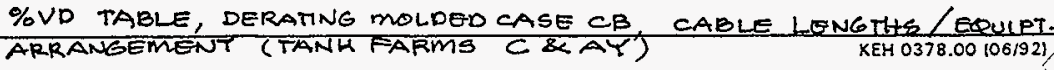




\section{DESIGN VERIFICATION SCREENING CRITERIA}

Project/Document No. W320/W320-31.003

When the design or design change affects hardware, formal design verification must be performed if one or more of the following questions must be answered affirmatively (YES).

YES NO

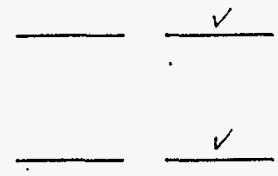

1. Does the design or desion change involved meet the established criteria to be considered Safety Class 1 ?

2. Does this design or design change cause or permit changes to Safety Class 1 instrument or alarm setpoints outside of previously approved operational limits?

3. Does this design or design change significantly affect the nuclear or environmental safety consequences of a malfunction or failure of the structure, system, or component?

4. Does this design or design change involve or change design that has previously undergone formal design verification?
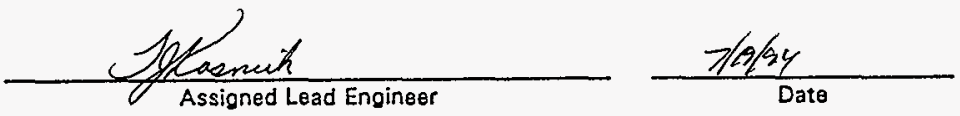

Responsible Discipline Manager

Date

Original Design Package Distribution:

Project Manager

Design Verification Officer

Engineering Document Control
HNF-2487, Rev. 0

Page C-3
Design Change Distribution:

Attach to Engineering Change Notice 


\section{KAISER ENGINEERS} HANFORD

S

Subject FEEDER CABLE SIZES
\& \% VOLTAE DROP

I OBJECTUE

\section{DATA:}

ASSUMPTIONS: INFO
DESIGN ANALYSIS
Calc. No. $W 320-31-003$

Revision

Page No.

WO/Job No. ER 4289

Client WHC WO/Job No. ER 4289

Location TANK FARM $C$ ZOO EAST AREARevised Checked $5 / 27 / 94$ By Dan fareon

DETERMINE THE FEEDER CABLE SIZE BASEDION 1993 NEC AMOACIT AND VOLTAGE DROP REQUIREMENTS THE FEEDER VOLDAGE DROP SHAL NOT BE MORO THAN $2 \%$ OF THE NOMNAL $400 \mathrm{VAC}$,OR $240 \mathrm{VAC}$ FOR THE CONSTRUCTION TRAILER SERUICES.

EQUIPMENT REMOUAL WILL BE DONE IN TWO WEEKS IN AUSUST BEFORE THE SLULCING PHASE, FAST INSTALATION OF ER TRALERS AND EQUIOMENTI NECESSITATES THE USE OF TEMPORARY SERVICE WIRINO METHOD THAT IS QULKLY AND EASILY INSTALED ANO REMOVED.

HOH PRESSLRE PUMP AND CONTROL TRAILERS CDESIGNED B. WHC) ARE PROV10EO WITH 150 A 30 O 460 Y CIRCUIT BREAKERS AND $200 A$, 3P DISCONNECT SW. WITH 2OOA EUSE AMBIENT TEMPERATURE $=115^{\circ} \mathrm{F}$ (AUSUST)

$3 / C$ COPPER TTPE MC CABLE WITH GROUNO LAPROVEO FOR DIRECT BUIRIAL INSTALCATION IS USEO

DEMAND CURRENT WILL BE CB RATING AS GIVEN BY WHC FOR INSTRUMONT IRALER \& HIGH PRESSURE PUMP IRAILER DEMANO LURPENT FOR STRONG BACK TRALER IS ASSUMEO 5OA. (THS FIGURE WWL BE CHECKED AGAINST VENDOR'S

THE FEEOER EABLE AMPACITIES FOR DRY $/ 90^{\circ}$ WWLL BE HNF-2487, Rev 0 ... DERATED FOR USE IN I15 F AMBIENT TEMP.

Page C-4 
KAISER ENGINEERS

HANFORD

Client WHC

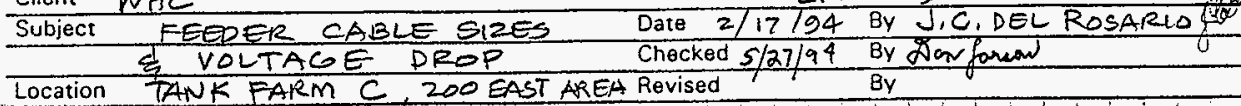

\section{Assomitons}

NO DEMAND EACTORS WLLBE APPUEO ON TRAUER LOAOS. $200 A$ FUSES WLLLLMT HPPT VE CONTROLLERIINPUT STARTING EUIRKENT AND ISOALB WILL LIMIT MOTOK RUNNING CURRENT. VE CONTROLLER IS TO"SOFTSTRRT"THE ASSUME $1 H O=1 K V A$ (SEEP3) AT IT TRALER WLTH $2-25 \mathrm{kVA} 480 \mathrm{~V}$ IQ TRANSFORIFR ASSUME: $25 \mathrm{KVA}, 480 \mathrm{VI} / \not$ LOAD ON UNLOADEO PHASE TS BALANCE THE LOADS. ACROSS THE THREE PHASES. ASSUME SOA MAXIMUM DEMAND ON THE SBT ON P3 D. METTOD TO BE USED MANUAL OALCULATLON

E REFERENCES/SOURCES

1. 1993 NEC NFPA I 70

2. WESTISITOUSE CET. $55=00001901-1992$

3 WEST. TRALER DWG H H 4. HIOH PRESSURE PUMO HP RATING \& PROTECTION

\section{CALCULATIONS}

\section{PETERMINE TRALER LOADS}

a) HiH PRESSURE FUMP TRALER LOAD EURNISHED BY WHC I (REF. L)

$1-1001 \mathrm{HP} 460 \mathrm{~V}$ PUMP motor WUTH VF CONTROLLER PROTECTED BU $150 \mathrm{~A}$ 46OV $3 P C B$

2.2HPmOTORS@34AEA H6O WLTHCB LONE $z H P$ motok OPERATEPAT A TIME)

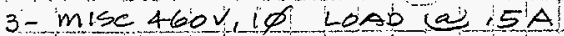

HNF-2487, Rev. 0 Page C-5

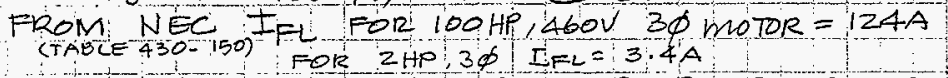
TOTAL IEL FROM HKA PRESSURE PUMF TRALER $=124+3.4 \mathrm{~A}+5=127.9 \mathrm{~A}$ HPP TRALLER HCLUDING PROTECTION - BY WESTINGHOO CAT SHEETS OF VF OONTROLLER WERE REQUESTEO FROM MR JACK DOWEL OF WESTING +OUSE. 
KAISER ENGINEERS

HANFORD

DESIGN ANALYSIS
Calc. No. W32s.31-003

Revision $\frac{0}{3 \text { of } 7}$

Client WHC WO/JOb NO. ER 4289

Subject FEEDER CABLE SIZES Date 2/17/94 BY J.G DEL ROSARCLYW

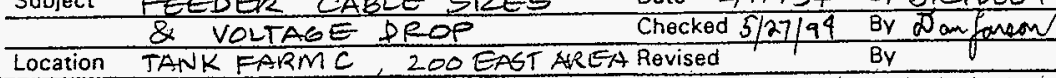

BRANCH CIRCULT PROTEUTION INSTAUED BY PUMP VENDOR $150 A C C B$ WITH $200 A$ FUSED DISCONWECT SWITCH.

ITOTAL + .25 I LARGEST MOTOR $=127.9+0.25 \times 124$

$$
=127.9+31=158.9 \mathrm{~A}
$$

USE 410 AWB, DERATE AMPACITTQ $120^{\circ}=-213 \mathrm{~A}$ SEE $\$$ H 5

FEODER CB - 225AT DERATEO FOR $122^{\circ} \mathrm{F}$ ( $50^{\circ} \mathrm{C}$ ) AMBIENT TEMP = 2054 SEE RESZ, P263

(LSED WEST TYPE FE \& JB BREAKERS FOR CALCS)

b. INSTROMENT TRALER (IT): 150 A 3 P $480 \mathrm{~V}$ (BY VENDOR) ODESIGNED BY WHE-REE 30

$3 \phi$ LOAD - - -12.5HP PUMP (ASSUME IHP $=1 \mathrm{KV}$ - 12ISKVA CINFO FROM WESTINGHOUS (IACK DOWELL)

2 25 KVA IQ $480-120 / 240 V$ TRANSF $3 \times 25=75$ KIA ASSUML BACANCES $\phi L O$

TOTA CONNECTED LOAD $=75+12.5=87.5 \mathrm{k}$

$E_{F L}=8 T I 5 \mathrm{kVA} / \sqrt{3} \times 148 \mathrm{KV}=105 \mathrm{~A}$

USE $2 / 0$ AWG. DDERATED AMP $=160 \mathrm{~A}$

USE 175 AT F225 MOLDED CASEICB, DERATED AMP $=159$. @1220 AmBIENT TEMP, REFZ P 263

C. STRONG BACK TRALLER:

2 - $10 \mathrm{HP}, 3 \phi$; $460 \mathrm{~V}$ PUMPS: OPERATING ALTER

HNF-2487, Rev. 0 NATELY: ASSUME: 50 A mAX LOAD

Page C-6 USE $3 / C$ \#2 AWG - DERATED AMPACITY

Q.1220 $=107 \mathrm{~A}$, USE 100 AT FEEOER CB, DERATED@1220\% TO 94A REF 2 Q 263 
KAISER ENGINEERS

DESIGN ANALYSIS
Calc. No. W/320-31-003

Revision 0

Page No. 4 of 7

Client WHC

WO/Job No. ER 4285
Date 2/17/94 BY IC. PEL ROSARLO \& VOLTACE DROP Checked $5 / 27 / 94$ BY Don far sow

Location

d.) CONSTRUCTION TRAILER POWER

BASED ON AVERAGE ESTIMATED CONN LOAD OF $15 \mathrm{KVA}$ PER TRAILER:

LOAD PER TRAILER = 15 KVA

TOTAL LOAD FOR 3 TRALERS $=45 \mathrm{KVA}$

ASSUME $100 \%$ DEMAND FACTOR.

ESTIMATED KRA DEMAND $=45 \mathrm{KVA}$

USE SOKVA, ID TRANSFORMER

IR $=104 A M P S$

$1 / 25 I_{P R T}=130 \mathrm{~A}, \quad$ USE $3 / \mathrm{C}$ VANG, $90^{\circ} \mathrm{C}$ INS AMPACITY@120E $=139 A$ SEE PAGE 5

USE 150 AA, $2 P$ CB TRANSFORMER PRIMARY CB BERATED AMPACITY@120 $@=138 A-R E F 2 \rho 2$

I RAILER $=\frac{15 \mathrm{KVA}}{.24 \mathrm{KV}}=62.5 \mathrm{~A}$

$=$

1:25 ITRAILER $=78,125 \mathrm{~A}$

USE \# AWE DERATEDAMP.@IZO $=78 \mathrm{~A}$ SEE PAGES

USE $70 \mathrm{~A}, 2 \mathrm{P}, 240 \mathrm{~V}$ SERVICE FEE PER PROTECTION

TOTAL CURRENT RATING OP TRANSF SEC, CBS $=3 \times 70=210$ AMPS

HNF-2487, Rev. 0

Page C-7

TRANSFORMER SEC, EFL $=\frac{50 \mathrm{KVA}}{.24 \mathrm{KV}}=208 \mathrm{~A}$

54-4300-037 KEH-0037.00 106/92 
KAISER ENGINEERS

DESIGN ANALYSIS
Call. No. W/320-31-00:

Revision $\frac{0}{5 \quad \text { of } \quad 7}$

Client WHC WO/Job No. ER 4289

SUbject FEEDER CABLE SIZES Date $2 / 17 / 94$ BY J.C, DEL ROSARIO OW

\& VOLTACE DROP Checked 5/27/94 BV D.E. Sorrow

Location TANK EARM C 200 EAST AREA Revised

DEBATING FACTOR FOR $120^{\circ}$ E AMBIENTITEMP. FOR $60-90^{\circ}$ COPPER CABLE =-82 REF I TABLE 31016

DERATEO AMPACITIES OF 90 C (DEY) COPPER CABLES W/XHHW INS.

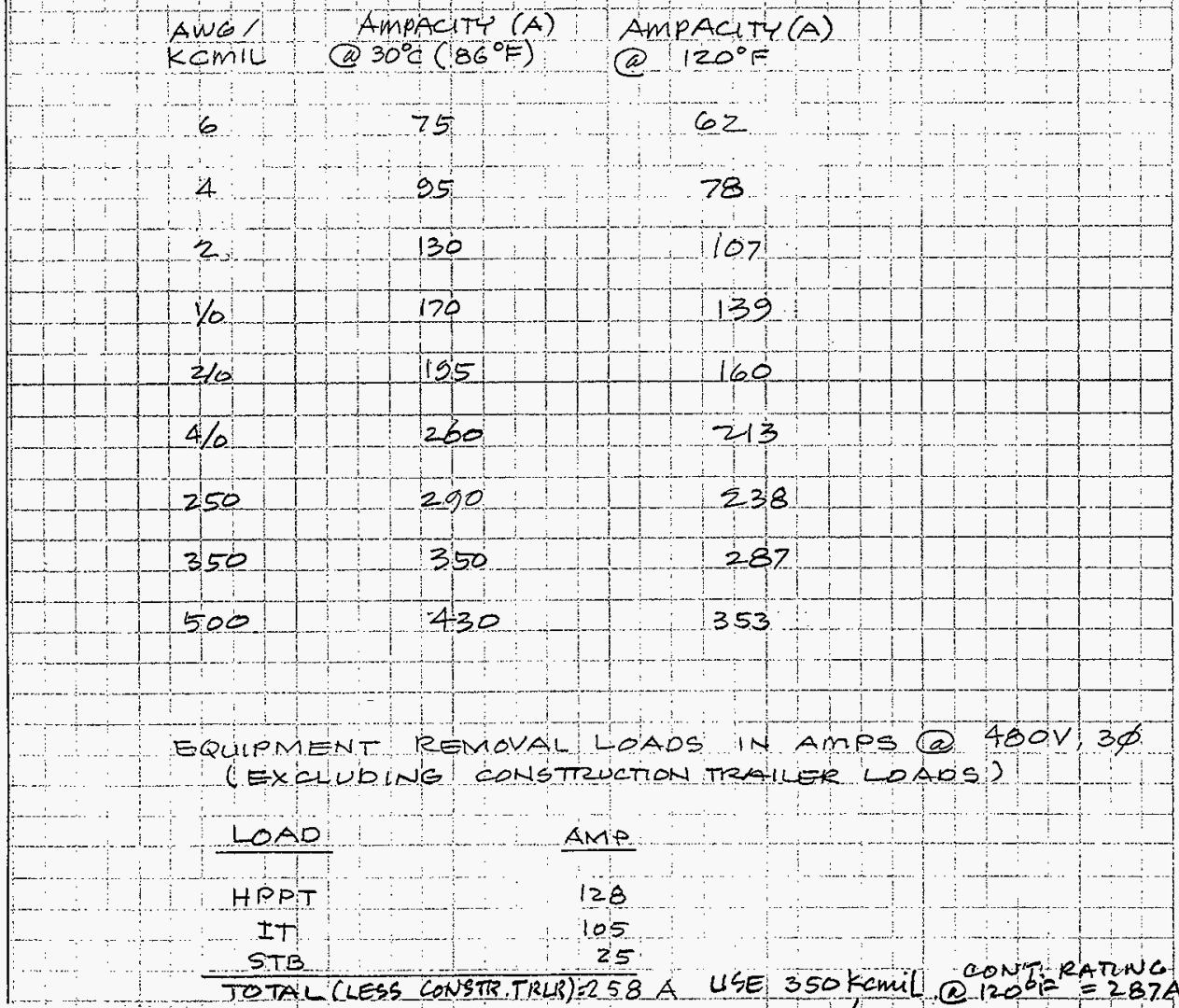

CONSTR. TRIER CONN, LOAD $=50,000 / 480=104 A$

HNF-2487, Rev. 0 - TOTAL CONN LOAD INCLUDING CONSTR TRAILERS $=362 A$

Page C-8 USE $2-350 \mathrm{kcmil} / \phi$ TRANSF SEC FDR TO POP AT TANKFARN CONTINUOUS CURRENT AMPACITY@ (20. $0_{54.4300-037}^{\circ}=574 \mathrm{~A}$ KEH-0037.00 
KAISER ENGINEERS

HANFORD

\section{DESIGN ANALYSIS}

Calc. No. $W=20-31-0 C$

Revision 0

Page No. $\frac{6}{6}$ of .7
Client

WHC

Subject FEEDER CABLE SIZES \& $\%$ VOLTAGE DROP

Location TANK EARA C 200 EAST
WO/Job No. ER, 4289

Date $2 / 17 / 94$ By J.C. DEL ROSARLO Checked 5727/94 Revised By By Dan farron
IASSUME TRANSF. SECONDARYIVOLTAGE AT TERMINAL $=480 \mathrm{~V}$ CABLE DATA: TRANSF EULU LOAD \% IZ = $4.43 \%$ (NOT INCLUDED)

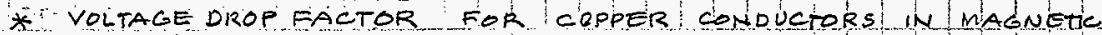

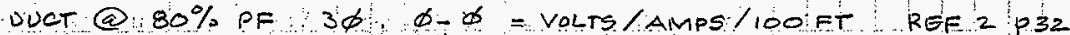

a SEE DWG H-Z- 820749 (COFARM) \& H-Z-820752 (AT-FARM) FOR CABLE LAYE

\begin{tabular}{|c|c|c|c|c|}
\hline & CABLETAG & FROM & 3 To & AMP \\
\hline & $R B_{--}^{-}$ & $-G E N$ & GPW & -2.58 \\
\hline-2 & $S B T-$ & $-P P P-1$ & SBT-IS & $\ldots 25$ \\
\hline 3 & $\because T$ & $-P O P-1$ & IT-TS & $\ldots-105 \ldots$ \\
\hline $4-$ & $\mathrm{HS}$ & $\therefore p$ & HPP-TS & 128 \\
\hline 5 & & HPP-TS & $P P F$ & $-128 \cdots$ \\
\hline & & $-7-7-\frac{1}{5}$ & & 105 \\
\hline & & $-S B F$ & SBF & -25 \\
\hline 8 & $\begin{array}{c}c 106-3011 \\
10.480 v\end{array}$ & $-P D P-1$ & SPT & -104 \\
\hline 9 & $\begin{array}{l}c 106-3 P_{1} 5-1 \\
\mid \phi, 240 \mathrm{~V}\end{array}$ & SPT & LRE & $-62,5$ \\
\hline 10 & $13,8 \mathrm{KVCABLE}$ & $\begin{array}{l}\text { POLE } \\
\text { E2ST }\end{array}$ & $\begin{array}{c}\text { TRANSF } \\
\text { TI }\end{array}$ & \\
\hline
\end{tabular}

YD $\phi-\phi(8)$ $\% \vee D(9)$

$=V D F A C T O R(\gamma)$ $=V D \phi \phi(8)$

\begin{tabular}{|c|c|}
\hline$\$ 12 \theta$ & $6 \angle 1$ \\
\hline Awo & $(F T)$ \\
\hline
\end{tabular}

UD FACTO $\$$. $\phi$ REF 2 \&32

350

60

+2

$22 \%$

$\$ 4 \%$

$4 \%$

$\# 4 / 0$

$+210$

$\$ 2$

$\$ 2$

$\$ 1 \%$

44

\#.

\#2

60

$0102-1.6-33$

\begin{tabular}{l|l|l|}
\hline 0323 & 5 & $1 \%$ \\
\hline & $1 \%$ & \\
\hline
\end{tabular}

$50 \mathrm{KVA}$ CONST.
LOAD $B \times C$

\begin{tabular}{r|r|r|r|}
-60 & 0187 & 1 & $25 \%$ \\
\hline
\end{tabular}

$60.0136 \quad 1.0 \% 2 \%$

MAX.C $2 \%$ RUN \begin{tabular}{rr|r|r|}
40 & 0136 & -8.18 & $-1 \%$
\end{tabular}

$4.460 *$

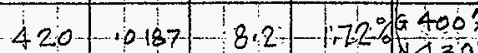

\begin{tabular}{lll:}
420 & $: 82$ \\
\hline
\end{tabular}

$540-0323-4 \div 31$

$-1.90$

1430

\begin{tabular}{|c|c|c|c|c|}
\hline 1 & 57 & & & SEEP \\
\hline 28 & 0.56 & & $4.08 \%$ & \\
\hline & & & $.94 \times 10^{-4}$ & 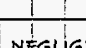 \\
\hline & & & & \\
\hline
\end{tabular}

$L(6)$

100

$100 \%$

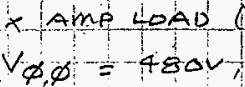

$(4)$

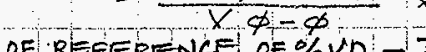

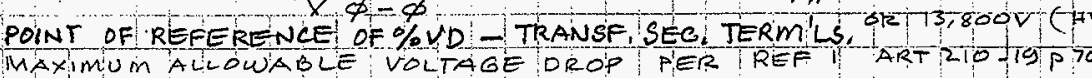
BRANCH - $3 \%$, COMBNED BRANCH \& FEEDER- $5 \%$ NORMA PSWER LOAP ON $-P D P_{1}-1=258^{(1,4)}+50,000 / 480=362$

\% VOLTAGE OROP ON TRANSF SEC FEEDER FROM TRANSF TO PDPEEZ3\% NORMAL POWER EEEOER \%O VOLTASE DROP: WEAE

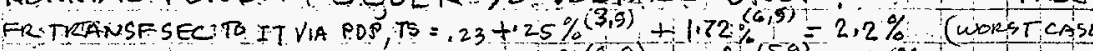
FR TRANSE SEC TD ITPT VIA PDP, TS $=23+2 \%(4,9)+1,2 \%(5,9)=2.13 \%$

FR TKANSF SEC TO SBTVIA: PDP, TS $=123+11(2,9)+.9(7,9)=1.23 \%$ OENERATOR POWER \% FEEOER VOLTAGE DROPS $.33 \%(1,0)+1.70 \% \%$ MAXIMUMICABLE LENGTH FOR MAX PERMISSRBLE $2 \%$ JEEÓ PROP (INLLUUDNG GEN FEEDER \% VD) FOR RUN BET TS \& HPPT: HNF-2487, Rev. $0=-(2.01 .33) \times 480=460^{\circ}$ of $=4 \%$

Page C-9 $128 \times .0136$ 
KAISER ENGINEERS HANFORD

DESIGN ANALYSIS

Talc. No. $\frac{W 320-31.00}{0}$
Revision $\frac{0}{7 \text { of } 7}$

Client $\quad W H C$

Subject FEEDER CABLE SIZES

WO/JOb No. ER 4289

Date $2 / 17 / 94$ By HiC. DEL ROSARLOA Checked $5 / 27 / 94$ By Danfarem

Location TANK FARM C

MAXIMUM I CABLE LENGTH BET IS \&HPPT ON NORMAL POWER

$=\frac{(2.0-23)}{128 \times 0130}, 480=4881$ USE 470

MAXIMUM CABLE LENGTH FOR TRAILER IT ON STBYGEN POWER $2 \%$ LENGTH OE CABLE RUN IN FT FO OM TS TO IT TRADER $=\frac{2.0-.33) 480}{105 \times .0187}=408^{\prime} \quad$ sAY 400 of $2 \%$ ON ON 105.0187 ON POL BET WEN C-PDP-1 ***

$$
=\frac{(20-13) 480}{105 \times 10187}=433^{1} \text { sAY } 430
$$

MAXIMUM CABLE LENGTH FOR SOT CON STOBYGEN PW K ASSUMWR MAXIMUM TOTAL \% VD OF $2 \%$, LESS $1 \%$ Y LENGTH OF CABLE RUN IN INT ROM TS TO TRALERSST

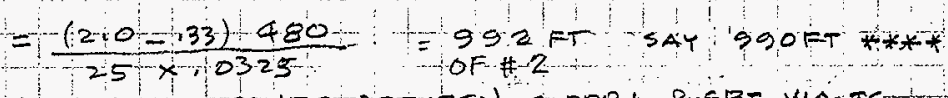

WAX RUN ON NORMA POWER BETWEEN C-PDP- \& SET VATS $=\frac{(2,0-23) 480}{25 \times .0323}=1050 . \mathrm{T}$

THE ABOVE CALCULATIONS LIMIT THE FEEDER DROP TO $2 \%$ MAXIMUM SINCE THE BRANCH CIRCUITS IN THE DRAWERS ARE SHORT THE BRANCH CIRCUIT VOLTAGE PROPS ARE NEGLiGIBLE $5 \%$ comBINED FEEDER AND BRANCH CIRCUIT VOLTAGE PROP NL NOT BE EXCEEDED EVEN ON STANDBY GENERATOR POWER.

TRANSFORMER SECONDARY FEEDER TO OPPP-I $2-350 \mathrm{kmLl} / \phi, \quad z$ per $1001=2005 \Omega$ FEEDER LENGTH $=60^{\circ}$

$$
\begin{aligned}
& \text { LOAD }=258^{(1,4)+50,000 / 480=362 \mathrm{~A}} \\
& \% V D=\frac{362 \times 6 \times \cdot 0051}{480} \equiv .23 \%
\end{aligned}
$$
IS: NEGLIGIBLE.

$\%$ VOLTAGE DROP AT FURTHEST CONS TRALEE ASSUMING $10 \% \%$ DE FACTOR $=23+.55+4.08=4.86 \%$ LESS THAN $5 \%$

FINDINGS AND CONCLUSIONS THE PRECEDING CALCULATING SHED THAT THE SIZES OE THE $3 /$ TYPE MU CABLES ARE ADEQU HNF-2487, Rev. O FOR THE LOAOS AND THE CALQULATEO $\%$ V VOLTAGE Page $\mathrm{C}-10$ DROPS ARE WITH IN THE MES PERMISSIBLE LM IT 


\section{Voltage Drop}

Voltage Drop

Volts per Amp per 100 Feet; 3-Phase, Phase-to-Phase

Copper Conductors

\begin{tabular}{|c|c|c|c|c|c|c|c|c|c|c|}
\hline \multirow{3}{*}{$\begin{array}{c}\text { Conductor } \\
\text { Size } \\
\text { AWG } \\
\text { or MCM }\end{array}$} & \multicolumn{5}{|c|}{$\begin{array}{c}\text { Mognetic Conduit } \\
\text { (Steel) }\end{array}$} & \multicolumn{5}{|c|}{$\begin{array}{l}\text { Nonmagnetic Conduit } \\
\text { (Aluminum or Nonmetallic) }\end{array}$} \\
\hline & \multicolumn{5}{|c|}{ Losd Power Foctor, $\%$} & \multicolumn{5}{|c|}{ Losd Power Factor, $\%$} \\
\hline & 60 & 70 & 80 & 90 & 100 & 60 & 70 & 80 & 90 & $\overline{1}$ \\
\hline $\begin{array}{r}14 \\
12 \\
10 \\
8 \\
\end{array}$ & $\begin{array}{r}.3390 \\
.2170 \\
.1390 \\
.0905 \\
\end{array}$ & $\begin{array}{r}.3910 \\
.2490 \\
.1590 \\
.1030 \\
\end{array}$ & $\begin{array}{r}.4430 \\
.2810 \\
.1790 \\
.1150 \\
\end{array}$ & $\begin{array}{l}.4940 \\
.3130 \\
.1980 \\
.1260 \\
\end{array}$ & $\begin{array}{l}.5410 \\
.3410 \\
.2150 \\
.1350 \\
\end{array}$ & $\begin{array}{l}.3370 \\
.2150 \\
.1370 \\
.0888 \\
\end{array}$ & $\begin{array}{l}.3900 \\
.2480 \\
.1580 \\
.1010 \\
\end{array}$ & $\begin{array}{l}.4410 \\
.2800 \\
.1780 \\
.1340 \\
\end{array}$ & $\begin{array}{l}.4930 \\
.3120 \\
.1970 \\
.1250 \\
\end{array}$ & $\begin{array}{l}.5 \\
.3 \\
.2 \\
.1: \\
\end{array}$ \\
\hline $\begin{array}{r}6 \\
\rightarrow-4 \\
-2 \\
\end{array}$ & $\begin{array}{l}.0595 \\
.0399 \\
.0275 \\
.0233 \\
\end{array}$ & $\begin{array}{l}.0670 \\
.0443 \\
.0300 \\
.0251 \\
\end{array}$ & $\begin{array}{r}.07421 \\
-0485 \\
-0323 \\
.0267 \\
\end{array}$ & $\begin{array}{r}.0809 \\
.0522 \\
.0342 \\
0.0279 \\
\end{array}$ & $\begin{array}{l}.0850 \\
.0534 \\
.0336 \\
.0267 \\
\end{array}$ & $\begin{array}{l}.0579 \\
.0384 \\
.0260 \\
.0298 \\
\end{array}$ & $\begin{array}{l}.0656 \\
.0430 \\
.0287 \\
.0238 \\
\end{array}$ & $\begin{array}{l}.0730 \\
.0473 \\
.0312 \\
.0256 \\
\end{array}$ & $\begin{array}{l}.0900 \\
.0513 \\
.0333 \\
.0270 \\
\end{array}$ & $\begin{array}{l}.04 \\
.0: \\
.0 \\
.0\end{array}$ \\
\hline $\begin{array}{r}\rightarrow 110 \\
+210 \\
310 \\
-4 / 0 \\
\end{array}$ & $\begin{array}{l}.0198 \\
.0171 \\
.0148 \\
.0130 \\
\end{array}$ & $\begin{array}{l}.0211 \\
.0180 \\
.0154 \\
.0134 \\
\end{array}$ & $\begin{array}{r}\rightarrow 0203 \\
\rightarrow 0187 \\
.0158 \\
-.0136 \\
\end{array}$ & $\begin{array}{r}00229 \\
.0190 \\
.0158 \\
.0133 \\
\end{array}$ & $\begin{array}{l}.0213 \\
.0170 \\
.0136 \\
.0109 \\
\end{array}$ & $\begin{array}{l}.0183 \\
.0156 \\
.0134 \\
.0116 \\
\end{array}$ & $\begin{array}{l}.0198 \\
.0167 \\
.0141 \\
.0121 \\
\end{array}$ & $\begin{array}{l}.0211 \\
.0176 \\
.0147 \\
.0124\end{array}$ & $\begin{array}{l}.0220 \\
.0181 \\
.0149 \\
.0124 \\
\end{array}$ & $\begin{array}{l}.0: \\
.0 \\
.0 \\
.01\end{array}$ \\
\hline $\begin{array}{r}250 \\
300 \\
-350 \\
500 \\
\end{array}$ & $\begin{array}{l}.0122 \\
.0111 \\
.0104 \\
.0100 \\
\end{array}$ & $\begin{array}{l}.0124 \\
.0112 \\
.0104 \\
.0091 \\
\end{array}$ & $\begin{array}{r}.0124 \\
.0111 \\
-0102 \\
.0087 \\
\end{array}$ & $\begin{array}{l}.0120 \\
.0106 \\
.0096 \\
.0080 \\
\end{array}$ & $\begin{array}{l}.0094 \\
.0080 \\
.0069 \\
.0053 \\
\end{array}$ & $\begin{array}{l}.0107 \\
.0097 \\
.0090 \\
.0078 \\
\end{array}$ & $\begin{array}{l}.0111 \\
.0099 \\
.0091 \\
.0077 \\
\end{array}$ & $\begin{array}{l}.0112 \\
.0098 \\
.0091 \\
.0075\end{array}$ & $\begin{array}{l}.0110 \\
.0096 \\
.0087 \\
.0070 \\
\end{array}$ & $\begin{array}{l}.06 \\
.06 \\
.06 \\
.06\end{array}$ \\
\hline $\begin{array}{r}600 \\
750 \\
1000 \\
\end{array}$ & $\begin{array}{l}.0088 \\
.0084 \\
.0080 \\
\end{array}$ & $\begin{array}{l}.0086 \\
.0081 \\
.0077 \\
\end{array}$ & $\begin{array}{l}.0082 \\
.0077 \\
.0072 \\
\end{array}$ & $\begin{array}{l}.0074 \\
.0069 \\
.0063 \\
\end{array}$ & $\begin{array}{l}.0046 \\
.0040 \\
.0035 \\
\end{array}$ & $\begin{array}{l}.0074 \\
.0069 \\
.0064 \\
\end{array}$ & $\begin{array}{l}.0072 \\
.0067 \\
.0062 \\
\end{array}$ & $\begin{array}{l}.0070 \\
.0054 \\
.0058 \\
\end{array}$ & $\begin{array}{l}.0064 \\
.0058 \\
.0052 \\
\end{array}$ & $\begin{array}{l}.06 \\
.00 \\
.00\end{array}$ \\
\hline
\end{tabular}

Aluminum Conductors

\begin{tabular}{|c|c|c|c|c|c|c|c|c|c|c|}
\hline \multirow{3}{*}{$\begin{array}{c}\text { Conductor } \\
\text { \$ize } \\
\text { AWG } \\
\text { or MCM }\end{array}$} & \multicolumn{5}{|c|}{$\begin{array}{l}\text { Magnetic Conduit } \\
\text { (Steel] }\end{array}$} & \multicolumn{5}{|c|}{$\begin{array}{l}\text { Nonmagnetic Conduit } \\
\text { (Aluminum or Nonmetallic) }\end{array}$} \\
\hline & \multicolumn{5}{|c|}{ Losd Power Factor, \% } & \multicolumn{5}{|c|}{ Load Power Factor, $\%$} \\
\hline & 60 & 70 & 80 & 90 & 100 & 60 & 70 & 80 & 90 & 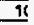 \\
\hline $\begin{array}{r}12 \\
10 \\
8 \\
\end{array}$ & $\begin{array}{r}.3296 \\
.2133 \\
.1305 \\
\end{array}$ & $\begin{array}{r}.3811 \\
.2429 \\
.1552 \\
\end{array}$ & $\begin{array}{l}.4349 \\
.2741 \\
.1758 \\
\end{array}$ & $\begin{array}{r}4848 \\
.3180 \\
.1951 \\
\end{array}$ & $\begin{array}{r}.5330 \\
.3363 \\
.2706 \\
\end{array}$ & $\begin{array}{r}.3312 \\
.2090 \\
.1286 \\
\end{array}$ & $\begin{array}{r}.3802 \\
.2410 \\
.1534 \\
\end{array}$ & $\begin{array}{r}.4328 \\
.2740 \\
.1745 \\
\end{array}$ & $\begin{array}{r}.4848 \\
.3052 \\
.1933 \\
\end{array}$ & $\begin{array}{r}.53 \\
.33 \\
.21 \\
\end{array}$ \\
\hline $\begin{array}{l}6 \\
4 \\
2 \\
1 \\
\end{array}$ & $\begin{array}{r}.0898 \\
.0595 \\
.0403 \\
.0332 \\
\end{array}$ & $\begin{array}{r}.1018 \\
.0660 \\
.0443 \\
.0357 \\
\end{array}$ & $\begin{array}{l}.1142 \\
.0747 \\
.0483 \\
.0396 \\
\end{array}$ & $\begin{array}{r}.1254 \\
.0809 \\
.0523 \\
.0423 \\
\end{array}$ & $\begin{array}{l}.1349 \\
.0862 \\
.0535 \\
.0428 \\
\end{array}$ & $\begin{array}{r}.0887 \\
.0583 \\
.0389 \\
.0318 \\
\end{array}$ & $\begin{array}{l}.1011 \\
.0654 \\
.0435 \\
.0349\end{array}$ & $\begin{array}{l}.1127 \\
.0719 \\
.0473 \\
.0391 \\
\end{array}$ & $\begin{array}{l}.1249 \\
.0800 \\
.0514 \\
.0411 \\
\end{array}$ & $\begin{array}{l}.13 \\
.08 \\
.05 \\
.04 \\
\end{array}$ \\
\hline $\begin{array}{l}110 \\
210 \\
310 \\
410 \\
\end{array}$ & $\begin{array}{l}.0286 \\
.0234 \\
.0209 \\
.0172 \\
\end{array}$ & $\begin{array}{l}.0305 \\
.0246 \\
.0220 \\
.0174 \\
\end{array}$ & $\begin{array}{l}.0334 \\
.0275 \\
.0234 \\
.0179 \\
\end{array}$ & $\begin{array}{r}.0350 \\
.0284 \\
.0241 \\
.0977 \\
\end{array}$ & $\begin{array}{l}.0341 \\
.0274 \\
.0217 \\
.0170 \\
\end{array}$ & $\begin{array}{l}.0263 \\
.0227 \\
.0160 \\
.0152 \\
\end{array}$ & $\begin{array}{l}.0287 \\
.0244 \\
.0171 \\
.0159 \\
\end{array}$ & $\begin{array}{l}.0322 \\
.0264 \\
.0218 \\
.0171 \\
\end{array}$ & $\begin{array}{l}.0337 \\
.0274 \\
.0233 \\
.0179 \\
\end{array}$ & $\begin{array}{l}.03 \\
.02 \\
.02 \\
.01 \\
\end{array}$ \\
\hline $\begin{array}{l}250 \\
300 \\
350 \\
500 \\
\end{array}$ & $\begin{array}{r}.0158 \\
.0137 \\
.0130 \\
.0112 \\
\end{array}$ & $\begin{array}{r}.0163 \\
.0139 \\
.0133 \\
.0111 \\
\end{array}$ & $\begin{array}{l}.0162 \\
.0143 \\
.0128 \\
.0114 \\
\end{array}$ & $\begin{array}{l}.0159 \\
.0144 \\
.0131 \\
.0099 \\
\end{array}$ & $\begin{array}{l}.0145 \\
.0122 \\
.0100 \\
.0076 \\
\end{array}$ & $\begin{array}{l}.0138 \\
.0126 \\
.0122 \\
.0093 \\
\end{array}$ & $\begin{array}{l}.0144 \\
.0128 \\
.0123 \\
.0094 \\
\end{array}$ & $\begin{array}{l}.0147 \\
.0133 \\
.0119 \\
.0094 \\
\end{array}$ & $\begin{array}{l}.0155 \\
.0132 \\
.0120 \\
.0091\end{array}$ & $\begin{array}{l}.01 \\
.01 \\
.01 \\
.00 \\
\end{array}$ \\
\hline $\begin{array}{r}600 \\
750 \\
1000 \\
\end{array}$ & $\begin{array}{l}.0101 \\
.0095 \\
.0085 \\
\end{array}$ & $\begin{array}{l}.0106 \\
.0094 \\
.0082 \\
\end{array}$ & $\begin{array}{r}.0097 \\
.0090 \\
.0078 \\
\end{array}$ & $\begin{array}{r}.0090 \\
.0084 \\
.0071 \\
\end{array}$ & $\begin{array}{r}.0063 \\
.0056 \\
.0043 \\
\end{array}$ & $\begin{array}{l}.0084 \\
.0081 \\
.0069 \\
\end{array}$ & $\begin{array}{r}.0085 \\
.0080 \\
.0068 \\
\end{array}$ & $\begin{array}{l}.0085 \\
.0078 \\
.0065 \\
\end{array}$ & $\begin{array}{r}.0081 \\
.0072 \\
.0058 \\
\end{array}$ & $\begin{array}{l}.00 \\
.00 \\
.00 \\
\end{array}$ \\
\hline
\end{tabular}

$$
\begin{aligned}
& \text { SEE IEEE REDBOOK } P Q S \\
& \text { FOR IQ MULTFLIER }(1.18)
\end{aligned}
$$

HNF-2487, Rev. 0

Page C-11 


\begin{tabular}{|l|l|l|}
\hline \multicolumn{2}{|l|}{ Ampere Rating At } \\
\hline $25^{\circ} \mathrm{C}$ & $50^{\circ} \mathrm{C}$ & $60^{\circ} \mathrm{C}$ \\
$\left(77^{\circ} \mathrm{F}\right)$ & $\left(122^{\circ} \mathrm{F}\right)$ & $\left(140^{\circ} \mathrm{F}\right)$ \\
\hline
\end{tabular}

\begin{tabular}{|l|l|l|l|}
\hline Breaker & Ampere Rating At \\
\cline { 2 - 4 } & & \\
Ampere & $25^{\circ} \mathrm{C}$ & $50^{\circ} \mathrm{C}$ & $60^{\circ} \mathrm{C}$ \\
Rating & $\left(77^{\circ} \mathrm{F}\right)$ & $\left(122^{\circ} \mathrm{F}\right)$ & $\left(140^{\circ} \mathrm{F}\right)$ \\
\hline at $40^{\circ} \mathrm{C}$ & & &
\end{tabular}

Molded Case Breakers for Application on Resistance Welding Circuits

Short circuit protection for resistance welding devices can be obtained by properly applying instantaneous trip molded case circuit breakers. These breakers permit normally high welding currents, but trip

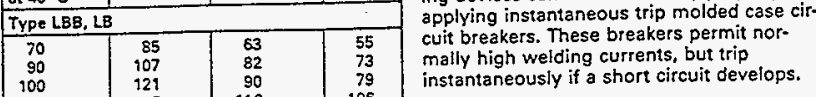

These breakers include standard molded case circuit breaker features such as tripfree operation, dead front and single phase protection. Because the breakers are resistible after tipping, replacement costs and downtime are minimized.

The proper breaker can be selected by using the instantaneous trip range tables (Table $L$ ) using the "during- weld" amperes and \% duty cycle.

Duty Cycle is based on the one minute averaging time of the breaker, and can be determined as follows:

\begin{tabular}{|c|c|c|c|c|c|}
\hline \multicolumn{4}{|c|}{ Trpe MA } & \multirow{2}{*}{\multicolumn{2}{|c|}{ mined as follows: }} \\
\hline 125 & 138 & $\begin{array}{l}115 \\
139\end{array}$ & $\begin{array}{l}103 \\
128\end{array}$ & & \\
\hline $\begin{array}{l}150 \\
175\end{array}$ & $\begin{array}{l}165 \\
192 \\
220\end{array}$ & $\begin{array}{l}139 \\
163 \\
186\end{array}$ & $\begin{array}{l}128 \\
151 \\
970\end{array}$ & $\%$ Duty Cycle $=$ & \\
\hline
\end{tabular}

Type FB

\begin{tabular}{|c|c|c|c|}
\hline 15 & 17 & 13 & 11 \\
20 & 22 & 18 & 16 \\
25 & 28 & 23 & 21 \\
30 & 33 & 28 & 26 \\
25 & 39 & 30 & 25 \\
40 & 44 & 37 & 34 \\
50 & 55 & 46 & 42 \\
60 & 66 & 56 & 52 \\
70 & 77 & 65 & 60 \\
90 & 99 & 84 & 78 \\
\hline 100 & 110 & 94 & 87 \\
125 & 137 & 116 & 105 \\
150 & 165 & 138 & 125 \\
\hline
\end{tabular}

\section{Type CA}

\begin{tabular}{|l|l|l|l|}
\hline 125 & 137 & 114 & 100 \\
150 & 165 & 136 & 120 \\
175 & 192 & 159 & 140 \\
200 & 220 & 182 & 160 \\
225 & 247 & 205 & 180 \\
\hline
\end{tabular}

ii. TYpe JB, KB

\begin{tabular}{|c|c|c|c|}
\hline 70 & 79 & 63 & 55 \\
90 & 102 & 81 & 71 \\
100 & 115 & 89 & 76 \\
125 & 140 & 114 & 102 \\
150 & 171 & 134 & 116 \\
175 & 200 & 156 & 134 \\
200 & 230 & 178 & 153 \\
225 & 252 & 205 & 183 \\
250 & 251 & 227 & 201 \\
\hline Type DA & & \\
\hline 250 & 275 & 235 & 220 \\
300 & 330 & 276 & 252 \\
350 & 385 & 325 & 301 \\
400 & 440 & 372 & 340 \\
\hline
\end{tabular}

372

340
200

225

250

300

350

400

450

500

600

700

800

\begin{tabular}{|l|l|l|l|}
\hline TYpe NB & \multicolumn{3}{l|}{} \\
\hline 700 & 770 & 658 & 613 \\
\hline
\end{tabular}

\begin{tabular}{|c|c|c|c|}
\hline 700 & 770 & 658 & 613 \\
800 & 880 & 754 & 704 \\
900 & 990 & 828 & 749 \\
1000 & 1100 & 900 & $\cdot$ \\
1200 & 1320 & 1090 & 825 \\
\hline Type PB & & 1000 \\
\hline
\end{tabular}

\begin{tabular}{|l|l|l|r|}
\hline TYpe PB & \multicolumn{3}{|l|}{} \\
\hline 600 & 660 & 540 & 474 \\
700 & 770 & 630 & 554 \\
800 & 880 & 728 & 632 \\
900 & 990 & 820 & 720 \\
1000 & 1100 & 920 & 800 \\
1100 & 1290 & 1010 & 891 \\
1200 & 1320 & 1115 & 972 \\
1400 & 1540 & 1304 & 1148 \\
1600 & 1760 & 1500 & 1320 \\
1800 & 1980 & 1690 & 1485 \\
2000 & 2200 & 1880 & 9650 \\
2500 & 2750 & 2350 & 2050 \\
3000 & 3300 & 2820 & 2470 \\
\hline
\end{tabular}

HINF-2487, Rev. 0

Page C-12
"During weld" amperes can be obtained from the welder manufacturer, or as follows:

\section{During-weid Amperes = During-weld KVA 1000 Voltage}

interrupting capacity of the breaker should be within the maximum available at the point of application.

For application information on Series $C$ breakers in welding circuits, refer to Westinghouse. 


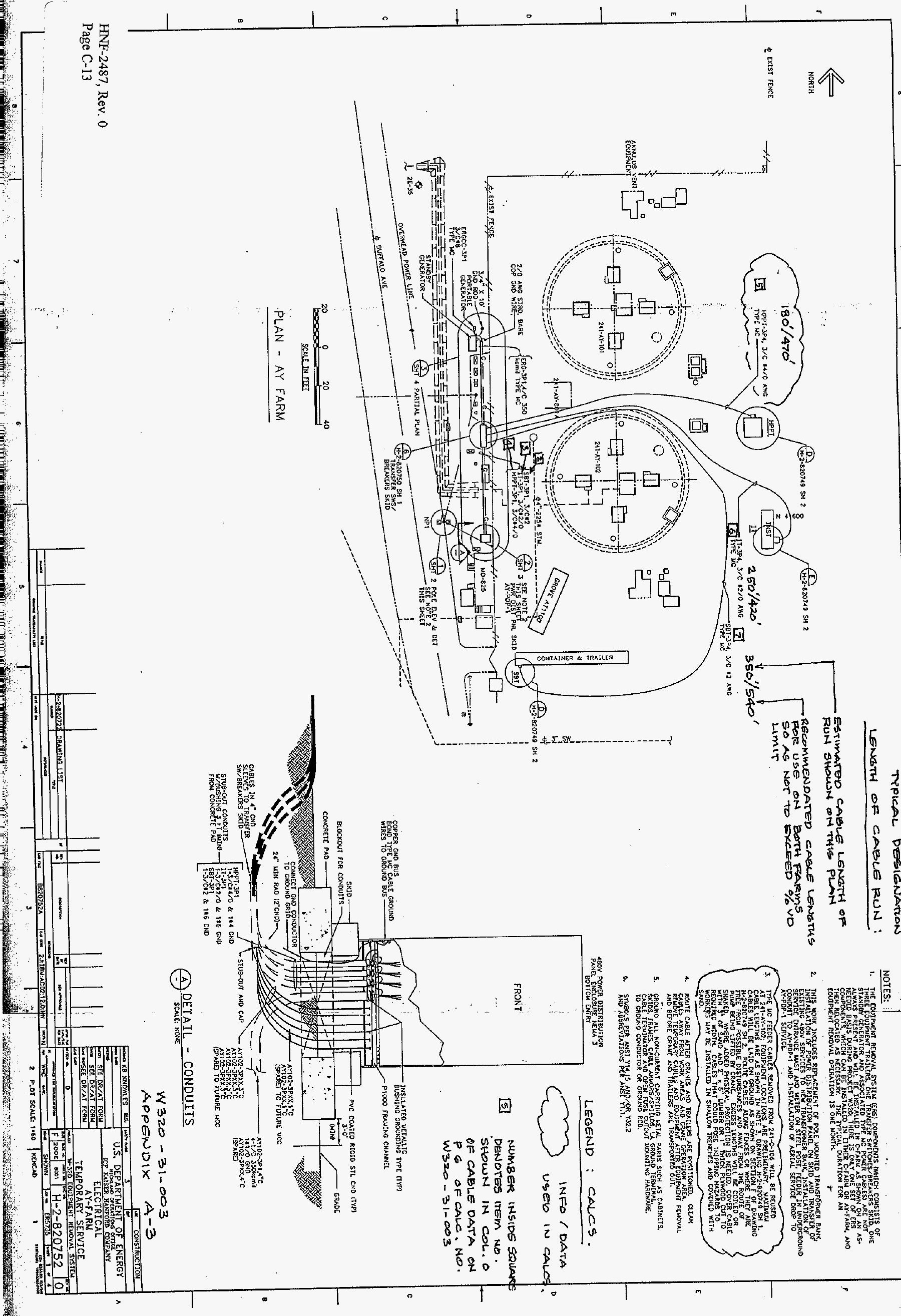




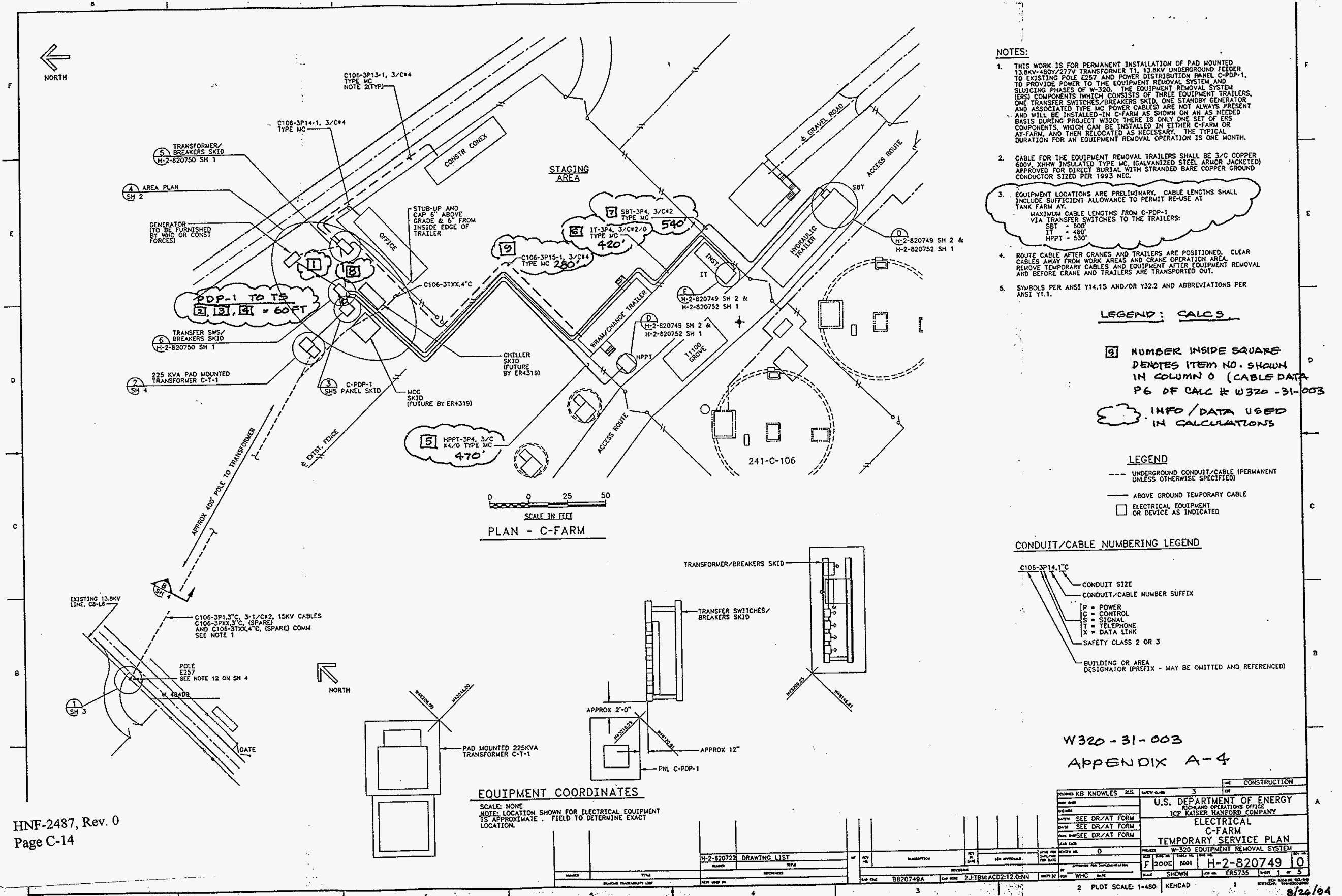


HNF-2487, Rev. 0

W320-31-010

C-106 Demand Load Analysis,

Size Feeders, Voltage Drop and Fault Study 
This sheet shows the status and description of the attached Design Analysis sheets.
Discipline Electrical
wo/Job No. Task Order N09
Calcutation No. W320-31-010

Project No. \& Name W320, Tank 241-C-106 Sluicing

Calculation Item C-106 Demand Load Analysis, Size Feeders, Voltage Drop, and Fault Study

These calculations apply to:

Dwg. No. H-2-818680, Sheet 1

Rev. No. 1

Dwg. No.

Rev. No.

other (Study, CDR)

The status of these calculations is:

[] Prel iminary calculations

[X] Final Calculations

[] Check Calculations (On Calculation Dated)

[] Void Calculation (Reason Voided)

Incorporated in Final Drawings?

[X] Yes [] No

This calculation verified by independent "check" calculations?

Original and Revised Calcutation Approvals:

\begin{tabular}{|l|c|c|c|}
\hline & $\begin{array}{c}\text { Rev. } \\
\text { signature/Date }\end{array}$ \\
\hline originator & $\mathrm{TJ} \mathrm{Kasnick} \mathrm{10/3/95}$ \\
\hline Checked by & $\mathrm{SA} \mathrm{Najjar} \mathrm{10/3/95}$ \\
\hline Approved by & $\mathrm{TJ} \mathrm{Kasnick} \mathrm{10/3/95}$ \\
\hline $\begin{array}{l}\text { Checked Against } \\
\text { Approved Vendor Data }\end{array}$ \\
$\begin{array}{l}\text { Design Analysis } \\
\text { Page No. }\end{array}$
\end{tabular}

\begin{tabular}{|c|l|}
\hline$i$ & Calculation Identification and Index \\
\hline ij & Calculation Cross Index \\
\hline 1 & Objective \\
\hline $2-3$ & Design Inputs \\
\hline 3 & Calculations \\
\hline 4 & Findings and Conclusions \\
\hline A l
\end{tabular}

A-1 to A-7 Attachment A: Study Input Report

B-1 to B-11 Attachment B: Demand Load Analys is

$C-1$ to C-9 Attachment C: Size Feeders and Transformers

D-1 to D-14 Attachment D: Load Flow and Voltage Drop

E-1 to E-36 Attachment E: Short Circuit Ana ys is

F Attachment $\mathrm{F}$ : Drawing $\mathrm{H}-2-818680$ 


\begin{tabular}{|c|c|c|c|c|c|c|c|c|c|c|}
\hline \multicolumn{10}{|c|}{$\begin{array}{l}\text { CALCULATION CROSS INDEX (Typical) } \\
\text { Subject Calculation No.: W320-31-010 }\end{array}$} & \multirow{3}{*}{$\begin{array}{l}\text { Page ii of } \underline{3} \\
\begin{array}{l}\text { Discipline manager's signature and } \\
\text { date indicating gvaluation } \\
\text { complete. }\end{array}\end{array}$} \\
\hline \multirow[t]{2}{*}{$\begin{array}{l}\text { Subject } \\
\text { Calculation } \\
\text { Revislon } \\
\text { No. }\end{array}$} & \multirow[t]{2}{*}{$\begin{array}{l}\text { Superceded } \\
\text { by } \\
\text { Calculation } \\
\text { No. }\end{array}$} & \multicolumn{2}{|c|}{ 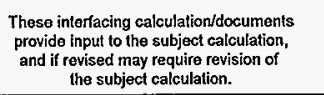 } & \multicolumn{2}{|c|}{$\begin{array}{l}\text { Results and conclusions of the subject } \\
\text { calculation aro used in these interacing } \\
\text { calculalions and/or documents }\end{array}$} & \multicolumn{2}{|c|}{$\begin{array}{l}\text { Doess the output } \\
\text { interface alaluctation } \\
\text { documents require } \\
\text { revision? }\end{array}$} & \multicolumn{2}{|c|}{$\begin{array}{l}\text { Has the output } \\
\text { interfacocacleutationl } \\
\text { documents been } \\
\text { revised? }\end{array}$} & \\
\hline & & Calculation/Document No. & $\begin{array}{c}\text { Revision } \\
\text { No. }\end{array}$ & CalculationDocument No. & $\begin{array}{l}\text { Revision } \\
\text { No. }\end{array}$ & Yes & No & Yes & & \\
\hline 0 & $N / A$ & $H-2-818680$ & 1 & $W 320-31-017$ & 0 & & $x$ & & $x$ & \\
\hline & & & & & & & & & & \\
\hline & & & & & & & & & & \\
\hline & & & & & & & & & & \\
\hline & & & & & & & & & & \\
\hline & & & & & & & & & & \\
\hline & & & & & & & & & & \\
\hline \multirow{2}{*}{\multicolumn{11}{|c|}{ 蛋 }} \\
\hline & & & & & & & & & & \\
\hline 怘 & & & & & & & & & & \\
\hline$\pi$ & & & & & & & & & & \\
\hline$\stackrel{2}{0}$ & & & & & & & & & & \\
\hline & & & & & & & & & & \\
\hline & & & & & & & & & & \\
\hline & & & & & & & & & & \\
\hline & & & & & & & & & & \\
\hline & & & & & & & & & & \\
\hline & & & & & & & & & & \\
\hline
\end{tabular}




\section{Fluor Daniel Northwest}

DESIGN ANALYSIS

client Numatec Hanford Corporation

subject C-106 Demand Load AnaTysis, Size

Feeders, Voltage Drop, Fault Study

Location 200E, C Tank Farm
Revision I

Page No. 1 of 3

1. OBJECTIVE

Determine the size of electrical equipment necessary to support the loads required for Project W320, Tank 241-C-106 Sluicing, at Tank $\mathrm{C}-106$.

\section{DESIGN INPUTS}

2.1 Function Design Criteria for Tank 241-C-106 Waste Retrieval, Project $W-320$, HNF-SD-W320-FDC-001 Rev. 5.

\subsection{Given or Known Data}

The Toads are shown on drawing H-2-818680, Sheet 1, Rev. 1. The size of motors and other equipment data are based on the Project $W-320$ vendor information file \#22668.

2.2.1 The Variable Speed Drives (VSD) are 95\% efficient.

2.2.2 Motor currents, full load amperes (FLA), which are based on the 1993 National Electrical Code (NEC) for the motor nameplate values except for the $250 \mathrm{HP}$ motor which is based on vendor information, are tabulated as follows:

1) In-line Booster Pump (P-1362), at $250 \mathrm{HP}$ (273A), driven by VSD.

2) Submersible (Immersibie) pump (P-1361), at $40 \mathrm{HP}(52 \mathrm{~A})$, driven by a soft starter.

3) Slurry Winch $(\mathrm{W}-1361)$, at $1.5 \mathrm{HP}(2.6 \mathrm{~A})$.

4) Exhaust Skid:

Exhaust Fan (FN-1362), at $7.5 \mathrm{HP}$ (11A), driven by VSD.

Vacuum pumps ( $P-1366$ \& $P-1367)$, at $3 \mathrm{HP}(4.8 \mathrm{~A})$ each.

5) Chiller Skid:

Circ Pumps (P-1364 \& P-1365), at $15 \mathrm{HP}$ (21A) each.

Compressor (R-1361), at 56 KVA.

6) Process Bldg: Recirc Fan (FN-1361), at $7.5 \mathrm{HP}$ (11A).

7) Service Bldg: Compressor (CPR-1361), at $7.5 \mathrm{HP}$ (11A).

8) Sluicer Pkg: Hydrautic Power Unit (SHC-1361), at $3 \mathrm{HP}$ (4.8A)

2.2.3 Other loads:

1) Process Bldg:

HVAC Process Heater (HC-1351), at $30 \mathrm{KW}$.

Space Heaters (UH-1361 \& UH-1362), at $4 \mathrm{KW}$ each.

2) Exhaust Skid: Process Heater $(\mathrm{HC}-1362)$, at $3 \mathrm{KW}$. 


\section{Fluor Daniel Northwest}

\section{DESIGN ANALYSIS}

client Numatec Hanford Corporation subject C-106 Demand Load Analysis, Size Feeders, Voltage Drop, Fault Study Location 200E, C Tank Farm

2.2.4 Mini-Power Panels are loaded to the transformer rating:
1) Cl06-PPl (Process Bldg) is $7.5 \mathrm{KVA}$.
2) C106-PP2 (EES) is 15 KVA.
3) C106-PP3 (Exhaust Skid) is 5 KVA.
4) Cl06-PP4 (Chiller Skid) is 3 KVA.
5) C106-PP5 (Service B1dg) is 5 KVA.
6) Sluicer Package is 0.3 KVA.

\subsection{Assumptions}

2.3.1 Miscellaneous loads:

1) Construction Support Trailers:

Office Trailer, at 16.6 KVA.

WRAM Trailer, at $16.6 \mathrm{KVA}$.

Conex Trailer, at 16.6 KVA.

2.3.2 Transformer impedances used are typical NEMA three phase values.

2.3.3 The utility fault current values are assumed typical values derived from previous projects and data obtained from the utilities department.

2.3.4 Dapper treats a17 loads as three phase loads: feeders for single phase loads must be considered separately (not part of this calculation).

2.4 Method to be used

2.4.1 The FDNW approved (for design use) software: SKM Power Tools, version 3.4.0.1, 1995-1997.

2.5 References/Sources

2.5.1 Drawing H-2-818680, Sheet 1, Rev. 1, Electrica] C-Farm One-Line Diagram

2.5.2 NFPA 70, National Electrical Code (NEC), 1993 edition.

2.5.3 ANSI C2, National Electrical Safety Code, (NESC) 1990.

2.5.4 U.S. Department of Energy (DOE), order DOE 6430.1A, General Design Criteria

2.5.5 Vendor information file \#22668.

HNF-2487, Rev. 0

Page D-4 
DESIGN ANALYSIS

client Numatec Hanford Corporation

subject C-106 Demand Load Analysis, Size

Feeders, Voltage Drop, Fault Study

Location 200E, C Tank Farm
Revision 1

Page No. 3 of 3

wo/Job No. Task Order Nog

Date $9 / 5 / 95$

By TJ Kasnick

checked $10 / 3 / 95$

By SA Najjar

Revised 5/14/98

By Cris Carlson

\section{CALCULATIONS}

3.1 Attachment A: Input Report: This is the feeder, transformer, and generation input data that is used by the studies below.

3.2 Attachment B: Demand Load Analysis: This analysis calculates the total system loads based upon system bus load inputs and summarizes them on a bus by bus basis, including total system load and power factor.

3.3 Attachment C: Size Feeders and Transformers: This analysis provides the NEC Code sized feeders and transformers to meet the minimum load requirements of the Demand Load Analysis Study - Total Connected Load, and the evaluation of a fixed-size feeder to the MCC. Impedance and capacity information for the feeders and transformers were taken directly from the Dapper library, which represents typical values found in the industry. Conduit sizes determined are the minimum size required.

3.4 Attachment D: Load Flow and Voltage Drop: This analysis calculates the exact system operating voltages, loads flows, and system losses.

3.5 Attachment E: Short Circuit Analysis: This analysis calculates the system short circuit fault currents. The calculations are based upon typical Hanford $13.8 \mathrm{Kv}$ utility fault current contributions, plus those from any motors running within the system.

4. FINDINGS AND CONCLUSIONS

4.1 Refer to Attachment C for system equipment sizing. Refer to other Attachments for various design information related to the distribution system equipment.

4.2 Refer to drawing $\mathrm{H}-2-818680$ for one-line diagram of the electrical distribution equipment necessary to support the loads. 
Calc. No. W-320-31-010

Revision 1

Attachment A

HNF-2487, Rev. 0

Page D-6 
Ca7c. No. W-320-31-010 Revision 1

Page No. A - 1

Project W-320 / Tank 241-C-106 STuicing

C-Farm Electrical Distribution

Calc. No. W320-31-010 (Reference Drawing H-2-818680, SH 1, Rev. 1)

Tue Ju] $21 \quad 17: 17: 491998$

ALL INFORMATION PRESENTED IS FOR REVIEW, APPROVAL

INTERPRETATION AND APPLICATION BY A REGISTERED ENGINEER ONLY

SKM POWER*TOOLS FOR WINDOWS

INPUT DATA REPORT

COPYRIGHT SKM SYSTEMS ANALYSIS, INC. 1995-1997

ALL PU VALUES ARE EXPRESSED ON A 100 MVA BASE.

HNF-2487, Rev. 0

Page D-7 
Ca]c. No. $W-320-31-010$

Revision 1

Page No. A - 2

Tue JuT $21 \quad 17: 17: 491998$

Project $W-320$ / Tank 241-C-106 S7uicing

Page 2

C-Farm Electrical Distribution

Ca7c. No. W320-31-010 (Reference Drawing H-2-818680, SH 1, Rev. 1)

FEEDER INPUT DATA

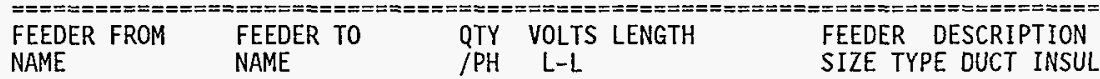

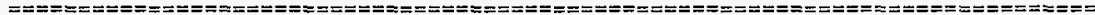

Ug Rsr P1 E257 XFMR C-T-1 Pri 113800260.0

$+1-$ Impedance: $0.2050+J \quad 0.0727$ OHMS/M Length

Zo Impedance: $0.6461+\mathrm{J} 0.1790$ OHMS/M Length

FT $2 \quad 2$ C $M \quad$ EPR

$0.0280+\mathrm{J} 0.0099 \mathrm{PU}$

$0.0882+\mathrm{J} 0.0244 \mathrm{PU}$

XFMR C-T-1 Sec C-PDP-1 $600 \mathrm{~A} \quad 2 \quad 480 \quad 25.0$ + - Impedance: $0.0250+\mathrm{J} 0.0480$ OHMS/M Length

20 Impedance: $0.0788+\mathrm{J} 0.11820 \mathrm{OHMS} / \mathrm{M}$ Length

FT $\quad 600$ C $M \quad$ THWN

$0.1356+\mathrm{J} 0.2604 \mathrm{PU}$

$0.4275+\mathrm{J} 0.6413 \mathrm{PU}$

$\begin{array}{llrrr}\text { C-PDP-1 } 600 \mathrm{~A} & \text { Xfmr Skid } & 1 & 480 & 50.0 \\ \text { +/- Impedance: } & 0.1200+\mathrm{J} & 0.0550 & \text { OHMS/M Length }\end{array}$

Z0 Impedance: $0.3782+\mathrm{J} 0.13550 \mathrm{HMS} / \mathrm{M}$ Length

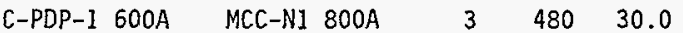

$+/-$ Impedance: $0.0390+\mathrm{J} 0.0500$ OHMS/M Length

Z0 Impedance: $0.1229+\mathrm{J} 0.1231$ OHMS/M Length

FT $\quad 1 / 0$ C $\quad M \quad$ MCXH

$2.60+\mathrm{J} \quad 1.19 \mathrm{PU}$

$8.21+\mathrm{J} 2.94 \mathrm{PU}$

Process B7dg C106-PP1 Bus 148015.0

$+/-$ Impedance: $0.7400+\mathrm{J} 0.0650$ OHMS/M Length

FT $\quad 350$ C $M \quad$ THWN

$0.1693+\mathrm{J} 0.2170 \mathrm{PU}$

$0.5335+\mathrm{J} 0.5345 \mathrm{PU}$

Zo Impedance: $2.33+J 0.16010 \mathrm{HMS} / \mathrm{M}$ Length

FT 8 C $M \quad$ THHN

$4.82+\mathrm{J} 0.4232 \mathrm{PU}$

MCC-N1 800A Process B7dg 14480240.0

$+/$ - Impedance: $0.1900+\mathrm{J} 0.0570$ OHMS/M Length

Zo Impedance: $0.5988+\mathrm{J} 0.1404$ OHMS/M Length

FT

$15.18+\mathrm{J}$

1.04

PU

MCC-N1 800A C106-PP2 Bus $14480 \quad 5.0$

+/- Impedance: $\quad 1.90+\mathrm{J} 0.0680$ OHMS/M Length

$19.79+\mathrm{J}$

C M THWN

$62.37+J$

5.94

14.62

PU

FT $\quad 12$ C $M \quad$ THWN

$4.12+\mathrm{J} 0.1476 \mathrm{PU}$

$12.99+\mathrm{J} 0.3634$ PU

Process BTdg FN-1361 Bus $\quad 1 \quad 48040.0$

+ - Impedance: $1.14+\mathrm{J} 0.0630$ OHMS/M Length

FT 10 C $M \quad$ THHN

ZO Impedance: $3.59+\mathrm{J} 0.1552$ OHMS/M Length

$19.79+\mathrm{J}$

$1.09 \mathrm{PU}$

$62.37+\mathrm{J} 2.69 \mathrm{PU}$

Process Bldg HC-1361 Bus 1448040.0

$+/-$ Impedance: $0.3000+\mathrm{J} 0.0600$ OHMS/M Length

FT

4

C M THHN

$5.21+\mathrm{J} 1.04 \mathrm{PU}$

Z0 Impedance: $0.9455+\mathrm{J} 0.1478$ OHMS/M Length

$16.41+\mathrm{J}$

$2.57 \mathrm{PU}$

HNF-2487, Rev. 0

Page D-8 
Ca]c. No. W-320-31-010

Revision 1

Page No. A - 3

Tue Jul 21 17:17:50 1998

Project W-320 / Tank 241-C-106 Sluicing

C-Farm Electrical Distribution

CaTc. No. W320-31-010 (Reference Drawing H-2-818680, SH 1, Rev. 1)

FEEDER INPUT DATA

\begin{tabular}{|c|c|c|c|c|c|c|}
\hline $\begin{array}{l}\text { FEFDER FROM } \\
\text { NAME }\end{array}$ & $\begin{array}{l}\text { FEEDER T0 } \\
\text { NAME }\end{array}$ & $\begin{array}{l}\text { QTY } \\
\text { /PH }\end{array}$ & $\begin{array}{l}\text { VOLTS LENGTH } \\
\text { L-L }\end{array}$ & $\begin{array}{l}\text { FEEDER } \\
\text { SIZE T }\end{array}$ & & \\
\hline $\begin{array}{l}\text { ocess Bldg } \\
\text { - Impedance: } \\
\text { Impedance: }\end{array}$ & $\begin{array}{c}\text { UH-1362 Bus } \\
1.90+\mathrm{J} \\
5.99+\mathrm{J}\end{array}$ & $\begin{array}{r}1 \\
0.0680 \\
0.1675\end{array}$ & $\begin{array}{l}48050.0 \\
\text { OHMS/M Length } \\
\text { OHMS/M Length }\end{array}$ & $\begin{array}{l}\text { FT } \quad 12 \\
41.23+\mathrm{J} \\
129.95+\mathrm{J}\end{array}$ & 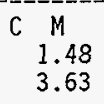 & $\mathrm{PU}$ \\
\hline
\end{tabular}

Process BTdg UH-1361 Bus $148030.0 \quad$ FT 12 C $\quad M \quad$ THHN

+ - Impedance: $1.90+\mathrm{J} 0.0680$ OHMS/M Length $24.74+\mathrm{J} 0.8854$ PU

20 Impedance: $5.99+\mathrm{J} 0.16750 \mathrm{HMS} / \mathrm{M}$ Length $77.97+\mathrm{J} 2.18 \mathrm{PU}$

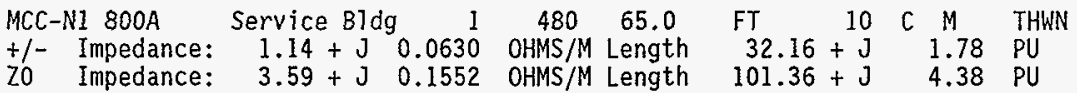

Service BTdg CPR-1361 Bus I 48015.0 FT 10 C $M$ THWN

$+/$ Impedance: $\quad 1.14+\mathrm{J} 0.0630$ OHMS/M Length $7.42+\mathrm{J} 0.4102 \mathrm{PU}$

ZO Impedance: $3.59+\mathrm{J} 0.1552$ OHMS/M Length $23.39+\mathrm{J} \quad 1.01 \mathrm{PU}$

Xfmr Skid Office Trl Bus $1480 \quad 50.0 \quad$ FT 4 C $M$ M

$+1-$ Impedance: $0.3000+\mathrm{J} 0.0600$ OHMS/M Length $6.51+\mathrm{J} 1.30$ PU

ZO Impedance: $0.9455+\mathrm{J} 0.1478$ OHMS/M Length $20.52+\mathrm{J} 3.21$ PU

Xfmr Skid Conex Tlr Bus 1 4 $480100.0 \quad$ FT 4 C $\quad M \quad$ MCXH

+ - Impedance: $0.3000+\mathrm{J} 0.0600$ OHMS/M Length $13.02+\mathrm{J} 2.60 \mathrm{PU}$

ZO Impedance: $0.9455+\mathrm{J} 0.1478$ OHMS/M Length $41.04+\mathrm{J} \quad 6.41 \mathrm{PU}$

MCC-N1 800A Chiller Skid $148030.0 \quad$ FT $4 / 0$ C $M$ THWN

$+/-$ Impedance: $0.0630+\mathrm{J} 0.0510$ OHMS/M Length $0.8203+\mathrm{J} 0.6641 \mathrm{PU}$

zo Impedance: $0.1985+\mathrm{J} 0.1256$ OHMS/M Length $2.59+\mathrm{J} \quad 1.64 \mathrm{PU}$

MCC-N1 800A Exhaust Skid $14480 \quad 320.0 \quad$ FT 10 C $M$ M THWN

$+/-$ Impedance: $1.14+\mathrm{J} 0.0630$ OHMS/M Length $158.33+\mathrm{J} \quad 8.75$ PU

ZO Impedance: $\quad 3.59+\mathrm{J} 0.1552$ OHMS/M Length $499.00+\mathrm{J} 21.55 \mathrm{PU}$

$\begin{array}{llllllllll}\text { MCC-N1 800A EXH Fan Vfd } & 1 & 480 & 10.0 & \text { FT } & 12 & C & M & \text { THWN }\end{array}$

$+/-$ Impedance: $1.90+\mathrm{J} 0.0680$ OHMS/M Length $8.25+\mathrm{J} 0.2951 \mathrm{PU}$

ZO Impedance: $5.99+\mathrm{J} 0.1675$ OHMS/M Length $25.99+\mathrm{J} 0.7269 \mathrm{PU}$

HNF-2487, Rev. 0

Page D-9 
Calc. No. W-320-31-010

Revision 1

Page No. A -4

Tue Jul $21 \quad 17: 17: 51 \quad 1998$

Project $W-320$ / Tank 241-C-106 Sluicing

Page 4

C-Farm Electrical Distribution

Calc. No. W320-31-010 (Reference Drawing H-2-818680, SH 1, Rev. 1)

FEEDER INPUT DATA

\begin{tabular}{|c|c|c|c|c|c|c|c|c|}
\hline $\begin{array}{l}\text { EDER FROM } \\
\text { AME }\end{array}$ & $\begin{array}{l}\text { FEEDER TO } \\
\text { NAME }\end{array}$ & $\begin{array}{l}\text { QTY } \\
\text { /PH }\end{array}$ & $\begin{array}{l}\text { VOLTS } \\
L-L\end{array}$ & LENGTH & \multicolumn{4}{|c|}{$\begin{array}{l}\text { FEEDER DESCRIPTION } \\
\text { SIZE TYPE DUCT INSUL }\end{array}$} \\
\hline $\begin{array}{l}:=== \pm======== \\
\text { mr Skjd } \\
- \text { Impedance: } \\
\text { Impedance: }\end{array}$ & $\begin{array}{l}=========== \\
\text { WRAM T1r Bus } \\
0.3000+\mathrm{J} \\
0.9455+\mathrm{J}\end{array}$ & $\begin{array}{l}======== \\
s \quad 1 \\
0.0600 \\
0.1478\end{array}$ & $\begin{array}{l}======= \\
480 \\
\text { OHMS } / M \\
\text { OHMS } / M\end{array}$ & $\begin{array}{l}220.0 \\
\text { Length } \\
\text { Length }\end{array}$ & $\begin{array}{l}==== \pm== \\
\mathrm{FT} \\
28.65 \\
90.28\end{array}$ & $\begin{array}{r}4 \\
+\mathrm{J} \\
+\mathrm{J}\end{array}$ & $\begin{array}{c}===== \\
C \quad M \\
5.73 \\
14.11\end{array}$ & $\begin{array}{l}\text { MCXH } \\
\text { PU } \\
\text { PU }\end{array}$ \\
\hline $\begin{array}{l}\mathrm{C}-\mathrm{N} 1 \mathrm{800 \textrm {A }} \\
\text { - Impedance: } \\
\text { Impedance: }\end{array}$ & $\begin{array}{l}\text { MCC-N2 } \\
0.0390+J \\
0.1229+J\end{array}$ & $\begin{array}{r}2 \\
0.0500 \\
0.1231\end{array}$ & $\begin{array}{c}480 \\
\text { OHMS } / M \\
\text { OHMS } / M\end{array}$ & $\begin{array}{l}70.0 \\
\text { Length } \\
\text { Length }\end{array}$ & $\begin{array}{l}\text { FT } \\
0.5924 \\
1.87\end{array}$ & $\begin{array}{l}350 \\
+J \\
+J\end{array}$ & $\begin{array}{rl}C & M \\
0.7595 \\
1.87\end{array}$ & $\begin{array}{l}\text { THWN } \\
\text { PU } \\
\text { PU }\end{array}$ \\
\hline $\begin{array}{l}\text { C-NI 800A } \\
-\quad \text { Impedance: } \\
\text { Impedance: }\end{array}$ & $\begin{array}{c}\text { STuicer Skid } \\
1.90+\mathrm{J} \\
5.99+\mathrm{J}\end{array}$ & $\begin{array}{l}1 \\
0.0680 \\
0.1675\end{array}$ & $\begin{array}{c}480 \\
\text { OHMS } / M \\
\text { OHMS } / M\end{array}$ & $\begin{array}{l}240.0 \\
\text { Length } \\
\text { Length }\end{array}$ & $\begin{array}{l}\mathrm{FT} \\
197.92 \\
623.74\end{array}$ & $\begin{array}{r}12 \\
+3 \\
+3\end{array}$ & $\begin{array}{c}\text { C } M \\
7.08 \\
17.44\end{array}$ & $\begin{array}{l}\text { THWN } \\
\text { PU } \\
\text { PU }\end{array}$ \\
\hline $\begin{array}{l}\mathrm{C}-\mathrm{N} 1 \text { 800A } \\
\text { Impedance: }\end{array}$ & $\begin{array}{c}\text { Slurry Winch } \\
1.90+\mathrm{J} \\
5.99+\mathrm{J}\end{array}$ & $\begin{array}{l}0.0680 \\
0.1675\end{array}$ & $\begin{array}{c}480 \\
\text { OHMS } / M \\
\text { OHMS } / M\end{array}$ & $\begin{array}{l}240.0 \\
\text { Length } \\
\text { Length }\end{array}$ & $\begin{array}{l}\text { FT } \\
197.92 \\
623.74\end{array}$ & $\begin{array}{r}12 \\
+\mathrm{J} \\
+\mathrm{J}\end{array}$ & $\begin{array}{l}\text { C }{ }^{7} \text { M.08 } \\
17.44\end{array}$ & $\begin{array}{l}\text { THWN } \\
\text { PU } \\
\text { PU }\end{array}$ \\
\hline $\begin{array}{l}\mathrm{C}-\mathrm{N} 2 \\
-\quad \text { Impedance: }\end{array}$ & $\begin{array}{l}\text { Booster Vfd } \\
0.0290+\mathrm{J} \\
0.0914+\mathrm{J}\end{array}$ & $\begin{array}{r}1 \\
0.0480 \\
0.1182\end{array}$ & $\begin{array}{c}480 \\
\text { OHMS } / M \\
\text { OHMS } / M\end{array}$ & $\begin{array}{l}10.0 \\
\text { Length } \\
\text { Length }\end{array}$ & $\begin{array}{l}\text { FT } \\
0.1259 \\
0.3967\end{array}$ & $\begin{array}{l}500 \\
+J \\
+J\end{array}$ & $\begin{array}{l}C \quad M \\
0.2083 \\
0.5131\end{array}$ & $\begin{array}{l}\text { THWN } \\
\text { PU } \\
\text { PU }\end{array}$ \\
\hline $\begin{array}{l}\mathrm{C}-\mathrm{N} 2 \\
\text { Impedance: }\end{array}$ & $\begin{array}{l}P-1361 \text { SS } \\
0.1200+J \\
0.1908+J\end{array}$ & $\begin{array}{l}1 \\
0.0440 \\
0.1119\end{array}$ & $\begin{array}{c}480 \\
\mathrm{OHMS} / \mathrm{M} \\
\mathrm{OHMS} / \mathrm{M}\end{array}$ & $\begin{array}{l}1.000 \\
\text { Length } \\
\text { Length }\end{array}$ & $\begin{array}{l}\text { FT } \\
0.0521 \\
0.0828\end{array}$ & $\begin{array}{l}1 / 0 \\
+\mathrm{J} \\
+\mathrm{J}\end{array}$ & $\begin{array}{l}C \quad N \\
0.0191 \\
0.0486\end{array}$ & $\begin{array}{l}\text { THWN } \\
\text { PU } \\
\text { PU }\end{array}$ \\
\hline $\begin{array}{l}-1361 \text { SS } \\
\text { Impedance: }\end{array}$ & $\begin{array}{l}P-1361 \text { Bus } \\
0.1200+J \\
0.3782+J\end{array}$ & $\begin{array}{l}0.1 \\
0.0550 \\
0.1355\end{array}$ & $\begin{array}{c}480 \\
\text { OHMS } / M \\
\text { OHMS/M }\end{array}$ & $\begin{array}{l}280.0 \\
\text { Length } \\
\text { Length }\end{array}$ & $\begin{array}{l}\text { FT } \\
14.58 \\
45.96\end{array}$ & $\begin{array}{l}1 / 0 \\
+J \\
+3\end{array}$ & $\begin{array}{r}\mathrm{C} \quad \mathrm{M} \\
6.68 \\
16.46\end{array}$ & $\begin{array}{l}\text { THWN } \\
\text { PU } \\
\text { PU }\end{array}$ \\
\hline $\begin{array}{l}\text { oster Vfd } \\
\text { Impedance: }\end{array}$ & $\begin{array}{l}\mathrm{P}-1362 \text { Bus } \\
0.0290+\mathrm{J} \\
0.0914+\mathrm{J}\end{array}$ & $\begin{array}{r}1 \\
0.0480 \\
0.1182\end{array}$ & $\begin{array}{c}480 \\
\text { OHMS } / M \\
\text { OHMS } / M\end{array}$ & $\begin{array}{l}330.0 \\
\text { Length } \\
\text { Length }\end{array}$ & $\begin{array}{l}\text { FT } \\
4.15 \\
13.09\end{array}$ & $\begin{array}{l}500 \\
+J \\
+J\end{array}$ & $\begin{array}{c}\text { C M } \\
6.88 \\
16.93\end{array}$ & $\begin{array}{l}\text { THWN } \\
\text { PU } \\
\text { PU }\end{array}$ \\
\hline $\begin{array}{l}\text { (H Fan Vfd } \\
-\quad \text { Impedance: } \\
\text { Impedance: }\end{array}$ & $\begin{array}{c}\mathrm{FN}-1362 \text { Bus } \\
1.90+\mathrm{J} \\
5.99+\mathrm{J}\end{array}$ & $\begin{array}{r}1 \\
0.0680 \\
0.1675\end{array}$ & $\begin{array}{c}480 \\
\text { OHMS/M } \\
\text { OHMS/M }\end{array}$ & $\begin{array}{l}320.0 \\
\text { Length } \\
\text { Length }\end{array}$ & $\begin{array}{l}\mathrm{FT} \\
263.89 \\
831.66\end{array}$ & $\begin{array}{r}12 \\
+\mathrm{J} \\
+\mathrm{J}\end{array}$ & $\begin{array}{c}\text { C } M \\
9.44 \\
23.26\end{array}$ & $\begin{array}{l}\text { THWN } \\
\text { PU } \\
\text { PU }\end{array}$ \\
\hline
\end{tabular}

HNF-2487, Rev. 0

Page D-10 
Carc. No, W-320-31-010

Revision 1

Page No. A - 5

Tue JuT 21 17:17:52 1998

Project W-320 / Tank 241-C-106 Sluicing

C-Farm Electrical Distribution

Calc. No. W320-31-010 (Reference Drawing H-2-818680, SH 1, Rev. 1)

FEEDER INPUT DATA

\begin{tabular}{|c|c|c|c|c|c|}
\hline $\begin{array}{l}\text { FEEDER FROM } \\
\text { NAME }\end{array}$ & $\begin{array}{l}\text { FEEDER TO } \\
\text { NAME }\end{array}$ & $\begin{array}{l}\text { QTY } \\
\text { /PH }\end{array}$ & $\begin{array}{l}\text { VOLTS LENGTH } \\
\text { L-L }\end{array}$ & $\begin{array}{l}\text { FEEDER DESCR } \\
\text { SIZE TYPE DUC }\end{array}$ & $\begin{array}{l}\text { IPTION } \\
T \text { INSUL }\end{array}$ \\
\hline $\begin{array}{l}\text { Service BIdg } \\
+/-\quad \text { Impedance: } \\
\text { ZO Impedance: }\end{array}$ & $\begin{array}{c}\text { C106-PP3 Bus } \\
1.14+\mathrm{J} \\
3.59+\mathrm{J}\end{array}$ & $\begin{array}{l}1 \\
0.0630 \\
0.1552\end{array}$ & $\begin{array}{cc}480 & 5.0 \\
\text { OHMS } / M & \text { Length } \\
\text { OHMS } / M \text { Length }\end{array}$ & 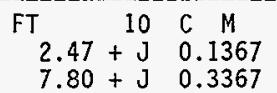 & $\begin{array}{l}\text { THWN } \\
\text { PU } \\
\text { PU }\end{array}$ \\
\hline
\end{tabular}

HNF-2487, Rev. 0

Page D-11 
Calc. No. W-320-31-010

Revision I

Page No. A - 6

Tue Ju1 21 17:17:52 1998

Project W-320 / Tank 241-C-106 Sluicing

C-Farm Electrical Distribution

Calc. No. W320-31-010 (Reference Drawing H-2-818680, SH 1, Rev. 1)

TRANSFORMER INPUT DATA

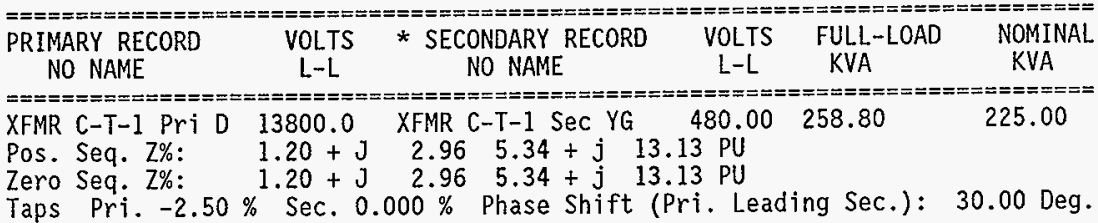

HNF-2487, Rev. 0

Page D-12 
Calc. No. W-320-31-010

Revision 1

Page No. A -7

Tue JuT $21 \quad 17: 17: 521998$

Project W-320 / Tank 241-C-106 Sluicing

Page 7

C-Farm Electrical Distribution

CaTc. No. W320-31-010 (Reference Drawing H-2-818680, SH 1, Rev. 1)

GENERATION DATA

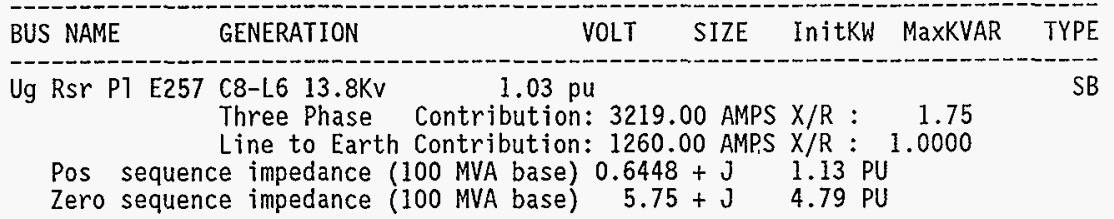

HNF-2487, Rev. 0

Page D-13 
Calc. No. W-320-31-010

Revision 1

\section{Attachment B}

HNF-2487, Rev. 0

Page D-14 
Calc. No. W-320-31-010 Revision 1

Page No. B - 1

Project W-320 / Tank 241-C-106 STuicing

C-Farm Electrical Distribution

Calc. No. W320-31-010 (Reference Drawing H-2-818680, SH 1, Rev. 1)

DATE: $7: 21: 1998$

TIME: $17: 17: 11.62$

ALL INFORMATION PRESENTED IS FOR REVIEW, APPROVAL, INTERPRETATION

AND APPLICATION BY A REGISTERED ENGINEER ONLY

SKM POWER*TOOLS FOR WINDOWS

DEMAND LOAD ANALYSIS REPORT

COPYRIGHT SKM SYSTEMS ANALYSIS, INC. 1995-1996

*** SOLUTION COMMENTS ***

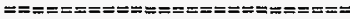

LOAD ANALYSIS INCLUDES ALL LOADS.

HNF-2487, Rev. 0

Page D-15 
HNF-2487, Rev. 0

Page D-16
Calc. No. $W-320-31-010$

Revision 1

Page No. B - 2

DATE: $7: 21: 1998$ TIME: $17: 17: 11.62$

PAGE 2

Project W-320 / Tank 241-C-106 Sluicing

C-Farm Electrical Distribution

Ca]c. No. W320-31-010 (Reference Drawing H-2-818680, SH 1, Rev. 1)

LOAD SUMMARY

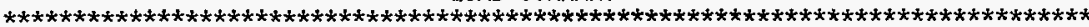

LOAD SCHEDULE FOR Ug RSP PT E257

13800. VOLTS LINE TO LINE

SOURCE OF PWR SOURCE BUS

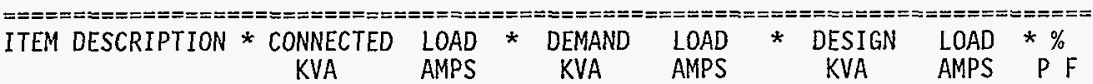

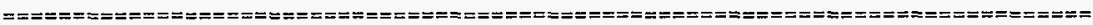

END USE LOADS

BRANCH LOADS

$\begin{array}{lllllll}\text { XFMR C-T-1 Pri } 501.1 & 21.0 & 501.1 & 21.0 & 566.9 & 23.7 & 89.63\end{array}$

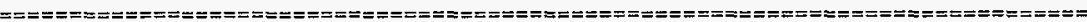

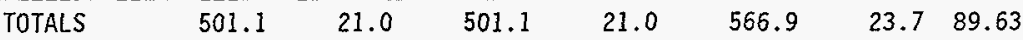

LOAD SCHEDULE FOR XFMR C-T-1 Pri

SOURCE OF PWR Ug RsY PT E257

13800. VOLTS LINE TO LINE

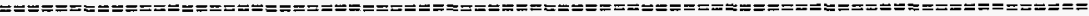

ITEM DESCRIPTION * CONNECTED LOAD * DEMAND LOAD * DESIGN LOAD * \%

KVA AMPS KVA AMPS KVA AMPS PF

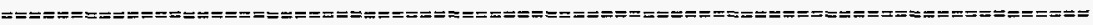
END USE LOADS

BRANCH LOADS

XFMR C-T-1 Sec $\quad 501.1 \quad 21.0 \quad 501.1 \quad 21.0 \quad 566.9 \quad 23.7 \quad 89.63$

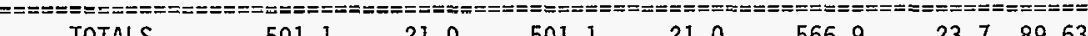
$\begin{array}{llllllll}\text { TOTALS } & 501.1 & 21.0 & 501.1 & 21.0 & 566.9 & 23.7 & 89.63\end{array}$

LOAD SCHEDULE FOR XFMR C-T-I SEC SOURCE OF PWR XFMR C-T-1 Pri

480. VOLTS LINE TO LINE

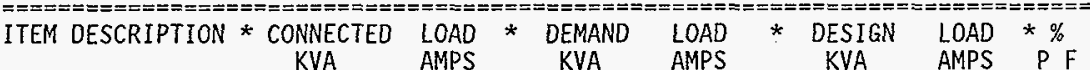

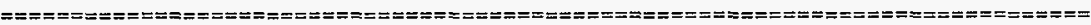

END USE LOADS

BRANCH LOADS

C-PDP-1 $600 \mathrm{~A}$

501.1

602.7

501.1

602.7

566.9

$681.9 \quad 89.63$

TOTALS

501.1

602.7

$501.1 \quad 602.7$

566.9

$681.9 \quad 89.63$

LOAD SCHEDULE FOR C-PDP-1 $600 \mathrm{~A}$

SOURCE OF PWR XFMR C-T-1 SEC

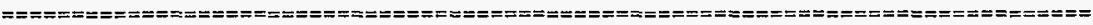
ITEM DESCRIPTION * CONNECTED LOAD * DEMAND LOAD * DESIGN LOAD * \%

KVA AMPS KVA AMPS KVA AMPS PF

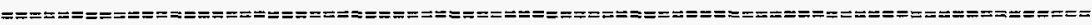

END USE LOADS

BRANCH LOADS

$X$ fmr Skid

49.8

59.9

49.8

59.9

49.8

$59.9 \quad 90.00$

MCC-N1 800A

451.3

542.8

451.3

542.8

517.2

$622.0 \quad 89.59$

TOTALS

$501.1 \quad 602.7$

$501.1 \quad 602.7$

566.9

681.9

89.63 
Calc. No. W-320-31-010 Revision 1

Page No. B - 3

DATE: $7: 21: 1998$ TIME: $17: 17: 11.62$

Project W-320 / Tank 241-C-106 Sluicing

PAGE 3

C-Farm Electrical Distribution

Calc. No. W320-31-010 (Reference Drawing H-2-818680, SH I, Rev. 1)

LOAD SUMMARY

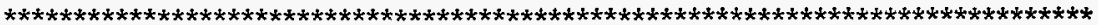

LOAD SCHEDULE FOR Xfmr Skid

SOURCE OF PWR C-PDP-1 600A

480. VOLTS LINE TO LINE

\begin{tabular}{|c|c|c|c|c|c|c|c|c|}
\hline ITEM DESCRIPTION & $\begin{array}{c}\text { * CONNECTED } \\
\text { KVA }\end{array}$ & $\begin{array}{l}\text { LOAD } \\
\text { AMPS }\end{array}$ & $\begin{array}{c}\text { DEMAND } \\
\text { KVA }\end{array}$ & $\begin{array}{l}\text { LOAD } \\
\text { AMPS }\end{array}$ & $\star$ & $\underset{\text { KVA }}{\text { DESIGN }}$ & $\begin{array}{l}\text { LOAD } \\
\text { AMPS }\end{array}$ & ${ }^{* \%} \%$ \\
\hline $\begin{array}{l}=====x==== \\
\text { END USE LOADS } \\
\text { BRANCH LOADS }\end{array}$ & & & & & & & & \\
\hline $\begin{array}{l}\text { Office Tri Bus } \\
\text { Conex T1r Bus } \\
\text { WRAM Tir Bus }\end{array}$ & $\begin{array}{l}16.6 \\
16.6 \\
16.6\end{array}$ & $\begin{array}{l}20.0 \\
20.0 \\
20.0\end{array}$ & $\begin{array}{l}16.6 \\
16.6 \\
16.6\end{array}$ & $\begin{array}{l}20.0 \\
20.0 \\
20.0\end{array}$ & & $\begin{array}{l}16.6 \\
16.6 \\
16.6\end{array}$ & $\begin{array}{l}20.0 \\
20.0 \\
20.0\end{array}$ & $\begin{array}{l}90.00 \\
90.00 \\
90.00\end{array}$ \\
\hline TOTALS & 49.8 & 59.9 & 49.8 & 59.9 & & 49.8 & 59.9 & 90 . \\
\hline
\end{tabular}

LOAD SCHEDULE FOR MCC-N1 $800 \mathrm{~A}$

SOURCE OF PWR C-PDP-1 600A

480. VOLTS LINE TO LINE

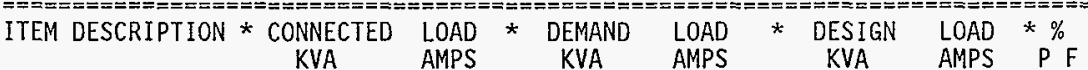

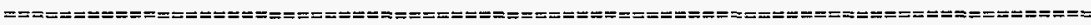

END USE LOADS

BRANCH LOADS

Process BTdg

C106-PP2 Bus

52.8

63.5

52.8

63.5

64.2

$\begin{array}{ll}77.3 & 98.77\end{array}$

Service BIdg

15.0

18.0

15.0

18.0

15.0

$18.0 \quad 90.00$

Chiller Skid

14.1

16.9

14.1

16.9

16.4

$19.7 \quad 83.69$

Exhaust Skid

76.4

91.9

76.4

91.9

90.4

$108.8 \quad 80.38$

EXH Fan Vfd

11.6

14.0

11.6

14.0

13.3

16.092 .11

MCC-N2

9.1

11.0

Sluicer Skid

269.5

9.1

11.0

11.4

$13.7 \quad 80.50$

Slurry Winch

4.3

324.1

2.2

5.2

269.5

324.1

326.2

$392.4 \quad 89.17$

$4.3 \quad 5.2$

5.3

6.480 .65

$=============$

2.6

2.2

2.6

3.280 .00

TOTALS

$451.3 \quad 542.8$

$451.3 \quad 542.8$

2.7

$3.2 \quad 80.00$
$6========$

$\begin{array}{llllll}542.8 & 451.3 & 542.8 & 517.2 & 622.0 & 89.59\end{array}$

LOAD SCHEDULE FOR C106-PP1 BUS SOURCE OF PWR Process Bldg

480. VOLTS LINE TO LINE

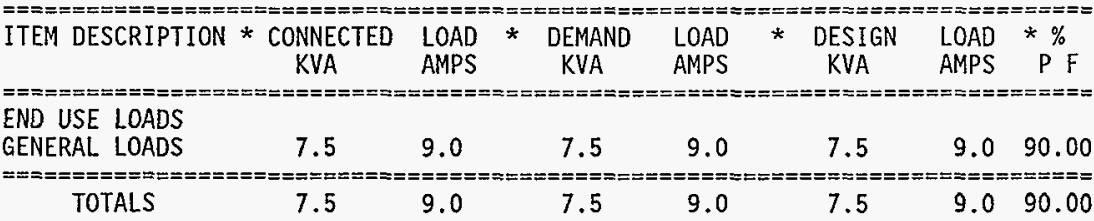

HNF-2487, Rev. 0

Page D-17 
LOAD SCHEDULE FOR ProcesS BIdg

SOURCE OF PWR MCC-N1 800A

480. VOLTS LINE TO LINE

\begin{tabular}{|c|c|c|c|c|c|c|}
\hline ITEM DESCRIPTION & * CONNECTED & $\begin{array}{l}\text { LOAD } \\
\text { AMPS }\end{array}$ & $\begin{array}{c}\text { * DEMAND } \\
\text { KVA }\end{array}$ & $\begin{array}{l}\text { LOAD } \\
\text { AMPS }\end{array}$ & * DESIGN & $\begin{array}{l}\text { LOAD } * \% \\
\text { AMPS } \quad P F\end{array}$ \\
\hline & $:=== \pm=:===$ & 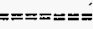 & $=-2$ & & & \\
\hline ND USE LOAOS & & & & & & \\
\hline BRANCH LOADS & & & & & & \\
\hline C106-PP1 Bus & 7.5 & 9.0 & 7.5 & 9.0 & 7.5 & 9.09 \\
\hline FN-1361 Bus & 9.1 & 11.0 & 9.1 & 11.0 & 11.4 & 13.7 \\
\hline HC-1361 Bus & 30.0 & 36.1 & 30.0 & 36.1 & 37.5 & 45.110 \\
\hline UH-1362 Bus & 4.0 & 4.8 & 4.0 & 4.8 & 5.0 & 6.010 \\
\hline UH-1361 Bus & 4.0 & 4.8 & 4.0 & 4.8 & 5.0 & 6.010 \\
\hline 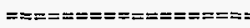 & $== \pm==== \pm==$ & $===== \pm$ & $========0$ & $===$ & $=== \pm===$ & \\
\hline TOTALS & 52.8 & 63.5 & 52.8 & 63.5 & 64.2 & 77.3 \\
\hline $\begin{array}{l}\text { LOAD SCHEDULE FOR } \\
\text { SOURCE OF PWR }\end{array}$ & $\begin{array}{l}\text { C106-PP2 } \\
M C C-N 1800\end{array}$ & & & & 480. VOLTS & INE TO LI \\
\hline ITEM DESCRIPTION & $\begin{array}{l}\text { CONNECTED } \\
\text { KVA }\end{array}$ & $\begin{array}{l}\text { LOAD } \\
\text { AMPS }\end{array}$ & * DEMAND & $\begin{array}{l}\text { LOAD } \\
\text { AMPS }\end{array}$ & $\begin{array}{l}\text { DESIGN } \\
\text { KVA }\end{array}$ & $\begin{array}{l}\text { LOAD } * \% \\
\text { AMPS } \quad P \%\end{array}$ \\
\hline
\end{tabular}

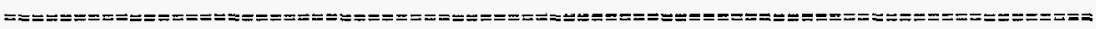
END USE LOADS

GENERAL LOADS

15.0

18.0

15.0

18.0

15.0

$18.0 \quad 90.00$ TOTALS

$15.0 \quad 18.0$

$15.0 \quad 18.0$

15.0

$18.0 \quad 90.00$

LOAD SCHEDULE FOR FN-1361 BUS SOURCE OF PWR Process BTdg

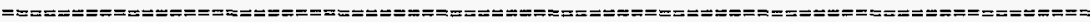
ITEM DESCRIPTION * CONNECTED LOAD * DEMAND LOAD * DESIGN LOAD * \% KVA AMPS KVA AMPS KVA AMPS P F

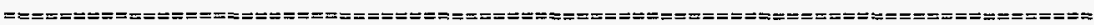
END USE LOADS

LARGEST KVA MTR $\quad 9.1 \quad 11.0 \quad 9.1 \quad 11.0 \quad 11.4 \quad 13.7 \quad 80.50$

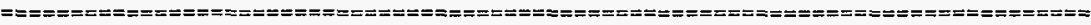
TOTALS 9.111 .0

9.1

11.0

11.4

13.780 .50

LOAD SCHEDULE FOR HC-1361 BUS SOURCE OF PWR ProcesS Bldg

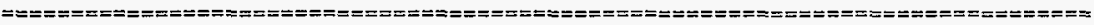

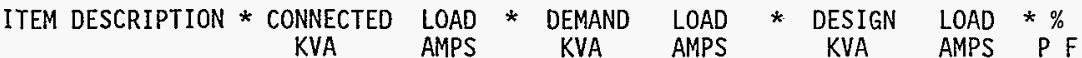

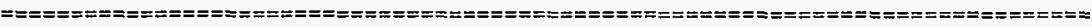
END USE LOADS

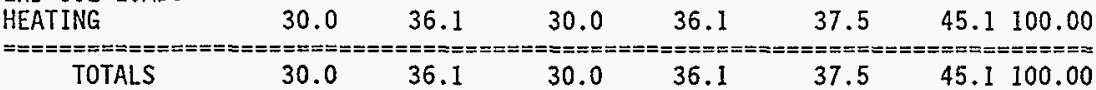


FNF-2487, Rev. 0

Page D-19

Calc. No. W-320-31-010

Revision 1

Page No. B - 5

DATE: $7: 21: 1998$ TIME: $17: 17: 11.62$

PAGE

Project $W-320$ / Tank 241-C-106 Sluicing

C-Farm Electrical Distribution

Calc. No. W320-31-010 (Reference Drawing H-2-818680, SH 1, Rev. 1)

LOAD SUMMARY

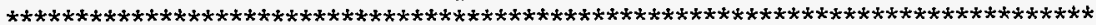

LOAD SCHEDULE FOR UH-1362 BUS

480. VOLTS LINE TO LINE SOURCE OF PWR Process Bldg

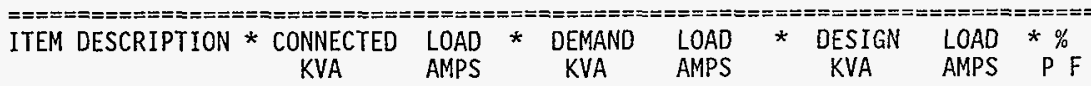

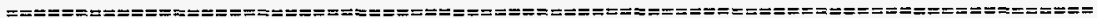

END USE LOADS

HEATING

4.0

4.8

4.0

4.8

5.0

6.0100 .00

$========= \pm P$

4.0

4.8

4.0

4.8

5.0

6.0100 .00

LOAD SCHEDULE FOR UH-136I BUS SOURCE OF PWR Process BTdg

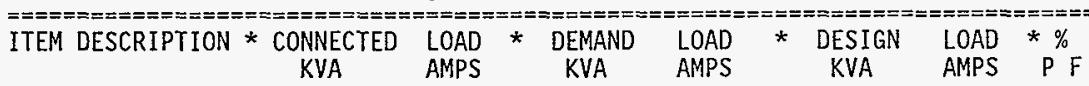

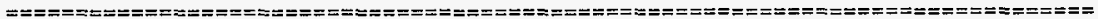
END USE LOADS

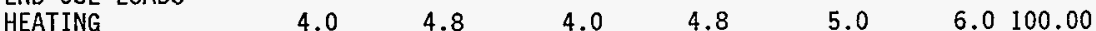

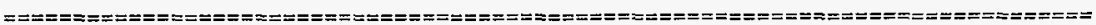
TOTALS

$4.0 \quad 4.8$

4.0

4.8

5.0

6.0100 .00

LOAD SCHEDULE FOR Service BTdg SOURCE OF PWR MCC-N1 800A

480. VOLTS LINE TO LINE

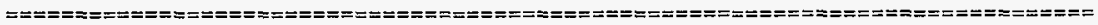

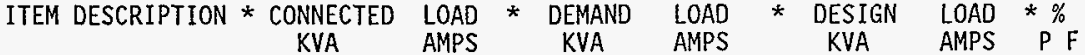

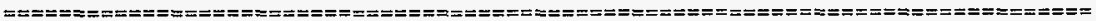

END USE LOADS

BRANCH LOADS

C106-PP3 Bus

CPR-1361 BUS

$5.0 \quad 6.0$

$6.0 \quad 5.0$

6.0

$9.1 \quad 11.0$

5.0

$6.0 \quad 90.00$

$== \pm===== \pm===$

$9.1 \quad 11.0$

$11.0 \quad 11.4$

$13.7 \quad 80.50$

TOTALS

14.1

16.9

14.1

16.9

16.4

$19.7 \quad 83.69$

LOAD SCHEDULE FOR C106-PP3 BUS SOURCE OF PWR Service BTdg

480. VOLTS LINE TO LINE

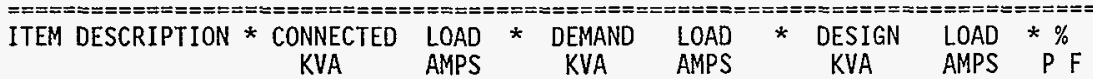

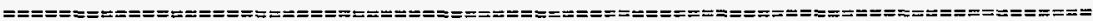
END USE LOADS

GENERAL LOADS

5.0

6.0

5.0

6.0

5.0

$6.0 \quad 90.00$

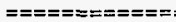

TOTALS

5.0

6.0

5.0

6.0

5.0

$6.0 \quad 90.00$ 
Project W-320 / Tank 24l-C-106 Sluicing

C-Farm Electrical Distribution

Calc. No. W320-31-010 (Reference Drawing H-2-818680, SH 1, Rev. 1)

LOAD SUMMARY

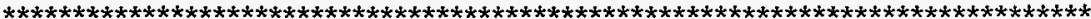

LOAD SCHEDULE FOR CPR-1351 BUS

480. VOLTS LINE TO LINE SOURCE OF PWR Service BTdg

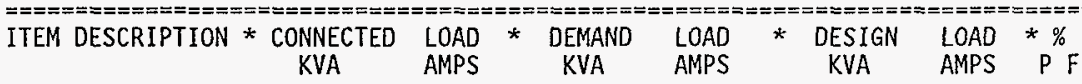

END USE LOADS

LARGEST KVA MTR

$9.1 \quad 11.0$

9.1

11.0

11.4

$13.7 \quad 80.50$

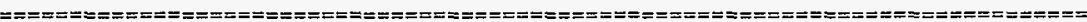
TOTALS

$9.1 \quad 11.0$

$9.1 \quad 11.0$

11.4

$13.7 \quad 80.50$

LOAD SCHEDULE FOR SOURCE OF PWR

Office Trl Bus Xfmr Skid

480. VOLTS LINE TO LINE

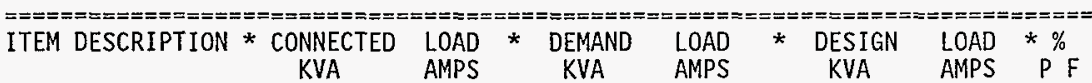

$========== \pm=$
END USE LOADS

GENERAL LOADS

16.6

20.0

16.6

20.0

16.6

$20.0 \quad 90.00$

$============$

$16.6 \quad 20.0$

16.6

20.0

16.6

$20.0 \quad 90.00$

LOAD SCHEDULE FOR Conex TTr Bus SOURCE OF PWR Xfmr Skid 480. VOLTS LINE TO LINE

\begin{tabular}{|c|c|c|c|c|c|c|c|}
\hline TEM DESCRIPTION & 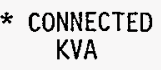 & $\begin{array}{l}\text { LOAD } \\
\text { AMPS }\end{array}$ & * DEMAND & $\begin{array}{l}\text { LOAD } \\
\text { AMPS }\end{array}$ & * $\underset{\text { KVSIGN }}{\text { DESIGN }}$ & $\begin{array}{l}\text { LOAD } \\
\text { AMPS }\end{array}$ & ${ }^{*} \%$ \\
\hline & & & & & & & \\
\hline IERAL LOADS & 16.6 & 20.0 & 16.6 & 20.0 & 16.6 & 20.0 & 10 \\
\hline TOTALS & 16.6 & 20.0 & 16.6 & 20.0 & 16.6 & 20.0 & 0. \\
\hline
\end{tabular}

LOAD SCHEDULE FOR

Chiller Skid 480. VOLTS LINE TO LINE SOURCE OF PWR MCC-N1 800A

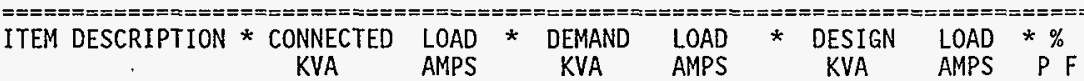

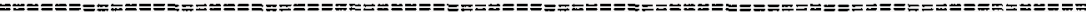
END USE LOADS

GENERAL LOADS

KVA TYPE MTR

3.0

3.6

3.0

3.6

3.0

3.6

90.00

LARGEST KVA MTR

17.5

21.0

17.5

21.0

17.5

$21.0 \quad 80.00$

$============0$

56.0

67.4

56.0

67.4

70.0

84.280 .00

91.9

76.4

91.9

90.4

108.8

80.38 
HNF-2487, Rev. 0

Page D-22

Calc. No. W-320-31-010

Revision 1

Page No. B -8

DATE: $7: 21: 1998$ TIME: $17: 17: 11.62$

Project W-320 / Tank 241-C-106 Sluicing

PAGE 8

C-Farm Electrical Distribution

Calc. No. W320-31-010 (Reference Drawing H-2-818680, SH 1, Rev. 1)

LOAD SUMMARY

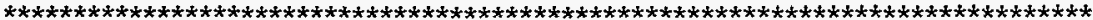

LOAD SCHEDULE FOR Sluicer SKId

480. VOLTS LINE TO LINE

SOURCE OF PWR MCC-NI 800A

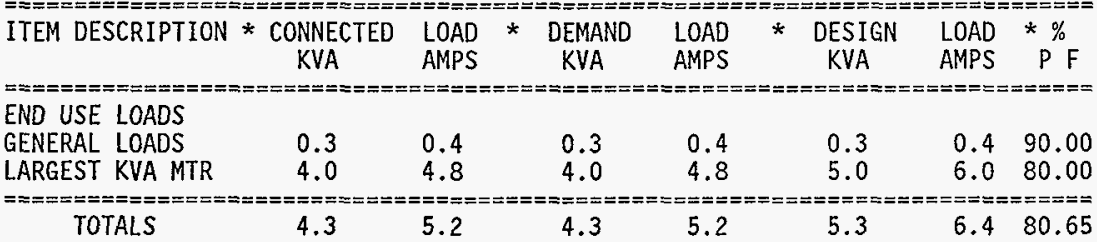

LOAD SCHEDULE FOR STurry Winch SOURCE OF PWR MCC-N1 800A

480. VOLTS LINE TO LINE

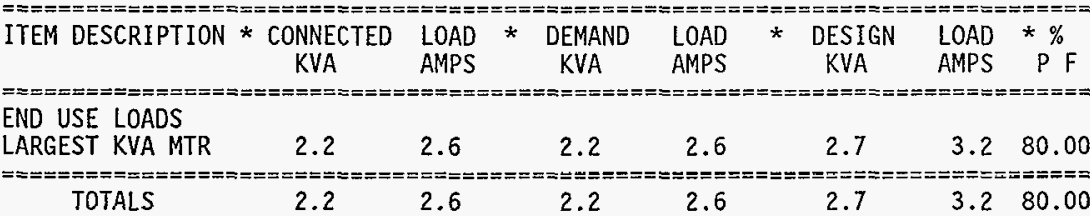

LOAD SCHEDULE FOR P-1361 SS

SOURCE OF PWR MCC-N2

480. VOLTS LINE TO LINE

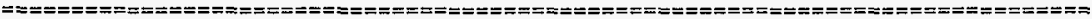

ITEM DESCRIPTION * CONNECTED LOAD * DEMAND LOAD * DESIGN LOAD *\%

KVA AMPS KVA AMPS KVA AMPS P F

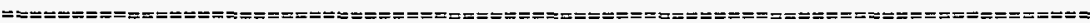

END USE LOADS

BRANCH LOADS

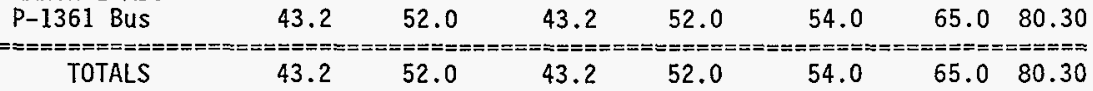

LOAD SCHEDULE FOR P-136] BUS

SOURCE OF PWR P-1361 SS

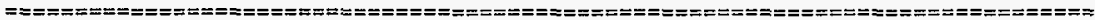

ITEM DESCRIPTION * CONNECTED LOAD * DEMAND LOAD * DESIGN LOAD * \%

KVA AMPS KVA AMPS KVA AMPS P F

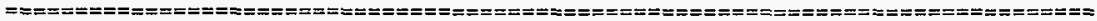

END USE LOADS

LARGEST KVA MTR $\quad 43.2 \quad 52.0 \quad 43.2 \quad 52.0 \quad 54.0 \quad 65.0 \quad 80.30$

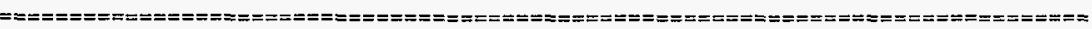

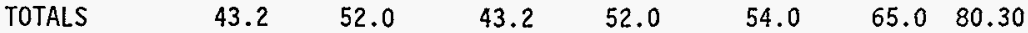


Calc. No. $W-320-31-010$

Revision 1

Page No. B - 9

DATE: $7: 21: 1998$ TIME: $17: 17: 11.62$

PAGE.

Project W-320 / Tank 241-C-106 Sluicing

C-Farm Electrical Distribution

Calc. No. W320-31-010 (Reference Drawing H-2-818680, SH 1, Rev, 1)

LOAD SUMMARY

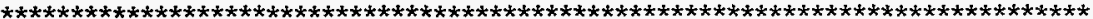

LOAD SCHEDULE FOR Booster Vfd

480. VOLTS LINE TO LINE

SOURCE OF PWR

MCC-N2

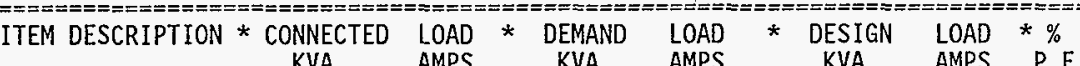

KVA AMPS KVA AMPS KVA AMPS PF

END USE LOADS

BRANCH LOADS

P-1362 Bus

$227.0 \quad 273.0$

227.0

273.0

$283.7 \quad 341.2 \quad 90.30$

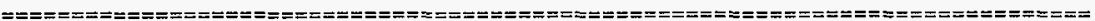
TOTALS

$227.0 \quad 273.0$

$227.0 \quad 273.0$

$\begin{array}{lll}283.7 & 341.2 & 90.30\end{array}$

LOAD SCHEDULE FOR P-1362 BUS SOURCE OF PWR Booster Vfd

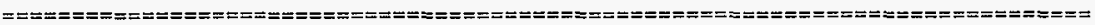
ITEM DESCRIPTION * CONNECTED LOAD * DEMAND LOAD * DESIGN LOAD * \% KVA AMPS KVA AMPS KVA AMPS P F

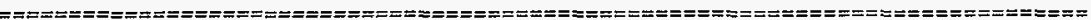

END USE LOADS

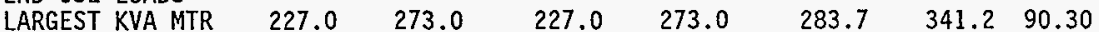

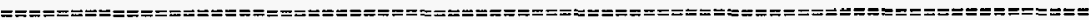
TOTALS

$227.0 \quad 273.0$

$227.0 \quad 273.0$

$\begin{array}{lll}283.7 & 341.2 & 90.30\end{array}$

LOAD SCHEDULE FOR FN-1362 BuS SOURCE OF PWR EXH Fan VFd

\begin{tabular}{|c|c|c|c|c|c|c|c|}
\hline ITEM DESCRIPTION & * CONNECTED & $\begin{array}{l}\text { LOAD } \\
\text { AMPS }\end{array}$ & * DEMAND & $\begin{array}{l}\text { LOAD } \\
\text { AMPS }\end{array}$ & * $\underset{\text { KVIG }}{\text { DESIGN }}$ & $\begin{array}{l}\text { LOAD } \\
\text { AMPS }\end{array}$ & ${ }^{*} \%$ \\
\hline $\begin{array}{l}\text { END USE LOADS } \\
\text { LARGEST KVA MTR }\end{array}$ & 9.1 & 11.0 & 9.1 & 11.0 & 11.4 & 13.7 & 80.50 \\
\hline TOTALS & 9.1 & 11.0 & 9.1 & 11.0 & 11.4 & 13.7 & 80.50 \\
\hline
\end{tabular}

HNF-2487, Rev. 0

Page D-23 
Calc. No. $W-320-31-010$

Revision 1

Page No. B - 10

DATE: $7: 21: 1998$ TIME: $17: 17: 11.62$

PAGE 10

Project $W-320$ / Tank 241-C-106 S7uicing

C-Farm Electrical Distribution

Calc. No. W320-31-010 (Reference Drawing H-2-818680, SH 1, Rev. 1)

TOTAL SOURCE LOAD SUMMARY

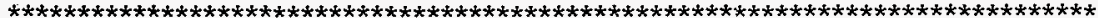

\begin{tabular}{|c|c|}
\hline $\begin{array}{l}\text { LOAD DESCRIPTION } \\
\text { TYPE }\end{array}$ & UNITS \\
\hline
\end{tabular}

TYPE

GENERAL LOADS

KW

77.0

37.3

77.0

77.0

KVAR

85.6

37.3

85.6

37.3

KVA

41.0

41.0

0.0

0.0

41.0

41.0

85.6

90.00 LAGGING

HEATING

KVAR

123.7

$\mathrm{KW}$

92.2

KVAR

154.3

123.7

92.2

154.3

51.2

KVA

204.9

204.9

97.5

97.5

227.0

0.0

51.2

100.00 UNITY

LARGEST KVA MTR

KW
KVAR

227.0

446.7

227.0

508.2

TOTAL LOADS

KW
KVAR
KVA
$\%$ PF

446.7

227.0

501.1

501.1

89.1

123.7

92.2

154.3

80.17 LAGGING

256.2

121.9

283.7

90.30 LAGGING

$\%$ PF

LAGGING

LAGGING

251.4

566.9

89.6

LAGGING

HNF-2487, Rev. 0

Page D-24 
Calc. No. W-320-31-010

Revision 1

Page No. B - 11

DATE: $7: 21: 1998$ TIME: $17: 17: 11.62$

PAGE 11

Project $W-320$ / Tank 241-C-106 STuicing

C-Farm Electrical Distribution

Calc. No. W320-31-010 (Reference Drawing H-2-818680, SH 1, Rev. 1)

LOAD DEMAND TABLE

\begin{tabular}{|c|c|c|c|c|c|c|}
\hline LOAD DESCRIPTION & $\begin{array}{l}\text { LOAD } \\
\text { TYPE }\end{array}$ & $\underset{\text { KVA }}{\text { FIRST }} \underset{\%}{\text { DEMAND }}$ & $\begin{array}{r}\text { KECOND } \\
\text { KVA }\end{array}$ & $\begin{array}{l}\text { DEMAND } \\
\%\end{array}$ & $\begin{array}{c}\text { THIRD DEMAND } \\
\text { KVA } \%\end{array}$ & $\begin{array}{c}\text { DESIGN } \\
\text { FACT }\end{array}$ \\
\hline-3 & - & $======= \pm===$ & - & 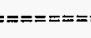 & $x^{2}$ & $===== \pm=$ \\
\hline $\begin{array}{l}\text { GENERAL LOADS } \\
\text { LIGHTING } \\
\text { RECEPTACLES } \\
\text { Load Not Defined } \\
\text { HEATING } \\
\text { OFFICE EQUIPMENT } \\
\text { WATER PUMPS } \\
\text { VAR SPEED DRIVE } \\
\text { SPARE } \\
\text { SPARE } \\
\text { HVAC MTR LOAD } \\
\text { HVAC LGST MTR } \\
\text { ACC MTR LOAD } \\
\text { ACC LGST MTR } \\
\text { SPARE } \\
\text { SPARE } \\
\text { SPARE } \\
\text { SPARE } \\
\text { SPARE } \\
\text { SPARE }\end{array}$ & $\begin{array}{l}Z \\
K \\
Z \\
K \\
Z \\
Z \\
K \\
K \\
K \\
K \\
K \\
K \\
K \\
K \\
K \\
K \\
K \\
K \\
K \\
K\end{array}$ & $\begin{array}{cc}100 . & 100 . \\
\text { ALL } 100 . \\
10 . & 100 . \\
\text { ALL } 0 . \\
\text { ALL } 100 . \\
\text { ALL } 100 . \\
\text { ALL } 100 . \\
\text { ALL } 5 . \\
\text { ALL } 100 . \\
\text { ALL } 100 . \\
\text { ALL } 100 . \\
\text { ALL } 100 . \\
\text { ALL } 100 . \\
\text { ALL } 100 . \\
\text { ALL } 100 . \\
\text { ALL } 100 . \\
\text { ALL } 100 . \\
\text { ALL } 100 . \\
\text { ALL } 100 . \\
\text { ALL } 100 .\end{array}$ & $\begin{array}{l}\text { ALL } \\
\text { ALL } \\
\text { ALL } \\
\text { ALL } \\
\text { ALL } \\
\text { ALL } \\
\text { ALL } \\
\text { ALL } \\
\text { ALL } \\
\text { ALL } \\
\text { ALL } \\
\text { ALL } \\
\text { ALL } \\
\text { ALL } \\
\text { ALL } \\
\text { ALL } \\
\text { ALL } \\
\text { ALL } \\
\text { ALL } \\
\text { ALL }\end{array}$ & $\begin{array}{r}50 . \\
100 . \\
50 . \\
0 . \\
100 . \\
100 . \\
100 . \\
5 . \\
100 . \\
100 . \\
100 \\
100 \\
100 \\
100 \\
100 \\
100 \\
100 \\
100 \\
100 \\
100\end{array}$ & $\begin{array}{lr}\text { ALL } & 50 . \\
\text { ALL } 100 . \\
\text { ALL } 50 . \\
\text { ALL } 0 . \\
\text { ALL } 100 . \\
\text { ALL } 100 . \\
\text { ALL } 100 . \\
\text { ALL } 5 . \\
\text { ALL } 100 . \\
\text { ALL } 100 . \\
\text { ALL } 100 . \\
\text { ALL } 100 . \\
\text { ALL } 100 . \\
\text { ALL } 100 . \\
\text { ALL } 100 . \\
\text { ALL } 100 . \\
\text { ALL } 100 . \\
\text { ALL } 100 . \\
\text { ALL } 100 . \\
\text { ALL } 100 .\end{array}$ & $\begin{array}{l}1.00 \\
1.25 \\
1.00 \\
1.00 \\
1.25 \\
1.00 \\
1.00 \\
1.00 \\
1.00 \\
1.00 \\
1.00 \\
1.25 \\
1.00 \\
1.25 \\
1.00 \\
1.00 \\
1.00 \\
1.00 \\
1.00 \\
1.00\end{array}$ \\
\hline
\end{tabular}

NOTES: LARGEST MOTOR CIRCUIT IDENTIFIED, AND USED TO CALCULATE DEMAND LOAD BASED ON NEC ART 430 DEMAND AND DESIGN FACTORS APPLIED AT EACH LOAD BUS AND ALL LOAD TOTALS ARE POWER FACTOR CORRECTED DESIGN LOAD VALUES BASED ON MOTOR RUNING OR STARTING CONDITION

HNF-2487, Rev. 0

Page D-25 
Ca1c. No. W-320-31-010 Revision 1

Attachment C

HNF-2487, Rev. 0

Page D-26 
CaTc. No. W-320-31-010 Revision 1

Page No. $C-1$

Project $W-320$ / Tank 241-C-106 Sluicing

C-Farm Electrical Distribution

Calc. No. W320-31-010 (Reference Drawing H-2-818680, SH 1, Rev. 1)

DATE: $\quad 7: 21: 1998$

TIME: $17: 17: 28.59$

ALL INFORMATION PRESENTED IS FOR REVIEW, APPROVAL, INTERPRETATION AND APPLICATION BY A REGISTERED ENGINEER ONLY

SKM POWER*TOOLS FOR WINDOWS

FEEDER AND TRANSFORMER SIZING REPORT

COPYRIGHT SKM SYSTEMS ANALYSIS, INC. 1995-1996

HNF-2487, Rev. 0

Page D-27 
Calc. No. W-320-31-010 Revision 1

Page No. C - 2

DATE 7:21:1998 TIME:17:17:28.59

PAGE 2

Project W-320 / Tank 241-C-106 Sluicing

C-Farm Electrical Distribution

Calc. No. W320-31-010 (Reference Drawing H-2-818680, SH 1, Rev. 1)

FEEDER AND TRANSFORMER STUDY CRITERIA

SIZE AND REPORT, DO NOT CHANGE THE DATA BASE

BRANCH VOLTAGE DROP CRITERIA $3.00 \%$

VOLTAGE DROP CALCULATIONS ARE PRELIMINARY

EXECUTE VOLTAGE DROP AND LOAD FLOW STUDY FOR MORE ACCURATE RESULTS

PRIMARY/SECONDARY TRANSFORMER FDRS SIZED AT 125. \% OF TX FULL LOAD RATING

$* * *$ NOTICE $* * *$ FEEDER SIZED TO 125. PERCENT OF TRANSFORMER SIZE

BRANCH FROM XFMR C-T-1 Pri TO XFMR C-T-I SeC

TX KVA: 501.1 TR FLA: 21.0

MINIMUM FEEDER AMPACITY: $\quad 26.2$

*** NOTICE $* * *$ FEEDER SIZED TO 125 . PERCENT OF TRANSFORMER SIZE

BRANCH FROM XFMR C-T-1 Pri TO XFMR C-T-1 SeC

TX KVA: $\quad 501.1$ TR FLA: 602.7

MINIMUM FEEDER AMPACITY: $\quad 753.3$

HNF-2487, Rev. 0

Page D-28 
Caic. No. $W-320-31-010$

Revision 1

Page No. C -3

DATE 7:21:1998 TIME: $17: 17: 28.59$

Project W-320 / Tank 241-C-106 S7uicing

PAGE 3

C-Farm Electrical Distribution

Calc. No. W320-31-010 (Reference Drawing H-2-818680, SH 1, Rev. 1)

FEEDER SIZE REPORT

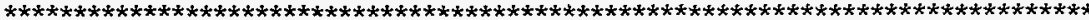

FEEDER SCHEDULE

\begin{tabular}{|c|c|c|c|c|c|c|c|c|c|c|c|}
\hline FEEDER & $\begin{array}{l}\text { ROUTING } \\
\text { NO NAME }\end{array}$ & $\begin{array}{r}\text { FEEDER } \\
\text { VOLTAGE }\end{array}$ & $\begin{array}{l}\mathrm{NO} \\
/ \mathrm{PH}\end{array}$ & $\begin{array}{l}\text { WIRE } \\
\text { QTY }\end{array}$ & $\begin{array}{l}\text { SIZE } \\
\text { FDR }\end{array}$ & $\begin{array}{l}\text { TYP } \\
\text { MA }\end{array}$ & & $\begin{array}{l}\text { INSUL } \\
\text { TYPE }\end{array}$ & $\begin{array}{l}\text { GROUND } \\
\text { WIRE }\end{array}$ & $\begin{array}{l}\text { RACEY } \\
\text { SIZE }\end{array}$ & $\begin{array}{l}\text { WAY } \\
\text { TY }\end{array}$ \\
\hline $20 M$ & $\underset{* * * * * * * * * * * * * * * * * * *}{\text { Ug Rsr PI E257 }}$ & 13800 & & & & & & & & & \\
\hline TO & XFMR C-T-1 Pri & EX & (1) & 3 & 2 & & CU & EPR & & $21 / 2^{\prime \prime}$ & $c$ \\
\hline $\begin{array}{l}\mathrm{EROM} \\
* * * * * *\end{array}$ & $\underset{* * * * * * *+* * * * * * * * * * *}{\text { XFMR C-T-1 Sec }}$ & 480. & & & & & & & & & \\
\hline TO & C-PDP-1 $600 \mathrm{~A}$ & EX & ( 2$)$ & 5 & 60 & & $\mathrm{cu}$ & THWN & $2 / 0$ & $31 / 2^{\prime \prime}$ & \\
\hline OM & $\underset{* * * * * * * * * * * * * * * * *}{\text { C-PDP-1 }}$ & 480 . & & & & & & & & & \\
\hline $\begin{array}{l}\text { TO } \\
\text { TO }\end{array}$ & $\begin{array}{l}\text { Xfmr Skid } \\
\text { MCC-N1 } 800 \mathrm{~A}\end{array}$ & $\begin{array}{l}E X \\
\text { EX }\end{array}$ & $\left(\begin{array}{l}1 \\
3\end{array}\right)$ & $\begin{array}{l}4 \\
5\end{array}$ & 35 & & $\begin{array}{l}C U \\
C U\end{array}$ & $\begin{array}{l}\text { MCXH } \\
\text { THWN }\end{array}$ & $2 / 0$ & $\begin{array}{l}D B \\
3^{\prime \prime} \mathrm{C}\end{array}$ & \\
\hline
\end{tabular}

FROM Process BTdg 480 .

$* * * * * * * * * * * * * * * * * * * * * * * * *$

TO C106-PP1 Bus EX

(1) 48 CU THHN $103 / 4^{\prime \prime} \mathrm{C}$

FROM MCC-N1 $800 \mathrm{~A}$

$* * * * * * * * * * * * * * * * * * * * * *$

TO Process B]dg

T0 C106-PP2 Bus

FROM Process Bidg $* * * * * * * * * * * * * * * * * * * * * * * * * *$

TO FN-1361 Bus

TO HC-1361 Bus

TO UH-1362 Bus

T0 UH-1361 Bus

FROM MCC-N1 800A

$* * * * * * * * * * * * * * * * * * * * * * * t$

TO Service Bldg

FROM Service BTdg $* * * * * * * * * * * * * * * * * * * * * * * *$

T0 CPR-1361 Bus

480.

EX (1) $4 \quad 2$ CU THWN 8 I $1 / 4^{\prime \prime}$ C M

$\begin{array}{lllllllllll} & \text { EX } & \text { ( 1) } & 4 & 12 & \mathrm{CU} & \mathrm{THWN} & 12 & 1 / 2^{\prime \prime} \mathrm{C} & \mathrm{M}\end{array}$

480 .

EX (1) 4 10 CU THHN $10 \quad 1 / 2^{\prime \prime} C \quad M$

EX (1) 4 4 4 CU THHN 8 I" $\mathrm{C} \quad M$

EX (1) 4 12 CU THHN 12 1/2" C M

EX (1) 4 12 CU THHN 12 1/2" C $M$

480 .

$\begin{array}{lllllllllll}\text { EX ( } 1) & 4 & 10 & \text { CU THWN } 10 & 1 / 2 " & C & M\end{array}$

480 .

EX

(1) 510 CU THWN $10 \quad 1 / 2^{11} \mathrm{C} \quad \mathrm{M}$

HNF-2487, Rev. 0

Page D-29 
Calc. No. W-320-31-010

Revision 1

Page No. C - 4

DATE $7: 21: 1998$ TIME:17:17:28.59

Project $W-320$ / Tank 241-C-106 Sluicing

PAGE 4

C-Farm Electrical Distribution

Ca1c. No. W320-31-010 (Reference Drawing H-2-818680, SH 1, Rev. 1)

FEEDER SIZE REPORT

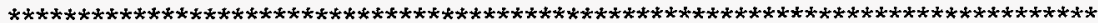

FEEDER SCHEDULE

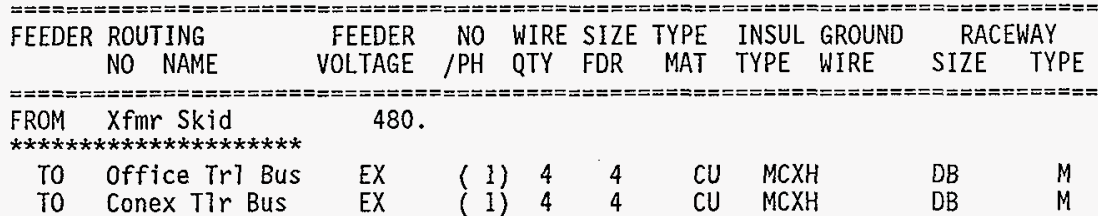

FR0M MCC-N1 800A $* * * * * * * * * * * * * * * * * * * * * * * *$

TO Chiller Skid

To Exhaust Skid

TO EXH Fan Vfd

480.

FROM Xfmr Skid

$* * * * * * * * * * * * * * * * * * * * * * *$

TO WRAM T1r Bus

$\begin{array}{llllllllll}\text { EX } & (1) & 4 & 4 / 0 & \text { CU } & \text { THWN } & 4 & 2^{\prime \prime} & \mathrm{C} & M \\ \text { EX } & (1) & 4 & 10 & \text { CU } & \text { THWN } & 10 & 1 / 2^{\prime \prime} & \mathrm{C} & M \\ \text { EX } & (1) & 4 & 12 & \text { CU } & \text { THWN } & 12 & 1 / 2^{\prime \prime} & \mathrm{C} & M\end{array}$

480.

$\begin{array}{llllllll}\text { EX (1) } & 4 & 4 & \mathrm{CU} & \mathrm{MCXH} & \mathrm{DB} & \mathrm{M}\end{array}$

FROM MCC-NI 800A

$* * * * * * * * * * * * * * * * * * * * * * * * *$

TO MCC-N2

TO Sluicer Skid

To Slurry Winch

FROM MCC-N2

$* * * * * * * * * * * * * * * * * * * * * * *$

TO Booster Vfd

TO P-1361 SS

480 .

$\begin{array}{llllllllll}\text { EX } & (2) & 5 & 350 & \text { CU } & \text { THWN } & 1 / 0 & 3 " C & M \\ \text { EX } & (1) & 4 & 12 & \text { CU } & \text { THWN } & 12 & 1 / 2 " & \text { C } & M \\ \text { EX } & (1) & 4 & 12 & \text { CU } & \text { THWN } & 12 & 1 / 2^{\prime \prime} & \text { C } & M\end{array}$

480 .

EX (1) 4500 CU THWN $3 \quad 3 " \mathrm{C} \quad M$

EX (1) 4 1/0 CU THWN 6 1 $1 / 2^{\prime \prime C N}$

FROM P-1361 SS

T0 P-1361 Bus

480.

EX (1) 4 1/0 CU THWN 6 1 $1 / 2^{\prime \prime C ~ M ~}$

FROM Booster Vfd

$* * * * * * * * * * * * * * * * * * * * * * *$

TO P-1362 Bus

480.

EX (1) 4500 CU THWN $3 \quad 3 " \mathrm{C} \quad M$

FROM EXH Fan Vfd

$* * * * * * * * * * * * * * * * * * * * * * * * *$

TO FN-1362 Bus

480 .

( 1) 312 CU THWN $1 / 2^{11} \mathrm{C} \quad M$

HNF-2487, Rev. 0

Page D-30 
Calc. No. H-320-31-010

Revision 1

Page No. C - 5

DATE $7: 21: 1998$ TIME: $17: 17: 28,59$

PAGE 5

Project W-320 / Tank 241-C-106 S7uicing

C-Farm Electrical Distribution

Calc. No. W320-31-010 (Reference Drawing H-2-818680, SH I, Rev. I)

FEEDER SIZE REPORT

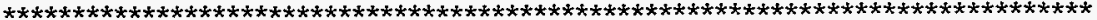

FEEDER SCHEDULE

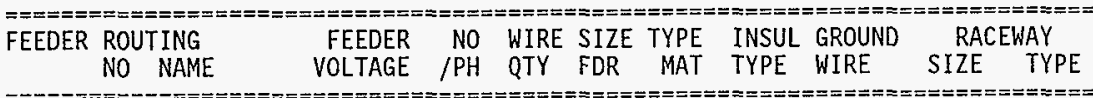

FROM Service B]dg 480 .

$* * * * * * * * * * * * * * * * * * * * * * * *$

TO C106-PP3 Bus

EX

(1) 410

CU THWN 10

$1 / 2^{\prime \prime C}$

M

HNF-2487, Rev. 0

Page D-31 
Calc. No. W-320-31-010

Revision 1

Page No. $C-6$

DATE $7: 21: 1998$ TIME: $17: 17: 28.59$

PAGE 6

Project W-320/Tank 24l-C-106 Sluicing

C-Farm Electrical Distribution

Calc. No. W320-31-010 (Reference Drawing H-2-818680, SH 1, Rev. I)

FEEDER EVALUATION

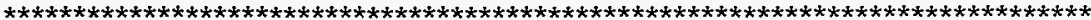

FEEDER DESIGN LOAD ANALYS I S

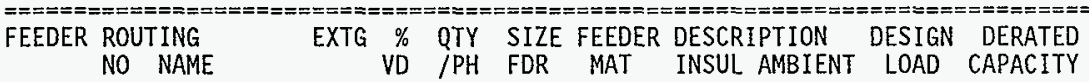

\begin{tabular}{|c|c|c|c|c|c|c|c|c|c|}
\hline $\begin{array}{l}\text { FROM } \\
* * * * * * *\end{array}$ & $\underset{* * * * * * * * * * * * * * * * * *}{\text { Ug Rsr P1 }}$ E257 & & 300. & & & & & & \\
\hline TO & XFMR C-T-1 Pri & EX & 0.02 & $I$ & $\mathrm{CU}$ & EPR & 30. & 24. $A$ & 150. A \\
\hline $\begin{array}{l}\text { FROM } \\
* * * * * * x\end{array}$ & 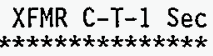 & & 480. & & & & & & \\
\hline 70 & $C-P D P-1 \quad 600 A$ & $E X$ & 0.13 & 2 & $\mathrm{CU}$ & THWN & 30. & 682. A & 840. A \\
\hline $\begin{array}{l}\text { FROM } \\
* * * * * * *\end{array}$ & 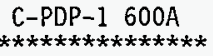 & & 480 . & & & & & & \\
\hline $\begin{array}{l}\text { T0 } \\
\text { T0 }\end{array}$ & $\begin{array}{l}\text { Xfmr skid } \\
\text { MCC-N1 } 800 \mathrm{~A}\end{array}$ & $\begin{array}{l}\text { EX } \\
\text { EX }\end{array}$ & $\begin{array}{l}0.14 \\
0.13\end{array}$ & $\begin{array}{l}1 \\
3\end{array}$ & $\begin{array}{l}\mathrm{CU} \\
\mathrm{CU}\end{array}$ & $\begin{array}{l}\text { MCXH } \\
\text { THWN }\end{array}$ & $\begin{array}{l}30 . \\
30 .\end{array}$ & $\begin{array}{r}\text { 60. A } \\
\text { 622. A }\end{array}$ & $\begin{array}{l}\text { 150. A } \\
\text { 930. A }\end{array}$ \\
\hline $\begin{array}{l}\text { FROM } \\
* * * * * * x\end{array}$ & 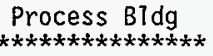 & & 480. & & & & & & \\
\hline T0 & C106-PP1 Bus & EX & 0.03 & 1 & $\mathrm{CU}$ & THHN & 70 . & 9. $A$ & 23. A \\
\hline $\begin{array}{l}\text { FROM } \\
* * * * * * x\end{array}$ & $\begin{array}{l}\text { MCC-N1 } 800 \mathrm{~A} \\
\text { M }\end{array}$ & & 480 . & & & & & & \\
\hline $\begin{array}{l}\text { T0 } \\
\text { TO }\end{array}$ & $\begin{array}{l}\text { Process Bidg } \\
\text { C106-PP2 Bus }\end{array}$ & $\begin{array}{l}E X \\
E X\end{array}$ & $\begin{array}{l}1.32 \\
0.06\end{array}$ & $\begin{array}{l}1 \\
1\end{array}$ & $\begin{array}{l}\mathrm{CU} \\
\mathrm{CU}\end{array}$ & $\begin{array}{l}\text { THWN } \\
\text { THWN }\end{array}$ & $\begin{array}{l}30 . \\
30 .\end{array}$ & $\begin{array}{l}\text { 77. A } \\
\text { 18. A }\end{array}$ & $\begin{array}{l}\text { 95. A } \\
\text { 20. A }\end{array}$ \\
\hline $\begin{array}{l}\text { FROM } \\
* * * * * * *\end{array}$ & $\underset{* * * * * * * * * * * * * * * * * * *}{\text { Process Bldg }}$ & & 480 . & & & & & & \\
\hline $\begin{array}{l}\text { T0 } \\
\text { TO } \\
\text { T0 } \\
\text { TO }\end{array}$ & $\begin{array}{ll}\text { FN-1361 } & \text { Bus } \\
\text { HC-1361 } & \text { Bus } \\
\text { UH-1362 } & \text { Bus } \\
\text { UH-1361 } & \text { Bus }\end{array}$ & $\begin{array}{l}E X \\
\text { EX } \\
E X \\
E X\end{array}$ & $\begin{array}{l}0.19 \\
0.20 \\
0.21 \\
0.12\end{array}$ & $\begin{array}{l}1 \\
1 \\
1 \\
1\end{array}$ & $\begin{array}{l}\mathrm{CU} \\
\mathrm{CU} \\
\mathrm{CU} \\
\mathrm{CU}\end{array}$ & $\begin{array}{l}\text { THHN } \\
\text { THHN } \\
\text { THHN } \\
\text { THHN }\end{array}$ & $\begin{array}{l}70 . \\
70 \\
70 \\
70\end{array}$ & $\begin{array}{r}\text { 14. A } \\
\text { 45. A } \\
\text { 6. A } \\
\text { 6. A }\end{array}$ & $\begin{array}{l}\text { 17. A } \\
\text { 41. A } \\
\text { 12. A } \\
\text { 12. A }\end{array}$ \\
\hline
\end{tabular}

FROM MCC-N1 800A 480.

$* * * * * * * * * * * * * * * * * * * * * *$

TO Service Bldg EX 0.4611 CU THWN 30. 20. A $30 . A$

FROM Service Bldg 480

$* * * * * * * * * * * * * * * * * * * * * * * *$

TO CPR-1361 Bus EX 0.0711 CU THWN 30. 14. A 30 . A

HNF-2487, Rev. 0

Page D-32 
Calc. No. W-320-31-010

DATE $7: 21: 1998$ TIME: $17: 17: 28.59$

PAGE 7

Project $\mathrm{H}-320$ / Tank $241-\mathrm{C}-106$ STuicing

C-Farm Electrical Distribution

Calc. No. W320-31-010 (Reference Drawing H-2-818680, SH 1, Rev. 1)

FEEDER EVALUATION

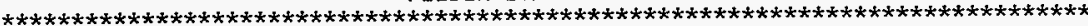

FEEDER DESIGN LOAD ANALYSIS

\begin{tabular}{|c|c|c|c|c|c|c|c|c|c|c|c|}
\hline FEEDER & $\begin{array}{l}\text { ROUTING } \\
\text { NO NAME }\end{array}$ & EXTC & $\%$ & $\begin{array}{l}\text { QTY } \\
\text { /PH }\end{array}$ & $\begin{array}{l}\text { SIZE } \\
\text { FDR }\end{array}$ & $\begin{array}{l}\text { FEEDER } \\
\text { MAT }\end{array}$ & $\begin{array}{l}\text { DESCRIP } \\
\text { INSUL A }\end{array}$ & $\begin{array}{l}\text { ION } \\
\text { BIENT }\end{array}$ & $\begin{array}{l}\text { DESIGN } \\
\text { LOAD }\end{array}$ & $C A$ & $\begin{array}{l}\text { RATED } \\
\text { ACITY }\end{array}$ \\
\hline & & & $==$ & & & $z==3$ & & & $=z$ & & \\
\hline $\operatorname{ROM}_{* * * * * *}$ & 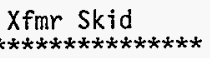 & & 480 . & & & & & & & & \\
\hline $\begin{array}{l}\text { TO } \\
\text { TO }\end{array}$ & $\begin{array}{l}\text { Office Trl Bus } \\
\text { Conex Tlr Bus }\end{array}$ & $\begin{array}{l}\text { EX } \\
\text { EX }\end{array}$ & $\begin{array}{l}0.11 \\
0.21\end{array}$ & $\begin{array}{l}1 \\
1\end{array}$ & & $\begin{array}{l}\mathrm{CU} \\
\mathrm{CU}\end{array}$ & $\begin{array}{l}\text { MCXH } \\
\text { MCXH }\end{array}$ & $\begin{array}{l}30 . \\
30 .\end{array}$ & $\begin{array}{l}20 . \\
20 .\end{array}$ & & $\begin{array}{l}\text { 70. A } \\
\text { 70. A }\end{array}$ \\
\hline $\begin{array}{l}\text { ROM } \\
* * * * *\end{array}$ & 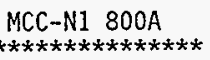 & & 480 . & & & & & & & & \\
\hline $\begin{array}{l}\text { TO } \\
\text { TO } \\
\text { TO }\end{array}$ & $\begin{array}{l}\text { Chiller Skid } \\
\text { Exhaust Skid } \\
\text { EXH Fan Vfd }\end{array}$ & $\begin{array}{l}\text { EX } \\
\text { EX } \\
\text { EX }\end{array}$ & $\begin{array}{l}0.10 \\
1.98 \\
0.08\end{array}$ & $\begin{array}{l}1 \\
1 \\
1\end{array}$ & & $\begin{array}{l}\mathrm{CU} \\
\mathrm{CU} \\
\mathrm{CU}\end{array}$ & $\begin{array}{l}\text { THWN } \\
\text { THWN } \\
\text { THWN }\end{array}$ & $\begin{array}{l}30 . \\
30 . \\
30\end{array}$ & $\begin{array}{r}109 . \\
16 . \\
14 .\end{array}$ & $\begin{array}{l}A \\
A \\
A\end{array}$ & $\begin{array}{r}230 \\
30 \\
20\end{array}$ \\
\hline
\end{tabular}

FROM Xfmr Skid 480.

$* * * * * * * * * * * * * * * * * * * * *$
TO WRAM TIr BUS EX $0.47 \quad 1$ CU MCXH 30. 20. A 70 . A

FROM MCC-N1 800A 480

$* * * * * * * * * * * * * * * * * * * * * * *$

TO MCC-N2 EX $0.28 \quad 2$

TO STuicer Skid EX $0.87 \quad 1$

TO S7urry Winch EX $0.44 \quad 1$

CU THWN 30. 392. A 620. A

CU THWN 30. 6. A 20. A

CU THWN 30. 3. A 20. A

FROM MCC-N2 480 .

$* * * * * * * * * * * * * * * * * * * * * * * *$

TO Booster Vfd EX 0.0611 CU THWN 30. 341. A 380. A

TO P-1361 SS EX $0.00 \quad 1$

CU THWN 30. 65. A 150. A

FROM P-1361 SS 480 .

$* * * * * * * * * * * * * * * * * * * * * *$
TO P-1361 BuS EX 0.8511 CU THWN 30. 65. A $150 . A$

FROM Booster Vfd 480.

$* * * * * * * * * * * * * * * * * * * * * * *$

TO P-1362 Bus EX 1.9011 CU THWN 44. 341. A 380. A

FROM EXH Fan Vfd 480.

$* * * * * * * * * * * * * * * * * * * * * * * *$

TO FN-1362 Bus

2.49112 CU THWN 30. 14. A 20. A

HNF-2487, Rev. 0

Page D-33 
Calc. No. H-320-31-010 Revision 1

Page No. C -8

DATE 7:21:1998 TIME:17:17:28.59

PAGE 8 Project $\mathrm{H}-320$ / Tank 241-C-106 Sluicing C-Farm Electrical Distribution

Calc. No. W320-31-010 (Reference Drawing H-2-818680, SH I, Rev. 1)

FEEDER EVALUATION

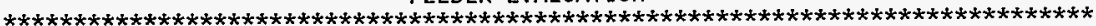
FEE D E R
DES I GN
$\angle O A D$
A N A L Y S I S

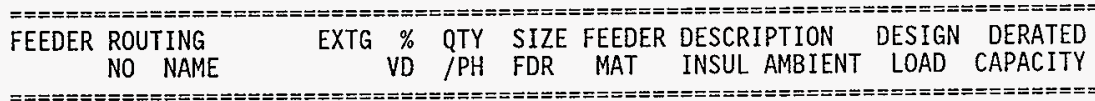

$========== \pm== \pm= \pm====== \pm= \pm=2$
FROM Service Bldg $\quad 480$.

$* * * * * * * * * * * * * * * * * * * * * * * * *$

TO C106 PP3 Bus EX $0.01 \quad 1 \quad$ CU THWN 30 . 6. A 30. A

HNF-2487, Rev. 0

Page D-34 
Calc. No. W-320-31-010

Revision 1

Page No. C -9

DATE 7:21:1998 TIME: 17:17:28.59

Project $W-320$ / Tank 241-C-106 Sluicing

PAGE

C-Farm Electrical Distribution

Calc. No. W320-31-010 (Reference Drawing H-2-818680, SH 1, Rev. 1)

TRANSFORMER SIZE REPORT

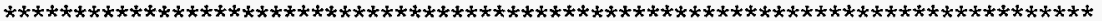

TRANSFORMER SCHEDULE

LOCATION DESCRIPTION VOLTAGE CONN PCT. TRANSFORMER DESCRIPTION
BUS NO. NAME
LEVELS CODE TAP

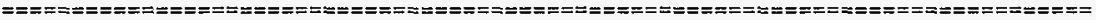

FROM XFMR C-T-1 Pri 13800. D -2.5 TYPE: <US SIZE:

TOr XFMR C-T-I SEC 480. YG DESCRIPTION: XFMR C-T-1

DEMAND LOAD: $501.1 \mathrm{KVA}$ EX $3.19 \% \mathrm{Z}$ NOMINAL RATING

0.0 KVA

DEMAND LOAD: 501.1 KVA

0.0 KVA

HNF-2487, Rev. 0

Page D.35 
Calc. No. W-320-31-010 Revision 1

Attachment D

HNF-2487, Rev. 0

Page D-36 
Calc. No. W-320-31-010

Revision 1

Page No. D - I

Project W-320 / Tank 241-C-106 Sluicing

C-Farm Electrical Distribution

Calc. No. W320-31-010 (Reference Drawing H-2-818680, SH 1, Rev. 1)

DATE: $7: 21: 1998$

TIME: $17: 17: 54.68$

ALL. INFORMATION PRESENTED IS FOR REVIEW, APPROVAL

INTERPRETATION AND APPLICATION BY A REGISTERED ENGINEER ONL $Y$

SKM POWER*TOOLS FOR WINDOWS

LOAD FLOW AND VOLTAGE DROP ANALYSIS REPORT

COPYRIGHT SKM SYSTEMS ANALYSIS, INC. 1995-1996

HNF-2487, Rev. 0

Page D-37 
Calc. No. W-320-31-010

Revision 1

Page No. D - 2

DATE: $7: 21: 1998$ TIME: $17: 17: 54.68$

Project $W-320$ / Tank 241-C-106 STuicing

PAGE 2

C-Farm Electrical Distribution

Calc. No. W320-31-010 (Reference Drawing H-2-818680, SH 1, Rev. 1)

\begin{tabular}{|c|c|}
\hline SOLUTION PARAMETERS & \\
\hline $\begin{array}{l}\text { BRANCH VOLTAGE CRITERIA } \\
\text { BUS VOLTAGE CRITERIA }\end{array}$ & $\begin{array}{l}\quad 3.00 \% \\
: \quad 5.00 \%\end{array}$ \\
\hline $\begin{array}{l}\text { ACCELERATION FACTOR FOR 'PV' GENERATORS } \\
\text { ACCELERATION FACTOR FOR CONSTANT KVA LOADS } \\
\text { EXACT (ITERATIVE) SOLUTION }\end{array}$ & $\begin{array}{l}1.00 \\
1.00 \\
\text { YES }\end{array}$ \\
\hline $\begin{array}{l}\text { UTILITY IMPEDANCE } \\
\text { TRANSFORMER PHASE SHIFT }\end{array}$ & $\begin{array}{ll}: & \text { YES } \\
\vdots & \text { NO }\end{array}$ \\
\hline
\end{tabular}

ALL PU VALUES ARE EXPRESSED ON A 100 MVA BASE

LOAD FLOW IS BASED ON CALCULATED DEMAND LOAD RESULTS

FROM THE DEMAND LOAD ANALYSIS STUDY.

LOAD ANALYSIS INCLUDES ALL LOADS.

〈<PERCENT VOLTAGE DROPS ARE BASED ON NOMINAL DESIGN VOLTAGES〉〉

SWING GENERATORS

$\begin{array}{llc}\text { SOURCE NAME } & \text { YOLTAGE } & \text { ANGLE } \\ =========================== \\ \text { C8-L6 } 13.8 \mathrm{KV} & 1.030 & 0.00\end{array}$

LARGEST LOAD:

CONVERGENCE CRITERIA:

LARGEST BUS MISMATCH

LARGEST BUS MISMATCH

LARGEST BUS MISMATCH

LARGEST BUS MISMATCH

0.011 KVA
0.96 KVA
P-1362 Bus
P-1362 Bus
P-1362 Bus
P-1362 Bus

$10.559 \mathrm{KVA}$

$0.766 \mathrm{KVA}$

$0.060 \mathrm{KVA}$

$0.005 \mathrm{KVA}$

HNF-2487, Rev. 0

Page D-38 
Calc. No, W-320-31-010

Revision 1

Page No. D - 3

DATE: $7: 21: 1998$ TIME: $17: 17: 54.68$

PAGE

3

Project W-320 / Tank 241-C-106 Sluicing

C-Farm Electrical Distribution

Calc. No. W320-31-010 (Reference Drawing H-2-818680, SH 1, Rev. 1)

BALANCED VOLTAGE DROP AND LOAD FLOW ANALYSIS (SWING GENERATORS)

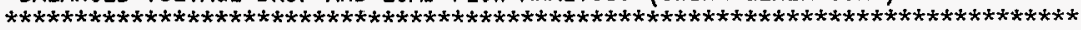

SOURCE VOLTAGE ANGLE KW KVAR VD\% (UTILITY IMPEDANCE)

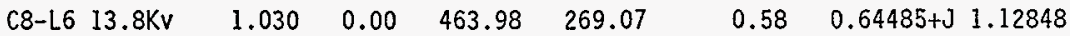

HNF-2487, Rev. 0

Page D-39 
HNF-2487, Rev. 0

Page D-40

Calc. No. W-320-31-010

Revision 1

Page No. D -4

DATE: $7: 21: 1998$ TIME: $17: 17: 54.68$

Project $W-320$ / Tank 241-C-106 Sluicing

PAGE

C-Farm Electrical Distribution

Calc. No. W320-31-010 (Reference Drawing H-2-818680, SH 1, Rev. 1)

BALANCED VOLTAGE DROP AND LOAD FLOW ANALYSIS

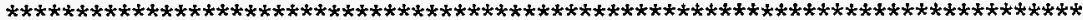

VOLTAGE EFFECT ON LOADS MODELED

VOLTAGE DROP CRITERIA: BRANCH $=3.00 \%$ BUS $=5.00$

$====$ BUS: Ug RSP PI E257 DESIGN VOLTS: 13800 BUS VOLTS: $14133 \%$ VD: -2.42

$========= \pm== \pm=========$ PU BUS VOLTAGE: 1.024 *** SWING GENERATOR: C8-L6 13.8KV

ANGLE: $\quad-0.2$ DEGREES

$464.0 \mathrm{KW} 259.1 \mathrm{KVAR}$

LOAD T0: XFMR C-T-1 Pri FEEDER AMPS: 21.7 VOLTAGE DROP: 2. \%VD: 0.02 PROJECTED POWER FLOW: $462.2 \mathrm{KW} 266.0$ KVAR $533.3 \mathrm{KVA}$ PF: 0.87 LAGGING LOSSES THRU FEEDER:

$0.1 \mathrm{KW} \quad 0.0 \mathrm{KVAR}$

$0.1 \mathrm{KVA}$

$====$ BUS: XFMR C - T-1 Pri DESIGN VOLTS: 13800 BUS VOLTS: $14131 \%$ VD: -2.40 $== \pm=== \pm== \pm== \pm===== \pm==== \pm$ PU BUS VOLTAGE: 1.024 ANGLE: -0.2 DEGREES

LOAD FROM: Ug Rsr P1 E257 FEEDER AMPS: 21.7 VOLTAGE DROP: 2. \%VD: 0.02 PROJECTED POWER FLOW: $462.2 \mathrm{KW} 266.0$ KVAR $533.2 \mathrm{KVA}$ PF: 0.87 LAGGING LOSSES THRU FEEDER: $\quad 0.1 \mathrm{KW} \quad 0.0 \mathrm{KVAR} \quad 0.1 \mathrm{KVA}$

LOAD TO: XFMR C-T-1 SeC TRANSF AMPS: 21.7 VOLTAGE DROP: $407 . \%$ VD: 2.95 PROJECTED POWER FLOW: $462.2 \mathrm{KW} 266.0 \mathrm{KVAR}$ 533.2 KVA PF: 0.87 LAGGING LOSSES THRU TRANSF: $\quad 13.8 \mathrm{KW} \quad 33.9$ KVAR $36.5 \mathrm{KVA}$

$= \pm==$ BUS: XFMR C-T-1 Sec DESIGN VOLTS: 480 BUS VOLTS: $477 \%$ VD: 0.55 $===================== \pm$ PU BUS VOLTAGE: 0.994 ANGLE: -2.7 DEGREES

LOAD FROM: XFMR C-T-1 Pri TRANSF AMPS: 20.6 VOLTAGE DROP: $14 . \%$ VD: 2.95 PROJECTED POWER FLOW: $462.2 \mathrm{KW} 266.0 \mathrm{KVAR} 533.2 \mathrm{KVA}$ PF: 0.87 LAGGING LOSSES THRU TRANSF: $\quad 13.8 \mathrm{KW} 33.9 \mathrm{KVAR} 36.5 \mathrm{KVA}$

LOAD TO: C-PDP-1 600A FEEDER AMPS: 610.6 VOLTAGE DROP: 1. \%VD: 0.12 PROJECTED POWER FLOW: $448.4 \mathrm{KW}$ 232.1 KVAR 504.9 KVA PF: 0.89 LAGGING LOSSES THRU FEEDER: $\quad 0.3 \mathrm{KW} \quad 0.7$ KVAR $0.8 \mathrm{KVA}$

$====$ BUS: C-PDP $-1 \quad 600 \mathrm{~A}$ DESIGN VOLTS: 480 BUS VOLTS: $477 \%$ VD: 0.67

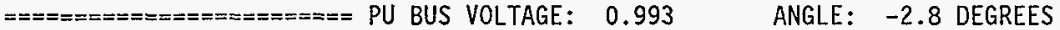

LOAD FROM: XFMR C-T-1 SeC FEEDER AMPS: 610.6 VOLTAGE DROP: $1 . \%$ VD: 0.12 PROJECTED POWER FLOW: $448.0 \mathrm{KW} 231.5 \mathrm{KVAR} 504.3 \mathrm{KVA}$ PF: 0.89 LAGGING LOSSES THRU FEEDER: $0.3 \mathrm{KW} \quad 0.7 \mathrm{KVAR} \quad 0.8 \mathrm{KVA}$

LOAD TO: Xfmr Skid PROJECTED POWER FLOW: LOSSES THRU FEEDER:
FEEDER AMPS: 59.2 VOLTAGE DROP: $44.1 \mathrm{KW} 21.3 \mathrm{KVAR}$ $0.1 \mathrm{KW}$

$0.0 \mathrm{KVAR}$

$0.1 \mathrm{KVA}$
1. \%VD: 0.14 PF: 0.90 LAGGING 
Calc. No. $W-320-31-010$

Revision 1

Page No. D - 5

DATE: $7: 21: 1998$ TIME: $17: 17: 54.68$

PAGE 5

Project $W-320$ / Tank 241-C-106 Sluicing

C-Farm Electrical Distribution

Calc. No. W320-31-010 (Reference Drawing H-2-818680, SH 1, Rev. 1)

BALANCED VOLTAGE DROP AND LOAD FLOW ANALYSIS

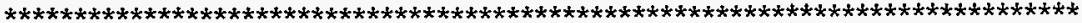

VOLTAGE EFFECT ON LOADS MODELED

VOLTAGE DROP CRITERIA: $B R A N C H=3.00 \%$ BUS $=5.00$

LOAD TO: MCC-NI 800A

FEEDER AMPS: 551.4 VOLTAGE DROP:

1. \%VD: 0.11

PROJECTED POWER FLOW: $404.0 \mathrm{KW}$ 210.2 KVAR 455.4 KVA PF: 0.89 LAGGING LOSSES THRU FEEDER:

$0.4 \mathrm{KW}$

0.5 KVAR

$0.6 \mathrm{KVA}$

$===$ BUS: Xfmr Skid

DESIGN VOLTS:

PU BUS VOLTAGE:

480 BUS VOLTS:

$476 \%$ VD: 0.81

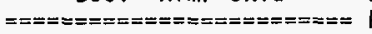

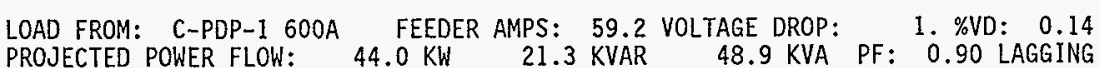

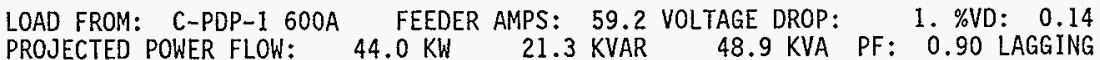
LOSSES THRU FEEDER: $\quad 0.1 \mathrm{KW} 0.0 \mathrm{KVAR} 0.1 \mathrm{kVA}$

LOAD TO: Office Tr] Bus FEEDER AMPS: 19.7 VOLTAGE DROP: $1 . \%$ VD: 0.11 PROJECTED POWER FLOW: $14.7 \mathrm{KW} \quad 7.1$ KVAR $16.3 \mathrm{KVA}$ PF: 0.90 LAGGING LOSSES THRU FEEDER: $\quad 0.0 \mathrm{KW} 0.0 \mathrm{KVAR} 0.0 \mathrm{KVA}$

LOAD TO: Conex TIr BuS FEEDER AMPS: 19.7 VOLTAGE DROP: $1 . \%$ VD: 0.21 PROJECTED POWER FLOW: $14.7 \mathrm{KW} 7.1 \mathrm{KVAR} 16.3 \mathrm{KVA}$ PF: 0.90 LAGGING LOSSES THRU FEEDER: $\quad 0.0 \mathrm{KW} 0.0 \mathrm{KVAR} \quad 0.0 \mathrm{KVA}$

LOAD TO: WRAM TTr BUS FEEDER AMPS: 19.7 VOLTAGE DROP: $2 . \%$ VD: 0.46 PROJECTED POWER FLOW: $14.6 \mathrm{KW} \quad 7.1$ KVAR $16.3 \mathrm{KVA}$ PF: 0.90 LAGGING LOSSES THRU FEEDER: $0.1 \mathrm{KW} 0.0 \mathrm{KVAR} \quad 0.1 \mathrm{KVA}$

$====$ BUS: MCC $-N 1800 \mathrm{~A}$ DESIGN VOLTS: 480 BUS VOLTS: $476 \%$ VD: 0.79 $==================== \pm$ PU BUS VOLTAGE: 0.992 ANGLE: -2.8 DEGREES

LOAD FROM: C-PDP-1 600A FEEDER AMPS: 551.4 VOLTAGE DROP: $1 . \%$ VD: 0.11 PROJECTED POWER FLOW: $403.6 \mathrm{KW}$ 209.7 KVAR 454.9 KVA PF: 0.89 LAGGING LOSSES THRU FEEDER: $0.4 \mathrm{KW} 0.5 \mathrm{KVAR} 0.6 \mathrm{KVA}$

LOAD T0: ProcesS BIdg FEEDER AMPS: 62.6 VOLTAGE DROP: $5 . \%$ VD: 1.07 PROJECTED POWER FLOW: $51.0 \mathrm{KW}$ 8.7 KVAR $51.7 \mathrm{KVA}$ PF: 0.99 LAGGING LOSSES THRU FEEDER: $\quad 0.5 \mathrm{~kW} 0.2 \mathrm{KVAR} 0.6 \mathrm{KVA}$

LOAD T0: C106-PP2 BuS FEEDER AMPS: 17.8 VOLTAGE DROP: $0 . \%$ VD: 0.06 PROJECTED POWER FLOW: $13.3 \mathrm{KW}$ 6.4 KVAR $14.8 \mathrm{KVA}$ PF: 0.90 LAGGING LOSSES THRU FEEDER: $\quad 0.0 \mathrm{KW} \quad 0.0 \mathrm{KVAR} \quad 0.0 \mathrm{KVA}$

LOAD TO: Service B]dg FEEDER AMPS: 17.0 VOLTAGE DROP: $2 . \%$ VD: 0.40 PROJECTED POWER FLOW: $11.8 \mathrm{KW} \quad 7.6$ KVAR $14.0 \mathrm{KVA}$ PF: 0.84 LAGGING LOSSES THRU FEEDER: $0.1 \mathrm{KW} 0.0 \mathrm{KVAR} \quad 0.1 \mathrm{KVA}$ 
Câlc. No. W-320-31-010

Revision 1

Page No. D - 6

DATE: $7: 21: 1998$ TIME: $17: 17: 54.68$

Project W-320 / Tank 241-C-106 Sluicing

PAGE 6

C-Farm Electrical Distribution

Calc. No. W320-31-010 (Reference Drawing H-2-818680, SH 1, Rev. 1)

BALANCED VOLTAGE DROP AND LOAD FLOW ANALYSIS

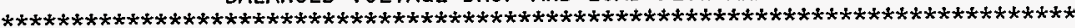

VOLTAGE EFFECT ON LOADS MODELED

VOLTAGE DROP CRITERIA: BRANCH $=3.00 \%$ BUS $=5.00$

LOAD TO: Chiller Skid FEEDER AMPS: 92.6 VOLTAGE DROP: $0 . \%$ VD: 0.08 PROJECTED POWER FLOW: $61.5 \mathrm{KW}$ 45.4 KVAR $76.4 \mathrm{KVA}$ PF: 0.80 LAGGING LOSSES THRU FEEDER: $\quad 0.0 \mathrm{KW} \quad 0.0 \mathrm{KVAR} \quad 0.1 \mathrm{KVA}$

LOAD TO: Exhaust Skid FEEDER AMPS: 13.8 VOLTAGE DROP: 8. \%VD: 1.72 PROJECTED POWER FLOW: $10.5 \mathrm{KW}$ 4.5 KVAR LOSSES THRU FEEDER:

$0.2 \mathrm{KW} \quad 0.0 \mathrm{KVAR}$

I1.4 KVA PF: 0.92 LAGGING

LOAD TO: EXH Fan Vfd

FEEDER AMPS: 11.3 VOLTAGE DROP:

0. \%VD: 0.06 PROJECTED POWER FLOW:

LOSSES THRU FEEDER:

$7.6 \mathrm{KW} \quad 5.4 \mathrm{KVAR}$

$0.0 \mathrm{KW} \quad 0.0 \mathrm{KVAR}$

9.3 KVA PF: 0.81 LAGGING

LOAD TO: MCC-N2

PROJECTED POWER FLOW:

FEEDER AMPS: 332.5 VOLTAGE DROP:

1. \%VD: 0.24

LOSSES THRU FEEDER:

$242.7 \mathrm{KW} 127.8 \mathrm{KVAR}$

$0.5 \mathrm{KW}$

0.6 KVAR

274.3 KVA PF: 0.88 LAGGING

LOAD TO: STuicer Skid PROJECTED POWER FLOW:

FEEDER AMPS:

5.2 VOLTAGE DROP:

3. \%VD: 0.71

LOSSES THRU FEEDER:

$3.5 \mathrm{KW}$

2.5 KVAR

$0.0 \mathrm{KW}$

0.0 KVAR

4.3 KVA PF:

$0.0 \mathrm{KVA}$

FEEDER AMPS: 2.6 VOLTAGE DROP:

2. \%VD: 0.35

LOAD TO: STurry Winch

PROJECTED POWER FLOW:

$1.7 \mathrm{KW}$

LOSSES THRU FEEDER:

$0.0 \mathrm{KW}$

1.3 KVAR

0.0 KVAR

2.2 KVA PF:

$0.0 \mathrm{KVA}$

0.80 LAGGING

$====$ BUS: C106-PPI BUS DESIGN VOLTS: 480 BUS VOLTS: $471 \%$ VD: 1.89

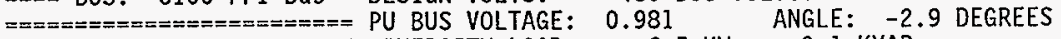
NET BRANCH DIVERSITY LOAD: $\quad 6.5 \mathrm{KW} \quad 3.1 \mathrm{KVAR}$

LOAD FROM: Process BIdg PROJECTED POWER FLOW:

LOSSES THRU FEEDER:

FEEDER AMPS: 8.8 VOLTAGE DROP:

$0 . \% \mathrm{VD}: 0.03$

$\begin{array}{lll}6.5 \mathrm{KW} & 3.1 \mathrm{KVAR} & 7.2 \mathrm{KVA} \text { PF: } 0.90 \text { LAGGING }\end{array}$ $0.0 \mathrm{KW}$

0.0 KVAR

0.0 KVA

$====$ BUS: Process Bldg DESIGN VOLTS: 480 BUS VOLTS: $471 \%$ VD: 1.86 $======== \pm=====0=======0$ PU BUS VOLTAGE: 0.981 ANGLE: -2.9 DEGREES

LOAD FROM: MCC-N1 800A PROJECTED POWER FLOW: LOSSES THRU FEEDER:
FEEDER AMPS: 62.6 VOLTAGE DROP: $50.4 \mathrm{KW}$ $0.5 \mathrm{KW}$ 8.6 KVAR 0.2 KVAR $0.6 \mathrm{KVA}$
5. \%VD: 1.07 0.99 LAGGING 
Calc. No. W-320-31-010

Revision 1

Page No. D - 7

DATE: $7: 21: 1998$ TIME: $17: 17: 54.68$

PAGE 7

Project $\mathrm{W}-320$ / Tank 24I-C-106 S7uicing

C-Farm Electrical Distribution

Calc. No. W320-31-010 (Reference Drawing H-2-818680, SH 1, Rev. 1)

BALANCED VOLTAGE DROP AND LOAD FLOW ANALYSIS

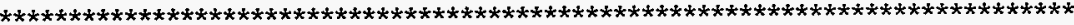

VOLTAGE EFFECT ON LOADS MODELED

VOLTAGE DROP CRITERIA: $B$ RANCH $=3.00 \%$ BUS $=5.00$

LOAD TO: C106-PPI BUS FEEDER AMPS: 8.8 VOLTAGE DROP: $0 . \%$ VD: 0.03 PROJECTED POWER FLOW: $\quad 6.5 \mathrm{KW} 3.1 \mathrm{KVAR} \quad 7.2 \mathrm{KVA}$ PF: 0.90 LAGGING LOSSES THRU FEEDER:

$0.0 \mathrm{KW} \quad 0.0 \mathrm{KVAR} \quad 0.0 \mathrm{KVA}$

LOAD T0: FN-1361 BUS

FEEDER AMPS: 11.2 VOLTAGE DROP:

1. \%VD: 0.15

PROJECTED POWER FLOW:

LOSSES THRU FEEDER:

$7.4 \mathrm{KW}$

5.4 KVAR

9.2 KVA PF: 0.8I LAGGING

$0.0 \mathrm{KW}$

0.0 KVAR

$0.0 \mathrm{KVA}$

LOAD TO: HC-1361 Bus

PROJECTED POWER FLOW:

FEEDER AMPS: 35.3 VOLTAGE DROP:

1. \%VD: 0.15

LOSSES THRU FEEDER:

$28.9 \mathrm{KW}$

0.0 KVAR

28.9 KVA PF: 1.00 UNITY

$0.0 \mathrm{KW}$

$0.0 \mathrm{KVAR}$

$0.0 \mathrm{KVA}$

LOAD TO: UH-1362 Bus

FEEDER AMPS: 4.7 VOLTAGE DROP:

1. \%VD: 0.16

PROJECTED POWER FLOW:

LOSSES THRU FEEDER:

$3.8 \mathrm{KW}$

0.0 KVAR

$0.0 \mathrm{KW}$

0.0 KVAR

3.8 KVA PF: 1.00 UNITY

LOAD TO: UH-1361 Bus

FEEDER AMPS: 4.7 VOLTAGE DROP:

$0 . \% V D: 0.10$ PROJECTED POWER FLOW:

$3.8 \mathrm{KW}$ 0.0 KVAR LOSSES THRU FEEDER:

$0.0 \mathrm{KW}$

$0.0 \mathrm{KVAR}$

3.8 KVA PF: 1.00 UNITY

$====$ BUS: C106-PP2 BuS DESIGN VOLTS:

$=====================$ PU BUS VOLTAGE: 0.992

480 BUS VOLTS:

$476 \%$ VD: 0.84 NET BRANCH DIVERSITY LOAD:

$13.3 \mathrm{KW}$

ANGLE: -2.8 DEGREES 6.4 KVAR

LOAD FROM: MCC-NI 800A

FEEDER AMPS: 17.8 VOLTAGE DROP:

$0 . \% \mathrm{VD}: 0.06$

PROJECTED POWER FLOW:

$13.3 \mathrm{KW}$

6.4 KVAR

LOSSES THRU FEEDER:

$0.0 \mathrm{KW}$

0.0 KVAR

14.7 KVA PF:

$0.0 \mathrm{KVA}$

0.90 LAGGING

$====$ BUS: FN-1361 BUS DESIGN VOLTS: 480 BUS VOLTS: $470 \%$ VD: 2.01

$===x=======0=========0$ PU BUS VOLTAGE: 0.980 ANGLE: -2.8 DEGREES NET BRANCH DIVERSITY LOAD: $\quad 7.4 \mathrm{KW} \quad 5.4$ KVAR

LOAD FROM: Process BIdg

PROJECTED POWER FLOW:

LOSSES THRU FEEDER:
FEEDER AMPS: 11.2 VOLTAGE DROP:

1. \%VD: 0.15

$7.4 \mathrm{KW} \quad 5.4 \mathrm{KVAR}$

$0.0 \mathrm{KW}$

0.0 KVAR

9.1 KVA PF: 0.81 LAGGING 0.0 KVA

HNF-2487, Rev. 0

Page D-43 
Calc. No. W-320-31-010

Revision 1

Page No. D - 8

DATE: $7: 21: 1998$ TIME: $17: 17: 54.68$

Project W-320 / Tank 241-C-106 Sluicing

PAGE 8

C-Farm Electrical Distribution

Calc. No. W320-31-010 (Reference Drawing H-2-818680, SH 1, Rev. 1)

BALANCED VOLTAGE DROP AND LOAD FLOW ANALYSIS

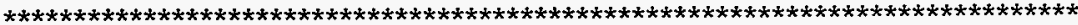

VOLTAGE EFFECT ON LOADS MODELED

VOLTAGE DROP CRITERIA: BRANCH $=3.00 \%$ BUS $=5.00$

$====$ BUS: HC -1361 BUS DESIGN VOLTS: 480 BUS VOLTS: $470 \%$ VD: 2.01

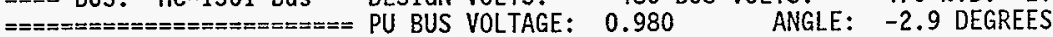

NET BRANCH DIVERSITY LOAD: $28.8 \mathrm{KW} 0.0 \mathrm{KVAR}$

LOAD FROM: Process BIdg FEEDER AMPS: 35.3 VOLTAGE DROP: 1 \%VD: 0.15 PROJECTED POWER FLOW: $28.8 \mathrm{KW}$ O.0 KVAR $28.8 \mathrm{KVA}$ PF: 1.00 UNITY LOSSES THRU FEEDER: $\quad 0.0 \mathrm{KW} \quad 0.0 \mathrm{KVAR} \quad 0.0 \mathrm{KVA}$

$====$ BUS: UH-1362 BUS DESIGN VOLTS: 480 BUS VOLTS: $470 \%$ VD: 2.02 $===== \pm================$ PU BUS VOLTAGE: 0.980 ANGLE: -2.9 DEGREES NET BRANCH DIVERSITY LOAD: $\quad 3.8 \mathrm{KW} \quad 0.0 \mathrm{KVAR}$

LOAD FROM: ProcesS Bldg FEEDER AMPS: 4.7 VOLTAGE DROP: $1 . \%$ VD: 0.16 PROJECTED POWER FLOW: $3.8 \mathrm{KW} \quad 0.0 \mathrm{KVAR} \quad 3.8 \mathrm{KVA}$ PF: 1.00 UNITY LOSSES THRU FEEDER: $\quad 0.0 \mathrm{KW} \quad 0.0 \mathrm{KVAR} \quad 0.0 \mathrm{KVA}$

$====$ BUS: UH-1361 BUS DESIGN VOLTS: 480 BUS VOLTS: $471 \%$ VD: 1.95 $===========0=========$ PU BUS VOLTAGE: 0.980 ANGLE: -2.9 DEGREES NET BRANCH DIVERSITY LOAD: $3.8 \mathrm{KW} 0.0 \mathrm{KVAR}$

LOAD FROM: Process BTdg FEEDER AMPS: 4.7 VOLTAGE DROP: $0 . \%$ VD: 0.10 PROJECTED POWER FLOW: $3.8 \mathrm{KW} \quad 0.0 \mathrm{KVAR} \quad 3.8 \mathrm{KVA}$ PF: 1.00 UNITY LOSSES THRU FEEDER: $\quad 0.0 \mathrm{KW} 0.0 \mathrm{KVAR} \quad 0.0 \mathrm{KVA}$

$====$ BUS: Service BTdg DESIGN VOLTS: 480 BUS VOLTS: $474 \%$ VD: 1.18

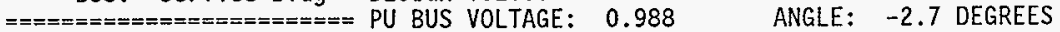

LOAD FROM: MCC-N1 800A FEEDER AMPS: 17.0 VOLTAGE DROP: 2 \%VD: 0.40 PROJECTED POWER FLOW: $11.8 \mathrm{KW} \quad 7.6 \mathrm{KVAR} 14.0 \mathrm{KVA}$ PF: 0.84 LAGGING LOSSES THRU FEEDER: $\quad 0.1 \mathrm{KW} 0.0 \mathrm{KVAR} 0.1 \mathrm{KVA}$

LOAD TO: C106-PP3 BuS FEEDER AMPS: 5.9 VOLTAGE DROP: $0 . \%$ VD: 0.01 PROJECTED POWER FLOW: $4.4 \mathrm{KW}$ 2.1 KVAR $4.9 \mathrm{KVA}$ PF: 0.90 LAGGING LOSSES THRU FEEDER: $\quad 0.0 \mathrm{KW} 0.0 \mathrm{KVAR} \quad 0.0 \mathrm{KVA}$

LOAD T0: CPR-1361 BUS FEEDER AMPS: 11.1 VOLTAGE DROP: $0 . \%$ VD: 0.06 PROJECTED POWER FLOW: $7.4 \mathrm{KW} \quad 5.4$ KVAR $9.2 \mathrm{KVA}$ PF: 0.81 LAGGING LOSSES THRU FEEDER: $0.0 \mathrm{KW} 0.0 \mathrm{KVAR} \quad 0.0 \mathrm{KVA}$

HNF-2487, Rev. 0

Page D-44 
Caic. No. W-320-31-010

Revision 1

Page No. D - 9

DATE: $7: 21: 1998$ TIME: $17: 17: 54.68$

PAGE 9

Project $W-320$ / Tank 241-C-106 Sluicing

C-Farm Electrical Distribution

Calc. No. W320-31-010 (Reference Drawing H-2-818680, SH 1, Rev. 1)

BALANCED VOLTAGE DROP AND LOAD FLOW ANALYSIS

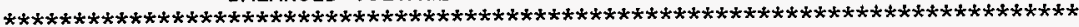

VOLTAGE EFFECT ON LOADS MODELED

VOLTAGE DROP CRITERIA: BRANCH $=3.00 \%$ BUS $=5.00$

$====$ BUS: C106-PP3 BUS DESIGN VOLTS: 480 BUS VOLTS: $474 \%$ VD: 1.20

$========== \pm== \pm==== \pm==0$ PU BUS VOLTAGE: 0.988 ANGLE: -2.7 DEGREES NET BRANCH DIVERSITY LOAD: $4.4 \mathrm{KW} 2.1 \mathrm{KVAR}$

LOAD FROM: Service BTdg FEEDER AMPS: 5.9 VOLTAGE DROP: $0 . \%$ VD: 0.01 PROJECTED POWER FLOW: $\quad 4.4 \mathrm{KW}$ 2.1 KVAR $4.9 \mathrm{KVA}$ PF: 0.90 LAGGING LOSSES THRU FEEDER: $\quad 0.0 \mathrm{KW} \quad 0.0 \mathrm{KVAR} 0.0 \mathrm{KVA}$

$====$ BUS: CPR-136I BUS DESIGN VOLTS: 480 BUS VOLTS: $474 \%$ VD: 1.24

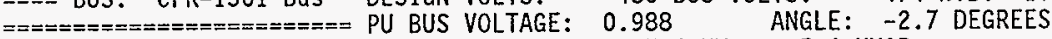
NET BRANCH DIVERSITY LOAD: $\quad 7.4 \mathrm{KW} \quad 5.4 \mathrm{KVAR}$

LOAD FROM: Service BTdg FEEDER AMPS: 11.1 VOLTAGE DROP: $0 . \%$ VD: 0.06 PROJECTED POWER FLOW: $7.4 \mathrm{KW}$ 5.4 KVAR $9.1 \mathrm{KVA}$ PF: 0.81 LAGGING LOSSES THRU FEEDER: $\quad 0.0 \mathrm{KW} \quad 0.0 \mathrm{KVAR} \quad 0.0 \mathrm{KVA}$

$====$ BUS: Office Trl BUS DESIGN VOLTS: 480 BUS VOLTS: $476 \%$ VD: 0.92 $======================$ PU BUS VOLTAGE: 0.991 ANGLE: -2.8 DEGREES NET BRANCH DIVERSITY LOAD: $14.7 \mathrm{KW} \quad 7.1 \mathrm{KVAR}$

LOAD FROM: Xfmr Skid PROJECTED POWER FLOW: LOSSES THRU FEEDER:
FEEDER AMPS: 19.7 VOLTAGE DROP: $14.7 \mathrm{KW} \quad 7.1 \mathrm{KVAR}$ $0.0 \mathrm{KW}$
0.0 KVAR
16.3 KVA PF:

1. \%VD: 0.11 $0.0 \mathrm{KVA}$

$====$ BUS: Conex T7r BuS DESIGN VOLTS: 480 BUS VOLTS: $475 \%$ VD: 1.03 $================== \pm===$ PU BUS VOLTAGE: 0.990 ANGLE: -2.8 DEGREES NET BRANCH DIVERSITY LOAD: $14.6 \mathrm{KW} \quad 7.1 \mathrm{KVAR}$

LOAD FROM: Xfmr Skid PROJECTED POWER FLOW: LOSSES THRU FEEDER:
FEEDER AMPS: 19.7 VOLTAGE DROP:

1. \%VD: 0.21 $14.6 \mathrm{KW}$ $0.0 \mathrm{KW}$
7.1 KVAR

0.0 KVAR
16.3 KVA $0.0 \mathrm{KVA}$

$====$ BUS: Chiller Skid DESIGN VOLTS: 480 BUS VOLTS: $476 \%$ VD: 0.87 $======================$ PU BUS VOLTAGE: 0.991 ANGLE: -2.8 DEGREES NET BRANCH DIVERSITY LOAD: $61.4 \mathrm{KW} \quad 45.4 \mathrm{KVAR}$

LOAD FROM: MCC-N1 800A FEEDER AMPS: 92.6 VOLTAGE DROP: $0 . \%$ VD: 0.08 PROJECTED POWER FLOW: $61.4 \mathrm{KW}$ 45.4 KVAR LOSSES THRU FEEDER: $\quad 0.0 \mathrm{KW} \quad 0.0 \mathrm{KVAR}$ 76.4 KVA PF: 0.80 LAGGING $0.1 \mathrm{KVA}$ 
Calc. No. $W-320-31-010$

Revision 1

Page No. D - 10

DATE: $7: 21: 1998$ TIME: $17: 17: 54.68$

PAGE 10

Project W-320 / Tank 241-C-106 Sluicing

C-Farm Electrical Distribution

Calc. No. W320-31-010 (Reference Drawing H-2-818680, SH 1, Rev. 1)

BALANCED VOLTAGE DROP AND LOAD FLOW ANALYSIS

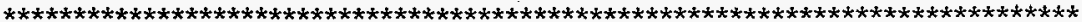

VOLTAGE EFFECT ON LOADS MODELED

VOLTAGE DROP CRITERIA: $B R A N C H=3.00 \%$ BUS $=5.00$

$====$ BUS: Exhaust Skid DESIGN VOLTS: 480 BUS VOLTS: $468 \%$ VD: 2.51

$================ \pm=====$ PU BUS VOLTAGE: 0.975 ANGLE: -2.5 DEGREES NET BRANCH DIVERSITY LOAD: $10.3 \mathrm{KW} \quad 4.5 \mathrm{KVAR}$

LOAD FROM: MCC-N1 800A FEEDER AMPS: 13.8 VOLTAGE DROP: $8 . \%$ VD: 1.72 PROJECTED POWER FLOW: $10.3 \mathrm{KW}$ 4.5 KVAR $11.3 \mathrm{KVA}$ PF: 0.92 LAGGING LOSSES THRU FEEDER: $\quad 0.2 \mathrm{KW} \quad 0.0 \mathrm{KVAR} \quad 0.2 \mathrm{KVA}$

$====$ BUS: EXH Fan Vfd DESIGN VOLTS: 480 BUS VOLTS: $476 \%$ VD: 0.85

$======================$ PU BUS VOLTAGE: 0.991 ANGLE: -2.8 DEGREES

LOAD FROM: MCC-N1 800A FEEDER AMPS: 11.3 VOLTAGE DROP: $0 . \%$ VD: 0.06 PROJECTED POWER FLOW: $\quad 7.6 \mathrm{KW} \quad 5.4 \mathrm{KVAR} \quad 9.3 \mathrm{KVA}$ PF: 0.81 LAGGING LOSSES THRU FEEDER: $\quad 0.0 \mathrm{KW} \quad 0.0 \mathrm{KVAR} \quad 0.0 \mathrm{KVA}$

LOAD TO: FN-1362 BuS FEEDER AMPS: 11.3 VOLTAGE DROP: 10. \%VD: 2.06 PROJECTED POWER FLOW: $7.6 \mathrm{KW} \quad 5.4$ KVAR $9.3 \mathrm{KVA}$ PF: 0.81 LAGGING LOSSES THRU FEEDER: $0.2 \mathrm{KW} 0.0 \mathrm{KVAR} \quad 0.2 \mathrm{KVA}$

$====$ BUS: WRAM TIr BUS DESIGN VOLTS: 480 BUS VOLTS: $474 \%$ VD: 1.28 $=====================$ PU BUS VOLTAGE: 0.987 ANGLE: -2.7 DEGREES NET BRANCH DIVERSITY LOAD: $14.6 \mathrm{KW} \quad 7.1 \mathrm{KVAR}$

LOAD FROM: Xfmr Skid PROJECTED POWER FLOW: LOSSES THRU FEEDER:
FEEDER AMPS: 19.7 VOLTAGE DROP:

2. \%VD: 0.46 $14.6 \mathrm{KW}$ $0.1 \mathrm{KW}$ 0.0 KVAR $0.1 \mathrm{KVA}$

$====$ BUS: MCC-N2 DESIGN VOLTS: 480 BUS VOLTS: $475 \%$ VD: 1.03

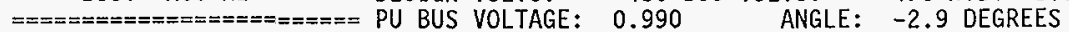

LOAD FROM: MCC-N1 800A PROJECTED POWER FLOW: LOSSES THRU FEEDER:

LOAD TO: P-1361 SS PROJECTED POWER FLOW: LOSSES THRU FEEDER:
FEEDER AMPS: 332.5 VOLTAGE DROP: $242.3 \mathrm{KW} \quad 127.2 \mathrm{KVAR}$ $0.5 \mathrm{KW}$ 0.6 KVAR 273.6 KVA 0.7 KVA

1. \%VD: 0.24 PF: 0.89 LAGGING

FEEDER AMPS: 52.8 VOLTAGE DROP: $35.0 \mathrm{KW}$ $0.0 \mathrm{KW}$ 25.9 KVAR 0.0 KVAR 43.5 KVA PF $0.0 \mathrm{KVA}$
$0 . \%$ VD: 0.00 PF: 0.80 LAGGING 
Calc. No. $W-320-31-010$

Revision 1

Page No. D - 11

DATE: $7: 21: 1998$ TIME: $17: 17: 54.68$

Project $W-320$ / Tank 241-C-106 Sluicing

PAGE 11

C-Farm Electrical Distribution

Calc. No. W320-31-010 (Reference Drawing H-2-818680, SH 1, Rev. 1)

BALANCED VOLTAGE DROP AND LOAD FLOW ANALYSIS

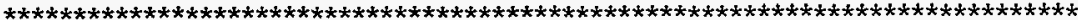

VOLTAGE EFFECT ON LOADS MODELED

VOLTAGE DROP CRITERIA: BRANCH $=3.00 \%$ BUS $=5.00$

LOAD TO: Booster Vfd FEEDER AMPS: 280.4 VOLTAGE DROP: $0 . \%$ VD: 0.05 PROJECTED POWER FLOW: $207.3 \mathrm{KW} 101.4 \mathrm{KVAR} 230.7 \mathrm{KVA}$ PF: 0.90 LAGGING LOSSES THRU FEEDER: $\quad 0.1 \mathrm{KW} \quad 0.1 \mathrm{KVAR} \quad 0.1 \mathrm{KVA}$

$====$ BUS: Sluicer Skid DESIGN VOLTS: 480 BUS VOLTS: $473 \%$ VD: 1.50

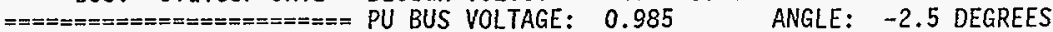
NET BRANCH DIVERSITY LOAD: $\quad 3.5 \mathrm{~kW} \quad 2.5 \mathrm{KVAR}$

LOAD FROM: MCC-N1 800A FEEDER AMPS: 5.2 VOLTAGE DROP: $3 . \%$ VD: 0.71 PROJECTED POWER FLOW: $3.5 \mathrm{KW} 2.5 \mathrm{KVAR} \quad 4.3 \mathrm{KVA}$ PF: 0.81 LAGGING LOSSES THRU FEEDER: $\quad 0.0 \mathrm{KW} \quad 0.0 \mathrm{KVAR} \quad 0.0 \mathrm{KVA}$

$====$ BUS: Slurry Winch DESIGN VOLTS: 480 BUS VOLTS: $\quad 475 \%$ VD: 1.14

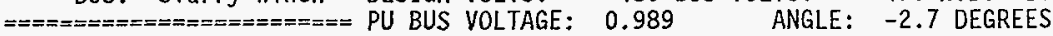
NET BRANCH DIVERSITY LOAD: $\quad 1.7 \mathrm{KW} \quad 1.3 \mathrm{KVAR}$

LOAD FROM: MCC-N1 800A FEEDER AMPS: 2.6 VOLTAGE DROP: 2 \% \%D: 0.35 PROJECTED POWER FLOW: $1.7 \mathrm{KW} \quad 1.3 \mathrm{KVAR} \quad 2.2 \mathrm{KVA}$ PF: 0.80 LAGGING LOSSES THRU FEEDER: $\quad 0.0 \mathrm{~kW} 0.0 \mathrm{KVAR} \quad 0.0 \mathrm{KVA}$

$===$ BUS: P-1361 SS DESIGN VOLTS: 480 BUS VOLTS: $475 \%$ VD: 1.03

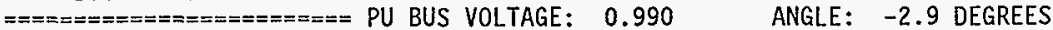

LOAD FROM: MCC-N2 FEEDER AMPS: 52.8 VOLTAGE DROP: $0 . \% \mathrm{VD}: 0.00$ PROJECTED POWER FLOW: $35.0 \mathrm{KW} \quad 25.9 \mathrm{KVAR} \quad 43.5 \mathrm{KVA}$ PF: 0.80 LAGGING LOSSES THRU FEEDER: $\quad 0.0 \mathrm{KW} \quad 0.0 \mathrm{KVAR} \quad 0.0 \mathrm{KVA}$

LOAD T0: P-1361 BuS

PROJECTED POWER FLOW:

LOSSES THRU FEEDER:

FEEDER AMPS: 52.8 VOLTAGE DROP:

3. \%VD: 0.69 $35.0 \mathrm{KW} \quad 25.9 \mathrm{KVAR}$ $0.3 \mathrm{KW}$

0.1 KVAR

43.5 KVA PF: 0.80 LAGGING 0.3 KVA

$====$ BUS: P-136I BuS DESIGN VOLTS: 480 BUS VOLTS: $472 \%$ VD: 1.72

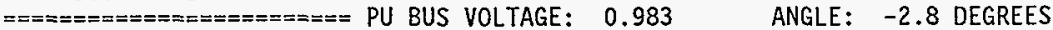
NET BRANCH DIVERSITY LOAD: $\quad 34.7 \mathrm{KW} \quad 25.8 \mathrm{KVAR}$

LOAD FROM: P-1361 SS PROJECTED POWER FLOW: LOSSES THRU FEEDER:
FEEDER AMPS: 52.8 VOLTAGE DROP: $\begin{array}{ll}34.7 \mathrm{KW} & 25.8 \mathrm{KVAR}\end{array}$ $0.3 \mathrm{KH}$

0.1 KVAR
3. $\%$ VD: 0.69 43.2 KVA PF: 0.80 LAGGING 0.3 KVA 
Calc. No. W-320-31-010

Revision 1

Page No. $0-12$

DATE: $7: 21: 1998$ TIME: $17: 17: 54.68$

Project $W-320$ / Tank 241-C-106 STuicing

PAGE 12

C-Farm Electrical Distribution

Calc. No. W320-31-010 (Reference Drawing H-2-818680, SH 1, Rev. 1)

BALANCED VOLTAGE DROP AND LOAD FLOW ANALYSIS

$* * * * * * * * * * * * * * * * * * * * * * * * * * * * * * * * * * * * * * * * * * * * * * * * * * * * * * * * * * * * * * * * * * * * * * * * * * * * * *$

VOLTAGE EFFECT ON LOADS MODELED

VOLTAGE DROP CRITERIA: $B R A N C H=3.00 \%$ BUS $=5.00$

$====$ BUS: Booster Vfd DESIGN VOLTS: 480 BUS VOLTS: $475 \%$ VD: 1.08

$=====================$ PU BUS VOLTAGE: 0.989 ANGLE: -2.9 DEGREES

LOAD FROM: MCC-N2

FEEDER AMPS: 280.4 VOLTAGE DROP:

$0 . \%$ VD: 0.05

PROJECTED POWER FLOW: $207.2 \mathrm{KW} 101.2$ KVAR $230.6 \mathrm{KVA}$ PF: 0.90 LAGGING LOSSES THRU FEEDER:

$0.1 \mathrm{KH} \quad 0.1 \mathrm{KVAR} \quad 0.1 \mathrm{KVA}$

LOAD TO: P-1362 BUS

FEEDER AMPS: 280.4 VOLTAGE DROP:

8. \%VD: 1.57 PROJECTED POWER FLOW: $207.2 \mathrm{KW} \quad 101.2 \mathrm{KVAR}$

LOSSES THRU FEEDER:

$2.3 \mathrm{KW}$

3.7 KVAR

230.6 KVA

4.4 KVA

$== \pm=$ BUS: P -1362 Bus

DESIGN VOLTS: $\quad 480$ BUS VOLTS:

$467 \%$ VD: 2.65

$==x=== \pm==x=== \pm==$

PU BUS VOLTAGE:

0.974 ANGLE: $\quad-3.5$ DEGREES NET BRANCH DIVERSITY LOAD: $204.9 \mathrm{KW} \quad 97.5 \mathrm{KVAR}$

LOAD FROM: Booster Vfd

FEEDER AMPS: 280.4 VOLTAGE DROP:

8. \%VD: 1.57

PROJECTED POWER FLOW: $204.9 \mathrm{KW} 97.5$ KVAR

LOSSES THRU FEEDER:

$2.3 \mathrm{KW}$

3.7 KVAR

227.0 KVA PF:

4.4 KVA

$==:=$ BUS: FN-1362 BuS DESIGN VOLTS: 480 BUS VOLTS: $466 \%$ VD: 2.92

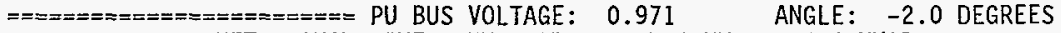
NET BRANCH DIVERSITY LOAD: $\quad 7.4 \mathrm{KW} \quad 5.4 \mathrm{KVAR}$

LOAD FROM: EXH Fan Vfd

PROJECTED POWER FLOW:

FEEDER AMPS: 11.3 VOLTAGE DROP:

10. \%VD: 2.06

LOSSES THRU FEEDER:

$7.4 \mathrm{KW} \quad 5.4$ KVAR

9.1 KVA PF: 0.80 LAGGING

$0.2 \mathrm{KW}$

0.0 KVAR

0.2 KVA

$====$ BUS: C8-L6 13.8KV DESIGN VOLTS: 1 BUS VOLTS: $1 \%$ VD: -3.00

$=======================$ PU BUS VOLTAGE: 1.030 ANGLE: 0.0 DEGREES *** SWING GENERATOR: C8-L6 $13.8 \mathrm{KV} \quad 464.0 \mathrm{KW} 269.1 \mathrm{KVAR}$

LOAD TO: Ug Rsr PI E257 FEEDER AMPS:291888 VOLTAGE DROP: $\quad 0 . \%$ VD: 0.58 PROJECTED POWER FLOW: $464.0 \mathrm{KW} 269.1$ KVAR $536.4 \mathrm{KVA}$ PF: 0.87 LAGGING LOSSES THRU FEEDER: $\quad 1.7 \mathrm{KW} \quad 3.1 \mathrm{KVAR} \quad 3.5 \mathrm{KVA}$

HNF-2487, Rev. 0

Page D-48 
Ca]c. No. $W-320-31-010$

Revision 1

Page No. D - 13

DATE: $7: 21: 1998$ TIME: $17: 17: 54.68$

Project $W-320$ / Tank 241-C-106 S7uicing

PAGE 13

C-Farm Electrical Distribution

Calc. No. W320-31-010 (Reference Drawing H-2-818680, SH 1, Rev. 1)

BALANCED VOLTAGE OROP AND LOAD FLOW BUS DATA SUMMARY

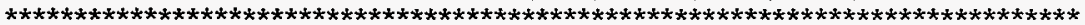

$\begin{array}{lrr}\text { BUS NAME } & \text { BASE VOLT } & \text { PU VOLT } \\ \text { Ug Rsr P1 E257 } & 13800.00 & 1.0242 \\ \text { XFMR C-T-1 Sec } & 480.00 & 0.9945 \\ \text { Xfmr Skid } & 480.00 & 0.9919 \\ \text { C106-PP1 Bus } & 480.00 & 0.9811 \\ \text { C106-PP2 Bus } & 480.00 & 0.9916 \\ \text { HC-1361 Bus } & 480.00 & 0.9799 \\ \text { UH-1361 Bus } & 480.00 & 0.9805 \\ \text { C106-PP3 Bus } & 480.00 & 0.9880 \\ \text { Office Tr1 Bus } & 480.00 & 0.9908 \\ \text { Chi1ler Skid } & 480.00 & 0.9913 \\ \text { EXH Fan Vfd } & 480.00 & 0.9915 \\ \text { MCC-N2 } & 480.00 & 0.9897 \\ \text { S1urry Winch } & 480.00 & 0.9886 \\ \text { P-1361 Bus } & 480.00 & 0.9828 \\ \text { P-1362 Bus } & 480.00 & 0.9735\end{array}$

BUS NAME

XFMR C-T-1 Pr

C-PDP-1 600A

MCC-N1 800A

Process Bldg

FN-1361 Bus

UH-1362 Bus

Service Bldg

CPR-1361 Bus

Conex Tir Bus

Exhaust Skid

WRAM TIr Bus

Sluicer Skid

P-1361 SS

Booster Vfd

FN-1362 Bus
BASE VOLT

13800.00

480.00

480.00

480.00

480.00

480.00

480.00

480.00

480.00

480.00

480.00

480.00

480.00

480.00

480.00
PU VOLT

1.0240

0.9933

0.9921

0.9814

0.9799

0.9798

0.9882

0.9876

0.9897

0.9749

0.9872

0.9850

0.9897

0.9892

0.9708

BALANCED VOLTAGE DROP AND LOAD FLOW BRANCH DATA SUMMARY

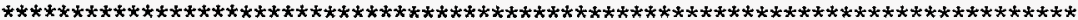

FROM NAME
Ug Rsr PT E257
XFMR C-T-1 Pri
XFMR C-T-1 SeC
C-PDP-1 600A
C-PDP-1 600A
Xfmr Skid
Xfmr Skid
Xfmr Skid
MCC-N1 $800 \mathrm{~A}$
MCC-N1 $800 \mathrm{~A}$
MCC-N1 $800 \mathrm{~A}$
MCC-N1 $800 \mathrm{~A}$
MCC-N1 $800 \mathrm{~A}$
MCC-N1 $800 \mathrm{~A}$
MCC-N1 $800 \mathrm{~A}$
MCC-N1 $800 \mathrm{~A}$
MCC-N1 $800 \mathrm{~A}$
Process Bldg
Process B1dg
Process B1dg
Process B1dg

TO NAME
XFMR C-T-1 Pri
XFMR C-T-1 SeC
C-PDP-1 600A
Xfmr Skid
MCC-N1 800A
Office Tr1 Bus
ConeX T1r Bus
WRAM T1r Bus
Process Bldg
C106-PP2 Bus
Service Bldg
Chiller Skid
EXhaust Skid
EXH Fan Vfd
MCC-N2
S1uicer Skid
Slurry Winch
C106-PP1 Bus
FN-1361 Bus
HC-1361 Bus
UH-1362 Bus

$\begin{array}{lrr}\text { TYPE } & \text { VD\% } & \text { AMPS } \\ \text { FDR } & 0.02 & 21.79 \\ \text { TX2 } & 2.95 & 21.79 \\ \text { FDR } & 0.12 & 610.69 \\ \text { FDR } & 0.14 & 59.26 \\ \text { FDR } & 0.11 & 551.45 \\ \text { FDR } & 0.11 & 19.78 \\ \text { FDR } & 0.21 & 19.76 \\ \text { FDR } & 0.46 & 19.71 \\ \text { FDR } & 1.07 & 62.69 \\ \text { FDR } & 0.06 & 17.89 \\ \text { FDR } & 0.40 & 17.02 \\ \text { FDR } & 0.08 & 92.68 \\ \text { FDR } & 1.72 & 13.88 \\ \text { FDR } & 0.06 & 11.33 \\ \text { FDR } & 0.24 & 332.56 \\ \text { FDR } & 0.71 & 5.23 \\ \text { FDR } & 0.35 & 2.62 \\ \text { FDR } & 0.03 & 8.85 \\ \text { FDR } & 0.15 & 11.23 \\ \text { FDR } & 0.15 & 35.36 \\ \text { FDR } & 0.16 & 4.71\end{array}$

HNF-2487, Rev. 0

Page D-49

\begin{tabular}{rr}
\multicolumn{1}{c}{ KVA } & RATING\% \\
533.31 & 14.52 \\
533.23 & 206.04 \\
504.91 & 72.70 \\
48.93 & 39.50 \\
455.38 & 59.30 \\
16.31 & 28.26 \\
16.30 & 28.23 \\
16.25 & 28.16 \\
51.71 & 65.99 \\
14.76 & 89.45 \\
14.03 & 56.72 \\
76.44 & 40.29 \\
11.45 & 46.27 \\
9.35 & 56.65 \\
274.31 & 53.64 \\
4.31 & 26.15 \\
2.16 & 13.09 \\
7.22 & 38.15 \\
9.16 & 64.52 \\
28.85 & 87.09 \\
3.85 & 40.64
\end{tabular}


Caic. No. $W-320-31-010$

Revision 1

Page No. D - 14

DATE: $7: 21: 1998$ TIME: $17: 17: 54.68$

Project W-320 / Tank 241-C-106 Sluicing

PAGE 14

C-Farm Electrical Distribution

Calc. No. W320-31-010 (Reference Drawing H-2-818680, SH 1, Rev. 1)

BALANCED VOLTAGE DROP AND LOAD FLOW BRANCH DATA SUMMARY

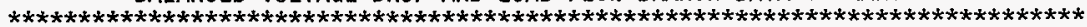

FROM NAME

Process Bldg

Service Bldg

Service Bldg

EXH Fan Vfd

MCC-N2

MCC-N2

P-1361 SS

Booster Vfd
TO NAME

UH-1361 BUS

C106-PP3 Bus

CPR-1361 Bus

FN-1362 Bus

P-1361 SS

Booster Vfd

$\mathrm{P}-1361$ Bus

P-1362 Bus

TYPE
FDR
FDR
FDR
FDR
FDR
FDR
FDR
FDR

VD\%

0.10

0.01

0.06

2.06

0.00

0.05

0.69

1.57
AMPS

4.72

5.94

11.14

11.33

52.88

280.41

52.88

280.41
KVA RATING\%

\subsection{5}

4.88

9.15

9.34

43.51

230.73

43.51

230.62
40.67

19.81

37.13

56.65

35.26

73.79

35.26

73.79

NOTE: FDR RATING\% $=\%$ AMPS RATING BASED ON LIBRARY FLA OR BRANCH INPUT FLA TX2 RATING\% $=\%$ KVA RATING BASED ON TRANSFORMER FL KVA

30 BUSES

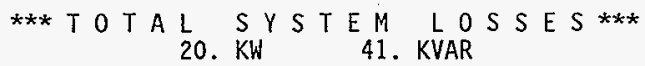

HNF-2487, Rev. 0

Page D-50 
Calc. No. W-320-31-010

Revision 1

Attachment E

HNF-2487, Rev. 0

Page D-51 
Calc. No. W-320-31-010

Revision 1

Page No. E - 1

Project W-320 / Tank 241-C-106 S7uicing C-Farm Electrical Distribution

Calc. No. W320-31-010 (Reference Drawing H-2-818680, SH 1, Rev. 1)

DATE: $7: 21: 1998$

TIME: $17: 18: 6.0$

ALL INFORMATION PRESENTED IS FOR REVIEW, APPROVAL.

INTERPRETATION AND APPLICATION BY A REGISTERED ENGINEER ONLY

SKM POWER*TOOLS FOR WINDOWS

SHORT CIRCUIT ANALYSIS REPORT

COPYRIGHT SKM SYSTEMS ANALYSIS, INC. 1995-1996

HNF-2487, Rev. 0

Page D-52 
Caic. No. W-320-31-010

Revision 1

Page No. E - 2

DATE: $7: 21: 1998$ TIME:17:18: 6.0

PAGE

Project W-320 / Tank 241-C-106 Sluicing

C-Farm Electrical Distribution

Calc. No. W320-31-010 (Reference Drawing H-2-818680, SH 1, Rev. 1)

ALL PU VALUES ARE EXPRESSED ON A 100 MVA BASE

SWING GENERATORS

\begin{tabular}{|c|c|}
\hline $\mathrm{C} 8-16 \quad 13.8 \mathrm{Kv}$ & 0.00 \\
\hline
\end{tabular}

HNF-2487, Rev. 0

Page D-53 
Calc. No. W-320-31-010

Revision 1

Page No. E - 3

DATE: $7: 21: 1998$ TIME: 17:18: 6.0

Project W-320 / Tank 241-C-106 STuicing

C-Farm Electrical Distribution

Calc. No. W320-31-010 (Reference Drawing H-2-818680, SH 1, Rev. 1)

PAGE 3

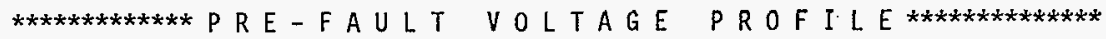

BUS\# NAME

Ug Rsr PI E257

XFMR C-T-1 Pri

XFMR $C-T-1$ SeC

C-PDP-1 600A

Xfmr Skid

MCC-N1 800A

C106-PPI Bus

Process Bldg

C106-PP2 Bus

FN-1361 Bus

HC-1361 Bus

UH-1362 Bus

UH-1361 Bus

Service Bldg

C106-PP3 Bus

CPR-136I Bus

office Trl Bus

Conex Tir Bus

chiller skid

Exhaust Skid

EXH Fan Vfd

WRAM TTr Bus

MCC-N2

Sluicer Skid

STurry Winch

P-1361 SS

P-1361 Bus

Booster Vfd

P-1362 Bus

FN-1362 Bus
BASE VOLTS

13800.00

13800.00

480.00

480.00

480.00

480.00

480.00

480.00

480.00

480.00

480.00

480.00

480.00

480.00

480.00

480.00

480.00

480.00

480.00

480.00

480.00

480.00

480.00

480.00

480.00

480.00

480.00

480.00

480.00

480.00
PU VOLTS

1.0300

1.0300

1.0564

1.0564

1.0564

1.0564

1.0564

1.0564

1.0564

1.0564

1.0564

1.0564

1.0564

1.0564

1.0564

1.0564

1.0564

1.0564

1.0564

1.0564

1.0564

1.0564

1.0564

1.0564

1.0564

1.0564

1.0564

1.0564

1.0564

1.0564
ANGLE (D)

0.

0.

-30 .

-30 .

-30 .

-30 .

-30 .

-30 .

-30 .

-30 .

-30 .

-30 .

-30 .

-30 .

-30 .

-30 .

-30 .

-30 .

-30 .

-30 .

-30 .

-30 .

-30 .

-30 .

$-30$.

-30 .

-30 .

-30 .

-30 .

-30 .

HNF-2487, Rev. 0

Page D-54 
Calc. No. W-320-31-010

Revision 1

Page No. E - 4

DATE: $7: 21: 1998$ TIME: $17: 18: 6.0$

Project $W-320$ / Tank 241-C-106 Sluicing

PAGE 4

C-Farm Electrical Distribution

Calc. No. W320-31-010 (Reference Drawing H-2-818680, SH 1, Rev. 1)

$* * * * * * * * * * * * * * * * *$ F A U L T A N A L Y S S R E POR T *****************

FAULT TYPE: $3 P H$

MODEL INDUCTION MOTOR CONTRIBUTION: YES

MODEL TRANSFORMER TAPS: YES

MODEL TRANSFORMER PHASE SHIFT: YES

Ug Rsr P1 E257 VOLTAGE BASE LL: 13800.0 (VOLTS)

INI. SYM. RMS FAULT CURRENT: $3338.1 /-60$. (AMPS/DEG)

THEVENIN EQUIVALENT IMPEDANCE: $0.638+j \quad 1.122(\mathrm{PU})$

THEVENIN IMPEDANCE X/R RATIO: 1.759

$$
\begin{array}{lccrr}
\text { ASYM } & \text { RMS } & \text { INTERRUPTING } & \text { AMPS } & \\
1 / 2 \text { CYCLES } & 3 \text { CYCLES } & 5 \text { CYCLES } & 8 \text { CYCLES } \\
3430.7 & 3338.1 & 3338.1 & 3338.1
\end{array}
$$

ASYM RMS FAULTED CURRENT ( AMPS )

$\begin{array}{rcc}\text { AT TIME } & 0.5 \text { CYCLES } & \\ \text {--PHASE A-- } & -- \text { PHASE B--- } & -- \text { PHASE C--- } \\ 3430.7 & 3430.7 & 3430.7\end{array}$

HNF-2487, Rev. 0

Page D-55 
Calc. No. W-320-31-010

Revision 1

Page No. E - 5

DATE: $7: 21: 1998$ TIME: $17: 18: 6.0$

PAGE 5

Project W-320 / Tank 241-C-106 Sluicing

C-Farm Electrical Distribution

Calc. No. W320-31-010 (Reference Drawing H-2-818680, SH 1, Rev. 1)

XFMR C-T-1 Pri VOLTAGE BASE LL: 13800.0 (VOLTS)

INI. SYM. RMS FAULT CURRENT: $3281.3 /-60$. (AMPS/DEG)

THEVENIN EQUIVALENT IMPEDANCE: $0.666+j 1.132$ (PU)

THEVENIN IMPEDANCE X/R RATIO: 1.701

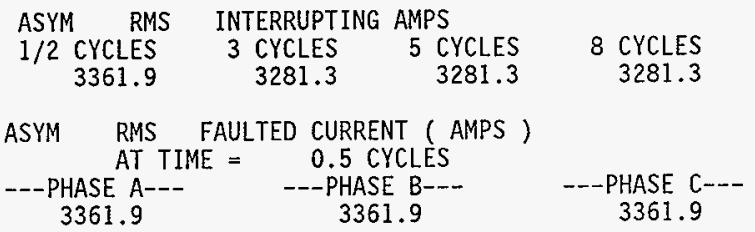

XFMR C-T-1 Sec VOLTAGE BASE LL:

480.0 (VOLTS)

INI. SYM. RMS FAULT CURRENT: $8872.2 /-98$. (AMPS/DEG)

THEVENIN EQUIVALENT IMPEDANCE: $\quad 5.342+j 13.288$ (PU)

THEVENIN IMPEDANCE X/R RATIO: 2.487

ASYM RMS INTERRUPTING AMPS

$\begin{array}{lrrr}1 / 2 \text { CYCLES } & 3 \text { CYCLES } & 5 \text { CYCLES } & 8 \text { CYCLES } \\ 9555.5 & 8872.2 & 8872.2 & 8872.2\end{array}$

ASYM RMS FAULTED CURRENT ( AMPS )

AT TIME $=0.5$ CYCLES

---PHASE A--- ---PHASE B---

9555.5

9555.4

9555.5

HNF-2487, Rev. 0

Page D-56 
Ca]c. No. W-320-31-010

Revision 1

Page No. E - 6

DATE: $7: 21: 1998$ TIME: $17: 18: 6.0$

Project W-320 / Tank 241-C-106 S7uicing

PAGE 6

C-Farm Electrical Distribution

Calc. No. W320-31-010 (Reference Drawing H-2-818680, SH 1, Rev. 1)

C-PDP-1 600A

VOLTAGE BASE LL:

480.0 (VOLTS)

INI. SYM. RMS FAULT CURRENT: $8722.2 /-98$. (AMPS/DEG)

THEVENIN EQUTVALENT IMPEDANCE: $\quad 5.448+j 13.511$ (PU)

THEVENIN IMPEDANCE X/R RATI0: $\quad 2.480$

ASYM RMS INTERRUPTING AMPS

$\begin{array}{ccrr}1 / 2 \text { CYCLES } & 3 \text { CYCLES } & 5 \text { CYCLES } & 8 \text { CYCLES } \\ 9389.1 & 8722.2 & 8722.2 & 8722.2\end{array}$

ASYM RMS FAULTED CURRENT ( AMPS)

AT TIME $=0.5$ CYCLES

---PHASE A--- ---PHASE B---

9389.1

9389.1

-.-PHASE C---

9389.1

Xfmr Skid

VOLTAGE BASE LL:

480.0 (VOLTS)

INI. SYM. RMS FAULT CURRENT: $7579.3 /-91$ ( AMPS/DEG)

THEVENIN EQUIVALENT IMPEDANCE: $8.052+\mathrm{j} 14.705$ (PU)

THEVENIN IMPEDANCE X/R RATIO: $\quad 1.826$

$\begin{array}{lccr}\text { ASYM } & \text { RMS } & \text { INTERRUPTING AMPS } & \\ 1 / 2 \text { CYCLES } & 3 \text { CYCLES } & 5 \text { CYCLES } & 8 \text { CYCLES } \\ 7818.4 & 7579.3 & 7579.3 & 7579.3\end{array}$

ASYM RMS FAULTED CURRENT (AMPS)

---PHASE AT TIME $=\quad 0.5$ CYCLES

7818.4

7818.4

---PHASE C---

7818.4

HNF-2487, Rev. 0

Page D-57 
Ca7c. No. W-320-31-010

Revision 1

Page No. E - 7

DATE: $7: 21: 1998$ TIME: $17: 18: 6.0$

PAGE 7

Project $\mathrm{W}-320$ / Tank 241-C-106 Sluicing

C-Farm Electrical Distribution

Calc. No. W320-31-010 (Reference Drawing H-2-818680, SH 1, Rev. 1)

MCC-N1 800A VOLTAGE BASE LL: 480.0 (VOLTS)

INI. SYM. RMS FAULT CURRENT: $8590.1 /-98$. ( AMPS/DEG)

THEVENIN EQUIVALENT IMPEDANCE: $5.582+j 13.698$ (PU)

THEVENIN IMPEDANCE X/R RATIO: $\quad 2.454$

$\begin{array}{lcrlr}\text { ASYM } & \text { RMS } & \text { INTERRUPTING } & \text { AMPS } & \\ 1 / 2 \text { CYCLES } & 3 \text { CYCLES } & 5 \text { CYCLES } & 8 \text { CYCLES } \\ 9230.0 & 8590.1 & 8590.1 & 8590.1\end{array}$

ASYM RMS FAULTED CURRENT ( AMPS)

-.-PHASE AT TIME $=0.5$ CYCLES

9230.0

PHASE B---

9230.0

9230.0

VOLTAGE BASE LL:

480.0 (VOLTS)

INI. SYM. RMS FAULT CURRENT: $3505.5 /-64$. (AMPS/DEG)

THEVENIN EQUIVALENT IMPEDANCE: $30.192+j 20.059$ (PU)

THEVENIN IMPEDANCE X/R RATIO: 0.664

$$
\begin{array}{lccr}
\text { ASYM } & \text { RMS } & \multicolumn{1}{l}{\text { INTERRUPTING AMPS }} & \\
1 / 2 \text { CYCLES } & 3 \text { CYCLES } & 5 \text { CYCLES } & 8 \text { CYCLES } \\
3505.8 & 3505.5 & 3505.5 & 3505.5
\end{array}
$$

ASYM RMS FAULTED CURRENT (AMPS)

-.-PHASE A---

AT TIME $=0.5$ CYCLES

3505.8

---PHASE B---

3505.8
---PHASE C---

3505.8

HNF-2487, Rev. 0

Page D-58 
Calc. No. W-320-31-010

Revision 1

Page No. E - 8

DATE: $7: 21: 1998$ TIME: $17: 18: 6.0$

PAGE 8

Project W-320 / Tank 241-C-106 Siuicing

C-Farm Electrical Distribution

Calc. No. W320-31-010 (Reference Drawing H-2-818680, SH 1, Rev. 1)

Process BIdg VOLTAGE BASE LL: 480.0 (VOLTS)

INI. SYM. RMS FAULT CURRENT: $3960.4 /-68$. ( AMPS/DEG)

THEVENIN EQUIVALENT IMPEDANCE: $25.374+j 19.636$ (PU)

THEVENIN IMPEDANCE X/R RATIO: 0.774

ASYM RMS INTERRUPTING AMPS

$\begin{array}{ccrr}1 / 2 \text { CYCLES } & 3 \text { CYCLES } & 5 \text { CYCLES } & 8 \text { CYCLES } \\ 3961.6 & 3960.4 & 3960.4 & 3960.4\end{array}$

ASYM RMS FAULTED CURRENT ( AMPS)

---PHASE A--. ---PHASE B---

3961.6

3961.6

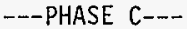

3961.6

C106-PP2 BuS VOLTAGE BASE LL:

480.0 (VOLTS)

INI. SYM. RMS FAULT CURRENT: 7514.8/ $/ 85$. ( AMPS/DEG)

THEVENIN EQUIVALENT IMPEDANCE: $9.706+\mathrm{j} 13.846$ (PU)

THEVENIN IMPEDANCE X/R RATIO: 1.427

ASYM RMS INTERRUPTING AMPS

$\begin{array}{ccrr}1 / 2 \text { CYCLES } & 3 \text { CYCLES } & 5 \text { CYCLES } & 8 \text { CYCLES } \\ 7506.1 & 7514.8 & 7514.8 & 7514.8\end{array}$

ASYM RMS FAULTED CURRENT ( AMPS )

-- PHASE A-- - - PHASE B---

7606.1

7606.1

7606.1

HNF-2487, Rev. 0

Page D-59 
Calc. No. W-320-31-010

Revision 1

Page No. $E-9$

DATE: $7: 21: 1998$ TIME: $17: 18: 6.0$

PAGE 9

Project $W-320$ / Tank 241-C-105 STuicing

C-Farm Electrical Distribution

Calc. No. W320-31-010 (Reference Drawing H-2-818680, SH 1, Rev. 1)

FN-1361 Bus

VOLTAGE BASE LL:

480.0 (VOLTS)

INI. SYM. RMS FAULT CURRENT: $2556.9 /-55$. (AMPS/DEG)

THEVENIN EQUIVALENT IMPEDANCE: $45.166+\mathrm{j} 20.730$ (PU)

THEVENIN IMPEDANCE X/R RATIO: 0.459

ASYM RMS INTERRUPTING AMPS

$\begin{array}{lrrr}1 / 2 \text { CYCLES } & 3 \text { CYCLES } & 5 \text { CYCLES } & 8 \text { CYCLES } \\ 2556.9 & 2556.9 & 2556.9 & 2556.9\end{array}$

ASYM RMS FAULTED CURRENT (AMPS)

AT TIME $=0.5$ CYCLES

---PHASE A-.--

---PHASE B----

2556.9

2556.9

HC-1361 Bus

VOLTAGE BASE LL:

480.0 (VOLTS)

INI. SYM. RMS FAULT CURRENT: $3442.0 /-64$. (AMPS/DEG)

THEVENIN EQUIVALENT IMPEDANCE: $30.582+\mathrm{j} 20.678$ (PU)

THEVENIN IMPEDANCE X/R RATIO: 0.676

$$
\begin{array}{lccrr}
\text { ASYM } & \text { RMS } & \text { INTERRUPTING } & \text { AMPS } & \\
1 / 2 \text { CYCLES } & 3 \text { CYCLES } & 5 \text { CYCLES } & 8 \text { CYCLES } \\
3442.3 & 3442.0 & 3442.0 & 3442.0
\end{array}
$$

ASYM RMS FAULTED CURRENT ( AMPS )

$\begin{array}{cc}\text { AT TIME }= & 0.5 \text { CYCLES } \\ \cdots \cdots-\text { PHASE A--- } & -\cdots \text {-PHASE B--- } \\ 3442.3 & 3442.3\end{array}$ 
Calc. No. W-320-31-010

Revision 1

Page No. E - 10

DATE: $7: 21: 1998$ TIME: $17: 18: 6.0$

PAGE 10

Project $W-320$ / Tank 241-C-106 Sluicing

C-Farm Electrical Distribution

Calc. No. W320-31-010 (Reference Drawing H-2-818680, SH 1, Rev. 1)

UH-1362 Bus

VOLTAGE BASE LL:

480.0 (VOLTS)

INI. SYM. RMS FAULT CURRENT: $1818.5 /-48$. ( AMPS/DEG)

THEVENIN EQUIVALENT IMPEDANCE: $66.607+\mathrm{j} 21.112$ (PU)

THEVENIN IMPEDANCE X/R RATIO: 0.317

$$
\begin{array}{lcrrr}
\text { ASYM } & \text { RMS } & \multicolumn{1}{c}{\text { INTERRUPTING }} & \text { AMPS } & \\
1 / 2 \text { CYCLES } & 3 \text { CYCLES } & 5 \text { CYCLES } & 8 \text { CYCLES } \\
1818.5 & 1818.5 & 1818.5 & 1818.5
\end{array}
$$

ASYM RMS FAULTED CURRENT ( AMPS)

$\begin{array}{ll} & \\ -- \text { PHASE A-- } & \text {-.PHASE B--- }\end{array}$ 1818.5 1818.5

UH-1361 Bus

VOLTAGE BASE LL:

INI. SYM. RMS FAULT CURRENT: $2346.5 /-52$. (AMPS/DEG)

THEVENIN EQUIVALENT IMPEDANCE: $50.114+j 20.521$ (PU)

THEVENIN IMPEDANCE X/R RATIO: 0.409

$$
\begin{array}{lcrrr}
\text { ASYM RMS } & \text { INTERRUPTING AMPS } & \\
1 / 2 \text { CYCLES } & 3 \text { CYCLES } & 5 \text { CYCLES } & 8 \text { CYCLES } \\
2346.5 & 2346.5 & 2346.5 & 2346.5
\end{array}
$$

ASYM RMS FAULTED CURRENT ( AMPS)

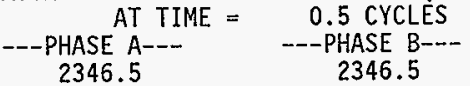

HNF-2487, Rev. 0

Page D-61 
Calc. No. W-320-31-010

Revision 1

Page No. E - 11

DATE: $7: 21: 1998$ TIME: $17: 18: 6.0$

PAGE II

Project $W-320$ / Tank 241-C-106 Sluicing

C-Farm Electrical Distribution

Ca]c. No. W320-31-010 (Reference Drawing H-2-818680, SH 1, Rev. 1)

Service Bldg VOLTAGE BASE LL:

480.0 (VOLTS)

INI. SYM. RMS FAULT CURRENT: $3114.9 /-52$. ( AMPS/DEG )

THEVENIN EQUIVALENT IMPEDANCE: $37.744+j 15.476$ (PU)

THEVENIN IMPEDANCE X/R RATIO: 0.410

$\begin{array}{lccr}\text { ASYM } & \text { RMS } & \text { INTERRUPTING } & \text { AMPS } \\ 1 / 2 \text { CYCLES } & 3 \text { CYCLES } & 5 \text { CYCLES } & 8 \text { CYCLES } \\ 3114.9 & 3114.9 & 3114.9 & 3114.9\end{array}$

ASYM RMS FAULTED CURRENT ( AMPS)

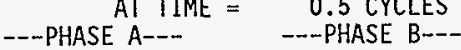

3114.9

3114.9

VOLTAGE BASE LL:

480.0 (VOLTS)

INI. SYM. RMS FAULT CURRENT: $2945.3 /-51$. ( AMPS/DEG)

THEVENIN EQUIVALENT IMPEDANCE: $40.218+\mathrm{j} 15.612$ (PU)

THEVENIN IMPEDANCE X/R RATIO: 0.388

$$
\begin{array}{lcrrr}
\text { ASYM } & \text { RMS } & \multicolumn{1}{l}{\text { INTERRUPTING }} & \text { AMPS } \\
1 / 2 \text { CYCLES } & 3 \text { CYCLES } & 5 \text { CYCLES } & 8 \text { CYCLES } \\
2945.3 & 2945.3 & 2945.3 & 2945.3
\end{array}
$$

ASYM RMS FAULTED CURRENT ( AMPS)

AT TIME $=0.5$ CYCLES
2945.3
-.-PHASE B---
2945.3

HNF-2487, Rev. 0

Page D-62 
Calc. No. W-320-31-010

Revision 1

Page No. E - 12

DATE: $7: 21: 1998$ TIME: 17:18: 6.0

PAGE 12

Project W-320 / Tank 241-C-106 STuicing

C-Farm Electrical Distribution

Calc. No. W320-31-010 (Reference Drawing H-2-818680, SH I, Rev. 1)

CPR-1361 Bus

VOLTAGE BASE LL:

INI. SYM. RMS FAULT CURRENT: 2654.0/ $/ 49$. ( AMPS/DEG)

THEVENIN EQUIVALENT IMPEDANCE: $45.166+\mathrm{j} 15.886$ (PU)

THEVENIN IMPEDANCE X/R RATIO: 0.352

$\begin{array}{lccr}\text { ASYM } & \text { RMS } & \text { INTERRUPTING } & \text { AMPS } \\ 1 / 2 \text { CYCLES } & 3 \text { CYCLES } & 5 \text { CYCLES } & 8 \text { CYCLES } \\ 2654.0 & 2654.0 & 2654.0 & 2654.0\end{array}$

ASYM RMS FAULTED CURRENT (AMPS )

$\begin{array}{cc}\text { AT TIME }= & 0.5 \text { CYCLES } \\ --- \text { PHASE A--- } & -- \text { PHASE B--- } \\ 2654.0 & 2654.0\end{array}$
PHASE C--2654.0

Office Tr] Bus VOLTAGE BASE LL: 480.0 (VOLTS)

INI. SYM. RMS FAULT CURRENT: $5871.9 /-78$, (AMPS/DEG)

THEVENIN EQUIVALENT IMPEDANCE: $14.562+\mathrm{j} 16.007$ (PU)

THEVENIN IMPEDANCE X/R RATIO: 1.099

ASYM RMS INTERRUPTING AMPS

$\begin{array}{lrrr}1 / 2 \text { CYCLES } & 3 \text { CYCLES } & 5 \text { CYCLES } & 8 \text { CYCLES } \\ 5891.2 & 5871.9 & 5871.9 & 5871.9\end{array}$

ASYM RMS FAULTED CURRENT (AMPS)

AT TIME $=0.5$ CYCLES

$5891.2 \quad 5891.2$

5891.2

HNF-2487, Rev. 0

Page D-63 
Calc. No. W-320-31-010

Revision 1

Page No. E - 13

DATE: $7: 21: 1998$ TIME: 17:18: 6.0

PAGE 13

Project $W-320$ / Tank 241-C-106 S7uicing

C-Farm Electrical Distribution

Calc. No. W320-31-010 (Reference Drawing H-2-818680, SH 1, Rev. 1)

Conex Tir Bus

VOLTAGE BASE LL:

480.0 (VOLTS)

INI. SYM. RMS FAULT CURRENT: $4659.5 /-69$. (AMPS/DEG)

THEVENIN EQUIVALENT IMPEDANCE: $21.073+\mathrm{j} 17.309$ (PU)

THEVENIN IMPEDANCE X/R RATIO: 0.821

ASYM RMS INTERRUPTING AMPS

$\begin{array}{ccrr}1 / 2 \text { CYCLES } & 3 \text { CYCLES } & 5 \text { CYCLES } & 8 \text { CYCLES } \\ 4661.8 & 4659.5 & 4659.5 & 4659.5\end{array}$

ASYM RMS FAULTED CURRENT ( AMPS)

AT TIME $=0.5$ CYCLES

---PHASE A--- ---PHASE B---

4661.8

4661.7

---PHASE C---

4661.7
Chiller skid

VOLTAGE BASE LL:

INI. SYM. RMS FAULT CURRENT:

THEVENIN EQUIVALENT IMPEDANCE:

THEVENIN IMPEDANCE $X / R$ RATIO:

ASYM RMS INTERRUPTING AMPS

$\begin{array}{rrrr}1 / 2 \text { CYCLES } & 3 \text { CYCLES } & 5 \text { CYCLES } & 8 \text { CYCLES } \\ 8607.9 & 8115.8 & 8115.8 & 8115.8\end{array}$

ASYM RMS FAULTED CURRENT ( AMPS)

$\begin{array}{cc}\text { AT TIME }= & 0.5 \text { CYCLES } \\ -- \text { PHASE A--- } & \text {--PHASE B--- } \\ 8607.9 & 8607.9\end{array}$

$\begin{array}{cc}\text { AT TIME }= & 0.5 \text { CYCLES } \\ -- \text { PHASE A--- } & \text {--PHASE B--- } \\ 8607.9 & 8607.9\end{array}$
480.0 (VOLTS)

$8115.8 /-96$. ( AMPS/DEG)

$6.322+j 14.324$ (PU)

2.266

HNF-2487, Rev. 0

Page D-64 
Calc. No. W-320-31-010

Revision 1

Page No. E - 14

DATE: $7: 21: 1998$ TIME: $17: 18: 6.0$

Project $W-320$ / Tank 241-C-106 Sluicing

PAGE 14

C-Farm Electrical Distribution

CaTc. No. W320-31-010 (Reference Drawing H-2-818680, SH 1, Rev. I)

Exhaust Skid

VOLTAGE BASE LL:

480.0 (VDLTS)

INI. SYM. RMS FAULT CURRENT: $768.0 /-38$. ( AMPS/DEG)

THEVENIN EQUIVALENT IMPEDANCE: $163.916+\mathrm{j} 22.448$ (PU)

THEVENIN IMPEDANCE X/R RATIO: 0.137

ASYM RMS INTERRUPTING AMPS

$\begin{array}{rrrr}1 / 2 \text { CYCLES } & 3 \text { CYCLES } & 5 \text { CYCLES } & 8 \text { CYCLES } \\ 768.0 & 768.0 & 768.0 & 768.0\end{array}$

ASYM RMS FAULTED CURRENT ( AMPS)

AT TIME $=0.5$ CYCLES

---PHASE A--- ---PHASE B--768.0

768.0

768.0

EXH Fan Vfd VOLTAGE BASE LL:

480.0 (VOLTS)

INI. SYM. RMS FAULT CURRENT: $6458.7 / \mathbf{- 7 5}$. (AMPS/DEG)

THEVENIN EQUIVALENT IMPEDANCE: $13.829+j 13.993$ (PU)

THEVENIN IMPEDANCE X/R RATIO: 1.012

ASYM RMS INTERRUPTING AMPS

$\begin{array}{ccrr}1 / 2 \text { CYCLES } & 3 \text { CYCLES } & 5 \text { CYCLES } & 8 \text { CYCLES } \\ 6471.6 & 6458.7 & 6458.7 & 6458.7\end{array}$

ASYM RMS FAULTED CURRENT ( AMPS)

AT TIME $=0.5$ CYCLES
- PHASE A-

6471.6

6471.6

6471.6

HNF-2487, Rev. 0

Page D-65 
Calc. No. W-320-31-010 Revision 1

Page No. E - 15

DATE: $7: 21: 1998$ TIME:17:18:6.0

PAGE $\quad 15$

Project $W-320$ / Tank 241-C-106 STuicing

C-Farm Electrical Distribution

Calc. No. W320-31-010 (Reference Drawing H-2-818680, SH 1, Rev. 1)

WRAM T7r BUS VOLTAGE BASE LL: $\quad 480.0$ (VOLTS)

INI. SYM. RMS FAULT CURRENT: $3025.2 /-59$. ( AMPS/DEG)

THEVENIN EQUIVALENT IMPEDANCE: $36.698+\mathrm{j} 20.434$ (PU)

THEVENIN IMPEDANCE X/R RATIO: 0.557

$$
\begin{array}{lcrrr}
\text { ASYM RMS } & \multicolumn{1}{c}{\text { INTERRUPTING }} & \text { AMPS } & \\
1 / 2 \text { CYCLES } & 3 \text { CYCLES } & 5 \text { CYCLES } & 8 \text { CYCLES } \\
3025.2 & 3025.2 & 3025.2 & 3025.2
\end{array}
$$

ASYM RMS FAULTED CURRENT ( AMPS )

--PHASE A- -. AT TIME $=0.5$ CYCLES

3025.2
-- PHASE B---

3025.2
-. PHASE C-.-

3025.2

$\mathrm{MCC}-\mathrm{N} 2$

VOLTAGE BASE LL:

480.0 (VOLTS)

INI. SYM. RMS FAULT CURRENT: 8121.7/ -97. ( AMPS/DEG)

THEVENIN EQUIVALENT IMPEDANCE: $6.111+j 14.402$ (PU)

THEVENIN IMPEDANCE X/R RATIO: 2.357

$\begin{array}{lccr}\text { ASYM } & \text { RMS } & \text { INTERRUPTING } & \text { AMPS } \\ 1 / 2 \text { CYCLES } & 3 \text { CYCLES } & 5 \text { CYCLES } & 8 \text { CYCLES } \\ 8667.9 & 8121.7 & 8121.7 & 8121.7\end{array}$

ASYM RMS FAULTED CURRENT ( AMPS )

-- PHASE A--- ---PHASE B---

8667.9

8667.9

HNF-2487, Rev. 0

Page D-66 
Calc. No. W-320-31-010

Revision 1

Page No. E - 16

DATE: $7: 21: 1998$ TIME: $17: 18: 6.0$

Project $W-320 /$ Tank 241-C-106 Sluicing

PAGE 16

C-Farm Electrical Distribution

Calc. No. W320-31-010 (Reference Drawing H-2-818680, SH 1, Rev. 1)

Sluicer Skid

VOLTAGE BASE LL:

480.0 (VOLTS)

INI. SYM. RMS FAULT CURRENT: 621.2/-36. ( AMPS/DEG)

THEVENIN EQUIVALENT IMPEDANCE: $203.499+j 20.782$ (PU)

THEVENIN IMPEDANCE X/R RATIO: 0.102

ASYM RMS INTERRUPTING AMPS

$\begin{array}{crrr}1 / 2 \text { CYCLES } & 3 \text { CYCLES } & 5 \text { CYCLES } & 8 \text { CYCLES } \\ 621.2 & 621.2 & 621.2 & 621.2\end{array}$

ASYM RMS FAULTED CURRENT (AMPS)

AT TIME $=0.5$ CYCLES

---PHASE A--- ---PHASE B---

621.2

621.2

---PHASE C-.-

621.2

Slurry winch

VOLTAGE BASE LL:

480.0 (VOLTS)

INI. SYM. RMS FAULT CURRENT: 621.2/ $/ 36$. ( AMPS/DEG)

THEVENIN EQUIVALENT IMPEDANCE: $203.499+\mathrm{j} 20.782$ (PU)

THEVENIN IMPEDANCE X/R RATI0: 0.102

$\begin{array}{lrrr}\text { ASYM } & \text { RMS } & \text { INTERRUPTING AMPS } & \\ 1 / 2 \text { CYCLES } & 3 \text { CYCLES } & 5 \text { CYCLES } & 8 \text { CYCLES } \\ 621.2 & 621.2 & 621.2 & 621.2\end{array}$

ASYM RMS FAULTED CURRENT ( AMPS )

$\begin{array}{ll}\text { AT TIME }= & 0.5 \text { CVCLES } \\ -- \text { PHASE A--- } & --- \text { PHASE B--- }\end{array}$

621.2

621.2

621.2

HNF-2487, Rev, 0

Page D-67 
Ca7c. No. $W-320-31-010$

Revision 1

Page No. E - 17

DATE: $7: 21: 1998$ TIME: $17: 18: 6.0$

PAGE 17

Project W-320 / Tank 241-C-106 Sluicing

C-Farm Electrical Distribution

Calc. No. W320-31-010 (Reference Drawing H-2-818680, SH 1, Rev. I)

$$
\text { P-1361 SS }
$$

VOLTAGE BASE LL:

480.0 (VOLTS)

INI. SYM. RMS FAULT CURRENT: $8103.4 /-97$. ( AMPS/DEG )

THEVENIN EQUIVALENT IMPEDANCE: $6.158+j 14.421$ (PU)

THEVENIN IMPEDANCE X/R RATI0: $\quad 2.342$

$$
\begin{array}{lcrrr}
\text { ASYM } & \text { RMS } & \text { INTERRUPTING AMPS } & \\
1 / 2 \text { CYCLES } & 3 \text { CYCLES } & 5 \text { CYCLES } & 8 \text { CYCLES } \\
8639.4 & 8103.4 & 8103.4 & 8103.4
\end{array}
$$

ASYM RMS FAULTED CURRENT ( AMPS )

AT TIME =
-- PHASE A--8639.4 8639.4

--- PHASE C--8639.4

$P-1361$ Bus

VOLTAGE BASE LL:

480.0 (VOLTS)

INI. SYM. RMS FAULT CURRENT: $4504.3 /-78$. (AMPS/DEG)

THEVENIN EQUIVALENT IMPEDANCE: $18.708+j 21.114$ (PU)

THEVENIN IMPEDANCE X/R RATI0: 1.129

ASYM RMS INTERRUPTING AMPS
$1 / 2$ CYCLES
3 CYCLES
4504.3
5 CYCLES
4504.3
8 CYCLES
4504.3

ASYM RMS FAULTED CURRENT ( AMPS )

---PHASE A--AT TIME $=0.5$ CYCLES

4521.5

---PHASE B---

4521.5

HNF-2487, Rev. 0

Page D-68 
Calc. No. W-320-31-010

Revision 1

Page No. E - 18

DATE: $7: 21: 1998$ TIME: $17: 18: 6.0$

PAGE 18

Project W-320 / Tank 241-C-106 Sluicing

C-Farm Electrical Distribution

Caic. No. W320-31-010 (Reference Drawing H-2-818680, SH 1, Rev. 1)

Booster Vfd VOLTAGE BASE LL:

480.0 (VOLTS)

INI. SYM. RMS FAULT CURRENT: $7998.5 /-97$. ( AMPS/DEG)

THEVENIN EQUIVALENT IMPEDANCE: $6.237+j 14.611$ (PU)

THEVENIN IMPEDANCE X/R RATIO: 2.342

$$
\begin{array}{lcccr}
\text { ASYM RMS } & \text { INTERRUPTING AMPS } & \\
1 / 2 \text { CYCLES } & 3 \text { CYCLES } & 5 \text { CYCLES } & 8 \text { CYCLES } \\
8528.1 & 7998.5 & 7998.5 & 7998.5
\end{array}
$$

ASYM RMS FAULTED CURRENT ( AMPS)

$\begin{array}{ll}\text { AT TIME }= & 0.5 \text { CYCLES } \\ --- \text { PHASE A--- } & -- \text { PHASE B--- }\end{array}$ 8528.1

8528.1

8528.1

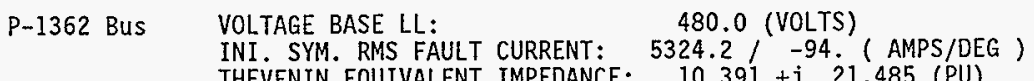

THEVENIN EQUIVALENT IMPEDANCE: $10.391+\mathrm{j} 21.485$ (PU)

THEVENIN IMPEDANCE X/R RATIO: 2.068

ASYM RMS INTERRUPTING AMPS

$\begin{array}{crrr}1 / 2 \text { CYCLES } & 3 \text { CYCLES } & 5 \text { CYCLES } & 8 \text { CYCLES } \\ 5573.4 & 5324.2 & 5324.2 & 5324.2\end{array}$

ASYM RMS FAULTED CURRENT ( AMPS)

AT TIME $=0.5$ CYCLES

PHASE A---

---PHASE B---

5573.4

PHASE C-D-

5573.4

HNF-2487, Rev. 0

Page D-69 
Calc. No. W-320-31-010

Revision 1

Page No. E - 19

DATE: $7: 21: 1998$ TIME: $17: 18: 6.0$

PAGE 19

Project W-320 / Tank 241-C-106 S1uicing

C-Farm Electrical Distribution

Calc. No. W320-31-010 (Reference Drawing H-2-818680, SH 1, Rev. 1)

FN-1362 Bus

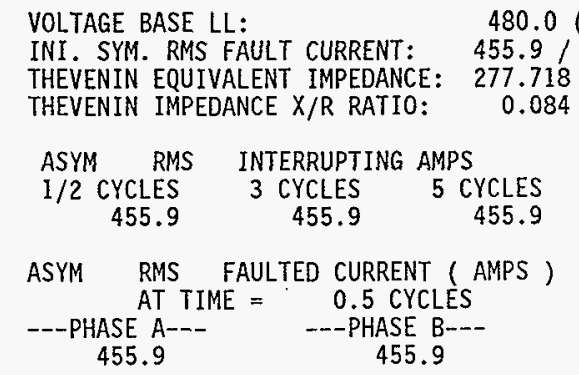
455.9
455.9

480.0 (VOLTS)

INI. SYM. RMS FAULT CURRENT: $455.9 /-35$. ( AMPS/DEG)

THEVENIN EQUIVALENT IMPEDANCE: $277.718+j 23.438$ (PU)

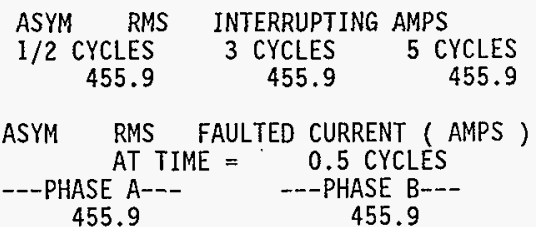

8 CYCLES

455.9

455.9

HNF-2487, Rev. 0

Page D-70 
Calc. No. W-320-31-010

Revision 1

Page No. E - 20

DATE: $7: 21: 1998$ TIME: $17: 18: 6.0$

PAGE 20

Project $W-320$ / Tank 241-C-106 STuicing

C-Farm Electrical Distribution

Calc. No. W320-31-010 (Reference Drawing H-2-818680, SH 1, Rev. 1)

$* * * * * * * * * * * * * * * * *$ F A U L T ANALYSIS REPORT

FAULT TYPE: SLG

MODEL INDUCTION MOTOR CONTRIBUTION: YES

MODEL TRANSFORMER TAPS: YES

MODEL TRANSFORMER PHASE SHIFT: YES

Ug Rsr P1 E257 VOLTAGE BASE LL:

13800.0 (VOLTS)

INI. SYM. RMS FAULT CURRENT: $1300.2 /-45$. (AMPS/DEG)

THEVENIN EQUIVALENT IMPEDANCE: $\quad 7.030+j 7.031$ (PU)

THEVENIN IMPEDANCE X/R RATIO:

1.000

SEQUENCE EQUIVALENT IMPEDANCE Z1: $0.638+\mathrm{j} 1.122$ (PU)

Z2: $\quad 0.638+j \quad 1.122(P U)$

Z0: $\quad 5.754+j$

4.787 (PU)

$$
\begin{array}{lcrrr}
\text { ASYM } & \text { RMS } & \text { INTERRUPTING AMPS } & \\
1 / 2 \text { CYCLES } & 3 \text { CYCLES } & 5 \text { CYCLES } & 8 \text { CYCLES } \\
1302.6 & 1300.2 & 1300.2 & 1300.2
\end{array}
$$

ASYM RMS FAULTED CURRENT ( AMPS )

AT TIME $=0.5$ CYCLES

---PHASE A--- ---PHASE B---

1302.6

0.0

PHASE C---

0.0

HNF-2487, Rev. 0

Page D-71 
Calc. No. W-320-31-010

Revision I

Page No. E - 21

DATE: $7: 21: 1998$ TIME: $17: 18: 6.0$

Project W-320 / Tank 241-C-106 Sluicing

PAGE 21

C-Farm Electrical Distribution

Calc. No. W320-31-010 (Reference Drawing H-2-818680, SH 1, Rev. 1)

XFMR C-T-1 Pri VOLTAGE BASE LL: 13800.0 (VOLTS)

INI. SYM. RMS FAULT CURRENT: $1283.1 /-45$. ( AMPS/DEG)

THEVENIN EQUIVALENT IMPEDANCE: $7.173+j \quad 7.075$ (PU)

THEVENIN IMPEDANCE X/R RATIO: 0.986

SEQUENCE EQUIVALENT IMPEDANCE Z1: $0.666+\mathrm{j} 1.132$ (PU)

Z2: $\quad 0.666+j \quad 1.132(P U)$

ZO: $\quad 5.842+j \quad 4.811$ (PU)

ASYM RMS INTERRUPTING AMPS

$\begin{array}{cccc}1 / 2 \text { CYCLES } & 3 \text { CYCLES } & 5 \text { CYCLES } & 8 \text { CYCLES } \\ 1285.3 & 1283.1 & 1283.1 & 1283.1\end{array}$

ASYM RMS FAULTED CURRENT ( AMPS)

AT TIME $=0.5 \mathrm{CYCLES}$

---PHASE A--- -.-PHASE B---

1285.3

0.0

---PHASE C---

0.0

XFMR C-T-1 SeC VOLTAGE BASE LL:

480.0 (VOLTS)

INI. SYM. RMS FAULT CURRENT: $8902.0 /-98$. (AMPS/DEG)

THEVENIN EQUTVALENT IMPEDANCE: $16.024+j 39.711$ (PU)

THEVENIN IMPEDANCE X/R RATI0: 2.478

SEQUENCE EQUIVALENT IMPEDANCE Z1: $5.342+j 13.288$ (PU)

$$
\text { Z2: } \quad 5.342+j 13.288 \text { (PU) }
$$

ZO: $\quad 5.340+j \quad 13.134$ (PU)

$\begin{array}{lccr}\text { ASYM } & \text { RMS } & \text { INTERRUPTING AMPS } & \\ 1 / 2 \text { CYCLES } & 3 \text { CYCLES } & 5 \text { CYCLES } & 8 \text { CYCLES } \\ 9581.3 & 8902.0 & 8902.0 & 8902.0\end{array}$

ASYM RMS FAULTED CURRENT ( AMPS)

$\begin{array}{ccc}\text { AT TIME }= & 0.5 \mathrm{CYCLES} & \\ -- \text { PHASE A--- } & -- \text { PHASE B--- } & \text {--PHASE C--- } \\ 9581.3 & 0.0 & 0.0\end{array}$

HNF-2487, Rev. 0

Page D-72 
Calc. No. W-320-31-010

Revision 1

Page No. E - 22

DATE: $7: 2 \mathrm{I}: 1998$ TIME: $17: 18: 6.0$

PAGE 22

Project $W-320$ / Tank 241-C-106 Sluicing

C-Farm Electrical Distribution

Calc. No. W320-31-010 (Reference Drawing H-2-818680, SH 1, Rev. 1)

C-PDP-1 600A VOLTAGE BASE LL: 480.0 (VOLTS)

INI. SYM. RMS FAULT CURRENT: $8649.9 /-98$. (AMPS/DEG)

THEVENIN EQUIVALENT IMPEDANCE: $16.663+j 40.798$ (PU)

THEVENIN IMPEDANCE X/R RATIO: 2.448

SEQUENCE EQUIVALENT IMPEDANCE Z1: $5.448+j 13.511$ (PU)

$$
\text { Z2: } \quad 5.448+j \quad 13.511 \text { (PU) }
$$

ZO: $\quad 5.768+j \quad 13.776(\mathrm{PU})$

\begin{tabular}{lcrr} 
ASYM & RMS & \multicolumn{1}{l}{ INTERRUPTING AMPS } & \\
$1 / 2$ CYCLES & 3 CYCLES & 5 CYCLES & 8 CYCLES \\
9290.7 & 8649.9 & 8649.9 & 8649.9
\end{tabular}

ASYM RMS FAULTED CURRENT ( AMPS )

---PHASE A--AT TIME $=\quad 0.5$ CYCLES 9290.7

---PHASE B---

0.0
-- PHASE C---
0.0

\section{Xfmr Skid}

VOLTAGE BASE LL:

INI. SYM. RMS FAULT CURRENT: $6922.5 /-87$. (AMPS/DEG)

THEVENIN EQUIVALENT IMPEDANCE: $30.079+\mathrm{j} \quad 46.126$ (PU)

THEVENIN IMPEDANCE X/R RATIO:

SEQUENCE EQUIVALENT IMPEDANCE $\mathrm{Zl}$ :

1.533

$\begin{array}{lrrr}\mathrm{Z1}: & 8.052+\mathrm{j} & 14.705(\mathrm{PU}) \\ \mathrm{Z2}: & 8.052+\mathrm{j} & 14.705(\mathrm{PU}) \\ \mathrm{Z0}: & 13.975+\mathrm{j} & 16.716(\mathrm{PU})\end{array}$

ASYM RMS INTERRUPTING AMPS
$1 / 2$ CYCLES
7036.6
3 CYCLES
6922.5
5 CYCLES
6922.5
8 CYCLES
6922.5

ASYM RMS FAULTED CURRENT (AMPS)

$\begin{array}{ccc}\text { AT TIME }= & 0.5 \text { CYCLES } & \\ -- \text { PHASE A--- } & \text {--PHASE B--- } & \text {--PHASE C--- } \\ 7036.6 & 0.0 & 0.0\end{array}$

HNF-2487, Rev. 0

Page D-73 
Calc. No. W-320-31-010

Revision 1

Page No. E - 23

DATE: $7: 21: 1998$ TIME: $17: 18: 6,0$

PAGE 23

Project $W-320$ / Tank 241-C-106 Sluicing

C-Farm Electrical Distribution

Calc. No. W320-31-010 (Reference Drawing H-2-818680, SH 1, Rev. 1)

MCC-N1 800A VOLTAGE BASE LL:

480.0 (VOLTS)

INI. SYM. RMS FAULT CURRENT: $8430.6 /-97$. (AMPS/DEG)

THEVENIN EQUIVALENT IMPEDANCE: $17.466+\mathrm{j} 41.707$ (PU)

THEVENIN IMPEDANCE X/R RATI0: 2.388

SEQUENCE EQUIVALENT IMPEDANCE Z1: $5.582+\mathrm{j} 13.698$ (PU)

Z2: $\quad 5.582+j \quad 13.698$ (PU)

Zo: $\quad 6.301+j 14.310$ (PU)

ASYM RMS INTERRUPTING AMPS
$1 / 2$ CYCLES
3 CYCLES
8430.6
5 CYCLES
8430.6
8 CYCLES
8430.6

ASYM RMS FAULTED CURRENT ( AMPS )

---PHASE A--AT TIME $=0.5$ CYCLES

9017.1

---PHASE B---

-- PHASE C---

0.0

C106-PP1 Bus

VOLTAGE BASE LL:

480.0 (VOLTS)

INI. SYM. RMS FAULT CURRENT: $2377.0 /-56$. ( AMPS/DEG)

THEVENIN EQUIVALENT IMPEDANCE: $144.242+\mathrm{j} 70.093$ (PU)

THEVENIN IMPEDANCE X/R RATIO: 0.486

SEQUENCE EQUIVALENT IMPEDANCE Z1: $30.192+j 20.059$ (PU)

Z2: $30.192+j \quad 20.059$ (PU)

$\mathrm{ZO}: \quad 83.859+\mathrm{j} 29.975$ (PU)

$\begin{array}{lcrrr}\text { ASYM } & \text { RMS } & \text { INTERRUPTING } & \text { AMPS } & \\ 1 / 2 \text { CYCLES } & 3 \text { CYCLES } & 5 \text { CYCLES } & 8 \text { CYCLES } \\ 2377.0 & 2377.0 & 2377.0 & 2377.0\end{array}$

ASYM RMS FAULTED CURRENT ( AMPS )

---PHASE A-..-

AT TIME $=0.5$ CYCLES

2377.0

-.--PHASE B---

0.0
PHASE C.--

0.0

HNF-2487, Rev. 0

Page D-74 
Calc. No. W-320-31-010

Revision 1

Page No. E - 24

DATE: $7: 21: 1998$ TIME: $17: 18: 6.0$

Project $W-320$ / Tank 241-C-106 Sluicing

PAGE 24

C-Farm Electrical Distribution

Calc. No. W320-31-010 (Reference Drawing H-2-818680, SH 1, Rev. 1)

Process Bldg

VOLTAGE BASE LL:

480.0 (VOLTS)

INI. SYM. RMS FAULT CURRENT: $2771.8 /-60$. (AMPS/DEG)

THEVENIN EQUIVALENT IMPEDANCE: $119.424+j 68.204$ (PU)

THEVENIN IMPEDANCE X/R RATIO: 0.571

SEQUENCE EQUIVALENT IMPEDANCE Z1: $25.374+j 19.636$ (PU)

Z2: $25.374+j \quad 19.636$ (PU)

ZO: $68.675+\mathrm{j} 28.932$ (PU)

ASYM RMS INTERRUPTING AMPS

$\begin{array}{rrrr}1 / 2 \text { CYCLES } & 3 \text { CYCLES } & 5 \text { CYCLES } & 8 \text { CYCLES } \\ 2771.8 & 2771.8 & 2771.8 & 2771.8\end{array}$

ASYM RMS FAULTED CURRENT ( AMPS )

---PHASE A---

AT TIME $=0.5$ CYCLES

2771.8

--DHASE B---

0.0
---PHASE C---

0.0

C106-PP2 Bus

VOLTAGE BASE LL:

480.0 (VOLTS)

INI. SYM. RMS FAULT CURRENT: $6642.8 /-78$. (AMPS/DEG)

THEVENIN EQUIVALENT IMPEDANCE: $\quad 38.707+\mathrm{j} 42.365$ (PU)

THEVENIN IMPEDANCE X/R RATIO: 1.095

SEQUENCE EQUIVALENT IMPEDANCE Z1: $9.706+\mathrm{j} 13.846$ (PU)

Z2: $\quad 9.706+j \quad 13.846$ (PU)

ZO: $19.296+j \quad 14.673$ (PU)

$\begin{array}{lcrr}\text { ASYM } & \text { RMS } & \text { INTERRUPTING } & \text { AMPS } \\ 1 / 2 \text { CYCLES } & 3 \text { CYCLES } & 5 \text { CYCLES } & 8 \text { CYCLES } \\ 6664.1 & 6642.8 & 6642.8 & 6642.8\end{array}$

ASYM RMS FAULTED CURRENT ( AMPS )

AT TIME $=0.5$ CYCLES

--- PHASE A--6664.1

-.-PHASE B--

0.0
- PHASE C---

0.0

HNF-2487, Rev. 0

Page D-75 
Calc. No. W-320-31-010

Revision 1

Page No. E - 25

DATE: $7: 21: 1998$ TIME: $17: 18: 6.0$

PAGE 25

Project $W-320$ / Tank 241-C-106 S7uicing

C-Farm Electrical Distribution

Calc. No. W320-31-010 (Reference Drawing H-2-818680, SH 1, Rev. 1)

FN-1361 Bus

VOLTAGE BASE LL:

480.0 (VOLTS)

INI. SYM. RMS FAULT CURRENT: $1635.1 /-48$. ( AMPS/DEG )

THEVENIN EQUIVALENT IMPEDANCE: $221.381+\mathrm{j} 73.085$ (PU)

THEVENIN IMPEDANCE X/R RATI0: 0.330

SEQUENCE EQUIVALENT IMPEDANCE $Z 1: 45.166+j 20.730$ (PU)

Z2: $45.166+j \quad 20.730(P U)$

$\mathrm{ZO}: 131.050+j 31.626(\mathrm{PU})$

ASYM RMS INTERRUPTING AMPS
$1 / 2$ CYCLES
3 CYCLES
1635.1
5 CYCLES
8 CYCLES
1635.1
1635.1
1635.1

ASYM RMS FAULTED CURRENT ( AMPS )

---PHASE A----

AT TIME $=\quad 0.5$ CYCLES

1635.1

-- PHASE B---

0.0

-- PHASE C--.

0.0

$H C-1361$ Bus

VOLTAGE BASE LL:

480.0 (VOLTS)

INI. SYM. RMS FAULT CURRENT: $2333.0 /-56$. ( AMPS/DEG)

THEVENIN EQUIVALENT IMPEDANCE: $146.255+\mathrm{j} 72.853$ (PU)

THEVENIN IMPEDANCE X/R RATIO: 0.498

SEQUENCE EQUIVALENT IMPEDANCE Z1: $30.582+j 20.678$ (PU)

$Z 2: \quad 30.582+j 20.678$ (PU)

$\mathrm{ZO}: \quad 85.090+\mathrm{j} \quad 3 \mathrm{l} .498$ (PU)

$$
\text { ASYM RMS INTERRUPTING AMPS }
$$

$\begin{array}{rrrr}1 / 2 \text { CYCLES } & 3 \text { CYCLES } & 5 \text { CYCLES } & 8 \text { CYCLES } \\ 2333.0 & 2333.0 & 2333.0 & 2333.0\end{array}$

ASYM RMS FAULTED CURRENT ( AMPS)

AT TIME $=0.5$ CYCLES

---PHASE A--- ---PHASE B---

2333.0

0.0

PHASE C---

0.0

HNF-2487, Rev. 0

Page D-76 
Calc. No. W-320-31-010

Revision 1

Page No. E - 26

DATE: $7: 21: 1998$ TIME: $17: 18: 6.0$

PAGE 26

Project $W-320$ / Tank 241-C-106 S7uicing

C-Farm Electrical Distribution

Calc. No. W320-31-010 (Reference Drawing H-2-818580, SH 1, Rev. 1)

UH-1362 Bus

VOLTAGE BASE LL:

480.0 (VOLTS)

INI. SYM. RMS FAULT CURRENT: $1120.6 /-43$. (AMPS/DEG)

THEVENIN EQUIVALENT IMPEDANCE: $331.836+\mathrm{j} 74.790$ (PU)

THEVENIN IMPEDANCE X/R RATIO: 0.225

SEQUENCE EQUIVALENT IMPEDANCE Z1: $66.607+j 21.112$ (PU)

Z2: $66.607+j 21.112$ (PU)

ZO: $198.622+j 32.567(\mathrm{PU})$

ASYM RMS INTERRUPTING AMPS

$\begin{array}{ccrr}1 / 2 \text { CYCLES } & 3 \text { CYCLES } & 5 \text { CYCLES } & 8 \text { CYCLES } \\ 1120.6 & 1120.6 & 1120.6 & 1120.6\end{array}$

ASYM RMS FAULTED CURRENT (AMPS)

---PHASE A---

AT TIME $=0.5$ CYCLES

1120.6

---PHASE B---

0.0

---PHASE C---

0.0

UH-1361 Bus

VOLTAGE BASE LL:

480.0 (VOLTS)

INI. SYM. RMS FAULT CURRENT: $1482.1 /-46$. ( AMPS/DEG)

THEVENIN EQUIVALENT IMPEDANCE: $246.871+j 72.156$ (PU)

THEVENIN IMPEDANCE X/R RATIO: 0.292

SEQUENCE EQUIVALENT IMPEDANCE Z1: $50.114+\mathrm{j} 20.521$ (PU)

Z2: $50.114+j 20.521$ (PU)

Z0: $146.644+j 31.113$ (PU)

$\begin{array}{lccr}\text { ASYM } & \text { RMS } & \text { INTERRUPTING } & \text { AMPS } \\ 1 / 2 \text { CYCLES } & 3 \text { CYCLES } & 5 \text { CYCLES } & 8 \text { CYCLES } \\ 1482.1 & 1482.1 & 1482.1 & 1482.1\end{array}$

ASYM RMS FAULTED CURRENT ( AMPS )

AT TIME $=$
- --PHASE A---

1482.1

0.0

--PHASE C---

0.0

HINF-2487, Rev. 0

Page D-77 
Calc. No. W-320-31-010

Revision 1

Page No. E - 27

DATE: $7: 21: 1998$ TIME: $17: 18: 6,0$

PAGE 27

Project $W-320 /$ Tank 241-C-106 Sluicing

C-Farm Electrical Distribution

Calc. No. W320-31-010 (Reference Drawing H-2-818680, SH 1, Rev. 1)

Service B1dg

VOLTAGE BASE LL:

INI. SYM. RMS FAULT CURRENT: 2008.9/-45. (AMPS/DEG)

THEVENIN EQUIVALENT IMPEDANCE: $183.147+j 49.639$ (PU)

THEVENIN IMPEDANCE X/R RATIO:

SEQUENCE EQUIVALENT IMPEDANCE 21 :

0.271

$37.744+j$

Z2: $37.744+j$

ZO: $107.660+j$

15.476 (PU)

15.476 (PU)

18.687 (PU)

ASYM RMS INTERRUPTING AMPS

$$
\begin{array}{ccrr}
1 / 2 \text { CYCLES } & 3 \text { CYCLES } & 5 \text { CYCLES } & 8 \text { CYCLES } \\
2008.9 & 2008.9 & 2008.9 & 2008.9
\end{array}
$$

ASYM RMS FAULTED CURRENT ( AMPS)

---PHASE A--AT TIME = 2008.9
0.5 CYCLES

---PHASE B---

0.0
-- PHASE C---

0.0

\section{C106-PP3 Bus}

VOLTAGE BASE LL:

480.0 (VOLTS)

INI. SYM. RMS FAULT CURRENT: $1884.9 /-44$. ( AMPS/DEG)

THEVENIN EQUIVALENT IMPEDANCE: $195.892+\mathrm{j} 50.249$ (PU)

THEVENIN IMPEDANCE X/R RATIO: 0.257

SEQUENCE EQUIVALENT IMPEDANCE Z1: $40.218+\mathrm{j} 15.612$ (PU)

Z2: $40.218+j \quad 15.612$ (PU)

ZO: $115.456+\mathrm{j} 19.024$ (PU)

ASYM RMS INTERRUPTING AMPS

$\begin{array}{rrrr}1 / 2 \text { CYCLES } & 3 \text { CYCLES } & 5 \text { CYCLES } & 8 \text { CYCLES } \\ 1884.9 & 1884.9 & 1884.9 & 1884.9\end{array}$

ASYM RMS FAULTED CURRENT (AMPS)

AT TIME $=0.5$ CYCLES

---PHASE A--- ---PHASE B---

1884.9

0.0

0.0

HNF-2487, Rev. 0

Page D-78 
Calc. No. $W-320-31-010$

Revision 1

Page No. E - 28

DATE: $7: 21: 1998$ TIME: $17: 18: 6.0$

PAGE 28

Project $W-320$ / Tank 241-C-106 Sluicing

C-Farm Electrical Distribution

Calc. No. W320-31-010 (Reference Drawing H-2-818680, SH 1, Rev. I)

CPR-1361 BuS VOLTAGE BASE LL: 480.0 (VOLTS)

INI. SYM. RMS FAULT CURRENT: $1677.2 /-43$. ( AMPS/DEG)

THEVENIN EQUIVALENT IMPEDANCE: $221.381+\mathrm{j} 51.469$ (PU)

THEVENIN IMPEDANCE X/R RATIO: 0.232

SEQUENCE EQUIVALENT IMPEDANCE $21: 45.166+j 15.886$ (PU)

$$
\text { Z2: } 45.166+j 15.885 \text { (PU) }
$$

Z0: $131.050+\mathrm{j} 19.697$ (PU)

ASYM RMS INTERRUPTING AMPS
$1 / 2$ CYCLES
1677.2
3 CYCLES
1677.2
5 CYCLES
1677.2
8 CYCLES
1677.2

ASYM RMS FAULTED CURRENT ( AMPS )

-..-PHASE A-בAT TIME $=0.5$ CYCLES

1677.2

-..-PHASE B-.-

--- PHASE C---

0.0

Office Tri Bus VOLTAGE BASE LL:

480.0 (VOLTS)

INI. SYM. RMS FAULT CURRENT: 4641.6/-69. (AMPS/DEG)

THEVENIN EQUIVALENT IMPEDANCE: $63.619+\mathrm{j} 51.937$ (PU)

THEVENIN IMPEDANCE X/R RATIO: 0.816

SEQUENCE EQUIVALENT IMPEDANCE $21: 14.562+j 16.007$ (PU)

Z2: $14.562+j \quad 16.007$ (PU)

ZO: $34.494+j \quad 19.924$ (PU)

\begin{tabular}{lcrrr} 
ASYM & RMS & \multicolumn{1}{l}{ INTERRUPTING } & AMPS & \\
$1 / 2$ CYCLES & 3 CYCLES & 5 CYCLES & 8 CYCLES \\
4643.7 & 4641.6 & 4641.6 & 4641.6
\end{tabular}

ASYM RMS FAULTED CURRENT (AMPS)

---PHASE A---

AT TIME $=0.5$ CYCLES

4643.7

-..PHASE B---

0.0
PHASE C---

0.0

HNF-2487, Rev. 0

Page D-79 
Calc. No. $W-320-31-010$ Revision 1

Page No. E - 29

DATE: $7: 21: 1998$ TIME: $17: 18: 6.0$

PAGE 29

Project $W-320$ / Tank 24l-C-106 Sluicing

C-Farm Electrical Distribution

Calc. No. W320-31-010 (Reference Drawing H-2-818680, SH 1, Rev. 1)

Conex T1r Bus VOLTAGE BASE LL:

480.0 (VOL.TS)

INI. SYM. RMS FAULT CURRENT: $3372.7 /-61$. ( AMPS/DEG)

THEVENIN EQUIVALENT IMPEDANCE: $97.158+\mathrm{j} 57.749$ (PU)

THEVENIN IMPEDANCE $X / R$ RATIO:

0.594

SEQUENCE EQUIVALENT IMPEDANCE Z1: $21.073+j$

Z2: $21.073+\mathbf{j}$

Z0: $\quad 55.012+j$

17.309 (PU)

$17.309(\mathrm{PU})$

23.131 (PU)

ASYM RMS INTERRUPTING AMPS

$\begin{array}{lrrr}1 / 2 \text { CYCLES } & 3 \text { CYCLES } & 5 \text { CYCLES } & 8 \text { CYCLES } \\ 3372.8 & 3372.7 & 3372.7 & 3372.7\end{array}$

ASYM RMS FAULTED CURRENT ( AMPS )

$\begin{aligned} \text { AT TIME } & =0.5 \text { CYCLES } \\ \ldots \text { PHASE A-- } & \text { - }\end{aligned}$

3372.8

0.0

-- PHASE C---

0.0

Chiller Skid

VOLTAGE BASE LL:

480.0 (VOLTS)

INI. SYM. RMS FAULT CURRENT: 7698.2/ -94. (AMPS/DEG)

THEVENIN EQUIVALENT IMPEDANCE: $21.530+j 44.593$ (PU)

THEVENIN IMPEDANCE X/R RATIO: 2.071

SEQUENCE EQUIVALENT IMPEDANCE Z1: $6.322+\mathrm{j} 14.324$ (PU)

Z2: $6.322+j \quad 14.324$ (PU)

$Z 0: \quad 8.886+j \quad 15.945$ (PU)

$\begin{array}{lccr}\text { ASYM } & \text { RMS } & \text { INTERRUPTING } & \text { AMPS } \\ 1 / 2 \text { CYCLES } & 3 \text { CYCLES } & 5 \text { CYCLES } & 8 \text { CYCLES } \\ 8060.3 & 7698.2 & 7698.2 & 7698.2\end{array}$

ASYM RMS FAULTED CURRENT (AMPS)

$\begin{array}{ccc}\text { AT TIME } & 0.5 \text { CYCLES } & \\ -- \text { PHASE A-_- } & \text {---PHASE B--- } & \text {---PHASE C--- } \\ 8060.3 & 0.0 & 0.0\end{array}$

HNF-2487, Rev. 0

Page D-80 
Calc. No. W-320-31-010

Revision 1

Page No. E - 30

DATE: $7: 21: 1998$ TIME: $17: 18: 6.0$

PAGE $\quad 30$

Project $W-320$ / Tank 241-C-106 Sluicing

C-Farm Electrical Distribution

Calc. No. W320-31-010 (Reference Drawing H-2-818680, SH 1, Rev. 1)

Exhaust Skid

EXH Fan Vfd
VOLTAGE BASE LL:

INI. SYM. RMS FAULT CURRENT:

THEVENIN EQUIVALENT IMPEDANCE:

THEVENIN IMPEDANCE X/R RATIO:

SEQUENCE EQUIVALENT IMPEDANCE
480.0 (VOLTS)

$455.4 /-36$. ( AMPS/DEG)

$833.128+j \quad 80.756$ (PU)

$$
0.097
$$

Z1: $163.916+j 22.448$ (PU)

Z2: $163.916+j \quad 22.448$ (PU)

ZO: $505.297+\mathrm{j} \quad 35.859$ (PU)

ASYM RMS INTERRUPTING AMPS

$1 / 2$ CYCLES

455.4

3 CYCLES

455.4

5 CYCLES

455.4

8 CYCLES

455.4

ASYM RMS FAULTED CURRENT (AMPS) AT TIME $=0.5$ CYCLES

---PHASE A--455.4
0.0
---PHASE C---
VOLTAGE BASE LL:

INI. SYM. RMS FAULT CURRENT: 5166.1/ -66. (AMPS/DEG)

THEVENIN EQUIVALENT IMPEDANCE: $59.948+j \quad 43.024$ (PU)

THEVENIN IMPEDANCE X/R RATIO: 0.718

SEQUENCE EQUIVALENT IMPEDANCE Z1: $13.829+\mathrm{j} 13.993$ (PU)

Z2: $13.829+\mathrm{j} 13.993(\mathrm{PU})$

ZO: $32.290+\mathrm{j} 15.037$ (PU)

$$
\begin{array}{lcrr}
\text { ASYM } & \text { RMS } & \text { INTERRUPTING } & \text { AMPS } \\
1 / 2 \text { CYCLES } & 3 \text { CYCLES } & 5 \text { CYCLES } & 8 \text { CYCLES } \\
5166.9 & 5166.1 & 5166.1 & 5166.1
\end{array}
$$

ASYM RMS FAULTED CURRENT ( AMPS )

-.-PHASE A---

5166.9

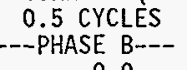

0.0
PHASE C---

0.0

HNF-2487, Rev. 0

Page D-81 
Calc. No. W-320-31-010 Revision 1

Page No. E - 31

DATE: $7: 21: 1998$ TIME: 17:18: 6.0

PAGE 31

Project W-320 / Tank 241-C-106 S7uicing

C-Farm Electrical Distribution

Calc. No. W320-31-010 (Reference Drawing H-2-818680, SH 1, Rev, 1)

WRAM TIr Bus

VOLTAGE BASE LL:

INI. SYM. RMS FAULT CURRENT: $1989.8 /-52$. (AMPS/DEG)

THEVENIN EQUIVALENT IMPEDANCE: $177.653+\mathrm{j} 71.697$ (PU)

THEVENIN IMPEDANCE X/R RATIO:

SEQUENCE EQUTVALENT IMPEDANCE $21: 36.698+j$

Z2: $36.698+j$

ZO: $104.257+j$

20.434 (PU)

20.434 (PU)

30.829 (PU)
ASYM RMS INTERRUPTING AMPS
$\begin{array}{rrrr}1 / 2 \text { CYCLES } & 3 \text { CYCLES } & 5 \text { CYCLES } & 8 \text { CYCLES } \\ 1989.8 & 1989.8 & 1989.8 & 1989.8\end{array}$

ASYM RMS FAULTED CURRENT ( AMPS )

---PHASE A--AT TIME $=0.5$ CYCLES

1989.8
PHASE $\mathrm{C}---$

0.0

MCC-N2

VOLTAGE BASE LL:

INI. SYM. RMS FAULT CURRENT: 7718.0/-96. ( AMPS/DEG)

THEVENIN EQUIVALENT IMPEDANCE: $20.391+\mathrm{j} 44.985$ (PU)

THEVENIN IMPEDANCE X/R RATIO: 2.206

SEQUENCE EQUIVALENT IMPEDANCE Z1:

6.111

Z2:

20:

$6.111+j$

$8.168+j$

14.402 (PU)

14.402 (PU)

16.180 (PU)

$\begin{array}{lcrr}\text { ASYM } & \text { RMS } & \text { INTERRUPTING AMPS } & \\ 1 / 2 \text { CYCLES } & 3 \text { CYCLES } & 5 \text { CYCLES } & 8 \text { CYCLES } \\ 8153.0 & 7718.0 & 7718.0 & 7718.0\end{array}$

ASYM RMS FAULTED CURRENT ( AMPS)

AT TIME $=\quad 0.5$ CYCLES
- PHASE A--

8153.0

0.0

PHASE C--

0.0

HNF-2487, Rev. 0

Page D-82 
Calc. No. W-320-31-010

Revision 1

Page No. E - 32

DATE: $7: 21: 1998$ TIME: $17: 18: 6.0$

PAGE 32

Project $W-320$ / Tank 241-C-106 S7uicing

C-Farm Electrical Distribution

Ca7c. No. W320-31-010 (Reference Drawing H-2-818680, SH I, Rev. 1)

STuicer Skid

VOLTAGE BASE LL:

INI. SYM. RMS FAULT CURRENT: $366.7 /-34$. ( AMPS/DEG)

THEVENIN EQUIVALENT IMPEDANCE: INFINITE

THEVENIN IMPEDANCE X/R RATIO:

SEQUENCE EQUIVALENT

$$
\begin{aligned}
& 0.071 \\
& \text { Z1: } 203.499+\mathrm{j} 20.782 \text { (PU) } \\
& \text { Z2: } 203.499+j 20.782 \text { (PU) } \\
& \text { ZO: } 630.045+j 31.754 \text { (PU) }
\end{aligned}
$$

ASYM RMS INTERRUPTING AMPS

$\begin{array}{rrrr}1 / 2 \text { CYCLES } & 3 \text { CYCLES } & 5 \text { CYCLES } & 8 \text { CYCLES } \\ 366.7 & 366.7 & 366.7 & 366.7\end{array}$

ASYM RMS FAULTED CURRENT ( AMPS)

---PHASE A--AT TIME $=0.5$ CYCLES 366.7
-- PHASE B-.--

0.0
-- PHASE C---
0.0
Slurry Winch

VOLTAGE BASE LL: INI. SYM. RMS FAULT CURRENT: $366.7 /-34$. ( AMPS/DEG) THEVENIN EQUIVALENT IMPEDANCE: INFINITE THEVENIN IMPEDANCE X/R RATIO: 0.071

SEQUENCE EQUIVALENT IMPEDANCE Z1: $203.499+j 20.782$ (PU) $Z 2: 203.499+j 20.782$ (PU) ZO: $630.045+j 31.754$ (PU)

ASYM RMS INTERRUPTING AMPS $\begin{array}{rrrr}1 / 2 \text { CYCLES } & 3 \text { CYCLES } & 5 \text { CYCLES } & 8 \text { CYCLES } \\ 366.7 & 366.7 & 366.7 & 366.7\end{array}$

ASYM RMS FAULTED CURRENT (AMPS)

---PHASE A--AT TIME $=0.5$ CYCLES 366.7
- PHASE C---

0.0

HNF-2487, Rev. 0

Page D-83 
Ca7c. No. W-320-31-010

Revision 1

Page No. E - 33

DATE: $7: 21: 1998$ TIME: $17: 18: 6.0$

PAGE

Project $W-320$ / Tank 241-C-106 Sluicing

C-Farm Electrical Distribution

Calc. No. W320-31-010 (Reference Drawing H-2-818680, SH 1, Rev. 1)

P-1361 SS

VOLTAGE BASE LL: $\quad 480.0$ (VOLTS)

INI. SYM. RMS FAULT CURRENT: $7694.5 /$-95. ( AMPS/DEG)

THEVENIN EQUIVALENT IMPEDANCE: $20.568+j \quad 45.070$ (PU)

THEVENIN IMPEDANCE X/R RATIO: 2.191

SEQUENCE EQUIVALENT IMPEDANCE Z1: $6.158+\mathrm{j} 14.421$ (PU)

22: $\quad 6.158+j \quad 14.421$ (PU)

ZO: $\quad 8.251+j \quad 16.229$ (PU)

ASYM RMS INTERRUPTING AMPS
$1 / 2$ CYCLES
3 CYCLES
7694.5
5 CYCLES
8 CYCLES
8120.1
7694.5
7694.5

ASYM RMS FAULTED CURRENT (AMPS)

$$
\text { AT TIME }=0.5 \text { CYCLES }
$$

---PHASE A---

8120.1

---PHASE B---

0.0

$-\cdots$ PHASE C---
0.0

P-1361 Bus

VOLTAGE BASE LL:

480.0 (VOLTS)

INI. SYM. RMS FAULT CURRENT: $3220.8 /-69$. (AMPS/DEG)

THEVENIN EQUIVALENT IMPEDANCE: $91.629+j 74.912$ (PU)

THEVENIN IMPEDANCE X/R RATI0: 0.818

SEQUENCE EQUIVALENT IMPEDANCE $\mathrm{Z1}: 18.708+\mathrm{j} 21.114$ (PU)

$Z 2: \quad 18.708+j \quad 21.114$ (PU)

Z0: $\quad 54.213+\mathrm{j} 32.685$ (PU)

$$
\begin{array}{lcrrr}
\text { ASYM } & \text { RMS } & \text { INTERRUPTING } & \text { AMPS } & \\
1 / 2 \text { CYCLES } & 3 \text { CYCLES } & 5 \text { CYCLES } & 8 \text { CYCLES } \\
3222.3 & 3220.8 & 3220.8 & 3220.8
\end{array}
$$

ASYM RMS FAULTED CURRENT ( AMPS)

AT TIME $=\quad 0.5$ CYCLES
-- PHASE A---

3222.3

0.0

---PHASE C---

0.0

HNF-2487, Rev. 0

Page D-84 
Ca]c. No. W-320-31-010

Revision 1

Page No. E - 34

DATE: $7: 21: 1998$ TIME: $17: 18: 6.0$

PAGE 34

Project $W-320$ / Tank 241-C-106 S7uicing

C-Farm Electrical Distribution

Calc. No. W320-31-010 (Reference Drawing H-2-818680, SH 1, Rev. 1)

Booster Vfd VOLTAGE BASE LL: 480.0 (VOLTS)

INI. SYM. RMS FAULT CURRENT: 7547.6/ $/ 95$. (AMPS/DEG)

THEVENIN EQUIVALENT IMPEDANCE: $21.040+\mathrm{j} 45.915$ (PU)

THEVENIN IMPEDANCE X/R RATIO: 2.182

SEQUENCE EQUIVALENT IMPEDANCE Z1: $6.237+\mathrm{j} 14.611$ (PU)

$$
\text { Z2: } \quad 6.237+\mathrm{j} 14.611 \text { (PU) }
$$

Z0: $8.565+\mathrm{j} \quad 16.694$ (PU)

$$
\begin{array}{lcrr}
\text { ASYM } & \text { RMS } & \text { INTERRUPTING AMPS } & \\
1 / 2 \text { CYCLES } & 3 \text { CYCLES } & 5 \text { CYCLES } & 8 \text { CYCLES } \\
7960.4 & 7547.6 & 7547.6 & 7547.6
\end{array}
$$

ASYM RMS FAULTED CURRENT ( AMPS )

---PHASE A---

$$
\text { AT TIME }=0.5 \text { CYCLES }
$$

7960.4

--- PHASE B---

0.0
---PHASE C---

0.0

\section{P-1362 Bus}

VOLTAGE BASE LL:

480.0 (VOLTS)

INI. SYM. RMS FAULT CURRENT: $4353.3 /-91$. (AMPS/DEG)

THEVENIN EQUIVALENT IMPEDANCE: $42.438+j 76.594$ (PU)

THEVENIN IMPEDANCE X/R RATIO: 1.805

SEQUENCE EQUIVALENT IMPEDANCE Z1: $10.391+\mathrm{j} 21.485$ (PU)

Z2: $10.391+j 21.485$ (PU)

ZO: $21.656+j$

33.624

(PU)

$$
\begin{array}{lcrr}
\text { ASYM } & \text { RMS } & \multicolumn{1}{c}{\text { INTERRUPTING AMPS }} & \\
1 / 2 \text { CYCLES } & 3 \text { CYCLES } & 5 \text { CYCLES } & 8 \text { CYCLES } \\
4485.3 & 4353.3 & 4353.3 & 4353.3
\end{array}
$$

ASYM RMS FAULTED CURRENT ( AMPS)

---PHASE A---

4485.3

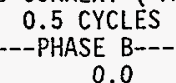

0.0
PHASE C---

0.0

HNF-2487, Rev. 0

Page D-85 
Ca7c. No. W-320-31-010

Revision 1

Page No. E - 35

DATE: $7: 21: 1998$ TIME: $17: 18: 6.0$

Project $W-320$ / Tank 241-C-106 Sluicing

C-Farm Electrical Distribution

Calc. No. W320-31-010 (Reference Drawing H-2-818680, SH 1, Rev. 1)

FN-1362 BuS VOLTAGE BASE LL: 480.0 (VOLTS)

INI. SYM. RMS FAULT CURRENT: 268.1 / -33. ( AMPS/DEG)

THEVENIN EQUIVALENT IMPEDANCE: INFINITE

THEVENIN IMPEDANCE X/R RATIO: 0.060

SEQUENCE EQUIVALENT IMPEDANCE $Z 1: 277.718+\mathrm{j} 23.438$ (PU)

Z2: $277.718+j \quad 23.438$ (PU)

ZO: $863.949+j 38.296$ (PU)

\begin{tabular}{ccrrr} 
ASYM & RMS & INTERRUPTING AMPS \\
$1 / 2$ CYCLES & 3 CYCLES & 5 CYCLES & 8 CYCLES \\
268.1 & 268.1 & 268.1 & 268.1 \\
ASYM & RMS FAULTED CURRENT ( AMPS ) \\
\multicolumn{4}{c}{ AT TIME $=$} & 0.5 CYCLES \\
--PHASE A--- & - -PHASE B--- & ---PHASE C--- \\
268.1 & 0.0 & 0.0
\end{tabular}

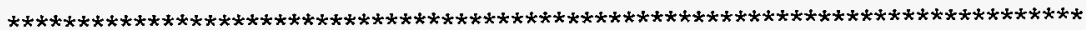

HNF-2487, Rev. 0

Page D-86 
Calc. No. $W-320-31-010$

Revision 1

Page No. E - 36

DATE: $7: 21: 1998$ TIME: $17: 18: 6.0$

PAGE 36

Project W-320 / Tank 241-C-106 Sluicing

C-Farm Electrical Distribution

Calc. No. W320-31-010 (Reference Drawing H-2-818680, SH 1, Rev. 1)

\begin{tabular}{|c|c|c|c|c|c|}
\hline BUS NAME & $\begin{array}{c}\text { VOLTAGE } \\
\text { L-L }\end{array}$ & $\begin{array}{l}\text { AVAILABLE } \\
3 \text { PHASE }\end{array}$ & $\begin{array}{l}\text { FAUL } \\
X / R\end{array}$ & $\begin{array}{l}\text { CURRENT } \\
\text { INE/GRND }\end{array}$ & $X / R$ \\
\hline $\begin{array}{l}\text { Ug Rsr P1 E257 } \\
\text { XFMR C-T-1 Pri } \\
\text { XFMR C-T-1 Sec } \\
\text { C-PDP-1 } 600 A \\
\text { Xfmr Skid }\end{array}$ & $\begin{array}{r}13800 \\
13800 \\
480 \\
480 \\
480\end{array}$ & $\begin{array}{l}3338.1 \\
3281.3 \\
8872.2 \\
8722.2 \\
7579.3\end{array}$ & $\begin{array}{l}1.8 \\
1.7 \\
2.5 \\
2.5 \\
1.8\end{array}$ & $\begin{array}{l}1300.2 \\
1283.1 \\
8902.0 \\
8649.9 \\
6922.5\end{array}$ & $\begin{array}{l}1.0 \\
1.0 \\
2.5 \\
2.4 \\
1.5\end{array}$ \\
\hline $\begin{array}{l}\text { MCC-N1 800A } \\
\text { Process Bldg } \\
\text { C106-PP1 Bus } \\
\text { C106-PP2 Bus } \\
\text { FN-1361 Bus }\end{array}$ & $\begin{array}{l}480 \\
480 \\
480 \\
480 \\
480\end{array}$ & $\begin{array}{l}8590.1 \\
3960.4 \\
3505.5 \\
7514.8 \\
2556.9\end{array}$ & $\begin{array}{l}2.5 \\
0.8 \\
0.7 \\
1.4 \\
0.5\end{array}$ & $\begin{array}{l}8430.6 \\
2771.8 \\
2377.0 \\
6642.8 \\
1635.1\end{array}$ & $\begin{array}{l}2.4 \\
0.6 \\
0.5 \\
1.1 \\
0.3\end{array}$ \\
\hline $\begin{array}{l}\text { HC-1361 Bus } \\
\text { UH-1362 Bus } \\
\text { UH-1361 Bus } \\
\text { Service Bldg } \\
\text { CPR-1361 Bus }\end{array}$ & $\begin{array}{l}480 \\
480 \\
480 \\
480 \\
480\end{array}$ & $\begin{array}{l}3442.0 \\
1818.5 \\
2346.5 \\
3114.9 \\
2654.0\end{array}$ & $\begin{array}{l}0.7 \\
0.3 \\
0.4 \\
0.4 \\
0.4\end{array}$ & $\begin{array}{l}2333.0 \\
1120.6 \\
1482.1 \\
2008.9 \\
1677.2\end{array}$ & $\begin{array}{l}0.5 \\
0.2 \\
0.3 \\
0.3 \\
0.2\end{array}$ \\
\hline $\begin{array}{l}\text { Office Trl Bus } \\
\text { Conex TTr Bus } \\
\text { Chiller Skid } \\
\text { Exhaust Skid } \\
\text { EXH Fan Vfd }\end{array}$ & $\begin{array}{l}480 \\
480 \\
480 \\
480 \\
480\end{array}$ & $\begin{array}{r}5871.9 \\
4659.5 \\
8115.8 \\
768.0 \\
6458.7\end{array}$ & $\begin{array}{l}1.1 \\
0.8 \\
2.3 \\
0.1 \\
1.0\end{array}$ & $\begin{array}{r}4641.6 \\
3372.7 \\
7698.2 \\
455.4 \\
5166.1\end{array}$ & $\begin{array}{l}0.8 \\
0.6 \\
2.1 \\
0.1 \\
0.7\end{array}$ \\
\hline $\begin{array}{l}\text { WRAM Tlr Bus } \\
\text { MCC-N2 } \\
\text { Sluicer Skid } \\
\text { Slurry Winch } \\
\text { Booster Vfd }\end{array}$ & $\begin{array}{l}480 \\
480 \\
480 \\
480 \\
480\end{array}$ & $\begin{array}{r}3025.2 \\
8121.7 \\
621.2 \\
621.2 \\
7998.5\end{array}$ & $\begin{array}{l}0.6 \\
2.4 \\
0.1 \\
0.1 \\
2.3\end{array}$ & $\begin{array}{r}1989.8 \\
7718.0 \\
366.7 \\
366.7 \\
7547.6\end{array}$ & $\begin{array}{l}0.4 \\
2.2 \\
0.1 \\
0.1 \\
2.2\end{array}$ \\
\hline $\begin{array}{l}P-1361 \text { SS } \\
P-1361 \text { Bus } \\
\text { P-1362 Bus } \\
\text { FN-1362 Bus } \\
\text { C106-PP3 Bus }\end{array}$ & $\begin{array}{l}480 \\
480 \\
480 \\
480 \\
480\end{array}$ & $\begin{array}{r}8103.4 \\
4504.3 \\
5324.2 \\
455.9 \\
2945.3\end{array}$ & $\begin{array}{l}2.3 \\
1.1 \\
2.1 \\
0.1 \\
0.4\end{array}$ & $\begin{array}{r}7694.5 \\
3220.8 \\
4353.3 \\
268.1 \\
1884.9\end{array}$ & $\begin{array}{l}2.2 \\
0.8 \\
1.8 \\
0.1 \\
0.3\end{array}$ \\
\hline
\end{tabular}

$* * * * * * * * * * * * * * * * * * * * * * *$ FAULT ANALYSIS REPORT COMPLETED $* * * * * * * * * * * * * * * * * * * * * * *$

HNF-2487, Rev. 0

Page D-87 


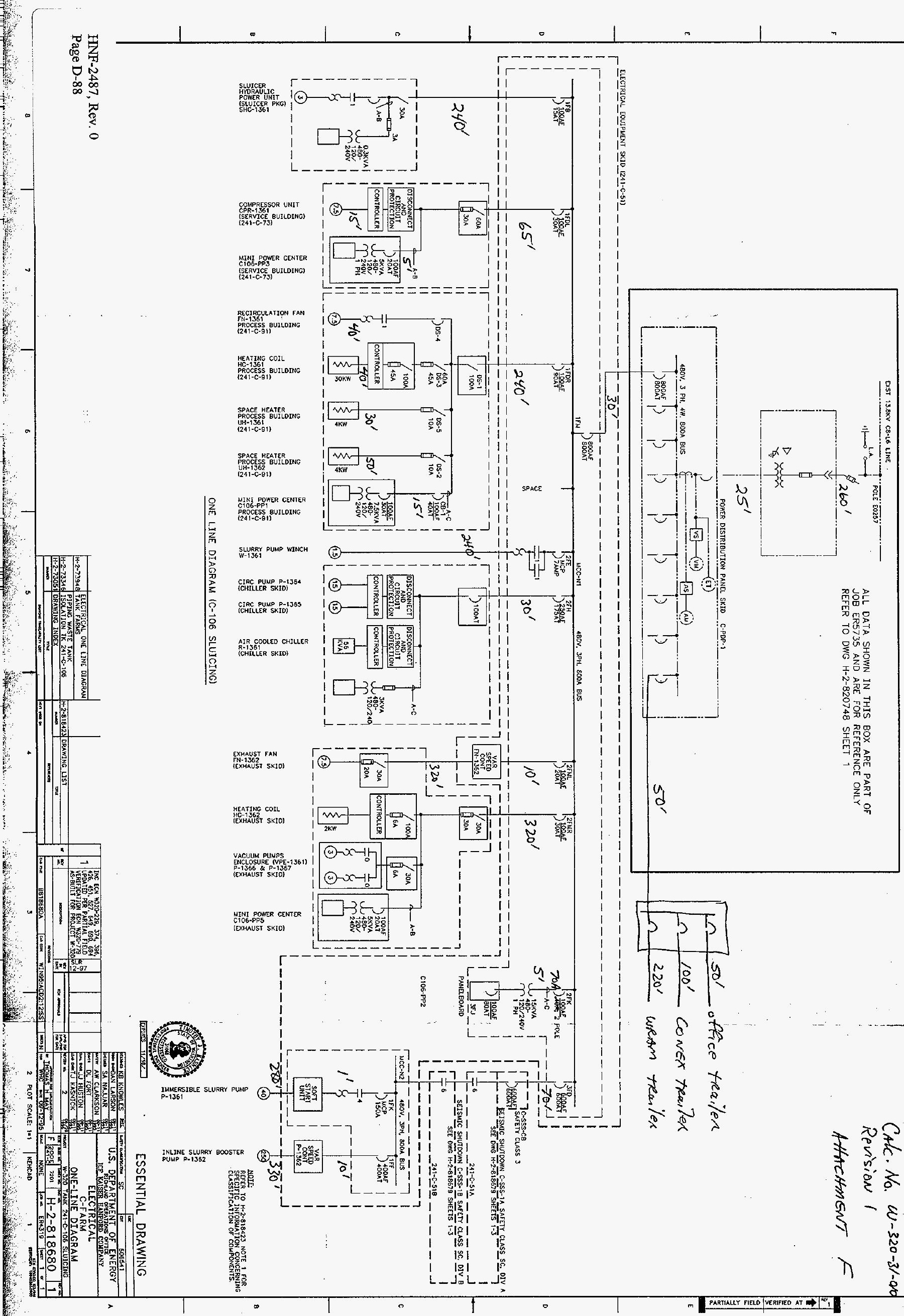


HNF-2487, Rev. 0

W320-31-011

Tank C-106 Load Determination, Feeder/Protection Sizing, Voltage

Drop, Lighting Calculations and Conduit sizing 


\section{Fluor Daniel Northwest}

This sheet shows the status and description of the attached Design Analysis sheets.
Discipline Electrical
wo/dob No. Task Order N09
Calculation No. W320-31-011

Project No. \& Name W-320 Tank 241-C-106 Sluicing

calculation Iten TANK C-106 LOAD DETERMINATION, FEEDER/PROTECTION SIZING, VOLTAGE DROP, LIGHTING CALCULATIONS, \& CONDUIT SIZING

These calculations apply to:

Dwg. No. See Continuation Sheet

Rev. No.

Dwg. No.

Rev. No.

other (study, CDR)

Rev. No.

The status of these calculations is:

[] prel iminary calculations

[X] Final Calculations

[] Check Calculations (On Catculation Dated)

[] Void Calculation (Reason Voided)

Incorporated in Final Drawings?

Calculation verified by independent "check" calculations?

$[X]$ Yes [] No

[] Yes $[X]$ No

original and Revised Calculation Approvals:

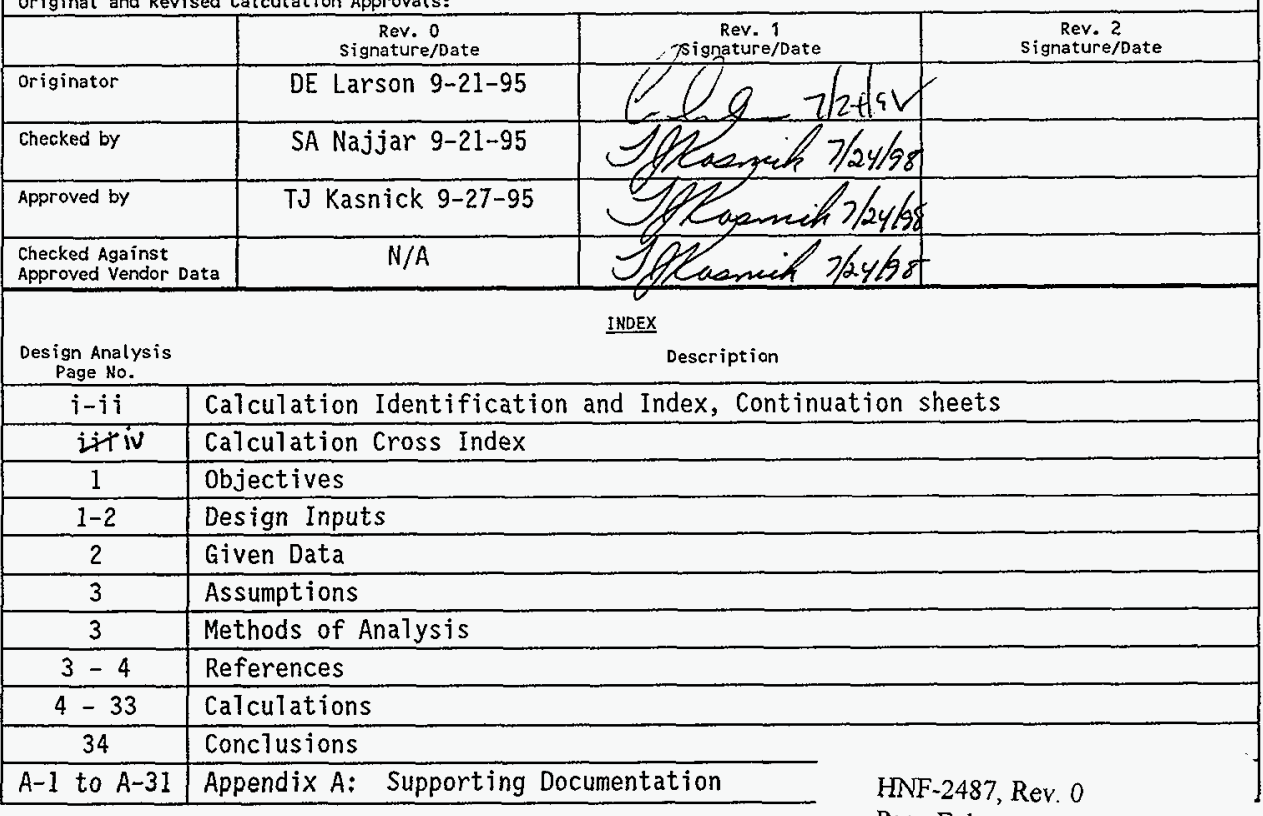

Page E-1 


\begin{tabular}{|c|c|c|c|}
\hline \multirow{2}{*}{\multicolumn{2}{|c|}{ Fluor Daniel Northwest }} & \multirow{2}{*}{$\begin{array}{l}\text { CALCULATION IDENTIFICATION AN } \\
\text { INDEX } \\
\text { Continuation sheet }\end{array}$} & Page $i j$ of 34 \\
\hline & & & $7 / 22 / 98$ \\
\hline \multicolumn{2}{|c|}{ Discipline Electrical } & Wo/Job No. Task Order N09 & No. $W 320-31-011$ \\
\hline & \multicolumn{2}{|c|}{$\begin{array}{c}\text { Rev. } 0 \\
\text { Signature/Date }\end{array}$} & $\begin{array}{c}\text { Rev. } 2 \\
\text { Signature/Date }\end{array}$ \\
\hline Originator & \multicolumn{2}{|c|}{ DE Larson 9-21-95 } & \\
\hline Checked by & \multicolumn{2}{|c|}{ SA Najjar 9-2I-95 } & \\
\hline
\end{tabular}

These calculations apply to:

H-2-818673, Sheet 1, Rev. 0 $\mathrm{H}-2-818674$, Sheet 1, Rev. 0

$\mathrm{H}-2-818674$, Sheet 2, Rev. 0

$\mathrm{H}-2-818674$, Sheet 3 , Rev. 0

$\mathrm{H}-2-818674$, Sheet 5, Rev. 0

$\mathrm{H}-2-818676$, Sheet 1 , Rev. 0

$\mathrm{H}-2-818676$, Sheet 2, Rev. 0

$\mathrm{H}-2-818676$, Sheet 3, Rev. 0

$\mathrm{H}-2-818676$, Sheet 4, Rev. 0

$\mathrm{H}-2-818676$, Sheet 5, Rev. 0

$\mathrm{H}-2-818677$, Sheet 1, Rev. 0

H-2-818677, Sheet 2, Rev. 0

$\mathrm{H}-2-818678$, Sheet 1 , Rev. 0

H-2-818678, Sheet 2, Rev. 0

$\mathrm{H}-2-818678$, Sheet 3, Rev. 0

$\mathrm{H}-2-818678$, Sheet 4 , Rev. 0

$\mathrm{H}-2-818678$, Sheet 5, Rev. 0

H-2-818679, Sheet 3, Rev. 0

$\mathrm{H}-2-818680$, Sheet 1, Rev. 0

$\mathrm{H}-2-818681$, Sheet 1, Rev. 0

$\mathrm{H}-2-818681$, Sheet 2, Rev. 0

$\mathrm{H}-2-818681$, Sheet 3, Rev. 0

H-2-818690, Sheet 1, Rev. 0

$\mathrm{H}-2-818702$, Sheet 1, Rev. 0

$\mathrm{H}-2-818702$, Sheet 2, Rev. 0

$\mathrm{H}-2-818704$, Sheet 1 , Rev. 0

$\mathrm{H}-2-818704$, Sheet 2, Rev. 0

H-2-818704, Sheet 3, Rev. 0

$\mathrm{H}-2-818704$, Sheet 4, Rev. 0

HNF-2487, Rev. 0

Page E-2 


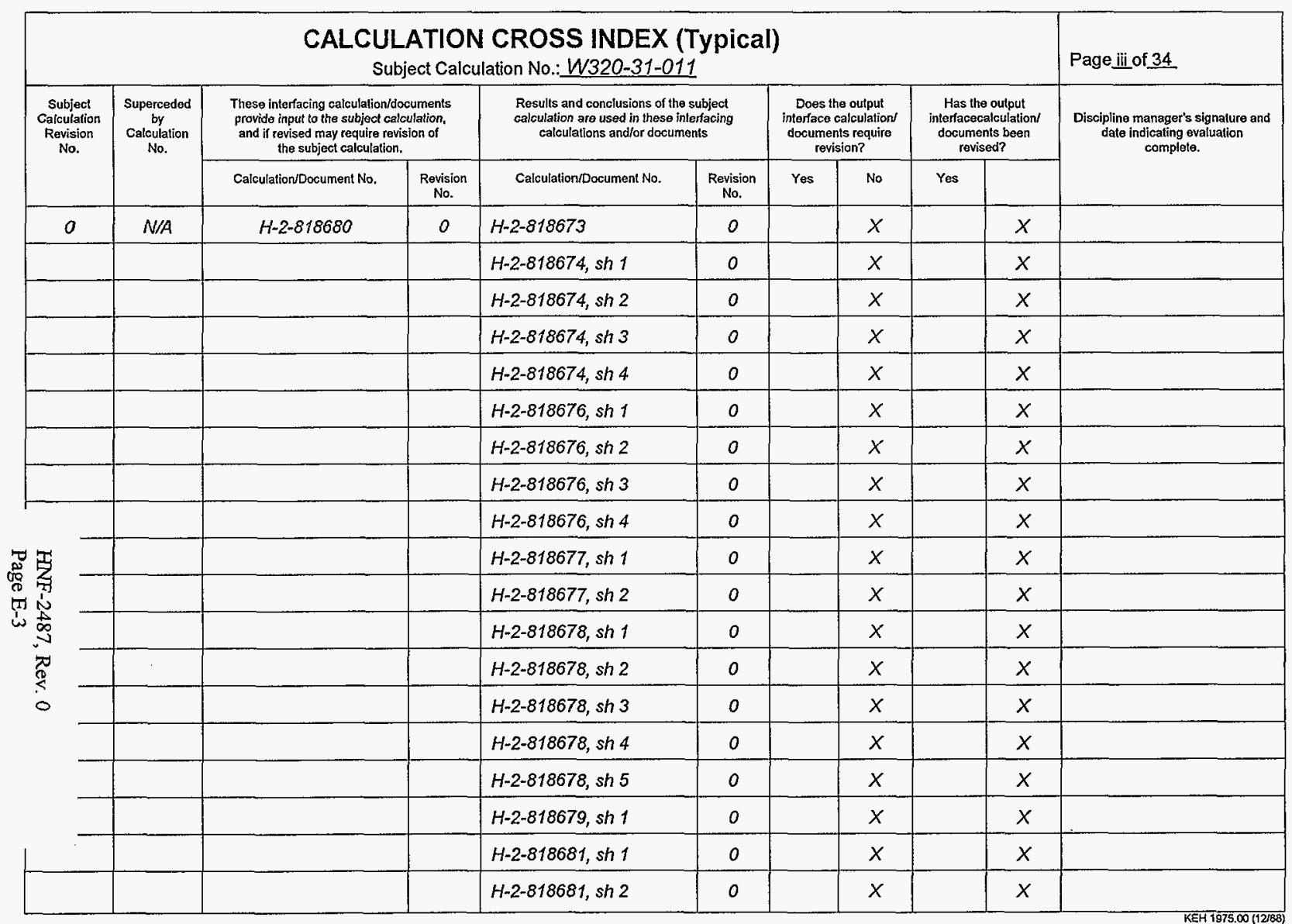




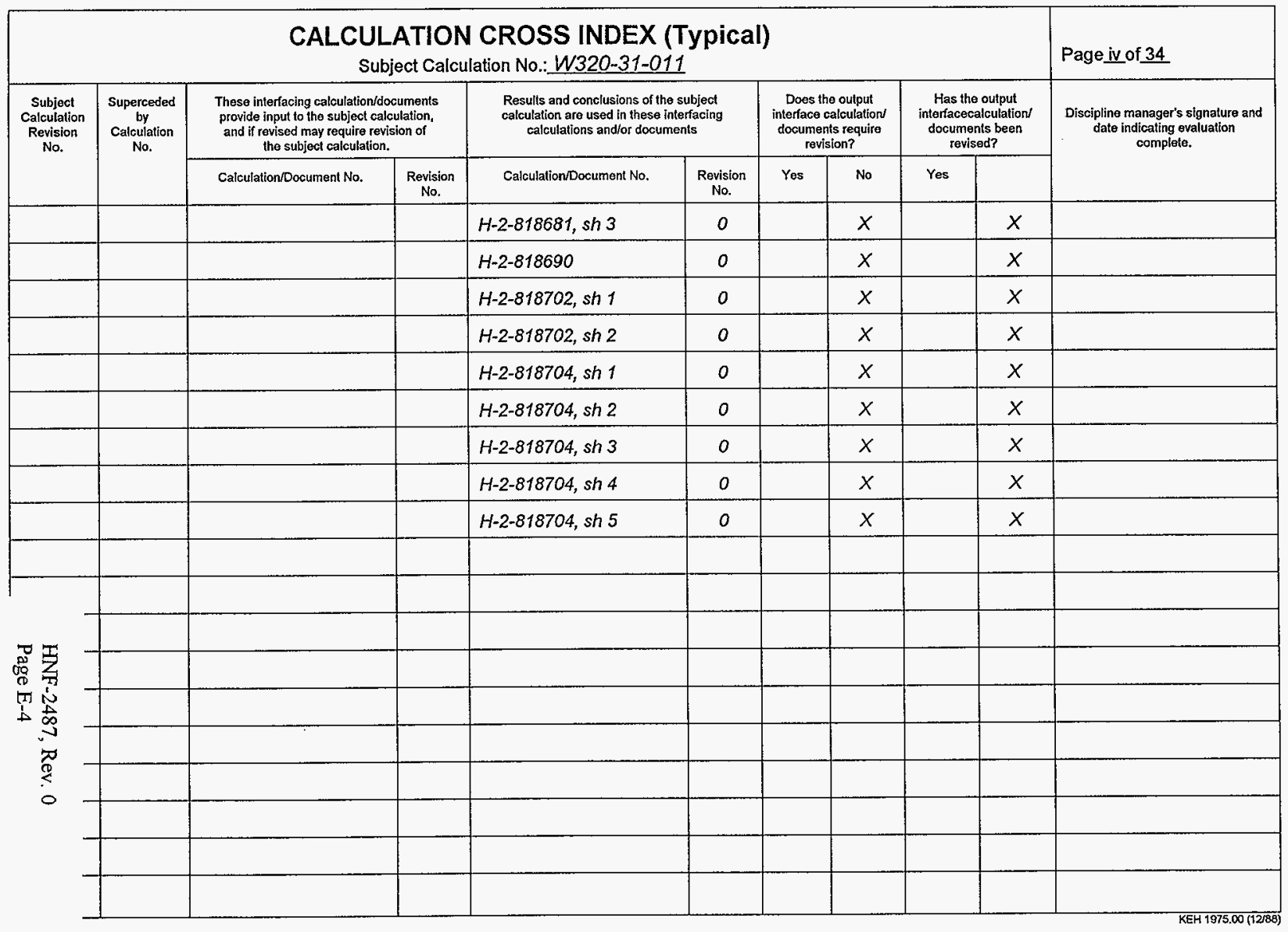




\section{Fluor Daniel Northwest}

\section{DESIGN ANALYSIS}

client Numatec Hanford Corporation

subject C-106 Load Determination, Feeder/Protection Sizing, Voltage Drop, Lighting Calculations, \& Conduit Sizing Location 200E, C Tank Farm
Revision 1

Page No. 1 of 34

wo/Job No. Task Order Nog

Date $9 / 13 / 95 \quad$ вy DE Larson

Checked $7 / 19 / 95$

By TJ Kasnick

Revised 5/6/98

\section{OBJECTIVES}

1.1. To determine the total connected load and estimate the maximum demand for the Tank 241-C-106 portion of Project $W-320$, Tank 241-C-106 Sluicing.

1.2 To size the feeder/branch circuit conductors, protection and conduits.

1.3 To calculate the voltages at motor terminals during normal running and starting conditions in order to ensure proper operation of pump motors.

1.4 To determine number of light fixtures required to provide adequate illumination levels.

1.5 To determine conduit sizes for cables used.

1.6 To determine adequacy of buried putiboxes (handholes).

2. DESIGN INPUTS

2.1 Function Design Criteria for Tank 241-C-106 Waste Retrieva1, Project W-320, HNF-SD-W320-FDC-001 Rev. 5.

2.2 Attached Electrical One-Line Diagram, Drawing (DWG) H-2-818680.

2.3 NFPA-70, National Electrical Code (NEC), 1993 edition.

a. Branch circuit conductors shall be sized to carry at least $125 \%$ of the largest load plus the sum of other loads, for multiple load circuits.

b. Single conductor copper wires, with $90^{\circ} \mathrm{C}$ XHHW, 600 VAC insulation in buried raceway and conductor ampacity per Reference 6.1, NEC, Table 31016 for $86^{\circ} \mathrm{F}\left(30^{\circ} \mathrm{C}\right)$ ambient temperature, shall be used.

c. Minimum conduit size shall be based on NEC Chapter 9, Tables 1 and $3 B$.

d. Motor rated full load amperes (FLA) shall be based on Reference 6.1, NEC, Table 430-150, except for the 250 HP motor which is based on vendor information, specifically that provided under Lawrence Pump P.0.961744/ Westinghouse P.0. W-78259-Z4 / Re1iance S.0. 897719.

e. Motor Circuit Protector (MCP) for motor protection and motor starters shall be sized per Reference 6.4 .

2.4 MCC N1 main 800 amp incoming circuit breaker shall provide the main service disconnecting means to the C-Farm loads supplied via the Electrical Equipment Skid (EES), Building 24l-C-5I. 
DESIGN ANALYSIS

client Numatec Hanford Corporation

subject C-106 Load Determination, Feeder/Protection Sizing, Voltage Drop, Lighting Calculations, \& Conduit Sizing Location 200E, C Tank Farm
Revision 1

Page No. 2 of 34

wo/Job No. Task Order N09

Date $9 / 13 / 95$

By DE Larson

checked $7 / 19 / 95$

By TJ Kasnick

Revised $5 / 6 / 98$
By Cris Carlson

2.5 Effective impedance $\left(Z_{\text {eff }}\right)$ at $85 \%$ Power Factor (PF), shown in NEC Chapter 9 Table 9 , shall be used for voltage drop calculations.

3. GIVEN DATA

3.1 The 225 KVA transformer bank installed by the Equipment Removal Phase of Project $\mathrm{H}-320$ is the source of power for these sluicing loads.

3.2 The 225 KVA distribution transformer $\% Z=3.19$, based on nameplate of the transformer.

3.3 Minimum allowable voltage at the motor terminals is 414 volts based on NEMA motor rating of $460 \mathrm{~V} \pm 10 \%$.

3.4 The EES housing 241-C-51 and Seismic Shutdown System missile shield enclosures 241-C-51A and 51B are air conditioned.

3.5 Motors shown on the Electrical One-Line Diagram, Drawing $\mathrm{H}-2-818680$ are induction motors rated $460 \mathrm{VAC}, 3 \phi, 60 \mathrm{~Hz}$. Motor FLA are from the NEC, except for the $250 \mathrm{HP}$ motor which is based on vendor information in CVI file \#22668.

3.6 The Variable Speed Drive (VSD) controller for the $250 \mathrm{HP}$ In-Line Booster pump motor has been furnished by Reliance Electric via Lawrence Pump (Procurement Specification W-320-P17). VSD controller maximum input current is $325 \mathrm{~A}$. (150\% Torque Limit for 60 SEC. maximum). See Attachment A, page A-8.

3.7 The $40 \mathrm{HP}$ submersible (immersible) pump is started before the booster pump. The submersible (immersible) pump is interlocked with the booster pump so that the booster pump can only be started with adequate NPSH detected as the pressure switch exceeds the pressure switch setpoint. A "Soft Start" unit is to be used to 7 imit torque effect to submersible pump hoses to prevent excess twisting.

3.8 Ambient temperature of pits 241-C-06A pump pit and 241-C-06C sluice pit are $59^{\circ} \mathrm{C}\left(138.2^{\circ} \mathrm{F}\right)$ based on HVAC calculations.

3.9 Ambient temperature of room 1 in the Process Building may reach $65.6^{\circ} \mathrm{C}\left(150^{\circ}\right.$ F) based on data provided by HVAC discipline. 


\section{Fluor Daniel Northwest}

DESIGN ANALYSIS

client Numatec Hanford Corporation

subject C-106 Load Determination,

Feeder/Protection Sizing, Voltage Drop,

Lighting Calculations, \& Conduit Sizing

Location 200E, C Tank Farm
Revision 1

Page No. 3 of 34

wo/sob ko. Task Order N09

Date $9 / 13 / 95$ By DE Larson

Checked $7 / 19 / 95$ By TJ Kasnick

Revised 5/6/98 By Cris Carlson

4. ASSUMPTIONS

4.1 Estimated duration for this project is two years.

4.2 Transformer primary taps are set at $100 \%$ and secondary terminal voltage at no-load conditions is $480 \mathrm{~V}$.

4.3 Demand factor (DF) for the booster and Immersible pumps motors is $80 \%$. 0ther assumed demand factors may be found within the calculation.

4.4 "Soft-Start" unit used for 40 HP submersible pump, P-1361, is considered to have the same efficiency as a conventional motor starter. The basis for this is the description given in the Cutler Hammer document, "User's Manual EasyStart Advantage EA Series." See Vendor Information file \#22668.

4.5 Unless otherwise stated, $\mathrm{PF}=.95$ and $\mathrm{EFF}=0.95$.

5. METHOD OF ANALYSIS

5.1 Hand calculations.

6. REFERENCES

6.1 Drawing H-2-818680, Sheet 1, Rev. 1, Electrical C-Farm One-Line Diagram.

6.2 NFPA-70, National Electrical Code, 1993 edition.

6.3 W320-31-010, C-106 Demand Load Analysis, Size Feeders, Voltage Drop and Fault study.

6.4 Westinghouse Cat. 25-000, Quick Selector 1993.

6.5 Additional References to Support C-Farm Demand Load Analysis:

a. Industrial Power Systems Handbook, Beeman, First Edition, Chapter 17 (Load and Cost Estimating Data).

b. Standard Handbook for Electrical Engineers, Fink and Beaty, Eleventh Edition, Chapter 18 (Demand and Diversity Factors).

c. The following assumptions were made for motor current calculations: $P F=.95, E f f=.95$. 


\section{Fluor Daniel Northwest}

\section{DESIGN ANALYSIS}

client Numatec Hanford Corporation

subject C-106 Load Determination,

Feeder/Protection Sizing, Voltage Drop, Lighting Calculations, \& Conduit Sizing Location 200E, C Tank Farm
Catc. No. W320-31-011

Revision 1

Page No. 4 of 34 wo/sob No. Task Order N09

Date $9 / 13 / 95$ By DE Larson

checked $7 / 19 / 95$

8y TJ Kasnick

Revised 5/6/98
By Cris Carison

d. ANSI C57, "Distribution, Power \& Regulating Transformers"

6.6 Vendor Information file \#22668.

7. CALCULATIONS

The following motor current values are from NEC Table 430-150, except for the $250 \mathrm{HP}$ motor which is based on vendor information. Single phase 480 volt toads are calculated as $I=V A / v o l t s$. Three phase 480 volt loads are calculated as $I=$ VA / (volts $\times \sqrt{3})$. 


\section{Fluor Daniel Northwest}

Calc. No. W320-31-011

DESIGN ANALYSIS

client Numatec Hanford Corporation

subject C-106 Load Determination,

Feeder/Protection Sizing, Voltage Drop,

Lighting Calculations, \& Conduit Sizing Location 200E, C Tank Farm
Revision 1

Page No. 5 of 34

wo/Job No. Task Order N09

Date $9 / 13 / 95$

By DE Larson

Checked $7 / 19 / 95$

By TJ Kasnick

Revised 5/6/98

7.1 Connected/Peak Load Determination for MCC-NI and MCC-N2

Load

Sluicer:

3 HP Hydraulic Power Unit (SHC-1361)

0.3 KVA Transformer (control power)

Service Building:

7.5 HP compressor unit (CPR-1361)

5 KVA Mini Power Center (C106-PP3)

Process Building:

7.5 HP Recirc Fan (FN-1361)

$30 \mathrm{KW}$ heating coil $(\mathrm{HC}-1361)$

$4 \mathrm{KW}$ Space Heater (UH-1361)

$4 \mathrm{KW}$ Space Heater (UH-1362)

7.5 KVA Mini Power Center (C106-PPl)

S1urry Pump Winch: $1.5 \mathrm{HP}$ (W-1361)

Chiller Skid:

15 HP Circ Pump (P-1364 or P-1365)

56 KVA Air cooled chiller (R-1361)

3.0 KVA Mini-Power Center (C106-PP4)
Amperes

4.8

.6 (ph A-B)

11,0

10.4 (ph A-B)

11,0

36.1

4.8

4.8

$15.6(\mathrm{ph} \mathrm{A}-\mathrm{C})$

2.6

21.0

70.3

$6.25(\mathrm{ph} \mathrm{A-C)}$

Exhaust Skid:

7.5 HP Exhaust Fan (VSD efficiency $=90 \%$ ) (FN-1362) 11.0

$3.0 \mathrm{KW}$ heating coil (HC-1362)

5 KVA Mini Power Center (C106-PP5)

3.0 HP Vacuum pump ( $\mathrm{P}-1366$ or $\mathrm{P}-1367$ )

3.6

10.4 (ph A-B)

4.8

15 KVA Mini Power Panel (C106-PP2) (PF = 95\%)

$32.9(\mathrm{ph} \mathrm{B-C)}$

MCC-N2:

40 HP Immersible S7urry Pump (P-1361)

$250 \mathrm{HP}$ In-Line Booster Pump (P-1362)

(with VSD efficiency $=95 \%$ )

$$
\text { Max Total Load }(C \text {-phase })=\ldots \ldots \text {. } 579.6 \mathrm{~A}
$$

Total Connected Load $=579.6 \mathrm{~A} \times 480 \mathrm{~V} \times \sqrt{3}=481.9 \mathrm{KVA}$

HNF-2487, Rev. 0

Page E-9 


\section{Fluor Daniel Northwest}

\section{DESIGN ANALYSIS}

client Numatec Hanford Corporation

\section{subject C-106 Load Determination,}

Feeder/Protection Sizing, Voltage Drop, Lighting Calculations, \& Conduit Sizing Location 200E, C Tank Farm
Revision 1

Page No. 6 of 34 wo/sob No. Task Order N09
Date $9 / 13 / 95$
By DE Larson
checked $7 / 19 / 95$
By TJ Kasnick
Revised 5/6/98

\subsection{Estimated Demand Determination}

The following discussion is for the determination of the adequacy of the planned transformer for the C-Farm portion of the project. Three situations are given in tabular form following. The first is the "normal" operating situation. This is the anticipated load that would be expected after sluicing operations are underway. The second situation given is the demand load under the "worst case" startup situation. This load will be experienced by the transformer for short durations only. The third situation described in the following tables is the loading that would be considered under "normal" demand operation. The method used for these three situations is that used by Kaiser Utilities to size transformers. It is based on ANSI C57. See attached Electric Service Request EUE95-054. 


\section{Fluor Daniel Northwest}

DESIGN ANALYSIS

client Numatec Hanford Corporation

subject C-106 Load Determination,

Feeder/Protection Sizing, Voltage Drop,

Lighting Calculations, \& Conduit Sizing

Location 200E, C Tank Farm
Calc. No. W320-31-011

Revision 1

Page No. 7 of 34

wo/Job No. Task Order N09

Date $9 / 13 / 95$ By DE Larson

checked $7 / 19 / 95$ By TJ Kasnick

Revised 5/6/98 By Cris Carison

\section{W-320 C-Farm Sluicing Operation Values - "Normal Demand"}

Load

Electrical Equipment Skid:

Mini-Power Center

Service Building:

Mini-Power Center

Compressor CPR-1361

Process Building:

Recirculation Fan

Heating Coil

Space Heating

Mini-Power Center

Chiller Skid:

Circulation Pump 1 or 2

Compressors/Fans

Control Power

\section{Connected}

Amps Load (KVA) DF Demand (AMP)

$32.9 \quad 15.0$

Vacuum pump

Heating Coit

Exhaust Fan w/VSD

Mini-Power Center

Sluicer Skid:

Hydraulic Pump

Mini-Power Center (control power)

Slurry Booster Pump w/VSD

Slurry Sub Pump w/soft-start

STurry Sub Pump Winch

Construction Office Trailers

Space Heating/Heat Pump

Lighting/Receptacles
4.8

0.6

287.0

52.0

2.6

(3):

30.1

30.1
5.0

8.8

11.0

11.0

36.0

9.6

15.6

21.0

70.3

6.3

3.6

11.0

10.4

4.8
8.8

30.0

8.0

7.5

16.7

56.0

3.0

3.0

8.8

5.0

3.8

3.8

0.3

.80

.30

.80

.80

.30

2.1

25.0

25.0
3.13

8.80

8.80

28.87

2.88

4.69

16.80

56.24

1.88

2.88

8.80

3.13

3.84

Total Demand Current $=\ldots \ldots \ldots 454.68$

Diversity Factor $=\ldots \ldots \ldots \ldots \ldots .61 .44$

Estimated Current $=\ldots \ldots \ldots \ldots .315 .75$

Estimated $K V A=\ldots \ldots \ldots \ldots \ldots 262.5$
3.84

0.19

229.60

41.60

0.78

9.03

9.03 


\section{Fluor Daniel Northwest}

DESIGN ANALYSIS

client Numatec Hanford Corporation

subject C-106 Load Determination,

Feeder/Protection Sizing, Voltage Drop,

Lighting Calculations, \& Conduit Sizing

Location 200E, C Tank Farm
Calc. No. W320-31-011

Revision 1

Page No. 8 of 34

wo/sob no. Task Order N09

Date $9 / 13 / 95$ By DE Larson

checked 7/19/95 By TJ Kasnick

Revised 5/6/98

\section{W-320 Transformer Demand Loading - Sluicing in Normal Operation}

Load

Electrical Equipment Skid:

Mini-Power Center

Service Building:

Mini-Power Center

Compressor CPR-136I

Process Building:

Recirculation Fan

Heating Coil

Space Heating

Mini-Power Center

Chiller Skid:

Circulation Pump 1 or 2

Compressors/Fans

Control Power

Exhaust Skid:

Heating Coil

Exhaust Fan w/VSD

Mini-Power Center

Vacuum pump

STuicer Skid:

Hydraul ic Pump

Mini-Power Center (control power)

S7urry Booster Pump w/VSD

STurry Sub Pump w/soft-start

Siurry Sub Pump Winch

Construction Office Trailers

Space Heating/Heat Pump

Lighting/Receptacles

(3):

\section{Connected}

Amps Load (KVA) DF Demand (AMP)

32.9

15.0

.30

9.87

10.4

5.0

.30

3.13

11.0

8.8

.80

8.80

11.0

36.0

9.6

15.6

8.8

1.0

.80

8.0

7.5

.30

.30

11.00

28.87

2.88

4.69

21.0

16.7

.80

1.0

3.0

.30

16.80

70.30

1.88

6.3

3.6

11.0

10.4

4.8

3.0

.80

8.8

1.0

5.0

.30

.30

2.88

11.00

3.13

3.84

4.8

3.8

1.0

4.80

0.3

.30

0.19

287.0

52.0

2.6

229.0

1.0

43.2

1.0

287.00

2.1

.00

52.00

0.00

30.1

25.0

.30

9.03

30.1

25.0

.30

9.03

Total Demand Current $=\ldots \ldots \ldots .541 .12$

Diversity Factor $=\ldots \ldots \ldots \ldots \ldots, \quad 1.44$

Estimated Current $=\ldots \ldots \ldots \ldots \ldots, 375.78$

Estimated $\mathrm{KVA}=\ldots \ldots \ldots \ldots \ldots \ldots, 312.42$ 


\section{Fluor Daniel Northwest}

Calc. No. W320-31-011

DESIGN ANALYSIS

client Numatec Hanford Corporation

subject C-106 Load Determination, Feeder/Protection Sizing, Voltage Drop, Lighting Calculations, \& Conduit Sizing Location 200E, C Tank Farm
Revision 1

Page No. 9 of 34

wo/Job No. Task Order NOg

Date 9/13/95 By DE Larson

checked $7 / 19 / 95$ By TJ Kasnick

Revised 5/6/98 By Cris Carlson

Summary of transformer loading:

"Norma7" Demand $\approx 262.5$ KVA $\div 225$ KVA $\approx 117 \%$ of Nameplate Rating.

"Peak" Demand on startup $\approx 481.9$ KVA $\div 225$ KVA $\approx 214 \%$ of Namepiate.

"Norma1" STuicing Operation $\approx 312.4 \div 225 \mathrm{KVA} \approx 139 \%$ of Nameplate.

Therefore, the 225 KVA transformer is adequate based on:

1) For "Normal" Operation, $130 \%$ of demand (or less) is acceptable per Electrical Utilities engineer, Dan Ford, and IEEE 57.91 \& 92 . See attachment.

2) Duration of "Worst Case" is less than 5 minutes for each occurrance. Project design 1 ife is only 2 years. Small losses of the transformer's 30 year design life expectancy are acceptabie.

\subsection{Breaker, Starter, Feeder and Conduit Sizing}

\subsubsection{Main feeder from C-PDP-1 into Electrical Equipment Skid (EES)}

A lockable 800 ampere circuit breaker (service entrance rated) at the input to MCC-NI is the main disconnect means for 241-C-51 (EES).

Feeder Sizing is in accordance with NEC 220-10, 240-21, 430-2, 430-22, 430-24 and 430-28 using actual loads listed in 7.1 .

Please see the following table for feeder size evaluation. Single phase $480 \mathrm{~V}$ loads are considered as adding equally to all three phases. This is a conservative allowance. Motor ampacity values have been obtained from NEC Table 430-150. Non-motor loads have been evaluated at 480 volts. Non-motor continuous loads have their full load ampacity values multiplied by 1.25 in accordance with NEC 220-3. 


\section{Fluor Daniel Northwest}

Calc. No. W320-31-011

DESIGN ANALYSIS

ctient Numatec Hanford Corporation

subject C-106 Load Determination,

Feeder/Protection Sizing, Voltage Drop,

Lighting Calculations, \& Conduit Sizing

Location 200E, C Tank Farm
Revision 1

Page No. 10 of 34

wo/sob No. Task Order No9

Date $9 / 13 / 95$ By DE Larson

checked $7 / 19 / 95$ By TJ Kasnick

Revised $5 / 6 / 98$ By Cris Carlson

\section{Load}

Sluicer:

3 HP Hydraulic Power Unit (SHC-1361)

0.3 KVA Transformer (control power)

Service Building:

7.5 HP compressor unit (CPR-1361)

5 KVA Mini Power Center (C106-PP3)

Process Building:

7.5 HP Recirc Fan (FN-1361)

$30 \mathrm{KW}$ heating $\mathrm{Coi1}(\mathrm{HC}-136 \mathrm{I})$

$4 \mathrm{KW}$ Space Heater (UH-1361)

$4 \mathrm{KW}$ Space Heater (UH-1362)

7.5 KVA Mini Power Center (C106-PPI)

STurry Pump Winch: $1.5 \mathrm{HP}$ (W-1361)

Chiller Skid:

15 HP Circ Pump (P-1364 or P-1365)

56 KVA Air cooled chiller (R-1361)

3.0 KVA Mini-Power Center (C106-PP4)
Amperes

4.8

.6 (ph A-B)

11.0

$10.4(p h \quad A-B)$

11.0

36.1

4.8

4.8

$15.6(\mathrm{ph} \mathrm{A}-\mathrm{C})$

2.6

21.0

70.3

$6.25(\mathrm{ph} \mathrm{A-C)}$

Exhaust Skid:

7.5 HP Exhaust Fan (VSD efficiency $=90 \%)(F N-1362) \quad 11.0$

$3.0 \mathrm{KW}$ heating coil

5 KVA Mini Power Center (C106-PP5)

3.0 HP Vacuum pump ( $P-1366$ or $P-1367)$

3.6

10.4 (ph A-B)

15 KVA Mini Power Panel (C106-PP2) (PF = 95\%)

$32.9(p h B-C)$

MCC-N2:

40 HP Immersible Slurry Pump ( $P-1361)$

250 HP In-Line Booster Pump (P-1362)

52.0

(with VSD efficiency $=95 \%$ )

287.0

Max Total Load (C-phase) $=\ldots \ldots .579 .6 \mathrm{~A}$

Total Connected Load $=579.6 \mathrm{~A} \times 480 \mathrm{~V} \times \sqrt{3}=481.9 \mathrm{KVA}$

An 800 ampere main breaker is required to protect the loads. The feeder must be capable of carrying 579.6 amperes. A choice has been made to use 3-350 kcmil conductors per phase from NEC Table 310-16. Install as three sets in 3 inch conduits to MCC $-\mathrm{Nl}$. Insta11 1/0 ground conductor in each conduit per NEC Table 250-95. 


\section{Fluor Daniel Northwest}

Calc. No. W320-31-011

DESIGN ANALYSIS

client Numatec Hanford Corporation

subject C-106 Load Determination,

Feeder/Protection Sizing, Voltage Drop,

Lighting Calculations, \& Conduit Sizing

Location 200E, C . Tank Farm
Revision 1

Page No. 11 of 34

Wo/Job No. Task Order Nog

$\begin{array}{ll}\text { Date } 9 / 13 / 95 & \text { By DE Larson } \\ \text { Checked } 7 / 19 / 95 & \text { By TJ Kasnick } \\ \text { Revised 5/6/98 } & \text { By Cris Carison }\end{array}$

Down-stream feeder conductors shall be 800 AMP rated $90^{\circ} \mathrm{C}$ :

1) Need 3-350 kcmil per phase.

2) Install three $350 \mathrm{kcmil}$ conductors and $1 \# 1 / 0$ ground, in each of three $3^{\prime \prime}$ conduits.

Note: The three 3 " conduits are extensions of conduits previously installed under ERS portion of project.

7.3.2 The 250 HP In-Line Booster Pump (P-1362) and VSD Controlier (VSD molded case disconnect included with controller provided by booster pump vendor).

VSD Feeder:

VSD controller maximum input current $=325 \mathrm{~A}$. (See Attachment " $A$ ", Page A-8) Per NEC 430-2, required feeder capacity $=1.0 \times 325 \mathrm{~A} \approx$ 325 A. Per NEC Table $310-16$, use $3-500$ kcmil conductors with $1 \# 1 / 0$ ground wire in a $3 \frac{1}{2}$ " conduit (upsized to $4^{\text {" }}$ conduit to aid in cable pulting).

Motor Feeder:

Booster pump motor $F L A=273 \mathrm{~A}$. Per $N E C$ 430-22, required feeder ampacity $=1.25 \times 273=341 \mathrm{~A}$. Per NEC Table 310-16, use $3-500$

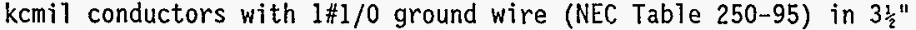
conduit (upsized to 4 " conduit to aid in cable pulling).

See 7.3.14 for "In-Pit" segment of motor feeder.

\subsubsection{The 40 HP Immersible STurry Pump (P-1361)}

Motor Feeder:

Immersibie pump motor $F L A=52.0 \mathrm{~A}$. The required feeder ampacity $=1.25 \times 52.0 \mathrm{~A}=65.0 \mathrm{~A}$. Use of $3 \# 6 \mathrm{AWG}$ (ampacity $=65 \mathrm{~A}$ ) is adequate based on ampacity. Use of $3 \# 1 / 0$ with $1 \# 4$ ground wire in 2 " conduit for conservatism will satisfy requirements.

Install 1\#1/0 AWG per phase from MCC-N2 to pump pit 241-C-06A.

Note: Larger conductor size was used because motor was originally planned to be larger. Moisture sensor cables are routed with $\mathrm{P}-1361$ wires so derating is required. Eighty per cent of $3 \# 1 / 0$ gives capability of 120 amps. (.8 X 150 amps $=120$ amps) 


\section{Fluor Daniel Northwest}

Calc. No. W320-31-011

\section{DESIGN ANALYSIS}

client Numatec Hanford Corporation

subject C-106 Load Determination, Feeder/Protection Sizing, Voltage Drop, Lighting Calculations, \& Conduit Sizing Location 200E, C Tank Farm
Revision 1

Page No. 12 of 34

wo/Job No. Task Order N09

Date $9 / 13 / 95$ By DE Larson

checked 7/19/95 . 8Y TJ Kasnjck

Revised $5 / 6 / 98$
By Cris Cartson

A reduced voltage solid-state starter mounted within MCC-N2 shall be used for immersible pump operation. See 7.3.14 for "In-Pit" segment of motor feeder.

\subsubsection{The 1.5 HP Winch $(W-1361)$}

Motor starter line and load side feeder conductors:

Winch motor FLA $=2.6 \mathrm{~A}$. The required feeder ampacity $=1.25 \times 2.6 \mathrm{~A}$ $=3.25 \mathrm{~A}$. Use $3 \# 10$ with $1 \# 12$ ground wire in $3 / 4^{\prime \prime}$ conduit (upsized to $I^{\prime \prime}$ conduit for conservatism). Note that this feeder will be routed with other feeders (See Power Conduit Block Diagram in the appendix).

Use 7 A MCP and NEMA Size 1, Full Voltage Reversing (FVR) starter.

\subsubsection{Chiller Skid}

Feeder circuit breaker "line and load side" Conductors:

The chiller load from above consists of the items listed below. Since the items are all mounted on a vendor supplied skid, there is no need to size individual load supply conductors. However, the feeder to the Chiller skid does need to be sized.

Loads:

15 HP Circ Pump (P-1364 or P-1365) FLA $=\quad 21.0 \mathrm{~A}$.

56 KVA Comp pkg (70.3 X 1.25) FLA $=\quad 87.9 \mathrm{~A}$.

3 KVA Mini-Power Panel (C106-PP4) (ph A-C) (1.25) $=7.8 \mathrm{~A}$.

$$
\text { Total }=\ldots \ldots \ldots \ldots 116.7 \mathrm{~A} \text {. }
$$

Per NEC Table 310-16, use 3\#1 AWG conductors with $1 \# 6$ AWG ground wire is adequate. For conservatism, a set of $3 \# 4 / 0$ conductors has been used (conduit C106-3P211). These conductors are capable of 230 amperes at $75^{\circ} \mathrm{C}$. Circuit breaker size has been se7ected as 225 amp frame with 175 amp trip.

\subsubsection{Minj-Power Panel}

No feeder required because unit is supplied with MCC-N1. 


\section{Fluor Daniel Northwest}

Calc. No. W320-31-011

\section{DESIGN ANALYSIS}

client Numatec Hanford Corporation

subject C-106 Load Determination,

Feeder/Protection Sizing, Voltage Drop,

Lighting Calculations, \& Conduit Sizing Location 200E, C Tank Farm
Revision 1

Page No. 13 of 34

wo/Job No. Task Order N09

Date $9 / 13 / 95 \quad$ By DE Larson

Checked 7/19/95 By TJ Kasnick

Revised 5/6/98 By Cris Carlson

\subsubsection{Service Building (241-C-73)}

Loads:

7.5 HP compressor motor (CPR-1361) (1.25)

$13.75 \mathrm{~A}$

5 KVA Mini-Power Center (C106-PP3) (oh A-B) (1.25) $13.00 \mathrm{~A}$

Total $=\ldots \ldots \ldots \ldots \ldots 26.75 \mathrm{~A}$

Per NEC Table 310-16, use 3\#10 AWG conductors with 1\#10 AWG ground wire in a 1" conduit. A $100 \mathrm{AF}$ breaker with a 30 AT will be used per NEC Table 310-16. The $7.5 \mathrm{HP}$ compressor motor will require a minimum of $3 \# 14$ AWG, but use $3 \# 10$ AWG with $1 \# 10$ ground wire exceeding requirements of DOE 6430.1a. Fused disconnect will house 30 amp fuses to protect the motor supply conductors. The Mini-Power Panel is existing. See calculation W-320-31-015.

\subsubsection{Process Building (241-C-91)}

Loads:

7.5 HP Recirc Fan (FN-1361) (1.25)

$30 \mathrm{KW}$ heating coil $(\mathrm{HC}-1361)(1.25)$

$4 \mathrm{KW} \mathrm{Space} \mathrm{Heater} \mathrm{(UH-1361)(1.25)}$

$4 \mathrm{KW}$ Space Heater (UH-1362)(1.25)

7.5 KVA Mini-Power Panel (C-106-PP1) (ph A-C) (1.25)

$$
\text { Total }=
$$

Per NEC Table 310-16, use 3\#3 AWG conductors. This has been upsized to the more common size of $3 \# 2$ with $1 \# 8$ ground wire in a $1.5^{\prime \prime}$ conduit. A circuit breaker of $100 \mathrm{AF}$ with a $90 \mathrm{AT}$ will be adequate. Process building loads and protection are summarized below:

$\begin{array}{llll}\text { Load } & \begin{array}{l}\text { Size of } \\ \text { Suppiy } \\ \text { Wire }\end{array} & \begin{array}{l}90^{\circ} \mathrm{C} \text { Amp } \\ \text { Rating } \\ \text { of Wire }\end{array} & \begin{array}{l}\text { Size of } \\ \text { Protection }\end{array} \\ \text { FN-1361 } & 3 \# 10 & 23.2 & \text { 100AF W/ 17.5AT } \\ \text { HC-1361 } & 3 \# 4 & 55.1 & 45 \text { A fuse } \\ \text { UH-1361 } & 3 \# 12 & 17.4 & 10 \text { A fuse } \\ \text { UH-1362 } & 3 \# 12 & 17.4 & 10 \text { A fuse } \\ \text { C106-PP-1 } & 3 \# 8 & 31.9 & \text { 10OAF/30AT CB }\end{array}$

Loads in room \#1 of the Process building are in a high ambient condition $\left(150^{\circ} \mathrm{F}\right)$. A derating factor of .58 has been applied to the $90^{\circ} \mathrm{C}$ ampacity rating of the supply conductors to these loads per NEC Table 310-16. 


\section{Fluor Daniel Northwest}

DESIGN ANALYSIS

ctient Numatec Hanford Corporation

subject C-106 Load Determination, Feeder/Protection Sizing, Voltage Drop, Lighting Calculations, \& Conduit Sizing Location 200E, C Tank Farm
Calc. No. W320-31-011

Revision 1

Page No. 14 of 34

Wo/Job No. Task Order NOg

Date $9 / 13 / 95$ By DE Larson

Checked 7/19/95 By TJ Kasnick

Revised 5/6/98 By Cris Carlson

\subsubsection{Sluicer Hydraulic Power Unit}

sluicer (SHC-1361):

3 HP motor (hydraulic power unit) (1.25)

0.3 KVA Trans (sluicer control power) (ph A-B)(1.25) 0.8 A

$$
\text { Total }=\ldots \ldots \ldots \ldots \ldots 6.8 \mathrm{~A}
$$

Per NEC Table 310-16, use 3\#12 with $1 \# 12$ ground wire in a $1.5^{\prime \prime}$ conduit. Loads on the sluicer skid are vendor supplied and wired. A 100 AF with 15 AT circuit breaker shall be used to protect the sluicer.

\section{3 .10 Exhaust Fan VSD (FN-1362)}

Exhaust Fan: $7.5 \mathrm{HP}$ (with VSD efficiency $=90 \%$ )

VSD controller maximum input current $=12.2 \mathrm{~A}$. Per NEC 430-2, required feeder capacity $=1.15 \times 12.2 \mathrm{~A} \approx 14 \mathrm{~A}$. Per NEC Table 31016 , use 3\#12 AWG conductors with $1 \# 12$ ground wire in a $3 / 4$ " conduit. A 100 AF with 20 AT shall be used to protect the wiring.

\subsubsection{Exhaust Skid}

Loads:

$3.0 \mathrm{KW}$ heating coil $0480 \mathrm{~V}(1.25)$

5 KVA Mini-Power Panel (C-106-PP5) (1.25)

$3.0 \mathrm{HP}$ vacuum pumps $(P-1366$ or $P-1367)(1.25)$

$$
\text { Total }=\ldots \ldots \ldots \ldots .23 .5 \mathrm{~A}
$$

Per NEC Table 310-16, use of $3 \# 10$ AWG sha71 be adequate with $1 \# 10$ ground wire in a minimum $1^{11}$ conduit. Loads on the exhaust skid are vendor supplied and wired. A circuit breaker with $100 \mathrm{AF}$ and $30 \mathrm{AT}$ shall be used to protect the wiring. 


\section{Fluor Daniel Northwest}

Calc. No. W320-31-011

\section{DESIGN ANALYSIS}

client Numatec Hanford Corporation

subject C-106 Load Determination,

Feeder/Protection Sizing, Voltage Drop,

Lighting Calculations, \& Conduit Sizing Location 200E, C Tank Farm
Revision 1

Page No. 15 of 34

wo/Job No. Task Order N09

Date $9 / 13 / 95$ By DE Larson

Checked $7 / 19 / 95$ By TJ Kasnick

Revised 5/6/98 By Cris Carlson

\subsubsection{Seismic Shutdown System Missile Enclosure}

Loads are air conditioner and receptacles:

$I=\frac{1320}{120}=11 \mathrm{~A} ; \quad 11 \mathrm{~A} \times 1.25=14 \mathrm{~A}$ capacity.

Use $2 \# 10$ with 1 \#10 ground wire in a 3/4" conduit per Calculation W32031-022 (upsized to 1" conduit for conservatism). The Seismic systems are supplied from C106-PP2 at the EES. $30 \mathrm{~A}$ circuit breakers shall be used to protect the wiring.

7.3.13 Iypical 120 VAC branch circuit conductor loaded up to 1.500 VA

$I=\frac{1500}{120}=12.5 \mathrm{~A} ; \quad 12.5 \mathrm{~A} \times 1.25=15.625 \mathrm{~A}$ capacity with a $20 \mathrm{~A}$

Use $2 \# 12$ with $1 \# 12$ ground wire in minimum $3 / 4$ " conduit.

7.3.14 "In-Pit" Cord Sizing

NEC article 40 defines the requirements for the "In Pit" cords. NEC article 430-22 states that branch circuit conductors supplying a single motor shall have an ampacity not less than 125 percent of the motor full-load current rating.

Per Reference Information $40 \mathrm{HP}$ motor $\mathrm{FLA}=52.0 \mathrm{~A}$. The $1993 \mathrm{NEC}$ Table 430-150 does not include values for motors of $250 \mathrm{HP}$. Reliance Electric information shows that the $250 \mathrm{HP}$ motor FLA is 273 amps.

The cords will be in free air within the pump pit. NEC Table 400-5(B) for Type G, portable power cord will be utilized. The Submersible and In-Line Booster pump cords are a thermoset cable that, if assumed equivalent to Type $G$, will need derating for high ambient conditions using similar correction factors to those in NEC Table 310-16.

Per Project $W-320$ Motor Cooling Evaluation dated $6 / 7 / 95$ (see attachments), the ambient temperature in the C-Farm pump pit will be $138^{\circ} \mathrm{F}\left(59^{\circ} \mathrm{C}\right)$. Per NEC Table $310-17$, a correction factor of 0.58 will apply. Since this is considered cord (not cable in conduit), the calculation of the desired derating factor is based on the cable manufacturer's recommendation. Per the Rockbestos company, the 


\section{Fluor Daniel Northwest}

DESIGN ANALYSIS

client Numatec Hanford Corporation

subject C-106 Load Determination, Feeder/Protection Sizing, Voltage Drop, Lighting Calculations, \& Conduit Sizing Location 200E, C Tank Farm
Revision 1

Page No. 16 of 34

wo/Job No. Task Order No9

Date $9 / 13 / 95$ By DE Larson

checked 7/19/95 By TJ Kasnick

Revised 5/6/98 By Cris Carlson

following formula is used for determining an acceptable derating factor (see Attachment).

$$
T C F=\sqrt{\frac{(T C 2-\operatorname{Ta} 2)}{(T C 1-T a 1)} \times \frac{(234.5+T C 1)}{(234.5+T C 2)}}
$$

Tcl = Maximum conductor temperature used to determine base ampacity.

$\mathrm{Tal}=$ Ambient temperature used to determine base ampacity $\left(30^{\circ} \mathrm{C}\right.$ per NEC Table 400-5(b).

Tc2 = Maximum conductor temperature used to determine adjusted ampacity $\left(90^{\circ} \mathrm{C}\right.$ for Rockbestos Firewall III for 40 year qual ified life).

Ta2 = Ambient temperature used to determine adjusted ampacity; $0.3^{\circ} \mathrm{C}$ additional is used as margin.

The cords for the pumps fall under the $F$ category, i.e. no more than 3 current carrying conductors. The $90^{\circ} \mathrm{C}$ column is used, because rating of the terminations in the pump terminal boxes will be defined to be $90^{\circ} \mathrm{C}$.

$$
T C F=\sqrt{\frac{(90-(59+0.3))}{(90-30)} \times \frac{(234.5+90)}{(234.5+90)}}=0.71
$$

(Since pump is rated for $90^{\circ} \mathrm{C}$ operation, we may safely use $90^{\circ}$ wire.)

For sizing the $40 \mathrm{HP}$ Immersible Pump cord:

$(52.0 \mathrm{~A} \times 1.25) / 0.71=91.6 \mathrm{~A}$; per NEC Table $400-5(\mathrm{~B})$ a minimum size of \#4 AWG is required, use \#4 AWG with $1 \# 4$ or larger ground wire.

For sizing the 250 HP Booster Pump cord:

(273 A X 1.25) / $0.71=480.6 \mathrm{~A}$; per NEC Tab7e 400-5(B), use twin sets of $\# 4 / 0$ with $1 \# 3$ or larger ground wire.

Rockbestos Firewall III XHHW P62-0104 is a \#1/0 AWG cable with an overal1 diameter of $1.22 "$ (Vendor furnished by procurement specification W-320-P17 for Immersible Pump). 


\section{Fluor Daniel Northwest}

\section{DESIGN ANALYSIS}

client Numatec Hanford Corporation subject C-106 Load Determination, Feeder/Protection Sizing, Voltage Drop, lighting Calculations, \& Conduit Sizing Location 200E, C Tank Farm
Calc, No. W320-31-011

Revision 1

Page No. 17 of 34

wo/Job No. Task Order N09

Date $9 / 13 / 95$

By DE Larson

Checked $7 / 19 / 95$

By TJ Kasnick

Revised $5 / 6 / 98$
By Cris Carlson

Rockbestos Firewall III XHHW P62-0404 is a \#4/0 cable with an overall diameter of 1.54,"

\subsection{Steady State Voltage Drop Evaluation}

Voltage drop considerations for the C-Farm portion of the $W-320$ project begin at the power source, C-PDP-1, and continue to the load. Calculations given below, which consider the supply transformer, are intended to validate that the transformer may be set at the $0 \%$ tap.

\subsection{1 \%VD on Transformer Bank}

$$
\begin{aligned}
& I_{P U}=\frac{312 \mathrm{KVA}}{225 \mathrm{KVA}}=1.39 \quad(\text { Total Worst Case Dem } / \mathrm{Xfmr} \mathrm{Sz}) \\
& \% V D=\% Z \times I_{\mathrm{PU}}=3.19 \times 1.39=4.4 \%
\end{aligned}
$$

7.4.2 \%VD on $250^{\prime}$ of \#2 AWG copper conductor from pole E257 in steel conduit

$$
\% V D=\frac{\sqrt{3} \times I \times \text { Zeff } / 1000^{\prime} \times L}{4.8}
$$

$$
\text { Where } I_{\text {SEC }}=\frac{312 \mathrm{KVA}}{\sqrt{3} \times 480 \mathrm{~V}} \approx 375 \mathrm{~A}
$$

$$
\begin{aligned}
& I_{P R I}=\frac{312 \mathrm{KVA}}{\sqrt{3} \times 13,800} \approx 13.1 \mathrm{~A} \\
& \left.\mathrm{Z}_{\text {eff }}=.20 ; \mathrm{L}=.250 \text { [Units of } 1000^{\prime}\right] \\
& \text { \%VD Service Drop }=\frac{\sqrt{3} \times 13.1 \times .20 \times .250}{13.8}=\underline{\underline{0.08 \%}}
\end{aligned}
$$

7.4.3 \%VD on Transformer to C-PDP-1: Parallel $600 \mathrm{kcmil}$ Feeders

$Z_{\text {eff }}=0.047 \div 2=0.0235, L=0.032 \mathrm{kft}$

\%VD $600 \mathrm{kcmil}$ feeder $=\frac{\sqrt{3} \times 375 \times 0.0235 \times .032}{4.8}=\underline{\underline{0.1 \%}}$ 


\section{Fluor Daniel Northwest}

DESIGN ANALYSIS

client Numatec Hanford Corporation

subject C-106 Load Determination,

Feeder/Protection Sizing, Voltage Drop,

Lighting Calculations, \& Conduit Sizing

Location 200E, C Tank Farm
Calc. No. W320-31-011

Revision 1

Page No. 18 of 34

wo/Job No. Task Order Nog

Date $9 / 13 / 95$ By DE Larson

Checked $7 / 19 / 95$ вy TJ Kasnick

Revised 5/6/98 By Cris Carlson

7.4.4 \%VD on C-PDP-1 to MCC-N1: Triple $350 \mathrm{kcmil}$ feeders

$Z_{\text {eff }}=0.06 \div 3=0.02, L=0.045 \mathrm{kft}$

\%VD $350 \mathrm{kcmil}$ feeder $=\frac{\sqrt{3} \times 375 \times 0.02 \times .045}{4.8}=\underline{\underline{0.12 \%}}$

\%VD for transformer primary feeder $=0.08 \%$

\%VD from transformer bank to EES $=4.4+0.1+0.12 \approx 4.62 \%$

Total \%VD from pole to EES $=0.08+4.62=\underline{\underline{4.70 \%}}$

Since the voltage drop under highest load conditions exceeds $5 \%$ prior to the evaluation of voltage drop across any branch circuit wiring, the supply transformer taps should be set at the lowest setting to boost the supply voltage by $5 \%$. This should be adequate since peak demand is expected very infrequentiy.

7.4.5 \%VD from MCC-N1 to SSA $(55 \mathrm{ft}$ of twin $350 \mathrm{kcmil}$ feeders)

$Z_{\text {eff }}=0.06 \div 2=0.03, L=0.055 \mathrm{kft}$

\%VD $350 \mathrm{kcmil}$ feeders $=\frac{\sqrt{3} \times(287+52) \times 0.03 \times .055}{4.8}=\underline{\underline{0.21 \%}}$

\subsection{6 \%VD from SSA to SSB (12 ft of twin $350 \mathrm{kcmi}$ feeders)}

$Z_{\text {eff }}=0.06 \div 2=0.03, L=0.012 \mathrm{kft}$

\%VD $350 \mathrm{kcmil}$ feeders $=\frac{\sqrt{3 \times(287+52) \times 0.03 \times .012}}{4.8}=0.04 \%$

7.4.7 \%VD from SSB to MCC-N2 $(50 \mathrm{ft}$ of twin $350 \mathrm{kcmil}$ feeders)

$Z_{\text {eff }}=0.06 \div 2=0.03, L=0.050 \mathrm{kft}$

$\%$ VD $350 \mathrm{kcmil}$ feeders $=\frac{\sqrt{3} \times(287+52) \times 0.03 \times .050}{4.8}=\underline{\underline{0.18 \%}}$

See Sketch on page following that summarizes voltage drops: 
FLUOR DANIEL NORTHWEST

\section{DESIGN ANALYSIS}

client Numatec Hanford Corporation

Subject C-106 Load Determination,

Feeder/Protection Sizing, Voltage Drop,

Lighting Calculations, \& Conóuit Sizing

Location 200E, C Tank Farm
Cale No. W320-31-011

Revision 1

Page No. 19 of 34

wo/Job No. Task Order N09

Date $9 / 13 / 95$

By DE Larson

Checked $7 / 19 / 95$

By $T J$ Kasnick

Revised $5 / 6 / 98$

\section{VOLTAGE DROP - C-FARM}
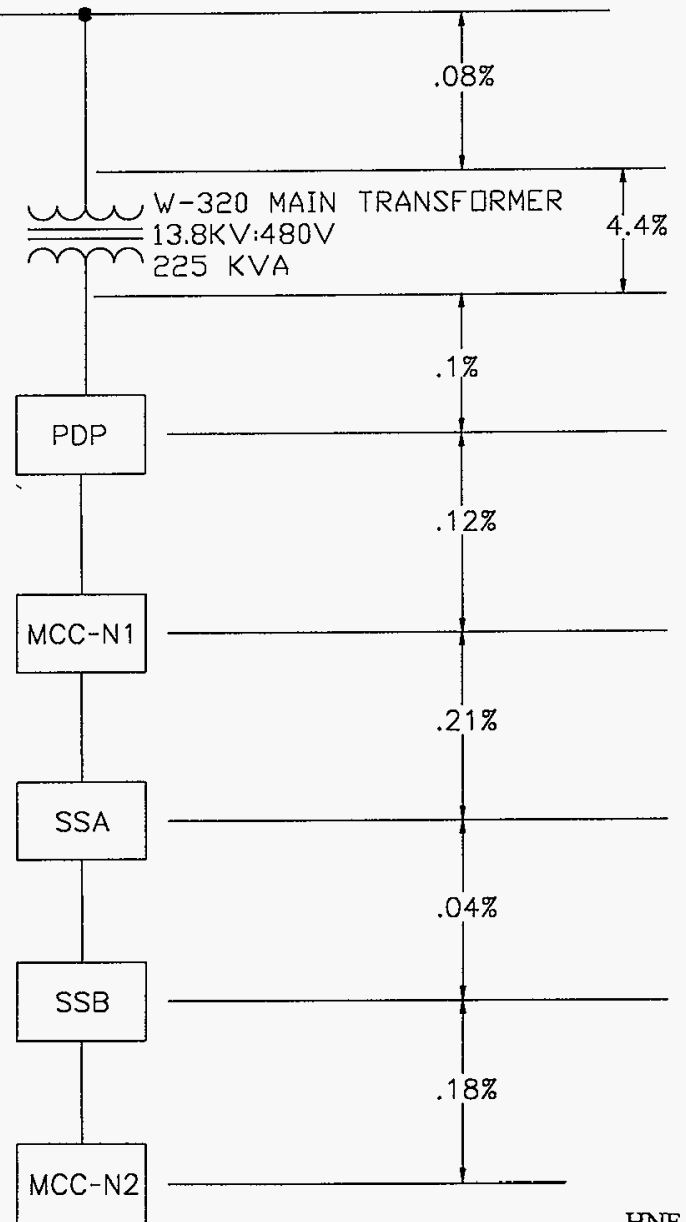

HNF-2487, Rev. 0 


\section{Fluor Daniel Northwest}

DESIGN ANALYSIS

client Numatec Hanford Corporation

subject C-106 Load Determination,

Feeder/Protection Sizing, Voltage Drop,

Lighting Calculations, \& Conduit Sizing

Location 200E, C Tank Farm
Calc. No. W320-31-011

Revision 1

Page No. 20 of 34

wo/ job No. Task Order No9

Date $9 / 13 / 95$ By DE Larson

Checked $7 / 19 / 95$ By TJ Kasnick

Revised 5/6/98 By Cris Carlson

$7.4 .8 \%$ VD from VSD Controller to Pump P-1362 Motor Terminal Block (300' of 500 kcmil copper motor feeder in steel conduit)

Motor FLA $=273 \mathrm{~A} ;$ Zeff $=.05 \mathrm{ohms} ; \mathrm{L}=0.3 \mathrm{kft}$

Total \%VD $=\frac{\sqrt{3} \times 273 \times .05 \times .3}{4.8}=1.48 \%$

\%VD from Pump P-1362 Motor Terminal Block to Pump P-1362 (20' of twin $4 / 0$ through pump pit cover block

Tota1 \%VD $=\frac{\sqrt{3} \times 273 \times .037 \times .02}{4.8}=0.07 \%$

Summary:

Tota] \%VD at motor terminals $=.12+.21+.04+.18+1.48+.07$

$=\underline{\underline{2.10 \%}}$

Motor terminal voltage $=480(1-0.0210) \approx 469.9 \mathrm{~V}$

Minimum permissible voltage $=414 \mathrm{~V}$

7.4.9 \%VD on Immersible Pump P-1361 Feeder (\#1/0 copper feeder in steel conduit - $300^{\prime}$ to pump pit and $60^{\prime}$ down into Tank $C-106$ )

Motor $\mathrm{FLA}=52 \mathrm{~A} ; \mathrm{Z}_{\text {eff }}$ for $\# 1 / 0 \mathrm{AWG}=.13 ; \mathrm{L}=.30+.06=.36$

$\% V D=\frac{\sqrt{3} \times 52 \times .13 \times .36}{4.8}=0.88 \%$

Total \%VD at motor terminals $=.12+.21+.04+.18+.88=1.43 \%$

Motor terminal voltage $=480(1-.0143) \approx 473.1 \mathrm{~V}$

Minimum permissible voltage $=414 \mathrm{~V}$ 


\section{Fluor Daniel Northwest}

Calc. ผo. W320-31-011

DESIGN ANALYSIS

client Numatec Hanford Corporation

subject C-106 Load Determination, Feeder/Protection Sizing, Voltage Drop, Lighting Calculations, \& Conduit Sizing Location 200E, C Tank Farm
Revision 1

Page No. 21 of 34

wo/ Job No. Task Order N09

Date 9/13/95 By DE Larson

Checked 7/19/95 By TJ Kasnick

Revised 5/6/98 By Cris Carison

\section{$7.5 \%$ Voltage Dip During Submersible Pump Startup}

Initially, all anticipated loads operating. "Peak" values used for current value.

7.5.1 Total Current Supplied:

$$
\begin{aligned}
& I_{\text {SURGE }}=\left(\begin{array}{llll}
6 & X & I_{P-1361}
\end{array}\right)+\left(I_{P_{\text {eak }}}-I_{P-1361}-I_{P-1362}\right) \\
& I_{\text {SURGE }}=(6 \times 52)+(375-52-273)=362 \mathrm{~A}
\end{aligned}
$$

\section{$7.5 .2 \%$ on Feeders up to EES (MCC-N2) with I $I_{\text {SURGE }}=362 \mathrm{~A}$}

$\%$ VD $350 \mathrm{kcmil}$ feeders to MCC-NI $=\frac{\sqrt{3} \times 362 \times 0.02 \times .045}{4.8}=\underline{\underline{0.12 \%}}$

Total feeder length of $350 \mathrm{kcmil}$ feeders MCC-N1 to MCC-N2 is $117 \mathrm{ft}$.

$\%$ VD $350 \mathrm{kcmil}$ feeders to MCC-N2 $=\frac{\sqrt{3} \times 362 \times 0.03 \times .117}{4.8}=0.46 \%$

Total \%VD to MCC-N2 $=.12 \%+.46 \%=.58 \%$

\subsection{3 \%VD on Immersible Pump Feeder}

$Z_{\text {eff }}$ for $\# I / 0=.13 ; L=.360 ; I=312 \mathrm{~A}$ (starting)

$\% \mathrm{VD}=\frac{\sqrt{3} \times 312 \times .13 \times .360}{4.8}=5.27 \%$

Total \%VD at submersible pump motor terminals = $5.27+.58=5.85 \%$

Motor terminal voltage $=480(1-.0585) \approx 452 \mathrm{~V}$

Minimum permissible voltage $=414 \mathrm{~V}$. The starting of the immersible pump ( $P-1361)$ represents the worst case condition that need be considered. The condition reperesented is very conservative since the immersible pump is started with a soft start unit and so should not have as large an inrush as was used in this calculation. No other motor starting need be considered. To be sure that starting will not be a problem we will consider voltage drop upstream of MCC-N1 as weII. 


\section{Fluor Daniel Northwest}

Calc. No. $\$ 320-31-011$

\section{DESIGN ANALYSIS}

client Numatec Hanford Corporation

subject C-106 Load Determination, Feeder/Protection Sizing, Voltage Drop, Lighting Calculations, \& Conduit Sizing Location 200E, C Tank Farm
Revision 1

Page No. 22 of 34

wo/sob No. Task Order Nog

Date $9 / 13 / 95$ By DE Larson

checked 7/19/95 By TJ Kasnick

Revised 5/5/98 By Cris Carlson

7.5.4 \%V on Submersible Pump Feeder including VD Across Supply Transformer

\%VD on pole to transformer feeder:

Primary Current $=$ Secondary Current $X X$ fmr turns ratio

$I=362 \times(480 / 13 ; 800)=12.6 \mathrm{amps}$

$\% \mathrm{VD}=\frac{\sqrt{3} \times 12.6 \times .20 \times .250}{13.8}=0.08 \%$

\section{8}

\%VD across supply transformer:

$I_{\text {BASE }}=$ Transformer $V_{A} /(480 \vee \times \sqrt{3})$

$I_{\text {BASE }}=225,000 /(480 \vee \times \sqrt{3})=270.6 \mathrm{~A}$

$I_{P U}=I / I_{B A S E}=362 / 270.6=1.34$

$\% V D=I_{P U} \times$ Transformer $\% Z=1.34 \times 3.19=4.27 \%$

\%VD across $600 \mathrm{kcmil}$ feeders to PDP:

$$
\% \text { VD }=\frac{\sqrt{3} \times 362 \times .024 \times .032}{4.8} \approx 0.10 \%
$$

Total \%VD $\approx 0.08 \%+4.27 \%+0.10 \%+5.85 \approx 10.3 \%$

Initially, the "soft start" unit was utilized to limit the torque effect of the submersible pump as it hangs down into the tank. Additionally, the "soft start" unit may be configured to limit the starting current to compensate for the high voltage drop across the supply transformer.

\section{$7.6 \%$ Voltage Dip With Booster Pump Starting}

7.6.1 \%VD to MCC N1 from PDP:

$$
\begin{aligned}
& I=(325)+(375-325)=375 \mathrm{~A} \\
& \% \mathrm{VD}=\frac{\sqrt{3} \times 375 \times .02 \times .045}{4.8} \approx 0.12 \%
\end{aligned}
$$$$
\text { [from } 7.3 .14 \& 7.4 .2]
$$ 


\section{Fluor Daniel Northwest}

Calc. No. W320-31-011

DESIGN ANALYSIS

client Numatec Hanford Corporation

subject C-106 Load Determination, Feeder/Protection Sizing, Voltage Drop, Lighting Calculations, \& Conduit Sizing Location 200E, C Tank Farm
Revision 1

Page No, 23 of 34 wo/sob No. Task Order No9

Date $9 / 13 / 95$

By DE Larson

checked $7 / 19 / 95$

By TJ Kasnick

Revised 5/6/98

7.6.2 \%VD to MCC N1 to MCC N2:

$$
\begin{aligned}
& I=(325)+(375-325)=375 \mathrm{~A} \\
& \% V D=\frac{\sqrt{3} \times 375 \times .03 \times .117}{4.8} \approx 0.47 \%
\end{aligned}
$$$$
\text { [from } 7.3 .2 \& 7.4 .2]
$$

\subsection{3\%VD on $500 \mathrm{kcmil}$ and \#4/0 Feeders:}

$$
\% \text { VD }=\frac{\sqrt{3} \times(325) \times[(.05 \times .3)+((.074 / 2) \times .02)]}{4.8}=1.85 \%
$$

Total $\%$ VD $\approx 0.12+.47+1.85 \approx 2.44 \%$

Motor terminal voltage $=480(1-0.0244) \approx 468 \mathrm{~V}$

Minimum permissible voltage $=414 \mathrm{~V}$

\subsection{Outdoor Lighting Calculations}

Each entrance needs 3 footcandles of lighting. Total area that needs to be illuminated is approximately $6^{\prime} \times 3^{\prime}=18 \mathrm{ft}^{2}$.

The fixture selected for outdoor use is the Voigt SOX-35 Wal1-Most. The manufacturer's data may be used to determine whether this fixture is adequate for our needs. The Footcandle Isolux diagram shows the footcandles available from Voight SOX -55 at a height of $15 \mathrm{ft}$. This must be adjusted to obtain values for the $\mathrm{SOX}-35$ at 10 feet.

To adjust for the lower lamp output, the footcandles will be multiplied by 0.6. This is obtained from the ratio of the Tumen output of the lamps. The Westinghouse Lighting Kandbook provides guidance for adjusting for the height difference. Per page 2-14 of the 1969 Westinghouse Lighting Handbook, "Footcandles for other mounting heights than the one shown are obtained by multiplying the values given on the curves by the ratio of the square of the present mounting height to the square of the new mounting height."

This adjustment factor will be $\left(15^{2} / 10^{2}\right)=2.25$.

We will consider an area $3 \mathrm{ft}$ away from the wall. The chart shows this as approximately 7 footcandles. 


\section{Fluor Daniel Northwest}

Calc. No. W320-31-011

DESIGN ANALYSIS

client Numatec Hanford Corporation

subject C-106 Load Determination,

Feeder/Protection Sizing, Voltage Drop, Lighting Calculations, \& Conduit Sizing Location 200E, C Tank Farm
Revision 1

Page No. 24 of 34

Wo/Job No. Task Order N09

Date $9 / 13 / 95$ By DE Larson

checked 7/19/95 By TJ Kasnick

Revised 5/6/98 By Cris Carlson

Using the adjustment factors, 7 footcandles $\times 2.25 \times 0.6=9.5$ footcandles.

A similar analysis for the S0X-18 (18W) lights used for the Process Bldg shows that there is adequate light at the many entrances by the Process B]dg.

The Tower lamp Tumen output is adjusted from the 55 watt data by multiplying by .225 , the ratio of the lamp lumens. Using the adjustment factors:

7 footcandles $\times 2.25 \times .225=3.543$ footcandles .

Outdoor fixture selected will give adequate light over doorways.

\subsection{Indoor Lighting Calculations}

Inside Lighting: Per IES, an average of 50 (approximately) footcandles is adequate. The Zonal-Cavity Method will be used to determine the number of fixtures. There are two buildings that need be considered here, the

Electrical Equipment Skid (241-C-51) and the Process Building (241-C-91).

Room Cavity Ratio $(R C R)=[5 \times h \times(L+W)] /(L \times W)$

where $\mathrm{h}=$ height of light

$\mathrm{L}=$ room length

$W=$ room width

$\operatorname{RCR}=[5 \times 8 \times(13+7)] /(13 \times 7)=8.8$

Using the Crouse-Hinds photometric data (see attachments), with an assumed cejling reflectance of $80 \%$ and an assumed wall reflectance of $50 \%$, the coefficient of utilization is 0.25 . Footcandle values of 20-30-50 will be utilized for areas of high contrast or large size per IESNA Figure 11.1 .

\subsubsection{Electrical Equipment Skid}

\# of fixtures $=($ FC $\times$ AREA $) /(\#$ of lamps $\times$ lumens $/ 1$ amp $\times$ CU $\times$ MF $)$

\# of fixtures $=(30 \times 11 \times 9) /(2 \times 3200 \times .25 \times .9)=2.06$

Use two fixtures. The actual size of the room is effectively reduced since the electrical cabinets are pseudo-walls. 


\section{Fluor Daniel Northwest}

Calc. No. W320-31-011

DESIGN ANALYSIS

client Numatec Hanford Corporation

subject C-106 Load Determination,

Feeder/Protection Sizing, Voltage Drop,

Lighting Calculations, \& Conduit Sizing
Revision 1

Page No. 25 of 34

wo/sob No. Task Order N09

Date $9 / 13 / 95$ By DE Larson

Checked $7 / 19 / 95$ By TJ Kasnick

Revised 5/6/98 By Cris Carlson

\subsubsection{Process Building}

Due to the geometry of the Process Building, two areas must be considered, Room 1 and Room 2. The dimensions of these two rooms may be obtained from drawings $\mathrm{H}-2-818455$ sheet 3 and $\mathrm{H}-2-818451$ sheet 1 .

Room 2:

$$
\begin{aligned}
\# \text { of fixtures } & =(\text { FC } \times \text { AREA }) /(\# \text { of lamps } \times \text { Tumens/lamp } \times \text { CU } \times \text { MF }) \\
\# \text { of fixtures } & =((30 \times 8 \times 4)+(30 \times 13 \times 5)) /(2 \times 3200 \times .25 \times .9) \\
& =2.02
\end{aligned}
$$

Therefore, use two fixtures in second part of Room 2 .

Room 1:

Room 1 of the Process Building needs less light. It is intended that this room be occupied on a rare basis. Visual tasks are only occasionally performed here. Fifteen footcandles should be adequate.

$\#$ of fixtures $=(F C \times$ AREA $) /(\#$ of lamps $\times$ lumens $/ 1$ amp $\times$ CU $\times$ MF $)$

\# of fixtures $=((15 \times 9 \times 8)+(15 \times 10 \times 13)) /$

$=2.1$

$(2 \times 3200 \times .25 \times .9)$

Therefore, use two fixtures in Room 1.

\subsection{Conduit Fill}

\subsubsection{General}

Three categories of conduits are used for the C-Farm installation,

Power, Instrument (signal) and Control. The three categories are

intended to be separate and distinct in the interests of safety and to minimize the propagation of electrical noise onto the signal wiring.

Conduit fill of power cabling shall be considered first. To minimize the number of conduits necessary, power cables will be combined. This is restricted by Note 8 to the ampacity tables of NEC article 310 . 


\section{Fluor Daniel Northwest}

Calc. No. W320-31-011

DESIGN ANALYSIS

client Numatec Hanford Corporation

subject C-106 Load Determination,

Feeder/Protection Sizing, Voltage Drop,

Lighting Calculations, \& Conduit Sizing

Location 200E, C Tank Farm
Revision 1

Page No. 26 of 34

wo/sob No. Task Order N09

Date $9 / 13 / 95$ By DE Larson

checked 7/19/95 By TJ Kasnick

Revised 5/6/98 By Cris Carlson

The attached conduit block diagrams shows how cables will be combined. Some variation exists between these documents and official design documents. Combining cable routes with \#10 AWG conductors within them will allow the use of less conduits.

\subsubsection{Specific Power Conduits}

Conduit Numbers C106-3P201, C106-3P202, and C106-3P203 are three inch conduits that are extensions of stub-outs installed under the ERS portion of the project. Each is intended to carry $4-350 \mathrm{kcmil}$ conductors of type XHHW-2. Per NEC Chapter 9 Table 3B, three inch conduits may carry up to $5-350 \mathrm{kcmil}$ conductors.

The following table shows the C-Farm loads, the size feeder to the load and the minimum required conduit size. Power conduits will also include a ground conductor to conform with DOE's 6430.1A, 1639-1. Those loads that share conduits will be evaluated separately.

Load

Sluicer (SHC-1361)

Service Building (241-C-73)

Process Building (241-C-91)

STurry Pump Winch (W-1361)

Chiller Skid

Exhaust Fan (FN-1362)

Exhaust Skid / Exhaust Fan

Immersible Slurry Pump

Booster Pump
Feeder Size

4-\#12 AWG

4-\#10 AWG

$3-\# 2, \quad 1-\# 6 \quad$ AWG

4-\#12 AWG

$3-\# 4 / 0,1-\# 2$ AWG

4-\#12 AWG

8-\#10 AWG

$3-\# 1 / 0$,

$1-\# 4,3-\# 4$ AWG

$3-500 \mathrm{kcmi}]$

I-\#2 AWG
Minimum

Conduit Size/Actual

\section{$1 / 2^{\prime \prime} \quad\left(3 P 2261^{11}\right)$}

$1 / 2^{\prime \prime}\left(3 P 2101^{\prime \prime}\right)$

$1-1 / 4^{\prime \prime}(3 P 001$ 1.5")

1/2" (3P224 1")

2" (3P211 2")

$1 / 2^{\prime \prime}\left(3 \mathrm{P} 213 \mathrm{I}^{\prime \prime}\right)$

$1 / 2^{\prime \prime}\left(3 \mathrm{P} 2251^{\prime \prime}\right)$

$1-1 / 2^{\prime \prime}\left(3 P 2232^{\prime \prime}\right)$

3" (3P222 4")

A11 conduits for each of these feeders is larger than the minimum.

\subsubsection{Shared Power Conduits}

The Exh Fan, Exhaust Skid, \& Winch all share a conduit. (C106-3P218)

The derating factor of $70 \%$ is applied. The conductors are checked to verify they have the required capacity.

Exh Fan: $70 \% \times 30 \mathrm{~A}=21 \mathrm{~A}$, which is satisfactory.

Exhaust Skid: $70 \% \times 40 \mathrm{~A}=28 \mathrm{~A}$; which is satisfactory.

Winch: $70 \% \times 30=21$, which is satisfactory. 


\section{Fluor Daniel Northwest}

DESIGN ANALYSIS

client Numatec Hanford Corporation

subject C-106 Load Determination, Feeder/Protection Sizing, Voltage Drop, Lighting Calculations, \& Conduit Sizing Location 200E, C Tank Farm
Calc. No. W320-31-011

Revision 1

Page No. 27 of 34

wo/sob No. Task Order N09

Date $9 / 13 / 95$

8y DE Larson

checked $7 / 19 / 95$

By TJ Kasnick

Revised 5/6/98

The Exhaust Fan \& Exhaust Skid share a conduit. (C106-3P225)

The derating factor of $80 \%$ is applied.

Exhaust Skid: $80 \% \times 40 \mathrm{~A}=32 \mathrm{~A}$, which is satisfactory.

Exhaust Fan: $80 \% \times 30 \mathrm{~A}=24 \mathrm{~A}$, which is satisfactory.

\subsubsection{Control Conduits}

The smallest control conduit used is $\frac{3}{4} "$ in diameter. Per NEC Chapter

9 TabTe 3B, this can carry 24-\#14 AWG conductors. See the attached Block Diagram. The most conductors in any conduit is 24-\#14 AWG and 3-\#12 for the winch limit switches. By inspection, these conductors will not overfill the $1.5^{\prime \prime}$ conduit (C106-3C018) planned for this purpose.

\subsubsection{Instrument Conduits}

The NEC defines the number of allowabie conductors in a conduit in Chapter 9 Table 1. This will be followed when evaluating the $\%$ fill of our instrument conduits. Refer to drawing $\mathrm{H}-2-818676$ sheets $1-4$ as an aid in following this evaluation.

The formula used to evaluate the fill of a conduit is:

$\% \mathrm{Fill}=$ sum of Cable Area(s) / conduit area $=$ sum of $\left(\pi \times 0^{2} / 4\right) /$ Conduit Area

Conduit Areas are in NEC Chapter 9 Table 4, third column.

The type of instrument cable being used is Rockbestos. See attached catalog sheet for specifics on each type. The following evaluation is done for each of the larger conduits.

Conduit C106-3S021: Wire Runs W106-90,91, 92 \& 93

1 triad $0.09 i n^{2}+1$ triad $0.09 \mathrm{in}^{2}+1$ pair $0.08 \mathrm{in}^{2}+2$ pair 0 $.31 \mathrm{in}^{2}=.57 \mathrm{in}^{2}$

$.57 \mathrm{in}^{2} / 40 \%=1.43 \mathrm{in}^{2}$

This translates to a diameter $=1.35^{\prime \prime}$; Use a $1.5^{\prime \prime}$ conduit.

$\mathrm{Fi11}=27.5 \%$

HNF-2487, Rev. 0

Page E-31 


\section{Fluor Daniel Northwest}

DESIGN ANALYSIS

client Numatec Hanford Corporation

subject C-106 Load Determination,

Feeder/Protection Sizing, Voltage Drop,

Lighting Calculations, \& Conduit Sizing Location 200E, C Tank Farm
Calc. No. W320-31-011

Revision 1

Page No. 28 of 34

wo/Job No. Task Order N09

Date 9/13/95 By DE Larson

checked $7 / 19 / 95$ By TJ Kasnick

Revised 5/6/98 By Cris Cartson

Conduit C106-35019: Wire Runs W106-92A, 93F, 96, 97A, 154B \& 154C

1 pair e.08 in $^{2}+2$ pair e. $.31+16$ pair e $1.13 \mathrm{in}^{2}+3$ pair e .34 $\mathrm{in}^{2}+2 \# 14$ e $.028 \mathrm{in}^{2}+2 \# 140.028 \mathrm{in}^{2}=1.92 \mathrm{in}^{2}$

$1.92 \mathrm{in}^{2} / 40 \%=4.79 \mathrm{in}^{2}$

This transiates to a diameter $=2.47^{\prime \prime}$; Use a $3^{\prime \prime}$ conduit.

$\mathrm{Fill}=25.6 \%$

Conduit C106-35027: Wire Runs W106-88, 88A \& 89A

1 triad $0.09 \mathrm{in}^{2}+1$ triad a $.09 \mathrm{in}^{2}+2$ pair $0.16 \mathrm{in}^{2}=.34 \mathrm{in}^{2}$

$.34 \mathrm{in}^{2} / 40 \%=.85 \mathrm{in}^{2}$

This translates to a diameter $=1.04 "$; Use a $2.5^{\prime \prime}$ conduit.

$\mathrm{Fi11}=7.0 \%$

Conduit C106-3S028: Wire Runs W106-95 \& 95A

Wire Run \#--95A is part of the 4 pair cable of Wire Run \#-95.

4 pair $.41 \mathrm{in}^{2}$

$.41 \mathrm{in}^{2} / .53=.77 \mathrm{in}^{2}$

This translates to a diameter of 1"; Use a $1 \frac{1}{2} "$ conduit.

$\mathrm{Fi11}=20.1 \%$

Conduit C106-35029: Wire Runs W106-95 \& 95A, \& 154

Wire Run \#-95A is part of the 4 pair cable of Wire Run \#-95.

4 pair $.41 \mathrm{in}^{2}+1$ pair $.08 \mathrm{in}^{2}=.49 \mathrm{in}^{2}$

$.49 \mathrm{in}^{2} / .31=1.58 \mathrm{in}^{2}$

This translates to a diameter $=1.41 "$; Use a $1.5^{\prime \prime}$ conduit.

Fill $=24.1 \%$

HNF-2487, Rev. 0

Page E-32 


\section{Fluor Daniel Northwest}

Calc. No. W320-31-011

\section{DESIGN ANALYSIS}

client Numatec Hanford Corporation

subject C-106 Load Determination,

Feeder/Protection Sizing, Voltage Drop, Lighting Calculations, \& Conduit Sizing Location 200E, C Tank Farm
Revision 1

Page No. 29 of 34

worsob No. Task Order N09

Date $9 / 13 / 95$

By DE Larson

checked $7 / 19 / 95$

By TJ Kasnick

Revised 5/6/98 By Cris Carlson

Conduit C106-3S031: Wire Runs W106-84, 85, 87, 87A, 87B, 97B, 154, $154 \mathrm{~A}, 154 \mathrm{~B}, 158,159$ \& 169

Wire Run \#-84 3 pair .24 in

Wire Run \#-85 2 pair $0.31 \mathrm{in}^{2}$

Wire Run \#-87 1 \#-14 .20 in

Wire Run $\#-87 \mathrm{~A} 8$ trd $0.64 \mathrm{in}^{2}$

Wire Run \#-87B 8 trd $0.64 \mathrm{in}^{2}$

Wire Run \#-97B 1 pair 0.08 in $^{2}$

Wire Run \#-154 1 pair e .08 in $^{2}$

Wire Run \#-154A 2\#14 @ .03 in

Wire Run \#-154B 2\#14 0.03 in

Wire Run $\#-1581$ pair 0.08 in $^{2}$

Wire Run $\#-159$ I pair 0.08 in $^{2}$

Wire Run \#-169 I pair 0.08 in $^{2}$

Total Area............2.49 in $^{2}$

$2.49 \mathrm{in}^{2} / .40=6.23 \mathrm{in}^{2}$

This translates to a diameter of 2.82 inches; Use a 3 " conduit.

$\mathrm{Fi11}=37.9 \%$

Conduit C106-35032: Wire Runs W106-81, 81A, 84, 85, 86, 137, 154A, $158 \& 159$

Wire Run \#-81 4\#14

Wire Run \#-81A 3\#14

Wire Run \#-84 3 pair

Wire Run \#-85 2 pair

Wire Run \#-137 Belden 1049A

Wire Run \#-154A 2\#14

Wire Run \#-158 1 pair

Wire Run \#-159 1 pair

Total Area.

$$
\begin{aligned}
& 0.06 \mathrm{in}^{2} \\
& 0.04 \mathrm{in}^{2} \\
& 0.24 \mathrm{in}^{2} \\
& 0.31 \mathrm{in}^{2} \\
& 0.00 \mathrm{in}^{2} \\
& 0.03 \mathrm{in}^{2} \\
& 0.08 \mathrm{in}^{2} \\
& 0.08 \mathrm{in}^{2} \\
& .1 .04 \mathrm{in}^{2}
\end{aligned}
$$

$1.04 \mathrm{in}^{2} / 40 \%=2.6 \mathrm{in}^{2}$

This translates to a diameter $=1.82$ "; Use a $3^{\text {" conduit. }}$

$\mathrm{Fill}=13.9 \%$ 


\section{Fluor Daniel Northwest}

DESIGN ANALYSIS

client Numatec Hanford Corporation subject C-106 Load Determination, Feeder/Protection Sizing, Voltage Drop, Lighting Calculations, \& Conduit Sizing Location 200E, C Tank Farm
Calc. No. W320-31-011

Revision 1

Page No. 30 of 34

HO/Job No. Task Order NO9

Date 9/13/95 By DE Larson

checked $7 / 19 / 95$ By TJ Kasnick

Revised 5/6/98 By Cris Carlson

Conduit C106-35036: Wire Runs W106-94, 95, \& 95A

Wire Run \#-95A is part of the 4 pair cable of Wire Run \#-95.

Wire Run \#-94 1 pair \#16 0.08 in $^{2}$

Wire Run \#-955 4 pair $0.41 \mathrm{in}^{2}$

Total area $=.49 \mathrm{in}^{2}$

$.49 \mathrm{in}^{2} / .31=1.58 \mathrm{in}^{2}$

This translates to a diameter $=1.41 "$; Use a $1.5^{\prime \prime}$ conduit.

$\mathrm{Fill}=24.1 \%$

Conduit C106-3S033: Wire Runs W106-82

2 pair $.31 \mathrm{in}^{2}$

Total area $=.31 \mathrm{in}^{2}$

$.31 \mathrm{in}^{2} / .53=.58 \mathrm{in}^{2}$

This translates to a diameter $=.85^{\prime \prime}$; Use a $1.5^{\prime \prime}$ conduit.

$\mathrm{Fi} 11=15 \%$

Conduit C106-35016: Wire Runs W106-70, 92A, 93F, 96, 97A, 97B, 154C \& 169

Wire Run $\#-70 \quad 1$ pair 0.08 in $^{2}$

Wire Run \#-92A 1 pair 0.08 in $^{2}$

Wire Run \#-93F 2 pair Q .31 in $^{2}$

Wire Run \#-96 16 pair 01.13 in $^{2}$

Wire Run \#-97A 3 pair .34 in $^{2}$

Wire Run \#-97B 1 pair .08 in $^{2}$

Wire Run \#-154C 2 \#14 @ .03 in

Wire Run \#-169 1 pair $\frac{.08 \mathrm{in}^{2}}{2.13 \mathrm{in}^{2}}$

$2.13 \mathrm{in}^{2} / .4=5.4 \mathrm{in}^{2}$

This translates to a diameter $=2.6^{\prime \prime}$; Use a $3^{\prime \prime}$ conduit.

$\mathrm{Fi11}=28.4 \%$

HNF-2487, Rev. 0

Page E-34 


\section{Fluor Daniel Northwest}

DESIGN ANALYSIS

client Numatec Hanford Corporation subject C-106 Load Determination, Feeder/Protection Sizing, Voltage Drop, Lighting Calculations, \& Conduit Sizing Location 200E, C Tank Farm
Revision 1

Page No. 31 of 34

wo/Job No. Task Order Nog

Date $9 / 13 / 95$

By DE Larson

checked $7 / 19 / 95$

вy TJ Kasnick

Revised 5/6/98 By Cris Carison

Conduit C106-3S018: Wire Runs W106-92A, 93F, 96, 97A \& 154C

Wire Run \#-92A 1 pair $e .08$ in $^{2}$

Wire Run \#-93F 2 pair $0.31 \mathrm{in}^{2}$

Wire Run \#-96 16 pair o 1.13 in $^{2}$

Wire Run \#-97A 3 pair o .34 in $^{2}$

Wire Run \#-154C 2\#14

Total Area................89 in $^{2}$

$1.89 \mathrm{in}^{2} / .4=4.73 \mathrm{in}^{2}$

This trans?ates to a diameter of 2.5"; Use a $3^{\prime \prime}$ conduit.

$\mathrm{FiTl}=25.2 \%$

Conduit C106-35030: Wire Runs W106-87, 87A, 87B, 137, \& 154B

Wire Run \#-87 $1 \# 14$

Wire Run \#-87A 8 triads

Wire Run \#-87B 8 triads

e. $.01 \mathrm{in}^{2}$

Wire Run \#-137 Beiden 1049A 0.20 in $^{2}$

Wire Run \#-154B $2 \# 14 \quad 0.03$ in $^{2}$

Total area.................52 $\mathrm{in}^{2}$

$1.52 \mathrm{in}^{2} / .4=3.8 \mathrm{in}^{2}$

This translates to a diameter $=2.2^{\prime \prime}$; Use a $3^{\prime \prime}$ conduit.

$\mathrm{Fi11}=20 \%$

Conduit C106-35037: Wire Runs W106-87, 87A \& 87B
Wire Run \#-87 $1 \# 14$
Wire Run \#-87A 8 triads
(0. $.01 \mathrm{in}^{2}$
Wire Run \#-87B 8 triads
$0.64 \mathrm{in}^{2}$
Total area.................
$\frac{.64 \mathrm{in}^{2}}{.1 .29 \mathrm{in}^{2}}$
$1.29 \mathrm{in}^{2} / .40=3.2 \mathrm{in}^{2}$

This translates to a diameter $=2.03^{\prime}$; Use a $3^{\prime \prime}$ conduit.

Fil1 $=17.2 \%$

Remaining conduit runs are short and contain few cables. 


\section{Fluor Daniel Northwest}

DESIGN ANALYSIS

client Numatec Hanford Corporation

subject C-106 Load Determination,

Feeder/Protection Sizing, Voltage Drop,

Lighting Calculations, \& Conduit Sizing

Location 200E, C Tank Farm
Calc. No. W320-3l-011

Revision 1

Page No. 32 of 34

Ho/Job No. Task Order N09

Date 9/13/95 By DE Larson

checked $7 / 19 / 95$ By TJ Kasnick

Revised 5/6/98 By Cris Carlson

\subsection{Pullbox Sizing}

Six pullboxes are used for the $W-320$ C-Farm installation work. They must all be checked to see whether they have been sized properly per NEC 370-28. The pullboxes will be tested to determine whether they satisfy the NEC rules for \# 4 and larger cables (NEC 370-16). This will be a conservative test. If this criteria is not satisfied, the electricians will need to add another row in the boxes. The boxes are rather deep and so this should present no problem. All pulls are considered as being made into the farm since this is necessary to avoid the spread of contamination.

\subsubsection{Power Pullboxes}

Refer to drawing $\mathrm{H}-2-818674$ Sheet 3 .

Pullbox 78

Al1 conduits are considered to be in one row. The conductors for the following size conduits pass straight through: $4 ", 2 ", 1.5^{\prime \prime}$, $1 "$ and 1 ".

$8 \times 4^{\prime \prime}=32^{\prime \prime}$

Box is 42" x 42"; pullbox has 42" available for pulling. Box size is satisfactory.

\section{Pul1box 8B}

There are angle pults into the C-Farm. Conduit sizes are $4^{\prime \prime}, 2^{\text {", }}$, $1.5^{\prime \prime}, 1^{\prime \prime}, 1^{\prime \prime}$. Straight pulls are for small conduits and need not be evaluated.

$6 \times 4^{\prime \prime}+2^{\prime \prime}+1.5^{\prime \prime}+1^{\prime \prime}+1^{\prime \prime}=29.5^{\prime \prime}$

Box is 42" x 42"; pullbox has $42 "$ available for pulling. Box size is satisfactory.

7.10.2 Control Pullboxes

\section{Pul1box 7A}

Both conduits are for straight pulls and for angle pulls. Conduit sizes are $1.5^{\prime \prime}$ and $1^{\prime \prime}$.

Straight Pu11: $8 \times 1.5^{\prime \prime}=12^{\prime \prime}$; minimum inside size is $2^{\prime}-0^{\prime \prime}$. 


\section{Fluor Daniel Northwest}

DESIGN ANALYSIS

client Numatec Hanford Corporation

subject C-106 Load Determination,

Feeder/Protection Sizing, Voltage Drop, Lighting Calculations, \& Conduit Sizing Location 200E, C Tank Farm
Revision 1

Page No. 33 of 34

wo/Job No. Task Order N09

Date $9 / 13 / 95$ By DE Larson

Checked 7/19/95 By TJ Kasnick

Revised 5/6/98

Angle pult: $6 \times 1.5^{\prime \prime}+1 "=10$." ; minimum inside size is $2^{\prime}-0^{\prime \prime}$.

Box is $24^{\prime \prime} \times 36^{\prime \prime}$; box size is satisfactory.

\section{Pullbox 8A}

Conduits are $1.5^{\prime \prime}$ or three 1".

Straight Pull: $8 \times 1.5^{\prime \prime}=12^{\prime \prime}$

Angle pul1: $6 \times 1^{\prime \prime}+1^{\prime \prime}+I^{\prime \prime}=8^{\prime \prime}$

Box is $24^{\prime \prime} \times 36^{\prime \prime}$; box size is satisfactory.

\subsubsection{Instrument Pullboxes}

Pullbox 7

Conduits are $3^{\prime \prime}, 1.5^{\prime \prime}, 1^{\prime \prime}$ and $.75^{\prime \prime}$.

Straight Pu11: $8 \times 3^{\prime \prime}=24^{\prime \prime}$

Angle PuT7: $6 \times 3^{\prime \prime}+1.5^{\prime \prime}+1^{\prime \prime}+.75^{\prime \prime}=21.25^{\prime \prime}\left(24^{\prime \prime}\right.$ is opposite dimension.)

Angle Pull: $6 \times 3^{\prime \prime}+3^{\prime \prime}+2^{\prime \prime}+1.5^{\prime \prime}+.75^{\prime \prime}=25.25^{\prime \prime}$ (36" is opposite dimension.) This does meet the test. Our largest instrument cable is a 16 pair. It's overall diameter is 1.20." The bending radius of this cable is our limiting factor.

$8 \times 1.20^{\prime \prime}=9.6^{\prime \prime}$

Box is $24^{\prime \prime} \times 36^{\prime \prime}$; There is room for the largest cable to bend. Box size is satisfactory.

\section{Pultbox 8}

Conduits are $2^{\prime \prime}, 3^{\prime \prime}, 3^{\prime \prime}$ and $1.5^{\prime \prime}$.

Straight PuTT: $8 \times 3^{\prime \prime}=24^{\prime \prime}$

Angle Pu11: $6 \times 3^{\prime \prime}+3^{\prime \prime}+1.5^{\prime \prime}+2^{\prime \prime}=24.5^{\prime \prime}$

Box is 24" $\times 36^{\prime \prime}$; Box size is unsatisfactory as oriented on the drawings unless another row is added for conduits in the box. 


\section{Fluor Daniel Northwest}

DESIGN ANALYSIS

client Numatec Hanford Corporation

subject C-106 Load Determination,

Feeder/Protection Sizing, Voltage Drop,

Lighting Calculations, \& Conduit Sizing Location 200E, C Tank Farm
Calc. No. W320-31-011

Revision 1

Page No. 34 of 34

Wo/Job No. Task Order N09

Date $9 / 13 / 95$

By DE Larson

checked $7 / 19 / 95$

By TJ Kasnick

Revised 5/6/98

8. CONCLUSIONS

8.1 Totai connected load $\approx 481.9$ KVA.

Maximum estimated demand for sluicing project $\approx 481.9$ KVA (Startup)

Estimated NORMAL demand on transformer bank $\approx 262.5$ KVA. (Operation)

8.2 The planned double $600 \mathrm{kcmil}$ feeder to C-PDP-1 and the triple $350 \mathrm{kcmil}$ feeders to the EES are adequate for the C-Farm sluicing demand.

$8.3500 \mathrm{kcmil}$ feeder for the $250 \mathrm{HP}$ VSD motor is adequate to maintain proper terminal voltage level during motor starting with the soft start option of VSD.

8.4 The C-Farm distribution to Submersible Pump P-1361 is adequate. Use of the soft start unit will aid in starting.

8.5 A single outdoor light fixture is adequate over the door. Four two-lamp fixtures are required inside the Process Building.

8.6 Pullbox sizes shown on the drawings are adequate.

HNF-2487, Rev. 0

Page E-38 
EUE 12 - UuT

\section{ELECTRIC SERVICE REQUEST}

Project Number $\mathrm{H} 320$

Project Engineer F.W. (Rick) Bradshaw Description (Attach Drakings)

Tank C-106 Sluicing: C-Farm electrical supply $\mathrm{H}-2 \sim 818680$
Phone 373-2692 Request Number EUE $93-264$ R.t

originator T.J. Kasnick Date 7/18/95

ritle/org. Lead Electrical Engineer

Telephone 373-3021

Facility Rep. Roger Taft

[X] WHC [X] KEH [] PNL [] BCS

Loc. MSIN S2-47

$\begin{array}{lll}\text { ADDITIOH [X] } & \text { REMOVAL [] } & \text { Service Location North side } \\ \text { Request For: } & \text { Normal Power [X] } & \text { Alternate Power [] } \\ & \text { Stand-by Power [] } & \text { Emergency Power [] }\end{array}$

(Stand-by or Emergency requests must be accompanied by applicable sections of PSE, PSAR, NSAR, SAR or OSR and must also include a completed ESR supplemental form.)

Dates Service Required:

August 1995

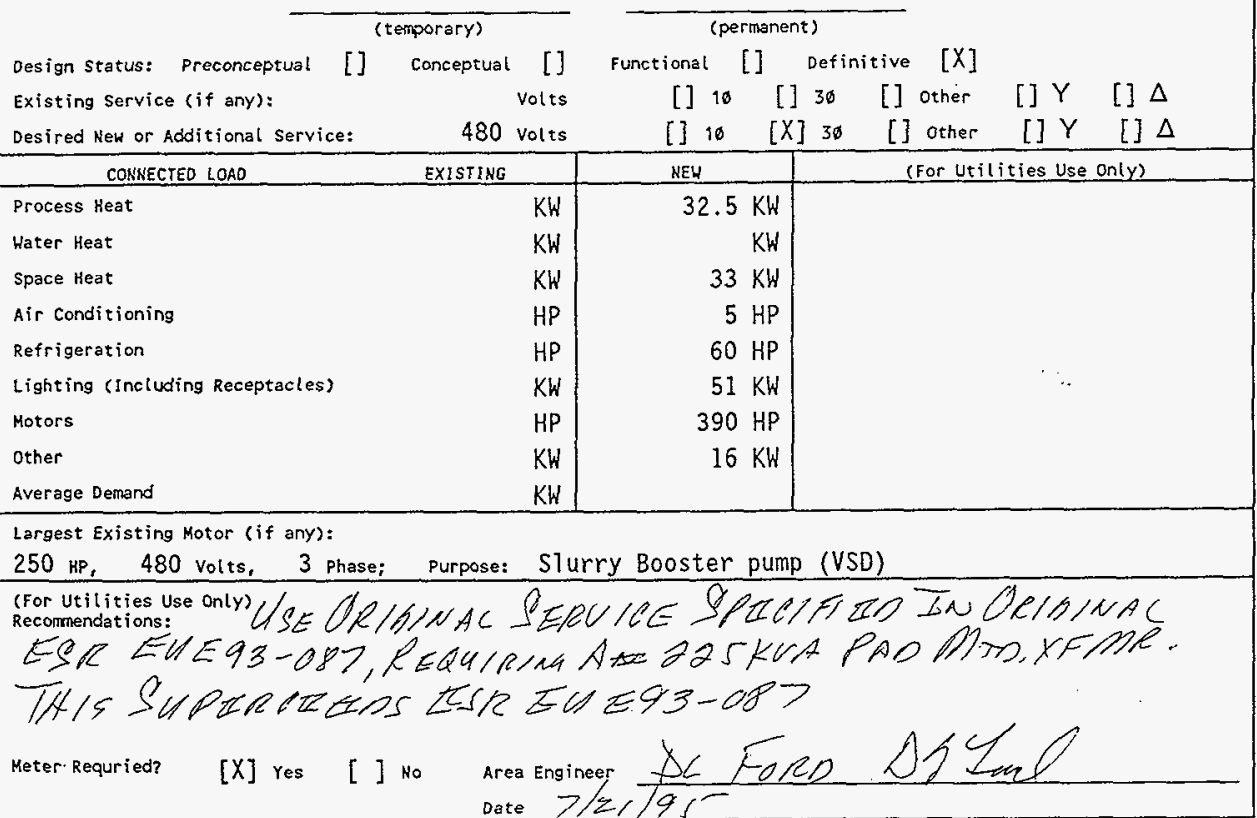

RETURN TO: ELECTRICAL UTILITIES ENGINEERING, MSIN S2-12, WHC

Calculation W320-31-oll
Page A-2




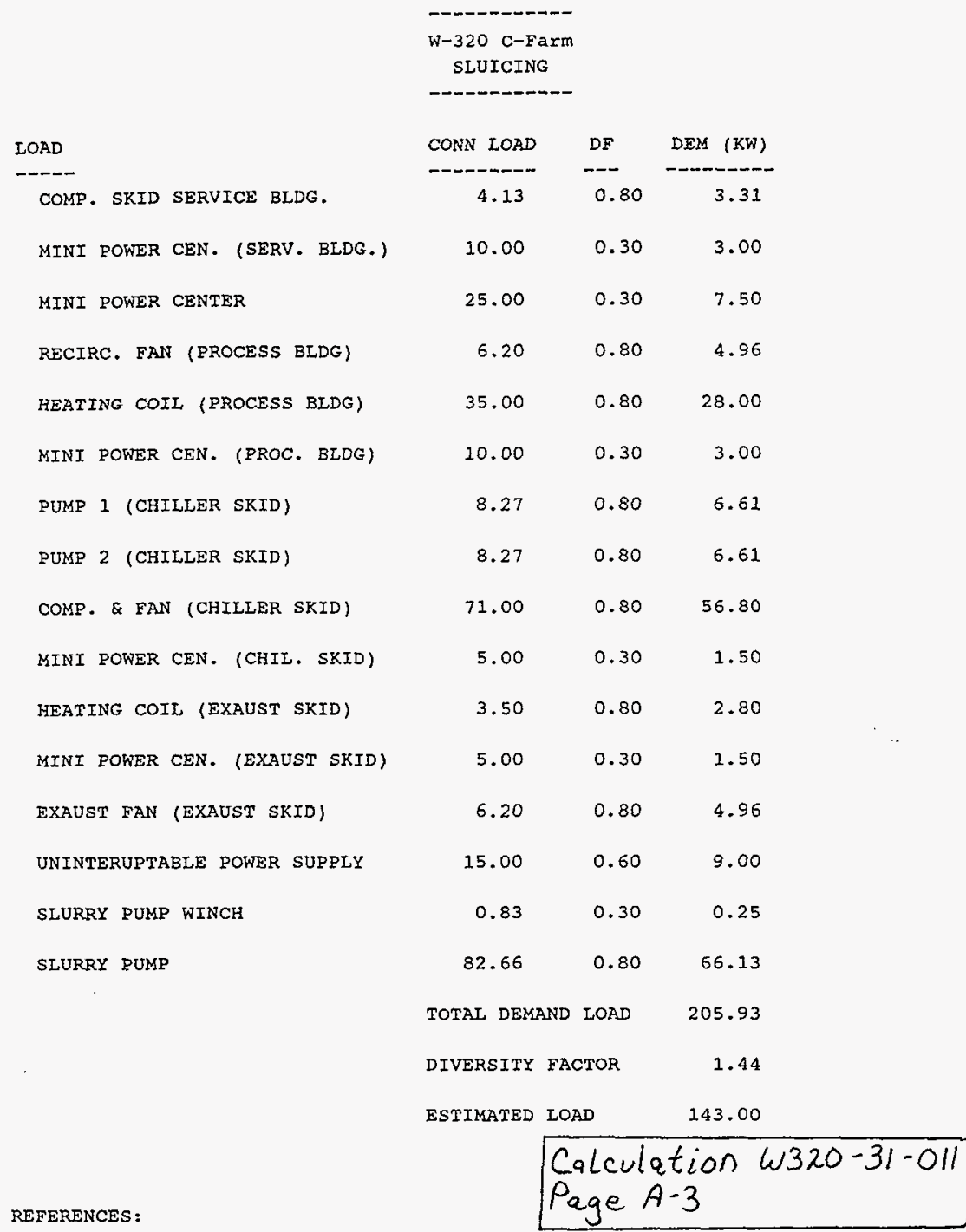

1. INDUSTRIAL POWER SYSTEMS HANDBOOK, BEEMAN, FIRST ED. CHAPTER 17 (LOAD AND COST ESTIMATING DATA

2. STANDARD HANDBOOK FOR ELECTRICAL ENGINEERS, FINK AND BEATY ELEVENTH EDITION, CHAPTER 18 (DEMAND AND DIVERSITY FACTORS)

TJ Kasnick 12/6/94

$C: \backslash W 320 \backslash E R S \backslash W-320-C F$. WKI

HNF-2487, Rev. 0

Page E-41 


\begin{tabular}{|c|c|c|c|c|}
\hline LOAD & HP & $\begin{array}{l}\text { CONN LOAD } \\
(X V A)\end{array}$ & $\mathrm{DF}$ & DEM (KVA) \\
\hline \multirow{2}{*}{\multicolumn{5}{|c|}{ ELECTRICAL EQUIPNENT SXID: }} \\
\hline & & & & \\
\hline MINI-POWER CENTER & & 15.00 & 0.30 & 4.50 \\
\hline \multicolumn{5}{|l|}{ SERVICE BUILDING: } \\
\hline MINI-POWER CENTER & & 5.00 & 0.30 & 1.50 \\
\hline COMPRESSOR SKID & 7.50 & 6.20 & 0.80 & 4.96 \\
\hline \multicolumn{5}{|l|}{ PROCESS BUILDING: } \\
\hline RECIRCULATION FAN & 7.50 & 6.20 & 0.80 & 4.96 \\
\hline HEATING COIL & & 30.00 & 0.80 & 24.00 \\
\hline SPACE HEATING & & 8.00 & 0.30 & 2.40 \\
\hline MINI-POWER CENTER & & 5.00 & 0.30 & 1.50 \\
\hline \multicolumn{5}{|l|}{ CHILLER SKID: } \\
\hline CIRCULATION PUMP 1 & 10 & 8.27 & 0.80 & 6.61 \\
\hline CIRCULATION PUMP 2 & 10 & 8.27 & 0.80 & 6.61 \\
\hline COMPRESSORS/FANS & & 71.00 & 0.80 & 56.80 \\
\hline MINI-POWER CENTER & & 5.00 & 0.30 & 1.50 \\
\hline \multicolumn{5}{|l|}{ EXHAUST SKID: } \\
\hline HEATING COIL & & 2.50 & 0.80 & 2.00 \\
\hline EXAUST FAN (WITH VSD) & 7.50 & 6.20 & 0.80 & 4.96 \\
\hline MINI-POWER CENTER & & 5.00 & 0.30 & $\cdot 1: 50$ \\
\hline VACUUM PUMP & 0.75 & 0.62 & 0.80 & 0.50 \\
\hline \multicolumn{5}{|l|}{ SLUICER SKID: } \\
\hline HYDRAULIC PUMP & 5 & 4.13 & 0.80 & 3.31 \\
\hline MINI-POWER CENTER & & 1.00 & 0.30 & 0.30 \\
\hline SLURRY BOOSTER PUMP (WITH VSD) & 250 & 206.65 & 0.80 & 165.32 \\
\hline SLURRY SUBMERSTBLE PUMP (SOFT START & 75 & 61.99 & 0.80 & 49.60 \\
\hline SLURRY SUBMERSIBLE PUMP WINCH & 2 & 1.65 & 0.30 & 0.50 \\
\hline \multicolumn{5}{|l|}{ CONSTRUCTION OFFICE TRAILERS (3) } \\
\hline SPACE HEATING/HEAT PUMP & & 25.00 & 0.30 & 7.50 \\
\hline IIGHTING/RECEPTACLES & & 25.00 & 0.30 & 7.50 \\
\hline Calculation w320-31-011 & & TOTAL DEMAN & ND LOAD & 358.32 \\
\hline Page $A-4$ & & DIVERSITY F & ACTOR & 1.44 \\
\hline
\end{tabular}

REFERENCES:

1. INDUSTRIAL POWER SYSTEMS HANDBOOK, BEEMAN, FIRST ED. CHAPTER 17 (LOAD AND COST ESTIMATING DATA)

2. STANDARD HANDBOOK FOR ELECTRICAL ENGINEERS, FINK AND BEATY ELEVENTH EDITION, CHAPTER 18 (DEMAND AND DIVERSITY FACTORS)

3. THE FOLLOWING ASSUMPTIONS WERE MADE: $\mathrm{PF}=.95$, EFF $=.95$ 


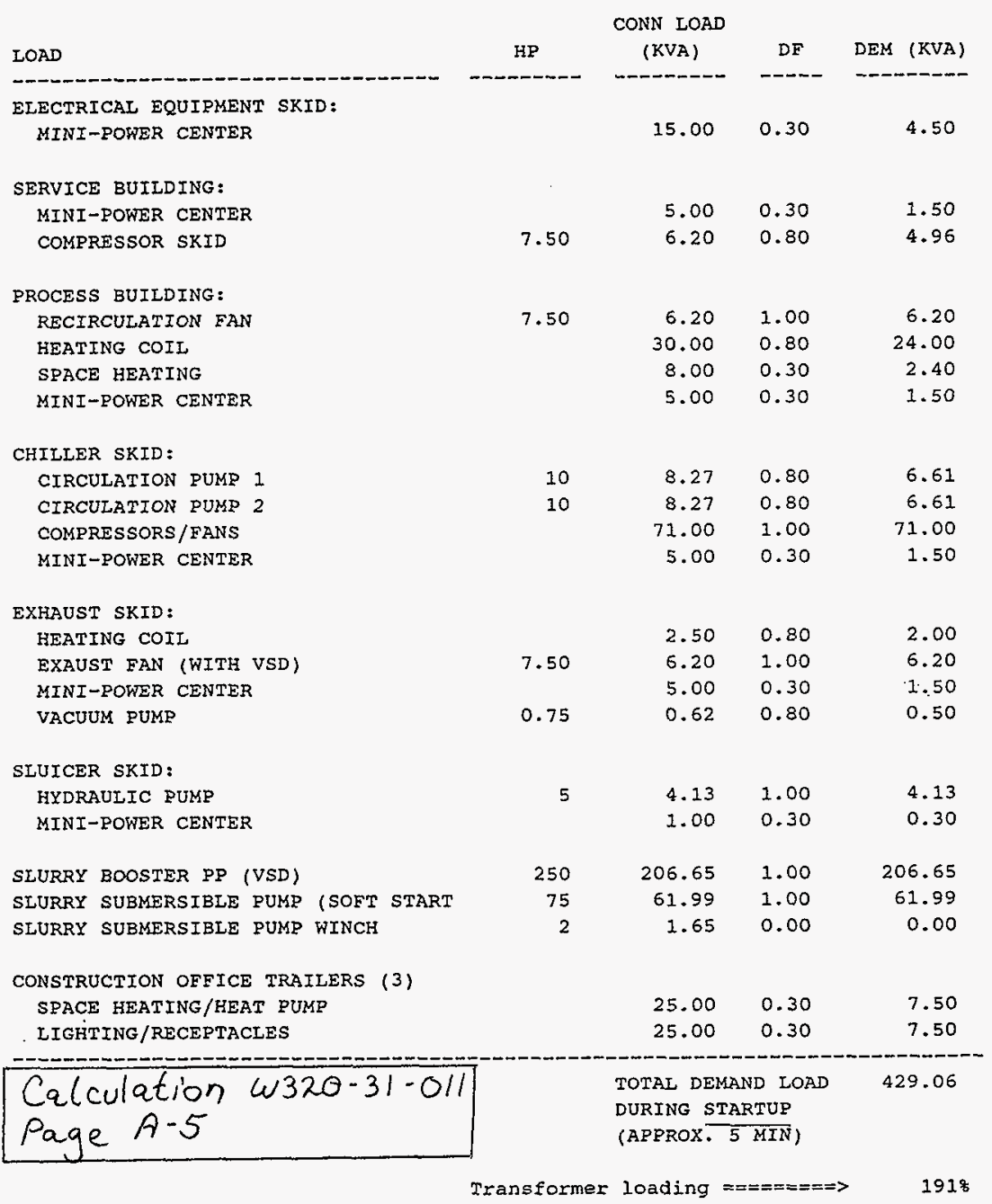

REFERENCES :

1. INDUSTRIAL POWER SYSTEMS HANDBOOK, BEEMAN, FIRST ED. CHAPTER 17 (LOAD AND COST ESTIMATING DATA)

2. STANDARD HANDBOOK FOR ELECTRICAL ENGINEERS, FINK AND BEATY ELEVENTH EDITION, CHAPTER 18 (DEMAND AND DIVERSITY FACTORS)

3. THE FOLLOWING ASSUMPTIONS WERE MADE: $\mathrm{PF}=.95, \mathrm{EFF}=.95$ 


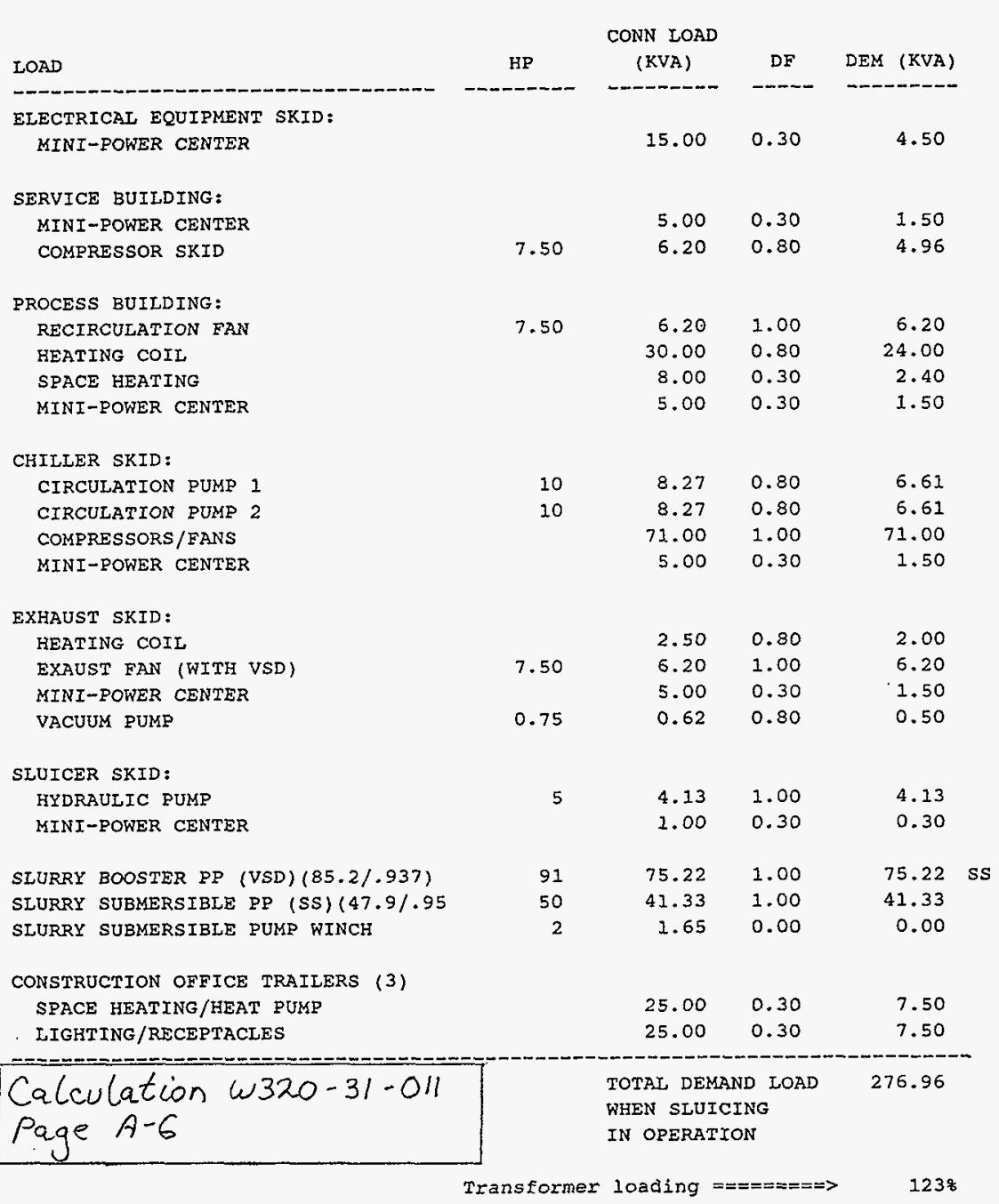

\section{REFERENCES :}

1. INDUSTRIAI POWER SYSTEMS HANDBOOK, BEEMAN, FIRST ED. CHAPTER 17 (LOAD AND COST ESTIMATING DATA)

2. STANDARD HANDBOOK FOR ELECTRICAI ENGINEERS, FINK AND BEATY ELEVENTH EDITION, CHAPTER 18 (DEMAND AND DIVERSITY FACTORS)

3. THE FOLLOWING ASSUMPTIONS WERE MADE: PF $=.95$, EFF $=.95$ 
Calculation w320-31-011

Page $A-7$

Jransformez Namb Plate Daja

KUA: 225 RISE $65^{\circ} \mathrm{C}$

SER: $95 J 877059$

STYLE: VSEES14LTN

HV: 13800 LW: 480 Y227 TAP:

HV:AL LV:AL L L 14490

HV:BIL: 95 LU BIL: 30

2. 14145

3. 13800

MFG. DATE: JUN. $q 5$

4. 13455

5..13110

IMREDRNCE 3.19

GAL.0IL 148

WT. LBS 3210

Class: $D A$ 60 Hert2 THREE PHASE

$B \triangle B$ CANE

323.6949 


\section{INMERTRON}

A-C DRIVES

\section{SPECIFICATIONS}

\section{Controller Ratings}

\begin{tabular}{|c|c|c|c|c|c|}
\hline $\begin{array}{l}\text { Controller } \\
\text { Mhodel } \\
\text { Number }\end{array}$ & $\begin{array}{l}\text { HPJKW } \\
\text { Rating(1) }\end{array}$ & $\begin{array}{c}\text { Input } \\
\text { volitage }\end{array}$ & $\begin{array}{l}\text { Input } \\
\text { KVA }\end{array}$ & $\begin{array}{l}\text { Input } \\
\text { Amps }\end{array}$ & $\begin{array}{l}\text { Maximum } \\
\text { Output } \\
\text { Amps }\end{array}$ \\
\hline $8 E 01125$ & $125 \mathrm{HP}$ & 460 & 131 & 160 & $150^{(2)}$ \\
\hline 8ED1150 & $150 \mathrm{HP}$ & $\overline{460}$ & $\overline{144}$ & 200 & $180^{(2)}$ \\
\hline $8 E D 1200$ & $200 \mathrm{HP}$ & 460 & 192 & 255 & $240^{(2)}$ \\
\hline $8 E D 1250$ & $250 \mathrm{HP}$ & 460 & 240 & 325 & $300^{(2)}$ \\
\hline BED1300 & $300 \mathrm{HP}$ & 460 & 288 & 380 & $360^{(2)}$ \\
\hline $8 E D 1350$ & $350 \mathrm{HP}$ & 460 & 336 & 435 & $420(3)$ \\
\hline $8 E 01400$ & $400 \mathrm{HP}$ & 460 & 394 & 490 & $480^{(4)}$ \\
\hline- & $500-1200 \mathrm{KP}$ & \multicolumn{4}{|c|}{ Contact Reliance Electric } \\
\hline $8 E D 3150$ & $90 \mathrm{KW}$ & 380 & 117 & 200 & $880^{22}$ \\
\hline $8 E 03200$ & $125 \mathrm{~kW}$ & 380 & 159 & 255 & $240^{(2)}$ \\
\hline $8 E 03250$ & $150 \mathrm{~kW}$ & 380 & 192 & 325 & $300^{(2)}$ \\
\hline $8 E 03300$ & $185 \mathrm{KW}$ & 380 & 243 & 380 & $360(2)$ \\
\hline $8 E D 3350$ & $220 \mathrm{KW}$ & 380 & 279 & 435 & $420^{(3)}$ \\
\hline $8 E D 3400$ & $250 \mathrm{KW}$ & 380 & 324 & 490 & $480(4)$ \\
\hline
\end{tabular}

\section{SERVICE CONDITIONS}

Elevation .............. to $3300 \mathrm{ft}$ (1000 meters $)$ Ambient Temperature Range:

- Cabinet .......... $10^{\circ} \mathrm{C}$ to $40^{\circ} \mathrm{C}\left(14^{\circ} \mathrm{F}\right.$ to $\left.104^{\circ} \mathrm{F}\right)$

- Chassis ........... $-10^{\circ} \mathrm{C}$ to $55^{\circ} \mathrm{C}\left(14^{\circ} \mathrm{r}\right.$ to $\left.131^{\circ} \mathrm{F}\right)$

Atmosphere ....... Non-Condensing Relative Humidity 5 to $95 \%$

A-C Line Voltage Variation plus or minus $10 \%$

A-C Line Frequency

Variation .......... $50 \mathrm{~Hz} \pm 2 \mathrm{~Hz}$ or $60 \mathrm{~Hz} \pm 2 \mathrm{~Hz}$

Storage Temperature ............ $-40^{\circ} \mathrm{C}$ to $65^{\circ} \mathrm{C}$

$\left(-40^{\circ} \mathrm{F}\right.$ to $\left.149^{\circ} \mathrm{F}\right)$

(1) NEMA $B$ induction motor reting onty. Application bast and speed requirements must be corsidered to property size the moier and controller.

(2) Load rating is $100 \%$ continuous, of $150 \%$ for $i$ minute.

(3) Load rating is $100 \%$ continuous, or $125 \%$ lot 1 minule.

(4) Load rating is $100 \%$ continuous, or $110 \%$ tor 1 minute.

(5) \% of base speed with proper adjusiment of stip compensation.

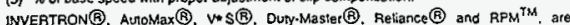
tradenarks of Reliance Electric Company of ins swosidiaries.

IBM(B) is a registered trademark of hiternational Business Machines Corp.

(C) Febrary. 1993 Reliance Elecric Company

\section{APPLICATION DATA}

Pulse Width Modulation (PWM) Flux regulated sine wave

Displacement Power Factor ................. 0.96

Long Term Frequency Stability:

With Digital Keypad ......... .01\% of base frequency

With Optional Analog

Input $\ldots \ldots \ldots \ldots \ldots \ldots \ldots \ldots$ of base frequency Communications ...............RS232/RS422, via any ASCII terminal (optional)

Speed Reference inputs....... 0-10 VDC [+/-1 Analog $0-20 \mathrm{~mA}$ Analog 4-20 $\mathrm{mA}$ Analog $2 \mathrm{KHz}-1 \mathrm{MHz}$ (Pulse Train)

Control Inputs .............24 VDC Standard 115 VAC Optional

\section{PROGRAMMABLE ADJUSTMENTS}

Acceleration Time .......... Linear 1 to $3277 \mathrm{sec}$. Deceleration Time .......... Linear 1 to 3277 sec. Voits/Hertz ..................... Selectable Minimum Frequency ................ $0 \mathrm{~Hz}$ Maximum Frequency ............... $200 \mathrm{~Hz}$ Slip Compensation ${ }^{(5)} \ldots \ldots \ldots .0 .5 \%$ Speed Regulation Current Limit . . . . . . . . . . . . . . . . . 20\%-i00\% Manual Torque Boost ..... 0 to $30 \%$ of Rated Voltage

\section{DIMENSIONS AND WEICHTS}

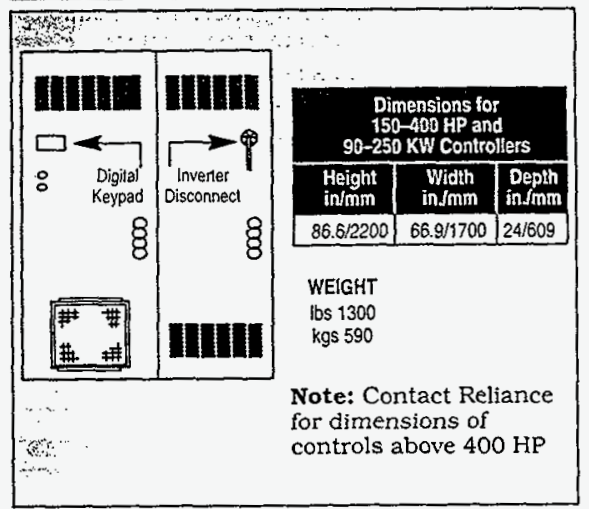




\section{TEFC}

\section{E•MASTER • THREE-PHASE}

\begin{tabular}{|c|c|c|c|c|c|c|c|c|c|c|c|}
\hline \multirow[b]{2}{*}{ HP } & \multirow[b]{2}{*}{$\begin{array}{l}\text { Synch } \\
\text { RPM }\end{array}$} & \multirow[b]{2}{*}{$\begin{array}{c}\text { Frame } \\
\text { Size }\end{array}$} & \multirow{2}{*}{$\begin{array}{l}\text { Full } \\
\text { Load } \\
\text { RPM }\end{array}$} & \multicolumn{2}{|c|}{ Amps $460 \mathrm{~V}$} & \multicolumn{3}{|c|}{ Torque (Ft-Lbs) } & \multicolumn{2}{|c|}{ Full Load } & \multirow[b]{2}{*}{$\begin{array}{c}\text { NEMA } \\
\text { Code }\end{array}$} \\
\hline & & & & $\begin{array}{l}\text { Full } \\
\text { Load }\end{array}$ & $\begin{array}{l}\text { Locked } \\
\text { Rotor }\end{array}$ & $\begin{array}{l}\text { Full } \\
\text { Load }\end{array}$ & $\begin{array}{l}\text { Break } \\
\text { Down }\end{array}$ & $\begin{array}{c}\text { Locked } \\
\text { Rotor }\end{array}$ & $\begin{array}{c}\text { Nom. } \\
\text { Eff. }\end{array}$ & $\begin{array}{l}\text { Power } \\
\text { Factor }\end{array}$ & \\
\hline 25 & $\begin{array}{r}3600 \\
1800 \\
1200 \\
900 \\
\end{array}$ & $\begin{array}{l}284 \mathrm{TS} \\
284 \mathrm{~T} \\
324 \mathrm{~T} \\
326 \mathrm{~T} \\
\end{array}$ & $\begin{array}{r}3533 \\
1766 \\
1178 \\
879 \\
\end{array}$ & $\begin{array}{l}29.3 \\
31.0 \\
32.2 \\
33.7 \\
\end{array}$ & $\begin{array}{l}181 \\
180 \\
174 \\
177 \\
\end{array}$ & $\begin{array}{c}37.2 \\
74.3 \\
111 \\
149 \\
\end{array}$ & $\begin{array}{r}98.0 \\
180 \\
250 \\
345 \\
\end{array}$ & $\begin{array}{r}62.0 \\
149 \\
205 \\
230 \\
\end{array}$ & $\begin{array}{l}91.0 \\
92.4 \\
91.7 \\
90.2 \\
\end{array}$ & $\begin{array}{l}87.5 \\
81.7 \\
79.1 \\
76.6 \\
\end{array}$ & $\begin{array}{l}G \\
G \\
F \\
G\end{array}$ \\
\hline 30 & $\begin{array}{r}3600 \\
1800 \\
1200 \\
900 \\
\end{array}$ & $\begin{array}{l}286 \mathrm{TS} \\
286 \mathrm{~T} \\
326 \mathrm{~T} \\
364 \mathrm{~T} \\
\end{array}$ & $\begin{array}{r}3529 \\
1764 \\
1178 \\
879 \\
\end{array}$ & $\begin{array}{l}35.7 \\
37.0 \\
39.2 \\
39.9 \\
\end{array}$ & $\begin{array}{l}217 \\
216 \\
214 \\
210 \\
\end{array}$ & $\begin{array}{c}44.6 \\
89.3 \\
134 \\
179 \\
\end{array}$ & $\begin{array}{r}120 \\
210 \\
305 \\
422 \\
\end{array}$ & $\begin{array}{l}75.0 \\
175 \\
255 \\
255 \\
\end{array}$ & $\begin{array}{l}91.0 \\
92.4 \\
91.7 \\
91.0 \\
\end{array}$ & $\begin{array}{l}86.2 \\
82.1 \\
78.0 \\
77.4 \\
\end{array}$ & $\begin{array}{l}G \\
G \\
G \\
F\end{array}$ \\
\hline 40 & $\begin{array}{r}3600 \\
1800 \\
1200 \\
900 \\
\end{array}$ & $\begin{array}{l}324 \mathrm{TS} \\
324 \mathrm{~T} \\
364 \mathrm{~T} \\
365 \mathrm{~T} \\
\end{array}$ & $\begin{array}{r}3551 \\
1773 \\
1182 \\
880 \\
\end{array}$ & $\begin{array}{l}48.4 \\
49.5 \\
49.4 \\
53.1 \\
\end{array}$ & $\begin{array}{l}280 \\
282 \\
289 \\
288 \\
\end{array}$ & $\begin{array}{r}59.2 \\
119 \\
178 \\
239 \\
\end{array}$ & $\begin{array}{l}144 \\
281 \\
415 \\
585 \\
\end{array}$ & $\begin{array}{r}4.0 \\
205 \\
335 \\
365 \\
\end{array}$ & $\begin{array}{l}91.7 \\
93.0 \\
93.0 \\
91.0 \\
\end{array}$ & $\begin{array}{l}84.0 \\
81.1 \\
81.2 \\
76.9 \\
\end{array}$ & $\begin{array}{l}F \\
G \\
G \\
G\end{array}$ \\
\hline 50 & $\begin{array}{r}3600 \\
1800 \\
1200 \\
900 \\
\end{array}$ & $\begin{array}{l}326 \mathrm{TS} \\
326 \mathrm{~T} \\
365 \mathrm{~T} \\
404 \mathrm{~T} \\
\end{array}$ & $\begin{array}{r}3550 \\
1769 \\
1181 \\
880 \\
\end{array}$ & $\begin{array}{l}59.3 \\
61.9 \\
61.9 \\
65.0 \\
\end{array}$ & $\begin{array}{l}357 \\
340 \\
362 \\
362 \\
\end{array}$ & $\begin{array}{c}74.0 \\
148 \\
222 \\
298 \\
\end{array}$ & $\begin{array}{l}183 \\
340 \\
520 \\
775 \\
\end{array}$ & $\begin{array}{l}130 \\
240 \\
425 \\
460 \\
\end{array}$ & $\begin{array}{l}92.4 \\
93.0 \\
93.0 \\
91.7 \\
\end{array}$ & $\begin{array}{l}84.9 \\
81.1 \\
81.1 \\
78.5 \\
\end{array}$ & $\begin{array}{l}G \\
F \\
G \\
G\end{array}$ \\
\hline 60 & $\begin{array}{r}3600 \\
1800 \\
1200 \\
900 \\
\end{array}$ & $\begin{array}{l}364 \mathrm{TS} \\
364 \mathrm{~T} \\
404 \mathrm{~T} \\
405 \mathrm{~T} \\
\end{array}$ & $\begin{array}{r}3556 \\
1778 \\
1182 \\
880 \\
\end{array}$ & $\begin{array}{l}69.8 \\
73.4 \\
70.5 \\
77.5 \\
\end{array}$ & $\begin{array}{l}433 \\
428 \\
415 \\
420 \\
\end{array}$ & $\begin{array}{r}88.6 \\
177 \\
267 \\
358 \\
\end{array}$ & $\begin{array}{l}250 \\
445 \\
655 \\
860 \\
\end{array}$ & $\begin{array}{r}154 \\
300 \\
410 \\
535 \\
\end{array}$ & $\begin{array}{l}93.0 \\
93.6 \\
93.6 \\
91.7 \\
\end{array}$ & $\begin{array}{l}86.3 \\
81.5 \\
85.1 \\
78.9 \\
\end{array}$ & $\begin{array}{l}G \\
G \\
F \\
F\end{array}$ \\
\hline 75 & $\begin{array}{r}3600 \\
1800 \\
1200 \\
900 \\
\end{array}$ & $\begin{array}{l}365 T S \\
365 T \\
405 T \\
444 T \\
\end{array}$ & $\begin{array}{r}3551 \\
1779 \\
1181 \\
886 \\
\end{array}$ & $\begin{array}{l}84.2 \\
87.0 \\
87.9 \\
94.0\end{array}$ & $\begin{array}{l}538 \\
542 \\
542 \\
542 \\
\end{array}$ & $\begin{array}{l}111 \\
221 \\
333 \\
444 \\
\end{array}$ & $\begin{array}{r}307 \\
555 \\
815 \\
1025 \\
\end{array}$ & $\begin{array}{l}186 \\
400 \\
530 \\
600 \\
\end{array}$ & $\begin{array}{l}93.0 \\
94.1 \\
93.6 \\
93.6\end{array}$ & $\begin{array}{l}89.7 \\
85.5 \\
85.2 \\
79.7 \\
\end{array}$ & $\begin{array}{l}G \\
G \\
G \\
G\end{array}$ \\
\hline 100 & $\begin{array}{r}3600 \\
1800 \\
1200 \\
900 \\
\end{array}$ & $\begin{array}{l}405 T S \\
405 T \\
444 T \\
445 T \\
\end{array}$ & $\begin{array}{r}3567 \\
1780 \\
1187 \\
886 \\
\end{array}$ & $\begin{array}{l}115 \\
116 \\
121 \\
123 \\
\end{array}$ & $\begin{array}{l}725 \\
710 \\
710 \\
695 \\
\end{array}$ & $\begin{array}{l}147 \\
145 \\
443 \\
593 \\
\end{array}$ & $\begin{array}{r}411 \\
735 \\
1115 \\
1335 \\
\end{array}$ & $\begin{array}{l}220 \\
475 \\
725 \\
825 \\
\end{array}$ & $\begin{array}{l}93.6 \\
94.5 \\
94.1 \\
93.6 \\
\end{array}$ & $\begin{array}{l}86.9 \\
85.3 \\
82.2 \\
81.0 \\
\end{array}$ & $\begin{array}{l}G \\
G \\
G \\
F\end{array}$ \\
\hline 125 & $\begin{array}{r}3600 \\
1800 \\
1200 \\
900 \\
\end{array}$ & $\begin{array}{l}44 \mathrm{TS} \\
444 \mathrm{~T} \\
445 \mathrm{~T} \\
445 \mathrm{~T} \\
\end{array}$ & $\begin{array}{r}3567 \\
1781 \\
1185 \\
885 \\
\end{array}$ & $\begin{array}{r}144 \\
140 \\
149 \\
154 \\
\end{array}$ & $\begin{array}{l}885 \\
902 \\
860 \\
885 \\
\end{array}$ & $\begin{array}{l}184 \\
369 \\
554 \\
741 \\
\end{array}$ & $\begin{array}{r}520 \\
930 \\
1303 \\
1700 \\
\end{array}$ & $\begin{array}{r}246 \\
595 \\
860 \\
1044 \\
\end{array}$ & $\begin{array}{l}94.5 \\
94.5 \\
94.1 \\
94.1 \\
\end{array}$ & $\begin{array}{l}85.7 \\
88.4 \\
83.3 \\
80.8 \\
\end{array}$ & $\begin{array}{l}G \\
G \\
F \\
G\end{array}$ \\
\hline 150 & $\begin{array}{r}3600 \\
1800 \\
1200 \\
900 \\
\end{array}$ & $\begin{array}{l}445 T S \\
445 T \\
445 T \\
447 T\end{array}$ & $\begin{array}{r}3567 \\
1785 \\
1186 \\
886 \\
\end{array}$ & $\begin{array}{l}168 \\
170 \\
176 \\
177 \\
\end{array}$ & $\begin{array}{l}1075 \\
1070 \\
1085 \\
1070 \\
\end{array}$ & $\begin{array}{l}221 \\
441 \\
664 \\
889 \\
\end{array}$ & $\begin{array}{r}600 \\
1120 \\
1628 \\
2085 \\
\end{array}$ & $\begin{array}{r}310 \\
650 \\
1097 \\
1295\end{array}$ & $\begin{array}{l}94.5 \\
95.0 \\
95.0 \\
94.5 \\
\end{array}$ & $\begin{array}{l}88.1 \\
86.8 \\
84.1 \\
83.8 \\
\end{array}$ & $\begin{array}{l}G \\
G \\
G \\
G\end{array}$ \\
\hline 200 & $\begin{array}{l}3600 \\
1800 \\
1200\end{array}$ & $\begin{array}{l}445 T S \\
445 T \\
447 T\end{array}$ & $\begin{array}{l}3573 \\
1784 \\
1188 \\
\end{array}$ & $\begin{array}{l}225 \\
225 \\
230\end{array}$ & $\begin{array}{l}1450 \\
1440 \\
1395\end{array}$ & $\begin{array}{l}294 \\
589 \\
884 \\
\end{array}$ & $\begin{array}{r}810 \\
1540 \\
2150 \\
\end{array}$ & $\begin{array}{r}445 \\
965 \\
1295 \\
\end{array}$ & $\begin{array}{l}95.0 \\
95.0 \\
95.0 \\
\end{array}$ & $\begin{array}{l}87.5 \\
87.3 \\
85.6 \\
\end{array}$ & $\begin{array}{l}G \\
G \\
F\end{array}$ \\
\hline 250 & $\begin{array}{l}3600 \\
1800 \\
1200 \\
\end{array}$ & $\begin{array}{l}447 \mathrm{TS} \\
447 \mathrm{~T} \\
449 \mathrm{~T} \\
\end{array}$ & $\begin{array}{l}3570 \\
1784 \\
1187 \\
\end{array}$ & $\begin{array}{r}278 \\
279 \\
280 \\
\end{array}$ & $\begin{array}{r}1825 \\
1820 \\
1825 \\
\end{array}$ & $\begin{array}{r}368 \\
736 \\
1105 \\
\end{array}$ & $\begin{array}{l}1025 \\
1900 \\
2800 \\
\end{array}$ & $\begin{array}{r}600 \\
1140 \\
1660 \\
\end{array}$ & $\begin{array}{l}95.0 \\
95.4 \\
95.4 \\
\end{array}$ & $\begin{array}{l}88.5 \\
87.9 \\
87.4 \\
\end{array}$ & $\begin{array}{l}G \\
G \\
G\end{array}$ \\
\hline 300 & 1800 & 449TS & 1783 & 335 & 2115 & 883 & 2356 & 1309 & 95.4 & 87.6 & $G$ \\
\hline
\end{tabular}

HNF-2487, Rev. 0

Page E-47 
HNF-2487, Rev. 0

Page E-48
Chapter

$\therefore:$

The following table shows the maximum heat dissipation at rated current for the controllers.

Figure 5.1

All soft start losses shall

Maximum Heat Dissipation

be as heat loss.

\begin{tabular}{|c|c|c|c|c|c|}
\hline $\begin{array}{c}\text { SMC PLUS Controller } \\
\text { Current Size }\end{array}$ & $24 \mathrm{~A}$ & $35 \mathrm{~A}$ & $54 \mathrm{~A}$ & $97 \mathrm{~A}$ & $135 \mathrm{~A}$ \\
\hline Maximum Watts & 110 & 150 & 200 & 285 & 410 \\
\hline
\end{tabular}

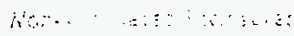

For NEMA Type 12 or non-ventilated enclosures, it is recommended that a by-pass contactor be used. This will allow the controller to start the motor. Once up to full voltage, the controller would be by-passed. Note that the features of the controller would no longer be available. See Page

Per NEC Table 430-150, a 75 fipmotor draws 96 amperes: $K V A$ total $=V I \sqrt{3}$. $K V A$ total $=(460$ volts $)(96$ amps $) \sqrt{3}=76,485.12 \cup A$

$$
\begin{aligned}
& E f f=\frac{\text { output }}{\text { Input }} \\
& E f f=\frac{76,485.12 \vee A}{76,485.12+285 \vee A}
\end{aligned}
$$

$E f f=.996$ or $99.6 \%$ eff
The controller is convection cooled except for the 97 A and 135A fan cooled unit. It is important to locate the controller in a position which allows free air flow vertically through the power module. The controller must be mounted with heatsink fins in a verticle plane and have a minimum of 6 inches free space above and below the controller. See Figures 5.2, 5.3 and 5.4.

Figure 5.2

Dimension Drawings (24A Controller)
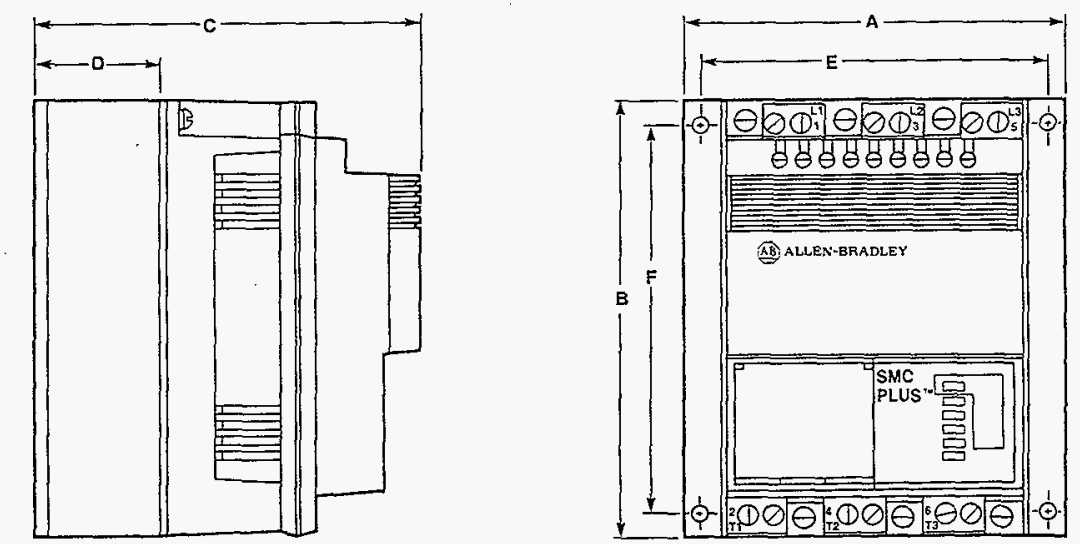

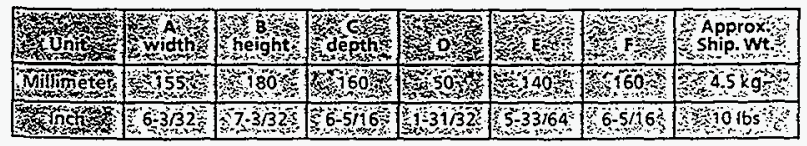

All dimensions are approximate and are not to be used for construction purposes. Refer to nearest Sales Office or the Sales Department at Milwaukee. Wisconsin, for complete dimension drawings. 


\section{THE FOCIKBESTOS COMPANY}

20 Enćley Perk Roso, P.0. 80x \{102, East Branby, CT 08028.1102 USA (203) 683.6300

Dar Larson

Hanford Nuclear

FAX: (S09) 373-3343
June $21 ; 1995$

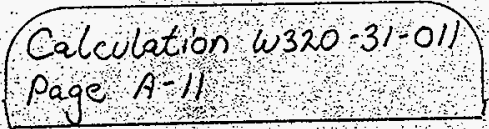

In response to your roquest for an ampacity conrection factor for anpacities calculated using the Neher McGTath Method (similar to that shown in NEC Table $310-16$ ), the following factor may be tised:

Formula derived from IEEE S-135 Vol 1 , equation 5 (See Attachment)

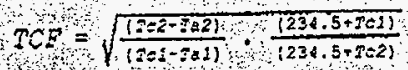

If you need ary additional information, plesse let me know.

Sincerely,

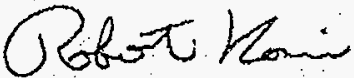

Robert Konrik

Applicetion/Design Engineer

RlK/ja

E005095

Te1 maximum conductor temperature used to defemine base ampacity

$T a 1=$ ambient tentseratito used to determine base ampacity

$\mathrm{Tc} 2=\mathrm{m}$ maximum conductor temperture used to determine adjusted ampacity

$\mathrm{T} z \mathrm{z}=$ anbient temperature used to deternine adjusted ampacity

HNF-2487, Rev. 0

Page E-49 
Calculation w320-31-011

Given

page $A-12$

$$
\begin{aligned}
& I=\sqrt{\frac{T C-(T A+D E L T A D)}{R D C(1+Y C) R C A}} \\
& I^{\prime}=\sqrt{\frac{T_{C}^{\prime}-\left(T_{A}+D \in L T T D^{\prime}\right)}{R D C\left(T^{\prime}+C_{C}^{\prime}\right) R C A^{\prime}}}
\end{aligned}
$$

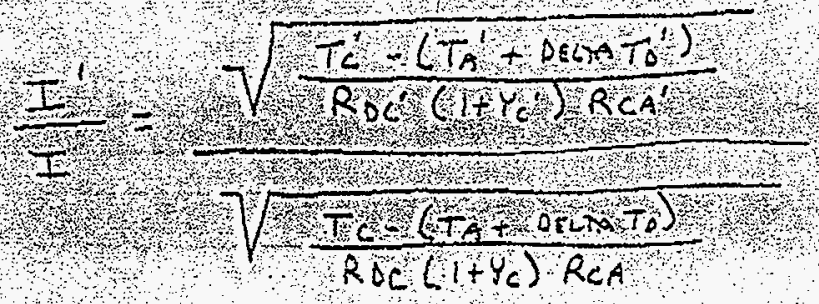

$$
\begin{aligned}
& \frac{I^{\prime}}{I}=\sqrt{\frac{T_{C}\left(-\left(T_{A}^{\prime}+\Delta B T_{A} T_{D}^{\prime}\right)\right.}{T_{C}-\left(T_{A}+\operatorname{DET} T_{D}\right)}} \times \frac{R_{D C}\left(1+Y_{C}\right) R_{C A}}{R_{B C}\left(1+Y_{C}^{\prime}\right) R_{C A^{\prime}}}
\end{aligned}
$$

HNF-2487, Rev. 0

Page E-50 
Calculation (320-31-01)

SPECIAL CASE 2

Page A-14

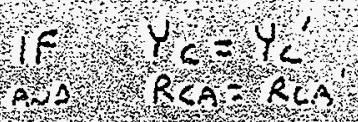

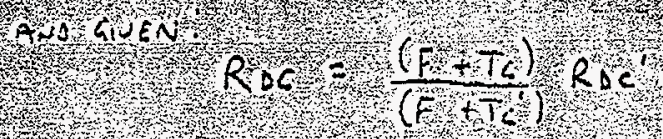

th then?

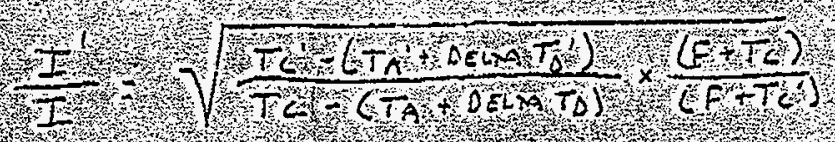

$$
\begin{aligned}
& \text { f }
\end{aligned}
$$

Titan:

$$
\frac{I}{I}=\sqrt{\frac{\left(T_{C}-T_{A}\right)}{\left(T_{C}-T_{A}\right)} \times \frac{\left(234, S+T_{C}\right)}{\left(234.5+T_{C}^{\prime}\right)}}
$$

$O R \quad\left(\right.$ Solving $\left.A_{R} T_{C}\right)$

$$
T_{G}=\left(\frac{I^{\prime}}{I}\right)^{2} \cdot\left(T_{C}-T_{A}\right) \cdot \frac{\left(234 . S+T_{C}\right)}{\left(239 . S+T_{C}\right)}+T_{A}^{\prime}
$$




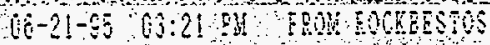

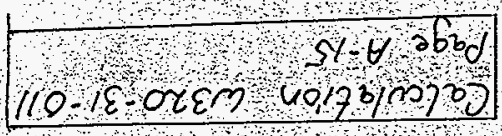

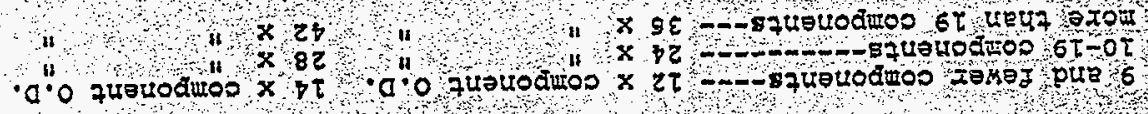

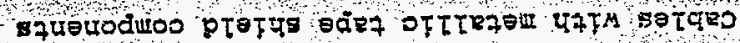

strotiodtos

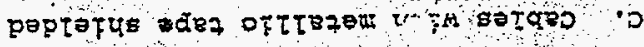

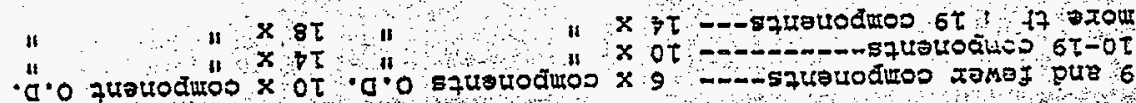
g7uotrositoo

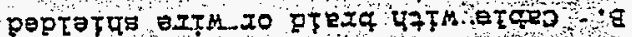

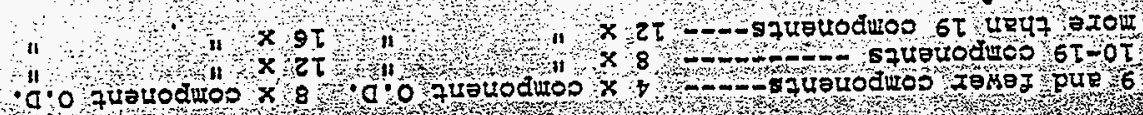

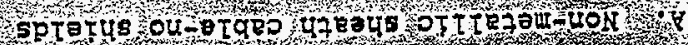

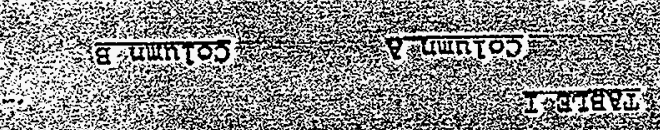

3.

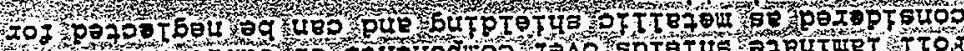
9907 oL 0 z

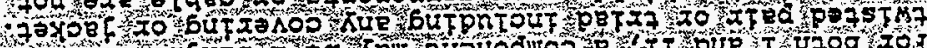

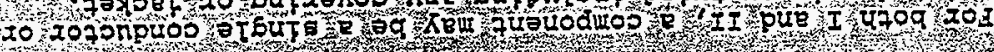

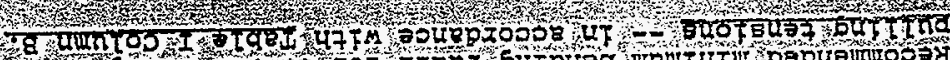

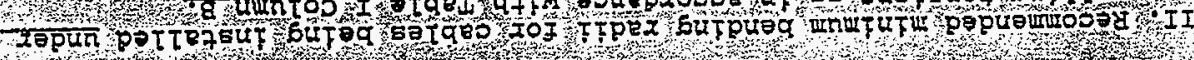

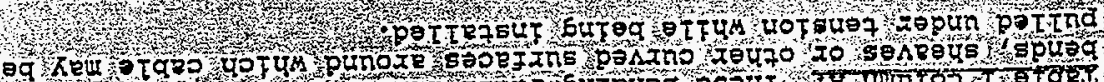

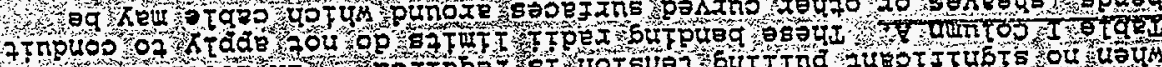

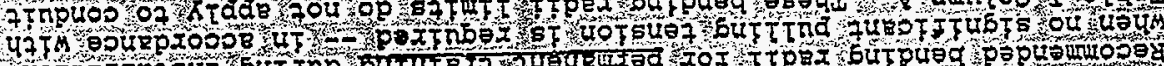

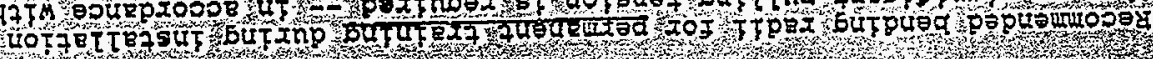

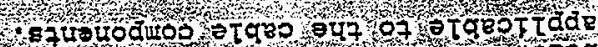

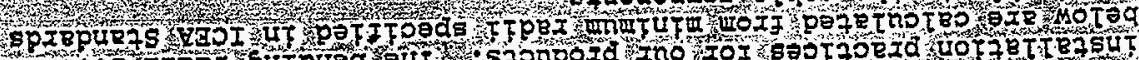

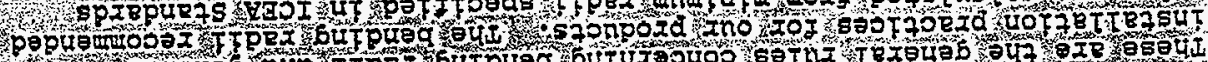

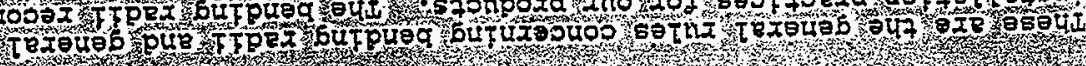

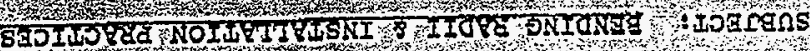


D. *cables w1th braid or wire shlelds over assembly (overall) -

$6 \times c a b l e 0.0 .10 \times c a b l e$. O.

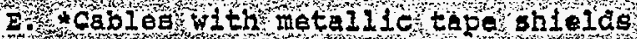
over assemb 2 y (overal1)

$8 \times \mathrm{Cable} 0 . \mathrm{D}$.

$12 \times$ cabie 0.0 . te

7. Hus (Gasder) cables o smooth

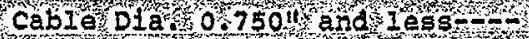

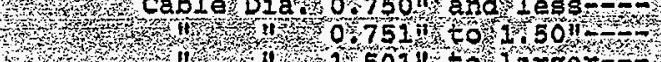

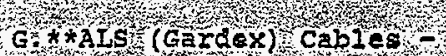

corrugated and Inter $10 \mathrm{ked}$

armored cables

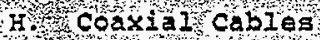

Jol composite cables

$10.0 a b 10.0 .0$

12 .

$15 \times$

$7 \mathrm{x}$ cable 0.0 .

$10 \times \operatorname{cable} 0.0$

8 x cable 0.D.

$12 \times$ cable 0 . D.

minimur tadidperditteo for largest component pithin the cable w111 abply.

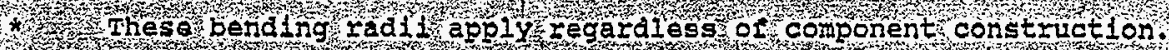

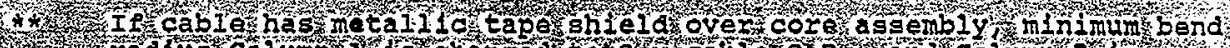

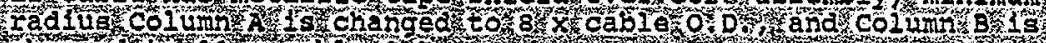

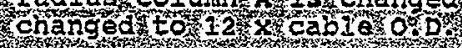

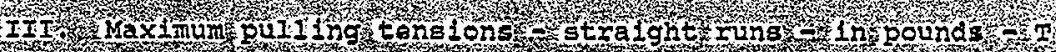

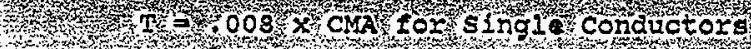

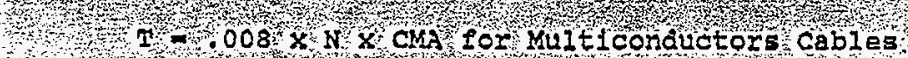

$T=008 \times N \times C M A \times R$ For MUltiple cables

CMA - ClreuIar MII Area of one Conalotor

$N$ - Number of Conductors

$x$ - Load sharing fector $=.8$

(Do not include ground whes, drain wires or control conaucter in povar cablesil

Specify calculated T or 10,000 Ibs., whlchever is less.

When calculating maximum puling tension tor cosxial cables with high strength inner conductor, the conductor is constlered as regular annealed copper of equal ajze. Recommended maximum puling tensions for our Firewall coaxial product lire has beoñ daloulated and aro 11 stadin Tabla II.

HNF-2487, Rev. 0

Calculation $1320-31-0 / 1$

Page E-54 
IV. Maximum side wall pressure - los per ft. of bend radius

coaxlal cable

solid dlalectric

200

- poam delectric.

150

Non meth 11 io sheath cables

- non shin $1 \mathrm{ded}$

1000

shielded

ALS (Gardex) smooth o corrugated

Steel Interlockedarmor

500

400

500

S11 1Cond 1 Insylated obble

300

V. Taxinun phing tens ion of III and IV above for ali product except

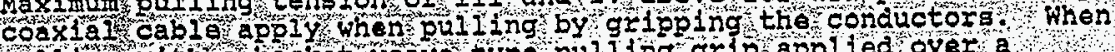

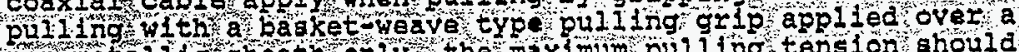
non-metalid oheathonly, the maximum puling tension ahould not excedd 1000 pounds.

Recommenat method of pulling coaxlal cable 1s by the baskat weave

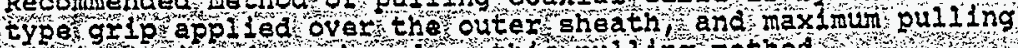
tensfons listedare based on this puling method.

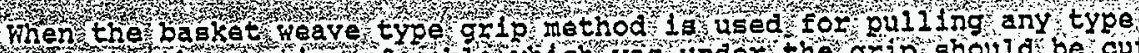
prodet the section of oblowhleh was underthe grip shoula be cut

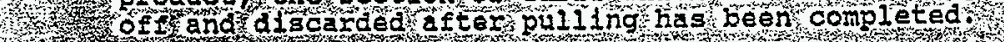

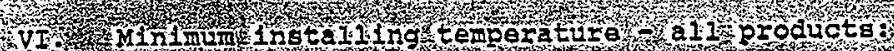

7 7.

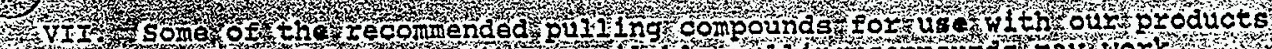

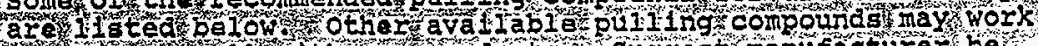

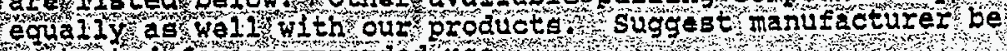

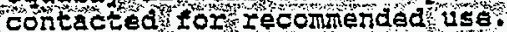

n Vololay Bentenlete whittakes y-er aAs alectro compound co. so, Plalnflela No
S1Ip-X-300-American collold polywater Iub, $A, C, G, J$ or $H$ Sinokie, ILI. a - - Polywater corp stili Water, im.

Ductiute - vtlifty Industries Flaxsoap - Sherwin-Williams Somerdale, NiJ.

Áqua-Ge1- Ideal Industries Inc.

$\therefore$ A 4 sycamore II

Wholvaterlalo may ory out and harden with age not recommended where subsequent removal of cable is anticipated.

HNF-2487, Rev. 0
Page E-55

Calculation $0320-31-077$
Page $A-170$ 
Aoregolng, such deviations ghould de revieweg ana apgrovou wy wa Teohnical tepartment.

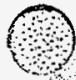

\section{TABLE II}

RECOMMENDED PUII ING TENSIONS

\section{SUO FOR COAXIAT CABLAS}

RSS

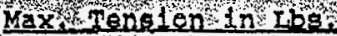

$3+6=10$

$6=100 / 2 \mathrm{I}$

$100 \mathrm{~A} / \mathrm{LB}$

$10 \mathrm{~T} / \mathrm{IT}$

$104 / \pm 3$

$105 / 2 \mathrm{E}$

$106 / \mathrm{IE}$

$107 / \pm \mathrm{E}$

$108 / \mathrm{LE}$

$109 / \mathrm{LE}$

$110 \mathrm{~A} / \mathrm{I} \mathrm{E}$

$111 / I \mathrm{E}$

$112 / I B$

$113 / 1 \mathrm{Z}$

$114 / \mathrm{LE}$

$115 / \mathrm{LE}$

$115 / 10$

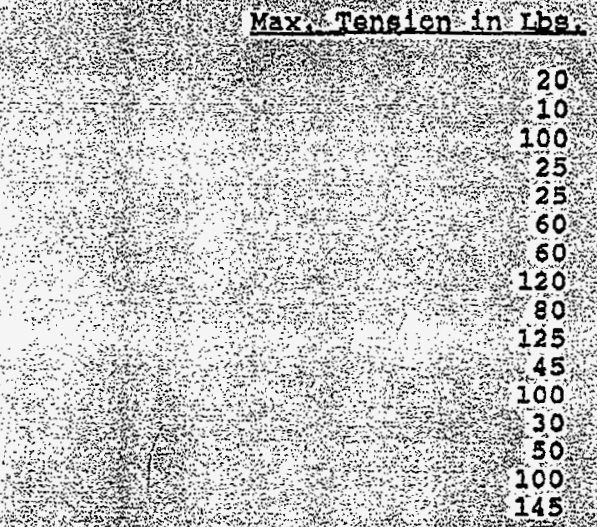

$+26 \% 200$

$0 \times 201$

202

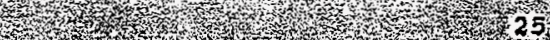

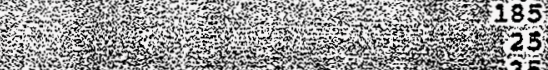

35

209

210

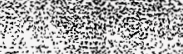

211

212

213

214

215

\section{the}

3

(a)

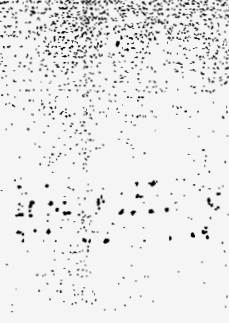

Frepared by: E. D'Agianno 


\section{INSTRUMENTATION CABLE}

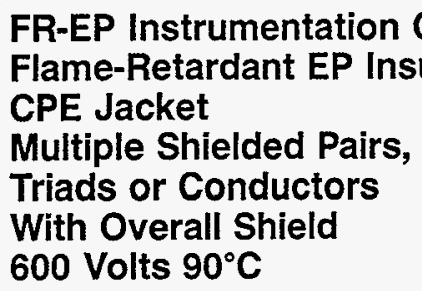

UL Type TC Flame-Retardant EP Ins
CPE Jacket
Multiple Shielded Pairs,
Triads or Conductors
With Overall Shield
600 Volts $90^{\circ} \mathrm{C}$

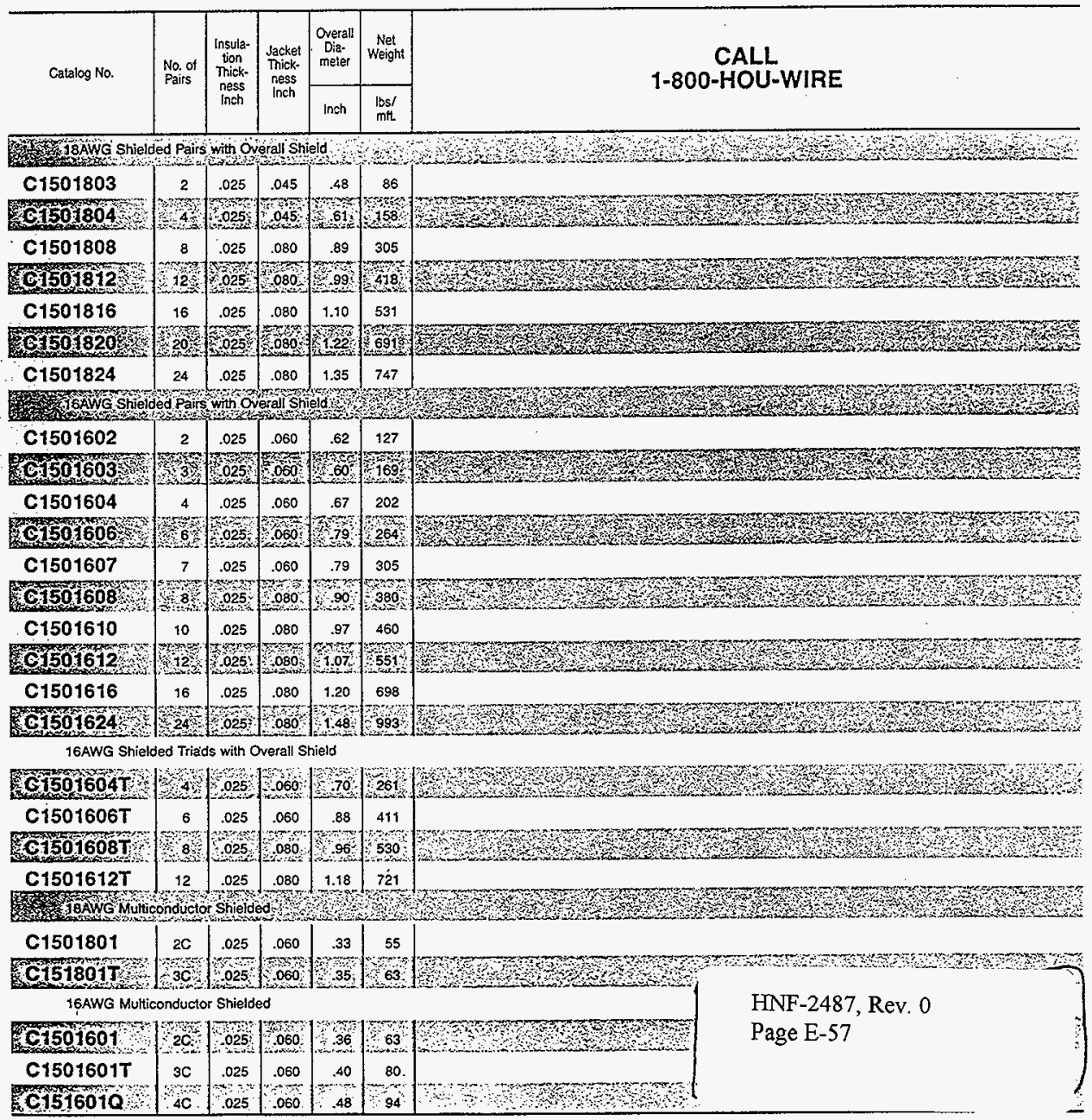

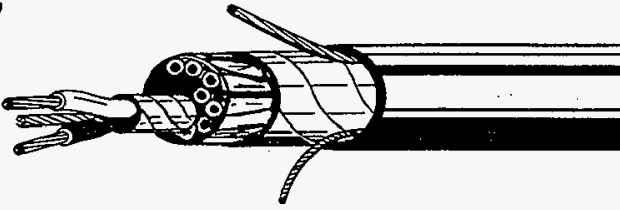




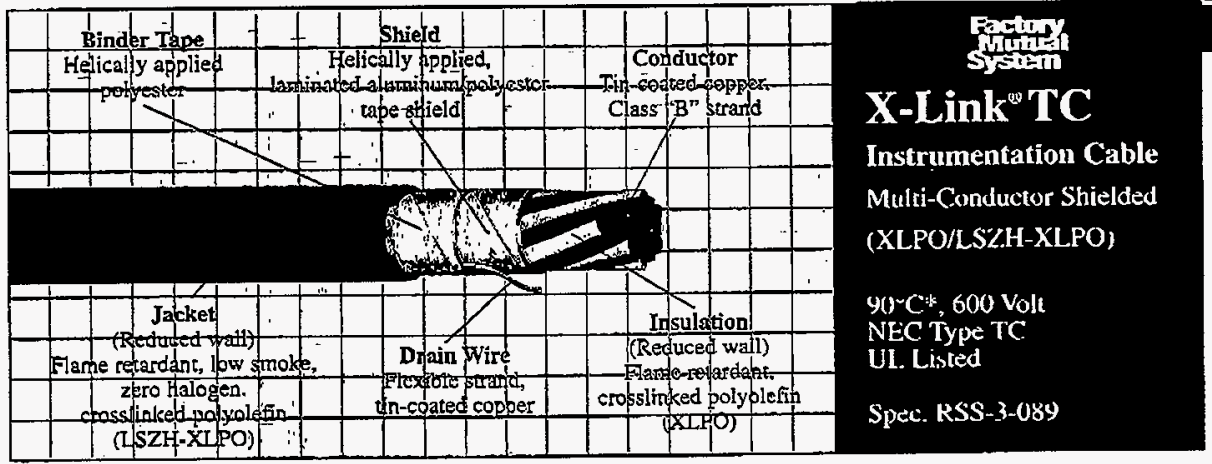

$\cdot \ddot{*}$

,ii,

.

and

Scope.

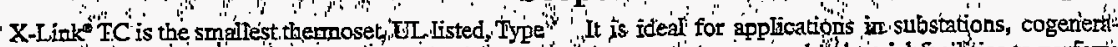

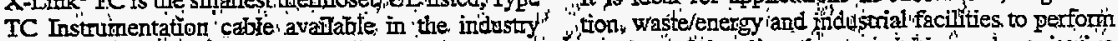

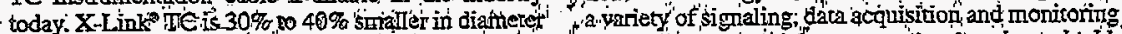

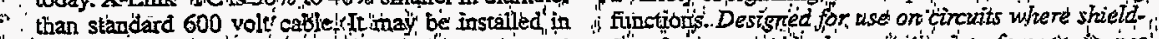

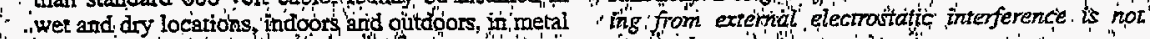

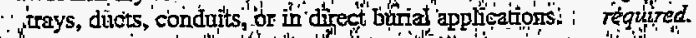

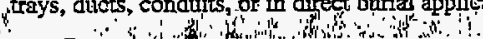

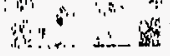

\section{Features}

- Thermoset insulation and jacket for enthenced thermal stability

- Small diameter \& light weight

- Econónical.

- More cubles per tray-or conduit

- 600 volt rating allowscables to be run in trays withuut separation ( 300 vs 600 volt)

- Flame retardane

- Flexible

- Heat, sunlight, oil and abresion resistant -

- Easily pulled (low fitiction jacker)

- Tin-coated conductors for improved terminations and corrosion resistance

- Jackets have printed sequential footage markers for improved inventory control

- Jacket surippability facilitates temination

- Reduced hulogen design -

- Low smoke jacket

- Lend free jacket

- Superior insulation and jacker moisture resistance

HNF-2487, Rev. 0

Pg $E-58$

\section{Performance Standards}

- UL listed, Type TC (UL 1277) in accordance with the NEC - Article 340

m.UL listed sunlight resistance

- Factory Murual Reserach Corp. group "I" fire rated per "Specification Test Standsrd for Cable Fire Propagation, Class 3972" $\dagger$

- Passer IEEE-383 70,000 BTU/hr vertical tray flame test and ICEA 70,000 BTU/hr vertical tray flame tesc (T-30-520)

- Single conductors pass UL VW.I flame test

- Single conductors in accordance with performance requirements of ICEA 5-66-524 and UL 44., Class XI

- Single conductors in accordance with AWM sryle No. 3502

- Jacket exceeds the requitements of UL - Class XL $/ 90^{\circ} \mathrm{C}$ and ICEA Publication T-33.655. Type II

- UL approved for $90^{\circ} \mathrm{C}$ operation in both wet and dry locations

- Cable components are in sompliance with the maximum leschable lead level required by the EPA in 40CFR. Part 261

- Meets the requirements of UL Standard No. 1685 to allow for i UL "LS" dexignation for limitedsmoke

\section{Construction}

Conductor:

Tin-conted copper conductors. Class " $\mathrm{B}$ " strand (ASTM B.8 \& B-33)

Insulation:

20 mils of flame retardant crosslinked polyolefin meating performance requirements of ICEA $5-66-524$ and UL 44 Class XL

Circuit Identification:

Colored insulation per ICEA Method 1. Table K.2, ${ }^{\text {w*** }}$

Fillers:

(Where required)

Shield System:

Helically applied aluminum/polyestcr laminated tape shield in continuous contact with flexible strund, tin-costed copper drain wire

Binder Tape: ${ }^{\text {*w* }}$

Helically applied polyester

Jaeket:

Reduced wall, black. flume recordant, low smoke, zero halogen, crosslinked polyolsfin jacket

* Rated $90^{\circ} \mathrm{C}$ for normal operation in wel and dry locations. $130^{\circ} \mathrm{C}$ for amersency overtoad onditions, and $250^{\circ} \mathrm{C}$ for short tircuit conditions.

** Not required on 2. 3, and 4 conductor configurations

$+2 / C \# 16$ and larger 
$X-\operatorname{Link}^{\oplus} \mathrm{TC}$

Instrumentation Cable

Multi-Conductor-Shielded

(XLPO/LSZH-XLPO)

$90^{\circ} \mathrm{C} * 600$ Volt

NEC Tȳpe TC

UL Listed
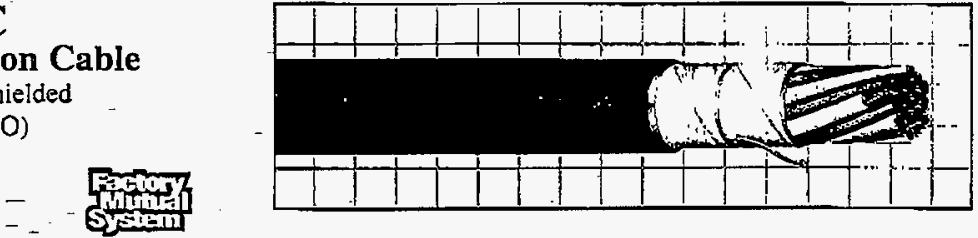

Spec. RSS-3-089

\section{AWG, 7 Strand}

\begin{tabular}{|c|c|c|c|c|c|c|c|c|c|c|}
\hline $\begin{array}{c}- \\
\text { Product } \\
\text { Code }\end{array}$ & $\begin{array}{c}\text { Number } \\
\text { of } \\
\text { Conductor: }\end{array}$ & - & $\begin{array}{c}- \\
\text { Insul } \\
\text { Thiel } \\
\text { - (Inch) }\end{array}$ & $\begin{array}{l}\text { tion } \\
\text { ness } \\
(\mathrm{mm})\end{array}$ & $\begin{array}{c}\text { Insulated } \\
\text { Conductor } \\
\text { Diameter } \\
\text { (Inch) }\end{array}$ & $\begin{array}{c}\text { Drain } \\
\text { Wire } \\
\text { Sizel } \\
\text { Stranding }\end{array}$ & $\begin{array}{c}\text { Jacket } \\
\text { Thickness } \\
\text { (Mils) }\end{array}$ & $\begin{array}{r}\text { Nor } \\
\text { Oy } \\
\text { Dia } \\
\text { (Inch) }\end{array}$ & $\begin{array}{l}\text { inal } \\
\text { all } \\
\text { eter } \\
(\mathrm{mm})\end{array}$ & $\begin{array}{c}\text { Approximate } \\
\text { Net Weight } \\
\text { (Lbs/M') }\end{array}$ \\
\hline $183-0021$ & 2 & & .020 & 5.08 & .10 & 18 AWG (16/s) & 35 & .27 & 6.86 & 4.5 \\
\hline [8,3-00031 & 3 & & .020 & 5.08 & .10 & 18 AWG (16/s) & 35 & .29 & 7.37 & 60 \\
\hline 185.0041 & 4 & & .020 & 5.08 & .10 & 18 AWG (16/s) & 35 & .31 & 7.87 & 70 \\
\hline [8.3-0.051 & 5 & - & .020 & 5.08 & .10 & 18 AWG (16/s) & 35 & .37 & 9.4 .0 & 95 \\
\hline$[83-007]$ & $7-$ & & .020 & 5.08 - & .10 & $18 \mathrm{AWG}(16 / \mathrm{s})$ & 35 & .40 & 10.16 & 120 \\
\hline $183-0091$ & $9^{-}$ & & .020 & 5.08 & .10 & 18 AWG $(16 / \mathrm{s})$ & 35 & .46 & 11.68 & 150 \\
\hline $18.3-0121$ & 12 & & .020 & 5.08 & $: 10$ & I8 AWG (16/:) & 35 & .51 & 12.95 & 190 \\
\hline เร.3-กเร। & 15 & & -.020 & 5.08 & .10 & 18 AWG (16/s) &.$\quad 45$ & .59 & 14.99 & 240 \\
\hline $183-0199$ & 19 & & .020 & 5.08 & $=.1 \overline{0}$ & I8 AWC $(16 / \mathrm{s})$ & 45 & $.6 !$ & 15.49 & 290 \\
\hline $18 \overline{5} .0271$ & 27 & & .020 & 5.08 & .10 & 18 AWG (16/s) & 45 & .73 & 18.34 & 395 \\
\hline$[83-0.77]$ & 37 & & .020 & 5.08 & .10 & I8 AWG (16/5) & 45 & .81 & 20.57 & 520 \\
\hline
\end{tabular}

\section{AWG, 7 Strand-}

\begin{tabular}{|c|c|c|c|c|c|c|c|c|c|c|}
\hline$[84-0021$ & 2 & & $.020^{\circ}$ & -5.08 & .09 & 20 AWG (10/s) & 35 & .25 & 6.35 & 35 \\
\hline$[84-003]$ & 3 & & .020 & 5.08 & .09 & 20 AWG $(10 / 3)$ & 35 & .26 & 6.60 & 45 \\
\hline 184-0041 & 4 & .. & .020 & S.os & .09 & 20 AWG (10/s) & 35 & .29 & 7.37 & 55 \\
\hline 184.0051 & s & & .020 & 5.08 & .09 & 20 AWG $(10 / \mathrm{s})$ & 35 & .33 & 8.38 & 70 \\
\hline$[84-0071$ & 7 & - & .020 & 5.08 & .09 & $20 \mathrm{AWG}(10 / \mathrm{s})$ & 35 & .36 & 9.14 & 90 \\
\hline 184-0091 & $9-$ & & .020 & 5.08 & $.09-$ & 20 AWG $(10 / 5)$ & 35 & $.4 !$ & 10.41 & 110 \\
\hline$[84.012]$ & 12 & & .020 & 5.08 & -.09 & $20 \mathrm{AWG}(10 / \mathrm{s})$ & 35 & .46 & 11.68 & 140 \\
\hline 184.0151. & 15 & & .020 & 5.08 & .09 & 20 AWG (10/s) & 35 & 51 & 12.95 & 165 \\
\hline$[84-0191$ & $15^{-}$ & & .020 & 5.08 & .09 & $20 \mathrm{AWG}(10 / \mathrm{s})$ & 45 & .55 & 13,97 & 210 \\
\hline [84-027I & 27 & & .020 & 5.08 & .09 & 20 AWG $(10 / \mathrm{s})$ & 45 & .65 & $|6.5|$ & 285 \\
\hline$[84.0371$ & 37 & _ & .020 & 5.08 & .09 & 20 AWG $(10 / \mathrm{s})$ & 45 & .72 & 18.29 & 375 \\
\hline
\end{tabular}

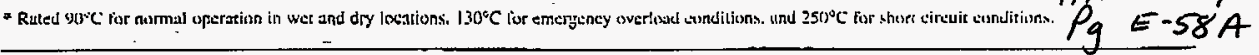




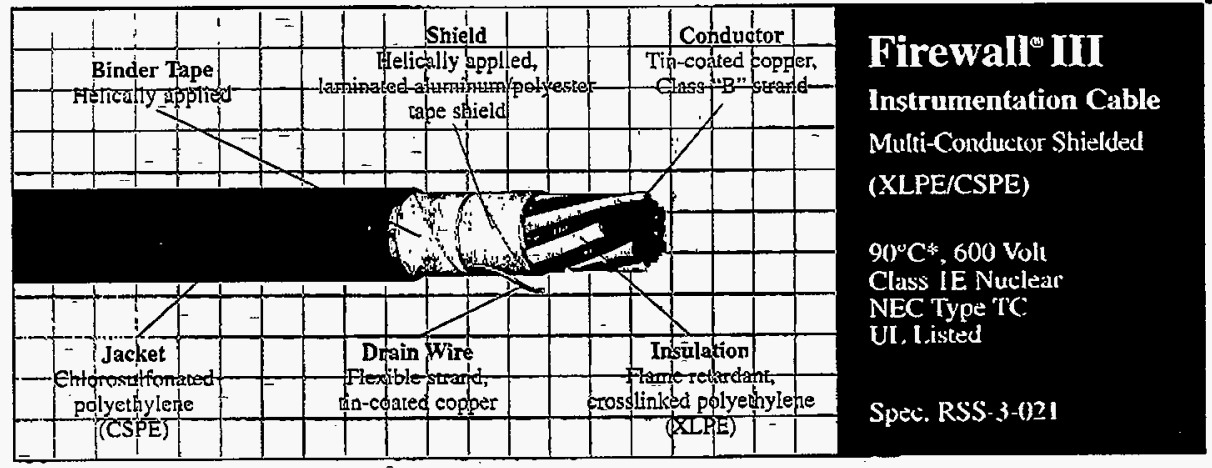

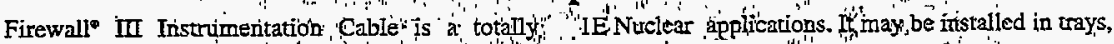

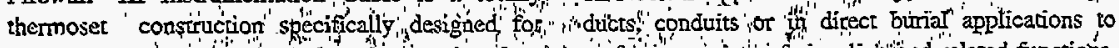
applications in power generation-plants, substations' perforni variety of signaling tand related functions.

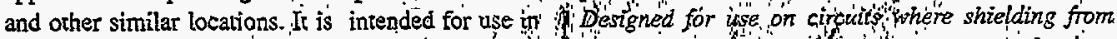

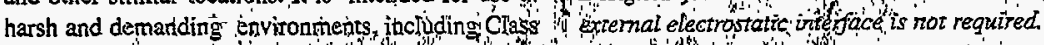

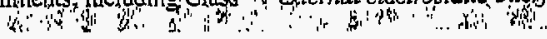

\section{Features}

- Thermoser insulation and jacket for enhunced thermal stability

- Specially tormulated insulation for superior long cerm waler resistanee

- Extremely flame recarclant

- Nuclear yūalified with a minimum 40year thermal life expectancy at $90^{\circ} \mathrm{C}$

- Rikdiution resistant (up to 200 megarads)

- Full traceabillicy

- Excellent méchanical properties

- Tin-couted copper conductors for improved terminations and corrosion resistance

- All singles pass a wet dielectric (tank) test prior to cabling to verify electrical integrity

- All jackets have printed sequential footage markers tor improved inventory control

- Eusy strippability for inctallation ease

\footnotetext{
+- Ruted $90^{\circ} \mathrm{C}$ for numbrnal upention in wet and dy

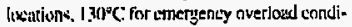
tions. and $350^{\circ} \mathrm{C}$ for shost cirtuil conditions.

5. Nat required on 2, 3 and 4 condector sonfigurallons
}

\section{Performance Standards}

- Insulation in accordance with ICEA Standard S-66-524 and UL approved for $90^{\circ} \mathrm{C}$ applications in both wer \& dry locations

- Jackets in accordance with ICEA Standard S-19-8I for heavy-duty chloposulfonated polyethylene (CSPE)

- Chass IE qualified in accordance with IEEE-383 and IEEE-323 (Rockbestos Reports QR-5804 or QR-5805)

- Cable pusses IEEE-383 70,000 BTU/hr vertical tray flame test as modified by NRC Reg. Guide 1.131

- Cable passes ICEA 210,000 BTU/hr vertical tray flame rest (Standard T.29.520)

- Single conductors pass the vertical flame tests specified in IEEE-383 para. 2.5.6 (ICEA S-19-81 Section 6.19.6) and UL VW-I

- Quality Assurance program in accordance with 10 CFR SO Appendix B

- UL. Listed Type TC for cable tray installations (UL 1277)

- In accordanee with the National Elecrical Code - Article 340 (approved for Class l, Division 2 hazardous locations)

- Cable components are in compliance with the maximum leachable lead level required by the EPA in 40 CFR. Part 261

\section{Construction}

Conductor:

Annealed, tin-conted copper. Class " $\mathrm{E}$ " sitand (ASTM B-8 \& B-33)

Insulation:

Proprictary hest, moisture and radiation resistant flame retardant crosslinked polyethylene

Circuit Identification:

Colored insulation per ICEA Mcthod I, Table K-!

Fillers:

(When required)

\section{Shield System:}

Helically applied aluminum/polyester laminated tape shield in continuous contuet with a flexible strand. tin-cualed sopper drain wire

Binder Tape: *:

Helically applied

Jacket:

Black, heavy-duty shloresulfonated polyethylene (also available in neoprene and FR-XLPE)

$$
\begin{aligned}
& \text { HNF }-2487, \text { Rev.O } \\
& \text { Pg } E-59
\end{aligned}
$$


Firewall ${ }^{\oplus}$ III

Instrumentation Cable

Multi-Conductor Shielded

(XLPE/CSPE)

$90^{\circ} \mathrm{C}^{*}, 600$ Volt

Class IE Nuclear

NEC Type TC

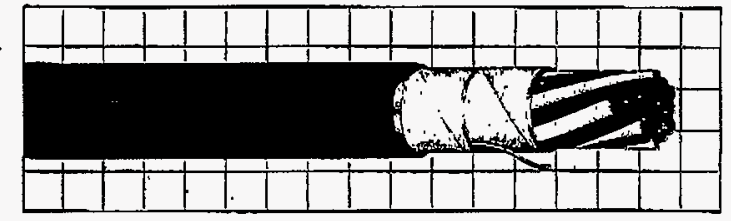

UL Listed

Spec. RSS-3-02!

\section{AWG, 7 Strand}

\begin{tabular}{|c|c|c|c|c|c|c|c|c|c|}
\hline $\begin{array}{c}\text { Product } \\
\text { Code }\end{array}$ & $\begin{array}{c}\text { Number } \\
\text { of } \\
\text { Conductors }\end{array}$ & $\begin{array}{l}\text { Insul } \\
\text { Thick } \\
\text { (Inch) }\end{array}$ & $\begin{array}{l}\text { tion } \\
(\mathrm{mm})\end{array}$ & $\begin{array}{c}\text { Insulated } \\
\text { Conductor } \\
\text { Diameter } \\
\text { (Inch) }\end{array}$ & $\begin{array}{c}\text { Drain } \\
\text { Wire } \\
\text { Size/ } \\
\text { Stranding }\end{array}$ & $\begin{array}{c}\text { Jacket } \\
\text { Thickness } \\
\text { (Mils) }\end{array}$ & $\begin{array}{r}\text { Nor } \\
\text { Ov } \\
\text { Dia } \\
\text { (Inch) }\end{array}$ & $\begin{array}{l}\text { inal } \\
\text { all } \\
\text { eter } \\
(\mathrm{mm})\end{array}$ & $\begin{array}{l}\text { Approximate } \\
\text { Net Weight } \\
\text { (Lbs/M') }\end{array}$ \\
\hline$[46-0021$ & 2 & .025 & 6.35 & .11 & 18 AWG (16/s) & 45 & .31 & 7.87 & 65 \\
\hline $146.003 !$ & 3 & .025 & .6 .35 & .11 & 18 AWG (16/s) & $4 j$ & .33 & 8.38 & 81 \\
\hline $146-3433$ & - & .025 & 6.35 & .11 & 18 AWG $(16 / \mathrm{s})$ & $45^{\circ}$ & .36 & 9.14 & 100 \\
\hline I46-3414 & 5 & -.025 & 6.35 & .11 & $18 \mathrm{AWG}(16 / \mathrm{s})$ & 45 & .42 & 10.76 & 135 \\
\hline $146-3425$ & 7 & .025 & 6.35 & .11 & $18 \mathrm{AWG}(16 / \mathrm{s})$ & 45 & .45 & 11.43 & 145 \\
\hline 140.3447 & - & .025 & 6.35 & .11 & $18 \mathrm{AWG}(16 / \mathrm{s})$ & 45 & .52 & 13.21 & 180. \\
\hline 146.3449 & 12 & -.025 & 6.35 & .11 & 18 AWG (16/s) & 60 & .61 & 15.49 & 250 \\
\hline 146.3450 & is & .025 & 6.35 & .11 & $18 \mathrm{AWG}(16 / 3)$ & 60 & .66 & 16.76 & 295 \\
\hline$|46-345|$ & $19^{-}$ & .025 & 6.35 & .11 & 18 AWG (16/s) & 60 & .70 & 17.78 & 345 \\
\hline $146-5979$ & 27 & .025 & $6.35^{\circ}$ & .11 & 18 AWG (16/s) & 60 & -.82 & 20.83 & 465 \\
\hline $146-5980$ & 37 & .025 & 6.35 & .11 & 18 AWG (16/s) & 80 & .95 & 24.13 & 650 \\
\hline
\end{tabular}

\section{AWG, 7 Strand}

\begin{tabular}{|c|c|c|c|c|c|c|c|c|c|c|}
\hline $157-0021$ & 2 & & .025 & 6.35 & .10 & 20 AWG (10/s) & 45 & .29 & 7.37 & 55 \\
\hline $157-0031$ & 3 & & .025 & 6.35 & .10 & 20 AWG $(10 / \mathrm{s})$ & 45 & .31 & 7.87 & 65 \\
\hline $157-0041$ & -4 & & .025 & 6.35 & .10 & $20 \mathrm{AWG}(10 / \mathrm{s})$ & 45 & .33 & 8.38 & 80 \\
\hline $157-0051$ & $\underline{5}$ & & $.02 s_{-}$ & 6.35 & .10 & $20 \mathrm{AWG}(10 / \mathrm{s})$ & 45 & .38 & 9.65 & 105 \\
\hline$\lfloor 57-007\rceil$ & 7 & - & $.025=$ & 6.35 & .10 & 20 AWG (10/s) & 45 & $.4 \mathrm{l}$ & 10.41 & 110 \\
\hline I57.0091 & 9 & & .025 & 6.35 & .10 & 20 AWG (10/5) & 45 & .47 & 11.94 & 140 \\
\hline 157.0121 & 12 & - & .025 & 6.35 & .10 & 20 AWG $(10 / 3)$ & 45 & .52 & -13.21 & 170 \\
\hline $157-0|5|$ & 15 & & .025 & 6.35 & .10 & $20 \mathrm{AWG}(10 / \mathrm{s})$ & 60 & .61 & 15.49 & 225 \\
\hline $157-0191$ & 19 & - & .025 & 6.35 & .10 & $20 \mathrm{AWG}(10 / 5)$ & 60 & .63 & 16.00 & 260 \\
\hline $157-0271$ & 27 & _ & .025 & 6.35 & .10 & $20 \mathrm{AWG}(10 / \mathrm{s})$ & 60 & .75 & 19.05 & 350 \\
\hline 157.0371 & 37 & & .025 & 6.35 & .10 & $20 \mathrm{AWG}(10 / \mathrm{s})$ & 60 & .83 & $16 . \infty$ & 450 \\
\hline
\end{tabular}

$$
\begin{aligned}
& \text { HNF. } 2487 \text { Rev. } \\
& \text { Pg E-59A }
\end{aligned}
$$

F Ruted $90^{\circ} \mathrm{C}$ lor normul operation in wet and dry locitions. $130^{\circ} \mathrm{C}$ for emergsency nverloud ennditions, snd $250^{\circ} \mathrm{C}$ for shor circuil conditions.

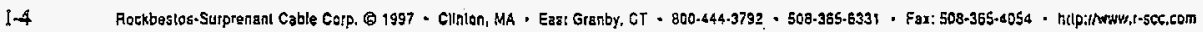




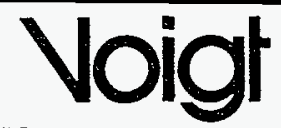

Page $A-22$ (acculatror
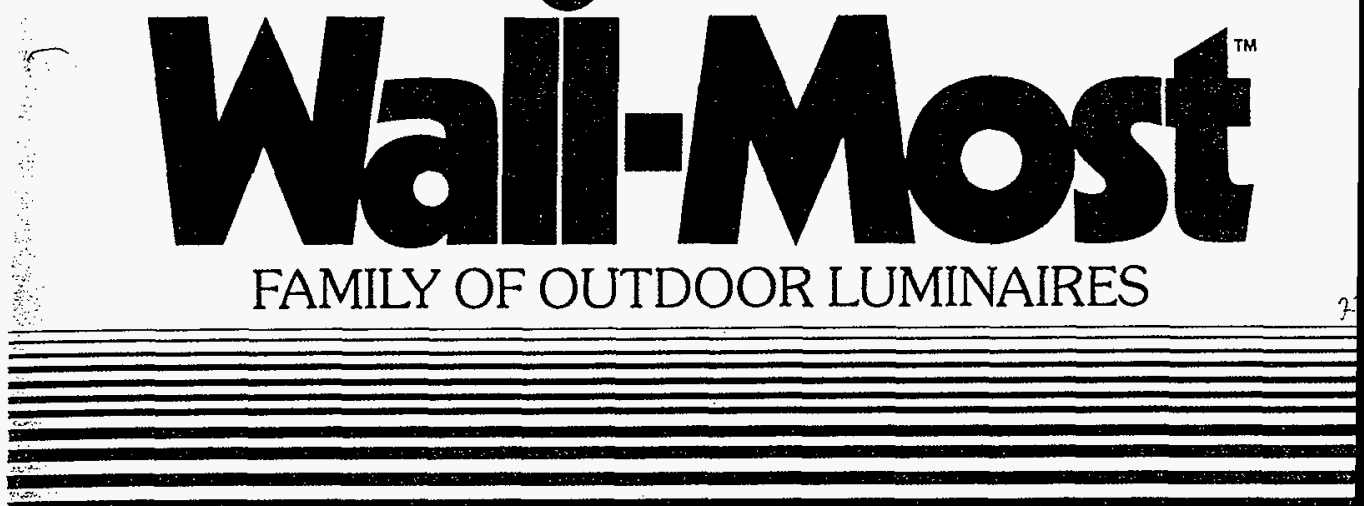

(3)

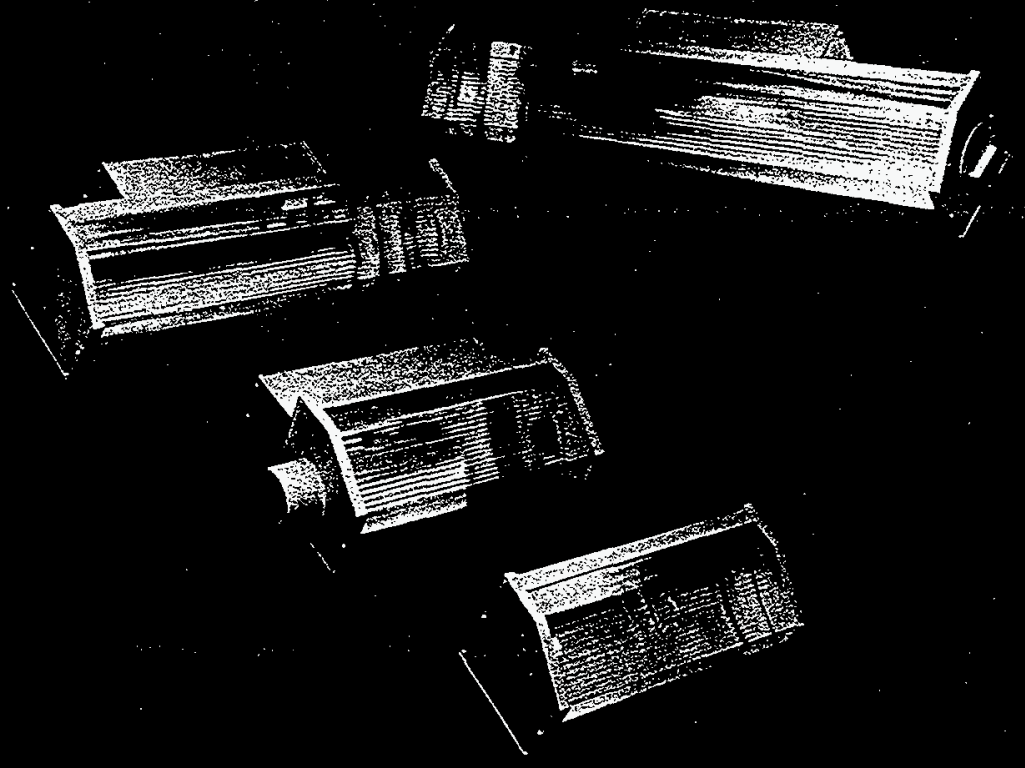

Aesthetically styled in pure architectural form for all low pressure sodium lamps (18 watt, 35 watt, 55 watt, 90 watt, 135 watt, 180 watt).

\section{(D) Voigt Lighling Industries, Inc.}




\section{Outdoor Energy-Dollar Saver Check-Chart}

\begin{tabular}{|c|c|c|c|}
\hline $\begin{array}{l}\text { If You Now Use } \\
\text { or Plan to Use }\end{array}$ & $\begin{array}{c}\text { Use Voigt } \\
\text { Wall-Most } \\
\text { for Equivalent } \\
\text { Lighting } \\
\text { Results - Instead }\end{array}$ & $\begin{array}{c}\text { Watts } \\
\text { Saved } \\
\text { for Each } \\
\text { Unit } \\
\text { Used } \\
\end{array}$ & \begin{tabular}{|c|} 
Annual \\
Dollar \\
Saving \\
for \\
Nighty \\
Use \\
$(6 \mathrm{f} /$ Kwhr \\
\end{tabular} \\
\hline $\begin{array}{l}\text { 1-300 Watt Incandescent } \\
\text { Fish Bowl Unit }\end{array}$ & $\begin{array}{l}\text { No. } 0.36 \\
\text { (35 watt) }\end{array}$ & 240 & 58 \\
\hline $\begin{array}{l}\text { 3-150 Watt Inc. Reflector } \\
\text { Lamp Cluster }\end{array}$ & $\begin{array}{l}\text { No. } 0.36 \\
\text { (55 watt) }\end{array}$ & 370 & 89 \\
\hline $\begin{array}{l}\text { 1-500 Wast Inc. } \\
\text { Quartz Floodlight }\end{array}$ & $\begin{array}{l}\text { No. } 0.36 \\
\text { (55 watt) }\end{array}$ & 420 & 101 \\
\hline $\begin{array}{l}\text { 1-1000 Watt Inc. } \\
\text { Quartz Light }\end{array}$ & $\begin{array}{l}\text { No. } 0.39 \\
\text { (180 watt) }\end{array}$ & 780 & 187 \\
\hline $\begin{array}{l}\text { 1-175 Watt Mercury } \\
\text { Vapor Wall-Unit }\end{array}$ & $\begin{array}{l}\text { No. } 0.36 \\
\text { (55 watt) }\end{array}$ & 125 & 30 \\
\hline $\begin{array}{l}\text { 1-400 Watt Mercury } \\
\text { Vapor Wall-Unit }\end{array}$ & $\begin{array}{l}\text { No. } 0-38 \\
\text { (135 wat1) }\end{array}$ & 275 & 66 \\
\hline $\begin{array}{l}\text { 1-400 Watt Metal Halide } \\
\text { Wall-Unit }\end{array}$ & $\begin{array}{l}\text { No. } 0-39 \\
\text { (180 wat } 1)\end{array}$ & 245 & 59 \\
\hline $\begin{array}{l}\text { 1-250 Watt H.P.S. } \\
\text { Wall-Unit }\end{array}$ & $\begin{array}{l}\text { No. } 0.38 \\
\text { (135 watt) }\end{array}$ & 132 & 32 \\
\hline
\end{tabular}

Whether you seek to

Protect visitors in a parking lot

Secure a fence against intruders

Highlight a building entrance

Provide visibility for your watchman

Pour light into a loading 18 -wheeler

Or

Assure proper T.V. surveillance lighting

The Wall-Most family does it exceptionally well and attractively using fewer watts and often for a much smaller dollar outlay.

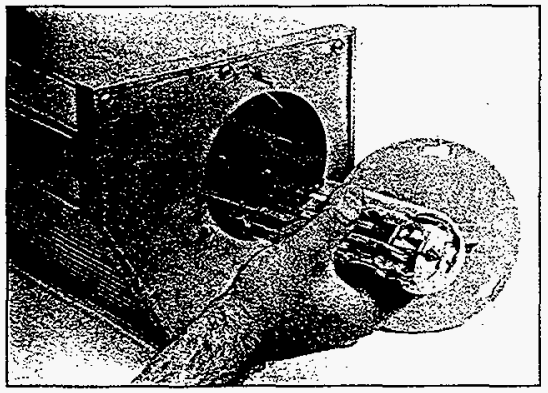

Relamping is accomplished through fully-gasketed door in end-keeping optical chamber sealed against bug and dirt entry.
Calculation w320-31-OHI)
Page A-23

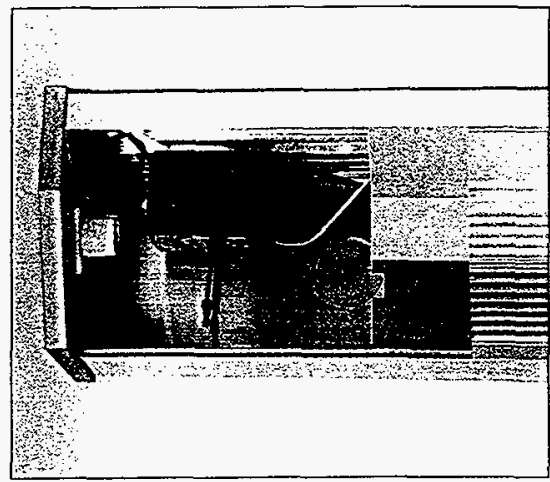

Installation, even under adverse outdoor conditions, is remarkably easy; with all mounting bolts and wire splices fully accessible as lens \& reflector slide out of the way.

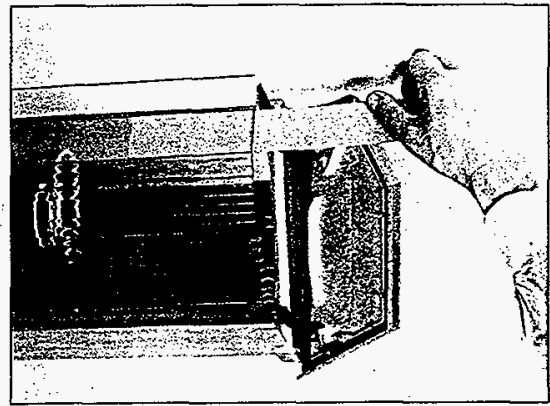

Internally mounted uplight shield can be field installed or removed at any time ( $S D-1$ shown with 0-36).

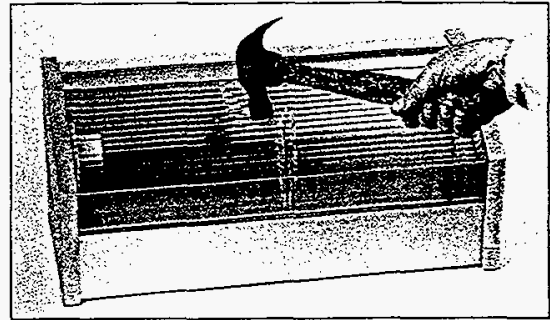

Polycarbonate lens resists breakage. Absence of ultraviolet in L.P.S. source eliminates lens embrittlement and yellowing over time.

HNF-2487, Rev. 0 


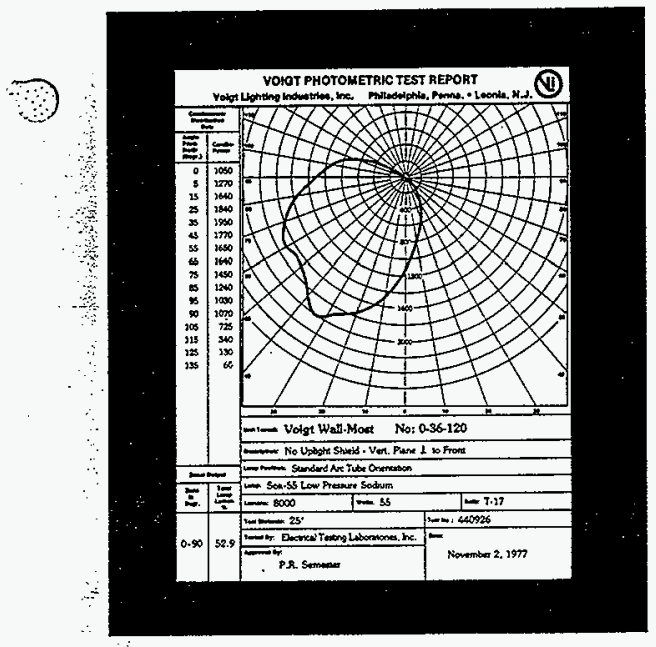

Wide beam spread of Wall-Most achieves coverage of large areas adjacent to buildings and mounting walls.
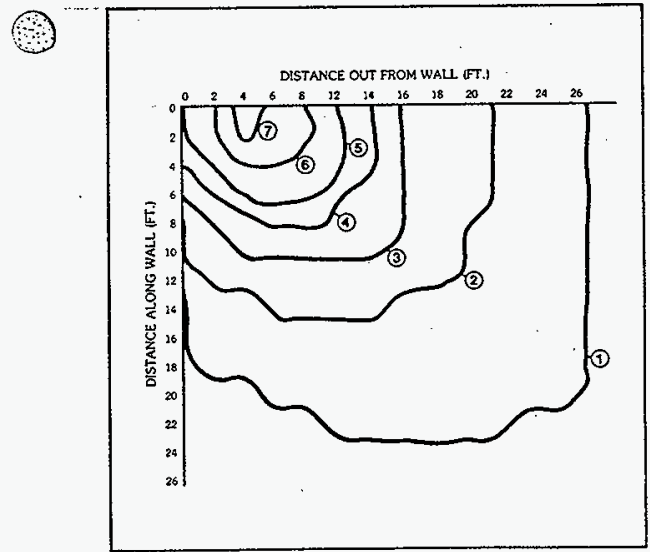

\section{Footcandle Isolux}

Top View of Footcandles on Ground From Single Unit.

Mounting Height $-15^{\prime}-0^{\prime \prime}$

S) From: Voigt Wall-Most ${ }^{\mathrm{TM}}$ No. 0-36-120 with 55 watt Low Pressure Sodium Lamp.

(Data: Electrical Testing Laboratories Test No. 440926$)$.

\section{Page $8-24$}

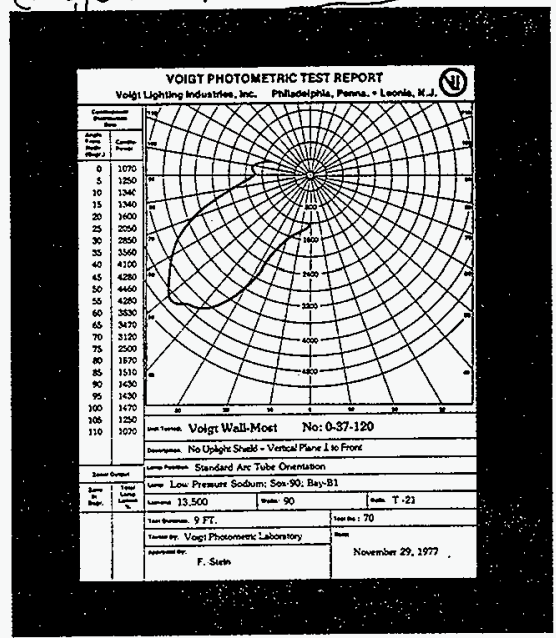

Larger (90 through 180 watts) Wall-Most have a more sharply defined distribution of light - to cover even larger areas. For 135 watts prorate data by multiplying factor of 1.67 . For 180 watts multiply by 2.44 .

Aesthetic elegance of WallMost can be enhanced by versions in black - or other architectural color finishes as desired. Simply specify color.

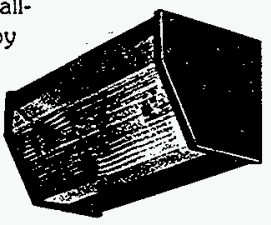

\section{Additional Wall-Most notes.}

Long luminous arc size, for all lamps, spreads the light over the entire WallMost lens face-reducing the viewing brightness-so that unit is comfortable to look ateven when viewed directly.

The yellow monochromatic light of Wall-Most does not attract bugs to your building. The light, however, does penetrate smoke, haze, fog \& smog more forcefully than other sources of illumination.

Unique prismatic lens structure precisely designed for twin Arc L.P.S. source, significantly aids in control of light into efficient beam pattern; and optically disperses source for greater comfort. 
Lamp Specifications and Ordering Information

\begin{tabular}{|c|c|c|c|c|}
\hline \multicolumn{5}{|c|}{ LOW PRESSURE SODJUM LAMPS ONLY } \\
(Order Separately)
\end{tabular}

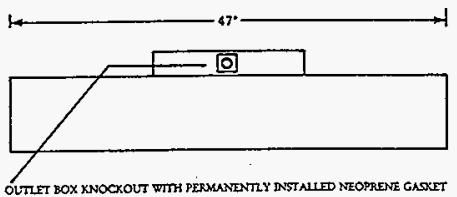

\section{Table of Electrical Characteristics for Wall-Most}

\begin{tabular}{|c|c|c|c|c|c|}
\hline Sodture Lempest & JSO WATTS & 135 WATTS & so WhTTS & 55 WhatTS & 35 WATIS \\
\hline Nomind permey & $120200240 \quad 2 \pi 400$ & 120203240274480 & $120206240 \quad 277490$ & $120200240 \mathrm{~mm} 400$ & $120208240 \quad 277490$ \\
\hline 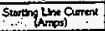 & $2.0 \quad 2,15 \quad 1.0 \quad 0.850 .5$ & $18 \quad 1.040 .908 \quad 0.45$ & 130.70 .6050 .4 & 0.950 .550 .480 .410 .3 & $\begin{array}{lllll}0.8 & 0.46 & 0.4 & 0.35 & 0.2\end{array}$ \\
\hline Optom & 1.91 .180950 .82 os & 1.640 .950 .820 .710 .4 & $1.16 \quad 67 \quad 58 \quad 50 \quad 29$ & 0.680 .430 .370 .320 .19 & 0.54032027 \\
\hline $\begin{array}{l}\text { Open Crat } \\
\text { Corres }(\text { Amposs }\end{array}$ & $\begin{array}{lllll}5.2 & 2.9 & 2.6 & 23 & 1.9 \\
\end{array}$ & $\begin{array}{llllll}52 & 2.9 & 2.6 & 2.3 & 1.9\end{array}$ & $\begin{array}{lllll}40 & 23 & 20 & 1.7 & 9\end{array}$ & $\begin{array}{llllll}25 & 1.45 & 1.25 & 1.7 & 6\end{array}$ & $\begin{array}{lllll}2.5 & 1.45 & 1.25 & 0.1 & .6\end{array}$ \\
\hline 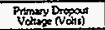 & $90 \quad 356 \quad 280208360$ & $66 \quad 114 \quad 135 \quad 352 \quad 354$ & $66 \quad 114 \quad 132 \quad 152 \quad 240$ & $66 \quad 314 \quad 332 \quad 152 \quad 250$ & $48 \quad 85 \times 111 \quad 185$ \\
\hline 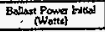 & $40 \quad 40 \quad 40 \quad 40 \quad 40$ & $\begin{array}{lllll}43 & 43 & 43 & 43 & 43\end{array}$ & $\begin{array}{lllll}35 & 35 & 35 & 35 & 35\end{array}$ & $252525 \quad 25$ & $25 \quad 25 \quad 25 \quad 25 \quad 25$ \\
\hline Toud Pout bolid & 200220200220220 & $\begin{array}{lllll}378 & 178 & 178 & 178 & 178\end{array}$ & $125125 \quad 22 \quad 125 \quad 225$ & 9080808080 & $60 \quad 60 \quad 60 \quad 60 \quad 60$ \\
\hline Dente fracer & $a=50 \%$ & Onst $x 05$ & av 500 & Ove 908 & Ond $x 00$ \\
\hline Lane Lasen & \multicolumn{5}{|c|}{ \pm 58 si $\pm 10 \%$ Voltage Reguletion } \\
\hline $\begin{array}{l}\text { Minkensm Ambident } \\
\text { Stut timg. }\end{array}$ & $.204 \mathrm{~F}$ & & Rower Tesperstu & on Sposedi Oriez) & \\
\hline
\end{tabular}

Wall-Most with exposed portions

Basic Wall-Most

Specification and Ordering Information wholly made of extruded and cast 0-39

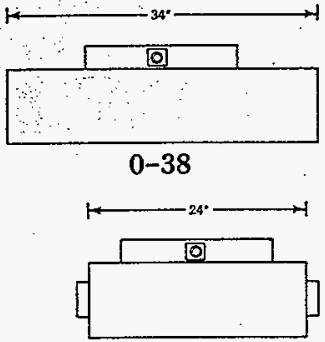

$0-37$

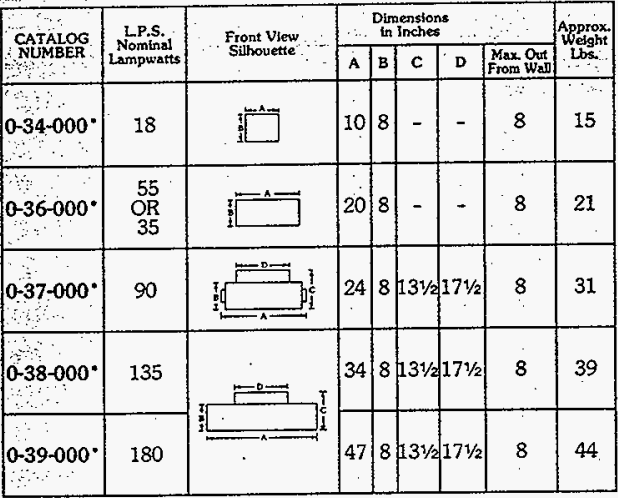

aluminum and transparent polycar-

bonate lens is the standard for strength and durability in outdoor wall mounted luminaires.

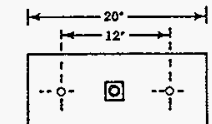

\section{Optional Accessories}

\begin{tabular}{|c|c|}
\hline $\begin{array}{c}\text { CATALOG } \\
\text { NUMBER }\end{array}$ & Description \\
\hline BLACK & Black Painted outside finish \\
\hline CA2 & Corner Adaptor for 0-34 +0-36 \\
\hline COLOR & Painted Finish - specify color \\
\hline ES & $\begin{array}{c}\text { Emergency Lighting Socket for } 15 \text { watt } \\
\text { Bayone Base, T-7 Inc. Lamp }\end{array}$ \\
\hline F & Single Fuse \\
\hline FF & Double Fuse \\
\hline NB & Unit without ballast to allow remote mounting \\
\hline PI & internally Mounted Photocell (120 volts only) \\
\hline PR & Twist-Lock Photocell Receptacle \\
\hline SD-1 & $\because$ Internal Shield for 0-36 \\
\hline SD-2 & Intemal Shied for 0-37 \\
\hline SD-3 & Internal Shield for 0-38 \\
\hline SD-4 & Internal Shield for 0-39 \\
\hline TP & Vandal Resistant, Tamper-Proof Screws \\
\hline
\end{tabular}

-Specify voltage required: $120,208,240,277$ or 480 t.e., No. $0.35-120$.

Listed by Underwriters Loboratories, Inc.

Units cary BEW label.

For additional technical or application data or actual demonstration of the Voigt Wall-Most contact Voigt Lighting Industries, ixc. Headquarters, or:

$$
\begin{aligned}
& \text { Calculation W320-31-011 } \\
& \text { Page A-25 }
\end{aligned}
$$

For additional accessories tniormation contact Volgt Engineming Depart ment. Cost for optional accessories available on request.
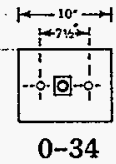

\footnotetext{
HNF-2487, Rev. 0

Page E-63
} 
Fig. 11.1. Currently Recommended Illuminance Categories and Illuminance Values for Lighting Design Targeted Maintained Levels

The following table is a consolidated listing of the IESNA's current illuminance recommendations. This listing is intended to guide the designer in selecting an appropriate iluminance.

Guidance is provided in two forms: (1), in Parts I, II and III as an Jluminance Category, representing a range of illuminances; and (2), in Parts $V, V$ and $V I$ as an Illuminance Value. Illuminance categories are represented by letter designations $A$ through I. Illuminance Values are given in lix with equivalents in footcandles and as such are intended as target values with deviations expected. These target values also represent maintainedvalues.
This table has been divided into six parts for ease of use. Part I lists both Illuminance Categories and Illuminance Values for generic types of interior activities and normally is to be used when Illuminance Categories for a specific Area/Activity cannot be found in Parts II and III. Parts IV, V and VI provide target maintained llluminance Values for outdoor facilities, sports and recreational areas, and transportation vehicles where special considerations apply as discussed.

In all cases the recommendations in this table are based on the assumption that the lighting will be properly designed to take into account the visual characteristics of the task.

I. Illuminance Categories and Illuminance Values for Generic Types of Activities in Interiors

\begin{tabular}{|c|c|c|c|c|}
\hline \multirow{2}{*}{ Type of Activity } & \multirow{2}{*}{$\begin{array}{c}\text { Illuminance } \\
\text { Category }\end{array}$} & \multicolumn{2}{|c|}{ Ranges of Illuminances } & \multirow{2}{*}{ Reterence Work-Plane } \\
\hline & & Lux & Footcandies & \\
\hline Public spaces with dark surroundings & A & $20-30.50^{\circ}$ & $2-3-5$ & \multirow{3}{*}{$\begin{array}{l}\text { General lighting } \\
\text { throughout spaces }\end{array}$} \\
\hline $\begin{array}{l}\text { Simple orientation for short temporany } \\
\text { visits }\end{array}$ & B & $50-75-100$ & $5-75-10$ & \\
\hline $\begin{array}{l}\text { Working spaces where visual tasks are } \\
\text { only occasionally performed }\end{array}$ & C & $100-150-200$ & $10-15-20$ & \\
\hline $\begin{array}{l}\text { Performance of visual tasks of high con. } \\
\text { trast or large size }\end{array}$ & D & $200-300-500$ & $20-30-50$ & \multirow{3}{*}{ illuminance on task } \\
\hline $\begin{array}{l}\text { Performance of visual tasks of medium } \\
\text { contrast or small size }\end{array}$ & $E$ & $500-750-1000$ & $50-75-100$ & \\
\hline $\begin{array}{l}\text { Performance of visual tasks of low con- } \\
\text { trast or very small size }\end{array}$ & $\mathbf{F}$ & $1000-1500-2000$ & $100-150-200$ & \\
\hline $\begin{array}{l}\text { Performance of visual tasks of low con- } \\
\text { trast and very small size over a pro- } \\
\text { longed period }\end{array}$ & G & $2000-3000-5000$ & $200-300-500$ & \multirow{3}{*}{$\begin{array}{l}\text { Illuminance on task, } \\
\text { obtained by a com- } \\
\text { bination of general } \\
\text { and local (supple. } \\
\text { mentary lighting) }\end{array}$} \\
\hline $\begin{array}{l}\text { Performance of very prolonged and ex- } \\
\text { acting visual task }\end{array}$ & $\bar{H}$ & $5000-7500-10000$ & $500-750-1000$ & \\
\hline $\begin{array}{l}\text { Performance of very special visual tasks } \\
\text { of extremely low contrast and small } \\
\text { size }\end{array}$ & 1 & $10000-15000-20000$ & $1000-1500-2000$ & \\
\hline
\end{tabular}

II. Commercial, Institutional, Residential and Public Assembly Interiors

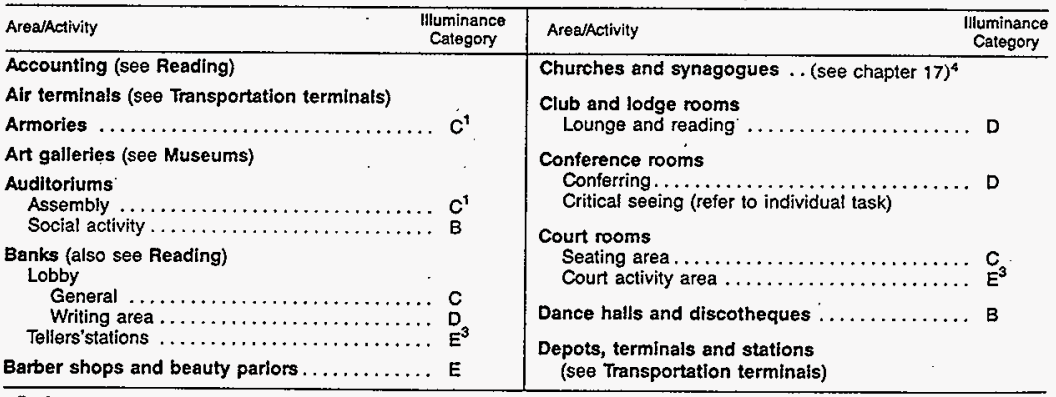

For footnotes, see end of table.

HNF-2487, Rev, 0

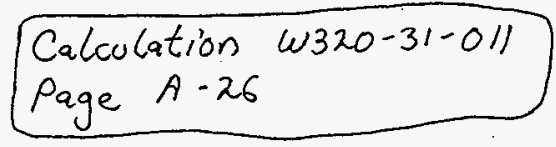

Page E-64 
NFW

\section{Fluorescent}

Industrial

\section{Lighting Fixtures}

\section{Application:}

NFW fixtures are used:

- in non hazardous locations where protection is required from wet, dirty and corrosive atmospheres

- where cleanliness and sanitation are prime factors, such as dairies, canneries, food processing plants, bottling plants,

laboratories

- in dock areas for protection against salt spray

\section{Features:}

- One-piece high impact tiberglass housing provides durability and corrosion resistance

- Energy efficient ballast is standard

- One-piece orismatic acrylic lens for

resistance to accidental damage

- Plastic latches apply positive, uniform pressure on gasketing to seal against dust and moisture. Six latches on four foot unit and twelve latches on eight foot unit

- Mounting lugs for ballast and channel are molded into housing

Standard Materials:

- Reflector housing and latches - reinforced fiberglass

- Prismatic lens - acrylic plastic

- Gaskel - closed cell neoprene

Standard Finishes:

- Fiberglass - pearl gray

- Acrylic plastic - clear

Size Ranges:

- 4 and 8 foot lengths

- 2 and 4 lamp

Electrical Rating Ranges:

. 120 and 277 volt

- 60 hertz

. 40,75, 110 watts

Compliances:

- UL Standard: 1570

\section{Options:}

The following special options are available from the factory by adding suffix to Cat. No: Suffix to be Acded to

Description Fixture Cat. \# - Chain mounting assembly $\S . . . \ldots .$. CMA

- Chain sets ................... CS - Low temperature ballast - rated $0^{\circ} \mathrm{F}$ ( 40 \& 75 watt) and $-20^{\circ} \mathrm{F}$ (110 watt) ......... BY - Fuse and holder (one per fixture) :...FB - Suitable for $220 \mathrm{v} 50 \mathrm{~Hz}$.......... Consult Crouse-Hinds

- Two pairs ol 4" lamps in tandem.

† 40 and 110 watt ballasts are capio start: 75 watt ballasis are instant start.

$\S$ Chain sets not incluved. Order ctadin sets by auding CS to Cal No.

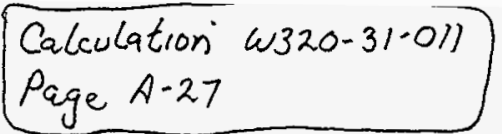

\section{Wet Locations}

\section{Crouse-Hinds $^{\circledast}$}

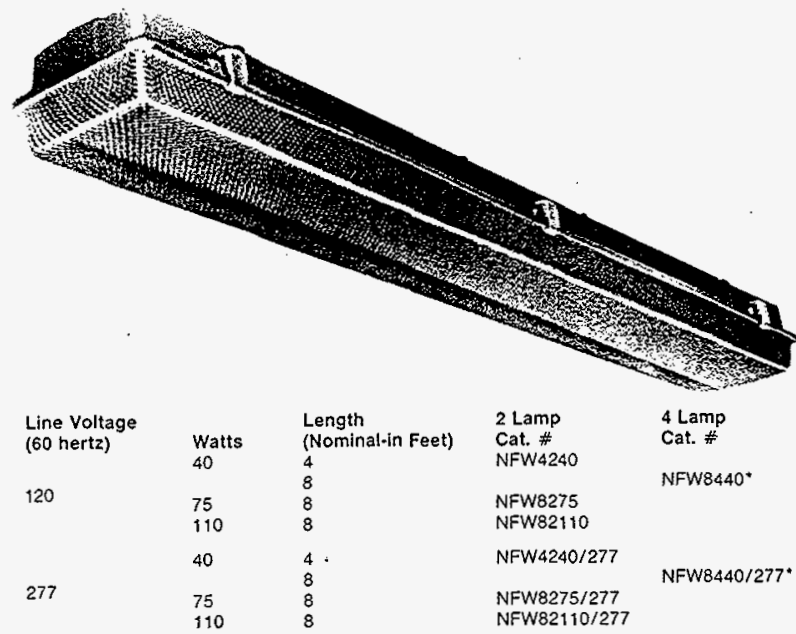

Lamps and Ballasts

$\begin{array}{lll}\text { Watts } & \text { Lamp } & \text { Ballast Typet } \\ 40 & \text { F40 (Med. Bi-Pin) } & 430 \mathrm{ma} \\ 75 & \text { F96T12 (Single Pin) } & 425 \mathrm{ma} \\ 110 & \text { F96T12HO (Recessed } \\ & \text { Double Contact) } & 800 \mathrm{ma}\end{array}$

\section{Dimensions}

Eight Foot

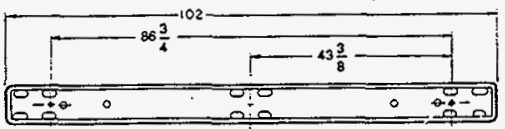

Top view

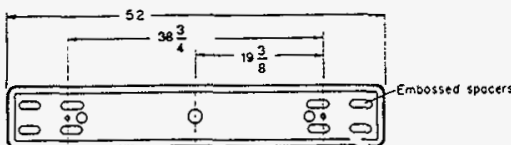

Four Foct
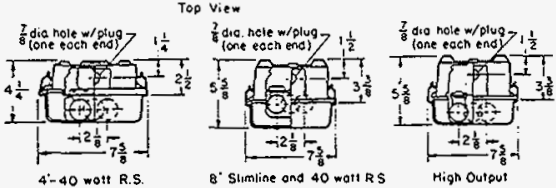

HNF-2487, Rev, 0

January 1992 
NFW \& FMR

Fluorescent

Industrial

Crouse-Hinds $^{\circledast}$

\section{Lighting Fixtures}

Photometric Data

Fixture: NFW4240

Lamp: 2-40/T-12

Zonal Degrees: $0-30 \quad 0-40 \quad 0-60 \quad 0-90 \quad 0-180$

Zonal Lumens: $857 \quad 1394 \quad 2478 \quad 3678 \quad 4211$

Total Bare Lamp Lumens: $3200 \times 2=6400$

To determine number and placement of fixtures,

see Lighting Selector Guide, pages L-7 to L-12.
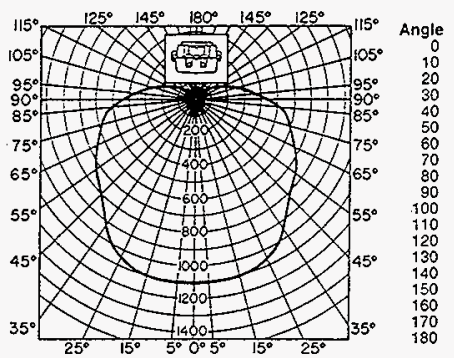

Candelas

Along Across

$1166 \quad 1166$

$1141 \quad 1170$

$1100 \quad 1192$

10131190

$841 \quad 1104$

$429 \quad 781$

$249 \quad 6: 6$

$107 \quad 497$

$\begin{array}{ll}18 & 350 \\ 14 & 220\end{array}$

1216

857

3
Coefficient of Utilization

Effective Floor Cavity Reflectance 20\%

$\%$ Reflectance Room Cavity Ratio

\begin{tabular}{cllllll} 
Eff. Ceil. & Wall & 9 & 2 & 3 & 4 & 5 \\
& 50 & .71 & .61 & .54 & .47 & .42 \\
80 & 30 & .68 & .56 & .47 & .40 & .35 \\
& 10 & .64 & .51 & .42 & .35 & .30 \\
\hline \multirow{3}{*}{70} & 50 & .69 & .60 & .52 & .46 & .41 \\
& 30 & .66 & .55 & .46 & .40 & .34 \\
& 10 & .63 & .50 & .41 & .35 & .30 \\
\hline \multirow{3}{*}{50} & 50 & .65 & .56 & .49 & .43 & .39 \\
& 30 & .62 & .52 & .44 & .38 & .33 \\
& 10 & .60 & .48 & .40 & .34 & .29 \\
\hline \multirow{3}{*}{30} & 50 & .61 & .53 & .46 & .41 & .37 \\
& 30 & .59 & .40 & .42 & .36 & .32 \\
& 10 & .57 & .46 & .38 & .33 & .28 \\
\hline \multirow{2}{*}{10} & 50 & .58 & .50 & .44 & .39 & .35 \\
& 30 & .56 & .47 & .40 & .35 & .30 \\
& 10 & .54 & .44 & .37 & .31 & .27 \\
\hline 0 & 0 & .52 & .42 & .35 & .30 & .25
\end{tabular}

$\%$ Reflectance Room Cavity Ratio

$\begin{array}{llllll}\text { Eff. Ceil. Wall } & 6 & 7 & 8 & 9 & 10\end{array}$

\begin{tabular}{lllllll}
\multirow{3}{*}{80} & 50 & .38 & .34 & .31 & .28 & .26 \\
& 30 & .31 & .27 & .25 & .22 & .20 \\
& 10 & .26 & .23 & .20 & .18 & .16 \\
\hline \multirow{3}{*}{70} & 50 & .37 & .33 & .30 & .28 & .25 \\
& 30 & .30 & .27 & .24 & .22 & .20 \\
& 10 & .26 & .23 & .20 & .18 & .16 \\
\hline \multirow{3}{*}{50} & 50 & .35 & .31 & .29 & .26 & .24 \\
& 30 & .29 & .26 & .23 & .21 & .19 \\
& 10 & .25 & .22 & .20 & .18 & .16 \\
\hline \multirow{2}{*}{30} & 50 & .33 & .30 & .27 & .25 & .23 \\
& 30 & .28 & .25 & .23 & .21 & .19 \\
& 10 & .24 & .21 & .19 & .17 & .16 \\
\hline \multirow{2}{*}{10} & 50 & .31 & .28 & .26 & .24 & .22 \\
& 30 & .27 & .24 & .22 & .20 & .18 \\
& 10 & .24 & .21 & .19 & .17 & .15 \\
\hline 0 & 0 & .22 & .19 & .17 & .15 & .14
\end{tabular}

Fixture: FMR4240

Lamp: $2 \cdot 40 / \mathrm{T}-12$

Zonal Degrees: $0-30 \quad 0-40 \quad 0-60 \quad 0.90 \quad 0-180$

Zonal Lumens: $761 \quad 1300 \quad 2508 \quad 3826 \quad 4601$

Total Bare Lamp Lumens: $3200 \times 2=6400$

To determine number and placement of fixtures,

see Lighting Selector Guide, pages L-7 $10 \mathrm{~L}-12$.

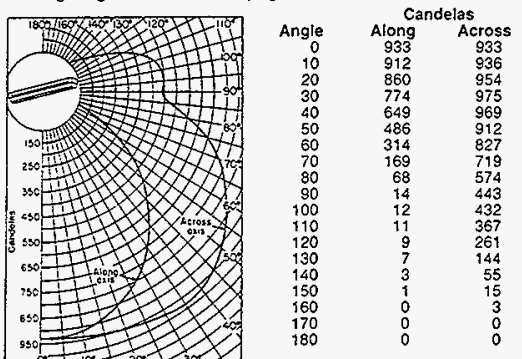

\section{Coefficient of Utilization}

Effective Floor Cavity Reflectance $20 \%$

\% Reflectance Room Cavity Ratio

\begin{tabular}{lllllll} 
Eff. Ceil. & Wall & 1 & 2 & 3 & 4 & 5 \\
& 50 & .69 & .59 & .51 & .45 & .40 \\
80 & 30 & .65 & .53 & .44 & .38 & .33 \\
& 10 & .62 & .48 & .39 & .33 & .28 \\
\hline \multirow{3}{*}{70} & 50 & .66 & .56 & .49 & .43 & .38 \\
& 30 & .63 & .51 & .43 & .37 & .32 \\
& 10 & .59 & .47 & .38 & .32 & .27 \\
\hline \multirow{3}{*}{50} & 50 & .61 & .52 & .45 & .39 & .35 \\
& 30 & .58 & .48 & .40 & .34 & .30 \\
& 10 & .55 & .44 & .36 & .30 & .25 \\
\hline \multirow{2}{*}{30} & 50 & .56 & .48 & .41 & .36 & .32 \\
& 30 & .54 & .44 & .37 & .32 & .28 \\
& 10 & .51 & .41 & .34 & .28 & .24 \\
\hline \multirow{2}{*}{10} & 50 & .51 & .44 & .38 & .33 & .30 \\
& 30 & .50 & .41 & .35 & .30 & .26 \\
& 10 & .48 & .38 & .32 & .27 & .23 \\
\hline 0 & 0 & .45 & .36 & .29 & .25 & .21
\end{tabular}

$\%$ Reflectance Room Cavity Ratio

\begin{tabular}{cllllll} 
Eff. Ceil. & Wall & 6 & 7 & 8 & 9 & 10 \\
& 50 & .35 & .32 & .29 & .26 & .24 \\
80 & 30 & .29 & .25 & .23 & .20 & .18 \\
& 10 & .24 & .21 & .18 & .16 & .15 \\
\hline \multirow{3}{*}{70} & 50 & .34 & .31 & .28 & .25 & .23 \\
& 30 & .28 & .25 & .22 & .20 & .18 \\
& 10 & .23 & .20 & .18 & .16 & .14 \\
\hline \multirow{3}{*}{50} & 50 & .31 & .28 & .26 & .24 & .22 \\
& 30 & .26 & .23 & .21 & .19 & .17 \\
& 10 & .22 & .19 & .17 & .15 & .14 \\
\hline \multirow{2}{*}{30} & 50 & .29 & .26 & .24 & .22 & .20 \\
& 30 & .24 & .22 & .19 & .18 & .16 \\
& 10 & .21 & .18 & .16 & .14 & .13 \\
\hline \multirow{2}{*}{10} & 50 & .27 & .24 & .22 & .21 & .19 \\
& 30 & .23 & .20 & .18 & .17 & .15 \\
& 10 & .20 & .17 & .15 & .14 & .12 \\
\hline 0 & 0 & .18 & .16 & .14 & .12 & .11
\end{tabular}




\section{-alculation w-320-31-0/l Page A-29}

POWER CONDUIT BLOCK DIAGRAM

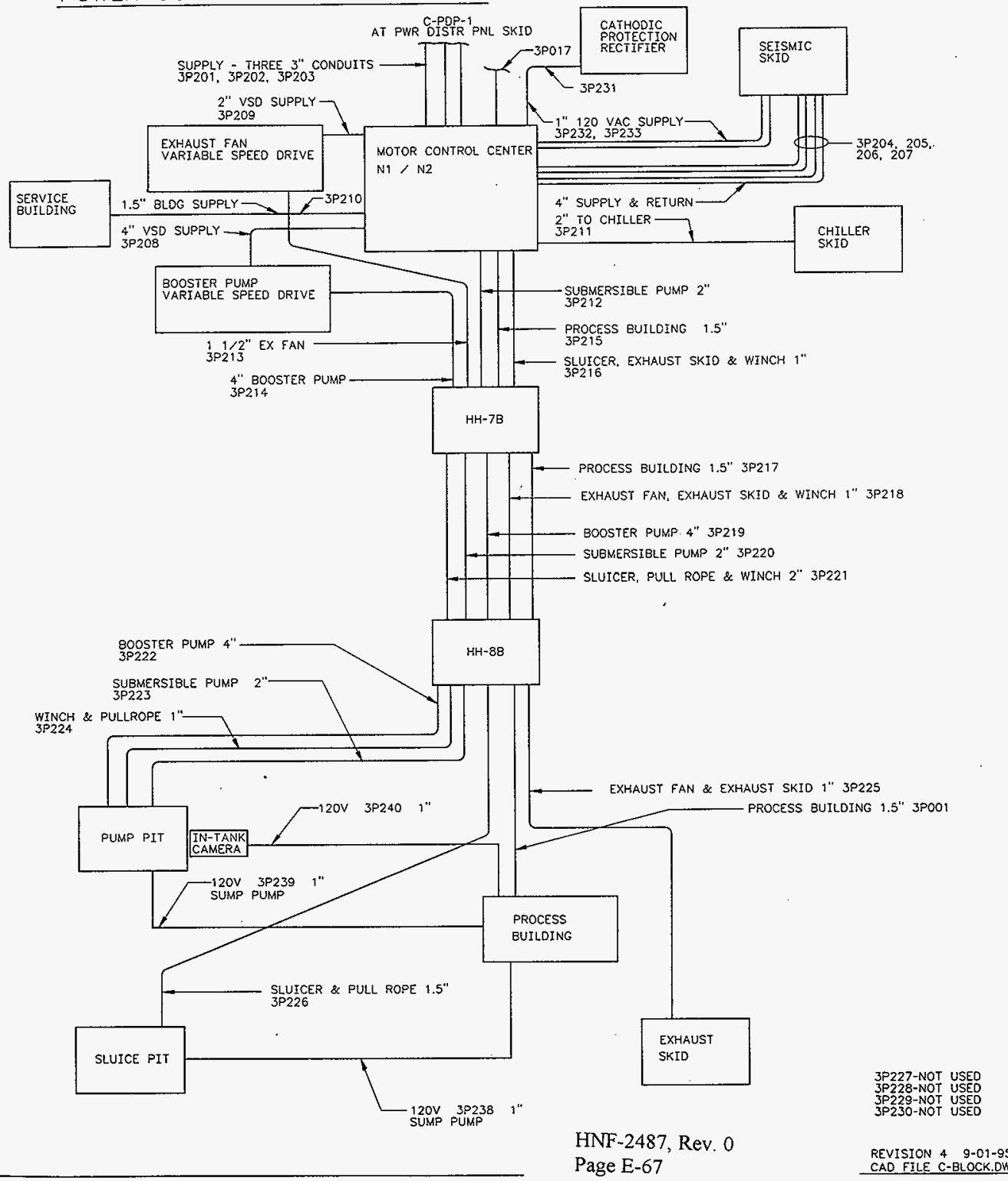


Calculation w320-31-071
page $A-30$

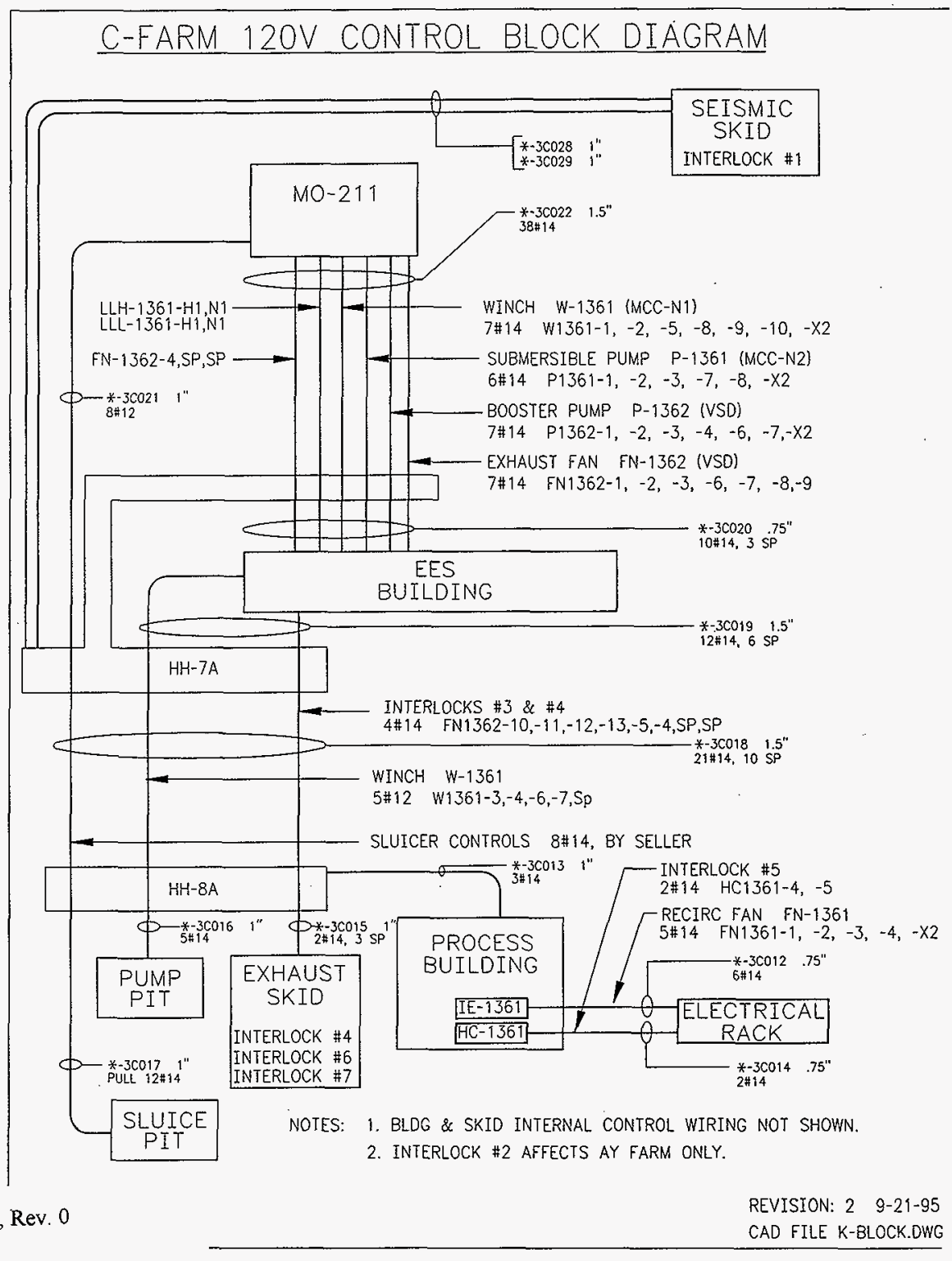

HNF-2487, Rev. 0 CAD FILE K-BLOCK.OWG 


\section{C-FARM INSTRUMENT CABLE BLOCK DIAGRAM}
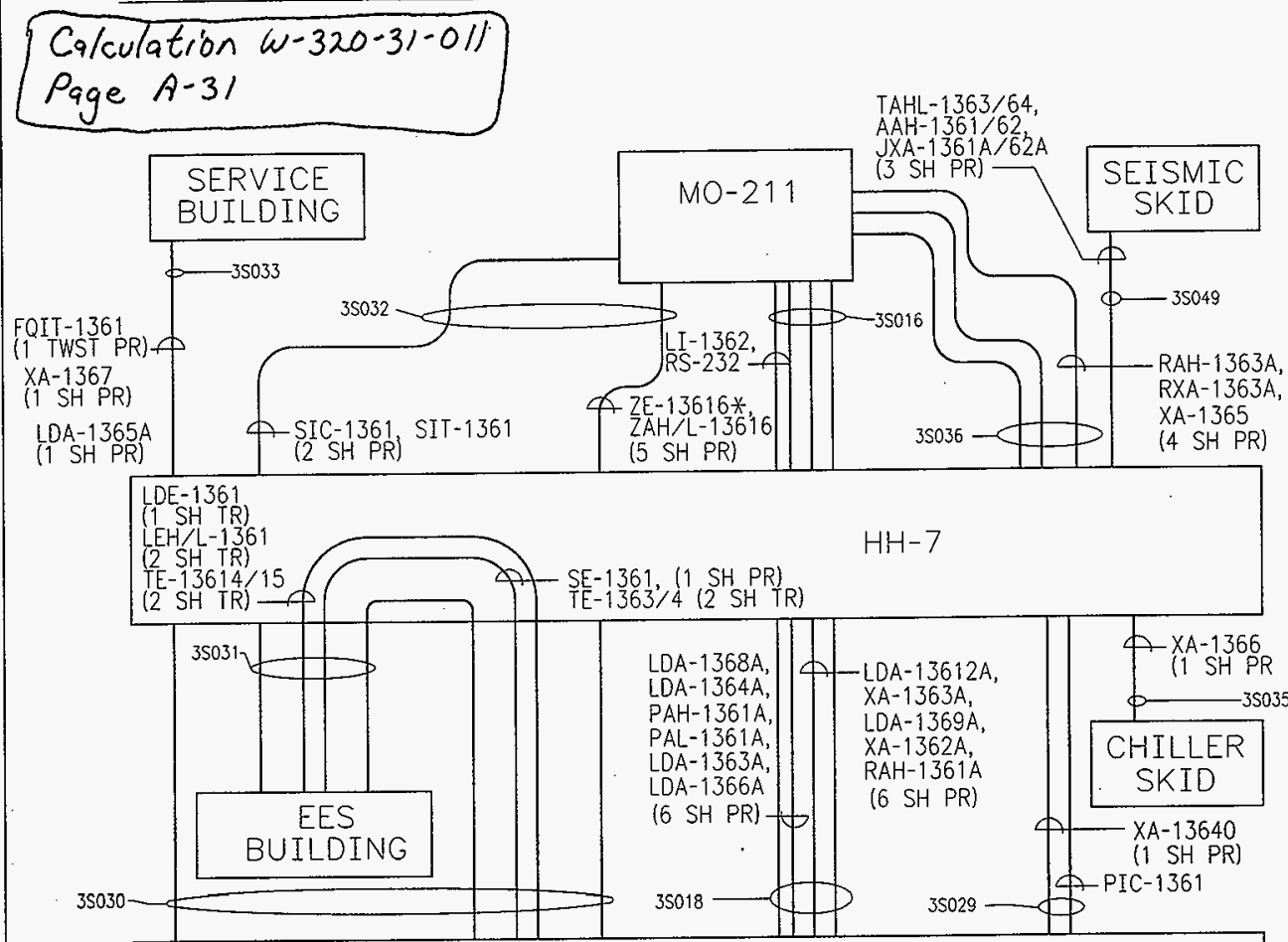

$\mathrm{HH}-7$

TAHL-1363/64

AAH $-1361 / 62$
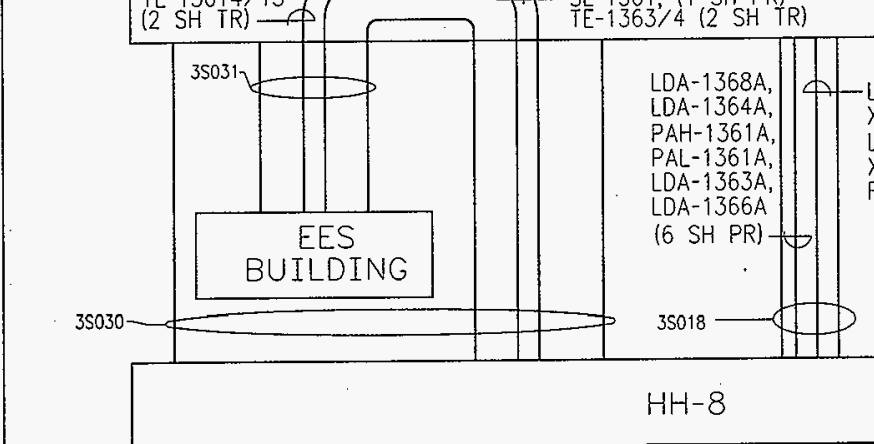

$\mathrm{HH}-8$

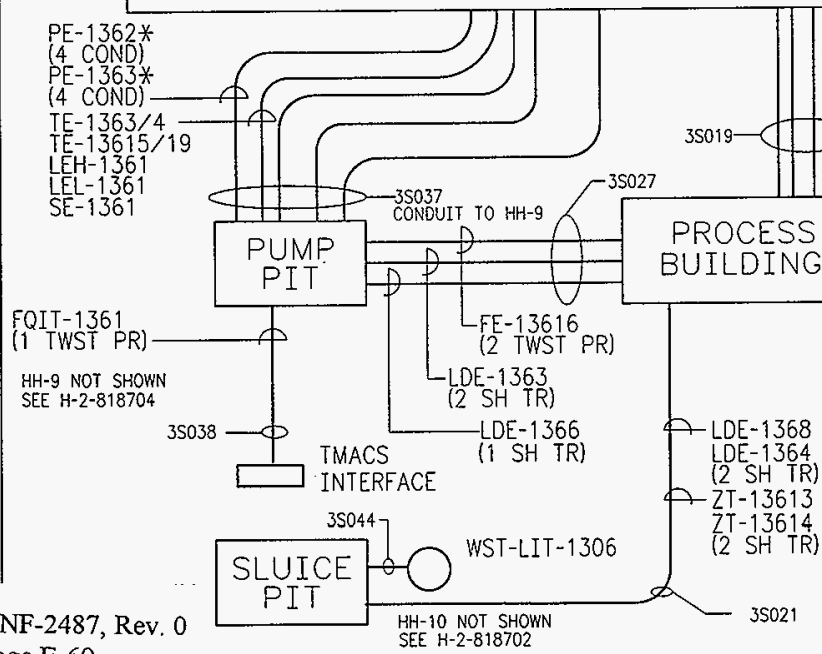

LDA- $13612 \mathrm{~A}$ $X A-1363 A_{1}$ LDA-1369A, $X A-1362 A$, RAH-1361A

(6 SH PR)

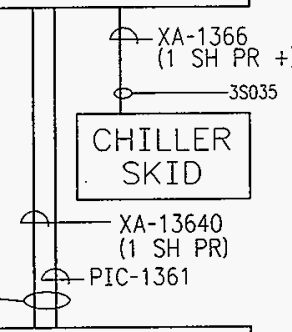
35029

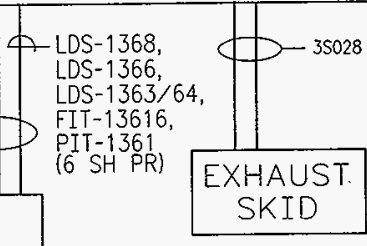

Page E-69

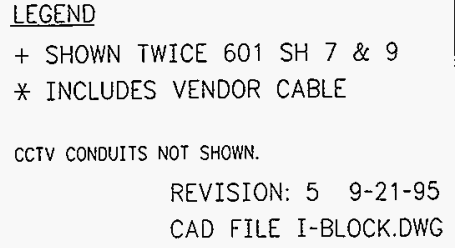

REVISION: $5 \quad 9-21-95$ CAD FILE I-BLOCK.DWG 
HNF-2487, Rev. 0

W320-31-016

AY-102 Demand Load Analysis, Size

Feeders, Voltage Drop and Fault Study 


\section{Fluor Daniel Northwest}

CALCULATION IDENTIFICATION AND

INDEX
Page $i$ of 3

$7 / 24 / 98$

This sheet shows the status and description of the attached Design Analysis sheets.

Discipline Electrical wo/sob No. Task Order N09 Calculation No. W320-31-016

Project No. \& Hame W320, Tank 241-C-106 STuicing

calculation item AY-102 Demand Load Analysis, Size Feeders, Voltage Drop, and Fault Study

These calculations apply to:

Dwg. No. H-2-818693, Sheet 1

Rev. No. 1

Dwg. No.

Rev. No.

other (Study, CDR)

Rev. No.

The status of these calculations is:

[] Preliminary Calculations

[X] Final Calculations

[] Check Calculations (On Calculation Dated)

[] Void Calculation (Reason Voided)

Incorporated in Final Drawings?

[X] Yes [] No

This calculation verified by independent "check" calculations? [] Yes [X] No

Original and Revised Calculation Approvals:

\begin{tabular}{|c|c|c|}
\hline & \multicolumn{1}{|c|}{$\begin{array}{c}\text { Rev, } 0 \\
\text { Signature/Date }\end{array}$} \\
\hline Originator & TJ Kasnick 8/7/95 \\
\hline Approved by & SA Najjar 8/7/95 \\
\hline $\begin{array}{c}\text { Checked Against } \\
\text { Approved Vendor Data }\end{array}$ & TJ Kasnick 8/7/95 \\
\hline \multicolumn{2}{|c|}{$\mathrm{N} / \mathrm{A}$} \\
\hline \\
Design Analysis \\
Page No.
\end{tabular}

HNF-2487, Rev. 0

KEH $0378.00(06 / 92)$ KEF072

Page F-1 


\begin{tabular}{|c|c|c|c|c|c|c|c|c|c|c|}
\hline \multicolumn{10}{|c|}{$\begin{array}{l}\text { CALCULATION CROSS INDEX (Typical) } \\
\text { Subject Calculation No.: W320-31-016 }\end{array}$} & \multirow{3}{*}{ 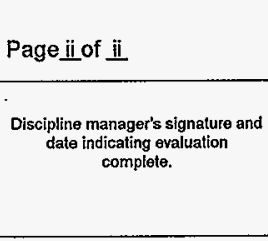 } \\
\hline \multirow[t]{2}{*}{$\begin{array}{l}\text { Subject } \\
\text { Calculation } \\
\text { Revision } \\
\text { No. }\end{array}$} & \multirow[t]{2}{*}{$\begin{array}{l}\text { Superceded } \\
\text { calculation } \\
\text { No. }\end{array}$} & \multicolumn{2}{|c|}{$\begin{array}{l}\text { These interfacing calculation/documents } \\
\text { provide inpul to the subject calculation, } \\
\text { and if revised may require revision of } \\
\text { the subject calcalculation. }\end{array}$} & \multicolumn{2}{|c|}{$\begin{array}{l}\text { Resulis and conclusions of the subject } \\
\text { calculation are used in these interfacing } \\
\text { calculations andior documents }\end{array}$} & \multicolumn{2}{|c|}{$\begin{array}{l}\text { Does the oulput } \\
\text { interface calculationt } \\
\text { documents require } \\
\text { revision? }\end{array}$} & \multicolumn{2}{|c|}{$\begin{array}{l}\text { Has the output } \\
\text { interfacecalculation } \\
\text { documments beon } \\
\text { revised? }\end{array}$} & \\
\hline & & Calculation/Document No. & $\begin{array}{c}\text { Revision } \\
\text { No. }\end{array}$ & Calculation/Document No. & $\begin{array}{c}\text { Revision } \\
\text { No. }\end{array}$ & Yes & No & Yes & & \\
\hline 0 & N/A & $H-2-818693$ & 1 & W320-31-017 & $\theta$ & KX & $x$ & & $x$ & \\
\hline & & & & & & & & & & \\
\hline & & & & & & & & & & \\
\hline & & & & & & & & & & \\
\hline & & & & & & & & & & \\
\hline & & & & & & & & & & \\
\hline & & & & & & & & & & \\
\hline & & & & & & & & & & \\
\hline & & & & & & & & & & \\
\hline$-\ldots$ & & & & & & & & & & \\
\hline & & & & & & & & & & \\
\hline 焉 & & & & & & & & & & \\
\hline 而 & & & & & & & & & & \\
\hline$N \underset{\sim}{\infty}$ & & & & & & & & & & \\
\hline 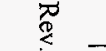 & & & & & & & & & & \\
\hline o & & & & & & & & & & \\
\hline & & & & & & & & & & \\
\hline & & & & & & & & & & \\
\hline & & & & & & & & & & \\
\hline
\end{tabular}




\section{Fluor Daniel Northwest}

\section{DESIGN ANALYSIS}

client Numatec Hanford Corporation

subject AY-102 Demand Load Analysis, Size

Feeders, Voitage Drop, and Fault Study

Location 200E, AY Tank Farm
Calc. No. W320-31-016

Revision 1

Page No. 1 of 3
Wo/Job No. Task Order N09

Date $8 / 3 / 95$

By TJ Kasnick

Checked $8 / 7 / 95$

Reviséd $7 / 24 / 98$
By SA Najjar

By TJ Kasnick

\section{OBJECTIVE}

Determine the size of electrical distribution equipment necessary to support the loads required for Project W320, Tank 241-C-106 Sluicing, at Tank AY-102.

2. DESIGN INPUTS

\subsection{Criteria and Source}

Function Design Criteria For Tank 241-C-106 Waste RetrievaT, Project W-320, HNF-SD-W320-FDC-001 Rev. 5.

\subsection{Given or Known Data}

The loads are shown on drawing H-2-818693, Sheet 1, Rev. 1. The size of motors and other equipment data are based on the Project W320 vendor information file $\# 22668$.

\subsubsection{The Variable Speed Drives (VSD) are 95\% efficient.}

2.2.2 Motor currents, Full Load Amperes (FLA), which are based on the 1993 National Electrical Code (NEC) for the motor nameplate values except for the $250 \mathrm{HP}$ motor which is based on vendor information, are tabulated as follows:

1) In-Line Booster pump ( $\mathrm{P}-0622)$, at $250 \mathrm{HP}(273 \mathrm{~A})$, driven by VSD.

2) Submersible (Immersible) pump (P-0621), at $40 \mathrm{HP}$ (52 A), driven by a soft starter.

3) Winch (W-0621), at $1.5 \mathrm{HP}(2.6 \mathrm{~A})$.

4) Pit O2E Chiller, at 10 amps.

2.2.3 Mini Power Panel is loaded to the 15 KVA transformer rating.

\subsection{Assumptions}

2.3.1 Transformer impedances used are typical NEMA three phase values.

2.3.2 The utility fault current values are assumed typical values derived from previous projects and data obtained from the utilities department.

HNF-2487, Rev. 0

Page F-3 


\section{Fluor Daniel Northwest}

\section{DESIGN ANALYSIS}

client Numatec Hanford Corporation

subject AY-102 Demand Load Analysis, Size

Feeders, Voltage Drop, and Fault Study

Location 200E, AY Tank Farm
Calc. No. W320-31-016

Revision 1

Page No. 2 of 3
wo/Job No. Task Order N09

Date 8/3/95 By TJ Kasnick

Checked 8/7/95 By SA Najjar

Revised $7 / 24 / 98$

By TJ Kasnick

2.4 Method to be Used

2.4.1 The FDNW approved (for design use) software: SKM Power Tools, version 3.4.0.1, 1995-1997.

\subsection{References/Sources}

2.5.1 Drawing H-2-818693, Sheet 1, Rev. 1, Electrical AY-Farm One-Line Diagram

2.5.2 NFPA 70, National Electrical Code (NEC), 1993 edition.

2.5.3 ANSI C2, National Electrical Safety Code, (NESC) 1990.

2.5.4 U.S. Department of Energy (DOE), order DOE 6430.1A, General Design Criteria

2.5.5 Vendor information fite \#22668.

3. CALCULATIONS

3.1 Attachment A: Input Report: This is the feeder, transformer, and generation input data that is used by the studies below.

3.2 Attachment B: Demand Load Analysis: This analysis calculates the total system loads based upon system bus load inputs and summarizes them on a bus by bus basis, including total system load and power factor.

3.3 Attachment C: Size Feeders and Transformers: This analysis provides the NEC Code sized feeders and transformers to meet the minimum load requirements of the Demand Load Analysis Study - Total Connected Load, and the evaluation of a fixed-size feeder to the MCC. Impedance and capacity information for the feeders and transformers were taken directly from the Dapper 1ibrary, which represents typical values found in the industry. Conduit sizes determined are the minimum size required.

3.4 Attachment D: Load Flow and Voltage Drop: This analysis calculates the exact system operating voltages, loads flows, and system losses.

3.5 Attachment E: Short Circuit AnaTysis: This analys is calculates the system short circuit fault currents. The calculations are based upon typical Hanford $13.8 \mathrm{Kv}$ utility fault current contributions, plus those from any motors running within the system. 


\section{Fluor Daniel Northwest}

DESIGN ANALYSIS

client Numatec Hanford Corporation

subject AY-102 Demand Load Analysis, Size

Feeders, Voltage Drop, and Fault Study

Location 200E, AY Tank Farm calc. No. W320-31-016

Revision 1

Page No. 3 of 3

h0/Job No. Task Order NO9

Date $8 / 3 / 95$

By TJ Kasnick

Checked $8 / 7 / 95$

By SA Najjar

Revised $7 / 24 / 98$

By TJ Kasnick

4. FINDINGS AND CONCLUSIONS

4.1 Refer to Attachment $C$ for system equipment sizing. Refer to other Attachments for various design information related to the distribution system equipment.

4.2 Refer to drawing $\mathrm{H}-2-818693$ for one-line diagram of the electrical distribution equipment necessary to support the loads. 
Ca7c. No. W-320-31-016

Revision 1

Attachment A

HNF-2487, Rev. 0

Page F-6 
Calc. No. W-320-31-016

Revision 1

Page No. A - 1

Project W-320 / Tank 241-C-106 sluicing

AY-Farm Electrical Distribution

Calc. No. W320-31-016 (Reference Drawing H-2-818693, SH 1, Rev. 1)

Fri Jul 24 08:35:17 1998

ALL INFORMATION PRESENTED IS FOR REVIEW, APPROVAL

INTERPRETATION AND APPLICATION BY A REGISTERED ENGINEER ONLY

SKM POWER*TOOLS FOR WINDOWS

INPUT DATA REPORT

COPYRIGHT SKM SYSTEMS ANALYSIS, INC. 1995-1997

ALL PU VALUES ARE EXPRESSED ON A 100 MVA BASE.

HNF-2487, Rev. 0

Page F-7 
Caic. No. W-320-31-016

Revision 1

Page No. A - 2

Fri Ju1 24 08:35:17 1998

Project W-320 / Tank 241-C-106 Sluicing

Page 2

AY-Farm Electrical Distribution

Calc. No. W320-31-016 (Reference Drawing H-2-818693, SH 1, Rev. 1)

FEEDER INPUT DATA

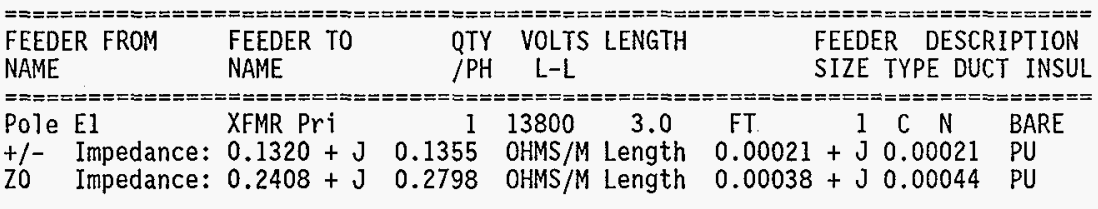

AY-PDP-1 $800 \mathrm{~A} \quad 2 \quad 480 \quad 50.0$

$+/-$ Impedance: $0.0390+J 0.0500$ OHMS/M Length

FT 350

$0.4232+\mathrm{J}$

C $M$

THWN

Z0 Impedance: $0.1229+\mathrm{J} 0.1231$ OHMS/M Length

$1.33+\mathrm{J}$

0.5425

$\mathrm{PU}$

AY-PDP-1 800A AY102-PP1 Bus 1

$+1-$ Impedance: $0.3000+J 0.0600$

$480 \quad 50.0$

FT

OHMS/M Length

$6.51+j$

1.34

PU

20 Impedance:

0.1478

OHMS/M Length

$20.52+J$

C M THWN

AY-PDP-1 800A Tap Bus Asmbty $2480 \quad 50.0$

+ - Impedance: $0.0390+\mathrm{J} 0.0500$ OHMS/M Length

ZO Impedance: $0.1229+\mathrm{J} 0.1231$ OHMS $/ M$ Length

FT $\quad 350$

$0.4232+\mathrm{J}$

1.30

PU

\section{Group CntTr}

Pit Chillr Bus

$+/-$ Impedance: $1.14+\mathrm{J}$

$480 \quad 110.0$

$1.33+\mathrm{J}$

3.21

PU

ZO Impedance: $\quad 3.59+\mathrm{J} 0.1552$

OHMS/M Length

FT

OHMS/M Length

$54.43+\mathrm{J}$

C $M$

THWN

Tap Bus Asmbly Group Cntir

$+/-$ Impedance: $0.1000+\mathrm{J}$

1

0.0540

$480 \quad 2.0$

OHMS/M Length

$171.53+$

0.5425

PU

1.34

PU

zo Impedance:

$0.3152+\mathrm{J}$

Group Cntlr Imrs SS

+1- Impedance: $0.1200+\mathrm{J}$

Z0 Impedance: $0.3782+\mathrm{J}$

Group Cntlr winch Bus

$+/-$ Impedance: $1.90+\mathrm{J}$

ZO Impedance: $5.99+\mathrm{J}$

0.1330

OHMS/M Length

$1480 \quad 1.000$

0.0550 OHMS/M Length

0.1355 OHMS/M Length

$480 \quad 120.0$

OHMS/M Length

OHMS/M Length

0.0680

0.1675

P-0622 Bus

Boostr VSD

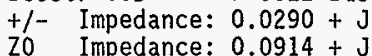

$\begin{array}{rcc}1 & 480 & 120.0 \\ 0.0480 & \text { OHMS } / M & \text { Length } \\ 0.1182 & \text { OHMS } / M \text { Length }\end{array}$
FT $2 / 0$

$0.0858+\mathrm{J}$

$0.2736+\mathrm{J}$

FT

$0.0521+\mathrm{J}$

$1 / 0$

$0.1641+\mathrm{J}$

C M THHN

0.0239 PU

0.0588 PU

FT

$98.96+\mathrm{J}$

$311.87+\mathrm{J}$

C M THHN

3.54 PU

$8.72 \mathrm{PU}$

FT

500
$1.51+\mathrm{J}$
$4.76+\mathrm{J}$
C M THWN

$2.50 \mathrm{PU}$

$6.16 \mathrm{PU}$

HNF-2487, Rev. 0

Page F-8 
Calc. No. W-320-31-016

Revision 1

Page No. A - 3

Fri JuT 24 08:35:18 1998

Project $W-320$ / Tank 241-C-106 Sluicing

AY-Farm Electrical Distribution

Calc. No. W320-31-016 (Reference Drawing H-2-818693, SH 1, Rev. 1)

FEEDER INPUT DATA

\begin{tabular}{|c|c|c|c|c|c|c|c|c|}
\hline \multirow[t]{2}{*}{$\begin{array}{l}\text { FEEDER FROM } \\
\text { NAME. }\end{array}$} & \multirow{2}{*}{$\begin{array}{l}\text { FEEDER TO } \\
\text { NAME } \\
============\end{array}$} & \multirow{2}{*}{$\begin{array}{l}\text { QTY } \\
/ P H\end{array}$} & \multirow{2}{*}{\multicolumn{2}{|c|}{$\begin{array}{l}\text { VOLTS LENGTH } \\
\text { L-L }\end{array}$}} & \multicolumn{4}{|c|}{$\begin{array}{l}\text { FEEDER DESCRIPTION } \\
\text { SIZE TYPE DUCT INSU }\end{array}$} \\
\hline & & & & & & & $=== \pm===$ & \\
\hline $\begin{array}{ll}\text { nrs } & \text { SS } \\
- & \text { Impedance: }\end{array}$ & $\begin{array}{l}\text { P-0621 Bus } \\
0.1200+J \\
0.3782+J\end{array}$ & $\begin{array}{r}1 \\
0.0550 \\
0.1355\end{array}$ & $\begin{array}{c}480 \\
\text { OHMS } / M \\
\text { OHMS } / M\end{array}$ & $\begin{array}{l}200.0 \\
\text { Length } \\
\text { Length }\end{array}$ & $\begin{array}{l}\mathrm{FT} \\
10 \\
32\end{array}$ & $\begin{array}{l}1 / 0 \\
+J \\
+J\end{array}$ & $\begin{array}{r}C M \\
4.77 \\
11.76\end{array}$ & $\begin{array}{l}\text { THWN } \\
\text { PU } \\
\text { PU }\end{array}$ \\
\hline $\begin{array}{ll}\text { ap } & \text { Bus Asmbly } \\
/- & \text { Impedance: } \\
0 & \text { Impedance: }\end{array}$ & $\begin{array}{l}\text { Boostr VSD } \\
0.0290+J \\
0.0914+J\end{array}$ & $\begin{array}{r}I \\
0.0480 \\
0.1182\end{array}$ & $\begin{array}{c}480 \\
\text { OHMS } / M \\
\text { OHMS } / M\end{array}$ & $\begin{array}{l}10.0 \\
\text { Length } \\
\text { Length }\end{array}$ & $\begin{array}{l}\text { FT } \\
0.1259 \\
0.3967\end{array}$ & $\begin{array}{l}500 \\
+J \\
+J\end{array}$ & $\begin{array}{l}C \quad M \\
0.2083 \\
0.5131\end{array}$ & $\begin{array}{l}\text { THWN } \\
\text { PU } \\
\text { PU }\end{array}$ \\
\hline
\end{tabular}

HNF-2487, Rev. 0

Page F-9 
Calc. No. W-320-31-016 Revision 1

Page No. A - 4

Fri JuT 24 08:35:18 1998

Project $W-320$ / Tank 241-C-106 Sluicing

Page 4

AY-Farm Electrical Distribution

Ca]c. No. W320-31-016 (Reference Drawing H-2-818693, SH 1, Rev. 1)

TRANSFORMER INPUT DATA

\begin{tabular}{|c|c|c|c|c|c|}
\hline $\begin{array}{l}\text { PRIMARY RECORD } \\
\text { NO NAME }\end{array}$ & $\begin{array}{c}\text { VOLTS } \\
L-L\end{array}$ & $\begin{array}{c}\text { * SECONDARY RECORO } \\
\text { NO NAME }\end{array}$ & $\begin{array}{l}\text { VOLTS } \\
L-L\end{array}$ & $\begin{array}{l}\text { FULL-LOAD } \\
\text { KVA }\end{array}$ & $\begin{array}{l}\text { NOMINAL } \\
\text { KVA }\end{array}$ \\
\hline 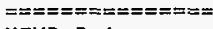 & $===== \pm= \pm==0=$ & 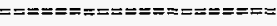 & $:=== \pm==0$ & 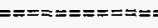 & $===\approx===$ \\
\hline $\begin{array}{l}\text { XFMR Pri } \\
\text { Pos. Seq. Z\%: } \\
\text { Zero Seq. Z\%: }\end{array}$ & D $\begin{array}{l}13800.0 \\
1.94+J \\
1.94+J\end{array}$ & 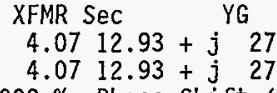 & $\begin{array}{l}480.00 \\
13 \mathrm{PU} \\
13 \mathrm{PU}\end{array}$ & 172.50 & 150.00 \\
\hline
\end{tabular}

HNF-2487, Rev. 0

Page F-10 
Calc. No. W-320-31-016 Revision 1

Page No. A - 5

Fri JuT 24 08:35:18 1998

Project W-320 / Tank 241-C-106 Sluicing

Page 5

AY-Farm Electrical Distribution

Calc. No. W320-31-016 (Reference Drawing H-2-818693, SH 1, Rev. 1)

GENERATION DATA

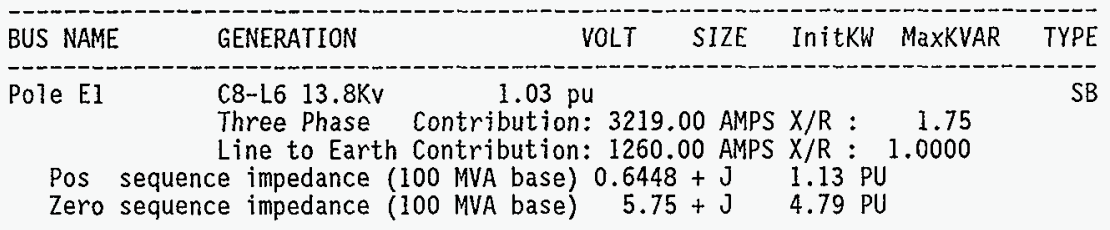

HNF-2487, Rev. 0

Page F-11 
Calc. No. W-320-31-016

Revision 1

Attachment B

HNF-2487, Rev. 0

Page F-12 
Calc. No. W-320-31-016

Revision 1

Page No. B - I

Project W-320 / Tank 241-C-106 S7uicing

AY-Farm Electrical Distribution

Calc. No. W320-31-016 (Reference Drawing H-2-818693, SH 1, Rev. 1)

DATE: $\quad 7: 24: 1998$

TIME: $8: 34: 53.83$

ALL INFORMATION PRESENTED IS FOR REVIEW, APPROVAL, INTERPRETATION AND APPLICATION BY A REGISTERED ENGINEER ONLY

SKM POWER*TOOLS FOR WINDOWS

DEMAND LOAD ANALYSIS REPORT

COPYRIGHT SKM SYSTEMS ANALYSIS, INC. 1995-1996

*** SOLUTION COMMENTS ***

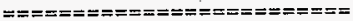

LOAD ANALYSIS INCLUDES ALL LOADS.

HNF-2487, Rev. 0

Page F-13 
Calc. No. W-320-31-016

Revision 1

Page No. B - 2

DATE: $7: 24: 1998$ TIME: $8: 34: 53.83$

PAGE 2

Project $W-320$ / Tank 241-C-106 Sluicing

AY-Farm Electrical Distribution

Calc. No. W320-31-016 (Reference Drawing H-2-818693, SH 1, Rev. 1)

LOAD SUMMARY

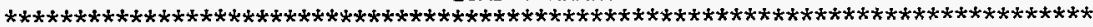

LOAD SCHEDULE FOR POIe E1

SOURCE OF PWR SOURCE BUS

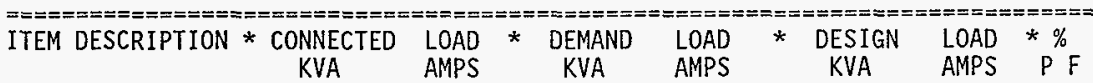

$============$
END USE LOADS

BRANCH LOADS

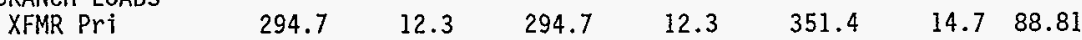

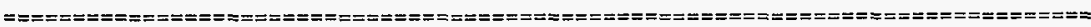

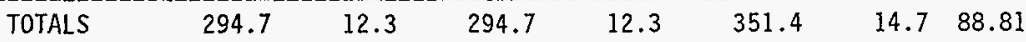

LOAD SCHEDULE FOR XFMR Pri

13800. VOLTS LINE TO LINE

SOURCE OF PWR POI El

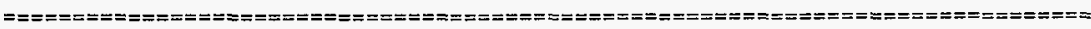

ITEM DESCRIPTION * CONNECTED LOAD * DEMAND LOAD * DESIGN LOAD * \%

KVA AMPS KVA AMPS KVA AMPS PF

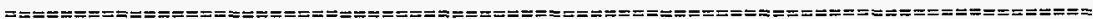

END USE LOADS

BRANCH LOADS

$\begin{array}{llllllll}\text { XFMR Sec } & 294.7 & 12.3 & 294.7 & 12.3 & 351.4 & 14.7 & 88.81\end{array}$

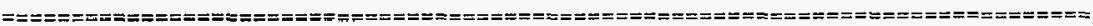

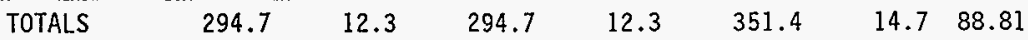

LOAD SCHEDULE FOR XFMR SEC

SOURCE OF PWR XFMR Pri

480. VOLTS LINE TO LINE

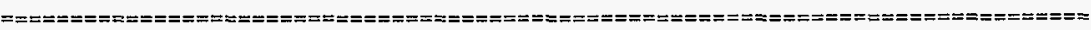

ITEM DESCRIPTION * CONNECTED LOAD * DEMAND LOAD * DESIGN LOAD *\%

KVA AMPS KVA AMPS KVA AMPS P F

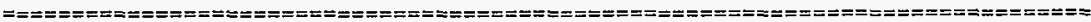

END USE LOADS

BRANCH LOADS

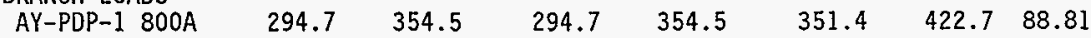

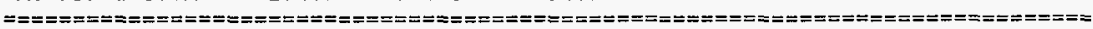
$\begin{array}{llllllll}\text { TOTALS } & 294.7 & 354.5 & 294.7 & 354.5 & 351.4 & 422.7 & 88.81\end{array}$

LOAD SCHEDULE FOR AY-PDP-1 $800 \mathrm{~A}$

SOURCE OF PWR . XFMR SEC

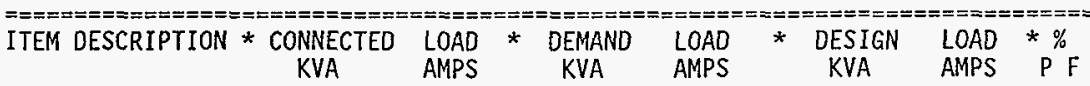

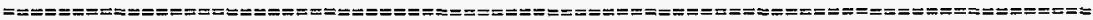

END USE LOADS

BRANCH LOADS

$\begin{array}{lrrrrrrr}\text { Tap Bus Asmbly } & 279.9 & 336.7 & 279.9 & 336.7 & 336.6 & 404.9 & 89.14\end{array}$

$\begin{array}{lrrrrr}\text { AYI02-PPI Bus } \quad 15.0 \quad 18.0 \quad 15.0 & 18.0 & 15.0 & 18.0 & 80.00\end{array}$

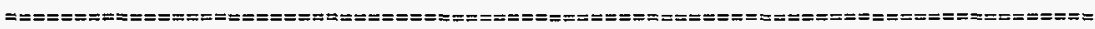

$\begin{array}{llllllll}\text { TOTALS } & 294.7 & 354.5 & 294.7 & 354.5 & 351.4 & 422.7 & 88.81\end{array}$

HNF-2487, Rev. 0

Page F-14 
Calc. No. W-320-31-016

Revision 1

Page No. B - 3

DATE: $7: 24: 1998$ TIME: $8: 34: 53.83$

Project $W-320$ / Tank 241-C-106 Sluicing

AY-Farm Electrical Distribution

CaTc. No. W320-31-016 (Reference Drawing H-2-818693, SH 1, Rev. 1)

LOAD SUMMARY

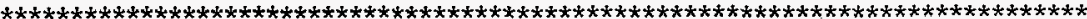

LOAD SCHEDULE FOR Tap BUS Asmbly

480. VOLTS LINE TO LINE

SOURCE OF PWR AY-PDP-1 800A

\begin{tabular}{|c|c|c|c|c|c|c|c|}
\hline 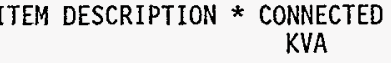 & $\begin{array}{l}\text { LOAD } \\
\text { AMPS }\end{array}$ & $*$ & $\begin{array}{l}\text { DEMAND } \\
\text { KVA }\end{array}$ & $\begin{array}{l}\text { LOAD } \\
\text { AMPS }\end{array}$ & $\star$ & $\begin{array}{l}\text { DESIGN } \\
\text { KVA }\end{array}$ & $\begin{array}{l}\text { LOAD } * \% \\
\text { AMPS } \quad \text { P F }\end{array}$ \\
\hline
\end{tabular}

$===:======= \pm=$
END USE LOADS

BRANCH LOADS

Group Cnt7r

Boostr VSD

53.6

54.4

53.6

64.4

64.4

$77.4 \quad 81.70$

$============$

$227.0 \quad 273.0$

227.0

273.0

283.7

$341.2 \quad 90.30$

TOTALS

$279.9 \quad 336.7$

$279.9 \quad 336.7$

$336.6 \quad 404.9$

89.14

LOAD SCHEDULE FOR Pit Chillr Bus

SOURCE OF PWR Group Cnt?r

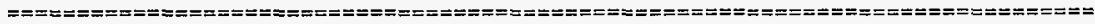

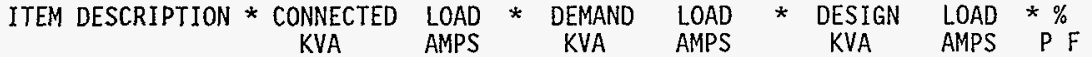

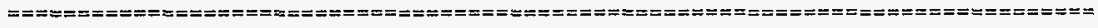

END USE LOADS

GENERAL LOADS

$8.3 \quad 10.0$

8.3

10.0

8.3

$10.0 \quad 90.00$

TOTALS

$8.3 \quad 10.0$

8.3

10.0

8.3

$10.0 \quad 90.00$

LOAD SCHEDULE FOR Group Cntir

SOURCE OF PUR Tap Bus Asmbly

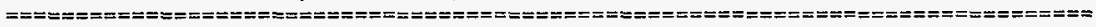

ITEM DESCRIPTION * CONNECTED LOAD * DEMAND LOAD * DESIGN LOAD * \%

KVA AMPS KVA AMPS KVA AMPS P F

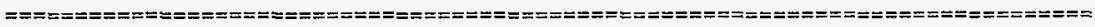

END USE LOADS

BRANCH LOADS

\begin{tabular}{|c|c|c|c|c|c|c|c|}
\hline $\begin{array}{l}\text { Pit Chilir Bus } \\
\text { Imrs SS } \\
\text { Winch Bus }\end{array}$ & $\begin{array}{r}8.3 \\
43.2 \\
2.2\end{array}$ & $\begin{array}{r}10.0 \\
52.0 \\
2.6\end{array}$ & $\begin{array}{r}8.3 \\
43.2 \\
2.2\end{array}$ & $\begin{array}{r}10.0 \\
52.0 \\
2.6\end{array}$ & $\begin{array}{r}8.3 \\
54.0 \\
2.7\end{array}$ & $\begin{array}{r}10.0 \\
65.0 \\
3.2\end{array}$ & $\begin{array}{l}90.00 \\
80.30 \\
80.00\end{array}$ \\
\hline & & & & & & & \\
\hline
\end{tabular}

53.6

64.4

64.4

$77.4 \quad 81.70$

HNF-2487, Rev. 0

Page F-15 
DATE: $7: 24: 1998$ TIME: $8: 34: 53.83$

Calc. No. W-320-31-016

Revision 1

Page No. $8-4$

Project $W-320$ / Tank 241-C-106 Sluicing

PAGE $\quad 4$

AY-Farm Electrical Distribution

Calc. No. W320-31-016 (Reference Drawing H-2-818693, SH 1, Rev. 1)

LOAD SUMMARY

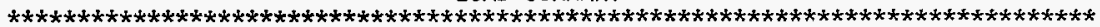

LOAD SCHEDULE FOR ImrS SS

SOURCE OF PWR Group Cnt7r

480. VOLTS LINE TO LINE

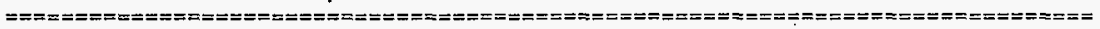
ITEM DESCRIPTION * CONNECTED LOAD * DEMAND LOAD * DESIGN LOAD * \% KVA AMPS KVA AMPS KVA AMPS PF

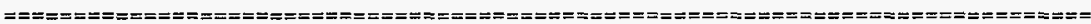
END USE LOAOS

BRANCH LOADS

P-0621 Bus

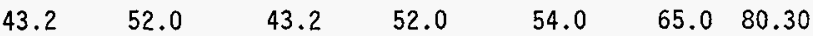

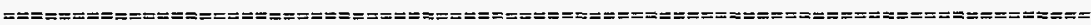

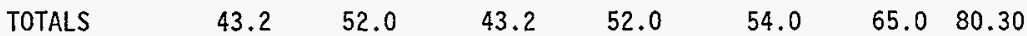

LOAD SCHEDULE FOR Winch Bus

SOURCE OF PWR Group Cntlr

480. VOLTS LINE TO LINE

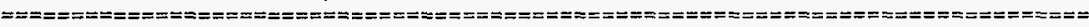

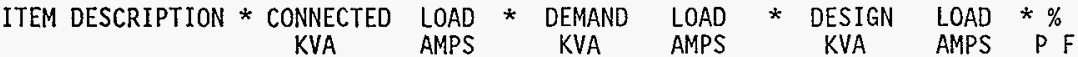

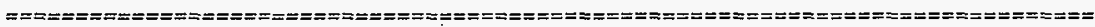

END USE LOADS

LARGEST KVA MTR

$2.2 \quad 2.6$

2.2

2.6

2.7

3.280 .00

TOTALS

2.2

2.6

2.2

2.6

2.7

3.280 .00

LOAD SCHEDULE FOR AYIO2-PPI BUS

SOURCE OF PWR AY-PDP-1 $800 \mathrm{~A}$

480. VOLTS LINE TO LINE

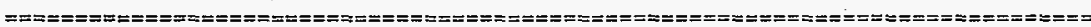

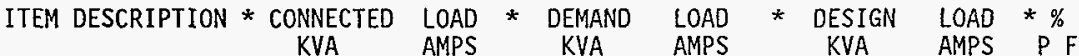

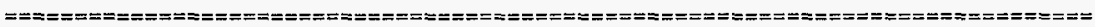
END USE LOADS

GENERAL LOADS

$15.0 \quad 18.0$

15.0

18.0

15.0

$18.0 \quad 80.00$

$==\approx=======$

$15.0 \quad 18.0$

$15.0 \quad 18.0$

15.0

$18.0 \quad 80.00$

LOAD SCHEDULE FOR Boostr VSD SOURCE OF PWR Tap Bus Asmbly

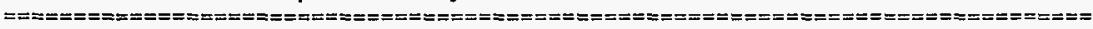

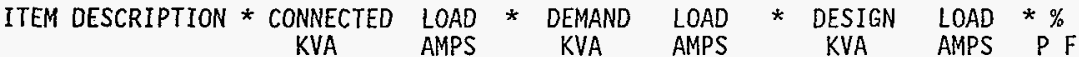

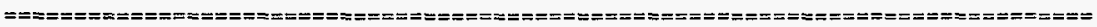
END USE LOADS

BRANCH LOADS

P-0622 BUS

$227.0 \quad 273.0$

227.0

273.0

283.7

$341.2 \quad 90.30$

TOTALS

$227.0 \quad 273.0$

227.0

273.0

283.7

$341.2 \quad 90.30$

HNF-2487, Rev. 0

Page F-16 
Calc. No. W-320-31-016

Revision 1

Page No. B - 5

DATE: $7: 24: 1998$ TIME: $8: 34: 53.83$

PAGE

5

Project W-320 / Tank 241-C-106 Sluicing

AY-Farm Electrical Distribution

Calc. No. W320-31-016 (Reference Drawing H-2-818693, SH 1, Rev. 1)

LOAD SUMMARY

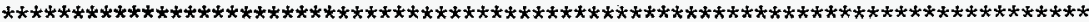

LOAD SCHEDULE FOR P-0622 BUS

480. VOLTS LINE TO LINE

SOURCE OF PWR

Boostr VSD

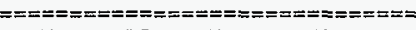

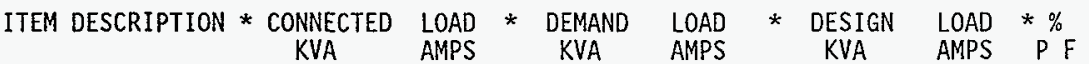

END USE LOADS

LARGEST KVA MTR

227.0

273.0

227.0

273.0

283.7

$341.2 \quad 90.30$

TOTALS

227.0

273.0

227.0

273.0

283.7

341.290 .30

LOAD SCHEDULE FOR

SOURCE OF PWR

P-0621 Bus

480. VOLTS LINE TO LINE

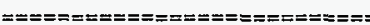

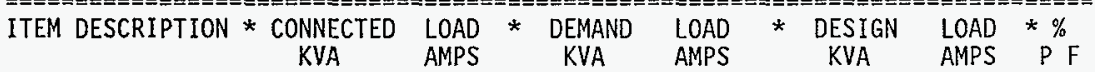

$=====\pi===== \pm$
END USE LOADS

LARGEST KVA MTR

43.2

52.0

43.2

52.0

54.0

$65.0 \quad 80.30$

TOTALS

43.2

52.0

43.2

52.0

54.0

$65.0 \quad 80.30$

HNF-2487, Rev. 0

Page F-17 
DATE: $7: 24: 1998$ TIME: $8: 34: 53.83$

Project $W-320$ / Tank $24 I-C-106$ sluicing

Calc. No. W-320-31-016

Revision 1

Page No. $8-6$

AY-Farm Electrical Distribution

Calc. No. W320-31-016 (Reference Drawing H-2-818693, SH 1, Rev. 1)

PAGE 6

TOTAL SOURCE LOAD SUMMARY

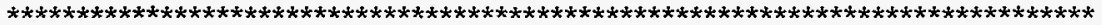

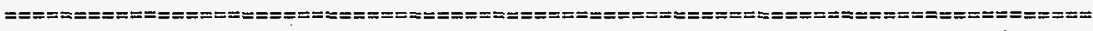

LOAD DESCRIPTION
TYPE $\begin{array}{ccccc}\text { UNITS } & \begin{array}{c}\text { CONNECTED } \\ \text { LOAD }\end{array} & \begin{array}{c}\text { DEMAND } \\ \text { LOAD }\end{array} & \begin{array}{c}\text { DESIGN } \\ \text { LOAD }\end{array} & \begin{array}{c}\text { POWER FACTOR } \\ \%\end{array}\end{array}$

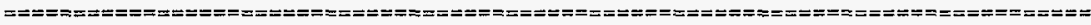

GENERAL LOADS

$\mathrm{KW}$

19.5

19.5

12.6

12.6

19.5

KVAR

23.2

23.2

12.6

KVA

36.4

36.4

27.0

83.92 LAGGING

KVA TYPE MTR

$\begin{array}{llll}\text { KW } & 36.4 & 36.4 & 36.4 \\ \text { KVAR } & 27.0 & 27.0 & 27.0 \\ \text { KVA } & 45.4 & 45.4 & 45.4\end{array}$

80.29 LAGGING

LARGEST KVA MTR

$\mathrm{KW}$

KVAR

204.9

97.5

204.9

256.2

KVA

227.0

97.5

121.9

283.7

90.30 LAGGING

TOTAL LOADS

$\mathrm{KW}$

KVAR

KVA

$\%$ PF

\section{8}

137.2

294.7

88.5

LAGGING

227.0

312.1

161.6

351.4

88.8

294.7

88.5

LAGGING

LAGGING

HNF-2487, Rev. 0

Page F-18 
Calc. No. W-320-31-016

Revision 1

Page No. B -7

DATE: $7: 24: 1998$ TIME: $8: 34: 53.83$

PAGE 7

Project $W-320$ / Tank 241-C-106 Sluicing

AY-Farm Electrical Distribution

Calc. No. W320-31-016 (Reference Drawing H-2-818693, SH 1, Rev. 1)

LOAD DEMAND TABLE

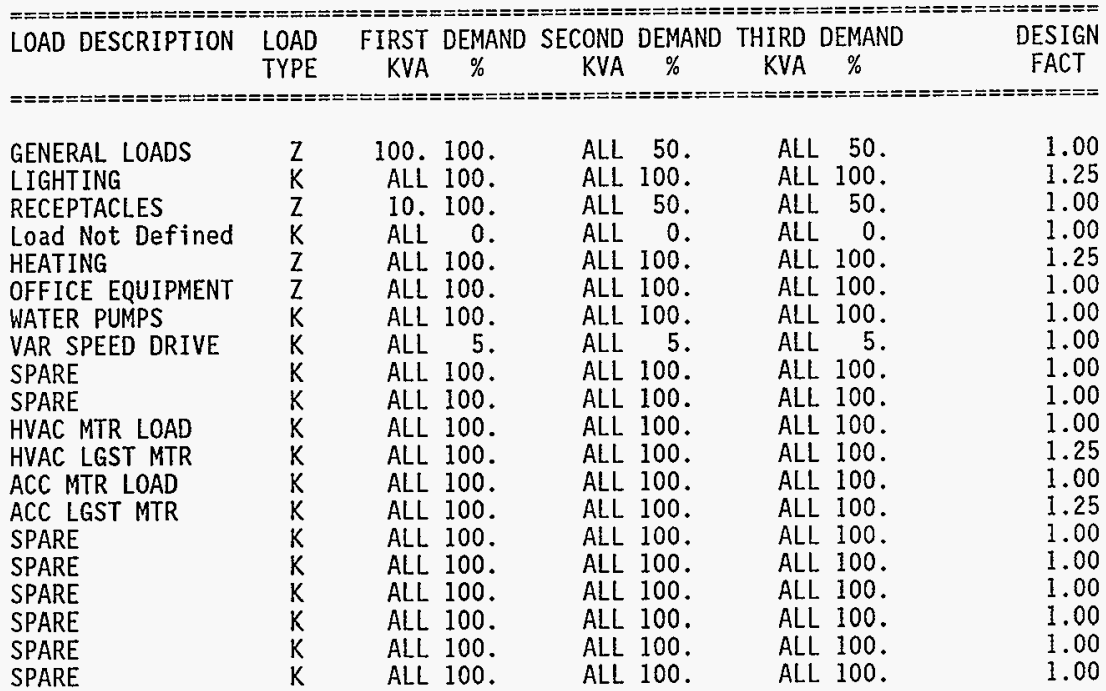

NOTES: LARGEST MOTOR CIRCUIT IDENTIFIED, AND USED TO CALCULATE DEMAND LOAD BASED ON NEC ART 430 DEMAND AND DESIGN FACTORS APPLIED AT EACH LOAD BUS

AND ALL LOAD TOTALS ARE POWER FACTOR CORRECTED

DESIGN LOAD VALUES BASED ON MOTOR RUNING OR STARTING CONDITION

HNF-2487, Rev. 0

Page $F-19$ 
Calc. No. W-320-31-016

Revision I

Attachment C

HNF-2487, Rev. O

Page F-20 
Calc. No. W-320-31-016

Revision 1

Page No. C - 1

Project $W-320$ / Tank 241-C-106 S7uicing

AY-Farm Electrical Distribution

Calc. No. W320-31-016 (Reference Drawing H-2-818693, SH 1, Rev. 1)

DATE: $\quad 7: 24: 1998$

TIME： $8: 34: 59.76$

ALL INFORMATION PRESENTED IS FOR REVIEW, APPROVAL, INTERPRETATION

AND APPLICATION BY A REGISTERED ENGINEER ONLY

SKM POWER*TOOLS FOR WINDOWS

FEEDER AND TRANSFORMER SIZING REPORT

COPYRIGHT SKM SYSTEMS ANALYSIS, INC. 1995-1996

HNF-2487, Rev. 0

Page F-21 
Calc. No. W-320-31-016

Revision 1

Page No. C - 2

DATE $7: 24: 1998$ TIME: $8: 34: 59.76$

PAGE

2

Project $W-320$ / Tank 24l-C-106 STuicing

AY-Farm Electrical Distribution

Calc. No. W320-31-016 (Reference Drawing H-2-818693, SH 1, Rev. 1)

FEEDER AND TRANSFORMER STUDY CRITERIA

SIZE AND REPORT, DO NOT CHANGE THE DATA BASE

BRANCH VOLTAGE DROP CRITERIA $3.00 \%$

VOLTAGE DROP CALCULATIONS ARE PRELIMINARY

EXECUTE VOLTAGE DROP AND LOAD FLOW STUDY FOR MORE ACCURATE RESULTS

PRIMARY/SECONDARY TRANSFORMER FDRS SIZED AT 125. \% OF TX FULL LOAD RATING

$* * *$ NOTICE *** FEEDER SIZED TO 125 . PERCENT OF TRANSFORMER SIZE

BRANCH FROM XFMR Pri TO XFMR SEC

TX KVA: 294.7 TR FLA: 12.3

MINIMUM FEEDER AMPACITY: $\quad 15.4$

$\star * *$ NOTICE $* * *$ FEEDER SIZED TO 125 . PERCENT OF TRANSFORMER SIZE

BRANCH FROM XFMR Pri TO XFMR SEC

TX KVA: 294.7 TR FLA: $\quad 354.5$

MINIMUM FEEDER AMPACITY: $\quad 443.1$

HNF-2487, Rev. 0

Page F-22 
Calc. No. W-320-31-016

Revision 1

Page No. C - 3

DATE 7:24:1998 TIME: $8: 34: 59.76$

PAGE 3

Project $W-320$ / Tank 24l-C-106 Sluicing

AY-Farm Electrical Distribution

Calc. No. W320-31-016 (Reference Drawing H-2-818693, SH 1, Rev. 1)

FEEDER SIZE REPORT

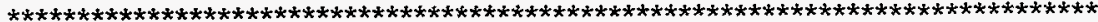

FEEDER SCHEDULE

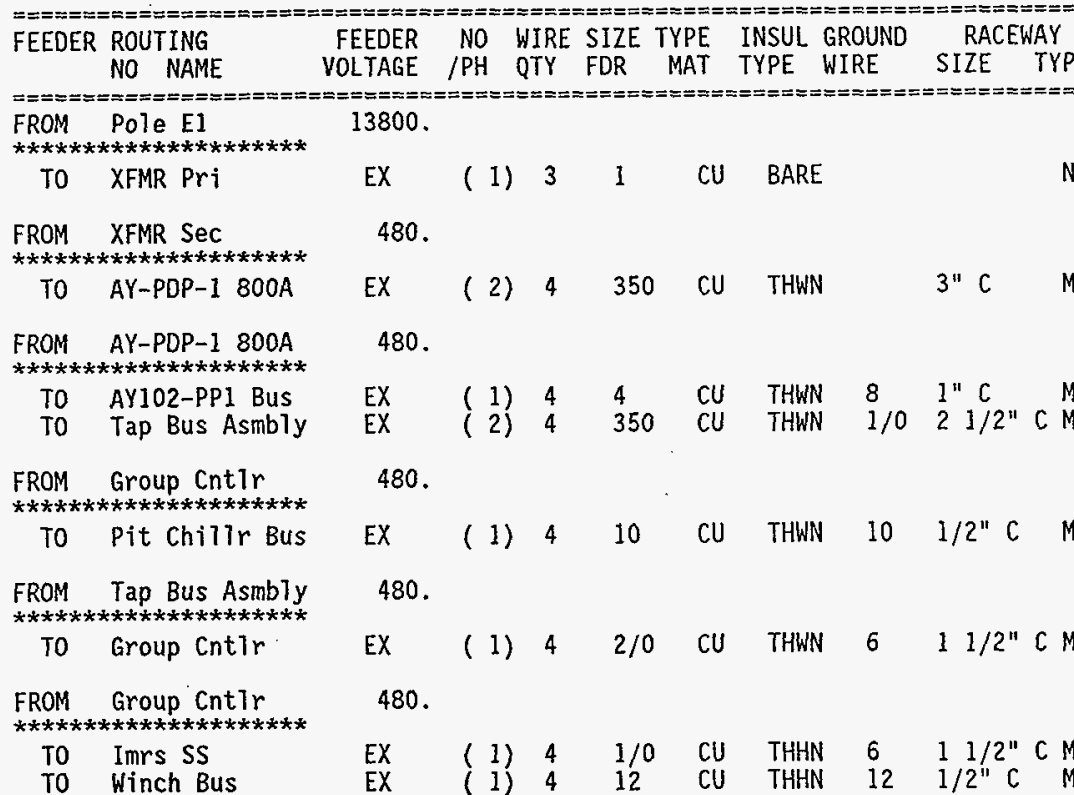

FROM Boostr VSD

$* \star * * * * * * * * * * * * * * * * * * * *$

T0 P-0622 Bus

FROM Imrs SS

$* * * * * * * * * * * * * * * * * * * * * *$

T0 P-0621 Bus

FROM Tap Bus Asmbiy $* * * * * * * * * * * * * * * * * * * * * *$

TO Boostr VSD
480.

EX

(1) 4

500

CU

THWN

$3 \quad 3 " C$

M

480.

EX (1) $4 \quad 1 / 0$ CU THWN $6 \quad 11 / 2 " C M$

480.

EX

(1) 4500

CU THWN 3 3"C

M
HNF-2487, Rev. 0

Page F-23 
Calc. No. W-320-31-016

Revision 1

Page No. C - 4

DATE $7: 24: 1998$ TIME: $8: 34: 59.76$

PAGE 4

Project $W-320$ / Tank 241-C-106 S7uicing

AY-Farm Electrical Distribution

Calc. No. W320-31-016 (Reference Drawing H-2-818693, SH 1, Rev. 1)

FEEDER EVALUATION

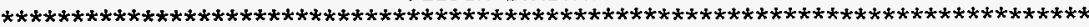

FEEDER DESI GN LOAD ANALYSIS

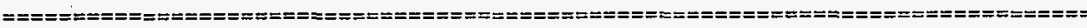
FEEDER ROUTING EXTG \% QTY SIZE FEEDER DESCRIPTION DESIGN DERATED NO NAME VD /PH FDR MAT INSUL AMBIENT LOAD CAPACITY

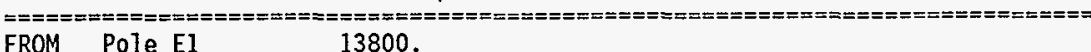

FROM Pole El 13800 .

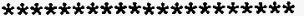

TO XFMR Pri EX 0.001 CU BARE 30. 15. A UNKNOWN $* * *$ WARNING $* * *$ ENGINEERING REVIEW OF FEEDER CAPACITY SUGGESTED

FROM XFMR Sec 480 .

$* * * * * * * * * * * * * * * * * * * * * * * *$

FROM AY-PDP-1 $800 \mathrm{~A} \quad 480$.

$* * * * * * * * * * * * * * * * * * * * * * * *$

TO AY102-PP1 BuS EX $0.09 \quad 1$

Tap Bus Asmbly EX 0.21

CU THWN 30. 18. A 70. A

CU THWN 30. 405. A 620. A

FROM Group Cntlr 480.

$* * * * * * * * * * * * * * * * * * * * * * * *$

TO Pit Chillr Bus EX 0.42

FROM Tap Bus Asmbly 480.

$* * * * * * * * * * * * * * * * * * * * * * *$

FROM Group Cntir 480

$* * * * * * * * * * * * * * * * * * * * * * * *$

TO Imrs SS EX $0.00 \quad 1$

TO Winch Bus

EX 0.221

CU THHN 30 .

65. A 150. A

CU THHN 70. 3. A 12. A

FROM Boostr VSD 480 .

$* * * * * * * * * * * * * * * * * * * * * * * *$

TO P-0622 Bus EX 0.69

FROM Imrs SS 480 .

$* * * * * * * * * * * * * * * * * * * * * * * * *$

TO P-0621 Bus EX 0.61

CU THWN 30. 65. A 150. A

FROM Tap Bus Asmbly 480.

************************

TO Boostr VSD

EX $0.06 \quad 1$

CU THWN 30, 341. A 380. A

HNF-2487, Rev. 0

Page F-24 
Calc. No. W-320-31-016

Revision 1

Page No. C - 5

DATE $7: 24: 1998$ TIME: $8: 34: 59.76$

PAGE 5

Project $\mathrm{W}-320$ / Tank 241-C-106 Sluicing

AY-Farm Electrical Distribution

Calc. No. W320-31-016 (Reference Drawing H-2-818693, SH 1, Rev. 1)

TRANSFORMER SIZE REPORT

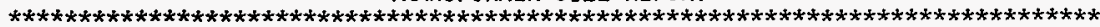

TRANSFORMER SCHEDULE

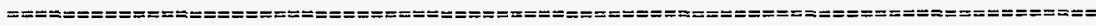
LOCATION DESCRIPTION VOLTAGE CONN PCT. TRANSFORMER DESCRIPTION

BUS NO. NAME

LEVELS CODE TAP

FROM XFMR Pri

TOr XFMR SEC

DEMAND LOAD:

294.7 KVA

13800. $\quad \mathrm{Y}_{\mathrm{H}}$

EX
$-5.0$

TYPE: OA
DESCRIPT

$4.51 \%$ Z NOMINAL RATING
$0.0 \mathrm{KVA}$

0.0 KVA

HNF-2487, Rev. 0

Page F-25 
CaTc. No. W-320-31-016

Revision 1

\section{Attachment D}

HNF-2487, Rev. 0

Page F-26 
Calc. No. W-320-31-016

Revision 1

Page No. D - 1

Project W-320 / Tank 241-C-106 Sluicing

AY-Farm Electrical Distribution

Calc. No. W320-31-016 (Reference Drawing H-2-818693, SH 1, Rev. 1)

DATE: $\quad 7: 24: 1998$

TIME: $8: 35: 19.70$

ALL INFORMATION PRESENTED IS FOR REVIEW, APPROVAL

INTERPRETATION AND APPLICATION BY A REGISTERED ENGINEER ONLY

SKM POWER*TOOLS FOR WINDOWS

LOAD FLOW AND VOLTAGE DROP ANALYSIS REPORT

COPYRIGHT SKM SYSTEMS ANALYSIS, INC. 1995-1996

HNF-2487, Rev. 0

Page F-27 
Calc. No. W-320-31-016

Revision 1

Page No. D - 2

DATE: $7: 24: 1998$ TIME: $8: 35: 19.70$

PAGE 2

Project $W-320$ / Tank 241-C-106 Sluicing

AY-Farm Electrical Distribution

Calc. No. W320-31-016 (Reference Drawing H-2-818693, SH 1, Rev. 1)

*** SOLUTION COMMENTS ***

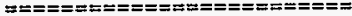

SOLUTION PARAMETERS

BRANCH VOLTAGE CRITERIA $: 3.00 \%$

BUS VOLTAGE CRITERIA : $5.00 \%$

ACCELERATION FACTOR FOR 'PV' GENERATORS : 1.00

ACCELERATION FACTOR FOR CONSTANT KVA LOADS: 1.00

EXACT(ITERATIVE) SOLUTION : YES

UTILITY IMPEDANCE

TRANSFORMER PHASE SHIFT

: YES

: NO

ALL PU VALUES ARE EXPRESSED ON A 100 MVA BASE

LOAD FLOW IS BASED ON CALCULATED DEMAND LOAD RESULTS

FROM THE DEMAND LOAD ANALYSIS STUDY.

LOAD ANALYSIS INCLUDES ALL LOADS.

<<PERCENT VOLTAGE DROPS ARE BASED ON NOMINAL DESIGN VOLTAGES〉>

SWING GENERATORS

SOURCE NAME

VOLTAGE ANGLE

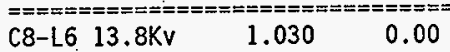

LARGEST LOAD:

CONVERGENCE CRITERIA:

LARGEST BUS MISMATCH

LARGEST BUS MISMATCH

LARGEST BUS MISMATCH

\begin{tabular}{ll}
\multicolumn{2}{c}{226.96 KVA } \\
P-0622 Bus & \\
P-0622 Bus & 0.681 KVA \\
P-0622 Bus & 0.057 KVA \\
& 0.005 KVA
\end{tabular}

HNF-2487, Rev. 0

Page F-28 
Calc. No. W-320-31-016

Revision 1

Page No. D - 3

DATE: $7: 24: 1998$ TIME: $8: 35: 19.70$

PAGE 3

Project W-320 / Tank 241-C-106 Sluicing

AY-Farm Electrical Distribution

Calc. No. W320-31-016 (Reference Drawing H-2-818693, SH 1, Rev. 1)

BALANCED VOLTAGE DROP AND LOAD FLOW ANALYSIS (SWING GENERATORS)

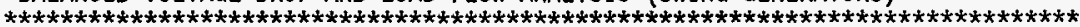

SOURCE VOLTAGE ANGLE KW KVAR VD\% (UTILITY IMPEDANCE)

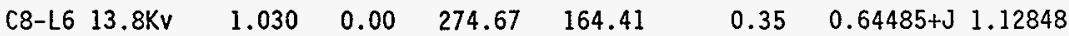

HNF-2487, Rev. 0

Page F-29 
Calc. No. W-320-31-016

Revision 1

Page No. D - 4

DATE: $7: 24: 1998$ TIME: $8: 35: 19.70$

PAGE 4

Project $W-320$ / Tank 24l-C-106 S7uicing

AY-Farm Electrical Distribution

Calc. No. W320-31-016 (Reference Drawing H-2-818693, SH 1, Rev. 1)

BALANCED VOLTAGE DROP AND LOAD FLOW ANALYSIS

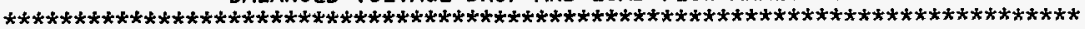

VOLTAGE EFFECT ON LOADS MODELED

VOLTAGE DROP CRITERIA: BRANCH $=3.00 \%$ BUS $=5.00$

$====$ BUS: Pole El DESIGN VOLTS: 13800 BUS VOLTS: $14165 \%$ VD: -2.65

$================== \pm===$ PU BUS VOLTAGE: 1.026 ANGLE: -0.1 DEGREES *** SWING GENERATOR: C8-L6 $13.8 \mathrm{KV} \quad 274.7 \mathrm{~kW} 164.4 \mathrm{KVAR}$

LOAD TO: XFMR Pri FEEDER AMPS: 13.0 VOLTAGE DROP: $0 . \%$ VD: 0.00 PROJECTED POWER FLOW: $274.0 \mathrm{KW} 163.3$ KVAR 319.0 KVA PF: 0.86 LAGGING LOSSES THRU FEEDER: $\quad 0.0 \mathrm{KH} \quad 0.0 \mathrm{KVAR} \quad 0.0 \mathrm{KVA}$

$====$ BUS: XFMR Pri DESIGN VOLTS: 13800 BUS VOLTS: $14165 \%$ VD: -2.65 $======0==============$ PU BUS VOLTAGE: 1.026 ANGLE: -0.1 DEGREES

LOAD FROM: PoTe El PROJECTED POWER FLOW: LOSSES THRU FEEDER:

LOAD TO: XFMR SEC PROJECTED POWER FLOW: LOSSES THRU TRANSF:

$== \pm=$ BUS: XFMR Sec

LOAD FROM: XFMR Pri

PROJECTED POWER FLOW: LOSSES THRU TRANSF:
FEEDER AMPS: 13.0 VOLTAGE DROP:

0 . \%VD: 0.00 $274.0 \mathrm{KW} 163.3 \mathrm{KVAR}$ 319.0 KVA PF: 0.86 LAGGING $0.0 \mathrm{KW} \quad 0.0 \mathrm{KVAR} \quad 0.0 \mathrm{KVA}$

TRANSF AMPS: 13.0 VOLTAGE DROP: 256. \%VD: 1.86 274.0 KW 163.3 KVAR 319.0 KVA PF: 0.86 LAGGING $11.3 \mathrm{KW} \quad 23.7 \mathrm{KVAR} \quad 26.2 \mathrm{KVA}$

DESIGN VOLTS: $\quad 480$ BUS VOLTS: $484 \%$ VD: -0.79 PU BUS VOLTAGE: 1.008 ANGLE: -2.9 DEGREES

LOAD TO: AY-PDP-1 800A FEEDER AMPS: 355.1 VOLTAGE DROP: $1 . \%$ VD: 0.19 PROJECTED POWER FLOW: $262.8 \mathrm{KW} 139.7 \mathrm{KVAR} 297.6 \mathrm{KVA}$ PF: 0.88 LAGGING LOSSES THRU FEEDER: $\quad 0.4 \mathrm{KW} \quad 0.5 \mathrm{KVAR} \quad 0.6 \mathrm{KVA}$

TRANSF AMPS: 12.1 VOLTAGE DROP: 9 . \%VD: 1.86 274.0 KW 163.3 KVAR 319.0 KVA PF: 0.86 LAGGING $11.3 \mathrm{KW} \quad 23.7 \mathrm{KVAR} \quad 26.2 \mathrm{KVA}$ 6.2 KVA

$==$ BUS: AY-PDP-I 800 A DESIGN VOLTS: 480 BUS VOLTS: $483 \%$ VD: -0.60 $=====================$ PU BUS VOLTAGE: 1.006 ANGLE: -3.0 DEGREES

LOAD FROM: XFMR Sec PROJECTED POWER FLOW: LOSSES THRU FEEDER:
FEEDER AMPS: 355.1 VOLTAGE DROP:

1. \%VD: 0.19 $262.4 \mathrm{KW}$ 139.2 KVAR $0.4 \mathrm{KW} \quad 0.5 \mathrm{KVAR}$ 297.0 KVA 0.6 KVA
0.88 LAGGING
1. \%VD: 0.18 LOAD TO: Tap Bus Asmbly FEEDER AMPS: 337.2 VOLTAGE DROP: PROJECTED POWER FLOW: $250.3 \mathrm{KW} 130.1 \mathrm{KVAR}$ LOSSES THRU FEEDER:
$0.3 \mathrm{KW}$

0.4 KVAR
282.1 KVA

$0.5 \mathrm{KVA}$
0.89 LAGGING 
Calc. No. W-320-31-016

Revision 1

Page No. D - 5

DATE: $7: 24: 1998$ TIME: $8: 35: 19.70$

PAGE 5

Project W-320 / Tank 241-C-106 Sluicing

AY-Farm Electrical Distribution

Calc. No. W320-31-016 (Reference Drawing H-2-818693, SH 1, Rev. 1)

BALANCED VOLTAGE DROP AND LOAD FLOW ANALYSIS

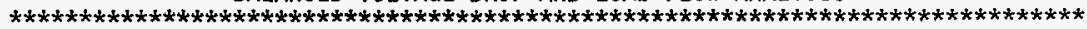

VOLTAGE EFFECT ON LOADS MODELED

VOLTAGE DROP CRITERIA: BRANCH $=3.00 \%$ BUS $=5.00$

LOAD TO: AY102-PP1 Bus FEEDER AMPS: 18.1 VOLTAGE DROP: $0 . \%$ VD: 0.09 PROJECTED POWER FLOW: $12.1 \mathrm{KW}$ 9.1 KVAR $15.2 \mathrm{KVA}$ PF: 0.80 LAGGING LOSSES THRU FEEDER: $\quad 0.0 \mathrm{KW} \quad 0.0 \mathrm{KVAR} \quad 0.0 \mathrm{KVA}$

$====$ BUS: Tap BuS Asmbly DESIGN VOLTS: 480 BUS VOLTS: $482 \%$ VD: -0.43 $==========x=====x====$ PU BUS VOLTAGE: 1.004 ANGLE: -3.0 DEGREES

LOAD FROM: AY-PDP-1 800A FEEDER AMPS: 337.2 VOLTAGE DROP: $1 . \%$ VD: 0.18 PROJECTED POWER FLOW: $249.9 \mathrm{KW} 129.7$ KVAR $281.6 \mathrm{KVA}$ PF: 0.89 LAGGING LOSSES THRU FEEDER: $\quad 0.3 \mathrm{KW} \quad 0.4$ KVAR $0.5 \mathrm{KVA}$

LOAD TO: Group Cnt7r FEEDER AMPS: 64.4 VOLTAGE DROP: $0 . \%$ VD: 0.01 PROJECTED POWER FLOW: $44.1 \mathrm{KW} 30.8 \mathrm{KVAR}$ 53.8 KVA PF: 0.82 LAGGING LOSSES THRU FEEDER:

$0.0 \mathrm{KW} \quad 0.0 \mathrm{KVAR} \quad 0.0 \mathrm{KVA}$

LOAD TO: Boostr VSD PROJECTED POWER FLOW:

FEEDER AMPS: 273.4 VOLTAGE DROP:

$0 . \%$ VD: 0.05 LOSSES THRU FEEDER: $205.8 \mathrm{KW} \quad 98.9 \mathrm{KVAR}$ $0.1 \mathrm{KW}$ 0.1 KVAR 0.1 KVA

$====$ BUS: Pit Chillr BuS DESIGN VOLTS: 480 BUS VOLTS: $480 \%$ VD: -0.01 $===m=s===m=====m===== \pm$ PU BUS VOLTAGE: 1.000 ANGLE: -2.9 DEGREES NET BRANCH DIVERSITY LOAD: $\quad 7.5 \mathrm{KW} \quad 3.6 \mathrm{KVAR}$

LOAD FROM: Group Cnt7r FEEDER AMPS: 10.0 VOLTAGE DROP: PROJECTED POWER FLOW: LOSSES THRU FEEDER: $7.5 \mathrm{KW} \quad 3.6 \mathrm{KVAR}$ $0.0 \mathrm{KW} \quad 0.0 \mathrm{KVAR}$ 8.3 KVA PF: 0.90 : 0.42 2. \%VD: 0.42
0.90 LAGGING $====$ BUS: Group Cnt7r DESIGN VOLTS: 480 BUS VOLTS: $482 \%$ VD: -0.42 $=====m=====m=====m====$ PU BUS VOLTAGE: 1.004 ANGLE: -3.0 DEGREES

LOAD FROM: Tap Bus AsmbTy FEEDER AMPS: 64.4 VOLTAGE DROP: $0 . \%$ VD: 0.01 PROJECTED POWER FLOW: $44.1 \mathrm{KW} \quad 30.8 \mathrm{KVAR}$ 53.8 KVA PF: 0.82 LAGGING LOSSES THRU FEEDER: $0.0 \mathrm{KW} 0.0 \mathrm{KVAR} \quad 0.0 \mathrm{KVA}$

LOAD T0: Pit Chillr BUS FEEDER AMPS: 10.0 VOLTAGE DROP: 2 . \%VD: 0.42 PROJECTED POWER FLOW: $\quad 7.5 \mathrm{KW} \quad 3.6 \mathrm{KVAR} \quad 8.3 \mathrm{KVA}$ PF: 0.90 LAGGING LOSSES THRU FEEDER: $\quad 0.0 \mathrm{KW} 0.0 \mathrm{KVAR} 0.0 \mathrm{KVA}$

HNF-2487, Rev. 0

Page F-31 
Calc. No. W-320-31-016

Revision 1

Page No. D - 6

DATE: $7: 24: 1998$ TIME: $8: 35: 19.70$

PAGE 6

Project $W-320$ / Tank 24l-C-106 Sluicing

AY-Farm Electrica] Distribution

Calc. No. W320-31-016 (Reference Drawing H-2-818693, SH 1, Rev. 1)

BALANCED VOLTAGE DROP AND LOAD FLOW ANALYSIS

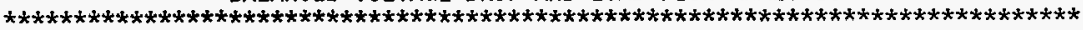

VOLTAGE EFFECT ON LOADS MODELED

VOLTAGE DROP CRITERIA: BRANCH $=3.00 \%$ BUS $=5.00$

LOAD TO: ImrS SS

PROJECTED POWER FLOW:

LOSSES THRU FEEDER:

LOAD TO: Winch Bus PROJECTED POWER FLOW: LOSSES THRU FEEDER:
FEEDER AMPS: 52.0 VOLTAGE DROP: $34.9 \mathrm{KW}$ $0.0 \mathrm{KW}$ 25.8 KVAR 0.0 KVAR

FEEDER AMPS: 2.5 VOLTAGE DROP:
43. $\%$. 0.00

43.4 KVA PF: 0.80 LAGGING $0.0 \mathrm{KVA}$

$====$ BUS: ImrS SS $\quad$ DESIGN VOLTS: 480 BUS VOLTS: $482 \%$ VD: -0.42

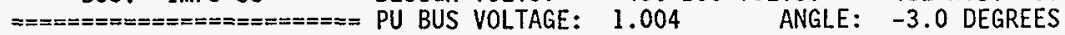

LOAD FROM: Group Cntlr PROJECTED POWER FLOW: LOSSES THRU FEEDER:

LOAD TO: P-0621 Bus PROJECTED POWER FLOW: LOSSES THRU FEEDER:
$1.7 \mathrm{KW} \quad 1.3 \mathrm{KVAR}$

$0.0 \mathrm{KW}$

0.0 KVAR
FEEDER AMPS: 52.0 VOLTAGE DROP:
$34.9 \mathrm{KW}$

$0.0 \mathrm{KW}$
25.8 KVAR 0.0 KVAR
2.2 KVA

0.0 KVA
1. \%VD: 0.17

FEEDER AMPS: 52.0 VOLTAGE DROP: 2. \%VD: 0.48 $34.9 \mathrm{KW} \quad 25.8 \mathrm{KVAR}$ $0.2 \mathrm{KW}$ $0.1 \mathrm{KVAR}$ 43.4 KVA PF: 0.80 LAGGING 0.2 KVA
0 . \%VD: 0.00 43.4 KVA PF: 0.80 LAGGING $0.0 \mathrm{KVA}$

$====$ BUS: Winch BuS $\quad$ DESIGN VOLTS: 480 BUS VOLTS: $481 \%$ VD: -0.25

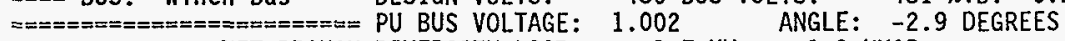
NET BRANCH DIVERSITY LOAD: $\quad 1.7 \mathrm{KW} \quad 1.3 \mathrm{KVAR}$

LOAD FROM: Group Cntlr PROJECTED POWER FLOW:

FEEDER AMPS: $\mathbf{2 . 5}$ VOLTAGE DROP:

1. \%VD: 0.17 LOSSES THRU FEEDER:

$1.7 \mathrm{KW}$

$0.0 \mathrm{KW}$
1.3 KVAR

0.0 KVAR
2.2 KVA 0.0 KVA

$====$ BUS: AY102-PP1 BUS DESIGN VOLTS: 480 BUS VOLTS: $482 \%$ VD: -0.51

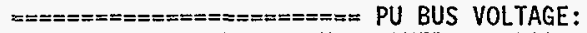
NET BRANCH DIVERSITY LOAD:

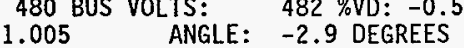

$12.1 \mathrm{KW}$ 9.1 KVAR

LOAD FROM: AY-PDP-1 800A FEEDER AMPS: 18.1 VOLTAGE DROP: $0 . \%$ VD: 0.09 PROJECTED POWER FLOW: $12.1 \mathrm{KW} \quad 9.1$ KVAR $15.2 \mathrm{KVA}$ PF: 0.80 LAGGING LOSSES THRU FEEDER:

$0.0 \mathrm{KW}$

0.0 KVAR 
Calc. No. W-320-31-016

Revision 1

Page No. D -7

DATE: $7: 24: 1998$ TIME: $8: 35: 19.70$

PAGE 7

Project W-320 / Tank 241-C-106 S7uicing

AY-Farm Electrical Distribution

Ca]c. No. W320-31-016 (Reference Drawing H-2-818693, SH 1, Rev. 1)

BALANCED VOLTAGE DROP AND LOAD FLOW ANALYSIS

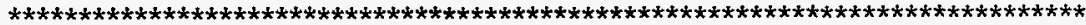

VOLTAGE EFFECT ON LOADS MODELED

VOLTAGE DROP CRITERIA: BRANCH $=3.00 \%$ BUS $=5.00$

$====$ BUS: Boostr VSD DESIGN VOLTS: 480 BUS VOLTS: $482 \%$ VD: -0.38 $==x====x===========x==$ PU BUS VOLTAGE: 1.004 ANGLE: -3.0 DEGREES

LOAD FROM: Tap BUS AsmbTy FEEDER AMPS: 273.4 VOLTAGE DROP: $\quad 0 . \%$ VD: 0.05 PROJECTED POWER FLOW: $205.7 \mathrm{KW}$ 98.8 KVAR 228.2 KVA PF: 0.90 LAGGING LOSSES THRU FEEDER: $\quad 0.1 \mathrm{KW} 0.1 \mathrm{KVAR} \quad 0.1 \mathrm{KVA}$

LOAD TO: P-0622 BUS FEEDER AMPS: 273.4 VOLTAGE DROP: $3 . \%$ VD: 0.55 PROJECTED POWER FLOW: $205.7 \mathrm{KW}$ 98.8 KVAR 228.2 KVA PF: 0.90 LAGGING LOSSES THRU FEEDER: $\quad 0.8 \mathrm{KW} \quad 1.3 \mathrm{KVAR} \quad 1.5 \mathrm{KVA}$

$====$ BUS: P-0622 BuS DESIGN VOLTS: 480 BUS VOLTS: $479 \%$ VD: 0.17 $=========== \pm====== \pm====$ PU BUS VOLTAGE: 0.998 ANGLE: -3.2 DEGREES NET BRANCH DIVERSITY LOAD: $204.9 \mathrm{KW} 97.5 \mathrm{KVAR}$

LOAD FROM: Boostr VSD PROJECTED POWER FLOW: LOSSES THRU FEEDER:

FEEDER AMPS: 273.4 VOLTAGE DROP:

3. \%VD: 0.55 $204.9 \mathrm{KW}$ 97.5 KVAR 227.0 KVA PF: 0.90 LAGGING $0.8 \mathrm{KW} \quad 1.3 \mathrm{KVAR} \quad 1.5 \mathrm{KVA}$

$====$ BUS: P-0621 BUS DESIGN VOLTS: 480 BUS VOLTS: $480 \% \mathrm{VD}: 0.06$ $====================$ PU BUS VOLTAGE: 0.999 ANGLE: -2.9 DEGREES NET BRANCH DIVERSITY LOAD: $34.7 \mathrm{KW} 25.8 \mathrm{KVAR}$

LOAD FROM: ImrS SS FEEDER AMPS: 52.0 VOLTAGE DROP: 2. \%VD: 0.48 PROJECTED POWER FLOW: $34.7 \mathrm{KW} 25.8$ KVAR 43.2 KVA PF: 0.80 LAGGING LOSSES THRU FEEDER: $\quad 0.2 \mathrm{KW} 0.1 \mathrm{KVAR} 0.2 \mathrm{KVA}$

$====$ BUS: C8-L6 13.8KV DESIGN VOLTS: I BUS VOLTS: $1 \%$ VD: -3.00 $=====x=====x======m=x==$ PU BUS VOLTAGE: 1.030 ANGLE: 0.0 DEGREES *** SWING GENERATOR: C8-L6 13.8KV $274.7 \mathrm{KW} 164.4 \mathrm{KVAR}$

LOAD TO: Pole E1 FEEDER AMPS: 174210 VOLTAGE DROP: $0 . \%$ VD: 0.35 PROJECTED POWER FLOW: $274.7 \mathrm{KW} 164.4 \mathrm{KVAR} 320.1 \mathrm{KVA}$ PF: 0.86 LAGGING LOSSES THRU FEEDER: $\quad 0.6 \mathrm{KW} \quad 1.1 \mathrm{KVAR} \quad 1.3 \mathrm{KVA}$

HNF-2487, Rev. 0

Page F-33 
Calc. No. W-320-31-016

Revision 1

Page No. D -8

DATE: $7: 24: 1998$ TIME: $8: 35: 19.70$

PAGE 8

Project $W-320$ / Tank 241-C-106 Sluicing

AY-Farm Electrical Distribution

Calc. No. W320-31-016 (Reference Drawing H-2-818693, SH 1, Rev. 1)

BALANCED VOLTAGE DROP AND LOAD FLOW BUS DATA SUMMARY

\begin{tabular}{|c|c|c|c|c|c|}
\hline $\begin{array}{l}\text { BUS NAME } \\
\text { Pole E1 } \\
\text { XFMR Sec } \\
\text { Tap Bus Asmbly } \\
\text { Group Cnt1r } \\
\text { Winch Bus } \\
\text { Boostr VSD } \\
\text { P-0621 Bus }\end{array}$ & $\begin{array}{r}\text { BASE VOLT } \\
13800.00 \\
480.00 \\
480.00 \\
480.00 \\
480.00 \\
480.00 \\
480.00\end{array}$ & $\begin{array}{l}\text { PU VOLT } \\
1.0265 \\
1.0079 \\
1.0043 \\
1.0042 \\
1.0025 \\
1.0038 \\
0.9994\end{array}$ & $\begin{array}{l}\text { BUS NAME } \\
\text { XFMR Pri } \\
\text { AY-PDP-1 } 800 A \\
\text { Pit Chillr Bus } \\
\text { Imrs SS } \\
\text { AY102-PPI Bus } \\
\text { P-0622 Bus }\end{array}$ & $\begin{array}{r}\text { BASE VOLT } \\
13800.00 \\
480.00 \\
480.00 \\
480.00 \\
480.00 \\
480.00\end{array}$ & $\begin{array}{r}\text { PU VOLT } \\
1.0265 \\
1.0060 \\
1.0001 \\
1.0042 \\
1.0051 \\
0.9983\end{array}$ \\
\hline
\end{tabular}

BALANCED VOLTAGE DROP AND LOAD FLOW BRANCH DATA SUMMARY

\begin{tabular}{|c|c|c|c|c|c|c|}
\hline $\begin{array}{l}\text { FROM NAME } \\
\text { Pole EI } \\
\text { XFMR Pri } \\
\text { XFMR Sec } \\
\text { AY-PDP-1 800A } \\
\text { AY-PDP-1 800A } \\
\text { Tap Bus Asmbiy } \\
\text { Tap Bus Asmbly } \\
\text { Group Cnt7r } \\
\text { Group Cnt7r } \\
\text { Group Cnt7r } \\
\text { Imrs SS } \\
\text { Boostr. VSD }\end{array}$ & $\begin{array}{l}\text { T0 NAME } \\
\text { XFMR Pri } \\
\text { XFMR Sec } \\
\text { AY-PDP-1 } 800 A \\
\text { Tap Bus Asmbly } \\
\text { AY102-PP1 Bus } \\
\text { Group Cntir } \\
\text { Boostr VSD } \\
\text { Pit Chil1r Bus } \\
\text { Imrs SS } \\
\text { Winch Bus } \\
\text { P-0621 Bus } \\
\text { P-0622 Bus }\end{array}$ & $\begin{array}{l}\text { TYPE } \\
\text { FDR } \\
\text { TX2 } \\
\text { FDR } \\
\text { FDR } \\
\text { FDR } \\
\text { FDR } \\
\text { FDR } \\
\text { FDR } \\
\text { FDR } \\
\text { FDR } \\
\text { FDR } \\
\text { FDR }\end{array}$ & $\begin{array}{l}\text { VD\% } \\
0.00 \\
1.86 \\
0.19 \\
0.18 \\
0.09 \\
0.01 \\
0.05 \\
0.42 \\
0.00 \\
0.17 \\
0.48 \\
0.55\end{array}$ & $\begin{array}{r}\text { AMPS } \\
13.00 \\
13.00 \\
355.13 \\
337.23 \\
18.13 \\
64.44 \\
273.46 \\
10.00 \\
52.01 \\
2.58 \\
52.01 \\
273.46\end{array}$ & $\begin{array}{r}\text { KVA } \\
319.02 \\
319.02 \\
297.58 \\
282.06 \\
15.17 \\
53.80 \\
228.33 \\
8.35 \\
43.42 \\
2.16 \\
43.42 \\
228.22\end{array}$ & $\begin{array}{r}\text { RATING\% } \\
\text { UNKNOWN } \\
184.94 \\
57.28 \\
54.39 \\
25.91 \\
36.82 \\
71.96 \\
33.34 \\
34.67 \\
22.26 \\
34.67 \\
71.96\end{array}$ \\
\hline
\end{tabular}

NOTE: FDR RATING\% $=\%$ AMPS RATING BASED ON LIBRARY FLA OR BRANCH INPUT FLA TX2 RATING\% $=\%$ KVA RATING BASED ON TRANSFORMER FL KVA

13 BUSES

$* * * T O T A \underset{13}{L}, \underset{K W}{S} Y S T E M \underset{27}{L}$ KVAR 0 S E S***

HNF-2487, Rev. 0

Page F-34 
Calc. No. W-320-31-016

Revision 1

Attachment E

HNF-2487, Rev. 0

Page F-35 
Calc. No. $W-320-31-016$ Revision 1

Page No. E - 1

Project $\mathrm{W}-320$ / Tank 241-C-106 Sluicing AY-Farm Electrical Distribution

Calc. No. W320-31-016 (Reference Drawing H-2-818693, SH 1, Rev. 1)

DATE: $\quad 7: 24: 1998$

TIME: $8: 35: 25.52$

ALL INFORMATION PRESENTED IS FOR REVIEW, APPROVAL

INTERPRETATION AND APPLICATION BY A REGISTERED ENGINEER ONLY

SKM POWER*TOOLS FOR WINDOWS

SHORT CIRCUIT ANALYSIS REPORT

COPYRIGHT SKM SYSTEMS ANALYSIS, INC. 1995-1996

HNF-2487, Rev, 0

Page F-36 
Calc. No. W-320-31-016

Revision 1

Page No. E - 2

DATE: $7: 24: 1998$ TIME: $8: 35: 25.52$

Project $W-320$ / Tank 241-C-106 sluicing

PAGE

2

AY-Farm Electrical Distribution

Caic. No. W320-31-016 (Reference Drawing H-2-818693, SH 1, Rev. 1)

ALL PU VALUES ARE EXPRESSED ON A 100 MVA BASE

SWING GENERATORS

SOURCE NAME VOLTAGE ANGLE

\begin{tabular}{|c|c|}
\hline $\mathrm{C} 8-16 \quad 13.8 \mathrm{KV}$ & 1.03 \\
\hline
\end{tabular}

HNF-2487, Rev. 0

Page F-37 
Calc. No. W-320-31-016

Revision 1

Page No. E - 3

DATE: $7: 24: 1998$ TIME: $8: 35: 25.52$

PAGE 3

Project $W-320$ / Tank 241-C-106 Sluicing

AY-Farm Electrical Distribution

Calc. No. W320-31-016 (Reference Drawing H-2-818693, SH 1, Rev. 1)

$* * * * * * * * * * * * *$ PRE-FAULT VOLTAGE PROFILE**************

BUS\# NAME

BASE VOLTS PU VOLTS

ANGLE (D)

Pole El

XFMR Pri

XFMR Sec

AY-PDP-1 800A

Tap Bus Asmbly

Pit Chillr Bus

Group Cntlr

Imrs SS

Winch Bus

AY102-PP1 Bus

Boostr VSD

P-0622 Bus

13800.00

1.0300

0.

13800.00

1.0300

480.00

1.0842

$480.00 \quad 1.0842$

480.00

1.0842

480.00

1.0842

480.00

1.0842

480.00

1.0842

480.00

1.0842

480.00

1.0842

480.00

1.0842

480.00

1.0842

480.00

1.0842

0.

-30 .

-30 .

-30 .

-30 .

-30 .

-30 .

-30 .

-30 .

-30 .

-30 .

-30 .

HNF-2487, Rev. 0

Page F-38 
Calc. No. W-320-31-016

Revision 1

Page No. E - 4

DATE: $7: 24: 1998$ TIME: $8: 35: 25.52$

Project $W-320$ / Tank 24l-C-106 Sluicing

PAGE 4

AY-Farm Electrical Distribution

Calc. No. W320-31-016 (Reference Drawing H-2-818693, SH 1, Rev. 1)

$* * * * * * * * * * * * * * * *$ F A U L T ANALYSIS RE PORT

FAULT TYPE: 3 PH

MODEL INDUCTION MOTOR CONTRIBUTION: YES

MODEL TRANSFORMER TAPS: YES

MODEL TRANSFORMER PHASE SHIFT: YES

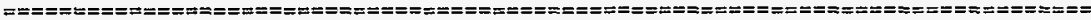

Pole EI

VOLTAGE BASE LL:

13800.0 (VOLTS)

INI. SYM. RMS FAULT CURRENT: $3363.7 /-61$. ( AMPS/DEG )

THEVENIN EQUIVALENT IMPEDANCE: $0.631+\mathrm{j} 1.115$ (PU)

THEVENIN IMPEDANCE X/R RATIO: 1.768

$$
\text { ASYM RMS INTERRUPTING AMPS }
$$

$\begin{array}{lrrr}1 / 2 \text { CYCLES } & 3 \text { CYCLES } & 5 \text { CYCLES } & 8 \text { CYCLES } \\ 3458.5 & 3363.7 & 3363.7 & 3363.7\end{array}$

ASYM RMS FAULTED CURRENT ( AMPS)

AT TIME $=0.5$ CYCLES
3458.5
---PHASE B-.-
3458.5
3458.5

HNF-2487, Rev. 0

Page F-39 
Ca7c. No. W-320-31-016

Revision 1

Page No. E - 5

DATE: $7: 24: 1998$ TIME: $8: 35: 25.52$

PAGE 5

Project $W-320$ / Tank 241-C-106 Sluicing

AY-Farm Electrical Distribution

Calc. No. W320-31-016 (Reference Drawing H-2-818693, SH 1, Rev. 1)

XFMR Pri

VOLTAGE BASE LL: $\quad 13800.0$ (VOLTS)

INI. SYM. RMS FAULT CURRENT: $3363.0 /-60$. ( AMPS/DEG )

THEVENIN EQUIVALENT IMPEDANCE: $0.631+j \quad 1.115$ (PU)

THEVENIN IMPEDANCE X/R RATIO: 1.767

$$
\begin{array}{lcrr}
\text { ASYM } & \text { RMS } & \text { INTERRUPTING AMPS } & \\
1 / 2 \text { CYCLES } & 3 \text { CYCLES } & 5 \text { CYCLES } & 8 \text { CYCLES } \\
3457.7 & 3363.0 & 3363.0 & 3363.0
\end{array}
$$

ASYM RMS FAULTED CURRENT ( AMPS )

$\begin{array}{cc}\text { AT TIME }= & 0.5 \text { CYCLES } \\ \text {--PHASE A--- } & --- \text { PHASE B--- } \\ 3457.7 & 3457.7\end{array}$

XFMR Sec

VOLTAGE BASE LL: 480.0 (VOLTS)

INI. SYM. RMS FAULT CURRENT: 6047.5/-100. (AMPS/DEG)

THEVENIN EQUIVALENT IMPEDANCE: $7.304+\mathrm{j} 20.290$ (PU)

THEVENIN IMPEDANCE X/R RATIO: 2.778

$$
\begin{array}{lccc}
\text { ASYM } & \text { RMS } & \text { INTERRUPTING AMPS } & \\
1 / 2 \text { CYCLES } & 3 \text { CYCLES } & 5 \text { CYCLES } & 8 \text { CYCLES } \\
6647.5 & 6047.5 & 6047.5 & 6047.5
\end{array}
$$

ASYM RMS FAULTED CURRENT ( AMPS )

AT TIME $=0.5$ CYCLES
6647.5
$\ldots$...PHASE B-..
6647.5

HNF-2487, Rev. 0

Page F-40 
DATE: $7: 24: 1998$ TIME: $8: 35: 25.52$

Calc. No. $W-320-31-016$

Revision 1

Page No. E - 6

Project W-320 / Tank 241-C-106 Sluicing

AY-Farm Electrical Distribution

Calc. No. W320-31-016 (Reference Drawing H-2-818693, SH 1, Rev. 1)

AY-PDP-1 800A VOLTAGE BASE LL: $\quad 480.0$ (VOLTS)

INI. SYM. RMS FAULT CURRENT: 5974.6/-100. (AMPS/DEG)

THEVENIN EQUIVALENT IMPEDANCE: $7.377+\mathbf{j} 20.543$ (PU)

THEVENIN IMPEDANCE X/R RATIO: 2.785

$$
\begin{array}{lcrr}
\text { ASYM } & \text { RMS } & \text { INTERRUPTING AMPS } & \\
1 / 2 \text { CYCLES } & 3 \text { CYCLES } & 5 \text { CYCLES } & 8 \text { CYCLES } \\
6570.6 & 5974.6 & 5974.6 & 5974.6
\end{array}
$$

ASYM RMS FAULTED CURRENT ( AMPS )

AT TIME $=0.5$ CYCLES

---PHASE A-- $\quad$-- PHASE B---

6570.6

6570.6

PHASE C---

6570.6

Tap Bus AsmbTy VOLTAGE BASE LL:

480.0 (VOLTS)

INI. SYM. RMS FAULT CURRENT: 5905.3/-100. (AMPS/DEG)

THEVENIN EQUIVALENT IMPEDANCE: $7.441+j 20.792$ (PU)

THEVENIN IMPEDANCE X/R RATIO: 2.794
ASYM RMS INTERRUPTING AMPS
$1 / 2$ CYCLES
3 CYCLES
5905.3
5 CYCLES
8 CYCLES
6498.7
5905.3
5905.3

ASYM RMS FAULTED CURRENT ( AMPS)

$$
\text { AT TIME }=0.5 \text { CYCLES }
$$

---PHASE A--- --PHASE B---

6498.7

6498.7

-.-PHASE C-.-

6498.7

HNF-2487, Rev. 0

Page F-41 
DATE: $7: 24: 1998$ TIME: $8: 35: 25.52$

Calc. No. W-320-3I-016

Revision 1

Page No. E - 7

Project $W-320$ / Tank 241-C-106 Sluicing

AY-Farm Electrical Distribution

Calc. No. W320-31-016 (Reference Drawing H-2-818693, SH 1, Rev. 1)

Pit Chillr Bus VOLTAGE BASE LL: $\quad 480.0$ (VOLTS)

INI. SYM. RMS FAULT CURRENT: $1964.8 /-51$. (AMPS/DEG )

THEVENIN EQUIVALENT IMPEDANCE: $61.944+\mathrm{j} 23.844$ (PU)

THEVENIN IMPEDANCE X/R RATIO: 0.385

ASYM RMS INTERRUPTING AMPS

$\begin{array}{rrrr}1 / 2 \text { CYCLES } & 3 \text { CYCLES } & 5 \text { CYCLES } & 8 \text { CYCLES } \\ 1964.8 & 1964.8 & 1964.8 & 1964.8\end{array}$

ASYM RMS FAULTED CURRENT ( AMPS )

AT .TIME $=0.5 \mathrm{CYCLES}$

---PHASE A--- -- PHASE B---

1964.8

1964.8

---PHASE C---

1964.8

Group Cntir

VOLTAGE BASE LL:

480.0 (VOLTS)

INI. SYM. RMS FAULT CURRENT: $5887.4 /-100$ ( AMPS/DEG)

THEVENIN EQUIVALENT IMPEDANCE: $7.517+\mathrm{j} 20.836$ (PU)

THEVENIN IMPEDANCE X/R RATIO: 2.772

$$
\begin{array}{llrrr}
\text { ASYM } & \text { RMS } & \multicolumn{2}{c}{\text { INTERRUPTING }} & \text { AMPS } \\
1 / 2 \text { CYCLES } & 3 \text { CYCLES } & 5 \text { CYCLES } & 8 \text { CYCLES } \\
6468.9 & 5887.5 & 5887.4 & 5887.4
\end{array}
$$

ASYM RMS FAULTED CURRENT ( AMPS)

0.5 CYCLES

$$
6468.9
$$

6468.9

HNF-2487, Rev. 0

Page F-42 
DATE: $7: 24: 1998$ TIME: $8: 35: 25.52$

Calc. No. W-320-31-016

Revision 1

Page No. E - 8

Project $W-320$ / Tank 241-C-106 Sluicing

PAGE 8

AY-Farm Electrical Distribution

Calc. No. W320-31-016 (Reference Drawing H-2-818693, SH 1, Rev. 1)

Imrs SS

VOLTAGE BASE LL:

480.0 (VOLTS)

INI. SYM. RMS FAULT CURRENT: $5877.7 /-100$ ( AMPS/DEG)

THEVENIN EQUIVALENT IMPEDANCE: $7.563+\mathrm{j} 20.859$ (PU)

THEVENIN IMPEDANCE X/R RATIO: 2.758

$$
\begin{array}{lcrrr}
\text { ASYM } & \text { RMS } & \text { INTERRUPTING } & \text { AMPS } & \\
1 / 2 \text { CYCLES } & 3 \text { CYCLES } & 5 \text { CYCLES } & 8 \text { CYCLES } \\
6451.9 & 5877.7 & 5877.7 & 5877.7
\end{array}
$$

ASYM RMS FAULTED CURRENT ( AMPS )

-.-PHASE A-- 0.5 CYCLES 6451.9 6451.9

6451.9

Winch Bus

VOLTAGE BASE LL:

480.0 (VOLTS)

INI. SYM. RMS FAULT CURRENT: $1198.9 /-44$. (AMPS/DEG)

THEVENIN EQUIVALENT IMPEDANCE: $105.692+\mathrm{j} 25.724$ (PU)

THEVENIN IMPEDANCE X/R RATI0: 0.243

$\begin{array}{lccrr}\text { ASYM } & \text { RMS } & \text { INTERRUPTING AMPS } & \\ 1 / 2 \text { CYCLES } & 3 \text { CYCLES } & 5 \text { CYCLES } & 8 \text { CYCLES } \\ 1198.9 & 1198.9 & 1198.9 & 1198.9\end{array}$

ASYM RMS FAULTED CURRENT ( AMPS)

AT TIME $=0.5$ CYCLES

---PHASE A--- ---PHASE B--1198.9

1198.9

HNF-2487, Rev. 0

Page F-43 
Caic. No. W-320-31-016

Revision 1

Page No. $E-9$

DATE: $7: 24: 1998$ TIME: $8: 35: 25.52$

PAGE 9

Project $W-320$ / Tank 241-C-106 Sluicing

AY-Farm Electrical Distribution

Calc. No. W320-31-016 (Reference Drawing H-2-818693, SH I, Rev. 1)

AY102-PP1 BUS VOLTAGE BASE LL: $\quad 480.0$ (VOLTS)

INI. SYM. RMS FAULT CURRENT: 5037.9/-88. ( AMPS/DEG)

THEVENIN EQUIVALENT IMPEDANCE: $13.888+\mathrm{j} 21.845$ (PU)

THEVENIN IMPEDANCE X/R RATIO: 1.573

$\begin{array}{lccrr}\text { ASYM } & \text { RMS } & \text { INTERRUPTING AMPS } & \\ 1 / 2 \text { CYCLES } & 3 \text { CYCLES } & 5 \text { CYCLES } & 8 \text { CYCLES } \\ 5129.8 & 5037.9 & 5037.9 & 5037.9\end{array}$

ASYM RMS FAULTED CURRENT ( AMPS)

$\begin{array}{ll}\text { AT TIME }= & 0.5 \text { CYCLES } \\ --- \text { PHASE A--.- } & -- \text { PHASE B-- }\end{array}$

5129.8

5129.8

PHASE C---

5129.8

Boostr VSD

VOLTAGE BASE LL:

480.0 (VOLTS)

INI. SYM. RMS FAULT CURRENT: $5875.3 /-100$. (AMPS/DEG)

THEVENIN EQUIVALENT IMPEDANCE: $\quad 7.471+j 20.901$ (PU)

THEVENIN IMPEDANCE X/R RATI0: 2.798

$$
\begin{array}{lcccc}
\text { ASYM } & \text { RMS } & \text { INTERRUPTING AMPS } & \\
1 / 2 \text { CYCLES } & 3 \text { CYCLES } & 5 \text { CYCLES } & 8 \text { CYCLES } \\
6467.3 & 5875.3 & 5875.3 & 5875.3
\end{array}
$$

ASYM RMS FAULTED CURRENT (AMPS)

AT TIME $=0.5$ CYCLES
- PHASE A---

6467.3

6467.3

HNF-2487, Rev. 0

Page F-44 
Calc. No. W-320-31-016

Revision 1

Page No. E - 10

DATE: $7: 24: 1998$ TIME: $8: 35: 25.52$

PAGE $\quad 10$

Project $W-320$ / Tank 241-C-106 Sluicing

AY-Farm Electrical Distribution

Calc. No. W320-31-016 (Reference Drawing H-2-818693, SH 1, Rev. 1)

P-0622 Bus

VOLTAGE BASE LL:

480.0 (VOLTS)

INI. SYM. RMS FAULT CURRENT: 5554.8/-101. (AMPS/DEG )

THEVENIN EQUIVALENT IMPEDANCE: $7.760+\mathrm{j} 22.157$ (PU)

THEVENIN IMPEDANCE X/R RATIO: $\quad 2.855$

$\begin{array}{lcrr}\text { ASYM } & \text { RMS } & \text { INTERRUPTING } & \text { AMPS } \\ 1 / 2 \text { CYCLES } & 3 \text { CYCLES } & 5 \text { CYCLES } & 8 \text { CYCLES } \\ 6139.2 & 5554.8 & 5554.8 & 5554.8\end{array}$

ASYM RMS FAULTED CURRENT ( AMPS )

AT TIME $=0.5$ CYCLES

6139.2

---PHASE B---

6139.2
-.-PHASE C-.-

6139.2

P-0621 BuS VOLTAGE BASE LL: 480.0 (VOLTS)

INI. SYM. RMS FAULT CURRENT: 4290.3/ -87. (AMPS/DEG)

THEVENIN EQUIVALENT IMPEDANCE: $16.480+\mathrm{j} 25.542$ (PU)

THEVENIN IMPEDANCE X/R RATIO: $\quad 1.550$

$\begin{array}{lccrr}\text { ASYM } & \text { RMS } & \text { INTERRUPTING } & \text { AMPS } & \\ 1 / 2 \text { CYCLES } & 3 \text { CYCLES } & 5 \text { CYCLES } & 8 \text { CYCLES } \\ 4364.1 & 4290.3 & 4290.3 & 4290.3\end{array}$

ASYM RMS FAULTED CURRENT ( AMPS )

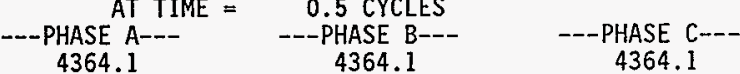

HNF-2487, Rev. 0

Page F 45 
Ca7c. No. W-320-31-016

Revision 1

Page No. E - 11

DATE: $7: 24: 1998$ TIME: $8: 35: 25.52$

Project $W-320$ / Tank 241-C-106 Sluicing

PAGE 11

AY-Farm Electrical Distribution

Calc. No. W320-31-016 (Reference Drawing H-2-818693, SH 1, Rev. 1)

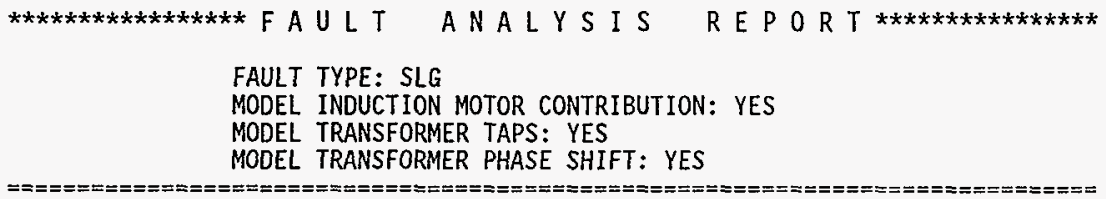

Pole E1

VOLTAGE BASE LL:

13800.0 (VOLTS)

INI. SYM. RMS FAULT CURRENT: $1302.9 /-45$. ( AMPS/DEG)

THEVENIN EQUIVALENT IMPEDANCE: $\quad 7.016+\mathrm{j} 7.017$ (PU)

THEVENIN IMPEDANCE X/R RATIO: 1.000

SEQUENCE EQUIVALENT IMPEDANCE Z1: $0.631+\mathrm{j} 1.115$ (PU)

Z2: $\quad 0.631+j \quad 1.115$ (PU)

ZO: $\quad 5.754+j$

4.787 (PU)

ASYM RMS INTERRUPTING AMPS

$\begin{array}{ccrr}1 / 2 \text { CYCLES } & 3 \text { CYCLES } & 5 \text { CYCLES } & 8 \text { CYCLES } \\ 1305.3 & 1302.9 & 1302.9 & 1302.9\end{array}$

ASYM RMS FAULTED CURRENT ( AMPS)

AT TIME $=0.5$ CYCLES

---PHASE A--- ---PHASE B---

1305.3

0.0

- PHASE C- -

0.0

HNF-2487, Rev. 0

Page F-46 
Calc. No. W-320-31-016

Revision 1

Page No. E - 12

DATE: $7: 24: 1998$ TIME: $8: 35: 25.52$

PAGE 12

Project $\mathrm{H}-320$ / Tank 241-C-106 Sluicing

AY-Farm Electrical Distribution

Calc. No. W320-31-016 (Reference Drawing H-2-818693, SH 1, Rev. 1)

XFMR Pri

VOLTAGE BASE LL: $\quad 13800.0$ (VOLTS)

INI. SYM. RMS FAULT CURRENT: $1302.7 /-45$. ( AMPS/DEG )

THEVENIN EQUIVALENT IMPEDANCE: $7.016+\mathrm{j} 7.018$ (PU)

THEVENIN IMPEDANCE X/R RATIO: 1.000

SEQUENCE EQUIVALENT IMPEDANCE Z1: $0.631+\mathrm{j} 1.115$ (PU)

Z2: $\quad 0.631+j \quad 1.115$ (PU)

Z0: $\quad 5.754+j \quad 4.787$ (PU)

ASYM RMS INTERRUPTING AMPS

$\begin{array}{lrrr}1 / 2 \text { CYCLES } & 3 \text { CYCLES } & 5 \text { CYCL.ES } & 8 \text { CYCLES } \\ 1305.2 & 1302.7 & 1302.7 & 1302.7\end{array}$

ASYM RMS FAULTED CURRENT ( AMPS )

AT TIME $=0.5$ CYCLES

$$
1305.2
$$

---PHASE B---

0.0

PHASE C---

0.0

XFMR Sec

VOLTAGE BASE LL: $\quad 480.0$ (VOLTS)

INI. SYM. RMS FAULT CURRENT: 5352.0/-98. (AMPS/DEG)

THEVENIN EQUIVALENT IMPEDANCE: $27.546+j 67.712$ (PU)

THEVENIN IMPEDANCE X/R RATIO: 2.458

SEQUENCE EQUIVALENT IMPEDANCE $21: \quad 7.304+\mathrm{j} 20.290$ (PU)

Z2: $\quad 7.304+j \quad 20.290$ (PU)

ZO: $12.937+j \quad 27.133$ (PU)

$\begin{array}{lcrrr}\text { ASYM } & \text { RMS } & \text { INTERRUPTING } & \text { AMPS } & \\ 1 / 2 \text { CYCLES } & 3 \text { CYCLES } & 5 \text { CYCLES } & 8 \text { CYCLES } \\ 5752.3 & 5352.0 & 5352.0 & 5352.0\end{array}$

ASYM RMS FAULTED CURRENT ( AMPS)

--PHASE AT TIME $=0.5$ CYCLES

5752.3

---PHASE B---

0.0

---PHASE C---

0.0

HNF-2487, Rev. 0

Page F-47 
Calc. No. W-320-31-016

Revision 1

Page No. E - 13

DATE: $7: 24: 1998$ TIME: $8: 35: 25.52$

PAGE 13

Project $W-320$ / Tank $241-C-106$ S7uicing

AY-Farm Electrical Distribution

Calc. No. W320-31-016 (Reference Orawing H-2-818693, SH 1, Rev. 1)

AY-PDP-1 800A VOLTAGE BASE LL: 480.0 (VOLTS)

INI. SYM. RMS FAULT CURRENT: $5190.9 /-97$ ( AMPS/DEG)

THEVENIN EQUIVALENT IMPEDANCE: $29.026+j 69.555$ (PU)

THEVENIN IMPEDANCE X/R RATIO: 2.396

SEQUENCE EQUIVALENT IMPEDANCE Z1: $7.377+j 20.543$ (PU)

Z2: $\quad 7.377+j \quad 20.543$ (PU)

Z0: $14.271+j \quad 28: 469$ (PU)

\begin{tabular}{lcrrr} 
ASYM & RMS & \multicolumn{1}{l}{ INTERRUPTING AMPS } & \\
$1 / 2$ CYCLES & 3 CYCLES & 5 CYCLES & 8 CYCLES \\
5555.3 & 5190.9 & 5190.9 & 5190.9
\end{tabular}

ASYM RMS FAULTED CURRENT (AMPS)

---PHASE A--- -.-PHASE B---

5555.3

0.0

$\begin{aligned}-- \text { PHASE } & \mathrm{C}-\cdots- \\ 0.0 & \end{aligned}$

Tap Bus Asmbly VOLTAGE BASE LL: 480.0 (VOLTS)

INI. SYM. RMS FAULT CURRENT: 5039.9/-97. (AMPS/DEG)

THEVENIN EQUIVALENT IMPEDANCE: $30.488+\mathrm{j} 71.389$ (PU)

THEVENIN IMPEDANCE X/R RATIO: 2.342

SEQUENCE EQUIVALENT IMPEDANCE Z1: $7.441+\mathrm{j} 20.792$ (PU)

Z2: $\quad 7.441+j 20.792$ (PU)

Z0: $\quad 15.605+\mathrm{j} 29.805$ (PU)

ASYM RMS INTERRUPTING AMPS
$1 / 2$ CYCLES
5373.3
3 CYCLES
5039.9
5 CYCLES
5039.9
8 CYCLES
5039.9

ASYM RMS FAULTED CURRENT ( AMPS)

$\begin{array}{ll}\text { AT TIME }= & 0.5 \text { CYCLES } \\ -- \text { PHASE A--- } & -- \text { PHASE B--- }\end{array}$

5373.3

0.0

HNF-2487, Rev. 0

Page F-48 
Calc. No. W-320-31-016

Revision 1

Page No. E - 14

DATE: $7: 24: 1998$ TIME: $8: 35: 25.52$

PAGE 14

Project W-320 / Tank 24l-C-106 Slujcing

AY-Farm Electrical Distribution

Calc. No. W320-31-016 (Reference Drawing H-2-818693, SH 1, Rev. 1)

Pit ChiT7r Bus VOLTAGE BASE LL: $\quad 480.0$ (VOLTS)

INI. SYM. RMS FAULT CURRENT: $1212.4 /-45$. ( AMPS/DEG)

THEVENIN EQUIVALENT IMPEDANCE: $311.297+\mathrm{j} 85.015$ (PU)

THEVENIN IMPEDANCE X/R RATI0: 0.273

SEQUENCE EQUIVALENT IMPEDANCE Z1: $61.944+\mathrm{j} 23.844$ (PU)

$\mathrm{Z2}: \quad 61.944+\mathbf{j} 23.844$ (PU)

ZO: $187.409+j 37.328$ (PU)

ASYM RMS INTERRUPTING AMPS

$\begin{array}{lrrr}1 / 2 \text { CYCLES } & 3 \text { CYCLES } & 5 \text { CYCLES } & 8 \text { CYCLES } \\ 1212.4 & 1212.4 & 1212.4 & 1212.4\end{array}$

ASYM RMS FAULTED CURRENT ( AMPS )

---PHASE A--- ---PHASE B---

1212.4

0.0

- - PHASE C---

0.0

Group Cntir

VOLTAGE BASE LL:

480.0 (VOLTS)

INI. SYM. RMS FAULT CURRENT: $5017.0 /-97$. (AMPS/DEG)

THEVENIN EQUIVALENT IMPEDANCE: $30.913+\mathrm{j} 71.592$ (PU)

THEVENIN IMPEDANCE X/R RATIO: 2.316

SEQUENCE EQUIVALENT IMPEDANCE Z1: $7.517+j 20.836$ (PU)

Z2: $\quad 7.517+j 20.836$ (PU)

ZO: $15.879+j 29.920$ (PU)

ASYM RMS INTERRUPTING AMPS

$\begin{array}{rrrr}1 / 2 \text { CYCLES } & 3 \text { CYCLES } & 5 \text { CYCLES } & 8 \text { CYCLES } \\ 5339.4 & 5017.0 & 5017.0 & 5017.0\end{array}$

ASYM RMS FAULTED CURRENT ( AMPS )

-- PHASE AT TIME $=0.5$ CYCLES

5339.4

0.0

---PHASE C---

0.0

HNF-2487, Rev. 0

Page F- 49 
Calc. No. W-320-31-016

Revision 1

Page No. E - 15

DATE: $7: 24: 1998$ TIME: $8: 35: 25.52$

PAGE 15

Project $W-320$ / Tank 241-C-106 Sluicing

AY-Farm Electrical Distribution

Calc. No. W320-31-016 (Reference Drawing H-2-818693, SH 1, Rev. 1)

Imrs SS VOLTAGE BASE LL: 480.0 (VOLTS)

INI. SYM. RMS FAULT CURRENT: $5004.3 /-97$. ( AMPS/DEG )

THEVENIN EQUTVALENT IMPEDANCE: $31.169+j 71.696$ (PU)

THEVENIN IMPEDANCE X/R RATIO: 2.300

SEQUENCE EQUIVALENT IMPEDANCE $\mathrm{Zl}: 7.563+\mathrm{j} 20.859$ (PU)

Z2: $\quad 7.563+j \quad 20.859$ (PU)

Z0: $16.043+\mathrm{j} 29.979$ (PU)

$\begin{array}{lcrrr}\text { ASYM } & \text { RMS } & \text { INTERRUPTING AMPS } & \\ 1 / 2 \text { CYCLES } & 3 \text { CYCLES } & 5 \text { CYCLES } & 8 \text { CYCLES } \\ 5320.2 & 5004.3 & 5004.3 & 5004.3\end{array}$

ASYM RMS FAULTED CURRENT ( AMPS )

---PHASE A---

AT TIME $=0.5$ CYCLES

5320.2

---PHASE B---

0.0

---PHASE C---

0.0

Winch Bus

VOLTAGE BASE LL: $\quad 480.0$ (VOLTS)

INI. SYM. RMS FAULT CURRENT: $715.9 /-39$. (AMPS/DEG)

THEVENIN EQUIVALENT IMPEDANCE: $539.053+j 89.960$ (PU)

THEVENIN IMPEDANCE X/R RATIO: 0.167

SEQUENCE EQUIVALENT IMPEDANCE Z1: $105.692+j 25.724$ (PU)

Z2: $105.692+j 25.724$ (PU)

ZO: $327.669+j 38.512$ (PU)

ASYM RMS INTERRUPTING AMPS

$\begin{array}{crrr}1 / 2 \text { CYCLES } & 3 \text { CYCLES } & 5 \text { CYCLES } & 8 \text { CYCLES } \\ 715.9 & 715.9 & 715.9 & 715.9\end{array}$

ASYM RMS FAULTED CURRENT ( AMPS)

---PHASE A--- $\quad$---PHASE B

715.9

0.0

0.0

HNF-2487, Rev. 0

Page F-50 
Calc. No. H-320-31-016

Revision 1

Page No. E - 16

DATE: $7: 24: 1998$ TIME: $8: 35: 25.52$

PAGE 16

Project $W-320$ / Tank $241-C-106$ Sluicing

AY-Farm Electrical Distribution

Calc. No. W320-31-016 (Reference Drawing H-2-818693, SH 1, Rev. 1)

AY102-PP1 BUS VOLTAGE BASE LL: $\quad 480.0$ (VOLTS)

INI. SYM. RMS FAULT CURRENT: $3994.2 /-80$. ( AMPS/DEG)

THEVENIN EQUIVALENT IMPEDANCE: $62.564+j 75.366$ (PU)

THEVENIN IMPEDANCE X/R RATIO: 1.205

SEQUENCE EQUIVALENT IMPEDANCE ZI: $13.888+\mathrm{j} 21.845$ (PU)

Z2: $13.888+j \quad 21.845$ (PU)

ZO: $\quad 34.789+\mathrm{j} 31.676$ (PU)

$\begin{array}{lcrr}\text { ASYM RMS } & \text { INTERRUPTING AMPS } & \\ 1 / 2 \text { CYCLES } & 3 \text { CYCLES } & 5 \text { CYCLES } & 8 \text { CYCLES } \\ 4015.8 & 3994.2 & 3994.2 & 3994.2\end{array}$

ASYM RMS FAULTED CURRENT ( AMPS )

AT TIME $=0.5$ CYCLES

$\begin{array}{cc}-- \text { PHASE A--- } & \text {--PHASE B--- } \\ 4015.8 & 0.0\end{array}$

---PHASE C---

0.0

Boostr VSD

VOLTAGE BASE LL: $\quad 480.0$ (VOLTS)

INI. SYM. RMS FAULT CURRENT: $4985.2 /-97$. (AMPS/DEG)

THEVENIN EQUIVALENT IMPEDANCE: $30.944+j 72.121$ (PU)

THEVENIN IMPEDANCE X/R RATIO: 2.331

SEQUENCE EQUIVALENT IMPEDANCE Z1: $7.471+\mathrm{j} 20.901$ (PU)

Z2: $\quad 7.471+j \quad 20.901$ (PU)

Z0: $16.002+\mathrm{j} 30.318$ (PU)

$\begin{array}{lcrrr}\text { ASYM } & \text { RMS } & \text { INTERRUPTING AMPS } & \\ 1 / 2 \text { CYCLES } & 3 \text { CYCLES } & 5 \text { CYCLES } & 8 \text { CYCLES } \\ 5311.0 & 4985.2 & 4985.2 & 4985.2\end{array}$

ASYM RMS FAULTED CURRENT ( AMPS )

AT TIME $=0.5$ CYCLES
-- PHASE A---

5311.0

0.0 PHASE C- -

0.0

HNF-2487, Rev. 0

Page F-51 
Calc. No. W-320-31-016

Revision 1

Page No. E - 17

DATE: $7: 24: 1998$ TIME: $8: 35: 25.52$

PAGE 17

Project $W-320$ / Tank 241-C-106 Sluicing

AY-Farm Electrical Distribution

Calc. No. W320-31-016 (Reference Drawing H-2-818693, SH 1, Rev. 1)

P-0622 BuS VOLTAGE BASE LL:

480.0 (VOLTS)

INI. SYM. RMS FAULT CURRENT: $4417.6 /-96$. ( AMPS/DEG)

THEVENIN EQUIVALENT IMPEDANCE: $36.283+\mathrm{j} 80.789$ (PU)

THEVENIN IMPEDANCE X/R RATI0: 2.227

SEQUENCE EQUIVALENT IMPEDANCE Z1: $7.760+\mathrm{j} 22.157$ (PU)

Z2: $\quad 7.760+j \quad 22.157$ (PU)

ZO: $20.763+\mathrm{j} 36.474$ (PU)

$\begin{array}{lccr}\text { ASYM } & \text { RMS } & \text { INTERRUPTING AMPS } & \\ 1 / 2 \text { CYCLES } & 3 \text { CYCLES } & 5 \text { CYCLES } & 8 \text { CYCLES } \\ 4673.0 & 4417.6 & 4417.6 & 4417.6\end{array}$

ASYM RMS FAULTED CURRENT (AMPS)

---PHASE A--- ---PHASE B

4673.0

0.0

--- PHASE C---
0.0

P-0621 Bus

VOLTAGE BASE LL:

480.0 (VOLTS)

INI. SYM. RMS FAULT CURRENT: $3161.7 /-79$. ( AMPS/DEG)

THEVENIN EQUIVALENT IMPEDANCE: $81.833+j 92.816$ (PU)

THEVENIN IMPEDANCE X/R RATIO: 1.134

SEQUENCE EQUIVALENT IMPEDANCE $21: 16.480+\mathrm{j} 25.542$ (PU)

Z2: $16.480+j \quad 25.542$ (PU)

ZO: $48.874+j \quad 41.732$ (PU)

$\begin{array}{lccr}\text { ASYM } & \text { RMS } & \text { INTERRUPTING AMPS } & \\ 1 / 2 \text { CYCLES } & 3 \text { CYCLES } & 5 \text { CYCLES } & 8 \text { CYCLES } \\ 3174.1 & 3161.7 & 3161.7 & 3161.7\end{array}$

ASYM RMS FAULTED CURRENT (AMPS)

-. PHASE A---
-

3174.1

0.0

-- PHASE C---

0.0

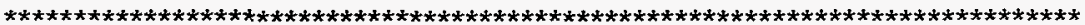

HNF-2487, Rev. 0

Page F-52 
Caic. No. W-320-31-016 Revision 1

Page No. E - 18

DATE: $7: 24: 1998$ TIME: $8: 35: 25.52$

PAGE 18

Project $W-320$ / Tank 241-C-106 Sluicing

AY-Farm Electrical Distribution

Calc. No. W320-31-016 (Reference Drawing H-2-818693, SH 1, Rev. 1)

$* * * * * * * * * * * * * * * * * *$ F A U T ANALYS I S SUMMARY****************

\begin{tabular}{|c|c|c|c|c|c|}
\hline BUS NAME & $\begin{array}{c}\text { VOLTAGE } \\
\text { L-L }\end{array}$ & $\begin{array}{l}\text { AVAIL } \\
3 \text { PHASE }\end{array}$ & $\begin{array}{l}\text { FAUL } \\
X / R\end{array}$ & $\begin{array}{r}\text { CURRENT } \\
\text { INE/GRND }\end{array}$ & $X / R$ \\
\hline $\begin{array}{l}\text { Pole E1 } \\
\text { XFMR Pri } \\
\text { XFMR Sec } \\
\text { AY-PDP-1 } 800 \mathrm{~A} \\
\text { AY102-PP1 Bus }\end{array}$ & $\begin{array}{r}13800 \\
13800 \\
480 \\
480 \\
480\end{array}$ & $\begin{array}{l}3363.7 \\
3363.0 \\
6047.5 \\
5974.6 \\
5037.9\end{array}$ & $\begin{array}{l}1.8 \\
1.8 \\
2.8 \\
2.8 \\
1.6\end{array}$ & $\begin{array}{l}1302.9 \\
1302.7 \\
5352.0 \\
5190.9 \\
3994.2\end{array}$ & $\begin{array}{l}1.0 \\
1.0 \\
2.5 \\
2.4 \\
1.2\end{array}$ \\
\hline $\begin{array}{l}\text { Tap Bus Asmbly } \\
\text { Group Cntlr } \\
\text { Pit Chillr Bus } \\
\text { Imrs SS } \\
\text { Winch Bus }\end{array}$ & $\begin{array}{l}480 \\
480 \\
480 \\
480 \\
480\end{array}$ & $\begin{array}{l}5905.3 \\
5887.4 \\
1964.8 \\
5877.7 \\
1198.9\end{array}$ & $\begin{array}{l}2.8 \\
2.8 \\
0.4 \\
2.8 \\
0.2\end{array}$ & $\begin{array}{r}5039.9 \\
5017.0 \\
1212.4 \\
5004.3 \\
715.9\end{array}$ & $\begin{array}{l}2.3 \\
2.3 \\
0.3 \\
2.3 \\
0.2\end{array}$ \\
\hline $\begin{array}{ll}\text { Boostr } & \text { VSD } \\
\text { P-0622 } & \text { Bus } \\
\text { P-0621 } & \text { Bus }\end{array}$ & $\begin{array}{l}480 . \\
480 \\
480\end{array}$ & $\begin{array}{l}5875.3 \\
5554.8 \\
4290.3\end{array}$ & $\begin{array}{l}2.8 \\
2.9 \\
1.5\end{array}$ & $\begin{array}{l}4985.2 \\
4417.6 \\
3161.7\end{array}$ & $\begin{array}{l}2.3 \\
2.2 \\
1.1\end{array}$ \\
\hline
\end{tabular}

HNF-2487, Rev. 0

Page F-53 


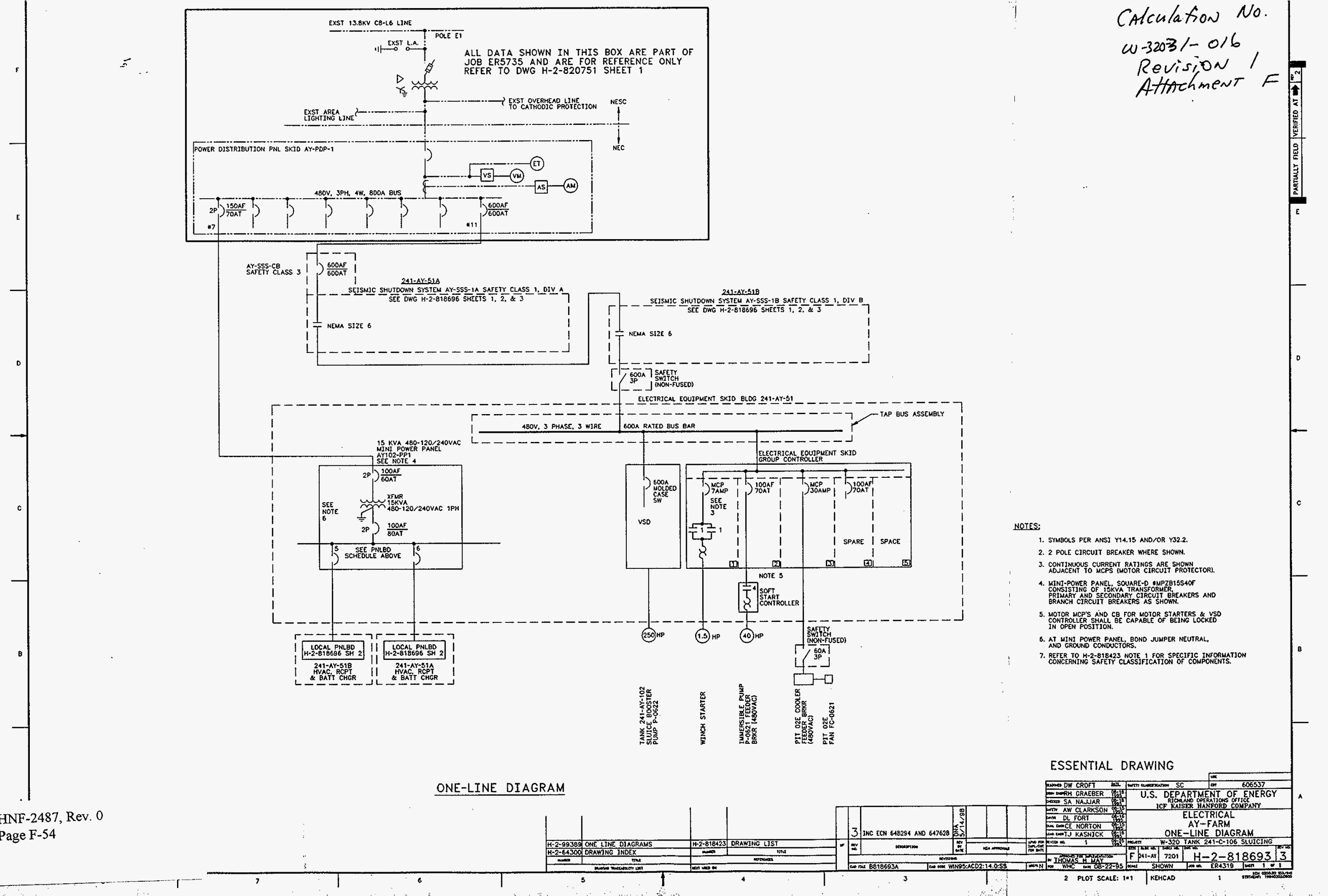


HNF-2487, Rev. 0

W320-31-017

Tank AY-102 Load Determination, Feeder/Protection Sizing, Voltage Drop, Lighting and Conduit Sizing Calculations 
This sheet shows the status and description of the attached Design Analysis sheets.

Discipline Electrical wo/Job No. Task Order N09 Calculation No. W320-31-017

Project No. \& Name W320 Tank 241-C-106 Sluicing

Calculation Item Tank AY-102 Load Determination, Feeder/Protection Sizing, Voltage Drop,

Lighting, and Conduit Sizing Calculations

These calculations apply to:

Dwg. No. See Continuation Sheet

Rev. No.

Dwg. No.

Rev. No.

Other (Study, CDR)

Rev. No.

The status of these calculations is:

[] Preliminary Calculations

[X] Final Calculations

[] Check Calculations (On Calculation Dated )

[] Void Calculation (Reason Voided)

Incorporated in final orawings?

[X] Yes [] No

This calculation verified by independent "check" calculations? [] Yes [X] No

Original and Revised Calculation Approvals:

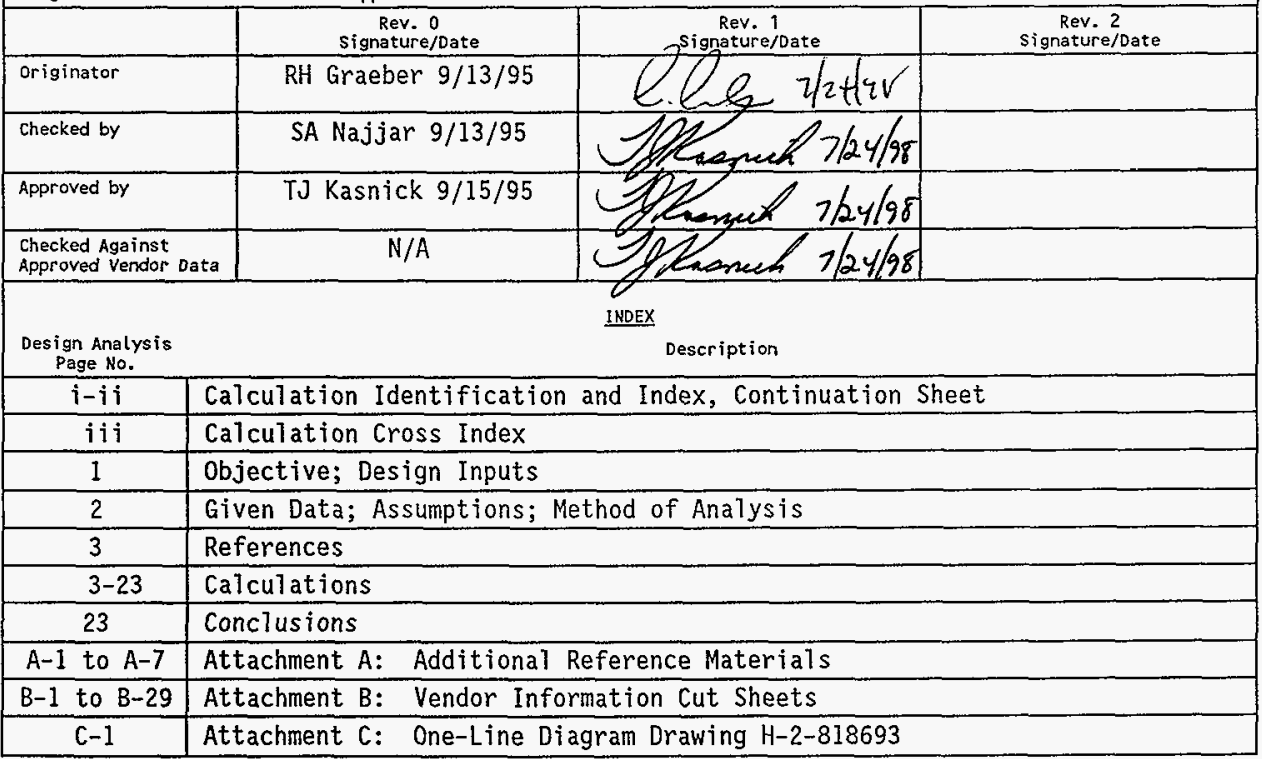

HNF-2487, Rev. 0

Page G-1 


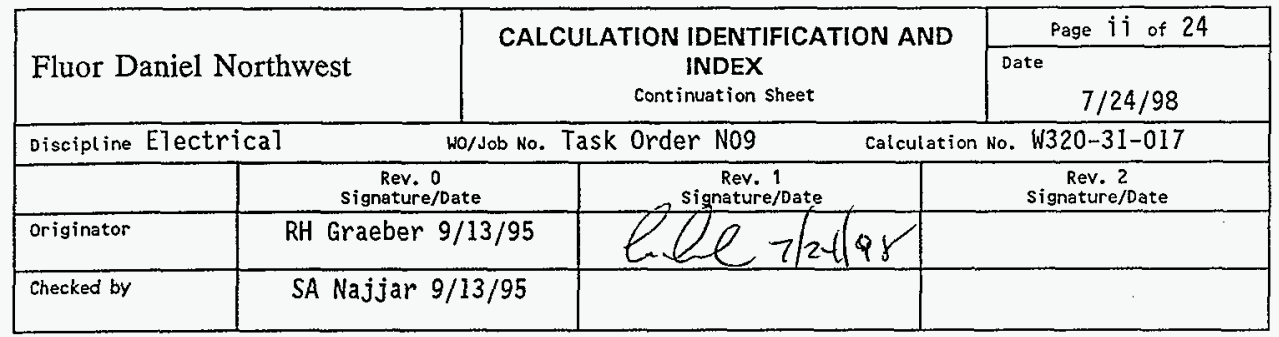

These calculations apply to:

H-2-818686 Sheet 1 , Rev. 0

H-2-818693 Sheet 1, Rev. 0

$\mathrm{H}-2-818694$ Sheet 1, Rev. 0

H-2-818695 Sheet 1, Rev. 1

H-2-818695 Sheet 2, Rev. 1

H-2-818695 Sheet 3, Rev. 1

H-2-818695 Sheet 4, Rev. 1

$\mathrm{H}-2-818695$ Sheet 5, Rev. 1

H-2-818696 Sheet 3, Rev. 0

H-2-818698 Sheet 1, Rev. 0

H-2-818698 Sheet 2, Rev. 0

H-2-818699 Sheet 1, Rev. O

H-2-818699 Sheet 2, Rev. 0

H-2-818700 Sheet 1 , Rev. 0

H-2-818700 Sheet 2, Rev. 0

H-2-818700 Sheet 3, Rev. 0

HNF-2487, Rev. 0

Page G-2 


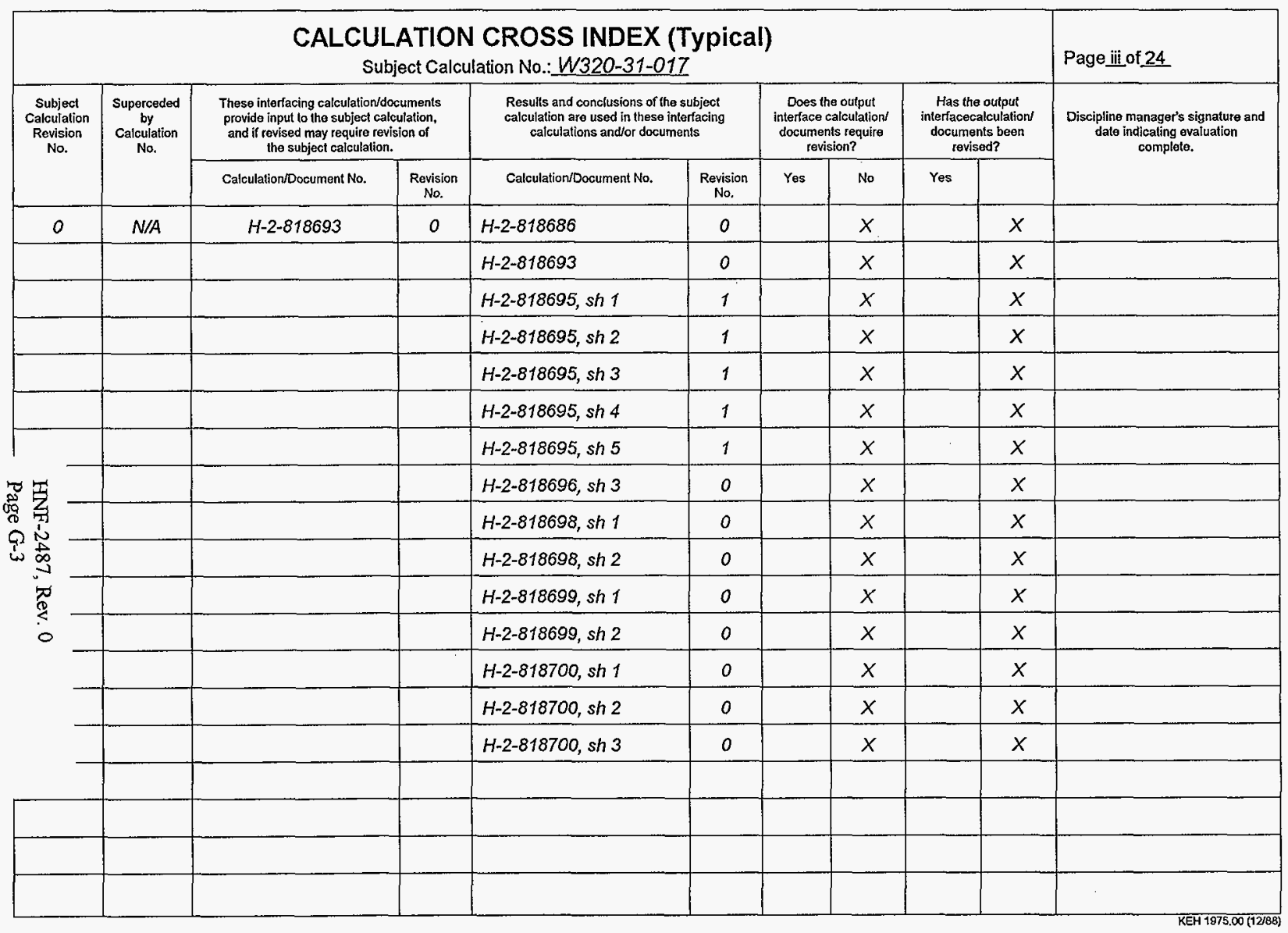




\section{DESIGN ANALYSIS}

client Numatec Hanford Corporation

subject TANK AY-102 LOAD DETERMINATION, FEEDER/PROTECTION SIZING, VOLTAGE DROP AND LIGHTING CALCULATIONS

Location 200E, AY Farm
Revision 1

Page No. 1 of 24

WO/Job No. Task Order N09

Date $9 / 13 / 95$

By RH Graeber

checked $9 / 13 / 95$

By SA Najjar

Revised $7 / 24 / 98$
By Cris Carlson

\section{OBJECTIVE}

1.1 To determine the total connected load and estimate the maximum demand for the Tank AY-102 partion of Project W-320, Tank 241-C-106 STuicing.

1.2 To size the power feeder/branch circuit conductors, circuit protection and conduits.

1.3 To calculate the voltages at motor terminals during normal running and starting conditions in order to ensure proper operation of pump motors.

1.4 To determine number of 1 ight fixtures required to provide adequate illumination levels.

1.5 To determine instrument and control conduit sizes.

1.6 Special situation equipment sizing and voltage drop checks.

\section{DESIGN INPUTS}

2.1 FunctionaT Design Criteria, HNF-SD-W320-FDC-001, Rev. 5.

2.2 One-Line Diagram, Drawing (DWG) H-2-818593.

2.3 NFPA-70, National Electrical Code (NEC), 1993 edition.

a. Branch circuit conductors shall be sized to carry at least $125 \%$ of the largest load plus the sum of other loads, for multiple load circuits.

b. Single conductor copper wires, with $90^{\circ} \mathrm{C}$ XHHW, 600 VAC insulation in buried raceway and conductor ampacity per Reference 6.1, Table 310-16 for $86^{\circ} \mathrm{F}\left(30^{\circ} \mathrm{C}\right)$ ambient temperature, sha1 1 be used.

c. Minimum conduit size shall be based on Reference 6.1, NEC Chapter 9 Table $3 \mathrm{~B}$.

d. Motor rated full load currents shall be based on Reference 6.1, 1993 NEC Table 430-150, Reliance Electric motor catalog and motor data sheets in vendor file \#22668. Vendor data for Booster pump motor (250 HP motor) shows full load amps is 273 amperes. See Reliance data sheet E06854-AA009.

e. Motor Circuit Protector (MCP) for motor protection and motor starters shall be sized per Reference 6.4 . 


\section{Fluor Daniel Northwest}

Calc. No. W320-31-017

\section{DESIGN ANALYSIS}

$\mathrm{Client}$ Numatec Hanford Corporation

subject TANK AY-102 LOAD DETERMINATION, FEEDER/PROTECTION SIZING, VOLTAGE DROP AND LIGHTING CALCULATIONS

Location 200E, AY Farm
Revision 1

Page No. 2 of 24

wo/sob No. Task Order NO9

Date $9 / 13 / 95$ By RH Graeber

checked $9 / 13 / 95$ By SA Najjar

Revised 7/24/98 By Cris Carlson

2.4 The lockable "Service Entrance Rated" safety switch shall provide the main service disconnecting means to the AY-Farm loads supplied via the Electrical Equipment Skid (EES), building 241-AY-51.

2.5 Effective impedance $\left(Z_{\text {eff }}\right)$ at $85 \%$ Power Factor (PF), shown in Reference 6.1 , NEC Chapter 9 Table 9 , shall be used for voltage calculations.

\section{GIVEN DATA}

3.1 The 150 KVA transformer bank installed by the Equipment Removal Phase of Project W-320 will be the source of power for these sluicing loads. Hanford Electrical Utilities Department estimates demand of the existing services is $22 \mathrm{KW} 0.8 \mathrm{PF}=27.5 \mathrm{KVA} \approx 28 \mathrm{KVA}$.

3.2 Distribution transformer $\% Z=2.6 \%$.

3.3 Minimum allowable voltage at the motor terminals is 414 volts based on NEMA motor rating of $460 \mathrm{~V} \pm 10 \%$.

3.4 Pit-02E, the EES housing 241-AY-51 and Seismic Shutdown System missile shield enclosures 24I-AY-51A and 51B are air conditioned.

3.5 Motors shown on the One-Line Diagram, Drawing $\mathrm{H}-2-818693$ are induction motors rated $460 \mathrm{VAC}, 36,60 \mathrm{~Hz}$. Motor FLA are from the NEC, except for the $250 \mathrm{HP}$ motor which is based on vendor information in CVI file \#22668.

3.6 The Variable Speed Drive (VSD) controller for the $250 \mathrm{HP}$ In-Line Booster pump motor has been furnished by pump vendor (Procurement Specification W-320P17). VSD controller maximum input current is $325 \mathrm{~A}$. (150\% Torque Limit for $60 \mathrm{sec}$. maximum).

3.7 The $40 \mathrm{HP}$ Immersible pump is started before the booster pump. The submersible pump is interlocked with the booster pump so that the booster pump can only be started after the submersible pump discharge pressure exceeds the pressure switch setpoint. A "Soft Start" unit is to be used to limit torque effect to submersible pump hoses to prevent excess twisting.

3.8 Pit chiller FLA is 8.1 A per Edwards Engineering Corp. Vendor data, see file \#22668. 


\section{Fluor Daniel Northwest}

\section{DESIGN ANALYSIS}

client Numatec Hanford Corporation

subject TANK AY-102 LOAD DETERMINATION,

FEEDER/PROTECTION SIZING, VOLTAGE DROP AND

LIGHTING CALCULATIONS

Location 200E, AY Farm
Calc. No. W320-31-017

Revision 1

Page No. 3 of 24

wo/Job No. Task Order N09

Date $9 / 13 / 95 \quad$ By RH Graeber

Checked 9/13/95 By SA Najjar

Revised 7/24/98 By Cris Carlson

\section{ASSUMPTIONS}

4.1 Estimated duration for this project is two years.

4.2 Transformer primary taps are set at $100 \%$ and secondary terminal voltage at no-load conditions is $480 \mathrm{~V}$.

4.3 Demand factor (DF) for the booster and submersible pump and pit cooler motors is $80 \%$; other loads at $30 \%$.

4.4 "Soft-Start" unit used for 40 HP submersible pump, P-0621, is considered to have the same efficiency as a conventional motor starter. The basis for this is the description given in the Cutler Hammer document, "User's Manual EasyStart Advantage EA Series." See Vendor Information file \#22668.

4.5 Unless otherwise stated, $P F=.95$ and EFF. $=0.95$

\section{METHOD OF ANALYSIS}

5.1 Hand calculations.

6. REFERENCES

6.1 NFPA-70, National ElectricaT Code (1993).

6.2 Drawing H-2-818693, Sheet 1, Rev. 1, Electrical AY-Farm One-Line Diagram.

6.3 W320-31-016, AY-102 Demand Load Analysis, Size Feeders, Voltage Drop and FauTt study.

6.4 Westinghouse Cat. 25-000, Quick Selector 1993.

6.5 Additional References to Support C-Farm Demand Load Anaiysis:

a. Industrial Power Systems Handbook, Beeman, First Edition, Chapter 17 (Load and Cost Estimating Data).

b. Standard Handbook for Electrical Engineers, Fink and Beaty, Eleventh Edition, Chapter 18 (Demand and Diversity Factors).

6.6 W-320 vendor data CVI file \#22668.

HNF-2487, Rev. 0

Page G-6 
DESIGN ANALYSIS

ctient Numatec Hanford Corporation

subject TANK AY-102 LOAD DETERMINATION,

FEEDER/PROTECTION SIZING, VOLTAGE DROP AND

LIGHTING CALCULATIONS

Location 200E, AY Farm
Revision 1

Page No. 4 of 24

worjob No. Task Order N09

Date 9/13/95 By RH Graeber

checked 9/13/95 By SA Najjar

Revised 7/24/98 By Cris Carlson

\section{CALCULATIONS}

7.1 Connected Load Determination

a) 250HP Booster Pump Motor: $273 \mathrm{~A}$

VSD with $250 \mathrm{HP}$ Input a $95 \%$ eff.

$287.4 \mathrm{~A}$

VSD maximum input: $325 \mathrm{~A}$

b) 40 HP Immersible Pump Motor: $52 \mathrm{~A}$

"Soft Start" Unit energized only during motor start. 52 A

c) $1.5 \mathrm{HP}$ Winch

d) Package Chiller

Subtotal 480V, 3 PH Motor Connected Load $\approx$

e) Mini-Power Pane1: $15 \mathrm{KVA}, 1 \mathrm{PH}(\mathrm{B}-\mathrm{C})$

Total Connected Load Phases $B \& C \approx$

Total Connected Load $\approx 381.4 \mathrm{~A} \times .480 \mathrm{KV} \times \sqrt{3} \approx 317.1 \mathrm{KVA}$

\subsection{Estimated Demand Determination}

7.2.1 ICF KH "Utilities" Method:

a) Existing Loads (Ave. Demand)

28 KVA

b) $250 \mathrm{HP}, 273 \mathrm{~A}$ ? $460 \mathrm{~V}$,

VSD efficiency $95 \%, 80 \%$ DF

c) $40 \mathrm{HP}, 52 \mathrm{~A} \bigcirc 460 \mathrm{~V}, 80 \% \mathrm{DF}$

183.2 KVA

d) $1.5 \mathrm{HP}, 2.6 \mathrm{~A} \cap 460 \mathrm{~V}, 30 \% \mathrm{DF}$

33.1 KVA

e) $15 \mathrm{KVA}$ - $95 \%$ eff, $30 \%$ DF

0.6 KVA

f) Pit Cooler $8.1 \mathrm{~A} \times .460 \mathrm{kV} \times 1.73 \times 80 \% \mathrm{DF}$

4.7 KVA

5.2 KVA

Subtotal $=$ 254.8 KVA

Diversity Factor

1.44

HNF-2487, Rev. 0

Total Estimated Demand $176.9 \mathrm{KVA}$ 


\section{Fluor Daniel Northwest}

DESIGN ANALYSIS

client Numatec Hanford Corporation

subject TANK AY-102 LOAD DETERMINATION, FEEDER/PROTECTION SIZING, VOLTAGE DROP AND LIGHTING CALCULATIONS

Location 200E, AY Farm
Calc. No. W320-31-017

Revision 1

Page No. 5 of 24

Ho/Job No. Task Order NO9

Date 9/13/95 By RH Graeber

checked $9 / 13 / 95$ By SA Najjar

Revised 7/24/98 By Cris Carlson

7.2.2 "Normal" Anticipated Method:
a) Existing Loads
28 KVA
b) $250 \mathrm{HP}$ running at $90 \mathrm{HP}$, extrapolated from motor data sheet, $100 \%$ DF
97.2 KVA
c) $40 \mathrm{HP}, 52 \mathrm{~A} 0460 \mathrm{~V}, 100 \% \mathrm{DF}$
41.4 KVA
d) $1.5 \mathrm{HP} @$ zero DF
0
e) $15 \mathrm{KVA}$ @ $95 \%$ eff \& $30 \%$ DF
4.7 KVA
f) Pit Cooler $8.1 \mathrm{~A} \times 460 \vee \times 1.73 \times 0.8$
$5.2 \mathrm{KVA}$

Tota7 Anticipated Demand $=$

$176.5 \mathrm{KVA}$

7.2.3 "Worst Case" Full Load Method:
a) Existing Loads
28 KVA
b) $250 \mathrm{HP}, 273 \mathrm{~A}$ \& $460 \mathrm{~V}, 95 \%$ VSD eff, $100 \%$ DF
$229.0 \mathrm{KVA}$
c) $40 \mathrm{HP} 552 \mathrm{~A} \odot 460 \mathrm{~V}, 100 \% \mathrm{DF}$
$41.4 \mathrm{KVA}$
d) $1.5 \mathrm{HP} @$ zero DF
0
e) $15 \mathrm{KVA} @ 95 \%$ eff, $30 \%$ DF
4.7 KVA
f) Pit cooter
$5.2 \mathrm{KVA}$

Total "Worst Case" Demand $=\ldots \ldots$ 308.3 KVA

S7uicing Project "Worst Case" demand $\approx 308.3$ KVA -28 KVA $\approx 280.3$ KVA

Three $50 \mathrm{KVA}$, Pole Mounted, 0il Filled Transformers are installed as a part of the Equipment Removal Phase of the project.

\subsubsection{Summary}
a) $176.9 \mathrm{KVA} \div 150 \mathrm{KVA} \approx 117.9 \%$ of nameplate.
b) $176.5 \mathrm{KVA} \div 150 \mathrm{KVA} \approx 117.7 \%$ of nameplate.
c) $308.3 \mathrm{KVA} \div 150 \mathrm{KVA} \approx 205.5 \%$ of nameplate. 


\section{Fluor Daniel Northwest}

DESIGN ANALYSIS

ctient Numatec Hanford Corporation

subject TANK AY-102 LOAD DETERMINATION,

FEEDER/PROTECTION SIZING, VOLTAGE DROP AND LIGHTING CALCULATIONS

Location 200E, AY Farm
Catc. No. W320-31-017

Revision 1

Page No. 6 of 24

wo/Job No. Task Order N09

Date $9 / 13 / 95$

By RH Graeber

Checked $9 / 13 / 95$

By SA Najjar

Revised $7 / 24 / 98$

d) Therefore, the 150 KVA transformer bank is adequate based on:

1) For "Normal" Operation, 130\% of demand (or 1ess) is acceptable per Electrical Utilities engineering and IEEE /ANSI C57.91 and 92.

2) Duration of "Worst Case" is less than 5 minutes. Project design life is only 2 years. Small losses of the transformer's 30 year design life expectancy are acceptable.

\subsection{Breaker, Starter. Feeder and Conduit Sizing}

7.3.1 Main feeder from AY-PDP-1 via the EES, Circuit Breaker, Seismic Contactors and Safety Switch, to the Tap Bus Assembly

The Lockable Non-Fused Safety Switch will be the main disconnect means for 241-AY-51 (EES).

Feeder Sizing Per NEC 220-10, 240-21, 430-2, 430-22, 430-24 and 43028 using actual loads listed in 7.1 .

a) Summation of motor loads Per VSD vendor \& NEC 430-24:

1) $250 \mathrm{HP}$ Booster Pump (VSD)

$287.4 \mathrm{~A}$

2) $40 \mathrm{HP}$ Immersible Pump (Soft Start)

$52 \mathrm{~A}$

3) $1.5 \mathrm{HP}$ Winch

4) Pit Chilier

5) Plus $25 \%$ of largest motor 1 )

Main Feeder Size Capacity $=\ldots \ldots$ 422.0 A

600AF/600AT circuit breaker used at AY-PDP-1.

Down-stream feeder conductors shall be rated $600 \mathrm{~A} \otimes 75^{\circ} \mathrm{C}$.

1) Need 2-350 kcmil per phase.

2) Requires $3-350 \mathrm{kcmil}$ conductors and $1 \# 1 / 0$ copper ground, in each of two $3 \frac{1}{2} "$ conduits.

HNF-2487, Rev. 0

Page G-9 


\section{Fluor Daniel Northwest}

\section{DESIGN ANALYSIS}

client Numatec Hanford Corporation

subject TANK AY-102 LOAD DETERMINATION,

FEEDER/PROTECTION SIZING, VOLTAGE DROP AND LIGHTING CALCULATIONS

Location 200E, AY Farm
Calc. No. W320-31-017

Revision 1

Page No. 7 of 24

WorJob No. Task Order N09

Date $9 / 13 / 95$ By RH Graeber

Checked 9/13/95 By SA Najjar

Revised $7 / 24 / 98$
By Cris Carlson

3) Wire runs W102-01A, 01B, 02, 02A, 03, 03A, 04, 04A, 19 and $19 \mathrm{~A}$.

4) Conduits AY102-3P0I, 01A, 04, 04A, 22, 22A and AY102 - 1P02, 02A, 03 and 03A.

Note: Up sized $3 \frac{1}{2}$ " conduits to $4^{\prime \prime}$ conduits based on constructability comment.

\subsubsection{The 250 HP Booster Pump and VSD Controller (P-0622)}

(VSD protective circuitry included with controller provided by booster pump vendor).

VSD Feeder:

VSO controller maximum input current $=325 \mathrm{~A}$ per vendor information. Per NEC 430-2, required feeder ampacity $=1.0 \mathrm{X}$ $325=325 \mathrm{~A}$. Per NEC Table $310-16$, use $3-500 \mathrm{kcmil} 90^{\circ} \mathrm{C}$ conductors with 1 \#1/0 ground wire in $3 \frac{1}{2} "$ conduit (Up sized to 4 " conduit to aid in cable pulling).

Wire run W102-05; conduit AY102-3P05

\section{Motor Feeder:}

Booster Pump motor (vendor information) $F L A=273 \mathrm{~A}$. Per NEC $430-22$, required feeder ampacity $=1.25 \times 273=341 \mathrm{~A}$. Per NEC Table $310-16$, use $3-500 \mathrm{kcmil} 90^{\circ} \mathrm{C}$ conductors with 1 \#1/0 ground wire in $3 \frac{1}{2}$ " conduit (Up sized to $4^{\prime \prime}$ conduit to aid in cable pulling).

Wire run W102-08; conduit AY102-3P08.

See 7.3.9 For "In Pit" segment of motor feeder.

VSO feeder is tapped off buss at "Tap Bus Assembly". Feeder length is approximately 7 feet. Feeder meets "10 foot" Tap Rule criteria (NEC 240-21 and 430-28). 


\section{Fluor Daniel Northwest}

\section{DESIGN ANALYSIS}

client Numatec Hanford Corporation

subject TANK AY-102 LOAD DETERMINATION, FEEDER/PROTECTION SIZING, VOLTAGE DROP AND LIGHTING CALCULATIONS Location 200E, AY Farm
Calc. No. W320-31-017

Revision 1

Page No. 8 of 24

Ho/Job No. Task Order N09

Date $9 / 13 / 95$

By RH Graeber

checked $9 / 13 / 95$

By SA Najjar

Revised $7 / 24 / 98$

\subsubsection{HP Submersible Pump (P-0621)}

Motor Feeder:

$\mathrm{FLA}=52 \mathrm{~A} ;$ required feeder ampacity $=1.25 \times 52 \mathrm{~A}=65 \mathrm{~A}$

3-\#4 conductors (ampacity $=70 \mathrm{~A}$ ) is adequate based on ampacity. Used 3-\#1/0 with 1-\#4 ground wire in 2" conduit.

Install 1\#1/0 AWG per phase from Group Controller Power

Terminal Block to "Soft Start" unit. Use 70 A MCP (mounted within Group Controller).

Wire run W102-09; conduit AY102-3P09.

Install 1\#1/0 AWG per phase from "soft start" to PTBX-0621.

Wire run W102-29; conduit AY102-3P26.

Use 150 A MCP and NEMA Size 4, Full Voltage Non-Reversing (FVNR) starter (mounted within Group Controller).

See 7.3.9 for "In Pit" Segment of Motor Feeder.

See 7.3.10 for "Group Controller" feeder and jumper sizing. Per Code Authority, power terminal block ties within Group Controller are not considered as taps. See Appendix page A-1.

\subsubsection{The 1.5 HP Winch (W-0621)}

\section{Motor starter line and load side feeder conductors:}

$F L A=2.6 \mathrm{~A}$; required feeder ampacity $=1.25 \times 2.6 \mathrm{~A}=3.25 \mathrm{~A}$

Use 7 A MCP and NEMA Size 1, Full Voltage Reversing (FVR) starter.

Use 3 -\#12 with 1 -\#12 ground wire in $3 / 4$ " conduit (Up sized to 1 " conduit for conservatism).

Wire run W102-10 \& 31; conduit AY102-3P10 \& 3P27.

Install 1\#12 AWG per phase from top of starter to Group Controller power terminal block. 


\section{Fluor Daniel Northwest}

DESIGN ANALYSIS

client Numatec Hanford Corporation

subject TANK AY-102 LOAD DETERMINATION,

FEEDER/PROTECTION SIZING, VOLTAGE DROP AND LIGHTING CALCULATIONS

Location 200E, AY Farm
Calc. No. W320-31-017

Revision 1

Page No. 9 of 24

wo/sob No. Task Order N09

Date $9 / 13 / 95$

By RH Graeber

checked $9 / 13 / 95$

By SA Najjar

Revised $7 / 24 / 98$ By Cris Carlson

Note: Out of PTBX on top of cover block, change to $3 / C$ cable. Change/upsize to $3 / \mathrm{C} \# 8$ with ground over to winch motor (manufacturer availability).

Wire run W102-28

\subsubsection{Packaged Pit Chiller (R-0621/FC-0621)}

Feeder circuit breaker "line and load side" Conductors:

Use $30 \mathrm{~A} \mathrm{CB}$, as recommended by MFR. Install in Group Controller."

Use 3-\#10 with $1-\# 10$ ground wire in $3 / 4 "$ conduit (Up sized to 1 " conduit for conservatism).

Wire run W102-11; conduits AY102-3P11 \& $3 P 17$.

Instal1 1\#10 AWG conductor per phase from circuit breaker within "Group Controller" to safety switch and to Group Controller power terminal blocks.

\subsubsection{Mini-Power Panel (AY102-PP1)}

Feed 15 KVA, 1ø, transformer, with $480 \mathrm{~V}$ primary, from circuit breaker in Power Distribution Panel.

$$
I=\frac{15,000 \mathrm{VA}}{480 \mathrm{~V}}=31.25 \mathrm{~A}
$$

Unit supplied with $60 \mathrm{~A}, 2$ pole primary circuit breaker by manufacturer.

Install 150AF/70AT, 2 Pole CB in AY-PDP-1, Ckt\# 7. Provide 70A, 1 phase circuit to unit.

Use 2-\#4 with 1-\#8 ground wire in $3 / 4$ " conduit.

Wire run W102-07; conduit AY102-3P39. 
DESIGN ANALYSIS

client Numatec Hanford Corporation

subject TANK AY-102 LOAD DETERMINATION, FEEDER/PROTECTION SIZING, VOLTAGE DROP AND LIGHTING CALCULATIONS Location 200E, AY Farm
Revision 1

Page No. 10 of 24

worJob No. Task Order N09

Date 9/13/95 By RH Graeber

Checked 9/13/95 By SA Najjar

Revised $7 / 24 / 98$ By Cris Carison

7.3.7 Seismic Shutdown Missile Enclosure Air Conditioning and Receptacles

$I=\frac{1320}{120}=11 \mathrm{~A} ; 11 \mathrm{~A} \times 1.25=14 \mathrm{~A}$ capacity (from AY102-PP1)

Calculation W102-31-022 requires \#10 AWG due to voltage drops.

Use 2-\#10 with 1-\#10 ground wire in 3/4" conduit (underground

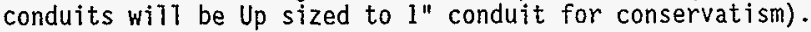

Wire runs W102-22 \& 23; conduits AV102-3P15 \& 3P16 will be 1".

7.3.8 Typical 120 VAC branch circuit conductor loaded up to 1,500 VA

$I=1500 \mathrm{VA} / 120 \mathrm{~V}=12.5 \mathrm{~A} ; 12.5 \mathrm{~A} \times 1.25=15.625 \mathrm{~A}$ capacity and a $20 \mathrm{~A}$ circuit breaker.

Use 2-\#12 with 1 -\#12 ground wire in $3 / 4^{\text {" }}$ conduit (underground conduits will be Up sized to $1^{1 "}$ conduit for conservatism).

Wire runs W102-12, 13, 14, 15, 16, 17, 18, 20, 21, 25, 32 \& 36 ; conduits AY102-3P13, 18, 19, 20, 21, 23, 25A, 28, 38 \& 41 will be $3 / 4^{11}$; conduits AY102-12, 14, 24, 25, 30 \& 34 will be $1 "$.

\subsection{9 "In Pit" Cord Sizing}

NEC article 40 defines the requirements for the "In-Pit" cords. NEC article 430-22 states that branch circuit conductors supplying a single motor shall have an ampacity not less than 125 percent of the motor full-load current rating.

$40 \mathrm{HP}$ Immersible pump motor has $\mathrm{FLA}=52 \mathrm{~A}$. The 1993 NEC Table 430-150 does not include values for motors of $250 \mathrm{HP}$. Reliance Electric information shows that the $250 \mathrm{HP}$ motor FLA is 273 amps.

The cables will be in free air within the pump pit. NEC Table 4005(B) for Type G, portable power cord will be utilized. The cable is a thermoset cabie that, if assumed equivalent to Type $G$, will need derating for high ambient conditions using the correction factors in Table 310-16.

Per Project $W-320$ Motor Cooling Evaluation dated $6 / 7 / 95$, the ambient temperature at the $\mathrm{AY}-102$ pump pit will be maintained below $120^{\circ} \mathrm{F}$

HNF-2487, Rev. 0 $\left(50^{\circ} \mathrm{C}\right)$ by the use of a pit cooter. Per NEC Table 310-17, a 


\section{DESIGN ANALYSIS}

client Numatec Hanford Corporation

subject TANK AY-102 LOAD DETERMINATION,

FEEDER/PROTECTION SIZING, VOLTAGE DROP AND LIGHTING CALCULATIONS

Location 200E, AY Farm
Revision 1

Page No. 11 of 24

wo/Job No. Task Order N09

Date $9 / 13 / 95$

By RH Graeber

checked $9 / 13 / 95$

By SA Najjar

Revised $7 / 24 / 98$

correction factor of 0.82 will apply. Since this is considered cord (not cable in conduit), the calculation of the desired derating factor is based on the cable manufacturer's recommendation.

Per the Rockbestos company, the following formula is used for determining an acceptable derating factor.

$$
T C F=\sqrt{\frac{(T C 2-\operatorname{Ta2})}{(\operatorname{TC1}-\operatorname{Ta} 1)} \times \frac{(234.5+T C 1)}{(234.5+T C 2)}}
$$

$T c 1=$ Maximum conductor temperature used to determine base ampacity.

$\mathrm{Ta} 1$ = Ambient temperature used to determine base ampacity $\left(30^{\circ} \mathrm{C} \mathrm{per}\right.$ NEC Table 400-5(b).

Tc2 = Maximum conductor temperature used to determine adjusted ampacity $\left(90^{\circ} \mathrm{C}\right.$ for Rockbestos Firewall III for 40 year qual ified 1ife.)

$\mathrm{Ta} 2$ = Ambient temperature used to determine adjusted ampacity; $0.3^{\circ} \mathrm{C}$ additional is used as margin.

The cords for the pumps falt under the $F$ category, i.e. no more than 3 current carrying conductors. The $90^{\circ}$ column is used, because of rating of the terminations in the pump terminal boxes will be defined to be $90^{\circ} \mathrm{C}$.

$$
T C F=\sqrt{\frac{(90-(50+0.3))}{(90-30)} \times \frac{(234.5+90)}{(234.5+90)}}=0.8134
$$

For sizing the $40 \mathrm{HP}$ Immersible Pump cord:

$(52 \mathrm{~A} \times 1.25) / .8134=79.9 \mathrm{~A}$; Per NEC Table 400-5(B), use a minimum \#6 conductor with ground wire.

Vendor supplied Wire Run W102-27.

\section{For sizing the 250 HP Booster Pump cord:}

$(273 \mathrm{~A} \times 1.25) / .8134=419.5 \mathrm{~A}$; per NEC Table $400-5(\mathrm{~B})$, use $500 \mathrm{kcmil}$ conductors with ground wire.

Wire Run W102-26. 


\section{Fluor Daniel Northwest}

DESIGN ANALYSIS

client Numatec Hanford Corporation

subject TANK AY-102 LOAD DETERMINATION,

FEEDER/PROTECTION SIZING, VOLTAGE DROP AND LIGHTING CALCULATIONS

Location 200E, AY Farm
Calc. No. W320-31-017

Revision 1

Page No. 12 of 24

wo/sob No. Task Order N09

Date $9 / 13 / 95$

By RH Graeber

Checked 9/13/95

BY SA Najjar

Revised $7 / 24 / 98$

Rockbestos Firewal7 III XHHW P62-3119 is a 3/C\#I AWG cable with an overall diameter of 1.16" (something similar will be vendor furnished by procurement specification W-320-P17).

Rockbestos Firewall III XHHW P62-0504 is a $500 \mathrm{kcmil}$ cable with an overal1 diameter of $2.26^{\prime \prime}$.

\subsubsection{Group Controller Feeder and "Jumper" (between all starters) Sizing}

a) Group Controller Total Load
1) Winch $(1.5 \mathrm{HP})$
$2.6 \mathrm{~A}$
2) Immersible Pump (40 HP)
$52 \mathrm{~A}$
3) Chitler motor loads
$8.1 \mathrm{~A}$
4) Plus $25 \%$ of largest motor 2)
$13 \mathrm{~A}$

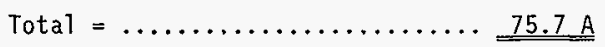

For $75^{\circ} \mathrm{C}$ rated conductors, use $3 \# 2 / 0$ and $1 \# 4$ ground wire in 2" conduit.

Wire run W102-06; conduit AY102-3P06.

Jumpers between power terminal blocks shall be $\# 2 / 0$.

NEC "10 Foot" Tap Rule applies and criteria is met.

\subsection{Steady State Voltage Drop Evaluation (Worst Case Full Load Method)}

7.4.1 On Transformer Bank

$$
\begin{aligned}
& I_{P U}=\frac{308.3 \mathrm{KVA}}{150 \mathrm{KVA}}=2.06 \\
& \% V D=\% Z \times I_{P U}=2.6 \times 2.06=5.3 \%
\end{aligned}
$$




\section{Fluor Daniel Northwest}

DESIGN ANALYSIS

client Numatec Hanford Corporation

subject TANK AY-102 LOAD DETERMINATION,

FEEDER/PROTECTION SIZING, VOLTAGE DROP AND LIGHTING CALCULATIONS

Location 200E, AY Farm
Calc. No. W320-31-017

Revision 1

Page No. 13 of 24

wo/sob No. Task Order N09

Date 9/13/95 By RH Graeber

checked $9 / 13 / 95$ By SA Najjar

Revised 7/24/98 By Cris Carlson

7.4.2 On 100' of Parallel $350 \mathrm{kcmil}$ copper feeders in steel conduits (AY-PDP-1 to Tap Bus Assembly). $\% V D=\frac{\sqrt{3} \times I \times Z e f f / 1000^{\circ} \times L}{4.8} ; 308.3 \mathrm{KVA}-28 \mathrm{KVA}=280.3 \mathrm{KVA}$ Where $\mathrm{I}=\frac{280.3 \mathrm{KVA}}{\sqrt{3} \times .480 \mathrm{KV}} \approx 337.2 \mathrm{~A}$

Parallel $350 \mathrm{kcmil}$ Feeders:

$Z_{\text {eff }}=0.06 \div 2=0.03, L=0.12$

$\%$ VD $350 \mathrm{kcmil}$ feeder $=\frac{\sqrt{3} \times 337.2 \times 0.03 \times .12}{4.8}=\underline{\underline{0.44 \%}}$

Total \%VD from transformer bank to Tap Buss Assemb7y $=0.44+5.3$

$$
=\underline{\underline{5.74 \%}}
$$

7.4.3 \%VD from VSD Controller to Motor $\left(100^{\prime}\right.$ of $500 \mathrm{kcmil}$ copper feeder in steel conduit)

Motor FLA $=273 \mathrm{~A}$; Zeff $=.05$ ohms, $L=.1$

$$
\text { Tota7 \%VD }=\frac{\sqrt{3} \times 273 \times .05 \times .1}{4.8}=\underline{\underline{0.49 \%}}
$$

Summary:

Total \%VD at motor terminaTs $=5.74+0.49=\underline{\underline{6.23 \%}}$

Motor terminal voltage $=480(1-0.0623) \approx 450.1$ Volts

Minimum permissible voltage $=414$ volts 


\section{Fluor Daniel Northwest}

Calc. No. W320-31-017

DESIGN ANALYSIS

client Numatec Hanford Corporation

subject TANK AY-102 LOAD DETERMINATION,

FEEDER/PROTECTION SIZING, VOLTAGE DROP AND

LIGHTING CALCULATIONS

Location 200E, AY Farm
Revision 1

Page No. 14 of 24

Wo/Job No. Task Order N09

Date 9/13/95 By RH Graeber

Checked 9/13/95 By SA Najjar

Revised $7 / 24 / 98 \quad$ By Cris Carison

7.4.4 \%V on Immersible Pump Feeder (\#1/0 copper feeder in steel conduit $80^{\prime}$ to Pump Pit and $60^{\prime} \# 4$ down into Tank $A Y-102$ )

Motor $F L A=52 A ; Z_{\text {eff }}$ for $\# 4 A W G=.30 ; L=.06$

$Z_{\text {eff }}$ for $\# 1 / 0=.13 ; L=.08$

$\%$ VD $=\frac{\sqrt{3} \times 52 \times((.13 \times .08)+(.30 \times .06))}{4.8}=0.53 \%$

Total \%vo at motor terminals $=5.74+0.53=6.27 \%$

Motor terminal voltage $=480(1-.0527) \approx 449.9$ Volts

Minimum permissible voltage $=414 \mathrm{~V}$

\section{$7.5 \%$ Voltage Dip During Immersible Pump Startup}

Initial conditions:

a) Booster Pump is off

b) Winch is off

c) Chiller is on

d) Normal Panelboard loads are on.

1) Chiller $\quad 8.1 \mathrm{~A}$

2) Mini-Power Panel (8 KVA)

Total

Existing Load: $28 \mathrm{KVA} \div 480 \div 1.73 \approx 34 \mathrm{~A}$

\subsection{1 \%VD on Transformer Bank}

$I=25.1+34+(6 \times F L A)=25.1+34+6(52)=25 . I+34+312$

$=371.1 \mathrm{~A}$

$\% \mathrm{VD}=\frac{371.1}{181} \times 2.6 \approx 5.33 \%$

Where 181 = transformer rated full load secondary current. 


\section{Fluor Daniel Northwest}

DESIGN ANALYSIS

client Numatec Hanford Corporation

subject TANK AY-102 LOAD DETERMINATION,

FEEDER/PROTECTION SIZING, VOLTAGE DROP AND LIGHTING CALCULATIONS

Location 200E, AY Farm
Calc. No. W320-31-017

Revision 1

Page No. 15 of 24

wo/Job no. Task Order N09

Date 9/13/95 By RH Graeber

Checked 9/13/95 By SA Najjar

Revised $7 / 24 / 98$ By Cris Carlson

7.5.2 \%VD on Service Drop and Feeder up to "Tap Bus Assembly" with $I=25.1$ $+6(52)=337.1 \mathrm{~A}$

Using direct proportionality:

$\% V D=1.24 \times \frac{337.1}{337.2}=1.24 \%$

7.5.3 \%VD on Submersible Pump Feeder

$Z_{\text {eff }}$ for $\# 1 / 0=.13 ; Z_{\text {eff }}$ for $\# 4=.30 ; L=.08$ for $\# 1 / 0$ and .05 for $\# 4$; $\mathrm{I}=336 \mathrm{~A}$ (starting)

$\% \mathrm{VD}=\frac{\sqrt{3} \times 312 \times((.13 \times .08)+(.30 \times .06))}{4.8}=3.20 \%$

Totaj \%VD up to Immersible Pump motor terminals $=$

$$
5.33+1.24+3.20=9.77 \%
$$

Motor terminal voltage $=480(1-.977) \approx 433.1 \mathrm{~V}$

Minimum permissible voltage $=414 \mathrm{~V}$

A single \#1/0 feeder is adequate. Initially, the "soft start" unit was utilized to limit the torque effect of the Immersible pump as it hangs down into the tank. Additionally, the "soft start" unit will be configured to limit starting current to aid in starting the Immersible pump..

7.6 \% Voltage Dip With Booster Pump Starting (Immersible Pump already running)

7.6.1 \%VD on Transformer Bank

With $150 \%$ current limit,

$$
\begin{aligned}
& I=(1.5 \times 273)+25.1+34+52=520.6 \mathrm{~A} \\
& \% V D=\frac{520.6}{181} \times 2.6=7.48 \%
\end{aligned}
$$

HNF-2487, Rev. 0 


\section{Fluor Daniel Northwest}

Calc. No. W320-31-017

DESIGN ANALYSIS

client Numatec Hanford Corporation

subject TANK AY-102 LOAD DETERMINATION, FEEDER/PROTECTION SIZING, VOLTAGE DROP AND LIGHTING CALCULATIONS

Location 200E, AY Farm
Revision 1

Page No. 16 of 24

wo/Job No. Task Order N09

Date 9/13/95 By RH Graeber

Checked 9/13/95 By SA Najjar

Revised 7/24/98 By Cris Carlson

\subsection{2 \%VD on parallel 350 xcmil Feeder}

$$
\% \mathrm{VD}=.44 \% \times 1.44=0.63 \%
$$

7.6.4 \%VD on $500 \mathrm{Kcmi1}$ motor feeder

$\% V D=.49 \% \times 1.44=0.63 \%$

[from 7.4.3]

Total $\%$ VD $=7.48 \%+0.63 \%+0.63 \%=8.74 \%$

Motor terminal voltage $=480(1-0.874)=438.1 \mathrm{~V}$

Minimum permissible voltage $=414 \mathrm{~V}$

\subsection{Outdoor Lighting Calculations}

Entrance lighting needs 3 footcandles. Total area that needs to be 1 it is approximately $6^{\prime} \times 3^{\prime}=18 \mathrm{ft}^{2}$

The fixture selected for outdoor use is the Voigt S0X-35 Wal1-Most. The manufacture's data may be used to determine whether this fixture is adequate for our needs. The Footcandle IsoTux diagram shows the footcandles available from Voigt SOX-55 at a height of $15 \mathrm{ft}$. This must be adjusted to obtain values for the SOX-35 at 10 feet.

To adjust for the lower lamp output, the footcandles will be multiplied by 0.6. This is obtained from the ratio of the lumen output of the lamps. The Westinghouse Lighting Handbook provides guidance for adjusting for the height difference. The following is quoted from page 2-14 of the 1969 Westinghouse Lighting Handbook, "Footcandles for other mounting heights than the one shown are obtained by multiplying the values given on the curves by the ratio of the square of the present mounting height to the square of the new mounting height."

This adjustment factor will be $\left(15^{2} / 10^{2}\right)=2.25$

We will consider an area $3 \mathrm{ft}$ out from the wall. The chart shows this as approximately 7 footcandles.

Using the adjustment factors: 7 footcandles $\times 2.25 \times .6=9.5$ footcandles Outdoor fixture selected will give adequate light over doorway. 


\section{Fluor Daniel Northwest}

DESIGN ANALYSIS

client Numatec Hanford Corporation

subject TANK AY-102 LOAD DETERMINATION,

FEEDER/PROTECTION SIZING, VOLTAGE DROP AND

LIGHTING CALCULATIONS

Location 200E, AY Farm
Calc. No. W320-31-017

Revision 1

Page No. 17 of 24

worsob No. Task Order N09

Date 9/13/95 By RH Graeber

Checked $9 / 13 / 95$ By SA Najjar

Revised $7 / 24 / 98$ By Cris Carlson

\subsection{Indoor Lighting Calculations - AY Electrical Equipment Skid}

Per IES, average of 50 (approximately) footcandles is adequate. The ZonalCavity Method will be used to determine the number of fixtures.

Room Cavity Ratio (RCR) $=[5 \times h \times(L+W)] /(L \times W)$

Where $h=$ height of light $=8^{\prime}$

$L=$ room length $=13^{\prime}$

$W=$ room width $\quad=7^{\prime}$

$\operatorname{RCR}=[5 \times 8 \times(13+7)] /(13 \times 7)=8.8$

Using the Crouse-Hinds photometric data (see Appendix $B$ ), with an assumed ceiling reflectance of $80 \%$ and an assumed wall reflectance of $50 \%$, the coefficient of utilization is 0.25 .

Number of fixtures $=(F C \times$ AREA $) /$ (number of lamps $\times 1$ umens $/ 7$ amp $\times C U \times M F)$

Number of fixtures $=(50 \times 13 \times 7) /(2 \times 3200 \times .25 \times .9)=3.16$ fixtures

Use three fixtures.

\subsection{Conduit Sizing for Control Wire Runs}

Wire runs W102-42, 85, 86, 86A, 87, 88, 90, 91, 94, 95 \& 104 are included.

Wire runs with Targest cross sections are W102-86 with 8\#14 and W102-87 with $4 \# 12$ and \#104 with 20\#14. Per NEC Table 3B, the first Wire Runs will "fit" into $\frac{3}{4} "$ conduits and the last wire run will fit into a $1 \frac{1}{2}$ " conduit which is $3 \mathrm{C} 11$.

AY102-3CO1, 3CO3, 3CO4, 3CO5, 3CO8, 3CO9 and $3 \mathrm{Cl0}$ wi11 be $\frac{3}{4} "$ conduits.

The following underground conduit runs will be Up sized to 1 " conduit for conservatism:

AY102-3C02 with wire run W102-86 and W102-86A

AY102-3C06 with wire run W102-87

AY 102-3C07 with wire run W102-88

Conduit AY102-3C11 with wire run W102-104 will be $1 \frac{1}{2} "$ conduit for to be conservative. 


\section{Fluor Daniel Northwest}

\section{DESIGN ANALYSIS}

client Numatec Hanford Corporation

subject TANK AY-102 LOAD DETERMINATION,

FEEDER/PROTECTION SIZING, VOLTAGE DROP AND LIGHTING CALCULATIONS

Location 200E, AY Farm
Revision 1

Page No. 18 of 24

wo/Job No. Task Order N09

Date $9 / 13 / 95$

By RH Graeber

checked $9 / 13 / 95$

By SA Najjar

Revised 7/24/98

\subsection{Conduit Sizing for Instrumentation (Signal) Wire Runs}

Instrument wiring used for $W-320$ (AY-Farm) is of the following sizes and types:

Type 1 - 3\#16 triad, diameter $<=.34 "$

Type 2 - same as Type 1 (in pit)

Type 3 - 2 pair \# 16, diameter $<=.63 "$

Type 4 - same as Type 3 (in pit)

Type 5 - 1 pair \#16, diameter $<=.34 "$

Type 13 - Vendor furnished wire with PE-0621/0622

Type $16-8 \mathrm{c} / \# 14$, diameter $<=.48^{\prime \prime}$

7.10.1 Conduit AY102-3S01

This conduit is a $1 "$ installed spare.

7.10.2 Wire Runs W102-48A, 48B, 48C, 49A, 498,50,51, 105, 106, $107 \& 109$ In Conduit AY102-3SO2

Ten Type 1, one Type 13 and one Vendor Wire Run (Nominal diameter .6").

Type 1: $(10)\left(3.14 \times(0.34 \div 2)^{2}\right)=$

Type 13: (1) $\left(3.14 \times(0.63 \div 2)^{2}\right)=$

Type 17 (vendor):

(1) $\left(3.14 \times(0.6 \div 2)^{2}\right)=$

$0.91 \mathrm{in}^{2}$

$0.16 \mathrm{in}^{2}$

$0.28 \mathrm{in}^{2}$

$1.35 \mathrm{in}^{2}$

$40 \%$ fill: $1.35 \div 0.4=3.38 \mathrm{in}^{2}$

$2 \frac{1}{2} "$ conduit cross-section area $=4.87 \mathrm{in}^{2}$

The minimum size needed for AY102-3SO2 is $2 \frac{1}{2} "$ conduit (Up sized to 3 " conduit for conservatism and because there is no other $2 \frac{1}{2}$ " conduit needed for the AY-102 installation).

7.10.3 Wire Runs W102-59, 61, 62, 63, \& 82 In Conduit AY102-3503

Five Type 1 and one Type 13.

Type 1: (4) $\left(3.14 \times\left(0.17^{2}\right)=0.36\right.$

Type 13: $(1)\left(3.14 \times\left(0.315^{2}\right)=0.31\right.$

$$
\overline{0.67 \text { in }^{2}}
$$




\section{Fluor Daniel Northwest}

DESIGN ANALYSIS

client Numatec Hanford Corporation

subject TANK AY-102 LOAD DETERMINATION,

FEEDER/PROTECTION SIZING, VOLTAGE DROP AND

LIGHTING CALCULATIONS

Location 200E, AY Farm
Calc. No. W320-31-017

Revision 1

Page No. 19 of 24

wo/sob No. Task Order NO9

Date 9/13/95 By RH Graeber

Checked $9 / 13 / 95$ By SA Najjar

Revised $7 / 24 / 98$ By Cris Carlson

$40 \%$ fill: $\quad 0.67 \div .4=1.68 \mathrm{in}^{2}$

$1 \frac{1}{2}$ " conduit area $=2.07$ in $^{2}$ from NEC Chapter 9 Table 4

AY102-3S03 Up sized to 2" conduit for conservatism.

7.10.4 Wire Runs W102-37A, 37B, 37C, 38A, 38B, 40,41, 43,115, 116, 117, 118 in Conduit AY102-3S19

Five Type 1, six Type 5, one Type 16

Types 1 \& 5: $(11)\left(3.14 \times .17^{2}\right)=1.00 \mathrm{in}^{2}$

Type 16:(1) $\left(3.14 \times .24^{2}\right)=.18 \mathrm{in}^{2}$

$\overline{1.18} \mathrm{in}^{2}$

$40 \%$ fill: $1.18 / 0.4=2.95 \mathrm{in}^{2}$

2" conduit cross sectional area $=3.41 \mathrm{in}^{2}$ from NEC Chapter 9 Table 4

Use a 2" conduit

7.10.5 Wire Runs W102-68, 69,70,71,72,73 \& 103 Routed Through Conduits AY102-3S08, 3S08A \& 3S10

A11 seven wire runs are Type 5 .

(7) $\times\left(3.14 \times 0.17^{2}\right)=0.64 \mathrm{in}^{2}$

$40 \%$ fill: $0.64 \div 0.4=1.60 \mathrm{in}^{2}$

$1 \frac{12}{2}$ conduit required (AY102-3508, 3508A, and 3510 Up sized to $2^{\prime \prime}$ conduit for conservatism).

7.10.6 Wire Run W102-80. In Conduit AY102-3SI4

Both are Type $3 \Rightarrow 0.63^{\prime \prime}$ diameter, radius $=.315^{\prime \prime}$

(3.14) $\times 0.315^{2}=0.31 \mathrm{in}^{2}$

$53 \%$ fil1: $0.31 \div 0.53=.59 \mathrm{in}^{2}$

$1^{1 "}$ conduit needed (AY102-3SI4 Up sized to 2" conduit for conservatism). 


\section{Fluor Daniel Northwest}

DESIGN ANALYSIS

client Numatec Hanford Corporation

subject TANK AY-102 LOAD DETERMINATION,

FEEDER/PROTECTION SIZING, VOLTAGE DROP AND

LIGHTING CALCULATIONS

Location 200E, AY Farm
Revision 1

Page No. 20 of 24

wo/sob No. Task Order N09

Date $9 / 13 / 95 \quad$ By RH Graeber

Checked 9/13/95 By SA Najjar

Revised $7 / 24 / 98$ вy Cris Cartson

7.10.7 Wire Runs W102-64, \& 67

Both are Type 5 .

Conduits AY102-3SO4, \& $3 \$ 13$ each contain only one of the above wire runs, i.e. one Type 5 .

(1) $\times\left(3.14 \times 0.17^{2}\right)=0.09 \mathrm{in}^{2}$

$53 \%$ fill applies: $0.09 \div 0.53=0.17 \mathrm{in}^{2}$

Minimum size of conduit required is $\frac{3}{4} "$. AY102-3504 \& $-3 \$ 13$ to be $\frac{3}{4} "$ conduit for conservatism.

7.10.8 Four Type 7 Wire Runs Per Conduit

W102-100 \& 101

AY102-3\$16 \& 3517

Per NEC Table 3B, either wire run fits into a $\frac{1}{2} "$ conduit.

(1) $\times\left(3.14 \times 0.315^{2}\right) \div 0.53=0.5879 \mathrm{in}^{2}$

$1^{\prime \prime}$. conduit area $=\left(3.14 \times(1.049 \div 2)^{2}=0.8638 \mathrm{in}^{2}\right.$

AY102-3\$16 \& $-3 \$ 17$ to be $1^{\text {" }}$ conduit for conservatism.

7.10.9 Wire Run W102-79. Type $142 / C$ \#16 Belden In Conduit AY102-3SO9

Type 5 - diameter $.34, "$ Cross-sectional area $=.091 \mathrm{sq"}$

1 " conduit is acceptable.

7.10,10 Wire Run W102-74, Belden 9953 (0.264" Diameter) in Conduit AY102$\underline{3515}$

$\left(3.14 \times(0.264 \div 2)^{2}\right)=0.05 \mathrm{in}^{2}$

$53 \%$ fill: $0.05 \div 53=0.10 \mathrm{in}^{2}$. Need minimum $\frac{3}{4}$ " conduit for AY102$3 S 15$. (Up sized to 1 " conduit for conservatism).

7.10.11 Wire Run W102-39, SE-0621 In Conduit AY102-3S07

1" installed spare. 


\section{Fluor Daniel Northwest}

DESIGN ANALYSIS

client Numatec Hanford Corporation

subject TANK AY-102 LOAD DETERMINATION,

FEEDER/PROTECTION SIZING, VOLTAGE DROP AND

LIGHTING CALCULATIONS

Location 200E, AY Farm

Calc. No. W320-31-017

Revision 1

Page No. 21 of 24

wo/Job No. Task Order N09

Date $9 / 13 / 95$

Checked $9 / 13 / 95$

Revised 7/24/98
By RH Graeber

By SA Najjar

By Cris Cartson

7.10.12 Wire Runs W102-65, 65A \& 66 In Conduits AY102-3S11 \& 3S12

3 or $4 \# 14$ Type 7

Use $\frac{3}{4} "$ conduit (Up sized to $1 "$ conduit for conservatism).

7.10.13 Miscellaneous Wire Runs W102-32 \& W102-33 ?? temperature ??

a) W102-32, Type 7 , in conduit AY102-3P30, 3\#12, vendor furnished:

Minimum conduit size for AY102-3P30 is $\frac{1}{2} "$, used $l^{\prime \prime}$.

b) W102-33, 3\#8, Type 7, in conduit AY102-3P3I:

Interfarm Cathodic Protection Rectifier to be fed from a 120 VAC, $20 \mathrm{~A}$ circuit. The $20 \mathrm{~A}$ circuit breaker will serve a $16 \mathrm{~A}$ circuit. Maximum rectifier input current is 4 Amps. Size circuit for 120VAC, 4 A minimum.

Circuit length is estimated at 225 LF $\times 2=450 \mathrm{ft}$.

1) $20 \mathrm{~A}$ circuit breaker dictates a \#12 conductor size (minimum).

$$
\begin{aligned}
& R_{a 55^{\circ} \mathrm{C}}=1.72 \times 1.115=1.92 \mathrm{ohm} / 1000 \mathrm{LF} \\
& \% \mathrm{VD}_{\max }=(4)(1.92 \times 0.45)=3.46 \text { volt }=2.9 \%
\end{aligned}
$$

$3 \%$ voltage drop is maximum allowable for branch circuit; the $2.9 \%$ is considered to be too high.

2) Try \#10 AWG: $R_{a 55^{\circ} \mathrm{C}}=1.1 \times 1.115=1.23$ ohms $/ 100 \mathrm{LF}$ $\% \mathrm{VD}_{\max }=4(1.23 \times 0.45)=2.21 \mathrm{volt}=1.8 \%$

This voltage drop is acceptable.

3) Since power is from an existing Panelboard, decided to upsize again to \#8 AWG (2\#8 with $1 \# 8$ ground wire).

$$
\begin{aligned}
& R_{a 55^{\circ} \mathrm{C}}=0.7 \times 1.115=0.78 \text { ohms } / 1000 \mathrm{LF} \\
& \% \mathrm{VD}=(4 \mathrm{~A})(0.78 \times 0.45)=1.4 \mathrm{~V}=1.2 \%
\end{aligned}
$$




\section{Fluor Daniel Northwest}

DESIGN ANALYSIS

client Numatec Hanford Corporation

subject TANK AY-102 LOAD DETERMINATION,

FEEDER/PROTECTION SIZING, VOLTAGE DROP AND LIGHTING CALCULATIONS

Location 200E, AY Farm
Calc. No. W320-31-017

Revision 1

Page No. 22 of 24

wo/Job No. Task Order N09

Date 9/13/95 By RH Graeber

Checked $9 / 13 / 95$ By SA Najjar

Revised $7 / 24 / 98$ By Cris Carison

$3 \# 8$ AWG requires a $\frac{1}{2} "$ conduit; Up sized AY102-3P31 to $1 "$ conduit for conservatism because it is a long underground run.

\subsection{Special Situations}

\subsubsection{P-0621 Motor Starter Control Power Transformer Sizing}

The Control Power Transformers are an integral part of seller supplied equipment. They are sized by the providers of the motor control equipment. No evaluation is needed here.

7.11.2 Winch (W-0621) Limit Switch - 120VAC Circuit Conductor Sizing (Wire Run Nos \#-86, \#-86A. \& \#-95)

Circuit limited to maximum of $0.8 \mathrm{~A}$ by CPT fuses, therefore \#14 AWG, Type 7, is acceptable.

Checking voltage drop during starting:

Size 1 starter inrush $=160$ VA with minimum

pickup voltage $85 \%(120 \mathrm{Vac} \times 0.85=102 \mathrm{~V})$.

CPT $\% Z=6.5 \%(\operatorname{cold}) ; 7.8 \%$ (hot). (typical values)

\#14 AWG R $55^{\circ} \mathrm{C}=2.73 \times 1.115$

$$
=3.04 \mathrm{ohm} / 1000 \mathrm{LF}
$$

Circuit Length: $80^{\prime} \times 2=160 \mathrm{LF}$

a) $\quad$ VD \#14 $=(160 \mathrm{VA} / 120 \mathrm{~V}) \times(3.04 \times 0.16)$

$$
=0.648 \text { Volt }=0.5 \%
$$

b) VD XFMR $=(6.5)(160 \mathrm{VA} / 120 \mathrm{~V})=8.7 \%$

$$
=(7.8)(1.333)=10.4 \% \text { (hot) }
$$

Total VD $=0.5+8.7=9.2 \%(\operatorname{col} d)$ and

$$
.5+10.4=10.9 \% \text { (hot) }
$$

Hot is worst case: (120V) $(1-0.109)=107 \mathrm{~V}$

Minimal allowable voltage $=102 \mathrm{~V}$

Will use \#14 AWG, Type 7 Wire.

HNF-2487, Rev. 0

Page G-25 


\section{Fluor Daniel Northwest}

\section{DESIGN ANALYSIS}

client Numatec Hanford Corporation

subject TANK AY-102 LOAD DETERMINATION,

FEEDER/PROTECTION SIZING, VOLTAGE DROP AND LIGHTING CALCULATIONS

Location 200E, AY Farm
Calc. No. W320-31-017

Revision 1

Page No. 23 of 24

Wo/Job No. Task Order N09

Date $9 / 13 / 95$

By RH Graeber

Checked $9 / 13 / 95$

By SA Najjar

Revised $7 / 24 / 98$

By Cris Carlson

\subsubsection{Seismic Safety System "Emergency Trip"/Remote Trip Circuit Conductor Sizing}

Normally would use 14 AWG - Due to FU3 secondary CPT

fuse size of 3 AMPS

Checking voltage drops during contactor pick up:

Size 6 Contactor In-Rush VA $=2900$

Pickup Volts $=70 \%(120 \mathrm{~V} \times 0.7=84 \mathrm{~V})$

Coit Voitage $=120$ VAC

750 VA CPT

CPT \%Z $=4.44$

Circuit Length $55^{\prime} \times 2=110 \mathrm{LF}$

\#14 AWG R $055^{\circ} \mathrm{C}=2.73 \times 1.115$

$$
=3.04 \mathrm{ohm} / 1000 \mathrm{LF}
$$

a) \%VD \#14 (inrush) $=(2900 \mathrm{VA} / 120 \mathrm{~V})(3.04 \mathrm{ohm} / 1000 \mathrm{LF} \times 110 \mathrm{ft})$

$$
=8.08 \mathrm{~V}=6.73 \%
$$

b) \%VD CPT (inrush) $=$ (I inrush / I base) (\%Z)

$I$ (inrush) $=2900 \mathrm{VA} / 120 \mathrm{~V}=24.17 \mathrm{~A}$

I base $=750 \mathrm{VA} / 120 \mathrm{~V}=6.25 \mathrm{~A}$

\%VD CPT $=24.17 / 6.25(4.44)=17.17 \%$

c) \#14 AWG combination: VD \#14+ VD CPT =

$$
6.74+17.17=23.91 \%
$$

$120 \mathrm{~V} \times(1-0.2391)=91.3 \mathrm{~V}$

Minimum allowable for pick-up $=84 \mathrm{~V}$

d) VD $\# 12$ (inrush) where $R$ o $55^{\circ} \mathrm{C}=$

$1.72 \mathrm{ohms} / 1000 \mathrm{LF} \times 1.115=1.92 \mathrm{ohm} / 1000 \mathrm{ft}$

VD \#12 (inrush) $=(2900 \mathrm{VA} / 120 \mathrm{~A})(1.92 \times .011)=$

$51 \mathrm{~V}=.43 \%$

e) \#12 AWG combination: 


\section{Fluor Daniel Northwest}

DESIGN ANALYSIS

client Numatec Manford Corporation

subject TANK AY-102 LOAD DETERMINATION,

FEEDER/PROTECTION SIZING, VOLTAGE DROP AND

LIGHTING CALCULATIONS

Location 200E, AY Farm calc. No. W320-31-017

Revision 1

Page No. 24 of 24

wo/sob No. Task Order N09

Date $9 / 13 / 95$

By RH Graeber

Checked $9 / 13 / 95$

By SA Najjar

Revised $7 / 24 / 98$

VD $\# 12+V D C P T=.43+17.17=17.6 \%$

$(120 \mathrm{~V}) \times(1-0.176)=98.9 \mathrm{~V}$

Minimum allowable for pick-up $=84 \mathrm{~V}$

Elected to use \#12 AWG, Type 7 Wire for W102-87 \& 88.

8. CONCLUSIONS

8.1 Total connected load is $317.1 \mathrm{KVA}$. [from 7.1]

Maximum estimated demand for sluicing project $=176.9 \mathrm{KVA}$. [from 7.2]

Estimated total non-coincident demand on transformer bank $=308.3 \mathrm{KVA}$. [from 7.2.3]

8.2 The existing parallel $350 \mathrm{kcmil}$ service entrance and feeder to AY-PDP-1 is adequate for the additional AY-102 Sluicing demand.

$8.3500 \mathrm{kcmil}$ feeder for the $250 \mathrm{HP}$ VSD motor is adequate to maintain proper terminal voltage level during motor starting.

8.4 To maintain adequate motor terminal voltage level for the $40 \mathrm{HP}$ Immersible Pump motor (using the \#1/0 feeder size indicated), the existing "soft start" witl be adequate.

8.5 A single outdoor Tight fixture is adequate over the door. Three "two-tube" fixtures are required inside the Electrical Equipment skid.

8.6 Controt and instrumentation conduit runs and misc. equipment are properly sized. 


\section{DISTRIBUTION SHEET}

\begin{tabular}{|c|c|c|c|c|c|}
\hline \multirow{2}{*}{$\begin{array}{l}\text { To } \\
\text { Distribution }\end{array}$} & \multirow{2}{*}{\multicolumn{3}{|c|}{$\begin{array}{l}\text { From } \\
\text { JW Bailey }\end{array}$}} & \multicolumn{2}{|l|}{ Page 1 of 1} \\
\hline & & & & \multicolumn{2}{|c|}{ Date 25 July 1998} \\
\hline \multicolumn{4}{|l|}{ Project Title/Work Order } & \multicolumn{2}{|c|}{ EDT No. 622245} \\
\hline \multicolumn{4}{|c|}{$\begin{array}{l}\text { Project W-320 Tank 106-C, HNF-2487, Electrical Calculations, } \\
\text { Vol. } 1\end{array}$} & \multicolumn{2}{|l|}{ ECN No. $n / a$} \\
\hline Name & MSIN & $\begin{array}{l}\text { Text } \\
\text { With All } \\
\text { Attach. }\end{array}$ & Text Only & $\begin{array}{l}\text { Attach./ } \\
\text { Appendix } \\
\text { Only } \\
\end{array}$ & $\begin{array}{l}\text { EDT/ECN } \\
\text { Only }\end{array}$ \\
\hline $\begin{array}{l}\text { JW Bailey } \\
\text { W-320 Project Files }\end{array}$ & $\begin{array}{l}\text { S2-48 } \\
\text { R1-29 }\end{array}$ & & & & \\
\hline
\end{tabular}

